\title{
MOBILITY IMPROVEMENT OF WHEELED MILITARY VEHICLES USING PREVIEW CONTROLLED ACTIVE RIDE HEIGHT SYSTEM
}

\author{
LASTIK TEKERLEKLİ ASKERİ ARAÇLARIN \\ ÖNIZLEMELİ AKTİF SÜRÜŞ YÜKSEKLİK DENETIMM \\ SISTEMI İLE HAREKET KABİLIYETININ \\ ARTIRILMASI
}

ALPEREN KALE

ASSOC. PROF. DR S. ÇAĞLAR BAŞLAMIŞLI

Supervisor

\begin{abstract}
Submitted to
Graduate School of Science and Engineering of Hacettepe University As a Partial Fulfillment to the Requirements for the Award of the Degree of Master of Science in Mechanical Engineering.
\end{abstract}





\begin{abstract}
MOBILITY IMPROVEMENT OF WHEELED MILITARY VEHICLES USING PREVIEW CONTROLLED ACTIVE RIDE HEIGHT SYSTEM
\end{abstract}

\author{
Alperen KALE \\ Master of Science Degree, Department of Mechanical Engineering \\ Supervisor: Assoc. Prof. Dr. S. Çağlar BAŞLAMIŞLI \\ June 2020, 250 pages
}

The aim of this thesis is to increase the mobility capabilities of wheeled military vehicles by designing a preview control strategy with Model Predictive Controller (MPC). Mobility capabilities of a military vehicle such as trench and obstacle crossing in the off-road are base design criteria that limit the design.

The study is basically divided into three parts. The target of the first part is to create a vehicle and create a co-simulation environment where MATLAB and ADAMS can run together. In the second part, the level of achievable improvement in vehicle's operational abilities is investigated. Developing a controller and optimizing vehicle ride height and position for better mobility operations are the main objectives of this part. The actuating system for the vehicle is its own hydropneumatic suspensions. All wheels are assumed to be independent and have height-adjustable springs/dampers. Many vehicles can adjust ride height passively according to off-road conditions. However, this passive adjustment can restrict mobility skills of the vehicle. In this thesis, measured road data is used to improve the controller performance and create an obstacle crossing algorithm.

At the end of the first two parts of the study, comparison with simulations for different land operation scenarios is given for both uncontrolled and controlled system. By this, scenario-based results are compared, and improvement levels are determined.

Keywords: Multi-Axle Vehicle, Active Suspension, MPC Controller, Preview Control, Ride-Height Control, Multi-Body Dynamics 


\title{
ÖZET
}

\section{LASTIK TEKERLEKLİ ASKERİ ARAÇLARIN ÖNIZLEMELI AKTIF SÜRÜŞ YÜKSEKLİK DENETIM SISTEMİ İLE HAREKET KABILIYYETININ ARTIRILMASI}

\author{
Alperen KALE \\ Yüksek Lisans, Makina Mühendisliği Bölümü \\ Danışman: Doç. Dr. S. Çağlar BAŞLAMIŞLI \\ Haziran 2020, 250 sayfa
}

Bu tezin amac1, Model Öngörülü Kontrol (Model Predictive Control- MPC) ile bir ön izleme denetim stratejisi tasarlayarak tekerlekli askeri araçların hareket kabiliyetini arttırmaktır. Askeri bir aracın arazi koşullarındaki hendek ve engel geçişi olarak örnek verilebilecek hareket kabiliyeti tasarımı sınırlayan temel tasarım kriterlerinden biridir.

Çalışma temel olarak üç bölüme ayrılmıştır. İlk bölümün hedefi, bir araç modeli kurmak ve MATLAB ile ADAMS'ın birlikte çalışabileceği bir benzetim ortamı oluşturmaktır. İkinci bölümde, aracın operasyonel yeteneklerinin ne kadar geliştirilebileceği araştırılmıştır. Daha iyi bir hareket kabiliyeti için bir kontrolcü geliştirmek ve araç sürüş yüksekliği ile pozisyonunu optimize etmek bu bölümün temel hedefleridir. Araç süspansiyon sistemindeki eyleyiciler aracın kendi hidropnömatik süspansiyonlarıdır. Araçtaki tüm tekerlekler bağımsızdır ve yüksekliği ayarlanabilir yaylara / amortisörlere sahiptir. Birçok araç pasif sürüş yüksekliğini arazi koşullarına göre ayarlayabilmektedir. Ancak bu pasif sürüş yüksekliği ayarı, aracın hareket kabiliyetini bazı durumlarda olumsuz yönde etkileyebilmektedir. Kontrolcü performansını artırmak ve bir engel geçiş algoritması oluşturmak için kullanılan ön izleme yol verisinin araca hazır olarak beslendiği varsayılmıştır. 
Çalışmanın ilk iki bölümünün sonunda, farklı arazi senaryoları için kontrol edilen ve edilmeyen sistemler simülasyonlarla test edilmiş, sonuçlar ortaya konmuştur. Sonuç olarak senaryo bazlı sonuçların karşılaştırılmasına dayanarak hareket kabiliyetindeki geliştirmeler son kısımda sunulmuştur.

Anahtar kelimeler: Çok Akslı Araçlar, Aktif Süspansiyon, Model Öngörülü Kontrol, Ön İzlemeli Kontrol, Sürüş Yüksekliği Kontrolü, Çoklu Cisim Dinamiği 


\section{ACKNOWLEDGEMENTS}

First of all, I would like to thank my supervisor, Assoc. Prof. Dr. S. Çağlar BAŞLAMIŞLI for his guidance and assistance during the entire graduate period. He consistently allowed this paper to be my own work but steered me in the right direction whenever he thought I needed it.

I would like to thank Cantürk SANAN and Fetihhan GÜRAN who have been with me throughout the thesis as well as from the beginning of my university life and who supported me in all matters.

I would also like to thank C. Bartu YAZICI from FNSS Defense Systems, who sacrificed valuable time during the study and helped and contributed to the solution of the problems I experienced while modelling the vehicle in ADAMS.

Finally, I present my endless gratitude to my mother and father, who made me come to these days, always with me and motivated me patiently under challenging times. Besides, I would also like to thank my brother, who helped me whenever I needed him. 


\section{TABLE OF CONTENTS}

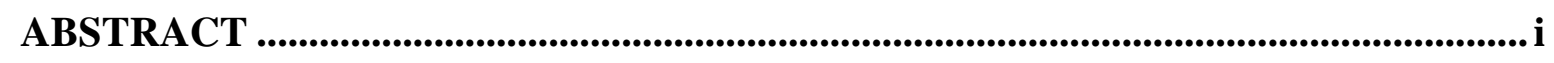

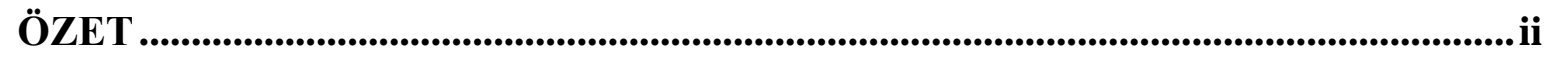

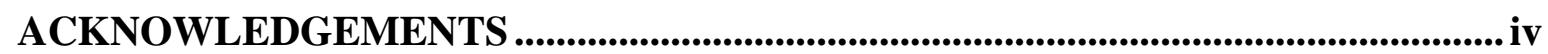

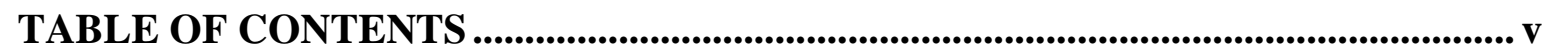

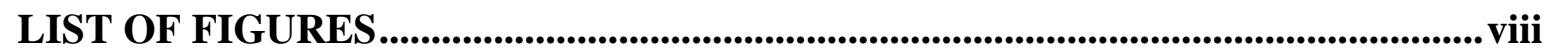

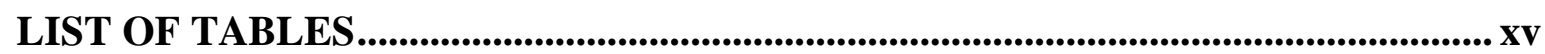

LIST OF SYMBOLS \& ABBREVIATIONS ..........................................................

1. INTRODUCTION ..................................................................................................................... 1

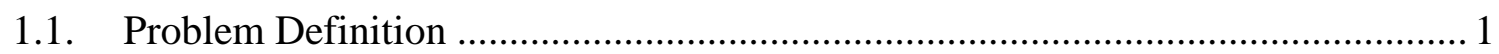

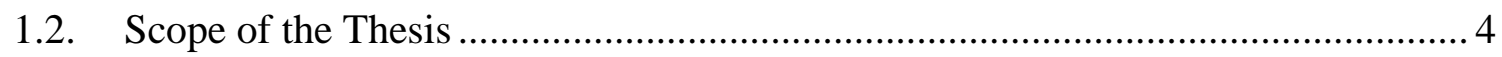

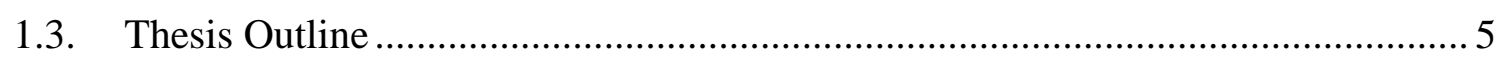

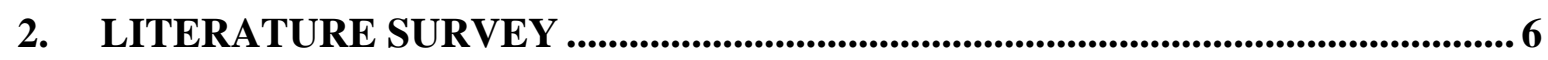

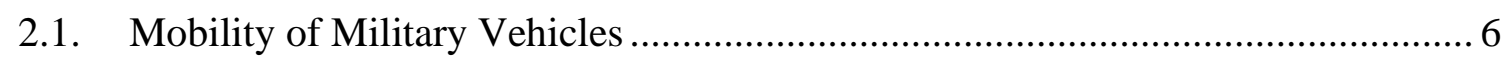

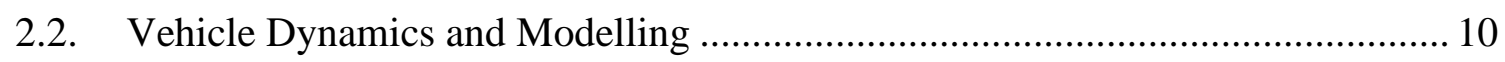

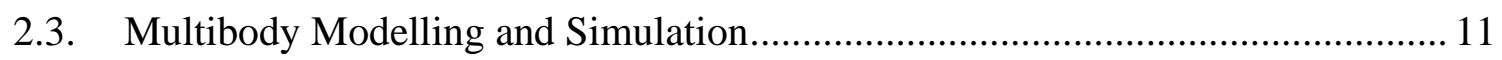

2.4. Model Predictive Controllers (MPC) .......................................................... 12

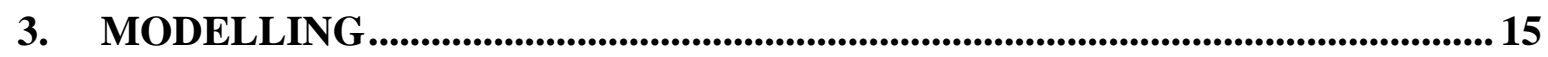

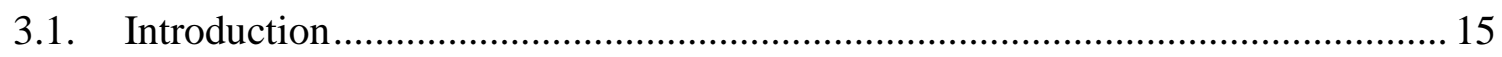

3.2. Full-Car Ride Model for Three-Axle Vehicle .................................................... 16

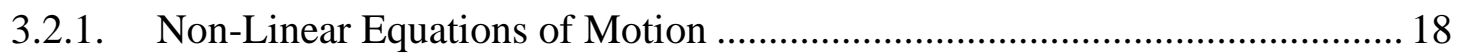

3.2.2. Linear State Space Representation of the System ..................................... 27

3.3. Multi-Body Dynamics Modelling of the Vehicle in ADAMS/Car...................... 28

3.3.1. Overview of Modelling in ADAMS .......................................................... 28

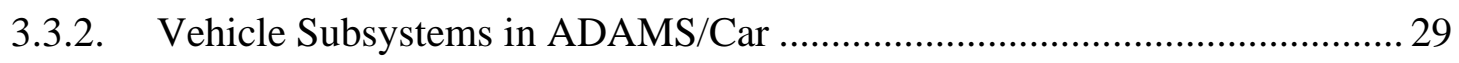


3.3.3. Creating the Full Vehicle and Simulation Environment in ADAMS/Car..... 34

3.4. Road and Obstacle Modelling in ADAMS ..................................................... 36

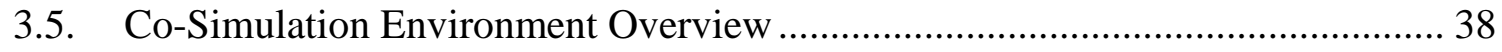

3.6. Preview Data Modelling in ADAMS …......................................................... 42

4. CONTROLLER DESIGN AND OBSTACLE CROSSING ALGORITHM ....... 44

4.1. Introduction to Controller Design and Algorithm ........................................... 44

4.2. Creating the LQR (Linear Quadratic Regulator) Controller .............................. 45

4.3. Creating MPC Controllers in Simulink with Linear Model ............................... 47

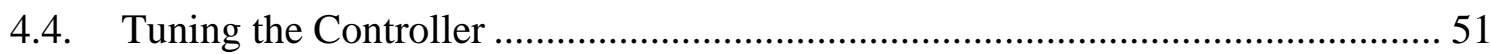

4.5. Creating Control System with ADAMS Plant................................................... 55

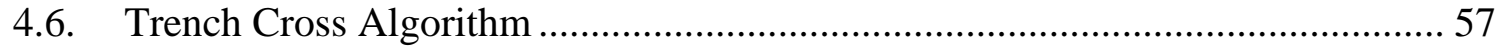

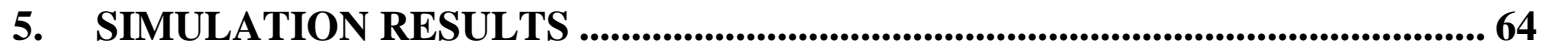

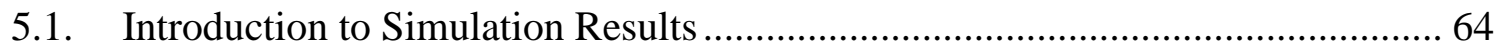

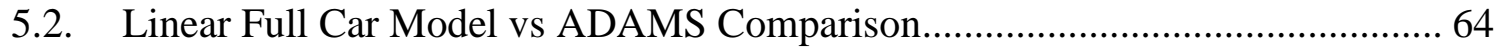

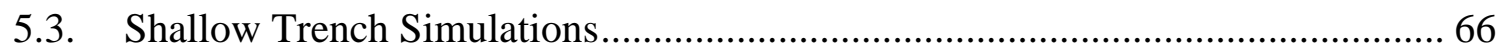

5.4. Deep Trench Passive System Simulations..................................................... 73

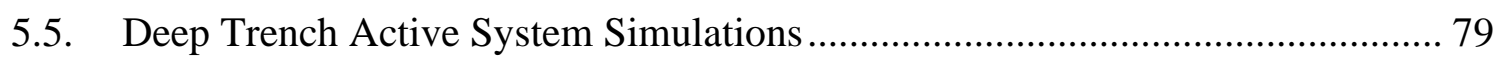

5.5.1. $\quad 1.4 \mathrm{~m}$ Trench, $9 \mathrm{kph}$, Controlled vs Uncontrolled System Comparison ........ 80

5.5.2. $\quad 1.5 \mathrm{~m}$ Trench, $1 \mathrm{kph}$, Controlled vs Uncontrolled System Comparison ........ 83

5.5.3. $\quad 1.5 \mathrm{~m}$ Trench, $3 \mathrm{kph}$, Controlled vs Uncontrolled System Comparison ....... 87

5.5.4. $\quad 1.7 \mathrm{~m}$ Trench, $6 \mathrm{kph}$, Controlled vs Uncontrolled System Comparison ....... 90

5.5.5. $1.9 \mathrm{~m}$ Trench, $1 \mathrm{kph}$, Controlled vs Uncontrolled System Comparison ....... 93

5.5.6. 2.0 m Trench, 9 kph, Controlled vs Uncontrolled System Comparison ....... 96

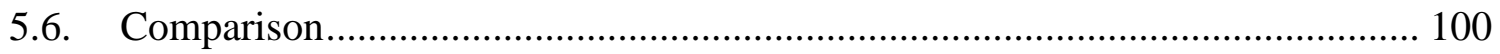

6. CONCLUSION AND FUTURE WORK........................................................ 110

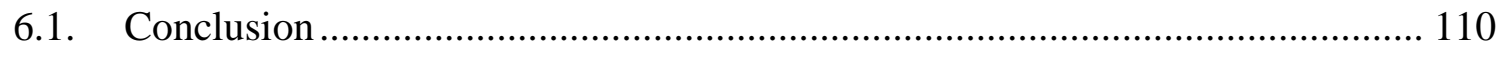

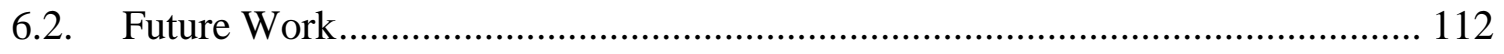




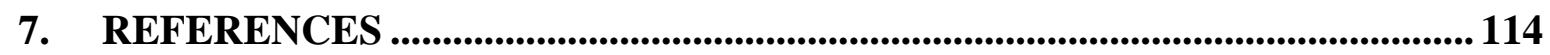

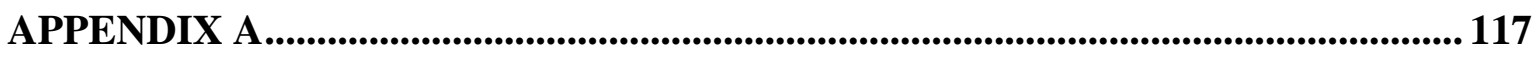
APPENDIX B ...................................................................................................................... 225

APPENDIX C........................................................................................................................ 244 


\section{LIST OF FIGURES}

Figure 1.1. Spring force vs displacement curves for various applications. [1] .................... 1

Figure 1.2. Different axle location options for $6 x 6$ and $8 x 8$ vehicles ............................... 2

Figure 1.3. Uphill and downhill operations.................................................................. 2

Figure 1.4. Obstacle and trench crossing operations..................................................... 2

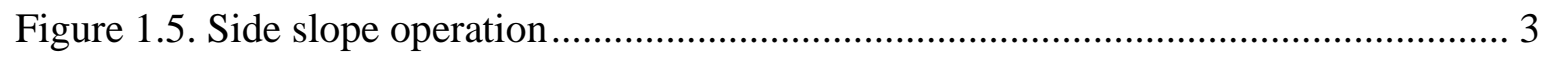

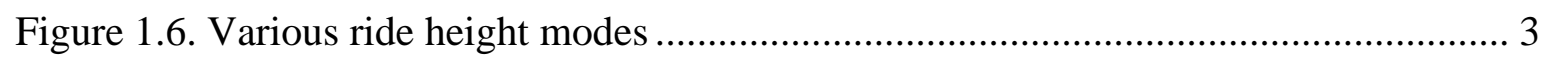

Figure 1.7. Trench crossing operation, $8 \times 8$ and $6 x 6$ vehicles ........................................... 4

Figure 2.1. PARS 6x6 SCOUT wheeled vehicle \& KAPLAN MEDIUM TANK tracked

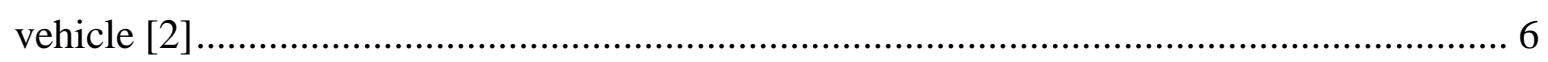

Figure 2.2. Hydropneumatic suspension schematic [4] ................................................ 7

Figure 2.3. Quarter Car (A), Half Car (B) and Full Car (C) models [12] ......................... 10

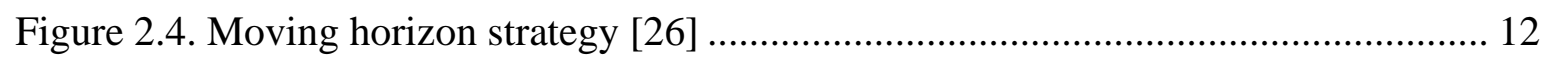

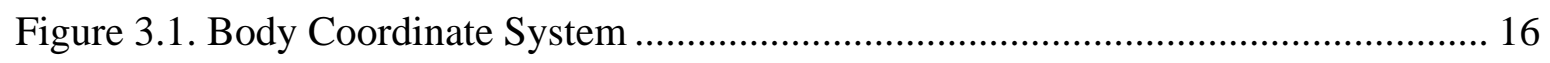

Figure 3.2. Nine-degrees of freedom full-car ride model of three-axle vehicle.................. 17

Figure 3.3. Force elements representation of the suspension and tire............................... 20

Figure 3.4. A simple full-car vehicle assembly in ADAMS ......................................... 29

Figure 3.5. A double-wishbone suspension template in ADAMS .................................. 30

Figure 3.6. Modified suspension subsystem in ADAMS ................................................ 30

Figure 3.7. Non-linear and linearized spring curves of hydropneumatic struts ................. 31

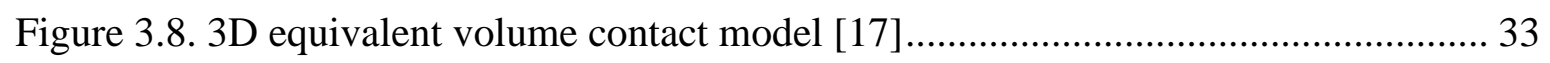

Figure 3.9. The body subsystem in ADAMS with front and rear bumpers ....................... 34

Figure 3.10. ADAMS specific event running (File Driven Events) ................................. 35

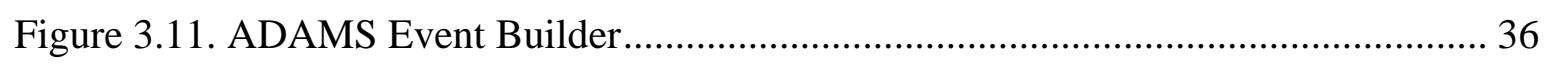

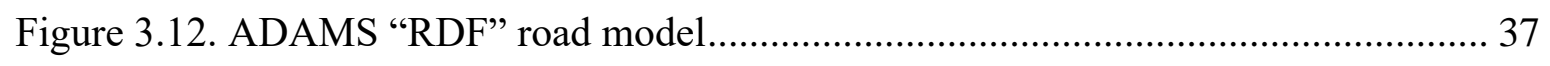

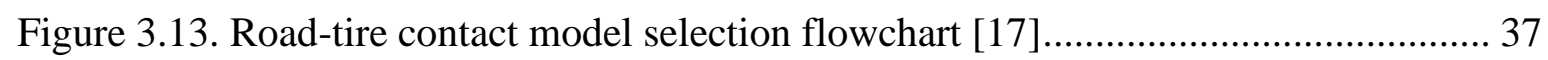

Figure 3.14. Vehicle body - ground interaction without contact model (A) and with contact

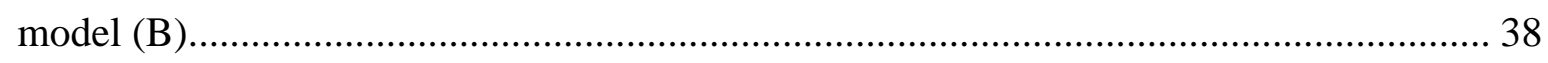

Figure 3.15. State-Variable definition in ADAMS/Template Builder .............................. 39

Figure 3.16. Exported ADAMS plant model in Simulink............................................... 41

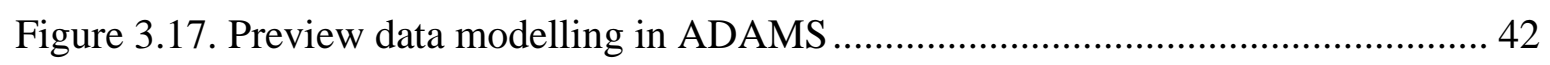

Figure 3.18. Nose Lidar marker modelled on the body .............................................. 42

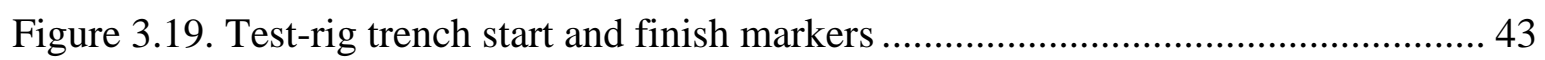


Figure 3.20. Tangent lines from nose lidar to each wheel.

Figure 4.1. Closed-loop MPC control system without preview

Figure 4.2. Closed-loop MPC control system with the preview 48

Figure 4.3. General overview of the MPC control system and ADAMS non-linear plant . 55

Figure 4.4. Detail overview of non-linear plant, trench crossing algorithm function ..........56

Figure 4.5. Detail overview of non-linear plant, ADAMS plant and its inputs/outputs...... 56

Figure 4.6. Detail overview of MPC controller block with inputs, outputs and online features

Figure 4.7. Inputs of the trench cross algorithm. 58

Figure 4.8. Preview information signals definition .58

Figure 4.9. Nose lidar to trench slopes and corrected slopes .59

Figure 4.10. Comparison of measured and corrected slopes from nose lidar to trench ...... 61

Figure 4.11. Flowchart of the trench cross algorithm 62

Figure 5.1. Linear Matlab model vs non-linear ADAMS model comparison, body vertical motion

Figure 5.2. Linear Matlab model vs non-linear ADAMS model comparison, pitch angle . 65 Figure 5.3. Linear Matlab model vs non-linear ADAMS model comparison, longitudinal velocity .65

Figure 5.4. Linear system test road..... 66

Figure 5.5. Uncontrolled vs controlled systems comparison, $5 \mathrm{kph}, 0.15 \mathrm{~m}$ depth, the horizontal axis is time, vertical axis is pitch angle [deg].

Figure 5.6. Uncontrolled vs controlled systems comparison, $10 \mathrm{kph}, 0.15 \mathrm{~m}$ depth, the horizontal axis is time, vertical axis is pitch angle [deg].

Figure 5.7. Uncontrolled vs controlled systems comparison, $20 \mathrm{kph}, 0.15 \mathrm{~m}$ depth, the horizontal axis is time, vertical axis is pitch angle [deg].

Figure 5.8. Uncontrolled vs controlled systems comparison, $5 \mathrm{kph}, 0.25 \mathrm{~m}$ depth, the horizontal axis is time, vertical axis is pitch angle [deg].

Figure 5.9. Uncontrolled vs controlled systems comparison, $10 \mathrm{kph}, 0.25 \mathrm{~m}$ depth, the horizontal axis is time, vertical axis is pitch angle [deg].

Figure 5.10. Uncontrolled vs controlled systems comparison, $10 \mathrm{kph}, 0.25 \mathrm{~m}$ depth, the horizontal axis is time, vertical axis is pitch angle [deg].

Figure 5.11. Uncontrolled vs controlled systems comparison, $5 \mathrm{kph}, 0.15 \mathrm{~m}$ depth, focused on disturbance section, the horizontal axis is time, vertical axis is pitch angle [deg] 
Figure 5.12. Uncontrolled vs controlled systems comparison, $5 \mathrm{kph}, 0.15 \mathrm{~m}$ depth, focused on reference tracking, 1

Figure 5.13. Uncontrolled vs controlled systems comparison, $5 \mathrm{kph}, 0.15 \mathrm{~m}$ depth, focused on reference tracking, 2, the horizontal axis is time, vertical axis is pitch angle [deg] ...... 71 Figure 5.14. Control inputs comparison of the linear simulations, the vertical axis is

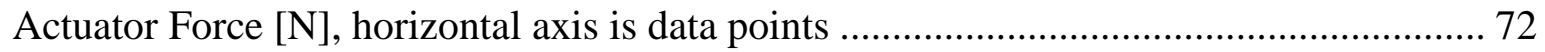

Figure 5.15. Uncontrolled trench crossing event, using bumpers 1.4m, $1 \mathrm{kph} . \ldots \ldots \ldots \ldots \ldots . . . . . . . .73$

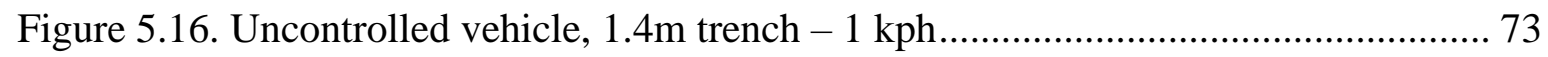

Figure 5.17. Uncontrolled vehicle, stuck in the trench, $1.4 \mathrm{~m}-9 \mathrm{kph}$............................. 74

Figure 5.18. Vehicle sub-systems modelled as solid blocks and longitudinal wheel hub

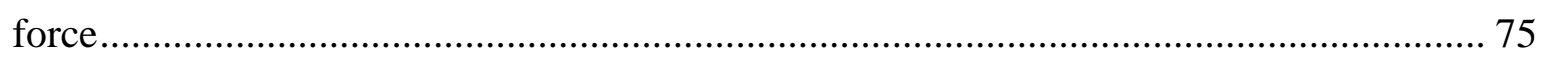

Figure 5.19. Uncontrolled trench crossing event, using bumpers $1.5 \mathrm{~m}-1 \mathrm{kph}$............... 78

Figure 5.20. Uncontrolled trench crossing event, using bumpers $1.6 \mathrm{~m}-1 \mathrm{kph}$................. 78

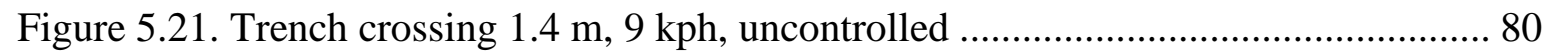

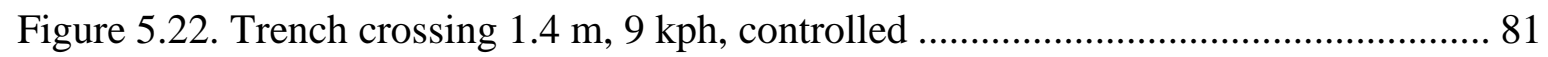

Figure 5.23. Trench crossing $1.4 \mathrm{~m}, 9 \mathrm{kph}$, controlled vs uncontrolled system results ...... 82

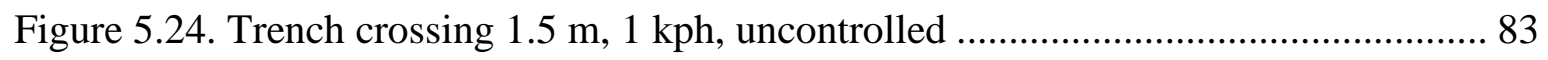

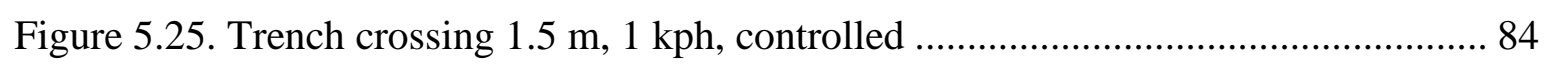

Figure 5.26. Trench crossing $1.5 \mathrm{~m}, 1 \mathrm{kph}$, controlled vs uncontrolled system results ...... 86

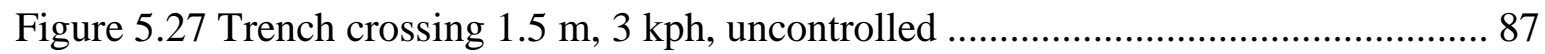

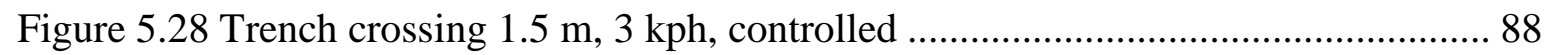

Figure 5.29. Trench crossing $1.5 \mathrm{~m}, 3 \mathrm{kph}$, controlled vs uncontrolled system results ...... 89

Figure 5.30 Trench crossing $1.7 \mathrm{~m}, 6 \mathrm{kph}$, uncontrolled ................................................. 90

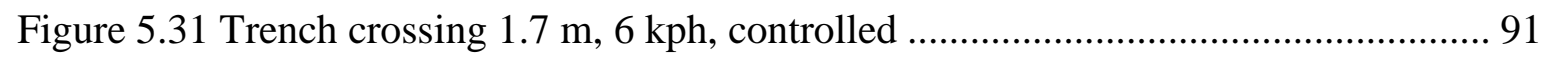

Figure 5.32 Trench crossing $1.7 \mathrm{~m}, 6 \mathrm{kph}$, body acceleration comparison of controlled vs

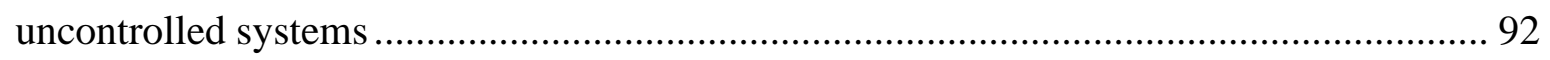

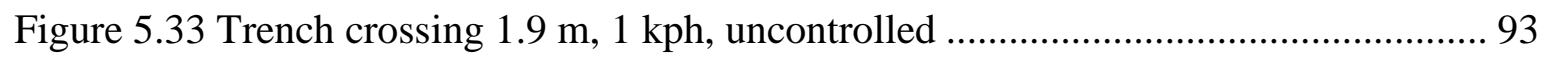

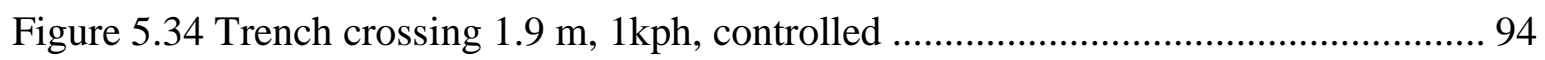

Figure 5.35 Trench crossing $1.9 \mathrm{~m}, 1 \mathrm{kph}$, body acceleration comparison of controlled vs

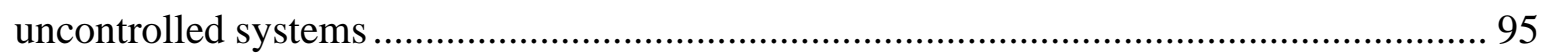

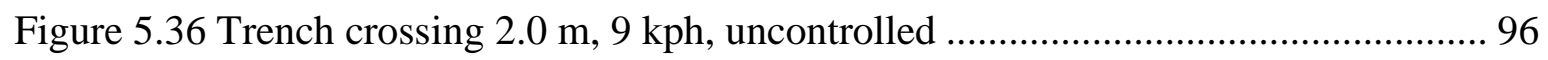

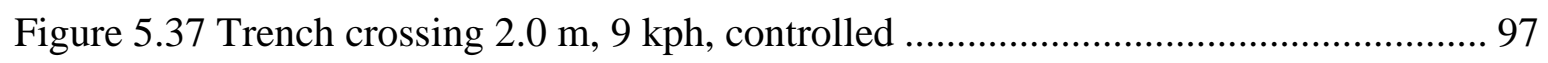

Figure 5.38 Trench crossing $2.0 \mathrm{~m}, 9 \mathrm{kph}$, body acceleration comparison of controlled vs

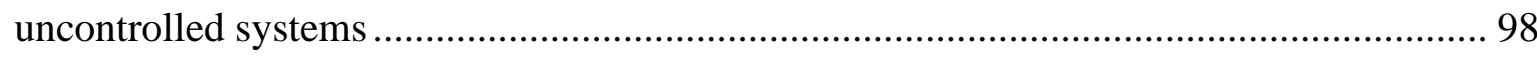


Figure 5.39. PSD Comparison example of controlled vs uncontrolled system, 1.8m, $6 \mathrm{kph}$

Figure 5.40. Contour plot of normalized cost function comparison of controlled vs uncontrolled systems 109

Figure 5.41. Surface plot of normalized cost function comparison of controlled vs uncontrolled systems 109

Figure A.1. Trench length $1.2 \mathrm{~m}, 1 \mathrm{kph}$, uncontrolled system. 117

Figure A.2. Trench length $1.2 \mathrm{~m}, 1 \mathrm{kph}$, controlled system ..... 118

Figure A.3. Trench length $1.2 \mathrm{~m}, 1 \mathrm{kph}$, controlled vs uncontrolled output comparison.. 119

Figure A.4. Trench length $1.2 \mathrm{~m}, 3 \mathrm{kph}$, uncontrolled system 120

Figure A.5. Trench length $1.2 \mathrm{~m}, 3 \mathrm{kph}$, controlled system 121

Figure A.6. Trench length $1.2 \mathrm{~m}, 3 \mathrm{kph}$, controlled vs uncontrolled output comparison.. 122

Figure A.7. Trench length 1.2 m, 6 kph, uncontrolled system ....................................... 123

Figure A.8. Trench length $1.2 \mathrm{~m}, 6 \mathrm{kph}$, controlled system ..... 124

Figure A.9. Trench length $1.2 \mathrm{~m}, 6 \mathrm{kph}$, controlled vs uncontrolled output comparison.. 125

Figure A.10. Trench length 1.2 m, 9 kph, uncontrolled system ...................................... 126

Figure A.11. Trench length $1.2 \mathrm{~m}, 9 \mathrm{kph}$, controlled system 127

Figure A.12. Trench length $1.2 \mathrm{~m}, 9 \mathrm{kph}$, controlled vs uncontrolled output comparison 128

Figure A.13. Trench length $1.3 \mathrm{~m}, 1 \mathrm{kph}$, uncontrolled system 129

Figure A.14. Trench length $1.3 \mathrm{~m}, 1 \mathrm{kph}$, controlled system . 130

Figure A.15. Trench length $1.3 \mathrm{~m}, 1 \mathrm{kph}$, controlled vs uncontrolled output comparison 131

Figure A.16. Trench length $1.3 \mathrm{~m}, 3 \mathrm{kph}$, uncontrolled system..... 132

Figure A.17. Trench length $1.3 \mathrm{~m}, 3 \mathrm{kph}$, uncontrolled system 133

Figure A.18. Trench length $1.3 \mathrm{~m}, 3 \mathrm{kph}$, controlled vs uncontrolled output comparison 134 Figure A.19. Trench length $1.3 \mathrm{~m}, 6 \mathrm{kph}$, uncontrolled system 135

Figure A.20. Trench length $1.3 \mathrm{~m}, 6 \mathrm{kph}$, controlled system . 136

Figure A.21. Trench length $1.3 \mathrm{~m}, 6 \mathrm{kph}$, controlled vs uncontrolled output comparison 137 Figure A.22. Trench length $1.3 \mathrm{~m}, 9 \mathrm{kph}$, uncontrolled system 138

Figure A.23. Trench length $1.3 \mathrm{~m}, 9 \mathrm{kph}$, controlled system 139

Figure A.24. Trench length $1.3 \mathrm{~m}, 9 \mathrm{kph}$, controlled vs uncontrolled output comparison 140 Figure A.25. Trench length $1.4 \mathrm{~m}, 1 \mathrm{kph}$, uncontrolled system 141

Figure A.26. Trench length $1.4 \mathrm{~m}, 1 \mathrm{kph}$, controlled system 142

Figure A.27. Trench length $1.4 \mathrm{~m}, 1 \mathrm{kph}$, controlled vs uncontrolled output comparison 143 Figure A.28. Trench length 1.4 m, 3 kph, uncontrolled system 144 
Figure A.29. Trench length $1.4 \mathrm{~m}, 3 \mathrm{kph}$, controlled system.

Figure A.30. Trench length 1.4 m, 3 kph, controlled vs uncontrolled output comparison 146

Figure A.31. Trench length $1.4 \mathrm{~m}, 6 \mathrm{kph}$, uncontrolled system

Figure A.32. Trench length $1.4 \mathrm{~m}, 6 \mathrm{kph}$, controlled system

Figure A.33. Trench length 1.4 m, 6 kph, controlled vs uncontrolled output comparison 149

Figure A.34. Trench length 1.4 m, 9 kph, uncontrolled system.................................... 150

Figure A.35. Trench length $1.4 \mathrm{~m}, 9 \mathrm{kph}$, controlled system

Figure A.36. Trench length 1.4 m, 9 kph, controlled vs uncontrolled output comparison 152

Figure A.37. Trench length $1.5 \mathrm{~m}, 1 \mathrm{kph}$, uncontrolled system

Figure A.38. Trench length $1.5 \mathrm{~m}, 1 \mathrm{kph}$, controlled system

Figure A.39. Trench length $1.5 \mathrm{~m}, 1 \mathrm{kph}$, controlled vs uncontrolled output comparison 155

Figure A.40. Trench length $1.5 \mathrm{~m}, 3 \mathrm{kph}$, uncontrolled system 156

Figure A.41. Trench length $1.5 \mathrm{~m}, 3 \mathrm{kph}$, controlled system. 157

Figure A.42. Trench length $1.5 \mathrm{~m}, 3 \mathrm{kph}$, controlled vs uncontrolled output comparison 158

Figure A.43. Trench length $1.5 \mathrm{~m}, 6 \mathrm{kph}$, uncontrolled system 159

Figure A.44. Trench length $1.5 \mathrm{~m}, 6 \mathrm{kph}$, controlled system

Figure A.45. Trench length $1.5 \mathrm{~m}, 6 \mathrm{kph}$, controlled vs uncontrolled output comparison 161

Figure A.46. Trench length $1.5 \mathrm{~m}, 9 \mathrm{kph}$, uncontrolled system. 162

Figure A.47. Trench length $1.5 \mathrm{~m}, 9 \mathrm{kph}$, uncontrolled system. 163

Figure A.48. Trench length $1.5 \mathrm{~m}, 9 \mathrm{kph}$, controlled vs uncontrolled output comparison 164

Figure A.49. Trench length $1.6 \mathrm{~m}, 1 \mathrm{kph}$, uncontrolled system 165

Figure A.50. Trench length $1.6 \mathrm{~m}, 1 \mathrm{kph}$, controlled system 166

Figure A.51. Trench length $1.6 \mathrm{~m}, 1 \mathrm{kph}$, controlled vs uncontrolled output comparison 167

Figure A.52. Trench length 1.6 m, 3 kph, uncontrolled system...................................... 168

Figure A.53. Trench length $1.6 \mathrm{~m}, 3 \mathrm{kph}$, controlled system

Figure A.54. Trench length $1.6 \mathrm{~m}, 3 \mathrm{kph}$, controlled vs uncontrolled output comparison 170

Figure A.55. Trench length $1.6 \mathrm{~m}, 6$ kph, uncontrolled system....................................... 171

Figure A.56. Trench length $1.6 \mathrm{~m}, 6 \mathrm{kph}$, controlled system 172

Figure A.57. Trench length $1.6 \mathrm{~m}, 6 \mathrm{kph}$, controlled vs uncontrolled output comparison 173 Figure A.58. Trench length $1.6 \mathrm{~m}, 9 \mathrm{kph}$, uncontrolled system.................................... 174

Figure A.59. Trench length $1.6 \mathrm{~m}, 9$ kph, controlled system....................................... 175

Figure A.60. Trench length $1.6 \mathrm{~m}, 9 \mathrm{kph}$, controlled vs uncontrolled output comparison 176

Figure A.61. Trench length 1.7 m, 1 kph, uncontrolled system.................................... 177

Figure A.62. Trench length 1.7 m, 1 kph, controlled system...................................... 178 
Figure A.63. Trench length $1.7 \mathrm{~m}, 1 \mathrm{kph}$, controlled vs uncontrolled output comparison 179

Figure A.64. Trench length 1.7 m, 3 kph, uncontrolled system .................................... 180

Figure A.65. Trench length 1.7 m, 3 kph, controlled system ........................................ 181

Figure A.66. Trench length $1.7 \mathrm{~m}, 3 \mathrm{kph}$, controlled vs uncontrolled output comparison 182

Figure A.67. Trench length 1.7 m, 6 kph, uncontrolled system ..................................... 183

Figure A.68. Trench length 1.7 m, 6 kph, controlled system ......................................... 184

Figure A.69. Trench length $1.7 \mathrm{~m}, 6 \mathrm{kph}$, controlled vs uncontrolled output comparison 185

Figure A.70. Trench length 1.7 m, 9 kph, uncontrolled system ...................................... 186

Figure A.71. Trench length 1.7 m, 9 kph, controlled system ....................................... 187

Figure A.72. Trench length $1.7 \mathrm{~m}, 9 \mathrm{kph}$, controlled vs uncontrolled output comparison 188

Figure A.73. Trench length $1.8 \mathrm{~m}, 1 \mathrm{kph}$, uncontrolled system ...................................... 189

Figure A.74. Trench length 1.8 m, 1 kph, controlled system ......................................... 190

Figure A.75. Trench length $1.8 \mathrm{~m}, 1 \mathrm{kph}$, controlled vs uncontrolled output comparison 191

Figure A.76. Trench length 1.8 m, 3 kph, uncontrolled system ..................................... 192

Figure A.77. Trench length 1.8 m, 3 kph, controlled system ........................................ 193

Figure A.78. Trench length $1.8 \mathrm{~m}, 3 \mathrm{kph}$, controlled vs uncontrolled output comparison 194

Figure A.79. Trench length 1.8 m, 6 kph, uncontrolled system ....................................... 195

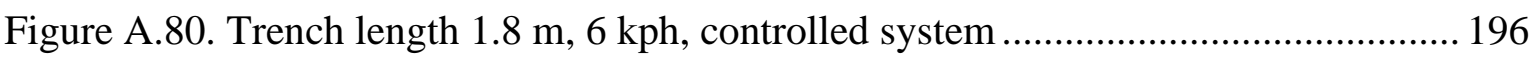

Figure A.81. Trench length $1.8 \mathrm{~m}, 6 \mathrm{kph}$, controlled vs uncontrolled output comparison 197

Figure A.82. Trench length 1.8 m, 9 kph, uncontrolled system ...................................... 198

Figure A.83. Trench length 1.8 m, 9 kph, controlled system ......................................... 199

Figure A.84. Trench length $1.8 \mathrm{~m}, 9 \mathrm{kph}$, controlled vs uncontrolled output comparison 200 Figure A.85. Trench length 1.9 m, 1 kph, uncontrolled system ....................................... 201

Figure A.86. Trench length 1.9 m, 1 kph, controlled system ......................................... 202

Figure A.87. Trench length $1.9 \mathrm{~m}, 1 \mathrm{kph}$, controlled vs uncontrolled output comparison 203

Figure A.88. Trench length 1.9 m, 3 kph, uncontrolled system ..................................... 204

Figure A.89. Trench length 1.9 m, 3 kph, controlled system ........................................ 205

Figure A.90. Trench length 1.9 m, 3 kph, controlled vs uncontrolled output comparison 206

Figure A.91. Trench length 1.9 m, 6 kph, uncontrolled system ..................................... 207

Figure A.92. Trench length 1.9 m, 6 kph, controlled system .......................................... 208

Figure A.93. Trench length 1.9 m, 6 kph, controlled vs uncontrolled output comparison 209

Figure A.94. Trench length 1.9 m, 9 kph, uncontrolled system ..................................... 210

Figure A.95. Trench length 1.9 m, 9 kph, controlled system ......................................... 211

Figure A.96. Trench length 1.9 m, 9 kph, controlled vs uncontrolled output comparison 212 
Figure A.97. Trench length $2.0 \mathrm{~m}, 1 \mathrm{kph}$, uncontrolled system 213

Figure A.98. Trench length $2.0 \mathrm{~m}, 1 \mathrm{kph}$, controlled system

Figure A.99. Trench length $2.0 \mathrm{~m}, 1 \mathrm{kph}$, controlled vs uncontrolled output comparison 215

Figure A.100. Trench length 2.0 m, 3 kph, uncontrolled system ................................. 216

Figure A.101. Trench length 2.0 m, 3 kph, controlled system.................................... 217

Figure A.102. Trench length $2.0 \mathrm{~m}, 3 \mathrm{kph}$, controlled vs uncontrolled output comparison

Figure A.103. Trench length $2.0 \mathrm{~m}, 6 \mathrm{kph}$, uncontrolled system 219

Figure A.104. Trench length $2.0 \mathrm{~m}, 6 \mathrm{kph}$, controlled system 220

Figure A.105. Trench length $2.0 \mathrm{~m}, 6 \mathrm{kph}$, controlled vs uncontrolled output comparison

Figure A.106. Trench length $2.0 \mathrm{~m}, 9 \mathrm{kph}$, uncontrolled system 222

Figure A.107. Trench length $2.0 \mathrm{~m}, 9 \mathrm{kph}$, controlled system. 223

Figure A.108. Trench length $2.0 \mathrm{~m}, 9 \mathrm{kph}$, controlled vs uncontrolled output comparison 224

Figure C.1. Controlled LQR block diagram vs uncontrolled system. 244

Figure C.2. Linear Uncontrolled system block diagram 244

Figure C.3. MPC Controller block diagram, linear shallow trench 245

Figure C.4. MPC Controller with preview block diagram, linear shallow trench 245

Figure C.5. Non-linear controlled system block diagram with MPC+preview 246

Figure C.6. Non-linear controlled system, manipulated variables sub-block 247

Figure C.7. Non-linear controlled system, ADAMS conversion sub-block 248

Figure C.8. Non-linear controlled system, ADAMS plant sub-block 249

Figure C.9. Non-linear controlled system, preview information sub-block 250 


\section{LIST OF TABLES}

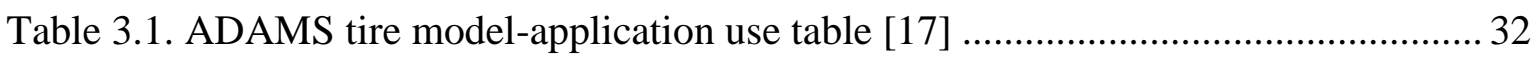

Table 3.2. Input variables of plant export for co-simulation .............................................. 39

Table 3.3. Output variables of plant export for co-simulation ............................................ 40

Table 4.1. Controller and plant combinations used ....................................................... 45

Table 4.2. Signal definitions and names used in MPC ..................................................... 49

Table 4.3. Scale factors of inputs and outputs for linear simulation .................................. 49

Table 4.4. Time step, prediction, and control horizon selection for linear simulation........ 50

Table 4.5. Hard constraints for MPC tuning for linear simulation ..................................... 52

Table 4.6. Constraint softening weights for linear simulation ........................................ 53

Table 4.7. Input and output weights for linear simulation .............................................. 54

Table 4.8. Hard constraint values of manipulated variables for different cases................. 63

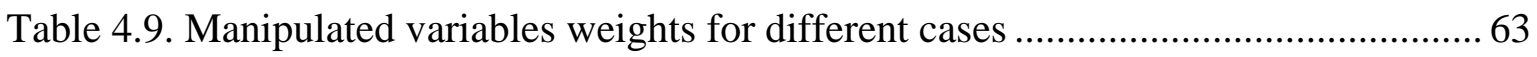

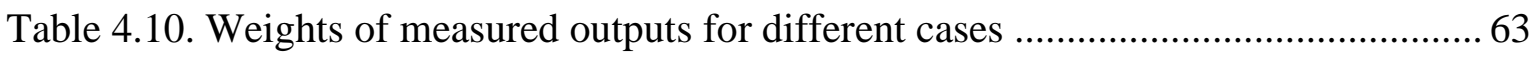

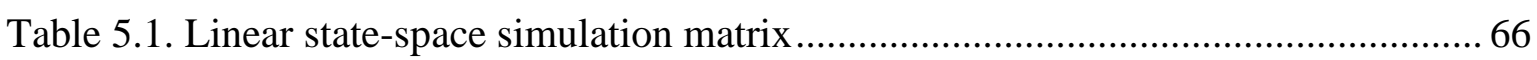

Table 5.2. Uncontrolled system simulation matrix, ADAMS plant .................................. 75

Table 5.3. Uncontrolled system trench cross criteria ….................................................... 76

Table 5.4. Comparison of all uncontrolled trench cross operations .................................... 77

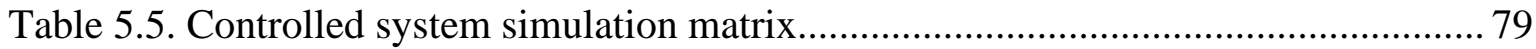

Table 5.6. Body vertical and longitudinal acceleration comparison of controlled and

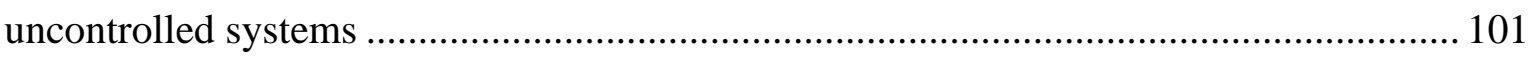

Table 5.7. Vehicle pitch angle comparison of controlled and uncontrolled systems ........ 102 Table 5.8. Body vertical and longitudinal PSD RMS acceleration comparison of controlled

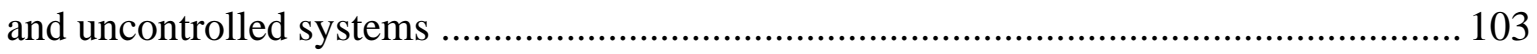

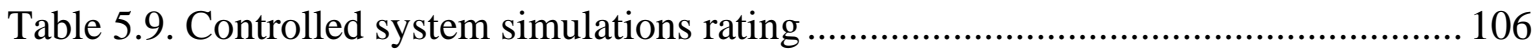

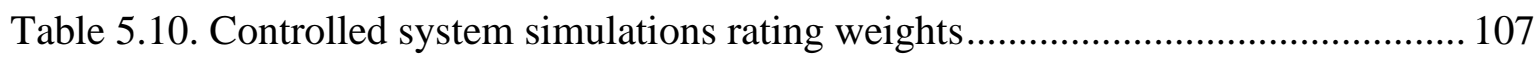

Table 5.11. Cost function comparison of controlled vs uncontrolled systems .................. 108 


\section{LIST OF SYMBOLS \& ABBREVIATIONS}

\section{List of Abbreviations}

$\begin{array}{ll}\text { ABS } & \text { Anti-Lock Brake System } \\ \text { CTIS } & \text { Central Tire Inflation System } \\ \text { ESP } & \text { Electronic Stability Program } \\ \text { LQG } & \text { Linear Quadratic Gaussian } \\ \text { LQR } & \text { Linear Quadratic Regulator } \\ \text { MIMO } & \text { Multiple Input, Multiple Output } \\ \text { MPC } & \text { Model Predictive Control } \\ \text { PID } & \text { Proportional, Integral, Derivative } \\ \text { PSD } & \text { Power Spectral Density } \\ \text { RMS } & \text { Root Mean Square }\end{array}$

\section{List of Symbols}

$t w_{i j} \quad$ Track Width of an $\mathrm{i}^{\text {th }}$ axle, left or right

$\operatorname{Co}_{X} \quad$ Center of Gravity Location on Longitudinal Axis

$z_{s} \quad$ Body vertical Displacement

$\gamma \quad$ Body Pitch Angle

$\theta \quad$ Body Roll Angle

$w b \quad$ Wheelbase

$m_{s} \quad$ Sprung Mass

$m_{u s i j} \quad$ Unsprung Mass of an $\mathrm{i}^{\text {th }}$ axle, left or right

$k_{s i j} \quad$ Stiffness of Suspension Springs

$k_{t i j} \quad$ Vertical Stiffness of Tires

$c_{s i j} \quad$ Damping Coefficient of Shock Absorbers

$c_{t i j} \quad$ Vertical Damping Coefficient of Tires 


$\begin{array}{ll}F_{t i j} & \text { Total Tire Force of an } \mathrm{i}^{\text {th }} \text { axle, left or right } \\ F_{s i j} & \text { Total Suspension Force of an } \mathrm{i}^{\text {th }} \text { axle, left or right } \\ J_{X} & \text { Moment of Inertia around the } \mathrm{x} \text {-axis } \\ J_{Y} & \text { Moment of Inertia around the y-axis } \\ F_{A C T i j} & \text { Actuator Force of an } \mathrm{i}^{\text {th }} \text { axle, left or right } \\ z_{s i j} & \text { Corner Sprung Mass Displacement of an } \mathrm{i}^{\text {th }} \text { axle, left or right } \\ z_{u s i j} & \text { Unsprung Mass Displacement of an } \mathrm{i}^{\text {th }} \text { axle, left or right } \\ z_{0 i j} & \text { Ground Displacement of an } \mathrm{i}^{\text {th }} \text { axle, left or right }\end{array}$





\section{INTRODUCTION}

\subsection{Problem Definition}

The mobility of military vehicles is of great importance in the off-road conditions as well as on-road conditions. For this reason, the right level of mobility in off-road conditions is to be provided with comfort and handling. In addition to proper handling and adequate comfort criteria, trying to improve mobility causes some difficulties in terms of vehicle design criteria. For example, in on-road conditions, spring constants used in the suspension system are working to be selected harder to reduce the rolling angle during cornering and to provide better handling, while trying to minimize the vertical wheel travel limits. Therefore, it is necessary to optimize the spring characteristics as soft enough to provide sufficient mobility and comfort and hard enough for a satisfactory road holding. Instead of conventional mechanical springs, air springs and hydropneumatic springs, which provide a more comprehensive design range, are more preferred today. To explain the more comprehensive design range, they can be adjusted more efficiently according to operating ranges. Getting all of these considerations, hydropneumatic suspensions (hydro-struts) are more privileged. Because these springs can be characterized in a nonlinear manner according to the desired spring behaviour. At the same time, hydropneumatic springs allow for the adjustment of ride height according to terrain type, and they are more advantageous in active suspension applications than mechanical springs.

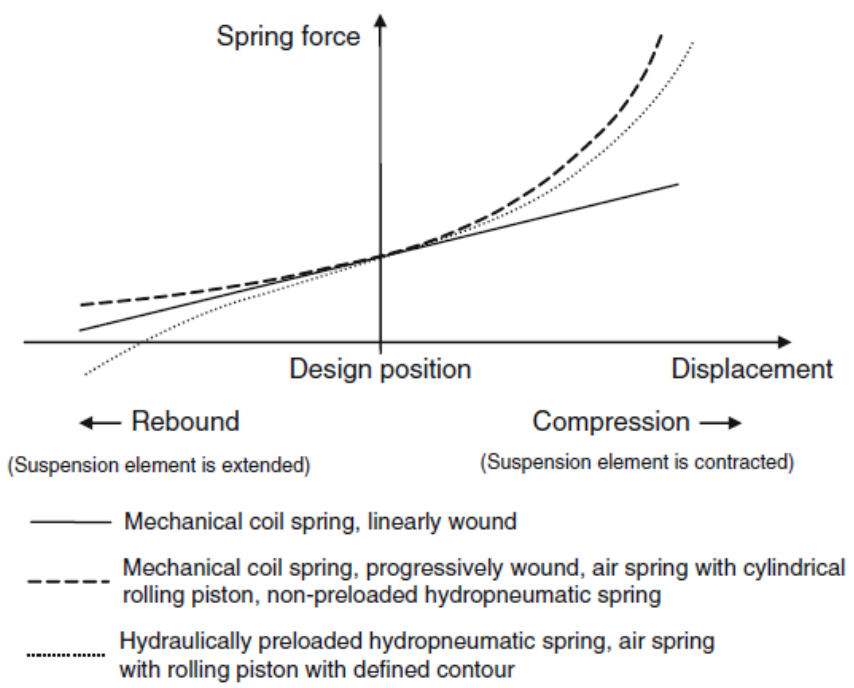

Figure 1.1. Spring force vs displacement curves for various applications. [1] 
Other factors affecting mobility are axle locations and load distributions. Different axle location options are shown below (Figure 1.2). Axle locations, mass, and centre of gravity location have a direct effect on mobility characteristics of the vehicle.

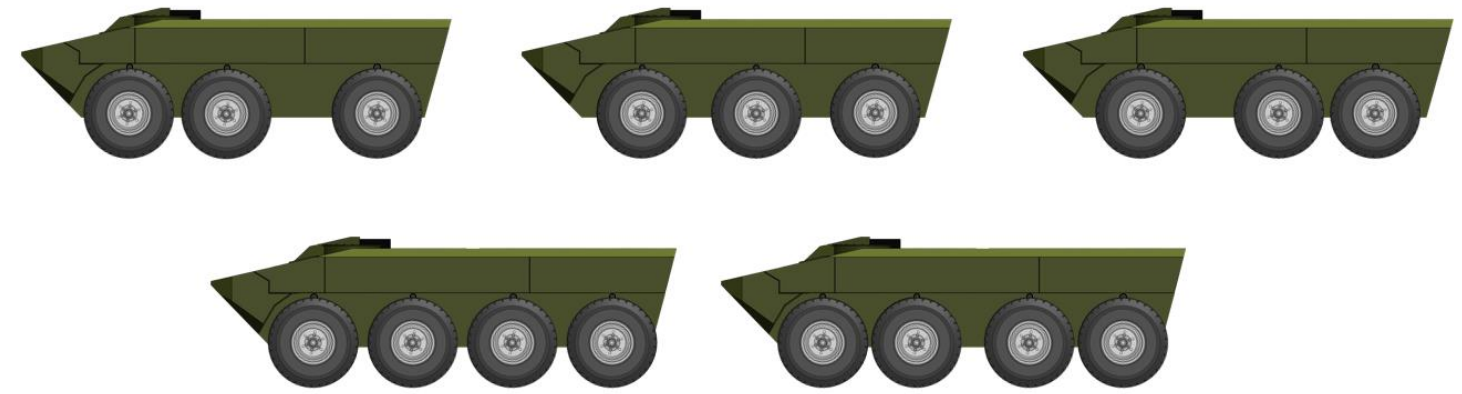

Figure 1.2. Different axle location options for $6 x 6$ and $8 x 8$ vehicles

Mobility can be divided into four different operations:

- Trench crossing (Figure 1.4)

- Obstacle crossing (Figure 1.4)

- Uphill and downhill operations (Figure 1.3)

- $\quad$ Side slope events (Figure 1.5)

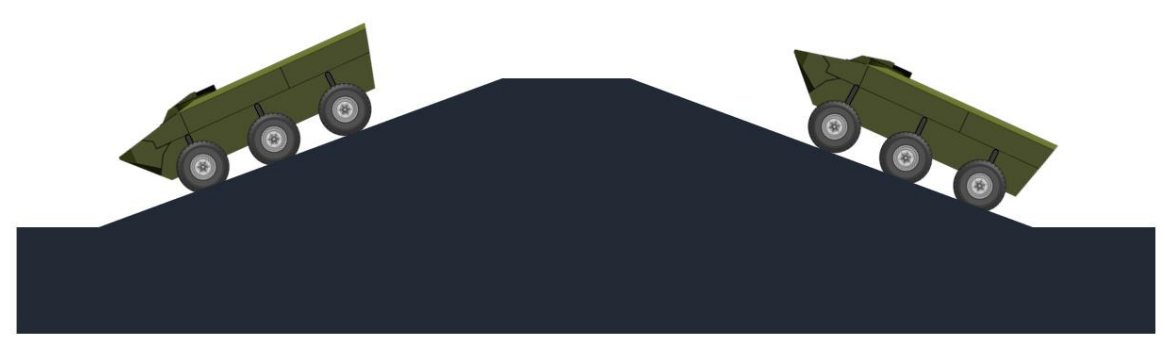

Figure 1.3. Uphill and downhill operations

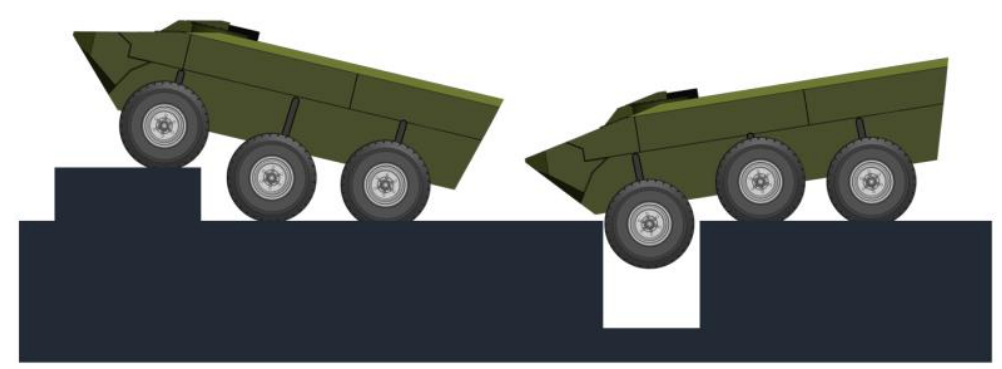

Figure 1.4. Obstacle and trench crossing operations 


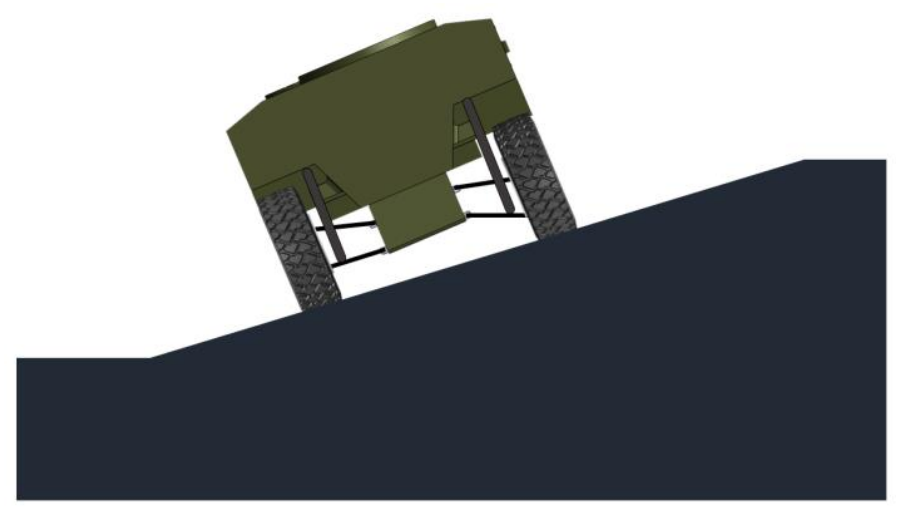

Figure 1.5. Side slope operation

For all these mobility operations, load distribution and ride height have a significant function in terms of rolling and pitching during the events. That is why using active suspension applications is critical. While active suspension applications usually focus on disturbance rejection in on-road vehicles, controlling the ride height also comes to the fore in military vehicles.

The main problem with crossing the trench in off-road condition is that the ride height control systems are used passively. For instance, the ride height is set by the driver to a specified value according to the terrain type, and the vehicle is driven with this ride height. Various ride height modes are given below. (Figure 1.6)

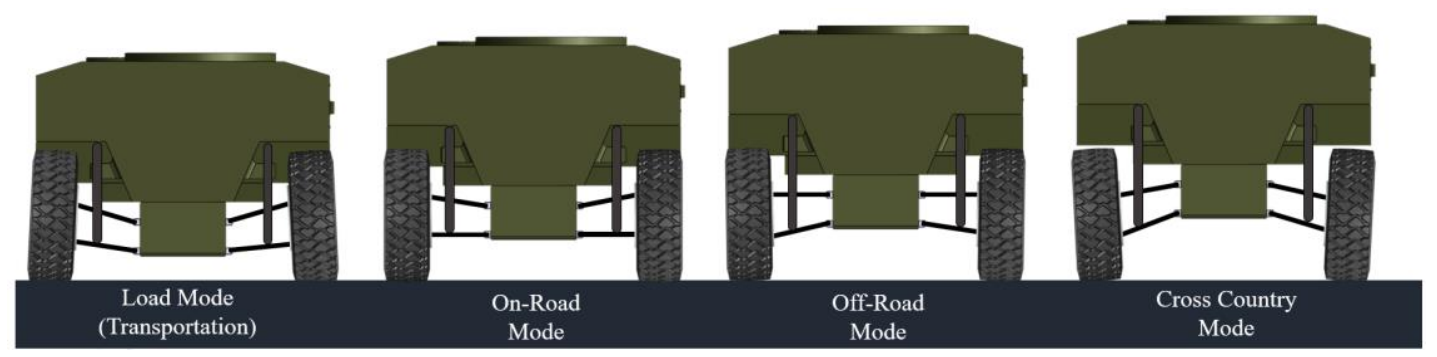

Figure 1.6. Various ride height modes

Using passive control does not always guarantee optimal ride height for mobility operations. Especially while crossing the obstacle encountered, different axles cannot be set to different ride heights. This implies that the physical limits of the vehicle cannot be fully utilized. 


\subsection{Scope of the Thesis}

The primary purpose of the thesis is to carry out a trend study on whether to increase the mobility of multi-axle vehicles by actively controlling ride heights of each axle during obstacle crossing events. Furthermore, another goal is to make mobility operations more productive by creating an obstacle-crossing algorithm.

Compared to $8 \times 8$ vehicles, $6 \times 6$ vehicles experience more difficulties in crossing trenches for passive systems. $8 \times 8$ vehicles can cross longer trenches than $6 \times 6$. As a matter of fact, 6x6 vehicles cannot pass the trenches at an acceptable level in terms of accelerations and forces acting on the vehicle body due to their structure without hitting their front and rear bumpers. Figure 1.7 shows the operation of $6 \times 6$ and $8 \times 8$ vehicles to a given trench below.

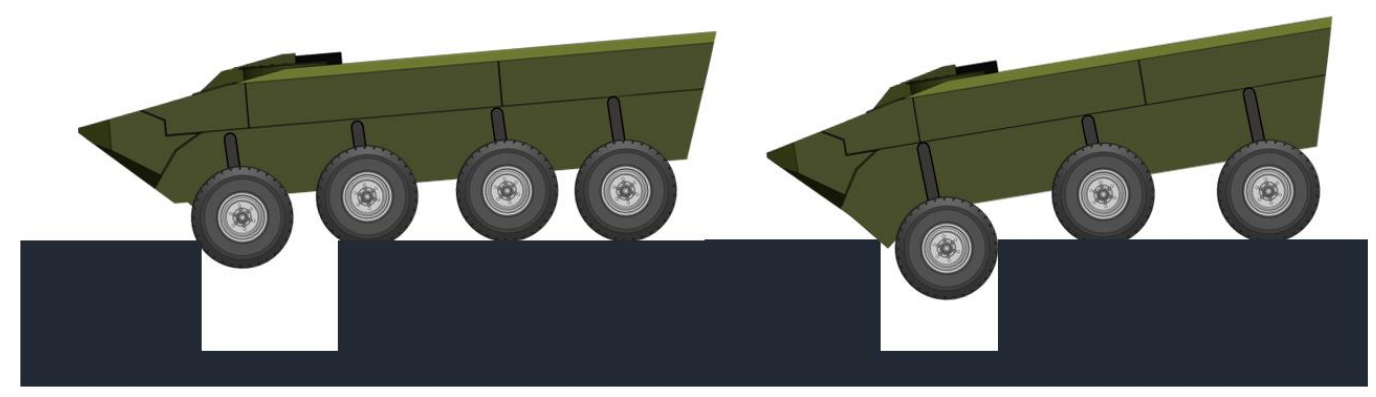

Figure 1.7. Trench crossing operation, $8 x 8$ and $6 x 6$ vehicles

$8 \times 8$ vehicles are generally more advantageous in trench passages since they have a more balanced weight distribution and higher number of wheels in contact with the ground during trench crossing. Since the trench cross of $8 \times 8$ vehicles is relatively easier than $6 \times 6$ vehicles, 6x6 vehicles were studied in this thesis. Two basic situations can be mentioned as a criterion for success. These can be listed as follows:

- Crossing a length of a trench without hitting any bumpers thanks to the activecontrolled system where 6x6 passive vehicles can only pass by hitting its bumpers.

- Another goal may be to cross longer trenches than passive systems can, despite hitting the bumpers with controlled system.

To sum up, the success criteria of the thesis are planned to be achieved by using an active ride height control system with preview data. In this thesis work, the preview road profile is assumed to be available. 


\subsection{Thesis Outline}

The first chapter consists of three sections describing the definition of problems, the purpose of the thesis and the general structure of the thesis.

In the following chapter, which is the literature survey, the previous research studies in the literature are given. These research topics include military vehicle mobility, vehicle dynamics, vehicle modelling, tire models, co-simulation environment, MPC controller, and preview control.

Vehicle modelling in ADAMS and MATLAB is given in the third chapter. This chapter also contains establishing a co-simulation environment. A linear vertical full car model was created and run in MATLAB. Then a multibody model of a $6 \times 6$ vehicle was established in ADAMS. Both models were validated and compared. After validation, the co-simulation environment was created according to required inputs and outputs between ADAMS and Simulink.

The fourth chapter states the controller design and the mobility algorithm. Some open-loop simulations were made to determine system limits which are the optimization parameters of the MPC controller. Then the controller was tuned according to desired response requirements. Finally, with the application of the preview road data, mobility algorithm was constructed.

The fifth chapter is the simulation and comparison part. The obstacle crossing scenarios were run according to different cases. Performance of the MPC controller with preview signal, the MPC controller without preview signal and conventional controllers were experienced and compared with each other.

In the last chapter, conclusion and discussion were made. The results of the obstacle crossing control algorithm were presented. Also, prospective future work was discussed. 


\section{LITERATURE SURVEY}

This section includes previous studies related to the topics mentioned in the content of this thesis. The literature research consists of four main parts which form the basis of the thesis. These are the mobility of military vehicles, vehicle dynamics and modelling, multibody vehicle modelling \& simulation and MPC controllers.

\subsection{Mobility of Military Vehicles}

First, two primary groups should be mentioned when talking about military vehicles. These are wheeled and tracked vehicles. There are many parameters to decide whether the military vehicle will be wheeled or tracked. The important ones are vehicle weight, operational conditions, technical specifications, payloads and logistics. Nevertheless, the critical part that interests us here is mobility.
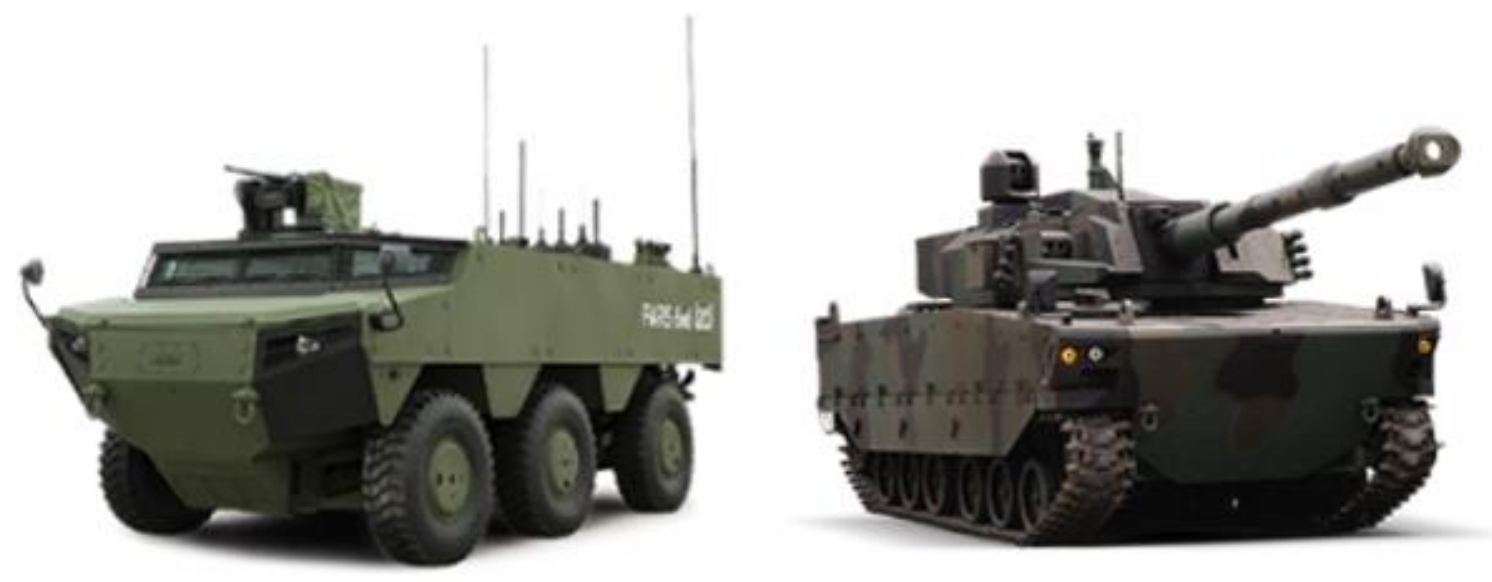

Figure 2.1. PARS 6x6 SCOUT wheeled vehicle \& KAPLAN MEDIUM TANK tracked vehicle [2]

To mention mobility, we can divide it into two, on-road and off-road mobility. Generally speaking, tracked vehicles offer better mobility than wheeled ones in off-road conditions. Vice versa, wheeled vehicles have greater mobility in on-road conditions. Tracked vehicles are also a better option for obstacle crossing events. Because harsh terrain conditions require a ground pressure that only tracked vehicles can produce [3]. However, the selection of a tracked or a wheeled vehicle is a trade-off and wheeled vehicles should provide a certain level of off-road mobility performance. 
In this study, since the mobility of wheeled vehicles is examined, the ability to increase off-road mobility capability is investigated. This part examines the mobility of wheeled vehicles.

In terms of mobility, a military vehicle is expected to have sufficient road holding and handling in on-road conditions as well as excellent off-road mobility. This leads to the necessity of different suspension characteristics in different road conditions. For example, in off-road or cross-country conditions, the vehicle needs higher ground clearance. Nonetheless in on-road conditions, the ground clearance must be minimized for a good road holding. Providing all these requirements together requires a sophisticated suspension design.

Active suspension systems are an effective way to ensure proper mobility and adequate road holding. These active suspension systems can be fully active and semi-active. Semiactive suspensions generally adjust damping coefficient according to road conditions to minimize body accelerations and enhance road holding. However in military vehicles, due to mobility requirements, the ride height adjustments can be required. Hydropneumatic suspensions come to the fore because these are an appropriate option for active and semiactive suspension applications in terms of ease of implementation. Sağlam and Ünlüsoy investigated comfort and ride height control with hydropneumatic suspensions in their work [4]. A basic schematic of a hydropneumatic suspension is given in Figure 2.2. There is also a master thesis about detailed work on hydropneumatic suspension written by Oscarsson which is useful for modelling the system with real hydrostrut dynamics [5].

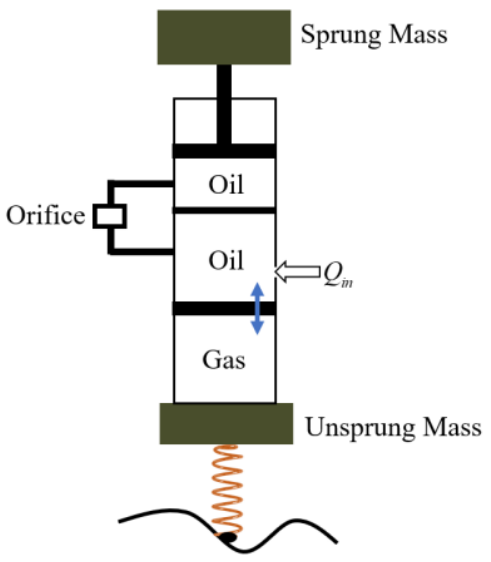

Figure 2.2. Hydropneumatic suspension schematic [4] 
There are limited studies in terms of wheeled vehicle obstacle crossing. In one of these studies [6]; Abhijeet, Senthilkumar and Vankudre examined a multi-axle combat vehicle model for its performance in off-road and ability to cross different terrain obstacles. Firstly, they build up a full car vehicle model in LMS Virtual Lab, which is a multi-body simulation tool. Then the vehicle model has been tested in 3 different obstacle scenarios, namely; Triangular Trench Crossing, Vertical Step Climbing and Straight Walled Trench Crossing. As a result, they have demonstrated a parametric study by comparing minimum and maximum body accelerations at different speeds against various obstacles.

Another study has been carried out by Trikande, Jagirdar, Rajamohan and Rao [7]. In this study, an 8x8 multi-axle armoured vehicle has been evaluated in terms of its performance against various semi-active suspension control strategies for improving ride quality and mobility capability over typical military obstacles. A co-simulation environment has been established; the full vehicle model has been modelled in MSC-ADAMS and control algorithms have been created in MATLAB/Simulink. They have considered three types of controllers; continuous skyhook, cascade loop control and cascade loop with ride control. Four types of different obstacle scenarios have been conducted; Step Climbing, Trench Crossing, Trapezoidal Bumps and Corrugated Track. Controllers have performed similar performances with the passive system on obstacle crossing scenarios, but cascade loop with ride control strategy was shown to be promising for ride quality.

Haou, Xu's and Zhoue have studied the effect of wheelbase crossing obstacles [8]. They analyzed the axle distance and the size of the obstacle mechanically and found numerical relations between them. In line with these relations, they developed a strategy and formulations that optimizes the axle distance. As a result, they showed that the performance of the obstacle passage increased by changing the longitudinal position of the middle wheel during the obstacle passage for a 6-wheel vehicle. 
In their another study, Jagirdar and Trikande examined the behavior of a $6 \times 6$ armored military vehicle in mobility operations [9]. For this, the 6x6 vehicle was modeled with the multi-body dynamics program (ADAMS) and simulated their passage through obstacles. They also provided similar vehicle parameters in the world as a comparison table. These parameters are parameters such as weight, tire dimensions and information, ride height, whether it is CTIS or not. In line with this information, they compared the mobility performance of vehicles for soft ground. In the continuation of the study, they simulated obstacle crossing scenarios for a $6 \times 6$ military vehicle. They presented the effects of vehicle features on mobility at the end of their studies.

Papunin, Belyakov and Makarov studied the trench transition performance of a lightweight $6 x 6$ vehicl [10]. They carried out mathematical modelling and experimental studies according to soil properties. The mathematical model they have established reveals the deformation of the soft ground during the trench transition and how many more times they can pass through the same trench. In addition, with these formulations, they obtained a model showing the trench width that a multi-axle vehicle can pass.

Thomas and Vantsevich did a very detailed study of the relationship between wheel, land and obstacles [11]. The focus of his work is to develop analytical tools that bring solutions to the following topics.

- to perceive the biggest obstacle length that the wheel can pass

- to be able to decide the size of the wheels that can overcome a certain obstacle

- to show a wheel model that can cross an obstacle on more types of terrain

- and studies on wheel dynamics with wheels to increase the mobility performance of an autonomous vehicle.

As a result, they have formulated formulations and calculations regarding the above issues. 


\subsection{Vehicle Dynamics and Modelling}

Vehicle dynamics is a subject that involves many sub-disciplines. These are basically longitudinal, lateral and vertical dynamics and other topics. To match these fundamental directional dynamics with the vehicle dynamics disciplines; longitudinal dynamics are related to vehicle performance. Similarly, lateral dynamics is also associated with handling and road holding where vertical dynamics are related to ride and comfort. Besides these, tire mechanics is another primary topic.

There are many books on vehicle dynamics in the literature, besides Jazar [12] and Gillespie [13] have written comprehensive books on vehicle dynamics. These books and many other studies generally contain quarter, half and full car ride models for two-axle vehicles. Nevertheless, there are fewer vertical dynamic models of multi-axle vehicles in the literature. These models are very similar and straight forward models. So, any multiaxle dynamic vehicle models can be easily derived from the given sources. Bayar [14], in his thesis, has established very detailed vehicle models for $4 \times 4,6 \times 6$ and $8 x 8$. These models include vertical, longitudinal and lateral dynamics of the vehicles with tire models and wheel dynamics. Another multi-axle vehicle model is studied by Önder and Başlamışlı [15]. Their model also includes vertical, lateral and longitudinal dynamics.

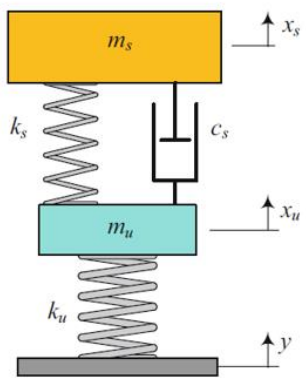

(A)

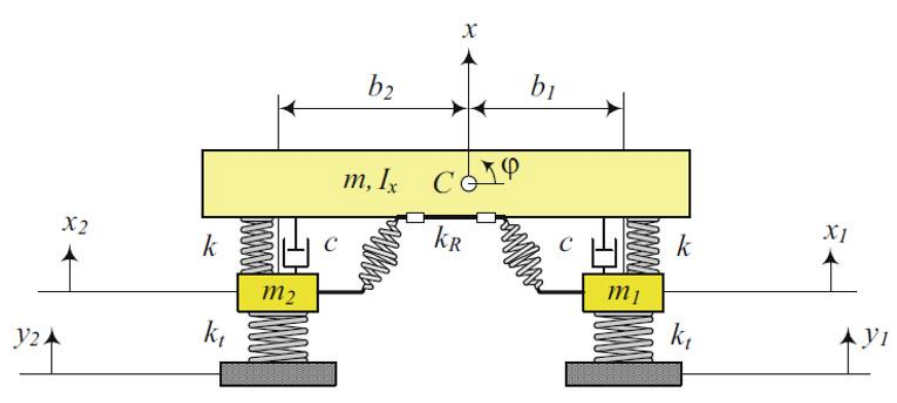

(B)

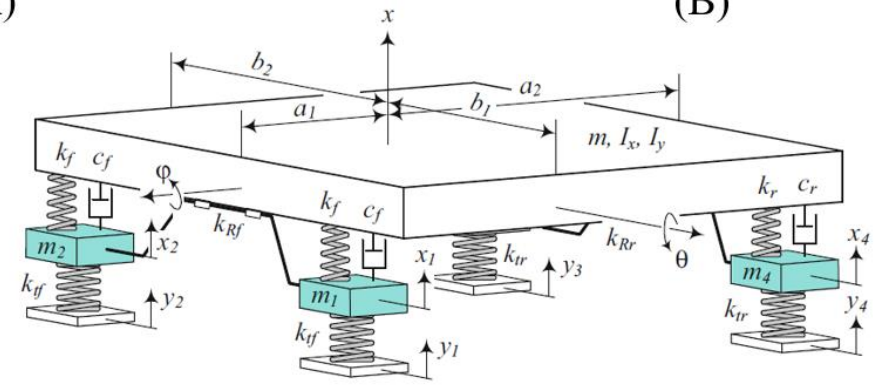

(C)

Figure 2.3. Quarter Car (A), Half Car (B) and Full Car (C) models [12] 


\subsection{Multibody Modelling and Simulation}

As British statistician George E.P. Box says, "Essentially, all models are wrong, but some are useful" [16] linear, and non-linear models used in vehicle dynamics and also in any other discipline contain specific errors. However, according to the type of application, many vehicle dynamics models converge to satisfactory results. In cases where these models are inadequate, multi-body dynamics modelling provides excellent convenience in the analysis of high degree of freedom and nonlinear systems and gives realistic results.

ADAMS is one of the most potent multi-body dynamics analysis programs, and ADAMS/Car is capable of performing many analyses and simulations on vehicles. It has many ready-made suspension and full vehicle simulations with test rigs. It also includes predefined subsystem and full vehicle templates with road and manoeuvring data. Besides, ADAMS has a powerful post-processing interface. Basic level controllers are also ready for use in ADAMS for many functions such as steering, gear shifting, acceleration and braking during a simulation [17]. In addition to all these, ADAMS is capable of cosimulation for complex control problems. In this way, sophisticated simulations can be performed by working simultaneously with different software [18].

Although ADAMS is widely used in automotive and vehicle dynamics analysis, there are generally more sources in the literature for passenger cars, sports vehicles and heavy commercial vehicles. Modelling and simulation studies of multi-axle military vehicles are more rare in the literature. In particular, simulation studies on large-scale obstacle clearance of such multi-axle vehicles are very few. In his thesis, Yazar [19] demonstrated the modelling in ADAMS and verification of a $6 \times 6$ military vehicle.

Many studies in the field of mechatronics and robotics using ADAMS/Controls are available in the literature. There are also co-simulation studies on vehicle systems. Li and He [20] studied the ESP system of a two-axle passenger car by using co-simulation of ADAMS and Simulink with fuzzy control to regulate the yaw angle. Xiu-qin, Chao and Guan-Neng analysed an ABS system of a multi-axle truck by using ADAMS and Simulink.[21] Yi, Min-min Jin-Yi and Hu worked on an active suspension application of a four-degree of freedom half-car model with LQG controller on ADAMS/Simulink cosimulation environment [22]. 


\subsection{Model Predictive Controllers (MPC)}

Model Predictive Controller (MPC) is an old control method dating back to the 1960s.

First applications of MPC are generally industrial process operations [23]. It is a method that is still actively used and researched today. Besides its usage area has expanded day by day. MPC is the control method with the most impact after PID. The trend of using MPC in process processes lasted until about 2000s, after which time it started to become widespread in areas such as automotive, aerospace, communication and energy sector [24].

The most important feature that distinguishes MPC from other controllers is its online optimization. It is basically a different kind of optimal controller, but the main difference and the advantage is that the MPC controller can solve the optimization problem on-line. Where offline optimization is difficult or not possible in multivariable systems, the problem-solving capability of MPC is superior [25].

The MPC is based on the following concepts:

i. Using a system model to predict the future behaviour of this system.

ii. Calculate the control process to optimize performance

iii. Receding horizon strategy, application of the first of the control signals, which slides forward in each time interval and calculated for the particular control horizon [26].

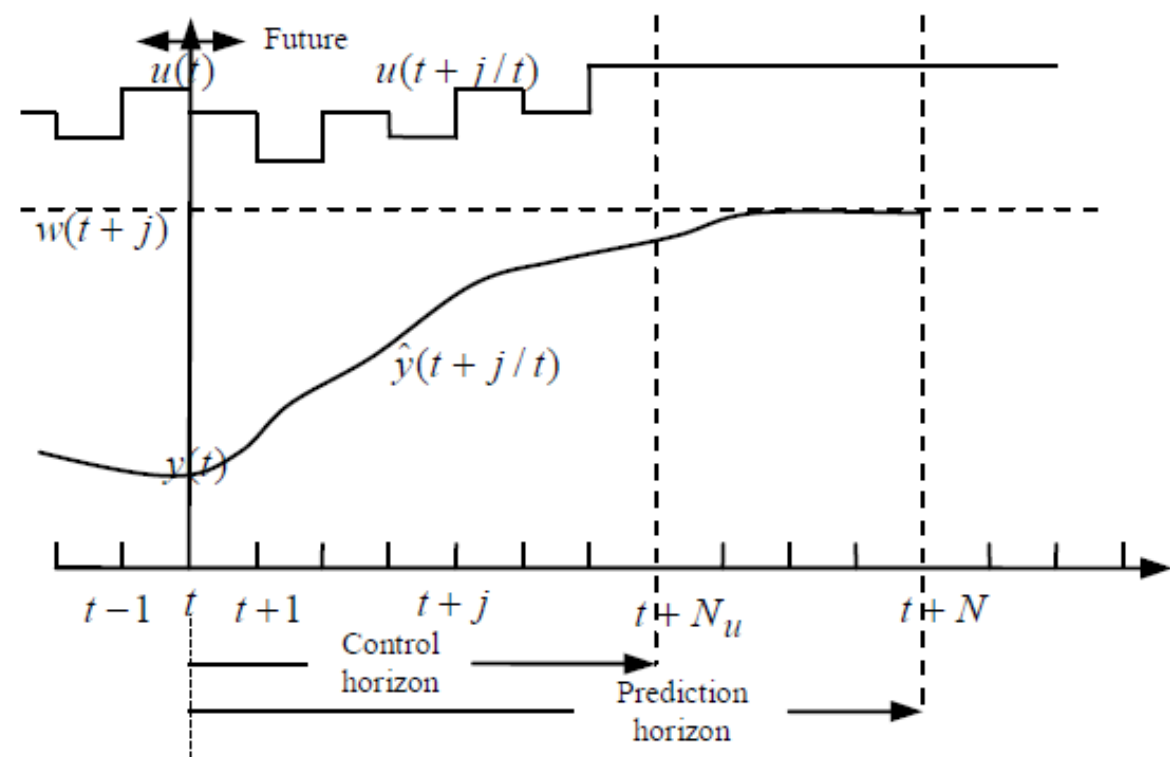

Figure 2.4. Moving horizon strategy [26] 
As shown in Figure 2.4, after the initial conditions are applied to the system, the new control input is not applied to the system directly. Instead, the data measured in the new time step are used to solve the new optimization problem. The first input calculated for the new trajectory predicted as a result of this optimization is applied to the system, and this process is repeated [27].

Model predictive controllers are an effective method for solving complex MIMO problems. However it requires a tremendous computational effort typically. Developments in optimization procedures in mathematics and processors pave the way for the development of MPCs too. Another exciting aspect of MPC controllers is their resemblance to the human brain. An example of the way the MPC works is that the driver can anticipate what is happening around and use the vehicle according to the predictions [28].

Another vital feature of MPC is that it can include limitations in a powerful way. They are prosperous in analysing the control process within or close to the specified limits [29]. MPC makes it easier to design a controller by allowing systematic combat with the limitations it faces. In addition, MPC can cope with troublesome situations such as structural system changes, sensor or actuator errors [30].

MPC's efficiency in the automotive field has increased noticeably in recent years. Powertrain applications as engine controls, automatized gearboxes and electric motors are one of the primary sub-disciplines of the automotive industry. Another crucial area is vehicle dynamics. With the increase of active suspension or steering applications and stability controls or traction controls, MPC becomes more widespread. Most importantly, in the studies about autonomous driving for the future, MPC is widely used. The advantage of using MPC in autonomous driving applications can be listed briefly as:

- It can combine longitudinal and lateral dynamics safely and comfortably.

- MPC can interconnect path planning and obstacle avoidance

- Stochastic prediction models can be used against model uncertainties, and these models can reflect a driver's behaviour [24]. 
In their study, Rasekhipour, Fadakar and Khajepour showed the motion plan and obstacle avoidance strategies of an autonomous vehicle using the MPC controller. What they do is to draw linear boundaries to the obstacles that come up and run optimization to stay outside these boundaries [31].

Another case study is the autonomous lane change assistant study using MPC toolbox and Automated Driving toolbox. A nonlinear MPC controller is used in this study. The reference signal is fed to the adaptive MPC with the help of an algorithm. The algorithm plans the trajectory of the vehicle when a vehicle is found in front of the vehicle. The vehicle follows the appropriate lane in this way. The reason for using a nonlinear MPC in this study is the vehicle model used in the controller is a nonlinear model [32].

For autonomous driving or obstacle avoidance manoeuvres, the desired control problem can be solved by feeding the reference signals or updating the input and output constraints also controller weights. In a sample case study using the controller weights and constraints, the vehicle manoeuvres to avoid the obstacle in front of it. While doing this manoeuvre, imaginary tangent lines are drawn from the vehicle to the corners of the obstacle. Also, tangent lines are drawn to a safe area around the obstacle. By changing the control parameters such as weights and constraints momentarily, the desired path to be followed determined in such a way that the tangent lines are outside the general boundaries [32]. 


\section{MODELLING}

\subsection{Introduction}

Various models are needed to be able to predict the behaviour of the vehicle and design a controller. The structure of these models can be simple or complex. While each model contains specific errors in its own right, the simplest model that meets the requirements will benefit from many aspects. For example, when a very detailed simulation is required, the model to be used is a high degree of freedom and nonlinear model. This often requires a multi-body dynamics model. However, linear models are often sufficient when there is a controller to work on any system actively, or when a fundamental analysis of a system is to be carried out.

In this thesis, since a controller system will be designed on a vehicle system, both a linear and very complex non-linear models are needed. The linear model is the model that will work fast in the controller. This is a full-car ride model for three-axle vehicle and capable of giving vehicle responses as body bounce, roll and pitch. The other model is the complex one which is close to the actual system and has the ability to test the controller performance. This model is a full vehicle assembly in ADAMS/Car.

In the following section, the modelling of roads and obstacles for mobility operations is described. Then an overview of the co-simulation environment between ADAMS and Matlab/Simulink is given. A more detailed description of this co-simulation environment will be given in the following sections. 


\subsection{Full-Car Ride Model for Three-Axle Vehicle}

A full-car ride model represents the vertical dynamics of the vehicle. This is a model with nine degrees of freedom which are vertical displacements of six unsprung masses, body bounce, body roll and body pitch. (Figure 3.2) This full-car model is actually non-linear because of containing trigonometric functions of body roll and pitch angles. However, in line with the physical limits of the vehicle, these angles are suitable for small-angle assumption, so the model can be linearized according to this assumption.

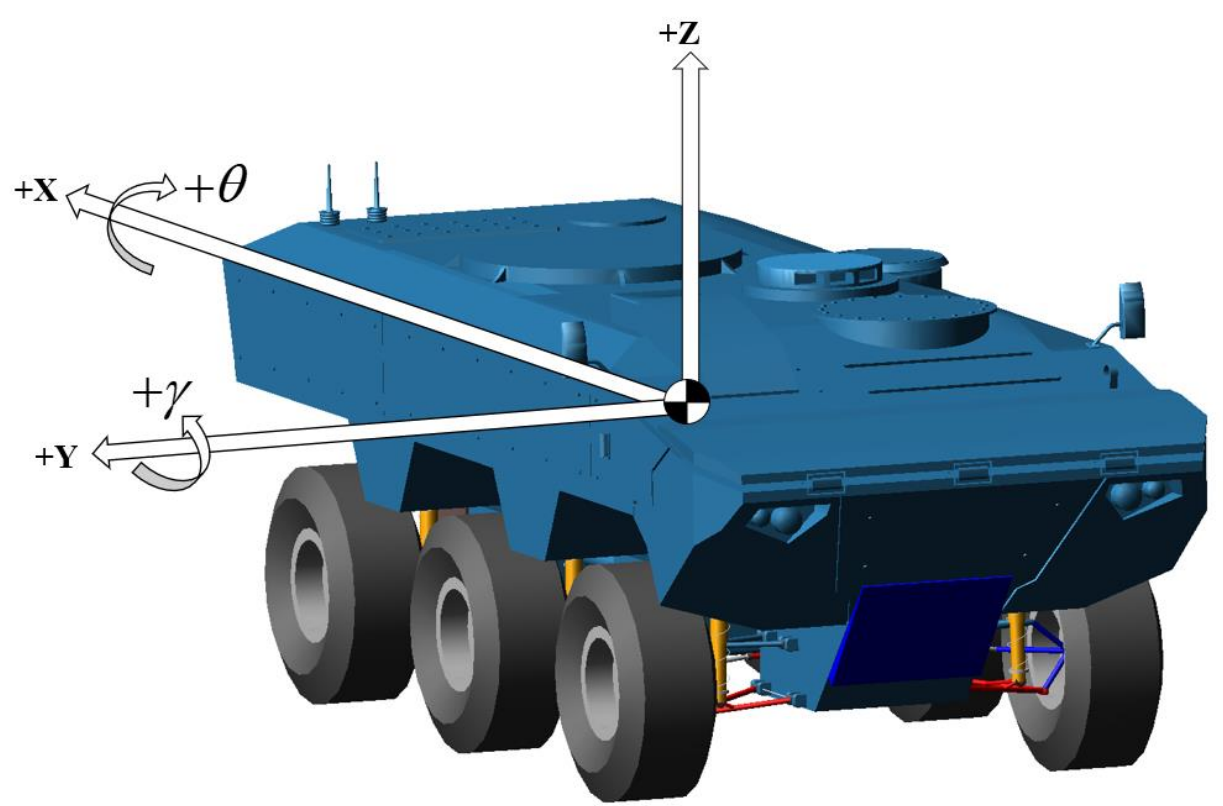

Figure 3.1. Body Coordinate System

Figure 3.1 shows the axis system of the models used in this thesis. A body has six degrees of freedom in space typically. The used degrees of freedom for the body are bounce, roll, and pitch. The body bounce is the vertical displacement of the vehicle (+Z-axis). The rotational motion about the vehicle's longitudinal axis is rolling, and about lateral axis is pitching. The positive roll means the vehicle leans to the left about $\mathrm{X}$-axis. The pitch angle is rotation about $\mathrm{Y}$-axis and positive when the vehicle pitches up. 


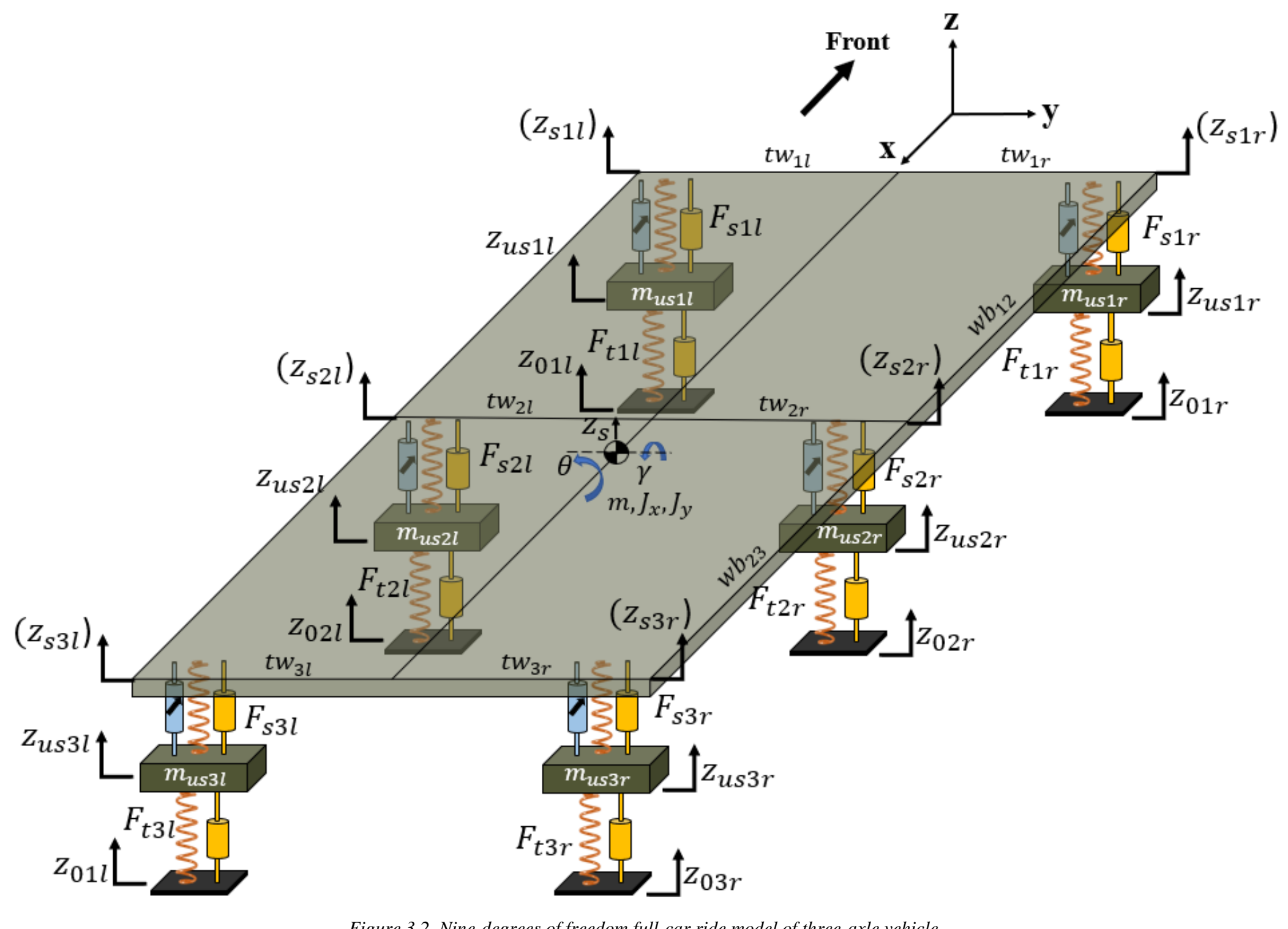

Figure 3.2. Nine-degrees of freedom full-car ride model of three-axle vehicle 
Figure 3.2 represents the full-car vertical dynamics model for a three-axle vehicle. It has ninedegrees of freedom. Six of them comes from vertical displacements of each unsprung masses. $\left(z_{u s .}\right)$ The other three-degrees of freedom belongs to the vehicle body. These are body bounce $\left(z_{s}\right)$, body roll $(\theta)$ and body pitch $(\gamma)$. There is no longitudinal or lateral dynamics in this vehicle model. Any disturbances coming from longitudinal or lateral motions are modelled in the multi-body dynamics environment. This linear full-car model is to design and tune controllers.

The terms in brackets $\left(z_{s . .}\right)$ refer to imaginary displacements that are not included in the final equations but are defined for convenience in expansions of equations. These terms are expressed to represent sprung mass displacements in each suspension element. However, these values are already expressed in terms of body vertical displacement, roll and pitch angles. The sprung mass is the mass supported by suspension elements as springs. Vehicle body, engine, payloads, and load in the body are sprung mass. Unsprung mass is the mass which is not supported by suspension springs. They are wheels, suspension arms, knuckle etc. High unsprung mass does not affect vehicle stability in on-road conditions. Nevertheless in off-road conditions, the higher unsprung mass has adverse effects and also unbalanced unsprung distribution influences the pitch dynamic of the vehicle negatively [33].

\subsubsection{Non-Linear Equations of Motion}

In this section, step by step derivation of the non-linear full vehicle model is explained based on fundamental force and moment equations. The directional assumptions are:

- Body bounce: $(+z)$

- Body roll: $(+\theta)$

- Body pitch: $(+\gamma)$

The imaginary sprung mass displacements denominated in body bounce, roll and pitch. The body motion relations:

$$
z_{s 1 r}=z_{s}+t w_{1 r} \sin \theta+\operatorname{CoG}_{X} \sin \gamma
$$




$$
\begin{gathered}
z_{s 1 l}=z_{s}-t w_{1 l} \sin \theta+\operatorname{CoG}_{X} \sin \gamma \\
z_{s 2 r}=z_{s}+t w_{2 r} \sin \theta+\left(\operatorname{CoG} G_{X}-w b_{12}\right) \sin \gamma \\
z_{s 2 l}=z_{s}-t w_{2 l} \sin \theta+\left(\operatorname{CoG} G_{X}-w b_{12}\right) \sin \gamma \\
z_{s 3 r}=z_{s}+t w_{3 r} \sin \theta-\left(w b_{12}+w b_{23}-C o G_{X}\right) \sin \gamma \\
z_{s 3 l}=z_{s}-t w_{3 l} \sin \theta-\left(w b_{12}+w b_{23}-C o G_{X}\right) \sin \gamma
\end{gathered}
$$

The basic form of equations of motion based on force and moment equilibriums are given below. There are nine equations that represent nine-degrees of freedom.

$$
\begin{gathered}
m_{u s 1 r} \ddot{z}_{u s 1 r}=F_{t 1 r}-F_{s 1 r}-W_{u s 1 r} \\
m_{u s 1 l} \ddot{z}_{u s 1 l}=F_{t 1 l}-F_{s 1 l}-W_{u s 1 l} \\
m_{u s 2 r} \ddot{z}_{u s 2 r}=F_{t 2 r}-F_{s 2 r}-W_{u s 2 r} \\
m_{u s 2 l} \ddot{z}_{u s 2 l}=F_{t 2 l}-F_{s 2 l}-W_{u s 2 l} \\
m_{u s 3 r} \ddot{z}_{u s 3 r}=F_{t 3 r}-F_{s 3 r}-W_{u s 3 r} \\
m_{u s 3 l} \ddot{z}_{u s 3 l}=F_{t 3 l}-F_{s 3 l}-W_{u s 3 l} \\
J_{X} \ddot{\theta}=F_{s 1 r} t w_{1 r}-F_{s 1 l} t w_{1 l}+F_{s 2 r} t w_{2 r}-F_{s 2 l} t w_{2 l}+F_{s 3 r} t w_{3 r}-F_{s 3 l} t w_{3 l} \\
m_{s} \ddot{z}_{s}=F_{s 1 r}+F_{s 1 l}+F_{s 2 r}+F_{s 2 l}+F_{s 3 r}+F_{s 3 l}-W_{s} \\
+F_{s 1 r} C_{O} G_{X}+F_{s 1 l} C_{o} G_{X}+F_{s 2 r}\left(C o G_{X}-w b_{12}\right) \\
+F_{s 2 l}\left(C o G_{X}-w b_{12}\right)-F_{s 3 r}\left(w b_{12}+w b_{23}-C o G_{X}\right) \\
-F_{s 3 l}\left(w b_{12}+w b_{23}-C o G_{X}\right)
\end{gathered}
$$

Here it should be noted that the weight terms (W) in the equations from (3.7) to (3.15) being neglected because these terms have no effect when dealing with dynamic behaviour. They are just statements showing static displacement. This static displacement will be considered in the following sections, where the controller design is described.

In order to facilitate the expansion of the equation, suspension and tire forces are included in the fundamental equations as single terms. In the following stages, each force elements as springs, dampers and actuator are expanded in the equations. (Figure 3.3) 


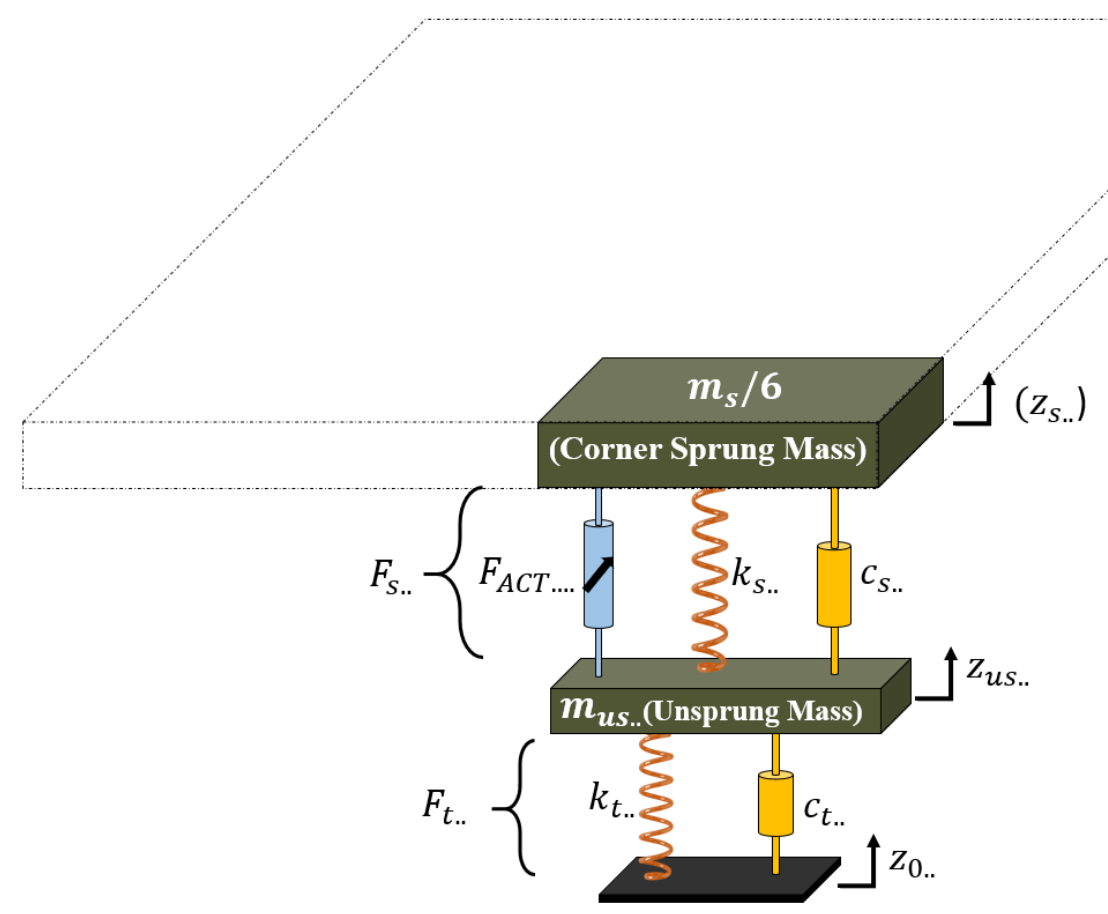

Figure 3.3. Force elements representation of the suspension and tire

Considering expansion of the force equations:

$$
\begin{gathered}
F_{t 1 r}=k_{t 1 r}\left(z_{01 r}-z_{u s 1 r}\right)+c_{t 1 r}\left(\dot{z}_{01 r}-\dot{z}_{u s 1 r}\right) \\
F_{s 1 r}=k_{s 1 r}\left(z_{u s 1 r}-z_{s 1 r}\right)+c_{s 1 r}\left(\dot{z}_{u s 1 r}-\dot{z}_{s 1 r}\right)+F_{A C T 1 r} \\
F_{t 1 l}=k_{t 1 l}\left(z_{01 l}-z_{u s 1 l}\right)+c_{t 1 l}\left(\dot{z}_{01 l}-\dot{z}_{u s 1 l}\right) \\
F_{s 1 l}=k_{s 1 l}\left(z_{u s 1 l}-z_{s 1 l}\right)+c_{s 1 l}\left(\dot{z}_{u s 1 l}-\dot{z}_{s 1 l}\right)+F_{A C T 1 l} \\
F_{t 2 r}=k_{t 2 r}\left(z_{02 r}-z_{u s 2 r}\right)+c_{t 2 r}\left(\dot{z}_{02 r}-\dot{z}_{u s 2 r}\right) \\
F_{s 2 r}=k_{s 2 r}\left(z_{u s 2 r}-z_{s 2 r}\right)+c_{s 2 r}\left(\dot{z}_{u s 2 r}-\dot{z}_{s 2 r}\right)+F_{A C T 2 r} \\
F_{t 2 l}=k_{t 2 l}\left(z_{02 l}-z_{u s 2 l}\right)+c_{t 2 l}\left(\dot{z}_{02 l}-\dot{z}_{u s 2 l}\right) \\
F_{s 2 l}=k_{s 2 l}\left(z_{u s 2 l}-z_{s 2 l}\right)+c_{s 2 l}\left(\dot{z}_{u s 2 l}-\dot{z}_{s 2 l}\right)+F_{A C T 2 l} \\
F_{t 3 r}=k_{t 3 r}\left(z_{03 r}-z_{u s 3 r}\right)+c_{t 3 r}\left(\dot{z}_{03 r}-\dot{z}_{u s 3 r}\right) \\
F_{s 3 r}=k_{s 3 r}\left(z_{u s 3 r}-z_{s 3 r}\right)+c_{s 3 r}\left(\dot{z}_{u s 3 r}-\dot{z}_{s 3 r}\right)+F_{A C T 3 r}
\end{gathered}
$$




$$
\begin{gathered}
F_{t 3 l}=k_{t 3 l}\left(z_{03 l}-z_{u s 3 l}\right)+c_{t 3 l}\left(\dot{z}_{03 l}-\dot{z}_{u s 3 l}\right) \\
F_{s 3 l}=k_{s 3 l}\left(z_{u s 3 l}-z_{s 3 l}\right)+c_{s 3 l}\left(\dot{z}_{u s 3 l}-\dot{z}_{s 3 l}\right)+F_{A C T 3 l}
\end{gathered}
$$

To linearize the system, small angle assumption is applied.

$$
\begin{aligned}
& \sin \theta \approx \theta \\
& \sin \gamma=\gamma
\end{aligned}
$$

So, the equation from (3.1) to (3.6) becomes:

$$
\begin{gathered}
z_{s 1 r}=z_{s}+t w_{1 r} \theta+\operatorname{CoG}_{X} \gamma \\
z_{s 1 l}=z_{s}-t w_{1 l} \theta+\operatorname{CoG}_{X} \gamma \\
z_{s 2 r}=z_{s}+t w_{2 r} \theta+\left(\operatorname{CoG} G_{X}-w b_{12}\right) \gamma \\
z_{s 2 l}=z_{s}-t w_{2 l} \theta+\left(\operatorname{Co} G_{X}-w b_{12}\right) \gamma \\
z_{s 3 r}=z_{s}+t w_{3 r} \theta-\left(w b_{12}+w b_{23}-\operatorname{Co} G_{X}\right) \gamma \\
z_{s 3 l}=z_{s}-t w_{3 l} \theta-\left(w b_{12}+w b_{23}-\operatorname{Co} G_{X}\right) \gamma
\end{gathered}
$$

Suspension and tire force terms in the equations from (3.16) to (3.27) are substituted into equations from (3.7) to (3.15). When all the sub-terms are rearranged according to statespace representation, the main equations of motion from (3.7) to (3.15) become: 


$$
\begin{aligned}
& \ddot{z}_{u s 1 r}=z_{01 r}\left(\frac{k_{t 1 r}}{m_{u s 1 r}}\right)+\dot{z}_{01 r}\left(\frac{c_{t 1 r}}{m_{u s 1 r}}\right)+z_{u s 1 r}\left(\frac{-k_{t 1 r}-k_{s 1 r}}{m_{u s 1 r}}\right)+\dot{z}_{u s 1 r}\left(\frac{-c_{t 1 r}-c_{s 1 r}}{m_{u s 1 r}}\right) \\
& +z_{s}\left(\frac{k_{s 1 r}}{m_{u s 1 r}}\right)+\dot{z}_{01 r}\left(\frac{c_{s 1 r}}{m_{u s 1 r}}\right)+\theta\left(\frac{k_{s 1 r} t w_{1 r}}{m_{u s 1 r}}\right)+\dot{\theta}\left(\frac{c_{s 1 r} t w_{1 r}}{m_{u s 1 r}}\right) \\
& +\gamma\left(\frac{k_{s 1 r} C o G_{X}}{m_{u s 1 r}}\right)+\dot{\gamma}\left(\frac{c_{s 1 r} C o G_{X}}{m_{u s 1 r}}\right)+F_{A C T 1 r}\left(\frac{-1}{m_{u s 1 r}}\right) \\
& \ddot{z}_{u s 1 l}=z_{01 l}\left(\frac{k_{t 1 l}}{m_{u s 1 l}}\right)+\dot{z}_{01 l}\left(\frac{c_{t 1 l}}{m_{u s 1 l}}\right)+z_{u s 1 l}\left(\frac{-k_{t 1 l}-k_{s 1 l}}{m_{u s 1 l}}\right)+\dot{z}_{u s 1 l}\left(\frac{-c_{t 1 l}-c_{s 1 l}}{m_{u s 1 l}}\right) \\
& +z_{s}\left(\frac{k_{s 1 l}}{m_{u s 1 l}}\right)+\dot{z}_{01 l}\left(\frac{c_{s 1 l}}{m_{u s 1 l}}\right)+\theta\left(\frac{-k_{s 1 l} t w_{1 l}}{m_{u s 1 l}}\right)+\dot{\theta}\left(\frac{-c_{s 1 l} t w_{1 l}}{m_{u s 1 l}}\right) \\
& +\gamma\left(\frac{k_{s 1 l} \operatorname{Co} G_{X}}{m_{u s 1 l}}\right)+\dot{\gamma}\left(\frac{c_{s 1 l} \operatorname{Co} G_{X}}{m_{u s 1 l}}\right)+F_{A C T 1 l}\left(\frac{-1}{m_{u s 1 l}}\right) \\
& \ddot{z}_{u s 2 r}=z_{02 r}\left(\frac{k_{t 2 r}}{m_{u s 2 r}}\right)+\dot{z}_{02 r}\left(\frac{c_{t 2 r}}{m_{u s 2 r}}\right)+z_{u s 2 r}\left(\frac{-k_{t 2 r}-k_{s 2 r}}{m_{u s 2 r}}\right) \\
& +\dot{z}_{u s 2 r}\left(\frac{-c_{t 2 r}-c_{s 2 r}}{m_{u s 2 r}}\right)+z_{s}\left(\frac{k_{s 2 r}}{m_{u s 2 r}}\right)+\dot{z}_{02 r}\left(\frac{c_{s 2 r}}{m_{u s 2 r}}\right)+\theta\left(\frac{k_{s 2 r} t w_{2 r}}{m_{u s 2 r}}\right) \\
& +\dot{\theta}\left(\frac{c_{s 2 r} t w_{2 r}}{m_{u s 2 r}}\right)+\gamma\left(\frac{k_{s 2 r}\left(C o G_{X}-w b_{12}\right)}{m_{u s 2 r}}\right)+\dot{\gamma}\left(\frac{c_{s 2 r}\left(C o G_{X}-w b_{12}\right)}{m_{u s 2 r}}\right) \\
& +F_{A C T 2 r}\left(\frac{-1}{m_{u s 2 r}}\right)
\end{aligned}
$$




$$
\begin{aligned}
& \ddot{z}_{u s 2 l}=z_{02 l}\left(\frac{k_{t 2 l}}{m_{u s 2 l}}\right)+\dot{z}_{02 l}\left(\frac{c_{t 2 l}}{m_{u s 2 l}}\right)+z_{u s 2 l}\left(\frac{-k_{t 2 l}-k_{s 2 l}}{m_{u s 2 l}}\right)+\dot{z}_{u s 2 l}\left(\frac{-c_{t 2 l}-c_{s 2 l}}{m_{u s 2 l}}\right) \\
& +z_{s}\left(\frac{k_{s 2 l}}{m_{u s 2 l}}\right)+\dot{z}_{02 l}\left(\frac{c_{s 2 l}}{m_{u s 2 l}}\right)+\theta\left(\frac{-k_{s 2 l} t w_{2 l}}{m_{u s 2 l}}\right)+\dot{\theta}\left(\frac{-c_{s 2 l} t w_{2 l}}{m_{u s 2 l}}\right) \\
& +\gamma\left(\frac{k_{s 2 l}\left(C o G_{X}-w b_{12}\right)}{m_{u s 2 l}}\right)+\dot{\gamma}\left(\frac{c_{s 2 l}\left(C o G_{X}-w b_{12}\right)}{m_{u s 2 l}}\right) \\
& +F_{A C T 2 l}\left(\frac{-1}{m_{u s 2 l}}\right) \\
& \ddot{z}_{u s 3 r}=z_{03 r}\left(\frac{k_{t 3 r}}{m_{u s 3 r}}\right)+\dot{z}_{03 r}\left(\frac{c_{t 3 r}}{m_{u s 3 r}}\right)+z_{u s 3 r}\left(\frac{-k_{t 3 r}-k_{s 3 r}}{m_{u s 3 r}}\right) \\
& +\dot{z}_{u s 3 r}\left(\frac{-c_{t 3 r}-c_{s 3 r}}{m_{u s 3 r}}\right)+z_{s}\left(\frac{k_{s 3 r}}{m_{u s 3 r}}\right)+\dot{z}_{03 r}\left(\frac{c_{s 3 r}}{m_{u s 3 r}}\right)+\theta\left(\frac{k_{s 3 r} t w_{3 r}}{m_{u s 3 r}}\right) \\
& +\dot{\theta}\left(\frac{c_{s 3 r} t w_{3 r}}{m_{u s 3 r}}\right)+\gamma\left(\frac{k_{s 3 r}\left(w b_{12}+w b_{23}-C o G_{X}\right)}{m_{u s 3 r}}\right) \\
& +\dot{\gamma}\left(\frac{c_{s 3 r}\left(w b_{12}+w b_{23}-C o G_{X}\right)}{m_{u s 3 r}}\right)+F_{A C T 3 r}\left(\frac{-1}{m_{u s 3 r}}\right) \\
& \ddot{z}_{u s 3 l}=z_{03 l}\left(\frac{k_{t 3 l}}{m_{u s 3 l}}\right)+\dot{z}_{03 l}\left(\frac{c_{t 3 l}}{m_{u s 3 l}}\right)+z_{u s 3 l}\left(\frac{-k_{t 3 l}-k_{s 3 l}}{m_{u s 3 l}}\right)+\dot{z}_{u s 3 l}\left(\frac{-c_{t 3 l}-c_{s 3 l}}{m_{u s 3 l}}\right) \\
& +z_{s}\left(\frac{k_{s 3 l}}{m_{u s 3 l}}\right)+\dot{z}_{03 l}\left(\frac{c_{s 3 l}}{m_{u s 3 l}}\right)+\theta\left(\frac{-k_{s 3 l} t w_{3 l}}{m_{u s 3 l}}\right)+\dot{\theta}\left(\frac{-c_{s 3 l} t w_{3 l}}{m_{u s 3 l}}\right) \\
& +\gamma\left(\frac{k_{s 3 l}\left(w b_{12}+w b_{23}-C o G_{X}\right)}{m_{u s 3 l}}\right)+\dot{\gamma}\left(\frac{c_{s 3 l}\left(w b_{12}+w b_{23}-C o G_{X}\right)}{m_{u s 3 l}}\right) \\
& +F_{A C T 3 l}\left(\frac{-1}{m_{u s 3 l}}\right)
\end{aligned}
$$




$$
\begin{aligned}
& \ddot{z}_{s}=z_{u s 1 r}\left(\frac{k_{s 1 r}}{m_{s}}\right)+\dot{z}_{u s 1 r}\left(\frac{c_{s 1 r}}{m_{s}}\right)+z_{u s 1 l}\left(\frac{k_{s 1 l}}{m_{s}}\right)+\dot{z}_{u s 1 l}\left(\frac{c_{s 1 l}}{m_{s}}\right)+z_{u s 2 r}\left(\frac{k_{s 2 r}}{m_{s}}\right)+\dot{z}_{u s 2 r}\left(\frac{c_{s 2 r}}{m_{s}}\right) \\
& +z_{u s 2 l}\left(\frac{k_{s 2 l}}{m_{s}}\right)+\dot{z}_{u s 2 l}\left(\frac{c_{s 2 l}}{m_{s}}\right)+z_{u s 3 r}\left(\frac{k_{s 3 r}}{m_{s}}\right)+\dot{z}_{u s 3 r}\left(\frac{c_{s 3 r}}{m_{s}}\right)+z_{u s 3 l}\left(\frac{k_{s 3 l}}{m_{s}}\right)+\dot{z}_{u s 3 l}\left(\frac{c_{s 3 l}}{m_{s}}\right) \\
& +z_{s}\left(\frac{-k_{s 1 r}-k_{s 1 l}-k_{s 2 r}-k_{s 2 l}-k_{s 3 r}-k_{s 3 l}}{m_{s}}\right)+\dot{z}_{s}\left(\frac{-c_{s 1 r}-c_{s 1 l}-c_{s 2 r}-c_{s 2 l}-c_{s 3 r}-c_{s 3 l}}{m_{s}}\right) \\
& +\theta\left(\frac{-k_{s 1 r} t w_{1 r}+k_{s 1 l} t w_{1 l}-k_{s 2 r} t w_{2 r}+k_{s 2 l} t w_{2 l}-k_{s 3 r} t w_{3 r}+k_{s 3 l} t w_{3 l}}{m_{s}}\right) \\
& +\dot{\theta}\left(\frac{-c_{s 1 r} t w_{1 r}+c_{s 1 l} t w_{1 l}-c_{s 2 r} t w_{2 r}+c_{s 2 l} t w_{2 l}-c_{s 3 r} t w_{3 r}+c_{s 3 l} t w_{3 l}}{m_{s}}\right) \\
& +\gamma\left(\frac{-k_{s 1 r} \operatorname{CoG}_{X}-k_{s 1 l} \operatorname{CoG}_{X}-k_{s 2 r}\left(C o G_{X}-w b_{12}\right)-k_{s 2 l}\left(C o G_{X}-w b_{12}\right)}{m_{s}}\right. \\
& \left.\ldots \frac{+k_{s 3 r}\left(w b_{12}+w b_{23}-C o G_{X}\right)+k_{s 3 l}\left(w b_{12}+w b_{23}-C o G_{X}\right)}{m_{s}}\right) \\
& +\dot{\gamma}\left(\frac{-c_{s 1 r} \operatorname{CoG}_{X}-c_{s 1 l} \operatorname{CoG}_{X}-c_{s 2 r}\left(C o G_{X}-w b_{12}\right)-c_{s 2 l}\left(C o G_{X}-w b_{12}\right)}{m_{s}}\right. \\
& \left.\ldots \frac{+c_{s 3 r}\left(w b_{12}+w b_{23}-C o G_{X}\right)+c_{s 3 l}\left(w b_{12}+w b_{23}-C o G_{X}\right)}{m_{s}}\right) \\
& +F_{A C T 1 r}\left(\frac{1}{m_{s}}\right)+F_{A C T 1 l}\left(\frac{1}{m_{s}}\right)+F_{A C T 2 r}\left(\frac{1}{m_{s}}\right)+F_{A C T 2 l}\left(\frac{1}{m_{s}}\right) \\
& +F_{A C T 3 r}\left(\frac{1}{m_{s}}\right)+F_{A C T 3 l}\left(\frac{1}{m_{s}}\right)
\end{aligned}
$$




$$
\begin{aligned}
& \ddot{\theta}=z_{u s 1 r}\left(\frac{k_{s 1 r} t w_{1 r}}{J_{X}}\right)+\dot{z}_{u s 1 r}\left(\frac{c_{s 1 r} t w_{1 r}}{J_{X}}\right)+z_{u s 1 l}\left(\frac{-k_{s 1 l} t w_{1 l}}{J_{X}}\right)+\dot{z}_{u s 1 l}\left(\frac{-c_{s 1 l} t w_{1 l}}{J_{X}}\right) \\
& +z_{u s 2 r}\left(\frac{k_{s 2 r} t w_{2 r}}{J_{X}}\right)+\dot{z}_{u s 2 r}\left(\frac{c_{s 2 r} t w_{2 r}}{J_{X}}\right)+z_{u s 2 l}\left(\frac{-k_{s 2 l} t w_{2 l}}{J_{X}}\right)+\dot{z}_{u s 2 l}\left(\frac{-c_{s 2 l} t w_{2 l}}{J_{X}}\right) \\
& +z_{u s 3 r}\left(\frac{k_{s 3 r} t w_{3 r}}{J_{X}}\right)+\dot{z}_{u s 3 r}\left(\frac{c_{s 3 r} t w_{3 r}}{J_{X}}\right)+z_{u s 3 l}\left(\frac{-k_{s 3 l} t w_{3 l}}{J_{X}}\right)+\dot{z}_{u s 3 l}\left(\frac{-c_{s 3 l} t w_{3 l}}{J_{X}}\right) \\
& +z_{s}\left(\frac{-k_{s 1 r} t w_{1 r}+k_{s 1 l} t w_{1 l}-k_{s 2 r} t w_{2 r}+k_{s 2 l} t w_{2 l}-k_{s 3 r} t w_{3 r}+k_{s 3 l} t w_{3 l}}{J_{X}}\right) \\
& +\dot{z}_{s}\left(\frac{-c_{s 1 r} t w_{1 r}+c_{s 1 l} t w_{1 l}-c_{s 2 r} t w_{2 r}+c_{s 2 l} t w_{2 l}-c_{s 3 r} t w_{3 r}+c_{s 3 l} t w_{3 l}}{J_{X}}\right) \\
& +\theta\left(\frac{-k_{s 1 r} t w_{1 r}{ }^{2}-k_{s 1 l} t w_{1 l}{ }^{2}-k_{s 2 r} t w_{2 r}{ }^{2}-k_{s 2 l} t w_{2 l}{ }^{2}-k_{s 3 r} t w_{3 r}{ }^{2}-k_{s 3 l} t w_{3 l}{ }^{2}}{J_{X}}\right) \\
& +\dot{\theta}\left(\frac{-c_{s 1 r} t w_{1 r}{ }^{2}-c_{s 1 l} t w_{1 l}{ }^{2}-c_{s 2 r} t w_{2 r}{ }^{2}-c_{s 2 l} t w_{2 l}{ }^{2}-c_{s 3 r} t w_{3 r}{ }^{2}-c_{s 3 l} t w_{3 l}{ }^{2}}{J_{X}}\right) \\
& +\gamma\left(\frac{-k_{s 1 r} \operatorname{CoG}_{X} t w_{1 r}+k_{s 1 l} \operatorname{CoG}_{X} t w_{1 l}-k_{s 2 r}\left(\operatorname{CoG}_{X}-w b_{12}\right) t w_{2 r}+k_{s 2 l}\left(C o G_{X}-w b_{12}\right) t w_{2 l}}{J_{X}}\right. \\
& \left.\ldots \frac{+k_{s 3 r}\left(w b_{12}+w b_{23}-C o G_{X}\right) t w_{3 r}-k_{s 3 l}\left(w b_{12}+w b_{23}-C o G_{X}\right) t w_{3 l}}{J_{X}}\right) \\
& +\dot{\gamma}\left(\frac{-c_{s 1 r} \operatorname{CoG}_{X} t w_{1 r}+c_{s 1 l} \operatorname{CoG}_{X} t w_{1 l}-c_{s 2 r}\left(C o G_{X}-w b_{12}\right) t w_{2 r}+c_{s 2 l}\left(C o G_{X}-w b_{12}\right) t w_{2 l}}{J_{X}}\right. \\
& \left.\ldots \frac{+c_{s 3 r}\left(w b_{12}+w b_{23}-C o G_{X}\right) t w_{3 r}-c_{s 3 l}\left(w b_{12}+w b_{23}-C o G_{X}\right) t w_{3 l}}{J_{X}}\right) \\
& +F_{A C T 1 r}\left(\frac{t w_{1 r}}{J_{X}}\right)+F_{A C T 11}\left(\frac{-t w_{1 l}}{J_{X}}\right)+F_{A C T 2 r}\left(\frac{t w_{2 r}}{J_{X}}\right)+F_{A C T 2 l}\left(\frac{-t w_{2 l}}{J_{X}}\right) \\
& +F_{A C T 3 r}\left(\frac{t w_{3 r}}{J_{X}}\right)+F_{A C T 3 l}\left(\frac{-t w_{3 l}}{J_{X}}\right)
\end{aligned}
$$




$$
\begin{aligned}
& \ddot{\gamma}=z_{u s 1 r}\left(\frac{k_{s 1 r} \operatorname{Co} G_{X}}{J_{Y}}\right)+\dot{z}_{u s 1 r}\left(\frac{c_{s 1 r} C_{0} G_{X}}{J_{Y}}\right)+z_{u s 1 l}\left(\frac{k_{s 1 l} C_{0} G_{X}}{J_{Y}}\right)+\dot{z}_{u s 1 l}\left(\frac{c_{s 1 l} C_{0} G_{X}}{J_{Y}}\right) \\
& +z_{u s 2 r}\left(\frac{k_{s 2 r}\left(C o G_{X}-w b_{12}\right)}{J_{Y}}\right)+\dot{z}_{u s 2 r}\left(\frac{c_{s 2 r}\left(C o G_{X}-w b_{12}\right)}{J_{Y}}\right) \\
& +z_{u s 2 l}\left(\frac{k_{s 2 l}\left(C o G_{X}-w b_{12}\right)}{J_{Y}}\right)+\dot{z}_{u s 2 l}\left(\frac{c_{s 2 l}\left(C o G_{X}-w b_{12}\right)}{J_{Y}}\right) \\
& +z_{u s 3 r}\left(\frac{-k_{s 3 r}\left(w b_{12}+w b_{23}-C o G_{X}\right)}{J_{Y}}\right)+\dot{z}_{u s 3 r}\left(\frac{-c_{s 3 r}\left(w b_{12}+w b_{23}-C o G_{X}\right)}{J_{Y}}\right) \\
& +z_{u s 3 l}\left(\frac{-k_{s 3 l}\left(w b_{12}+w b_{23}-C o G_{X}\right)}{J_{Y}}\right)+\dot{z}_{u s 3 l}\left(\frac{-c_{s 3 l}\left(w b_{12}+w b_{23}-C o G_{X}\right)}{J_{Y}}\right) \\
& +z_{s}\left(\frac{-k_{s 1 r} C_{o} G_{X}+k_{s 1 l} C_{o} G_{X}-k_{s 2 r}\left(C o G_{X}-w b_{12}\right)-k_{s 2 l}\left(C o G_{X}-w b_{12}\right)}{J_{Y}} \ldots\right. \\
& \left.\ldots \frac{+k_{s 3 r}\left(w b_{12}+w b_{23}-C o G_{X}\right)+k_{s 3 l}\left(w b_{12}+w b_{23}-C o G_{X}\right)}{J_{Y}}\right) \\
& +\dot{z}_{s}\left(\frac{-c_{s 1 r} \operatorname{CoG}_{X}+c_{s 1 l} C_{o} G_{X}-c_{s 2 r}\left(C o G_{X}-w b_{12}\right)-c_{s 2 l}\left(C o G_{X}-w b_{12}\right)}{J_{Y}} \ldots\right. \\
& \left.\ldots \frac{+c_{s 3 r}\left(w b_{12}+w b_{23}-C o G_{X}\right)+c_{s 3 l}\left(w b_{12}+w b_{23}-C o G_{X}\right)}{J_{Y}}\right) \\
& +\theta\left(\frac{-k_{s 1 r} t w_{1 r} \operatorname{CoG}_{X}-k_{s 1 l} t w_{1 l} \operatorname{CoG}_{X}-k_{s 2 r} t w_{2 r}\left(C o G_{X}-w b_{12}\right)-k_{s 2 l} t w_{2 l}\left(C o G_{X}-w b_{12}\right)}{J_{Y}} \ldots\right. \\
& \left.\ldots \frac{k_{s 3 r} t w_{3 r}\left(w b_{12}+w b_{23}-C o G_{X}\right)+k_{s 3 l} t w_{3 l}\left(w b_{12}+w b_{23}-C o G_{X}\right)}{J_{Y}}\right) \\
& +\dot{\theta}\left(\frac{-c_{s 1 r} t w_{1 r} \operatorname{Co} G_{X}-c_{s 1 l} t w_{1 l} \operatorname{CoG}_{X}-c_{s 2 r} t w_{2 r}\left(C o G_{X}-w b_{12}\right)-c_{s 2 l} t w_{2 l}\left(C o G_{X}-w b_{12}\right)}{J_{Y}} \ldots\right. \\
& \left.\ldots \frac{c_{s 3 r} t w_{3 r}\left(w b_{12}+w b_{23}-C o G_{X}\right)+c_{s 3 l} t w_{3 l}\left(w b_{12}+w b_{23}-C_{o} G_{X}\right)}{J_{Y}}\right) \\
& +\gamma\left(\frac{-k_{s 1 r} \operatorname{CoG}_{X}{ }^{2}-k_{s 1 l} \operatorname{CoG}_{X}{ }^{2}-k_{s 2 r}\left(C o G_{X}-w b_{12}\right)^{2}-k_{s 2 l}\left(C o G_{X}-w b_{12}\right)^{2}}{J_{Y}}\right. \\
& \left.\ldots \frac{+k_{s 3 r}\left(w b_{12}+w b_{23}-C o G_{X}\right)^{2}+k_{s 3 l}\left(w b_{12}+w b_{23}-C o G_{X}\right)^{2}}{J_{Y}}\right) \\
& +\dot{\gamma}\left(\frac{-c_{s 1 r} \operatorname{CoG}_{X}{ }^{2}-c_{s 1 l} \operatorname{CoG}_{X}{ }^{2}-c_{s 2 r}\left(C o G_{X}-w b_{12}\right)^{2}-c_{s 2 l}\left(C o G_{X}-w b_{12}\right)^{2}}{J_{Y}}\right. \\
& \left.\ldots \frac{+c_{s 3 r}\left(w b_{12}+w b_{23}-C o G_{X}\right)^{2}+c_{s 3 l}\left(w b_{12}+w b_{23}-C o G_{X}\right)^{2}}{J_{Y}}\right) \\
& +F_{A C T 1 r}\left(\frac{C o G_{X}}{J_{Y}}\right)+F_{A C T 1 l}\left(\frac{C o G_{X}}{J_{Y}}\right)+F_{A C T 2 r}\left(\frac{C o G_{X}-w b_{12}}{J_{Y}}\right)+F_{A C T 2 l}\left(\frac{C o G_{X}-w b_{12}}{J_{Y}}\right) \cdots
\end{aligned}
$$




$$
\ldots+F_{A C T 3 r}\left(\frac{-\left(w b_{12}+w b_{23}-C o G_{X}\right)}{J_{Y}}\right)+F_{A C T 3 l}\left(\frac{-\left(w b_{12}+w b_{23}-C o G_{X}\right)}{J_{Y}}\right)
$$

\subsubsection{Linear State Space Representation of the System}

System equations for the controller to be designed in Matlab/Simulink are arranged in statespace form. The general state-space form can be shown as:

$$
\dot{x}=A x+B u+G w
$$

where the $\boldsymbol{x}$ is the state matrix consists of states of the system which is;

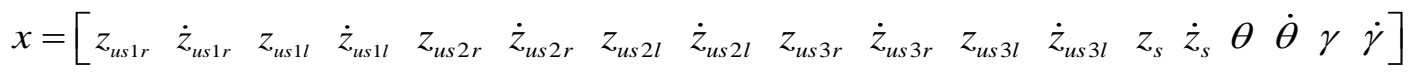

$\boldsymbol{u}$ is the input matrix;

$$
u=\left[\begin{array}{llllll}
F_{A C T 1 r} & F_{A C T 1 l} & F_{A C T 2 r} & F_{A C T 2 l} & F_{A C T 3 r} & F_{A C T 3 l}
\end{array}\right]
$$

Finally, $\boldsymbol{w}$ is the disturbance matrix:

$$
w=\left[\begin{array}{llllllllllll}
z_{01 r} & \dot{z}_{01 r} & z_{01 l} & \dot{z}_{01 l} & z_{02 r} & \dot{z}_{02 r} & z_{02 l} & \dot{z}_{02 l} & z_{03 r} & \dot{z}_{03 r} & z_{03 l} & \dot{z}_{03 l}
\end{array}\right]
$$

The last eight terms of $\mathbf{w}$ matrix are the same as the first four terms as signals but are shifted in the time axis depending on the vehicle speed.

Then the state space matrix representation becomes;

$$
[\dot{x}]_{18 x 1}=[A]_{18 x 18}[x]_{18 x 1}+[B]_{18 x 6}[u]_{6 x 1}+[G]_{18 x 12}[w]_{12 x 1}
$$

Row and column elements of A, B and G matrices are the coefficients of related terms of equations from (3.36) to (3.44). 


\subsection{Multi-Body Dynamics Modelling of the Vehicle in ADAMS/Car}

The linear full car model is enough for controller design and is useful for the analysis of vehicle's response to road disturbances and actuator inputs. However, this linear model has some deficiencies such as tire and body interaction with ground and joint elements. A more complex non-linear model is needed, as mentioned in the previous sections to overcome these shortcomings as tire behaviour in trench corners. A multi-body dynamics model created in simulation software like ADAMS is required. Because and solid body interactions can be simulated in ADAMS. Also, it has good capability in terms of tire mechanics and suspension kinematics. In this sub-section, modelling full vehicle assembly and subsystems of the vehicle in ADAMS are described. The View and Controller infrastructure is used where necessary and will be discussed later.

\subsubsection{Overview of Modelling in ADAMS}

ADAMS is a software which includes many modules such as Car, Controller, Chassis, Driveline, View etc. ADAMS / Car module is used in modelling and simulations.

In ADAMS/Car, the full vehicle assembly consists of many sub-systems as suspension, body, wheel, powertrain, brake, and steering. According to the scope of the simulation, not all of them need to be used all the time. In this work, the following subsystems are used to simulate obstacle crossing events:

- Suspension

- Wheel

- Body

- $\quad$ Steering

Unnecessary subsystems such as powertrain, driveline and brake systems are not included in the model because the vehicle's acceleration, deceleration performance or lateral dynamics are not of interest. Besides the speed of the vehicle is controlled by the PID controller in ADAMS. 
In addition, road profile and obstacle types required for simulation and some required bodyground contact models are modelled.

\subsubsection{Vehicle Subsystems in ADAMS/Car}

In ADAMS, subsystems use basic templates or data files of related parts/systems. Some subsystems are included in the system in the form of external import models. For example, suspension subsystems use suspension templates and also spring $\&$ damper curve data. Finally, all subsystems to be used are brought together in a specific systematic. Templates are the essential elements that reflect the architecture of a subsystem in accordance with the general working principle.

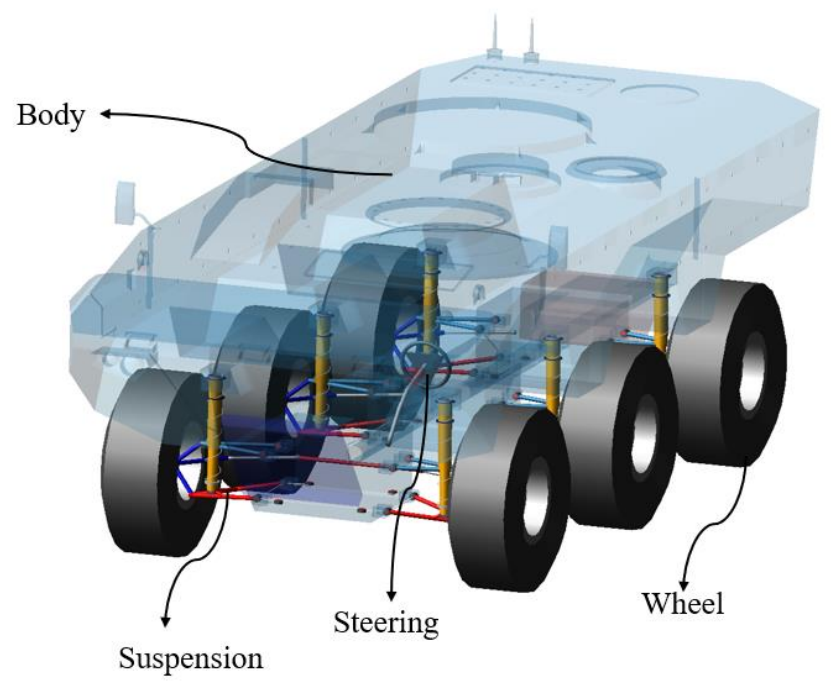

Figure 3.4. A simple full-car vehicle assembly in ADAMS

\subsubsection{Suspension Modelling}

Suspension subsystem is one of the most used in ADAMS. A suspension system is available as a template in ADAMS template builder module. According to simulation needs, the template can be modified. In Figure 3.5, a standard double-wishbone template is given. 


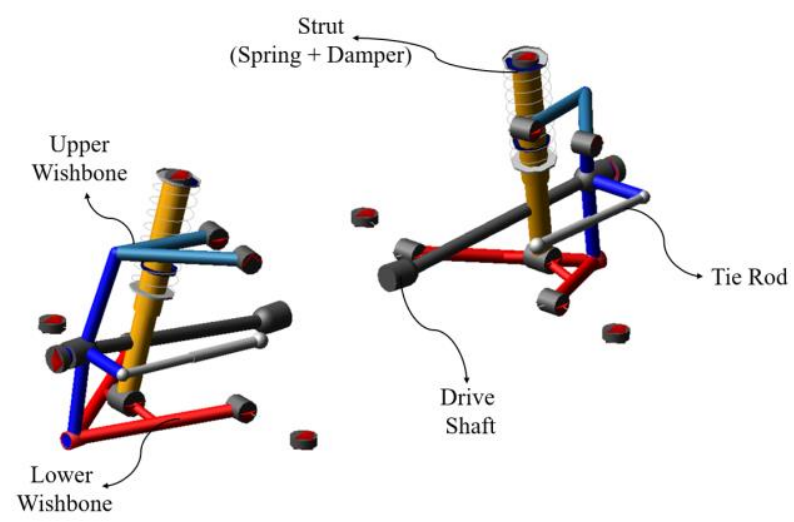

Figure 3.5. A double-wishbone suspension template in ADAMS

In this thesis model, some modifications are applied to the suspension template. (Figure 3.6) The first one is removing the drive shafts. Because powerpack and drivetrain components are not used in the vehicle model. It is needed here to define motion to the wheels. Then hardpoints are updated for the vehicle design. Then actuator forces are defined to the struts. Finally, spring and damper curves are defined in the suspension subsystem.

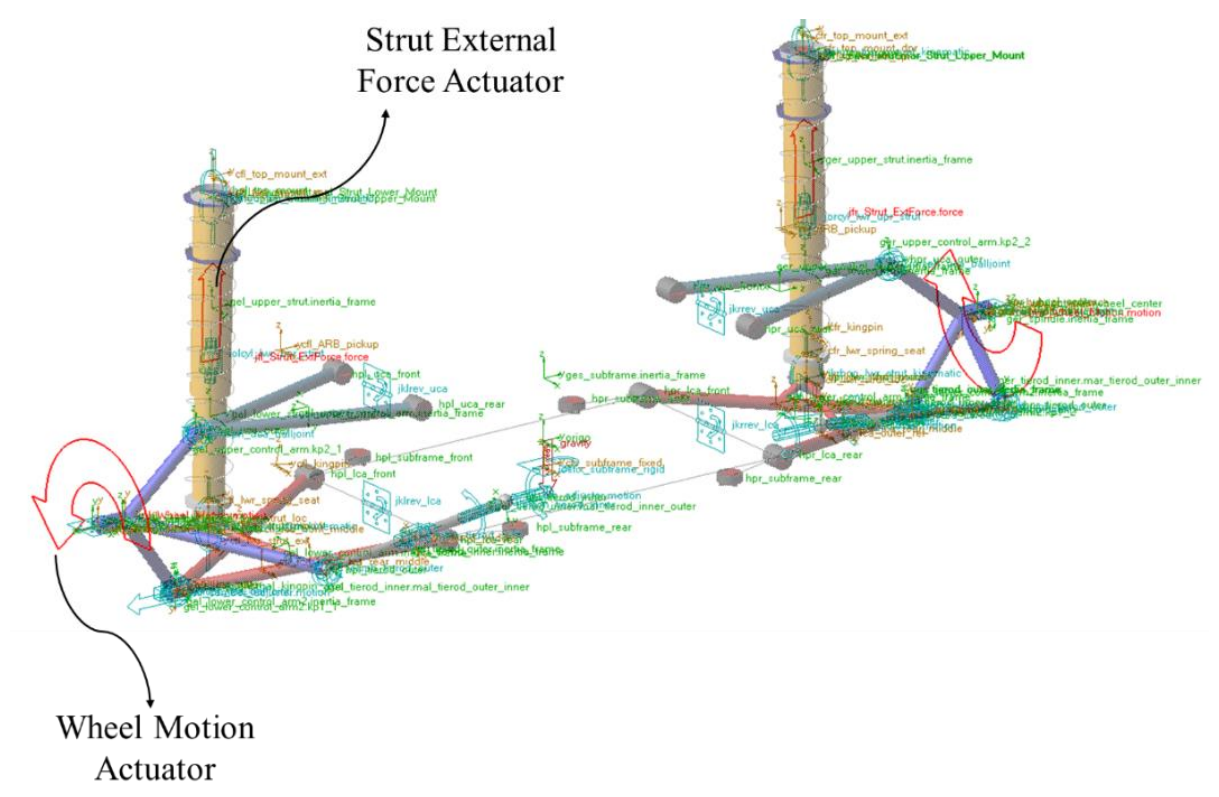

Figure 3.6. Modified suspension subsystem in ADAMS

The passive hydro strut has a non-linear behaviour according to drive modes as on-road, offroad and cross country. In this work, the spring curve modelled as a linear spring in both ADAMS and MATLAB/Simulink model (Figure 3.7) to keep the ADAMS model as close as possible to linear one and to be in a safe zone. Because linear spring has worse behaviour in mobility operations. 


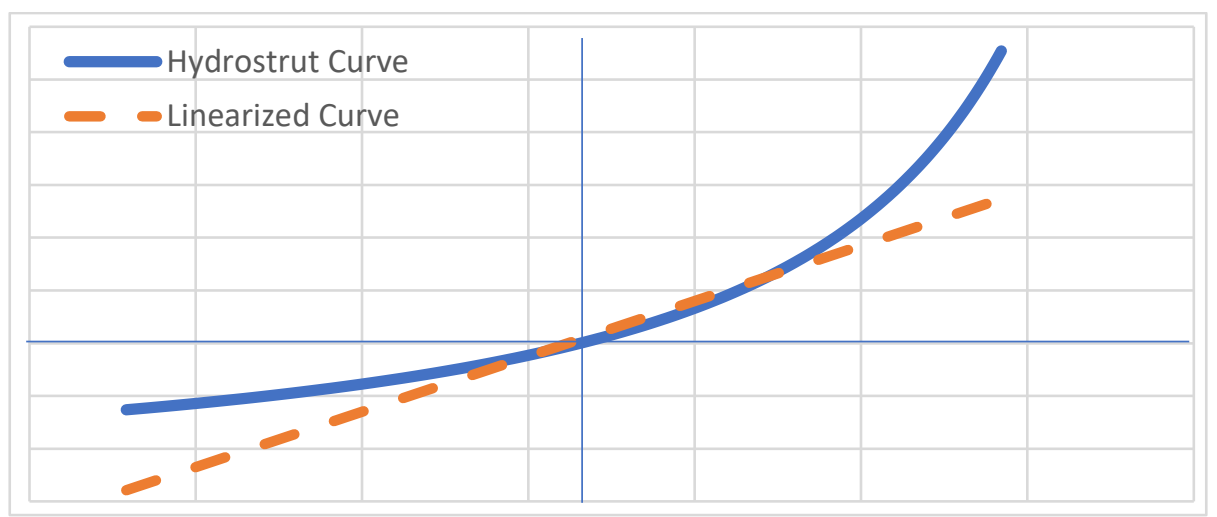

Figure 3.7. Non-linear and linearized spring curves of hydropneumatic struts

\subsubsection{Wheel / Tire Modelling}

The other critical subsystem is wheel subsystem. This is basically a tire. Wheel subsystem consists of tire, rim and any other mounting data inside. ADAMS advises using the right tire model according to the simulation application. For this reason, simulation events are divided into several groups and subgroups. The table given below shows the typical applications for different tire models. 
Table 3.1. ADAMS tire model-application use table [17]

\begin{tabular}{|c|c|c|c|c|c|c|c|c|c|}
\hline \multirow{2}{*}{$\begin{array}{c}\text { MD } \\
\text { Adams }\end{array}$} & \multirow[t]{2}{*}{ Event / Maneuver } & \multicolumn{8}{|c|}{ ADAMS/ Handling Tire } \\
\hline & & PAC2002 & PAC2002 & PAC.TIME & PAC89 & PAC94 & FIALA & 5.2.1. & UA Tire \\
\hline \multirow{9}{*}{ 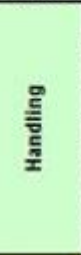 } & Stand still and start & + & + & ol+ + & $0 /+$ & olt & o/* & $\mathrm{ol}+$ & ol* \\
\hline & Parking (standing steering effort) & + & + & - & - & - & - & $\cdot$ & - \\
\hline & Standing on tilt table & + & + & + & + & + & + & + & + \\
\hline & Steady state cornering & + & + & + & ol* & + & $\circ$ & $\circ$ & o/* \\
\hline & Lane change & + & + & + & $0 / \cdot$ & * & 0 & 0 & ol* \\
\hline & ABS braking distance & $\cdot$ & + & o/* & o/t & $0 / \cdot$ & 0 & 0 & ol* \\
\hline & Braking/power-off in a turn & + & + & + & 0 & 0 & $\circ$ & 0 & 0 \\
\hline & Vehicle Roll-over & + & + & $\circ$ & o & 0 & $\circ$ & $\circ$ & $\circ$ \\
\hline & On-line scaling tire properties & + & + & $=$ & $\overline{-}$ & $=$ & $\cdot$ & $=$ & - \\
\hline \multirow{5}{*}{$\frac{}{x}$} & Cornering on uneven roads ${ }^{1}$ & o/t+ & + & 0 & o & 0 & 0 & 0 & 0 \\
\hline & Braking on uneven roads ${ }^{1}$ & o/t+ & + & 0 & 0 & $\circ$ & $\circ$ & 0 & $\circ$ \\
\hline & Crossing cleats / obstacles &. & + & . & + & 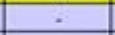 & + & - & - \\
\hline & Driving over uneven road & - & + & - & - & 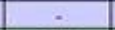 & . & 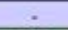 & - \\
\hline & 4 post rig (A/Ride) & - & + & o/t & o/* & o/t & o/* & o/* & o/* \\
\hline \multirow{6}{*}{ 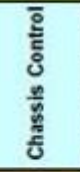 } & ABS braking control & o/t+ & + & 0 & 0 & 0 & 0 & 0 & 0 \\
\hline & Shimmy ${ }^{2}$ & $\mathrm{o} /+$ & + & 0 & 0 & 0 & $\circ$ & $\circ$ & o \\
\hline & Steering system vibrations & o/* & + & $\circ$ & $\circ$ & $\circ$ & $\circ$ & $\circ$ & $\circ$ \\
\hline & Real-time & + & $\therefore$ & 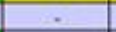 & $\overline{-}$ & - & $\cdot$ & $\overline{-}$ & 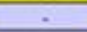 \\
\hline & Chassis control systems $>8 \mathrm{~Hz}$ & olt & + & - & - & 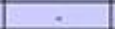 & . & $\overline{-}$ &. \\
\hline & Chassis control with ride & - & + & 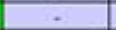 & $\overline{-}$ & - & $\overline{-}$ & $\overline{-}$ & - \\
\hline \multirow{4}{*}{ 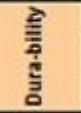 } & Driving over curb & - & olt & 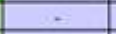 & + & + & $\cdot$ & 0 & 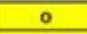 \\
\hline & Driving over curb with rim impact & $\circ$ & o/t & - & $\cdot$ & - & - & o & 0 \\
\hline & Passing pothole & $\cdot$ & ol* & - & - & - & $\cdot$ & 0 & 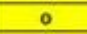 \\
\hline & Load cases & - & ol* & - & - & - & + & $\circ$ & $\circ$ \\
\hline \multirow{2}{*}{$\begin{array}{l} \\
\\
\end{array}$} & Design of Experiments & - & + & + & - & - & - & 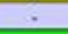 & - \\
\hline & SMP parallel & + & + & + & + & + & + & + & + \\
\hline \multirow{2}{*}{$\begin{array}{c}\text { MD } \\
\text { Adams }\end{array}$} & \multirow[t]{2}{*}{ Event / Maneuver } & \multicolumn{3}{|c|}{ Specific Models } & \multicolumn{3}{|c|}{ Aircraft } & & \\
\hline & & PAC-AAC & FTire & SoftSoil & Basic & Enhanced & TRR64 & & \\
\hline \multirow{9}{*}{$\begin{array}{l}\text { 总 } \\
\text { 言 } \\
\text { 至 } \\
\text { I. }\end{array}$} & Stand still and start & o/* & + & - & olt+ & ol+ & o/* & & \\
\hline & Parking (standing steering effort) & . & + & $\cdot$ & $\cdot$ & - & - & & \\
\hline & Standing on tilt table & + & + & - & + & * & * & & \\
\hline & Steady state cornering & + & olt & + & $\circ$ & + & o/* & & \\
\hline & Lane change & $\cdot$ & o/* & + & 0 & - & o/* & & \\
\hline & ABS braking distance & olt+ & + & olt & 0 & olt & o/* & & \\
\hline & Braking/power-off in a turn & + & olt & + & - & $\circ$ & $\circ$ & & \\
\hline & Vehicle Roll-over & $\circ$ & + & ol* & $\circ$ & - & - & & \\
\hline & On-line scaling tire properties & - & 0 & 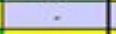 & + & $\cdot$ & $\cdot$ & & \\
\hline \multirow{5}{*}{$\frac{\stackrel{0}{0}}{\alpha}$} & Cornering on uneven roads ${ }^{1}$ & 0 & + & 0 & 0 & 0 & $\circ$ & & \\
\hline & Braking on uneven roads ${ }^{1}$ & $\circ$ & + & 0 & 0 & 0 & 0 & & \\
\hline & Crossing cleats / obstacles & - & + & 0 & - & 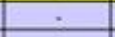 & - & & \\
\hline & Driving over uneven road & - & + & $\circ$ & 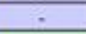 & . & 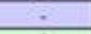 & & \\
\hline & 4 post rig (A/Ride) & olt+ & + & - & olt & olt+ & $0 /+$ & & \\
\hline & ABS braking control & $\circ$ & + & + & $\circ$ & $\circ$ & $\circ$ & & \\
\hline E & Shimmy ${ }^{2}$ & 0 & + & $=$ & $\circ$ & $\circ$ & $\circ$ & & \\
\hline o & Steering system vibrations & $\circ$ & + & + & $\circ$ & $\circ$ & 0 & & \\
\hline$\frac{n}{n}$ & Real-time & - & 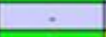 & . & - & . & . & & \\
\hline है & Chassis control systems $>8 \mathrm{~Hz}$ & - & + & $\circ$ & - & - & - & & \\
\hline & Chassis control with ride & - & + & - & - & $\cdot$ & - & & \\
\hline 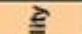 & Driving over curb & - & + & $\circ$ & - & $\circ$ & $\circ$ & & \\
\hline 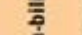 & Driving over curb with rim impact & - & + & 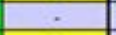 & + & $\circ$ & $\circ$ & & \\
\hline$\stackrel{5}{3}$ & Passing pothole & - & + & $\circ$ & + & $\circ$ & $\circ$ & & \\
\hline ○ & Load cases & - & + & 0 & - & 0 & 0 & & \\
\hline$\ddot{q}$ & Design of Experiments & - & + & - & - & - & - & & \\
\hline $\bar{z}$ & SMP parallel & + & + & + & + & + & + & & \\
\hline- & Not possible/Not realistic & ; & & wavelengtt & road ob & stacles $>$ tire & diameter & & \\
\hline$\circ$ & Possible & ${ }^{2}$ & & wheel yaw & ng vibrat & ion due to & & & \\
\hline olt & Better & & & supension & lexibility & and tire dyn & amic resp & & \\
\hline+ & Best to use & tire model: & s assumed & $\begin{array}{l}\text { to be used } \\
\text { PAC2002 w }\end{array}$ & $\begin{array}{l}\text { n transie } \\
\text { th belt d }\end{array}$ & $\begin{array}{l}\text { nt and comb } \\
\text { namics }\end{array}$ & ined slip & & \\
\hline
\end{tabular}

Regarding Table 3.1, although the second model seems to be recommended to be used, in this thesis, the PAC2002 tire model is used. The reason below can be explained as follows; the FTire is very complicated, expensive to use and required more computational time. This model requires lots of detailed test data. So, in this work PAC2002 tire model is defined to the wheel subsystems.

The PAC2002 tire model based on Pacejka's magic formula and contains the latest developments given in [34]. Normally PAC2002 model is suitable for handling manoeuvres. 
However extended PAC2002 model is suitable for cars, trucks and aircraft. This model also contains the capability of turn-slip torque and parking torque.

The cleats or obstacles defined in Table 3.1 are small wavelengths. The obstacle dimensions are higher than tires for this thesis work, and these obstacle profiles are even. So, at this point, the vertical characteristics of the tires stand out. Also, lateral dynamics or handling are not focused. That's why PAC2002 tire model is good enough for obstacle crossing events.

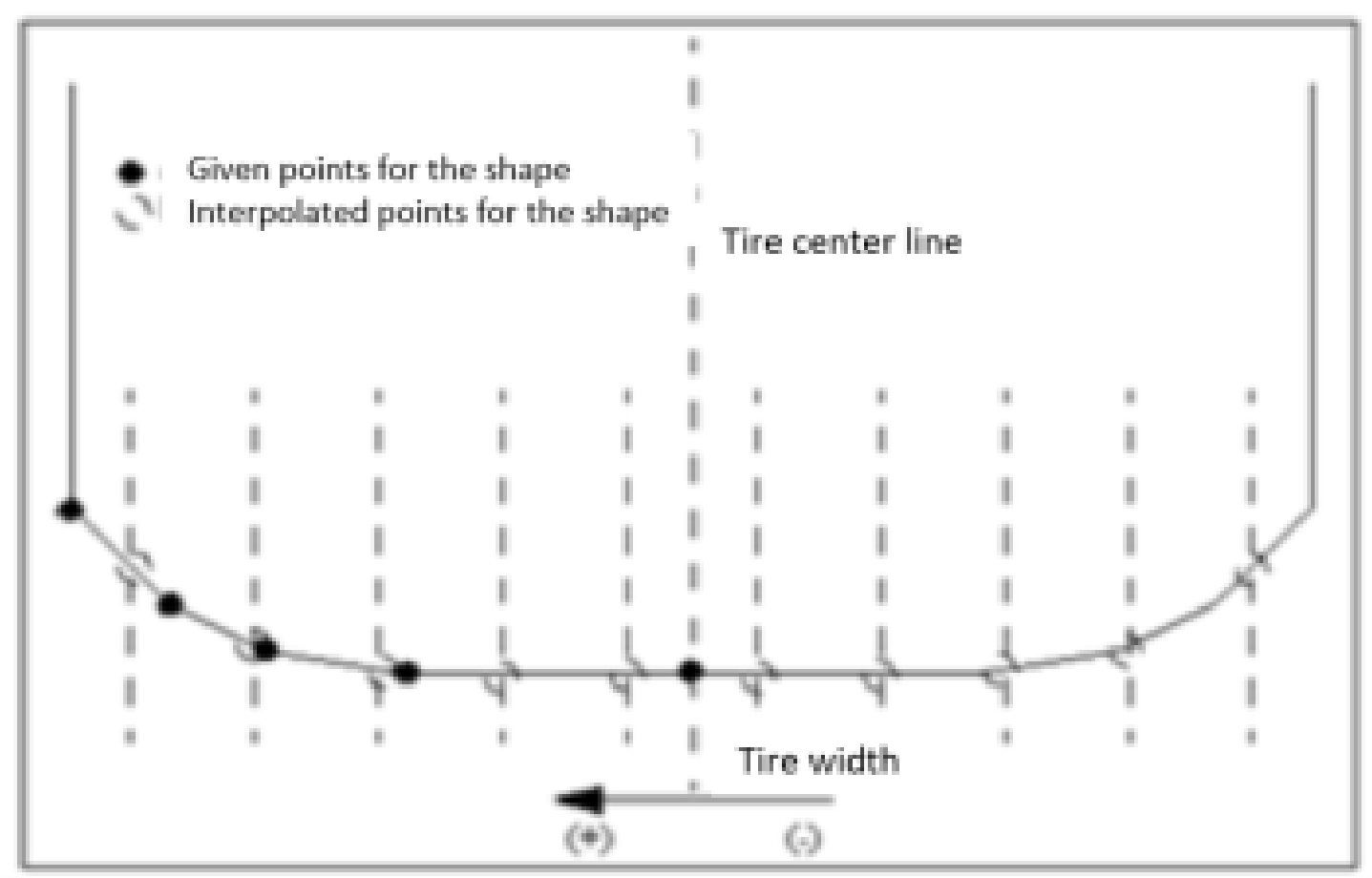

Figure 3.8. 3D equivalent volume contact model [17]

The 3D equivalent volume contact tire model is used with 3D shell road models. This is a 3D tire-road contact model. An intersection volume between road and tire is computed then this volume is converted into equivalent tire information. These are tire normal load, penetration, contact point and effective friction. The road model is discrete triangular meshes [17].

\subsubsection{Body Modelling}

The other subsystem is the body. The body is the base mass and inertia element in the full vehicle model, and this is imported from the CAD model of the vehicle. The only 
modification in the body subsystem is adding front and rear bumper and their contact models with the ground.

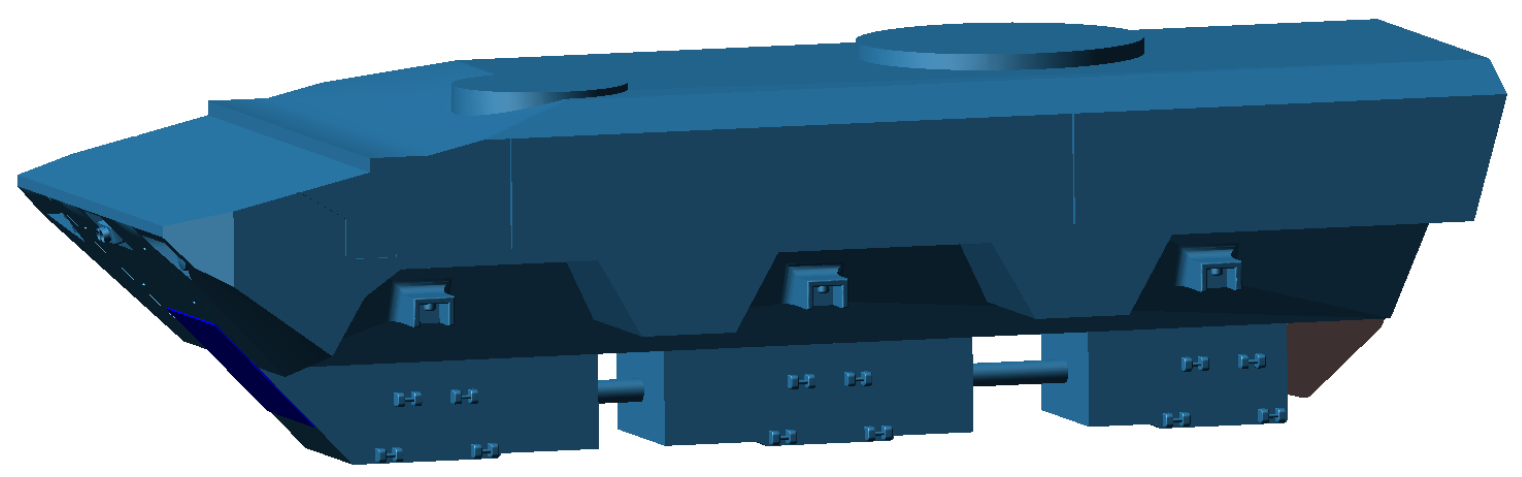

Figure 3.9. The body subsystem in ADAMS with front and rear bumpers

The last primary subsystem for the vehicle model is the steering subsystem. The steering is not required for the obstacle crossing events, but it is required to assemble the full vehicle. So, the steering subsystem is included and locked during simulations.

\subsubsection{Creating the Full Vehicle and Simulation Environment in ADAMS/Car}

After modifying the subsystems, they are gathered in a full vehicle assembly in ADAMS/Car Standard interface. In this interface suspension, various full vehicle simulations and specific events can be run. Two main files must be defined to establish a specific event. These are road and event data. 


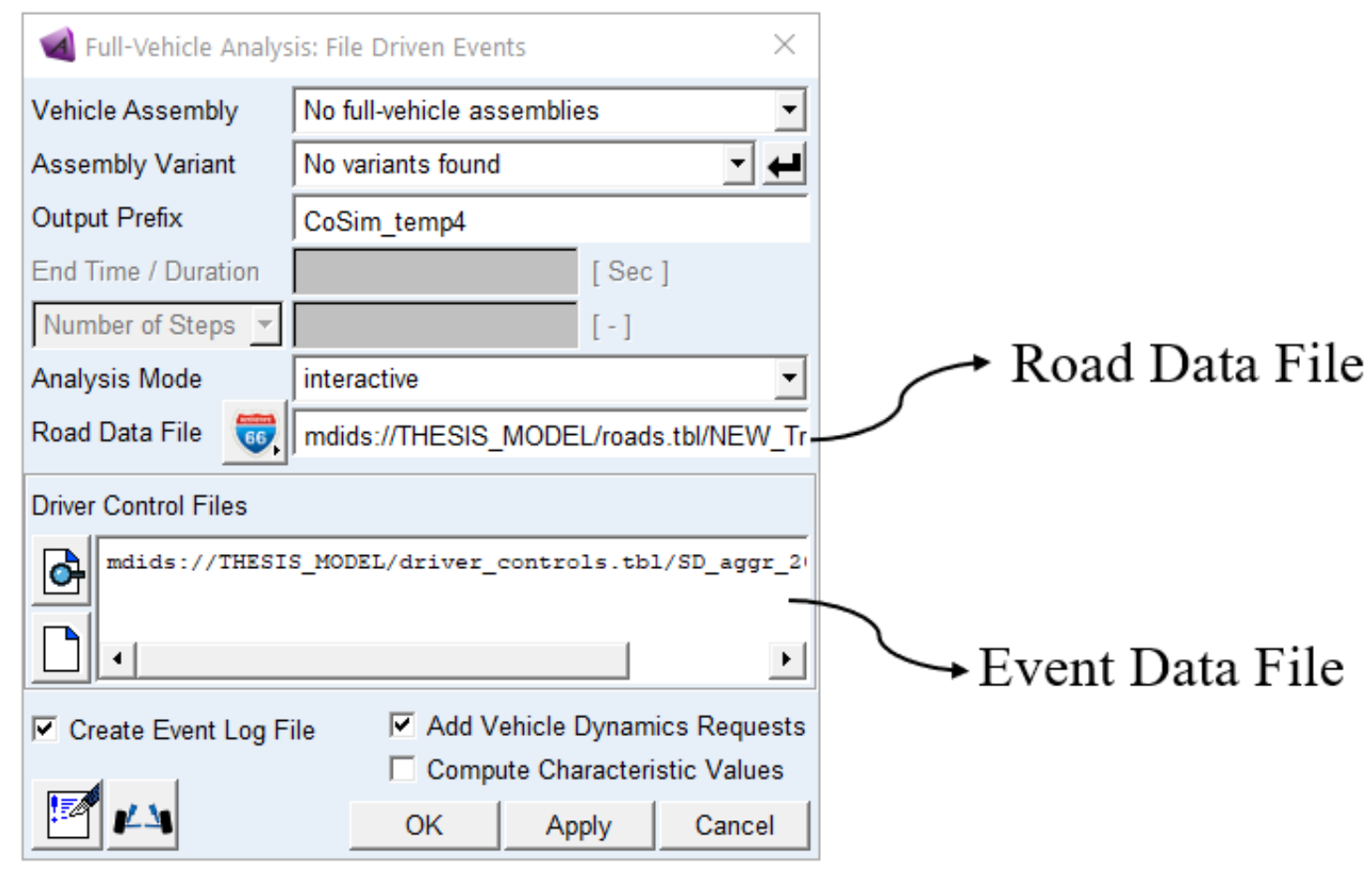

Figure 3.10. ADAMS specific event running (File Driven Events)

The road modelling and data file will be explained in the next part. The event data file contains initial settings and vehicle control information. For example, this file controls the vehicle's path and speed in the desired profiles. If it is needed, gear and clutch usage can also be included. Path and steering controls can be open or closed loop. If ADAMS is to control the vehicle, PID controllers can be tuned in this event builder. (Figure 3.11) The controller can also be used from an external source by co-simulation. 


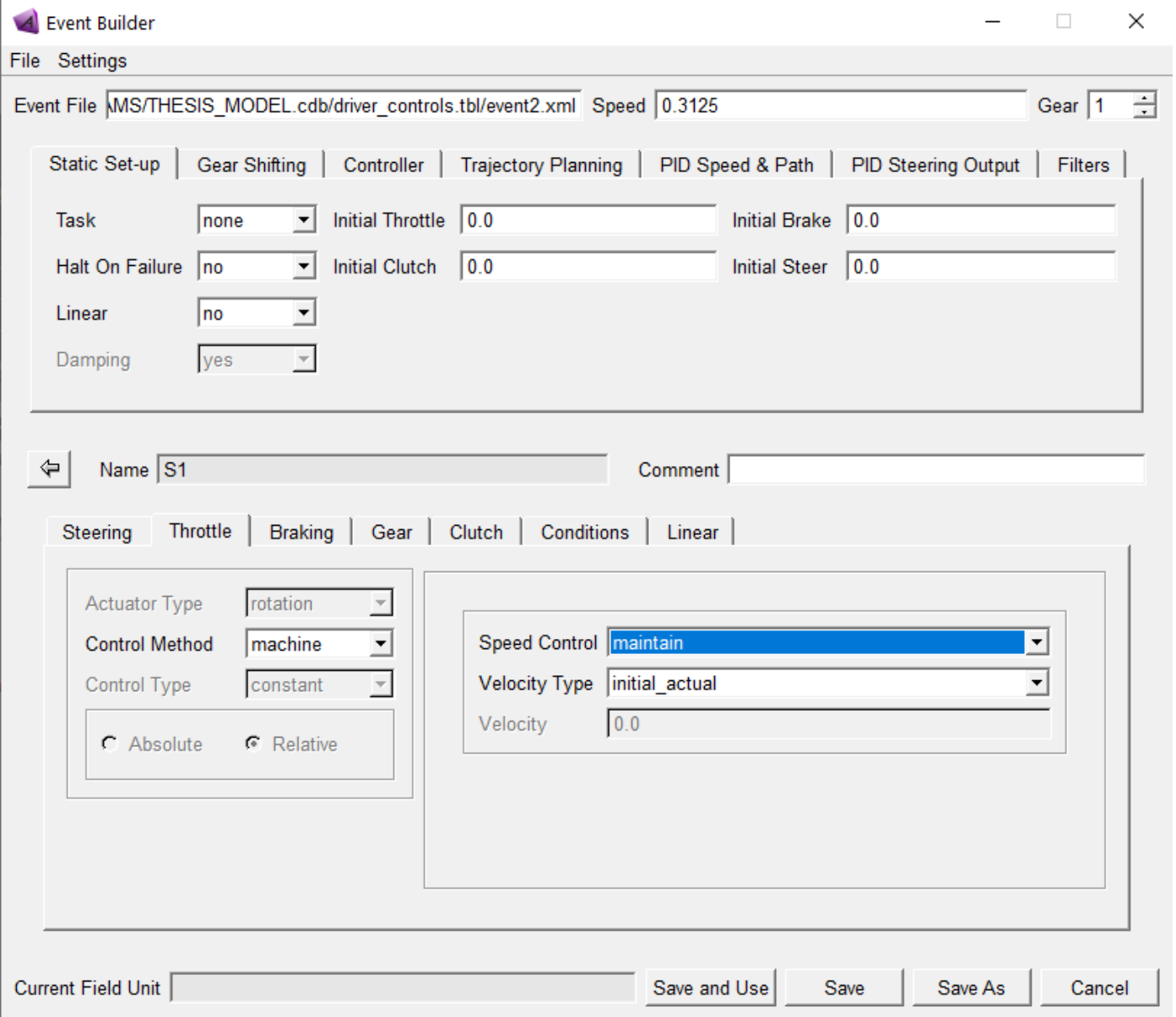

Figure 3.11. ADAMS Event Builder

\subsection{Road and Obstacle Modelling in ADAMS}

The default road data files in ADAMS are based on shells. To create a road model road builder interface of ADAMS can be used. This module creates 2D surfaces. Using this surface model makes tire-ground interaction to be $2 \mathrm{D}$ point contact. In this contact model, only the bottom contact point of the tire is recognized by road. All surfaces of the tire must be able to contact with the ground for extensive obstacle crossing simulations.

So, the tire contact model must be converted to a $3 \mathrm{D}$ equivalent contact model. This requires using the road models as “.rdf” road data. (Figure 3.13) This road data contains mesh elements of the road. In the road data file, the nodes and mesh element coordinates are defined. (Figure 3.12) It is a proper road and obstacle profile, so mesh and node numbers can be as little as possible. 


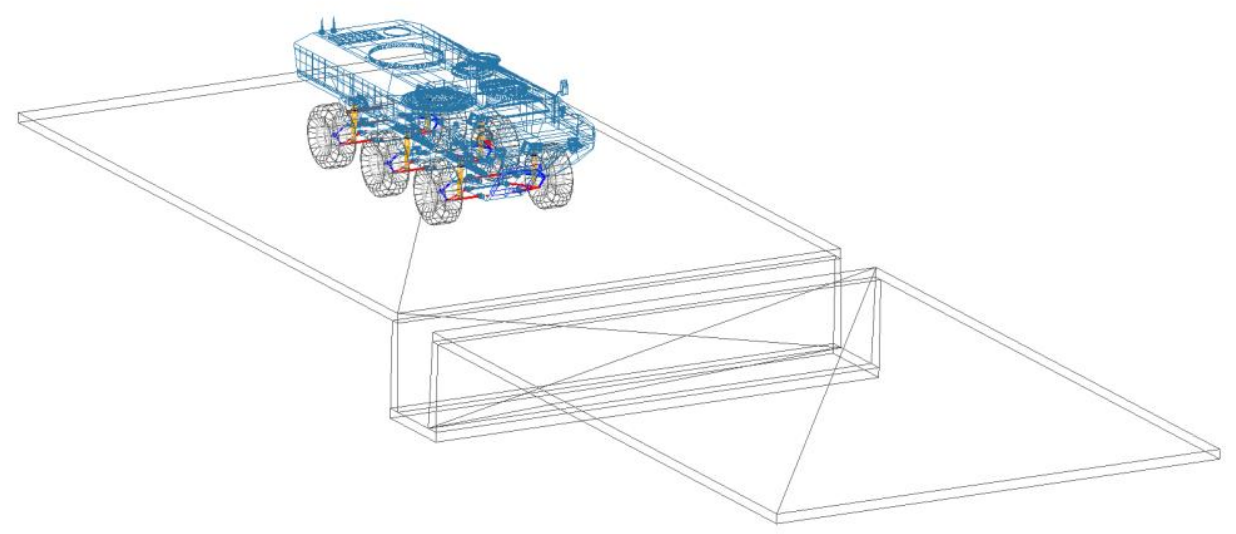

Figure 3.12. ADAMS “RDF” road model

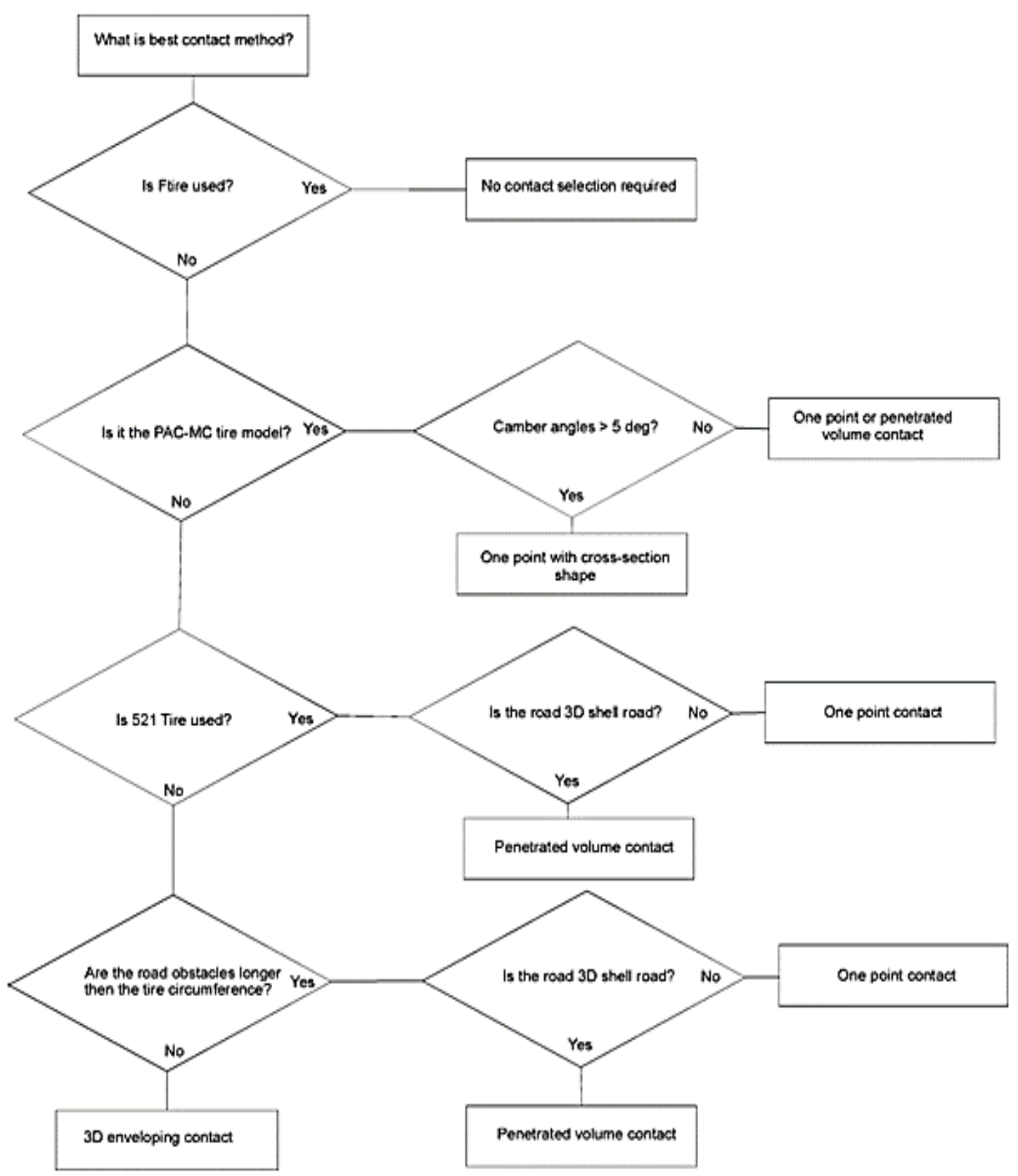

Figure 3.13. Road-tire contact model selection flowchart [17] 
Due to the natural behaviour of 6x6 vehicle during trench crossing, the vehicle must hit its bumpers to the ground. In ADAMS, the road can only recognize the tires. To model interaction between body and ground, a contact model is required. In previous parts, the front and rear bumpers in body subsystem are defined. Here, a solid part attached to the ground is needed. So, in ADAMS/View module, solid parts with the same shape with road profile are added to the ground. In this thesis work, there are some road profiles that tire ground clearance is cut off entirely, and the tire hangs in the air. In this case, the 3D Volume Contact tire model is still valid. Also, other tire models can be used for a hanging situation but as it is explained in section 3.3.2, the focus regarding tyre in this thesis is vertical stiffness. ADAMS gives tire forces and deflections in its radial direction for tire-road interaction. When the contact is gone, the normal tire force is zero.

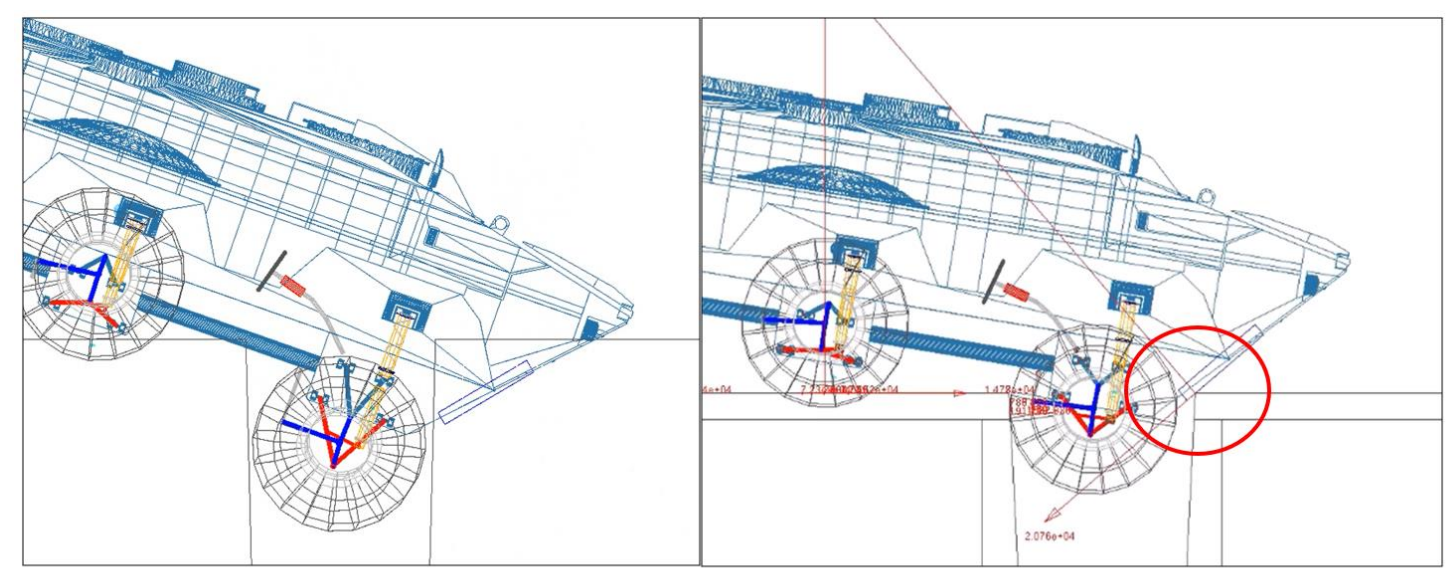

(A)

(B)

Figure 3.14. Vehicle body - ground interaction without contact model (A) and with contact model (B)

\subsection{Co-Simulation Environment Overview}

In this part, a basic overview of the co-simulation environment is given. More detailed sections will be covered in the next section. To create the co-simulation environment, first, the inputs and outputs of the co-simulation model are to be determined. This inputs and outputs can be pre-defined system variables or custom defined state variables. In our model, strut external forces, displacements and wheel angular velocity actuators are defined as the state variables. (Figure 3.15) 


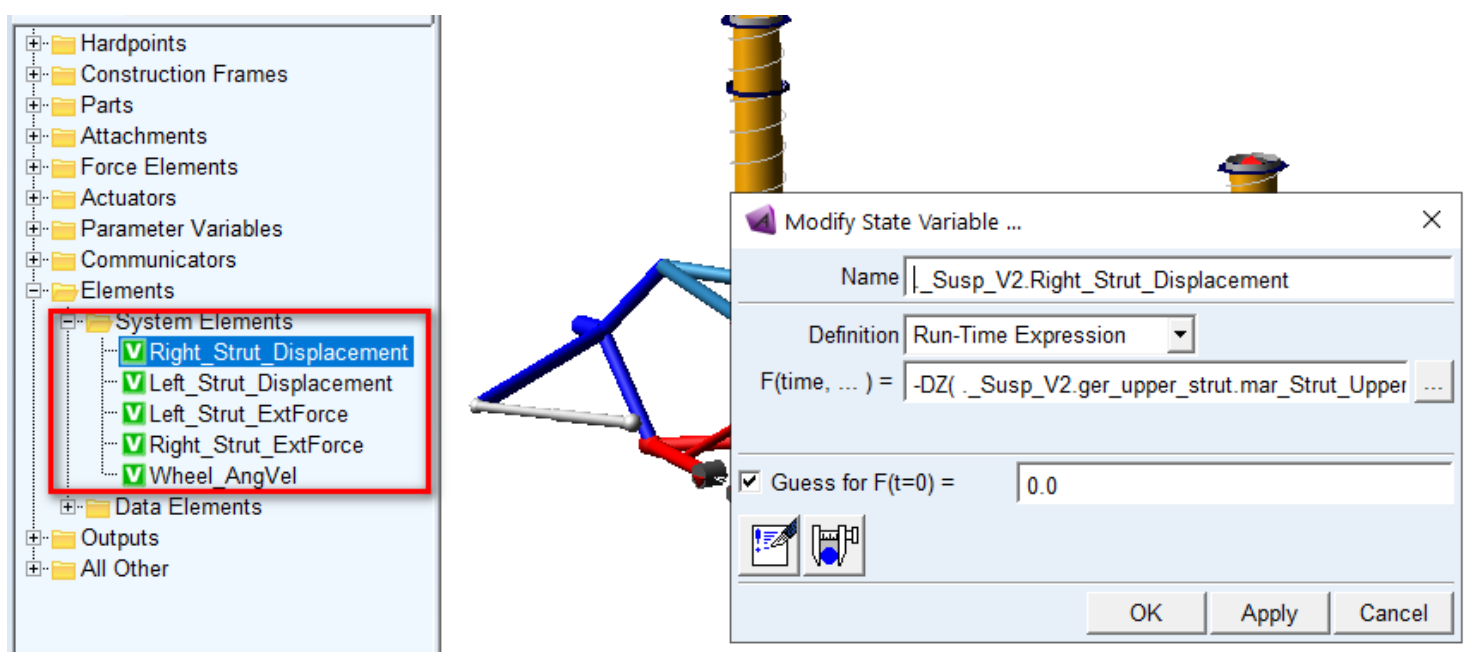

Figure 3.15. State-Variable definition in ADAMS/Template Builder

When the required inputs and outputs are defined, the model plant needs to be exported to Matlab. ADAMS/Controls module is used to export the plant. The input and output variables used for co-simulation are given below.

Table 3.2. Input variables of plant export for co-simulation

\begin{tabular}{|l|l|}
\hline \multicolumn{1}{|c|}{ Input Variables } & \multicolumn{1}{c|}{ Explanation } \\
\hline .Vehicle1.Front_Suspension_V2.Right_Strut_ExtForce & $\begin{array}{l}\text { Front Suspension Right } \\
\text { Suspension Actuator Force }\end{array}$ \\
\hline .Vehicle1.Front_Suspension_V2.Left_Strut_ExtForce & $\begin{array}{l}\text { Front Suspension Left } \\
\text { Suspension Actuator Force }\end{array}$ \\
\hline .Vehicle1.Middle_Suspension_V2.Right_Strut_ExtForce & $\begin{array}{l}\text { Middle Suspension Right } \\
\text { Suspension Actuator Force }\end{array}$ \\
\hline .Vehicle1.Middle_Suspension_V2.Left_Strut_ExtForce & $\begin{array}{l}\text { Middle Suspension Left } \\
\text { Suspension Actuator Force }\end{array}$ \\
\hline .Vehicle1.Rear_Suspension_V2.Right_Strut_ExtForce & $\begin{array}{l}\text { Rear Suspension Right } \\
\text { Suspension Actuator Force }\end{array}$ \\
\hline .Vehicle1.Rear_Suspension_V2.Left_Strut_ExtForce & $\begin{array}{l}\text { Rear Suspension Left } \\
\text { Suspension Actuator Force }\end{array}$ \\
\hline .Vehicle1.Front_Suspension_V2.Wheel_AngVel & $\begin{array}{l}\text { Front Wheels Angular } \\
\text { Velocity }\end{array}$ \\
\hline .Vehicle1.Middle_Suspension_V2.Wheel_AngVel & $\begin{array}{l}\text { Middle Wheels Angular } \\
\text { Velocity }\end{array}$ \\
\hline .Vehicle1.Rear_Suspension_V2.Wheel_AngVel & $\begin{array}{l}\text { Rear Wheels Angular } \\
\text { Velocity }\end{array}$ \\
\hline .Vehicle1.testrig.desired_velocity & Desired Vehicle Velocity \\
\hline
\end{tabular}


Table 3.3. Output variables of plant export for co-simulation

\begin{tabular}{|l|l|}
\hline \multicolumn{1}{|c|}{ Output Variables } & \multicolumn{1}{c|}{ Explanation } \\
\hline .Vehicle1.testrig.body_acce_z & Body Vertical Acceleration \\
\hline .Vehicle1.testrig.body_roll_angle & Body Roll Angle \\
\hline .Vehicle1.testrig.body_pitch_angle & Body Pitch Angle \\
\hline .Vehicle1.testrig.Wheel_1R_PosZ & $\begin{array}{l}\text { Front Right Wheel Center Vertical } \\
\text { Position }\end{array}$ \\
\hline .Vehicle1.testrig.Wheel_1L_PosZ & $\begin{array}{l}\text { Front Left Wheel Center Vertical } \\
\text { Position }\end{array}$ \\
\hline .Vehicle1.testrig.Wheel_2R_PosZ & $\begin{array}{l}\text { Middle Right Wheel Center Vertical } \\
\text { Position }\end{array}$ \\
\hline .Vehicle1.testrig.Wheel_2L_PosZ & $\begin{array}{l}\text { Middle Left Wheel Center Vertical } \\
\text { Position }\end{array}$ \\
\hline .Vehicle1.testrig.Wheel_3R_PosZ & $\begin{array}{l}\text { Rear Right Wheel Center Vertical } \\
\text { Position }\end{array}$ \\
\hline .Vehicle1.testrig.Wheel_3L_PosZ & $\begin{array}{l}\text { Rear Left Wheel Center Vertical } \\
\text { Position }\end{array}$ \\
\hline .Vehicle1.testrig.NoseLidar_to_TrenchStart_X & $\begin{array}{l}\text { Longitudinal Distance from Nose } \\
\text { Lidar to Trench Start }\end{array}$ \\
\hline .Vehicle1.testrig.NoseLidar_to_TrenchStart_Z & $\begin{array}{l}\text { Vertical Distance from Nose Lidar to } \\
\text { Trench Start }\end{array}$ \\
\hline .Vehicle1.testrig.NoseLidar_to_TrenchFinish_X & $\begin{array}{l}\text { Longitudinal Distance from Nose } \\
\text { Lidar to Trench Finish }\end{array}$ \\
\hline .Vehicle1.testrig.NoseLidar_to_TrenchFinish_Z & $\begin{array}{l}\text { Vertical Distance from Nose Lidar to } \\
\text { Trench Finish } \\
\text { Body Vertical Displacement }\end{array}$ \\
\hline Vehicle1.testrig.body_disp_z & Body Longitudinal Velocity \\
\hline Vehicle1.testrig.body_velocity_x &
\end{tabular}

After exporting the plant, ADAMS generates a Matlab script. A Simulink system model can be created by using this script. The generated Simulink model is given in Figure 3.16 

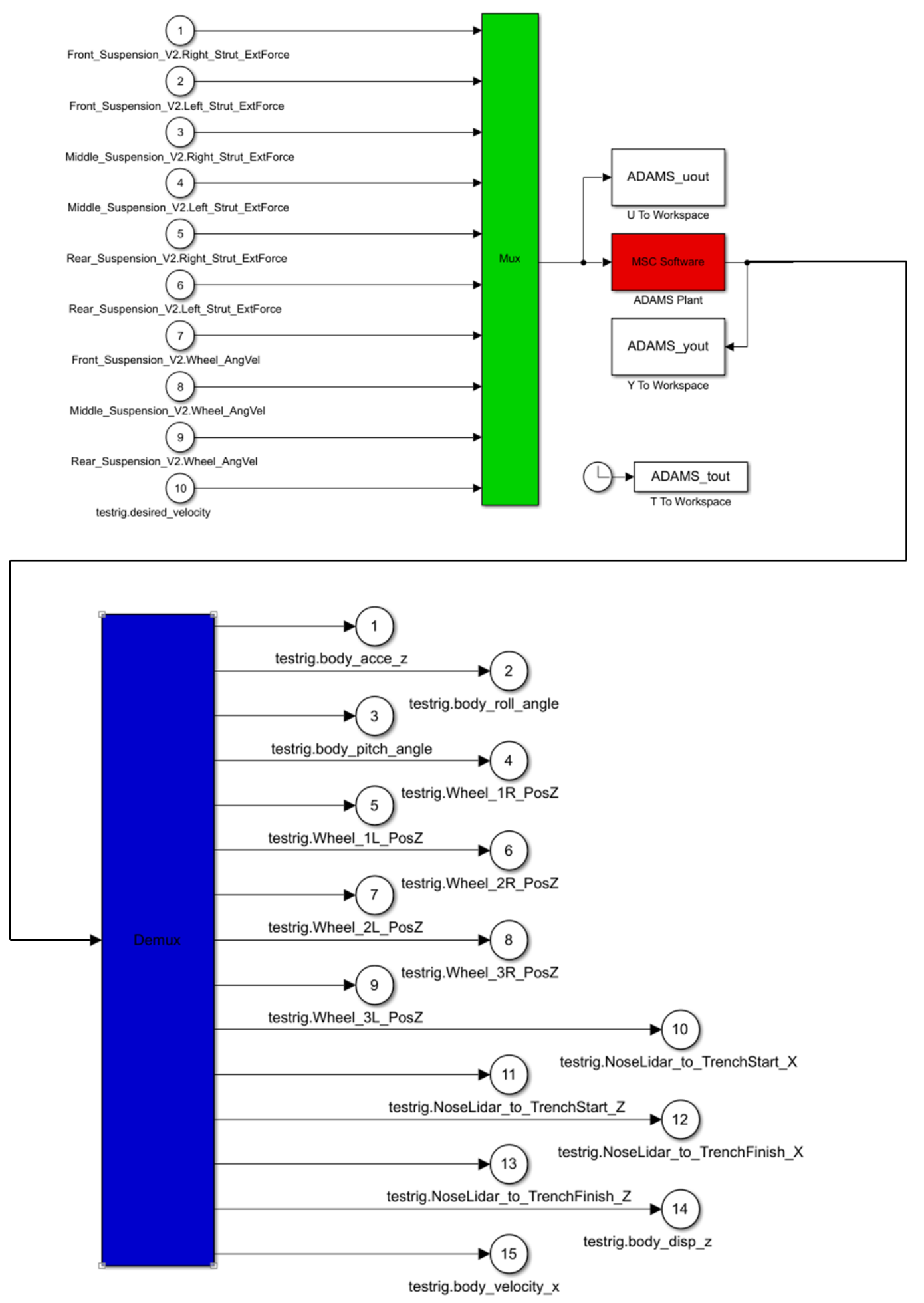

Figure 3.16. Exported ADAMS plant model in Simulink 


\subsection{Preview Data Modelling in ADAMS}

The preview information was assumed to be ready for use, as stated in the previous sections. This section shows how road data, that is, the position of the trench's start and end points relative to the vehicle, is modelled for co-simulation. (Figure 3.17)

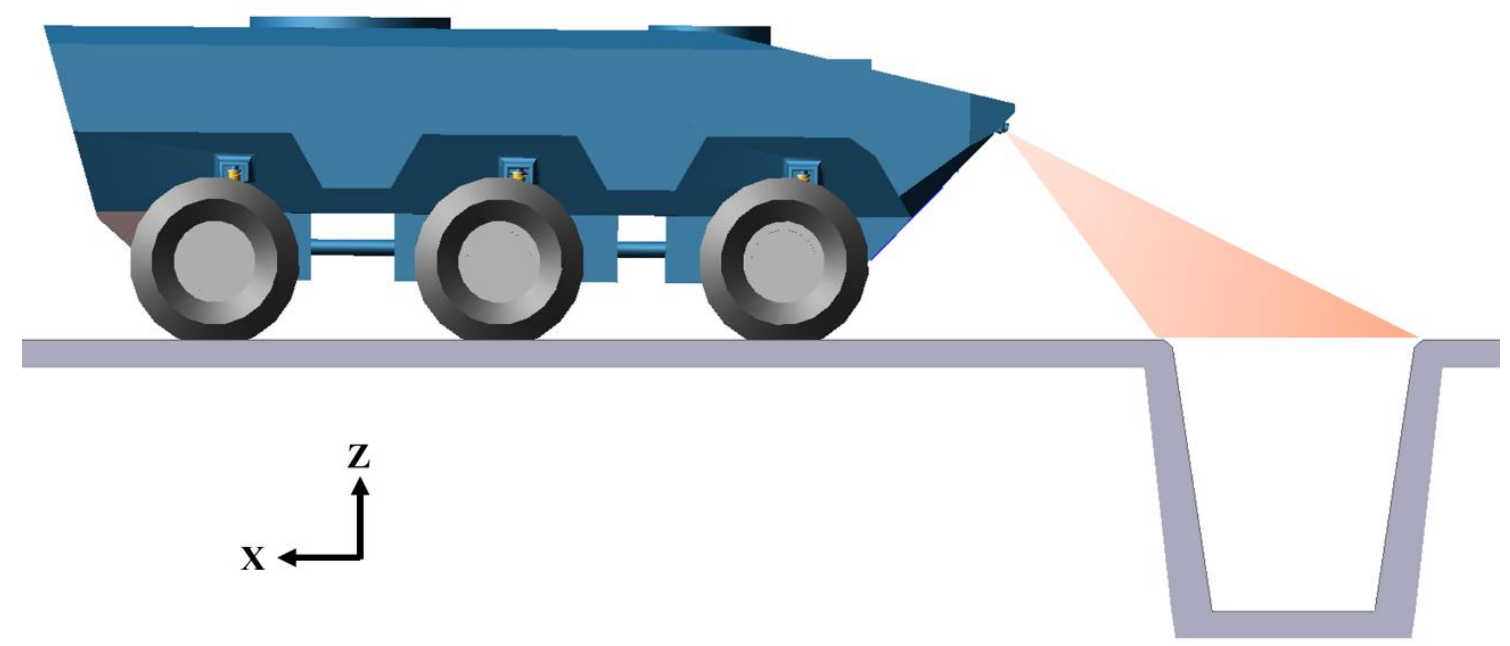

Figure 3.17. Preview data modelling in ADAMS

Markers that give vehicle-trench relationship and positions relative to each other are modelled in body and test-rig template files. Figure 3.18 shows the lidar marker on the front of the body (nose). In Figure 3.19, markers showing the beginning and end of the trench are modelled on the test-rig.

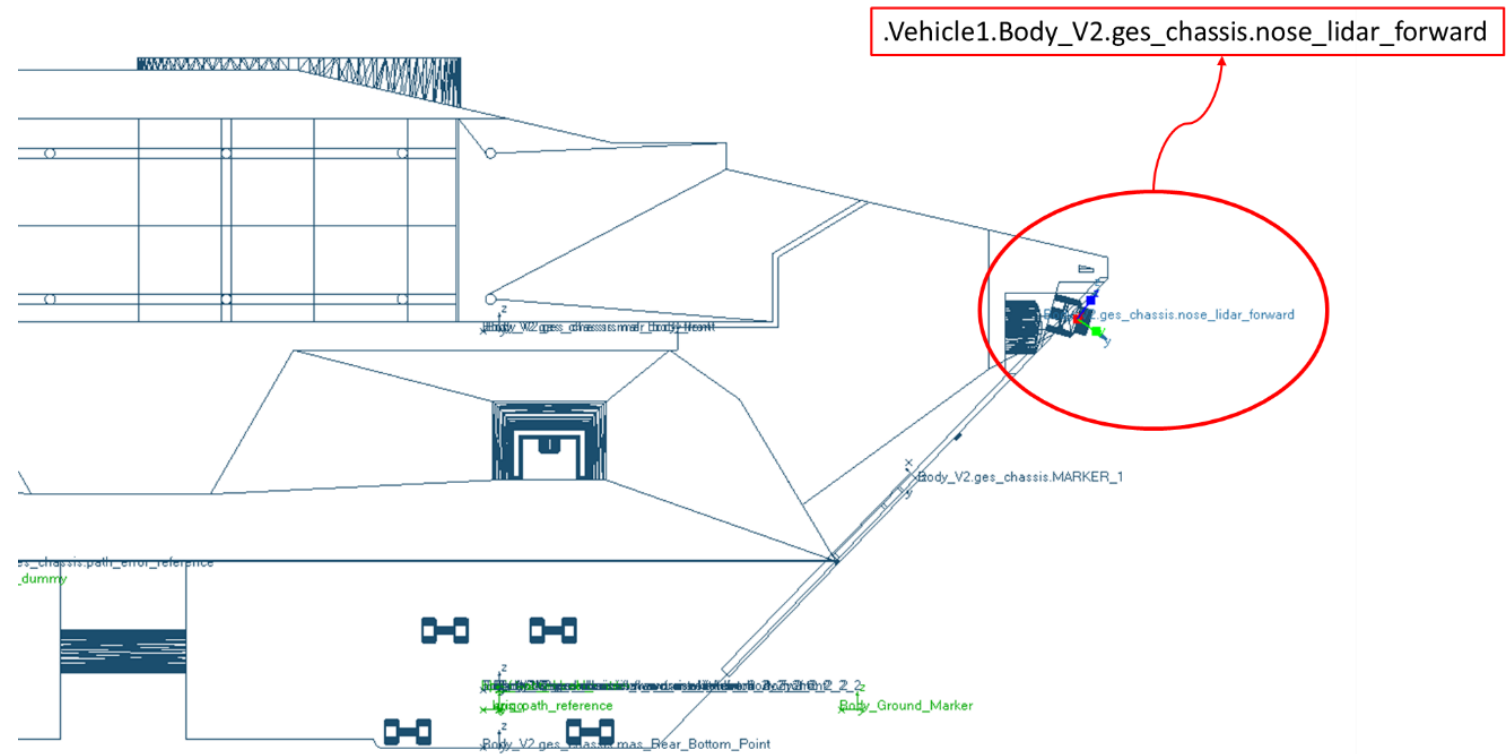

Figure 3.18. Nose Lidar marker modelled on the body 


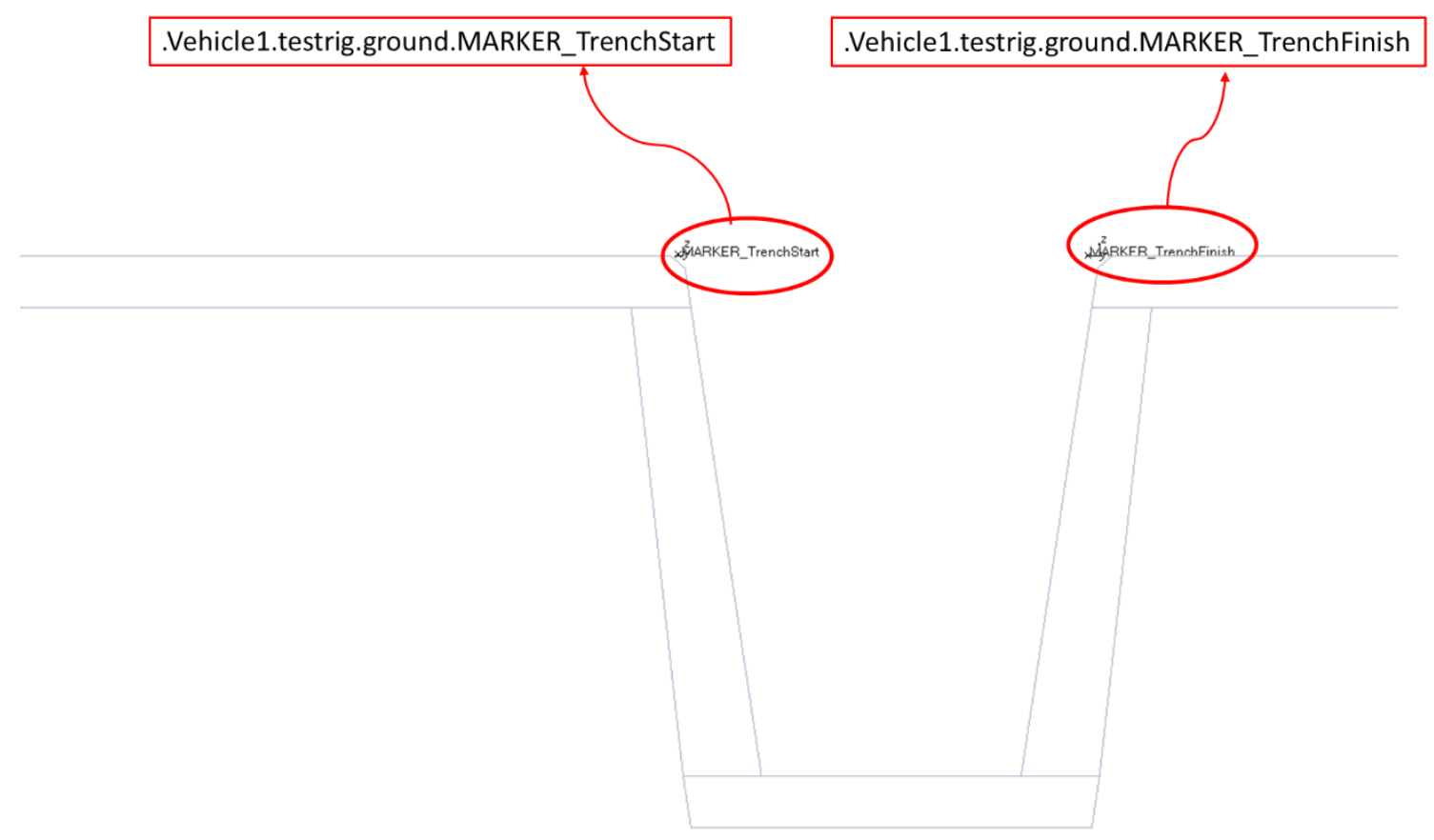

Figure 3.19. Test-rig trench start and finish markers

In the trench cross algorithm, the vehicle-specific lidar-to-wheel tangent lines and angles (Figure 3.20) to be used for transition between cases have been predetermined. The angle values shown are used to understand the position of the wheels relative to the trench. Details on the use and calculations of the specified angles are described in the next sections.

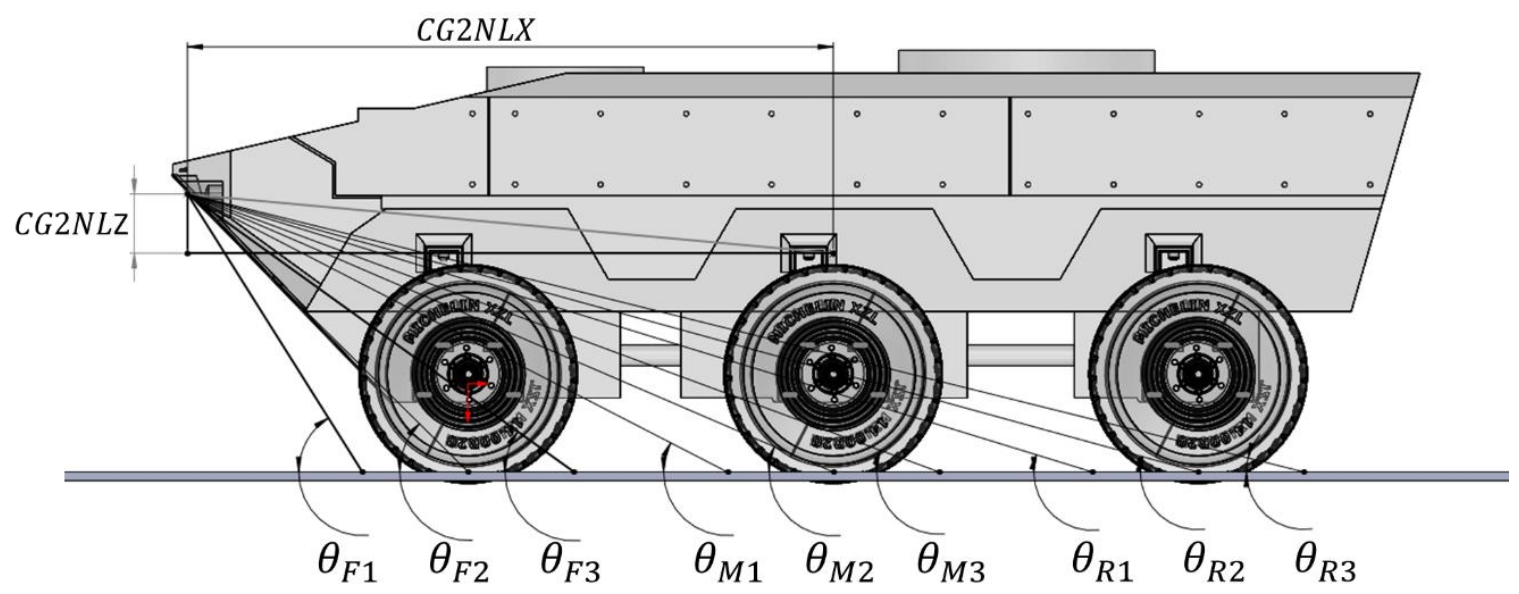

Figure 3.20. Tangent lines from nose lidar to each wheel

ADAMS plant feeds the distances from the lidar in the nose to the starting and ending points of the trench as an output to the co-simulation environment in $\mathrm{X}$ and $\mathrm{Z}$ axes. (Figure 3.17) 


\section{CONTROLLER DESIGN AND OBSTACLE CROSSING ALGORITHM}

\subsection{Introduction to Controller Design and Algorithm}

In this section, the controllers design is discussed. The controllers are created with the full-car ride model and tuned accordingly. Then it tested with the non-linear ADAMS plant. There are three different controllers used in this thesis. They are:

- MPC controller without preview

- MPC controller with preview

- LQR (Linear Quadratic Regulator) Full State Feedback controller

In the first part, the LQR controller is defined. The cost function and the control problem are given. Then the structure of the LQR controller is shown. Then, the creation of the MPC control system with a linear model is explained. Later, with this linear model, it is shown that the controller is adjusted within the performance criteria and system limits. After that, implementation of the preview control to MPC is explained. Finally, the linear state-space model is replaced by the non-linear ADAMS plant.

The LQR controller is for comparing the MPC controllers with a different control strategy. The main focus of the work is MPC controllers. This section then focuses on integrating the preview signal into the MPC. Using the preview data has three main reasons:

- Forecast in advance whether the vehicle can cross the obstacle or not

- Decide what should be body and suspension positions while crossing the obstacle

- Improve the controller prediction and performance

By using preview data, an obstacle crossing algorithm is created and tested with the nonlinear ADAMS plant. 
Different controllers are compared with each other. The controller-plant matrix used in this work is given in the table below.

Table 4.1. Controller and plant combinations used

\begin{tabular}{|c|c|c|c|c|c|c|}
\hline & \multicolumn{2}{|c|}{ LQR } & \multicolumn{2}{c|}{ MPC } & \multicolumn{2}{c|}{ MPC + Preview } \\
\hline Plant & $\begin{array}{c}\text { State-Space } \\
\text { Model }\end{array}$ & $\begin{array}{c}\text { ADAMS } \\
\text { Plant }\end{array}$ & $\begin{array}{c}\text { State-Space } \\
\text { Model }\end{array}$ & $\begin{array}{c}\text { ADAMS } \\
\text { Plant }\end{array}$ & $\begin{array}{c}\text { State-Space } \\
\text { Model }\end{array}$ & $\begin{array}{c}\text { ADAMS } \\
\text { Plant }\end{array}$ \\
\hline
\end{tabular}

The state-space model is used for tuning and comparing the controllers. So, the in-depth trench crossing simulations are not run with this model. The ADAMS plant is used for these significant obstacle crossing events.

\subsection{Creating the LQR (Linear Quadratic Regulator) Controller}

Regarding equation (3.45), the general state-space formulation is shown like;

$$
\dot{x}=A x+B u
$$

The controller gain for LQR controller and cost function can be considered as:

$$
\begin{gathered}
u(t)=K(t) x(t) \\
J=\int_{0}^{t_{f}}\left(x^{T} Q x+u^{T} R u\right) d t
\end{gathered}
$$

The solution for the $\boldsymbol{K}$ matrix is the solution of a Riccati equation;

$$
-Q=A^{T} P+P A-P B R^{-1} B^{T} R
$$

where,

$$
K=R^{-1} B^{T} P
$$


The $\boldsymbol{Q}$ matrix is the weight of the states, and the $\boldsymbol{R}$ matrix is the weight of control inputs in the cost function.

$$
\begin{aligned}
Q & =\left[\begin{array}{ccccc}
q_{1} & 0 & 0 & \cdots & 0 \\
0 & q_{2} & & & 0 \\
0 & & \ddots & & \vdots \\
\vdots & & & q_{17} & 0 \\
0 & 0 & \cdots & 0 & q_{18}
\end{array}\right] \\
R & =\left[\begin{array}{ccccc}
r_{1} & 0 & 0 & \cdots & 0 \\
0 & r_{2} & & & 0 \\
0 & \ddots & & \vdots \\
\vdots & & & r_{5} & 0 \\
0 & 0 & \cdots & 0 & r_{6}
\end{array}\right]
\end{aligned}
$$

where the coefficients of the weight matrices,

$$
q_{1} \rightarrow q_{18}=1.0 E+05, q_{17}=50.0 E+05
$$

The state representing the pitch angle has higher weight to be penalized more. Because the pitch angle is to be regulated more. The order of magnitudes are related to magnitudes of outputs and control signals.

$$
r_{1} \rightarrow r_{6}=1
$$




\subsection{Creating MPC Controllers in Simulink with Linear Model}

A discrete state-space system model is required for using the MPC toolbox. The system matrices used in this controller are A and B matrices defined in Section 3.2.2. The output matrices $\mathrm{C}$ is not an $18 \times 18$ identity matrix; it is custom 9x18 matrix according to outputs of body acceleration, roll, pitch, and unsprung mass positions. When the state-space model is defined, it is imported into MPC toolbox.

In Figure 4.1, a general overview of the closed-loop MPC control system without a preview. The plant used in the loop is the state-space full-car ride model created in Section 3.2.2.

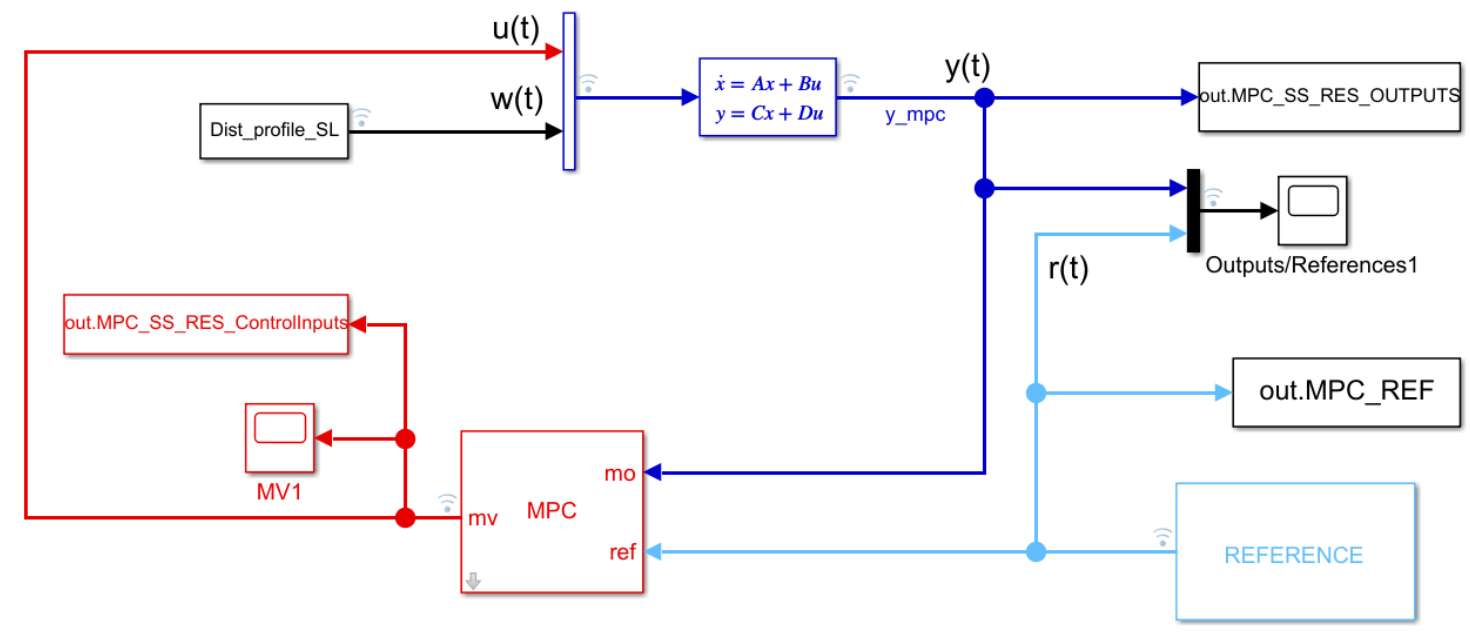

Figure 4.1. Closed-loop MPC control system without preview

In order to see the effect and difference of preview control, two MPC systems are created. Figure 4.1 shows the MPC without preview, and the figure given below shows the block diagram of MPC with preview control. (Figure 4.2)

The MPC control system without preview has signals of;

- 9 Measured Outputs (MO)

- 6 Manipulated Variables (MV) (Control Inputs)

- 12 unmeasured disturbances 


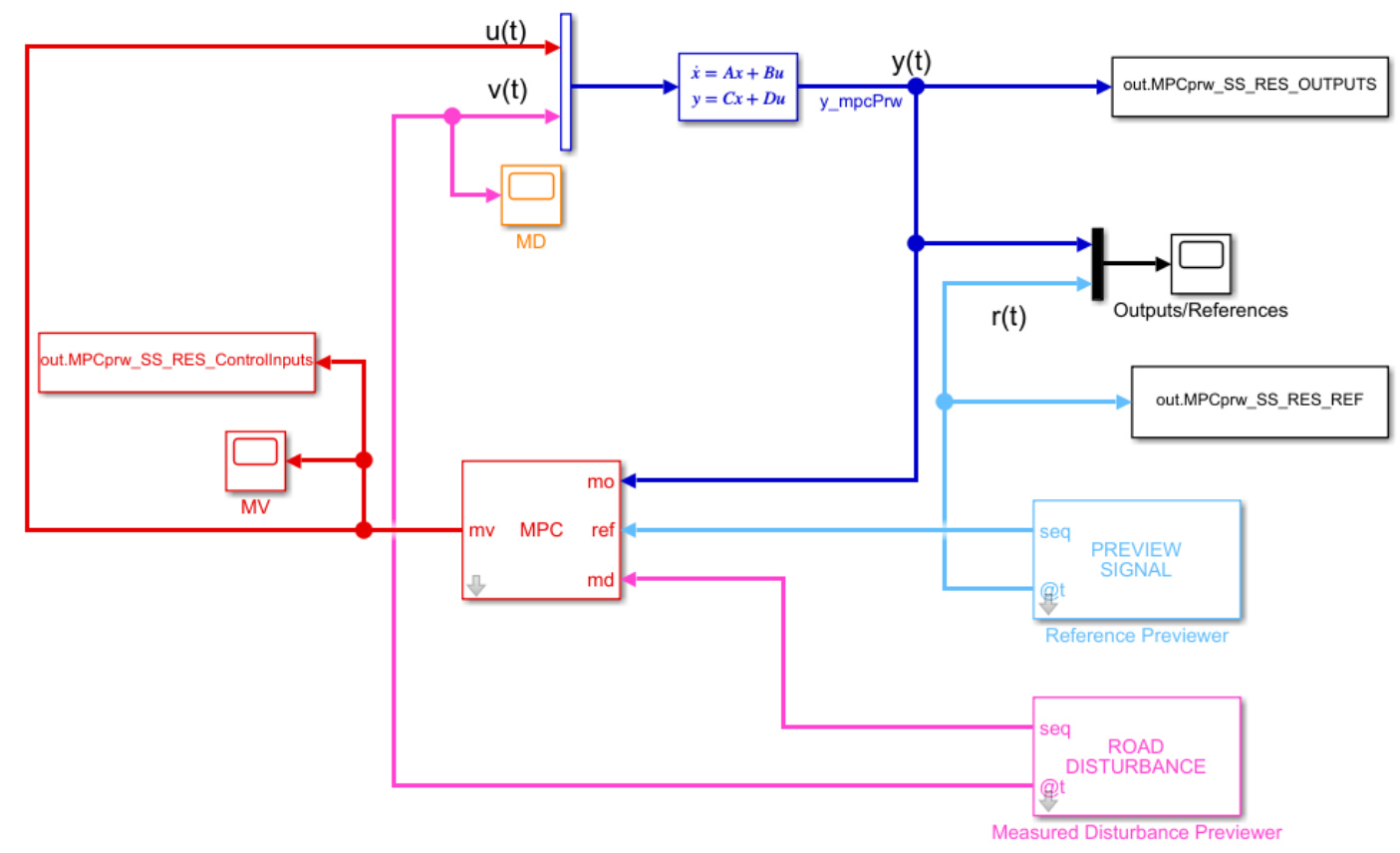

Figure 4.2. Closed-loop MPC control system with the preview

The MPC control system with preview has signals of;

- 9 Measured Outputs (MO)

- 6 Manipulated Variables (MV) (Control Inputs)

- 12 Measured Disturbances (MD)

After importing the model, the MPC structure is defined. Manipulated variables are actuator forces on the suspension units. The measured outputs are body displacement, roll and pitch angles and wheel positions. Table 4.2 shows the signal definitions and their names.

The used model is a linear state-space model. If the imported model is non-linear, it must be linearized around a specific point of interest while importing the model into MPC structure. 
Table 4.2. Signal definitions and names used in MPC

\begin{tabular}{|c|c|l|}
\hline MPC Signal & Signal & Signal Name \\
\hline MV1 & $F_{1 R}$ & Front Axle Right Actuator \\
\hline MV2 & $F_{1 L}$ & Front Axle Left Actuator \\
\hline MV3 & $F_{2 R}$ & Middle Axle Right Actuator \\
\hline MV4 & $F_{2 L}$ & Middle Axle Left Actuator \\
\hline MV5 & $F_{3 R}$ & Rear Axle Right Actuator \\
\hline MV6 & $F_{3 L}$ & Rear Axle Left Actuator \\
\hline MO1 & $\ddot{z}_{S}$ & Body Acceleration \\
\hline MO2 & $\theta$ & Body Roll Angle \\
\hline MO3 & $\gamma$ & Body Pitch Angle \\
\hline MO4 & $z_{u s 1 r}$ & Front Axle Right Wheel Position \\
\hline MO5 & $z_{u s 1 l}$ & Front Axle Left Wheel Position \\
\hline MO6 & $z_{u s 2 r}$ & Middle Axle Right Wheel Position \\
\hline MO7 & $z_{u s 2 l}$ & Middle Axle Left Wheel Position \\
\hline MO8 & $z_{u s 3 r}$ & Rear Axle Right Wheel Position \\
\hline MO9 & $z_{u s 3 l}$ & Rear Axle Left Wheel Position \\
\hline
\end{tabular}

Scale factors should be added to facilitate tuning since the ranks of the inputs and outputs differ before starting the controller design. Adding these scale factors eliminate the dimension difference between input and outputs. Also, this scaling reduces the impact of numerical errors. The scale factor is basically the upper bound of the magnitude if it is known. If not, setting the scale factor to 1 is recommended. In the table given below, MPC Toolbox warns the user about scale factors [35]. (Table 3.1)

Table 4.3. Scale factors of inputs and outputs for linear simulation

\begin{tabular}{|c|c|c|c|c|}
\hline Signal & $\begin{array}{c}\text { Scale } \\
\text { Factor }\end{array}$ & Signal & $\begin{array}{c}\text { Scale } \\
\text { Factor }\end{array}$ \\
\hline MV1 & $3.00 \mathrm{E}+05$ & & MO1 & $4.00 \mathrm{E}+00$ \\
\hline MV2 & $3.00 \mathrm{E}+05$ & & MO2 & $2.00 \mathrm{E}-02$ \\
\hline MV3 & $3.00 \mathrm{E}+05$ & & MO3 & $2.00 \mathrm{E}-01$ \\
\hline MV4 & $3.00 \mathrm{E}+05$ & & MO4 & $1.00 \mathrm{E}+00$ \\
\hline MV5 & $3.00 \mathrm{E}+05$ & & MO5 & $1.00 \mathrm{E}+00$ \\
\hline MV6 & $3.00 \mathrm{E}+05$ & & MO6 & $1.00 \mathrm{E}+00$ \\
\hline & & & MO7 & $1.00 \mathrm{E}+00$ \\
\hline & & & MO8 & $1.00 \mathrm{E}+00$ \\
\hline & & & MO9 & $1.00 \mathrm{E}+00$ \\
\hline
\end{tabular}


Before tuning the controller, prediction and control horizon must be defined according to system dynamic behaviour. Then it is not recommended changing horizons for tuning the controller. The prediction horizon $\left(N_{p}\right)$ is the length that wanted to be predicted. This prediction is related to time step and desired response time of the controlled system. The future state variables can be expressed:

$$
x\left(k_{i}+1 \mid k_{i}\right), x\left(k_{i}+2 \mid k_{i}\right), \ldots, x\left(k_{i}+m \mid k_{i}\right), \ldots, x\left(k_{i}+N_{p} \mid k_{i}\right)
$$

$x\left(k_{i}+m \mid k_{i}\right)$ is the predicted state variable at $k_{i}+m[36]$

For the prediction horizon, the following approach can be used;

$$
T \approx N_{p} T_{s}
$$

where $T$ is desired settling time of the closed-loop system and $T_{s}$ is the time step. Regarding the prediction horizon, the effect of making it larger decreases as increasing the control horizon. It can be a valid method to increase it until its effect is getting a minor impact. The recommended value of the prediction horizon is less than 50. The exception is that if the time step is too small, the prediction horizon can be selected as more massive.

The control horizon $\left(N_{c}\right)$ is the number of steps that are included in the optimization problem. It should be decidedly smaller than the prediction horizon. The smaller the control horizon means that faster computation. Also, the small control horizon increases the tendency of the controller to be stable.

The time step, prediction horizon and control horizons are defined as follows:

Table 4.4. Time step, prediction, and control horizon selection for linear simulation

\begin{tabular}{|r|c|}
\hline Sample Time & 0.005 \\
\hline Prediction Horizon & 50 \\
\hline Control Horizon & 3 \\
\hline
\end{tabular}




\subsection{Tuning the Controller}

The MPC controller is an optimization process. If it is assumed that there are no constraints on the controller, the linear prediction model and input-state relations can be defined as:

$$
\begin{gathered}
x_{k+1}=A x_{k}+B u_{k} \\
y_{k}=C x_{k} \\
x_{k}=A^{k} x_{0}+\sum_{j=0}^{k-1} A^{j} B u_{k-1-j}
\end{gathered}
$$

The linear MPC is a linear state-feedback controller if there are no constraints [24].

The MPC controller includes the input and output constraint into the optimization process. Also, the rates of the input constraints can be defined. If there is no constraint softening, MPC solves the problem with hard constraints. That means the input and output signals will not go out of range.

Although there are different functions for various purposes for MPC controllers, the standard form is:

$$
J\left(z_{k}\right)=J_{y}\left(z_{k}\right)+J_{u}\left(z_{k}\right)+J_{\Delta u}\left(z_{k}\right)+J_{\varepsilon}\left(z_{k}\right)
$$

Each term focus on particular aspects, and the $z_{k}$ term is the decision for Quadratic Programming (QP), in other words, optimization problem.

For output reference tracking MPC follows the cost function given below [35]:

$$
J_{y}\left(z_{k}\right)=\sum_{j=1}^{n_{y}} \sum_{i=1}^{p}\left\{\frac{w_{i, j}^{y}}{s_{j}^{y}}\left[r_{j}(k+i \mid k)-y_{j}(k+i \mid k)\right]\right\}^{2}
$$

where,

$k$ is the current control interval

$p$ is the prediction horizon

$n_{y}$ is the number of the plant output variables 
$z_{k}$ is QP decision defined by,

$$
x_{k}^{T}=\left[u(k \mid k)^{T} u(k+1 \mid k)^{T} \cdots u(k+p-1 \mid k)^{T} \varepsilon_{k}\right]
$$

- $y_{j}(k+1 \mid k)$ is the prediction value of $\mathrm{j}^{\text {th }}$ plant output at $\mathrm{i}^{\text {th }}$ horizon step

- $\quad r_{j}(k+1 \mid k)$ is the reference value of $\mathrm{j}^{\text {th }}$ plant output at $\mathrm{i}^{\text {th }}$ horizon step

- $\quad s_{j}^{y}$ is the scale factor for $\mathrm{j}^{\text {th }}$ plant output

- $\quad w_{j, j}^{y}$ is the tuning weight for $\mathrm{j}^{\text {th }}$ plant output at $\mathrm{i}^{\text {th }}$ horizon step

Table 4.5. Hard constraints for MPC tuning for linear simulation

\begin{tabular}{|c|c|c|c|c|}
\hline & \multicolumn{4}{|c|}{ Hard Constraints } \\
\cline { 2 - 5 } & Min & Max & RateMin & RateMax \\
\hline MV1 & $-1.50 \mathrm{E}+05$ & $1.50 \mathrm{E}+05$ & $1.20 \mathrm{E}+06$ & $1.20 \mathrm{E}+06$ \\
\hline MV2 & $-1.50 \mathrm{E}+05$ & $1.50 \mathrm{E}+05$ & $1.20 \mathrm{E}+06$ & $1.20 \mathrm{E}+06$ \\
\hline MV3 & $-1.50 \mathrm{E}+05$ & $1.50 \mathrm{E}+05$ & $1.20 \mathrm{E}+06$ & $1.20 \mathrm{E}+06$ \\
\hline MV4 & $-1.50 \mathrm{E}+05$ & $1.50 \mathrm{E}+05$ & $1.20 \mathrm{E}+06$ & $1.20 \mathrm{E}+06$ \\
\hline MV5 & $-1.50 \mathrm{E}+05$ & $1.50 \mathrm{E}+05$ & $1.20 \mathrm{E}+06$ & $1.20 \mathrm{E}+06$ \\
\hline MV6 & $-1.50 \mathrm{E}+05$ & $1.50 \mathrm{E}+05$ & $1.20 \mathrm{E}+06$ & $1.20 \mathrm{E}+06$ \\
\hline MO1 & $-2.00 \mathrm{E}+00$ & $2.00 \mathrm{E}+00$ & N/A & N/A \\
\hline MO2 & $-1.00 \mathrm{E}-02$ & $1.00 \mathrm{E}-02$ & N/A & N/A \\
\hline MO3 & $-1.00 \mathrm{E}-01$ & $1.00 \mathrm{E}-01$ & N/A & N/A \\
\hline MO4 & $-3.00 \mathrm{E}-01$ & $3.00 \mathrm{E}-01$ & N/A & N/A \\
\hline MO5 & $-3.00 \mathrm{E}-01$ & $3.00 \mathrm{E}-01$ & N/A & N/A \\
\hline MO6 & $-3.00 \mathrm{E}-01$ & $3.00 \mathrm{E}-01$ & N/A & N/A \\
\hline MO7 & $-3.00 \mathrm{E}-01$ & $3.00 \mathrm{E}-01$ & N/A & N/A \\
\hline MO8 & $-3.00 \mathrm{E}-01$ & $3.00 \mathrm{E}-01$ & N/A & N/A \\
\hline MO9 & $-3.00 \mathrm{E}-01$ & $3.00 \mathrm{E}-01$ & N/A & N/A \\
\hline
\end{tabular}


Table 4.6. Constraint softening weights for linear simulation

\begin{tabular}{|l|c|c|c|c|}
\hline & \multicolumn{5}{|c|}{ Constraint Softening } \\
\cline { 2 - 5 } & minECR & maxECR & RateMinECR & RateMaxECR \\
\hline MV1 & $1.00 \mathrm{E}-03$ & $1.00 \mathrm{E}-03$ & $1.00 \mathrm{E}-03$ & $1.00 \mathrm{E}-03$ \\
\hline MV2 & $1.00 \mathrm{E}-03$ & $1.00 \mathrm{E}-03$ & $1.00 \mathrm{E}-03$ & $1.00 \mathrm{E}-03$ \\
\hline MV3 & $1.00 \mathrm{E}-03$ & $1.00 \mathrm{E}-03$ & $1.00 \mathrm{E}-03$ & $1.00 \mathrm{E}-03$ \\
\hline MV4 & $1.00 \mathrm{E}-03$ & $1.00 \mathrm{E}-03$ & $1.00 \mathrm{E}-03$ & $1.00 \mathrm{E}-03$ \\
\hline MV5 & $1.00 \mathrm{E}-03$ & $1.00 \mathrm{E}-03$ & $1.00 \mathrm{E}-03$ & $1.00 \mathrm{E}-03$ \\
\hline MV6 & $1.00 \mathrm{E}-03$ & $1.00 \mathrm{E}-03$ & $1.00 \mathrm{E}-03$ & $1.00 \mathrm{E}-03$ \\
\hline MO1 & $1.00 \mathrm{E}-01$ & $1.00 \mathrm{E}-01$ & N/A & N/A \\
\hline MO2 & $1.00 \mathrm{E}-02$ & $1.00 \mathrm{E}-02$ & N/A & N/A \\
\hline MO3 & $5.00 \mathrm{E}-01$ & $5.00 \mathrm{E}-01$ & N/A & N/A \\
\hline MO4 & $1.00 \mathrm{E}-01$ & $1.00 \mathrm{E}-01$ & N/A & N/A \\
\hline MO5 & $1.00 \mathrm{E}-01$ & $1.00 \mathrm{E}-01$ & N/A & N/A \\
\hline MO6 & $1.00 \mathrm{E}-01$ & $1.00 \mathrm{E}-01$ & N/A & N/A \\
\hline MO7 & $1.00 \mathrm{E}-01$ & $1.00 \mathrm{E}-01$ & N/A & N/A \\
\hline MO8 & $1.00 \mathrm{E}-01$ & $1.00 \mathrm{E}-01$ & N/A & N/A \\
\hline MO9 & $1.00 \mathrm{E}-01$ & $1.00 \mathrm{E}-01$ & N/A & N/A \\
\hline
\end{tabular}

The input limits, rate limits and softening settings are defined in Table 4.5 and Table 4.6. If the softening coefficients are zero, it means there are only hard constraints. The controller will not produce larger than these constraints. The softening coefficients are between 0-20. The more significant coefficient makes the controller generate control inputs with a higher tolerance. The softening coefficients defined minimal numbers to allow the controller using just a little tolerance.

The output constraints are also defined in Table 4.5 and Table 4.6 The body bounce and roll angle have lower constraints than pitch angle because the controller will not regulate the pitch angle around zero. The obstacle crossing algorithm can set higher pitch angles about $-5^{\circ}$ to $+5^{\circ}$ as reference. If lower values are set as pitch constraint, the controller cannot set the vehicle's pitch angle to desired values. Also, the pitch angle has a larger softening (tolerance).

Lastly, the input and output weights are defined according to desired penalizing outputs and inputs. The weights are given in Table 4.7 
Table 4.7. Input and output weights for linear simulation

\begin{tabular}{|l|c|c|}
\hline & Weight & $\begin{array}{c}\text { Rate } \\
\text { Weight }\end{array}$ \\
\hline MV1 & 0.1 & 0.1 \\
\hline MV2 & 0.1 & 0.1 \\
\hline MV3 & 0.1 & 0.1 \\
\hline MV4 & 0.1 & 0.1 \\
\hline MV5 & 0.1 & 0.1 \\
\hline MV6 & 0.1 & 0.1 \\
\hline MO1 & 10 & N/A \\
\hline MO2 & 0.5 & N/A \\
\hline MO3 & 100 & N/A \\
\hline MO4 & 0.4 & N/A \\
\hline MO5 & 0.4 & N/A \\
\hline MO6 & 0.4 & N/A \\
\hline MO7 & 0.4 & N/A \\
\hline MO8 & 0.4 & N/A \\
\hline MO9 & 0.4 & N/A \\
\hline
\end{tabular}




\subsection{Creating Control System with ADAMS Plant}

When the controller tuning is complete, it is tested with non-linear ADAMS plant. The exported control system plant given in section 3.5 is replaced with the linear state-space full-car model. The general overview of the control system with the ADAMS plant is given below in Figure 4.3.

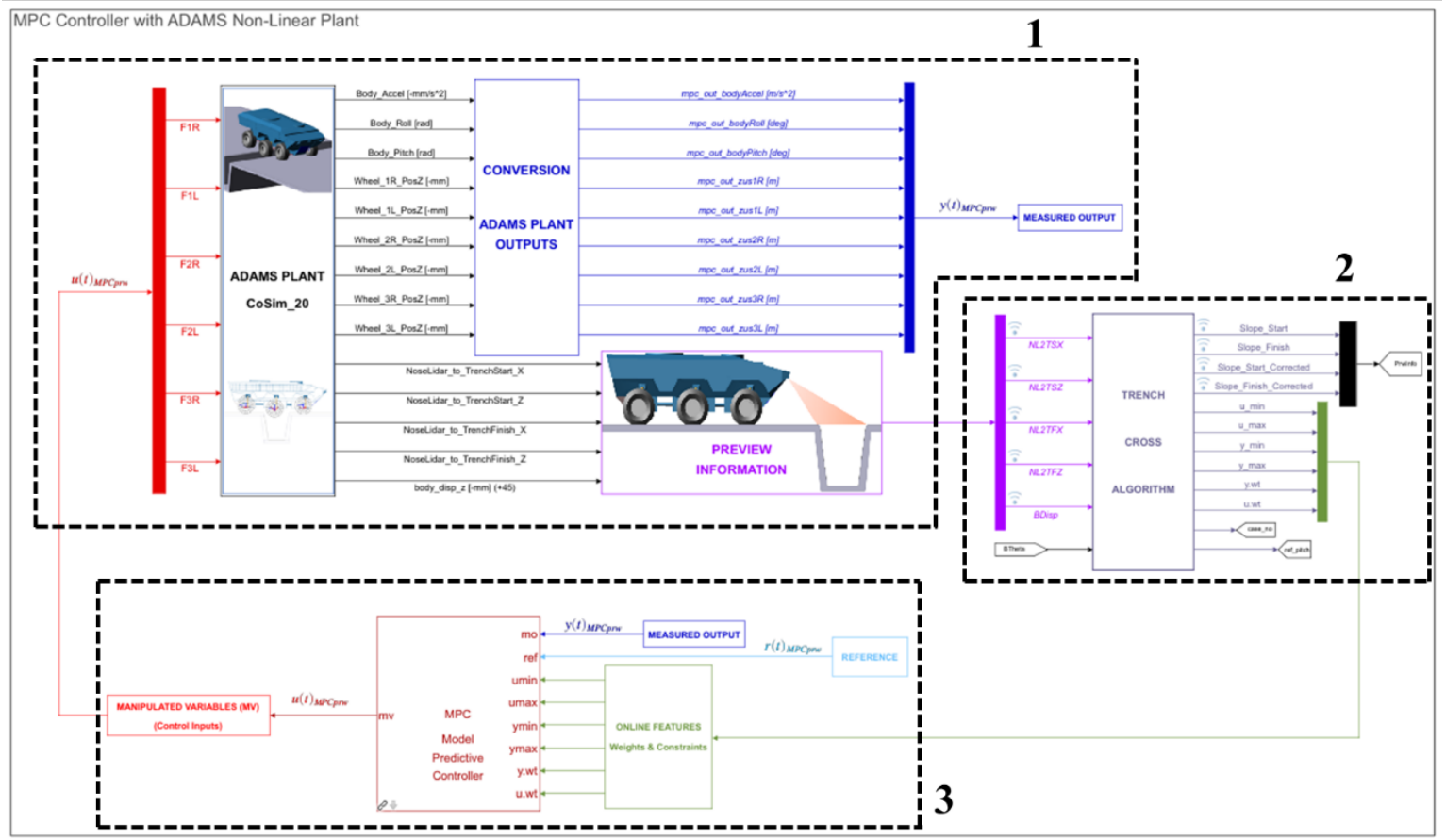

Figure 4.3. General overview of the MPC control system and ADAMS non-linear plant

Figure 4.3 is a block diagram showing plant and controller input and output of the trenching algorithm with MPC + preview controller. Block details and signals are given in more detail both in the previous section and in the appendices. 
To look at the plant subsystem in more detail;

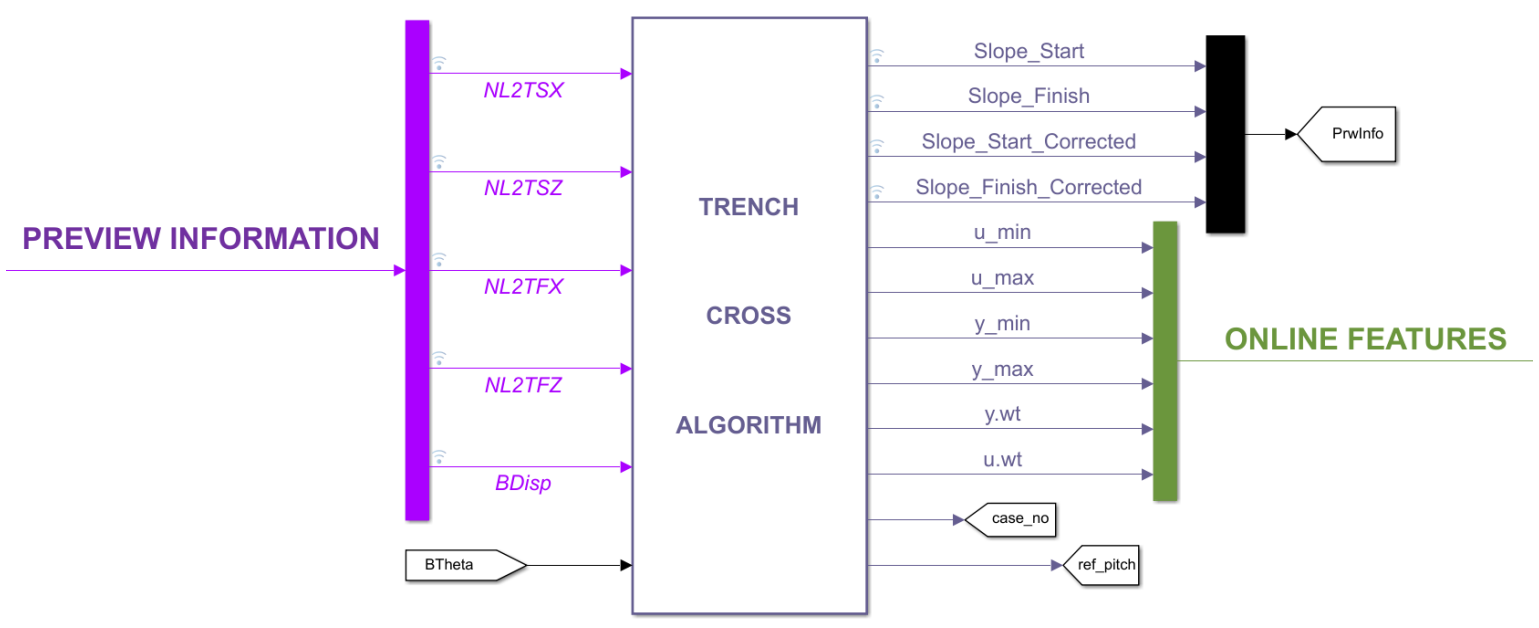

Figure 4.4. Detail overview of non-linear plant, trench crossing algorithm function

Figure 4.4 shows the Part 2 of the system block given in Figure 4.3. It contains strut displacements data and obstacle crossing algorithm. The algorithm is a Matlab function which determines the reference pitch angle of the vehicle while crossing the obstacle. It checks the strut displacements, and according to displacements value, the function predicts the vehicle position roughly with respect to the obstacle. Then it gives the reference pitch angle to the controller.

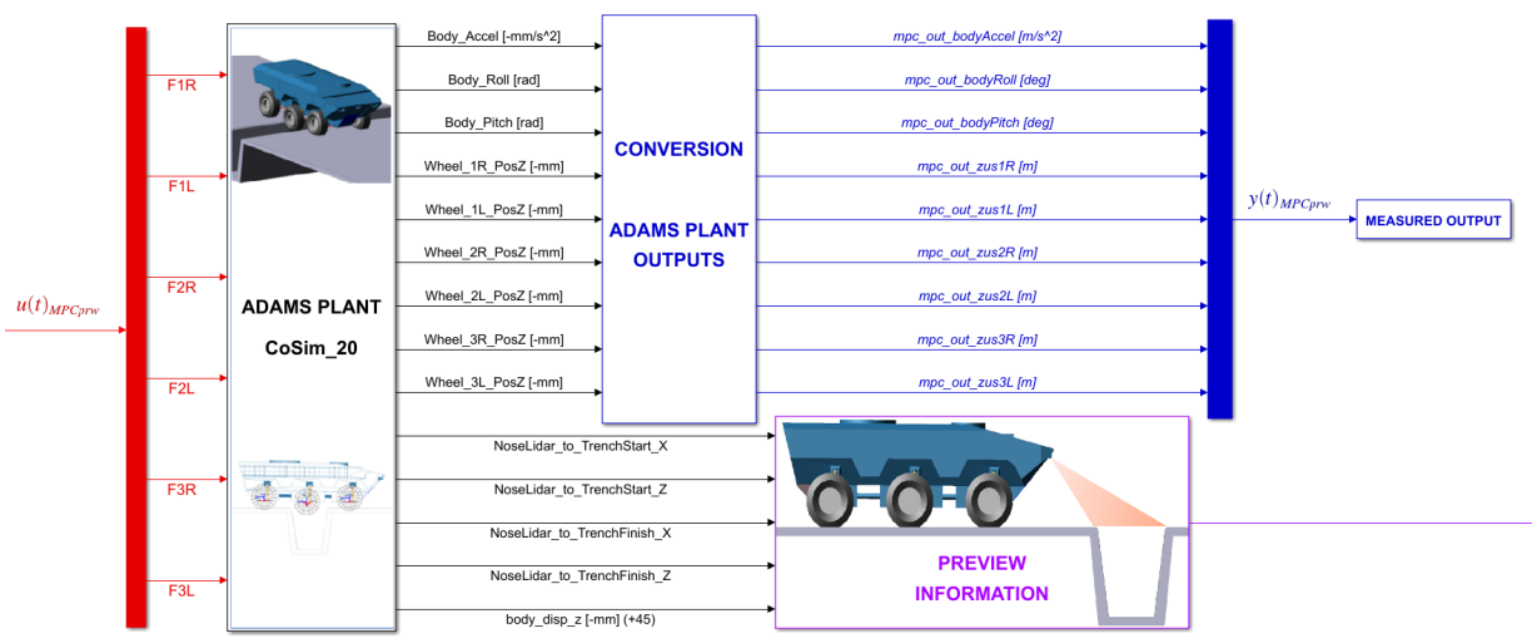

Figure 4.5. Detail overview of non-linear plant, ADAMS plant and its inputs/outputs 
In Figure 4.5, the ADAMS plant part (Part 1) of the system block given in Figure 4.3 is represented. This is the block which is exported from the ADAMS/Controls Plant Export module according to given inputs and outputs. Also, there is conversion block which includes some mathematical unit conversions and corrections. The preview information block is a block that converts incoming preview road signals into more meaningful numbers that the trenching algorithm function can use.

To take a closer look at Part 3, the part showing the input and output of the MPC controller is given in Figure 4.6.

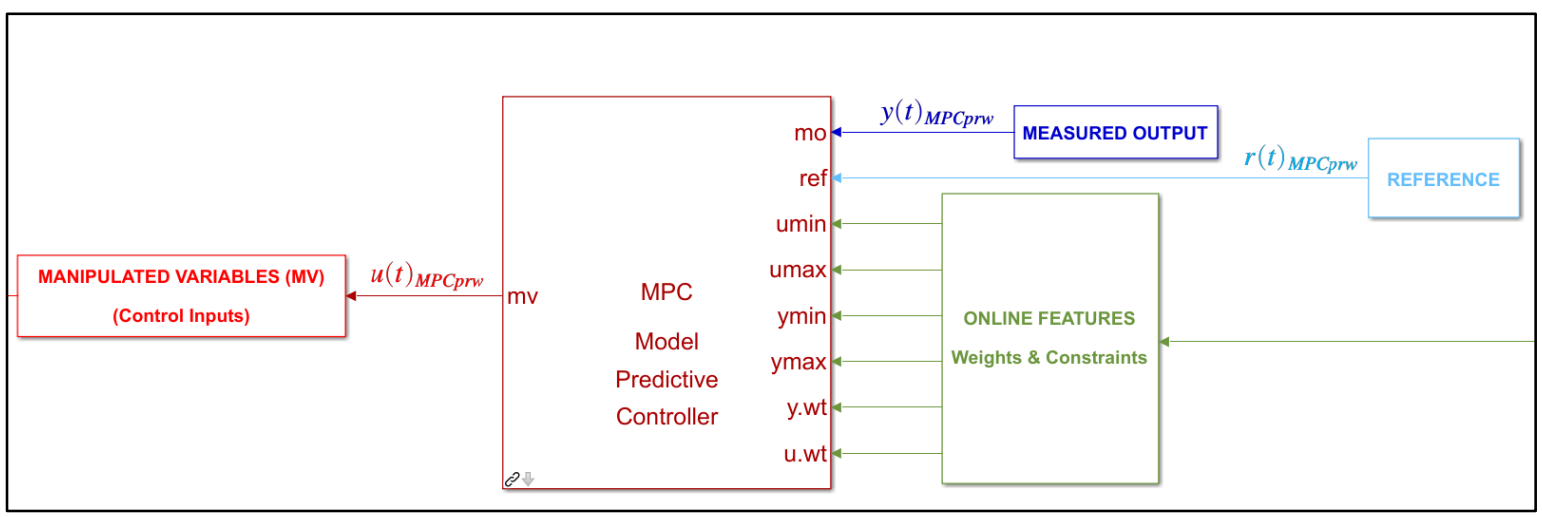

Figure 4.6. Detail overview of MPC controller block with inputs, outputs and online features

\subsection{Trench Cross Algorithm}

This section describes the operating principle of the trench cross algorithm, which is an essential step in the MPC controller's ability to work with preview support. What the preview information signals are is shown in Table 3.3 in the previous sections. Again, these signals are as follows. Figure 4.7 shows the inputs signal names of the trench cross algorithm. Also Figure 4.8 shows the definition of these signals on the vehicle and ground. 


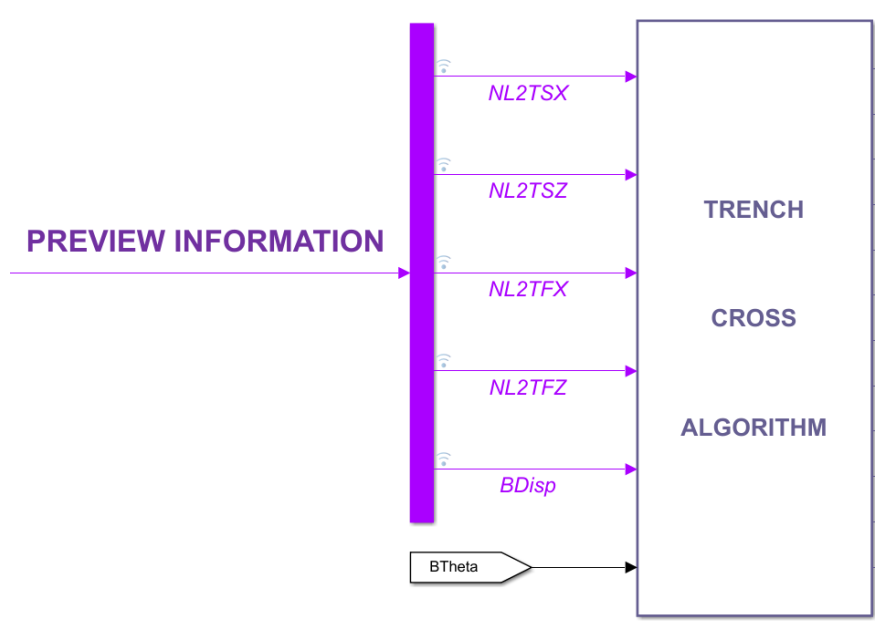

Figure 4.7. Inputs of the trench cross algorithm

- NL2TSX: Longitudinal Distance from Nose Lidar to Trench Start

- NL2TSZ: Vertical Distance from Nose Lidar to Trench Start

- NL2TFX: Longitudinal Distance from Nose Lidar to Trench Finish

- NL2TFZ: Vertical Distance from Nose Lidar to Trench Finish

- BDisp: Body Vertical Displacement

- BTheta: Body Pitch Angle

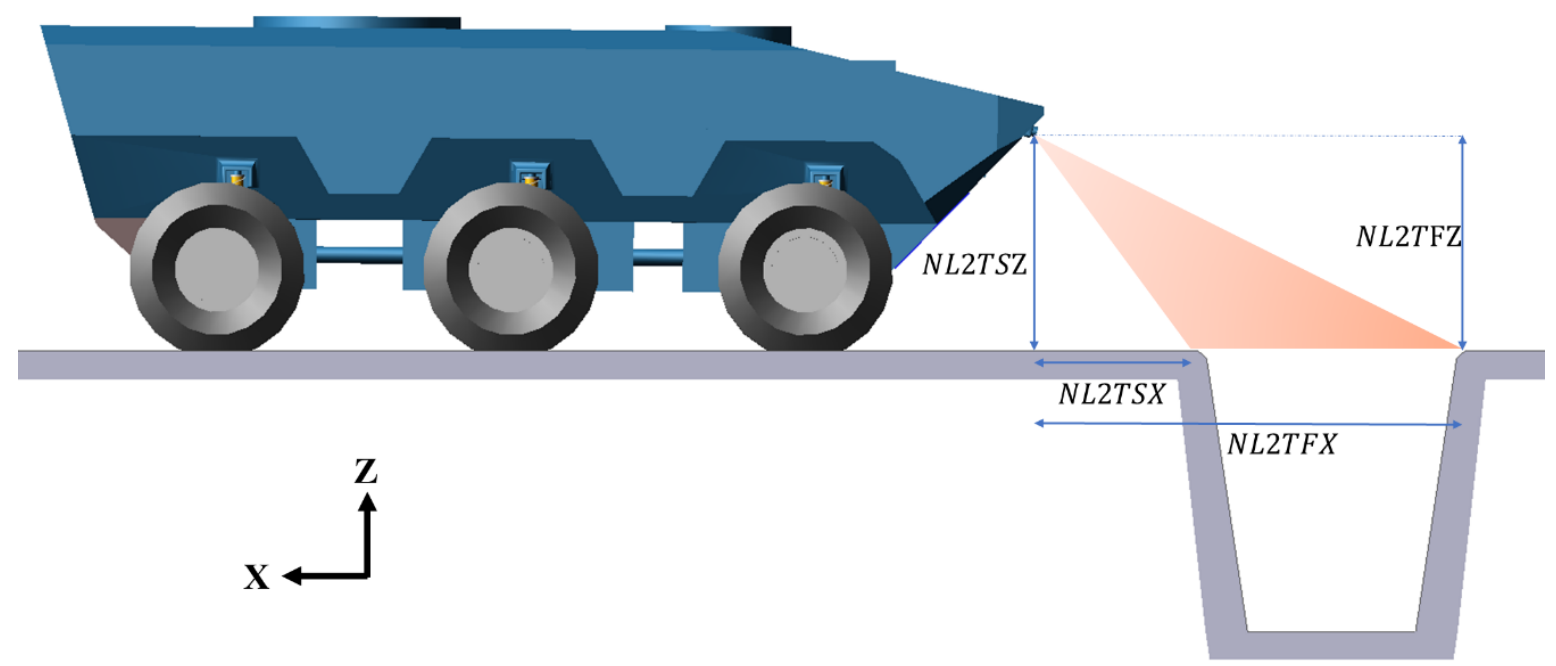

Figure 4.8. Preview information signals definition 
Since the lidar sensor on the body is naturally affected by the dynamic movements of the vehicle, it is necessary to make corrections to avoid any errors in the measurements. The angles of the curves drawn from the nose lidar to the trench are corrected. This correction is done by using fundamental geometric relations according to the vertical movement and pitch angle of the vehicle. In this way, the position of the vehicle wheels relative to the trench is determined with minimalized errors.

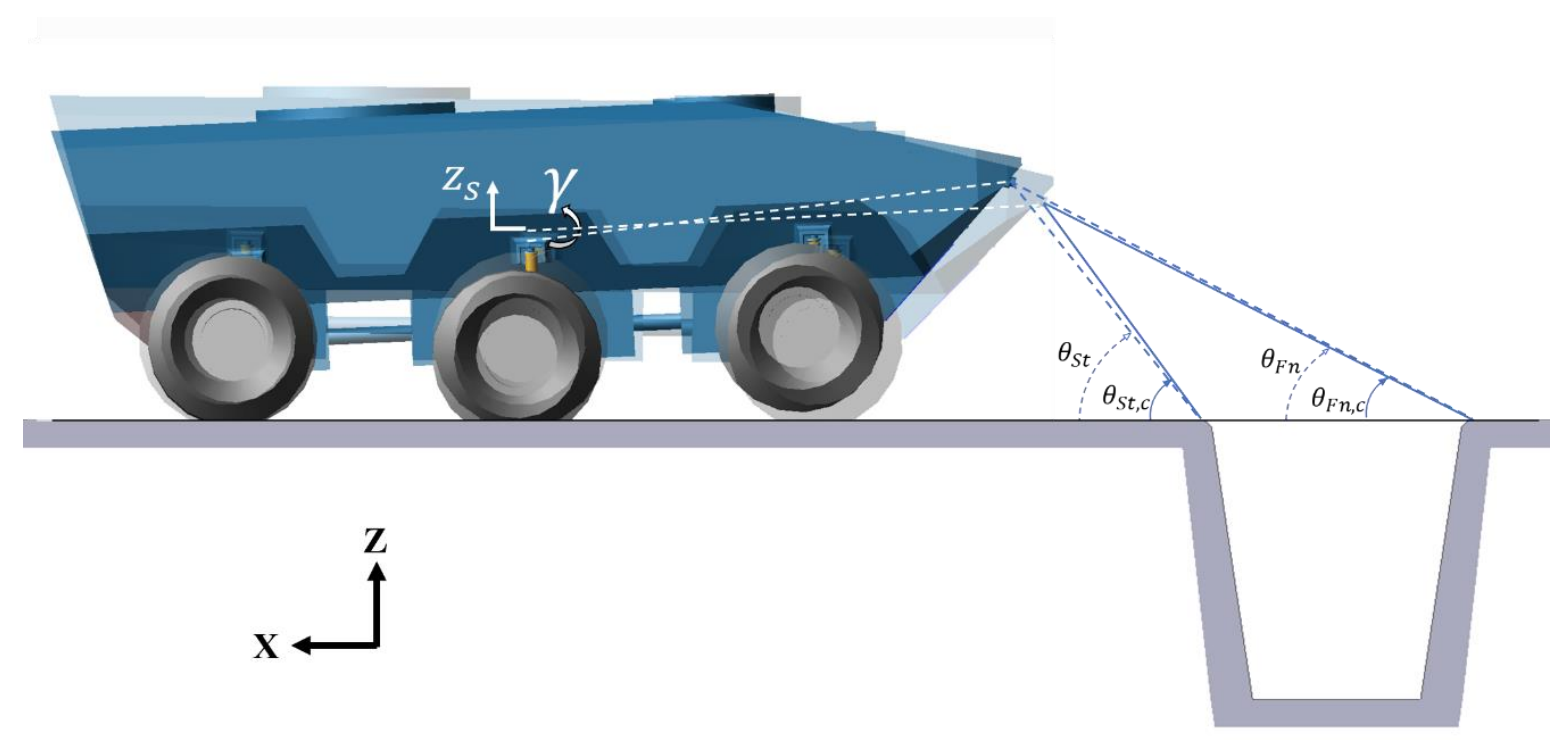

Figure 4.9. Nose lidar to trench slopes and corrected slopes

The real time slope coming from direct measurements for trench start:

$$
\theta_{S t}=\tan ^{-1}\left(\frac{N L 2 T S Z}{N L 2 T S X}\right)
$$

Changes of longitudinal and vertical distance of nose lidar position due to body bounce and pitch angle are:

$$
\begin{aligned}
& \Delta_{N L 2 T S X}=(\cos \gamma \cdot C G 2 N L X-\sin \gamma \cdot C G 2 N L Z)-C G 2 N L X \\
& \Delta_{N L 2 T S Z}=(\sin \gamma \cdot C G 2 N L X+\cos \gamma \cdot C G 2 N L Z)-C G 2 N L Z
\end{aligned}
$$

Corrected distances from nose lidar to trench start:

$$
\begin{gathered}
N L 2 T S X_{\text {corrected }}=N L 2 T S X+\Delta_{N L 2 T S X} \\
N L 2 T S Z_{\text {corrected }}=N L 2 T S Z-\Delta_{N L 2 T S Z}-z_{S}
\end{gathered}
$$


Corrected slope of trench start:

$$
\theta_{S t, c}=\tan ^{-1}\left(\frac{N L 2 T S Z_{\text {corrected }}}{N L 2 T S X_{\text {corrected }}}\right)
$$

The real time slope coming from direct measurements for trench finish:

$$
\theta_{F n}=\tan ^{-1}\left(\frac{N L 2 T F Z}{N L 2 T F X}\right)
$$

Changes of longitudinal and vertical distance of nose lidar position due to body bounce and pitch angle are:

$$
\begin{aligned}
\Delta_{N L 2 T F X} & =(\cos \gamma \cdot C G 2 N L X-\sin \gamma \cdot C G 2 N L Z)-C G 2 N L X \\
\Delta_{N L 2 T F Z} & =(\sin \gamma \cdot C G 2 N L X+\cos \gamma \cdot C G 2 N L Z)-C G 2 N L Z
\end{aligned}
$$

Corrected distances from nose lidar to trench finish:

$$
\begin{gathered}
N L 2 T F X_{\text {corrected }}=N L 2 T F X+\Delta_{N L 2 T F X} \\
N L 2 T F Z_{\text {corrected }}=N L 2 T F Z-\Delta_{N L 2 T F Z}-z_{S}
\end{gathered}
$$

Corrected slope of trench finish:

$$
\theta_{F n, c}=\tan ^{-1}\left(\frac{N L 2 T F Z_{\text {corrected }}}{N L 2 T F X_{\text {corrected }}}\right)
$$

The expressions given in equations (4.17) and (4.23) are the slope angle values indicated by the preview information in real-time. The equations given to correct them according to the movements of the body are used. As a result, the adjusted slope values in equations (4.22) and (4.28) are used in the trench cross algorithm. 


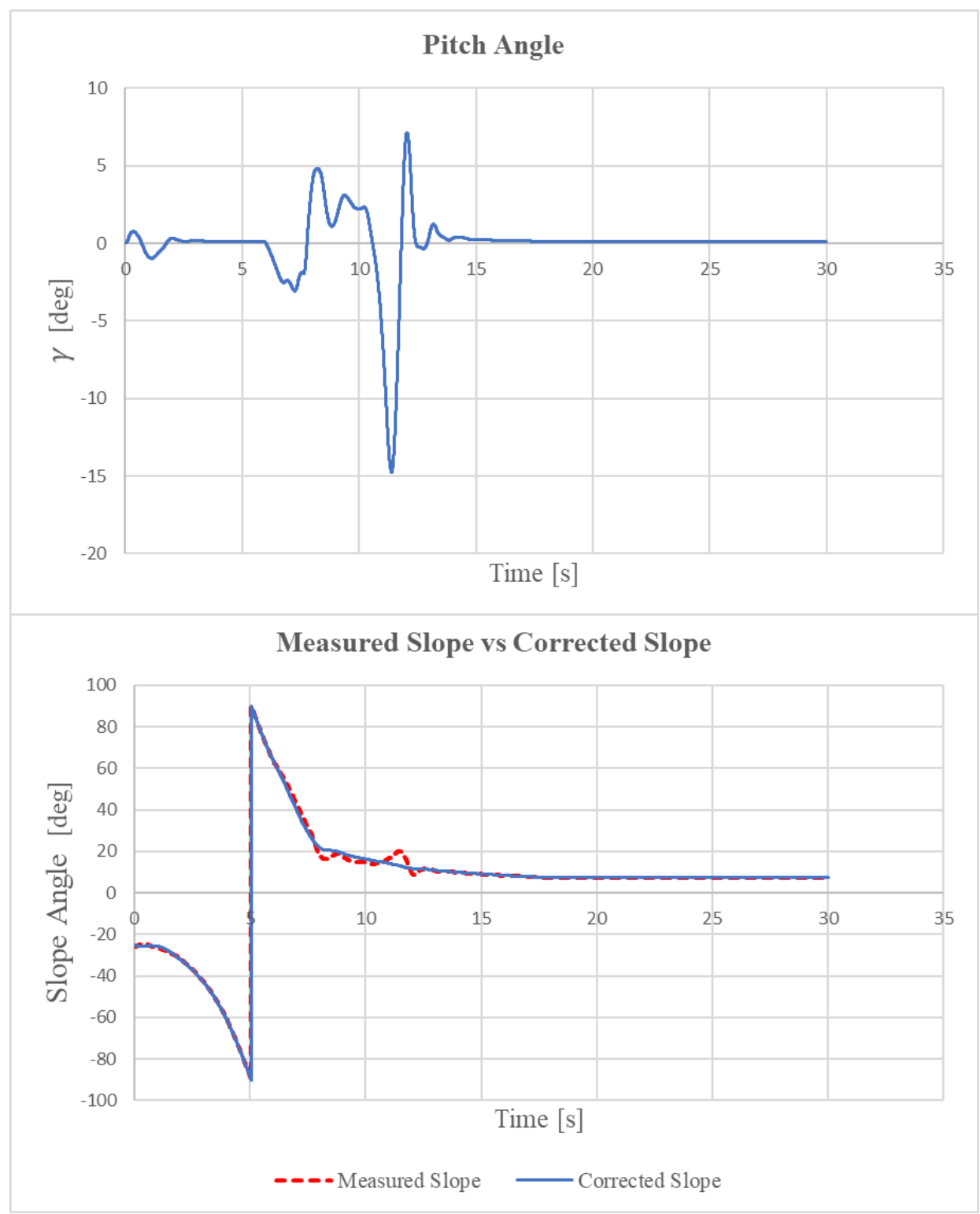

Figure 4.10. Comparison of measured and corrected slopes from nose lidar to trench

Figure 4.10 shows how the slope angle and the corrected slope angle change concerning the body pitch angle. It can be seen that the small-angle assumption starts to deteriorate at angles above about $5^{\circ}$ and the size of the errors will increase if measurements are taken directly.

Flowchart of the trench cross algorithm is shown in Figure 4.11. The inputs of this algorithm are corrected trench start and finish slope angles. These angles are used to detect the positions of each axle relative to the trench. So, the algorithm determines the transition between different cases. 


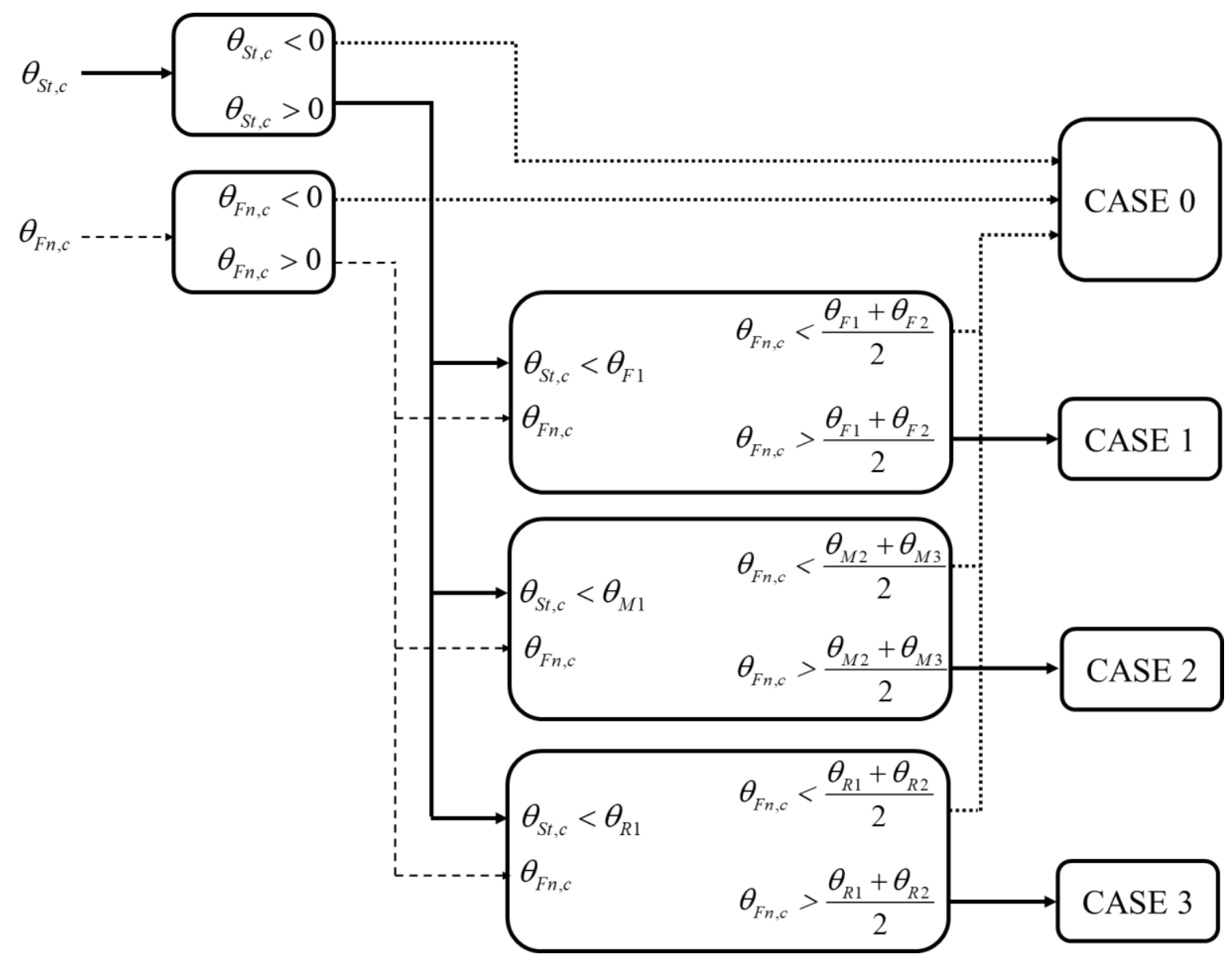

Figure 4.11. Flowchart of the trench cross algorithm

Case 0 represents that all wheels are in contact with the ground outside the trench. In this case, the controller parameters are kept constant at the designed nominal values. These values are given in Section 4.4. Case 1 represents the situation from the moment the first axle starts to enter the start point of the trench until it leaves the end of the trench. Similarly, the states of the second and third axles in the trench are Case 2 and Case 3, respectively. In the continuation of this section, tables are showing the change of hard constraint, constraint softening and weights for each case. Scale factor and controller sample time and horizon values were kept constant for each case. Besides, constraint softening and ECR parameters are more inclusive, so these values are not changed in case transitions. Constraint values for control inputs during trench cross are given in Table 4.8 and weight values in Table 4.9. Similarly, the weights of the outputs are shown in Table 4.10. 
Table 4.8. Hard constraint values of manipulated variables for different cases

\begin{tabular}{|c|c|c|c|c|c|c|}
\hline \multirow{2}{*}{} & \multicolumn{6}{|c|}{ HARD CONSTRAINTS } \\
\cline { 2 - 7 } & \multicolumn{2}{|c|}{ Case 1 } & \multicolumn{2}{c|}{ Case 2 } & \multicolumn{2}{c|}{ Case 3 } \\
\cline { 2 - 7 } & Min & Max & Min & Max & Min & Max \\
\hline MV1 & $5.00 \mathrm{E}+04$ & $7.00 \mathrm{E}+04$ & $-1.50 \mathrm{E}+05$ & $1.50 \mathrm{E}+05$ & $-1.50 \mathrm{E}+05$ & $1.50 \mathrm{E}+05$ \\
\hline MV2 & $5.00 \mathrm{E}+04$ & $7.00 \mathrm{E}+04$ & $-1.50 \mathrm{E}+05$ & $1.50 \mathrm{E}+05$ & $-1.50 \mathrm{E}+05$ & $1.50 \mathrm{E}+05$ \\
\hline MV3 & $-1.50 \mathrm{E}+05$ & $1.50 \mathrm{E}+05$ & $5.00 \mathrm{E}+04$ & $7.00 \mathrm{E}+04$ & $-1.00 \mathrm{E}+05$ & $9.00 \mathrm{E}+04$ \\
\hline MV4 & $-1.50 \mathrm{E}+05$ & $1.50 \mathrm{E}+05$ & $5.00 \mathrm{E}+04$ & $7.00 \mathrm{E}+04$ & $-1.00 \mathrm{E}+05$ & $9.00 \mathrm{E}+04$ \\
\hline MV5 & $-1.50 \mathrm{E}+05$ & $1.50 \mathrm{E}+05$ & $-1.50 \mathrm{E}+05$ & $1.50 \mathrm{E}+05$ & $6.00 \mathrm{E}+04$ & $8.00 \mathrm{E}+04$ \\
\hline MV6 & $-1.50 \mathrm{E}+05$ & $1.50 \mathrm{E}+05$ & $-1.50 \mathrm{E}+05$ & $1.50 \mathrm{E}+05$ & $6.00 \mathrm{E}+04$ & $8.00 \mathrm{E}+04$ \\
\hline
\end{tabular}

Table 4.9. Manipulated variables weights for different cases

\begin{tabular}{|c|c|c|c|c|c|c|}
\hline \multirow{2}{*}{} & \multicolumn{3}{|c|}{ WEIGHTS 1 } & \multicolumn{2}{c|}{ Case 2 } & \multicolumn{2}{c|}{ Case 3 } \\
\cline { 2 - 7 } & Weight & $\begin{array}{c}\text { Rate } \\
\text { Weight }\end{array}$ & Weight & $\begin{array}{c}\text { Rate } \\
\text { Weight }\end{array}$ & Weight & $\begin{array}{c}\text { Rate } \\
\text { Weight }\end{array}$ \\
\hline MV1 & 0.5 & 0.1 & 2.0 & 0.1 & 0.5 & 0.1 \\
\hline MV2 & 0.5 & 0.1 & 2.0 & 0.1 & 0.5 & 0.1 \\
\hline MV3 & 2.0 & 0.1 & 0.5 & 0.1 & 0.1 & 0.1 \\
\hline MV4 & 2.0 & 0.1 & 0.5 & 0.1 & 0.1 & 0.1 \\
\hline MV5 & 5.0 & 0.1 & 2.0 & 0.1 & 2.0 & 0.1 \\
\hline MV6 & 5.0 & 0.1 & 2.0 & 0.1 & 2.0 & 0.1 \\
\hline
\end{tabular}

Table 4.10. Weights of measured outputs for different cases

\begin{tabular}{|c|c|c|c|}
\hline \multirow{2}{*}{} & \multicolumn{3}{|c|}{ WEIGHTS } \\
\cline { 2 - 4 } & Case 1 & Case 2 & Case 3 \\
\cline { 2 - 4 } & Weight & Weight & Weight \\
\hline MO1 & 10.0 & 10.0 & 10.0 \\
\hline MO2 & 0.0 & 0.0 & 0.0 \\
\hline MO3 & 30.0 & 100.0 & 30.0 \\
\hline MO4 & 0.0 & 0.0 & 0.0 \\
\hline MO5 & 0.0 & 0.0 & 0.0 \\
\hline MO6 & 0.0 & 0.0 & 0.0 \\
\hline MO7 & 0.0 & 0.0 & 0.0 \\
\hline MO8 & 0.0 & 0.0 & 0.0 \\
\hline MO9 & 0.0 & 0.0 & 0.0 \\
\hline
\end{tabular}




\section{SIMULATION RESULTS}

\subsection{Introduction to Simulation Results}

In this section, first, the model comparisons are made. The full-car ride model is run in MATLAB and compared with ADAMS model. Then passive system simulations are carried out for different road/obstacle scenarios. In the end, the closed-loop simulations are presented. According to all these passive and active systems simulations, the improvement of mobility is discussed by comparison in the last section.

\subsection{Linear Full Car Model vs ADAMS Comparison}

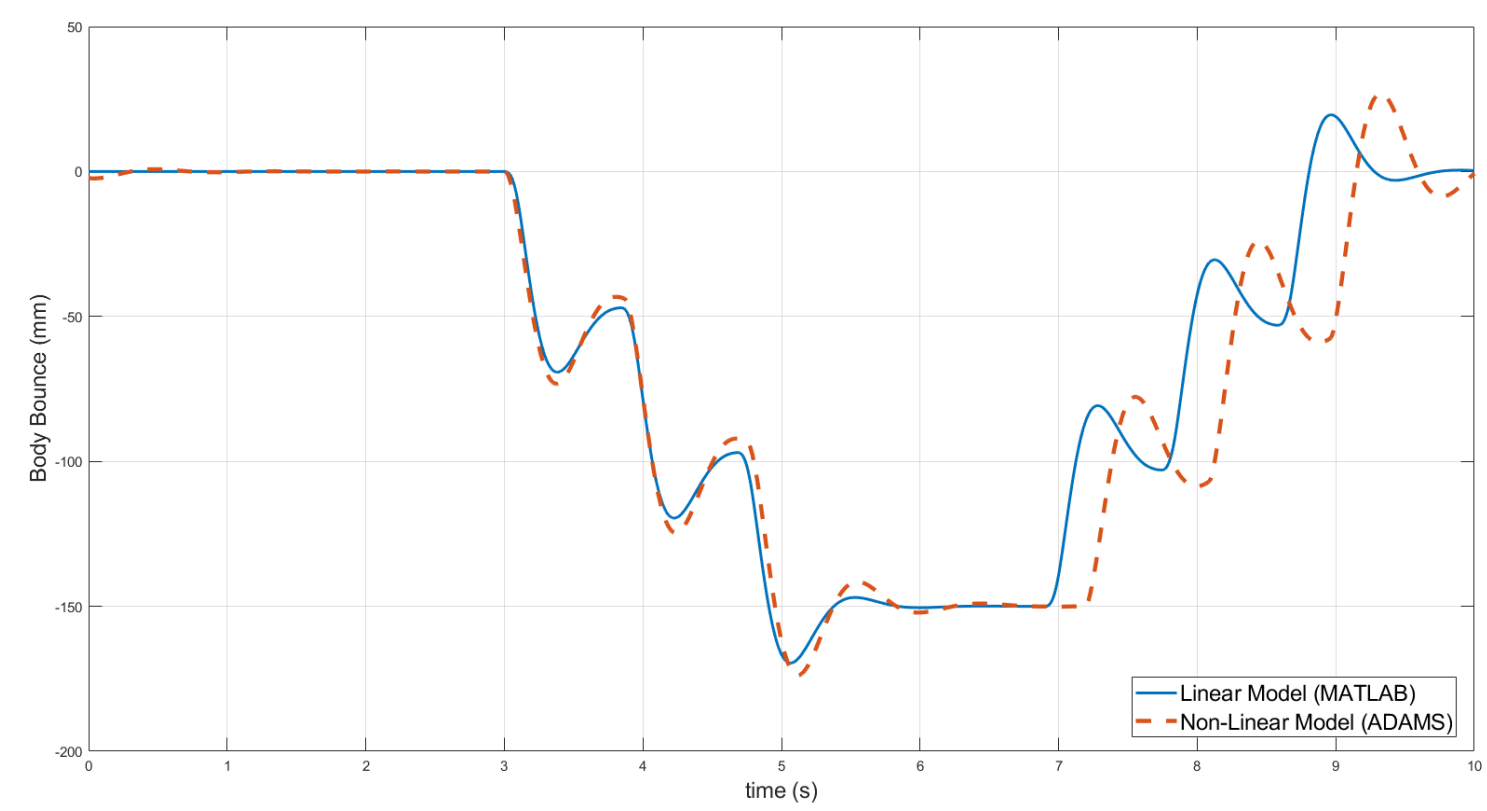

Figure 5.1. Linear Matlab model vs non-linear ADAMS model comparison, body vertical motion

Figure 5.1 shows the comparison of the vertical body displacement, and Figure 5.2 shows the pitch angle comparison of linear and non-linear models. There seems a significant difference, but they can be considered as close to each other. The source of the difference is that the longitudinal velocity is constant for the linear Matlab model while running in Simulink. However, in ADAMS, there is realistic road and obstacle, and the ADAMS speed controller cannot keep the vehicle's speed precisely in the desired value. (Figure 5.3) 


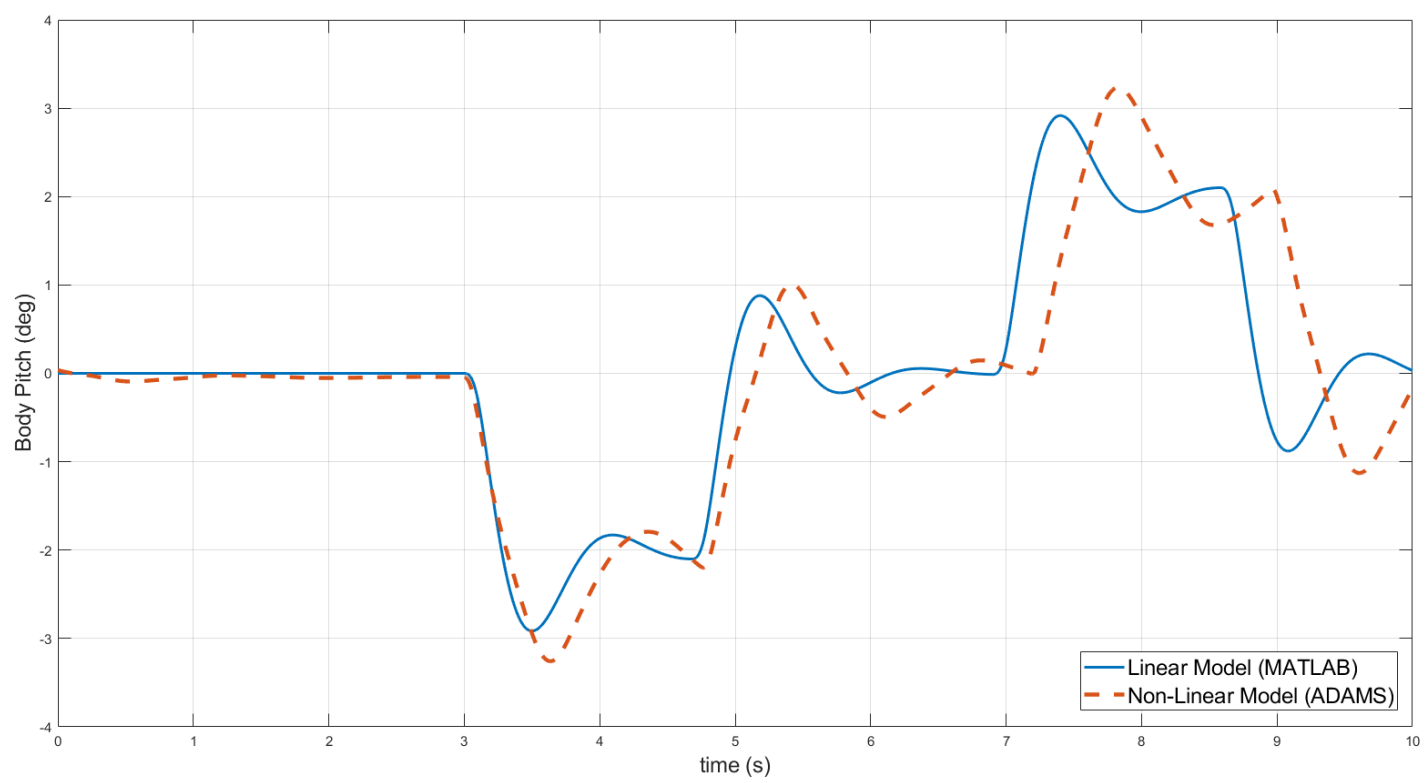

Figure 5.2. Linear Matlab model vs non-linear ADAMS model comparison, pitch angle

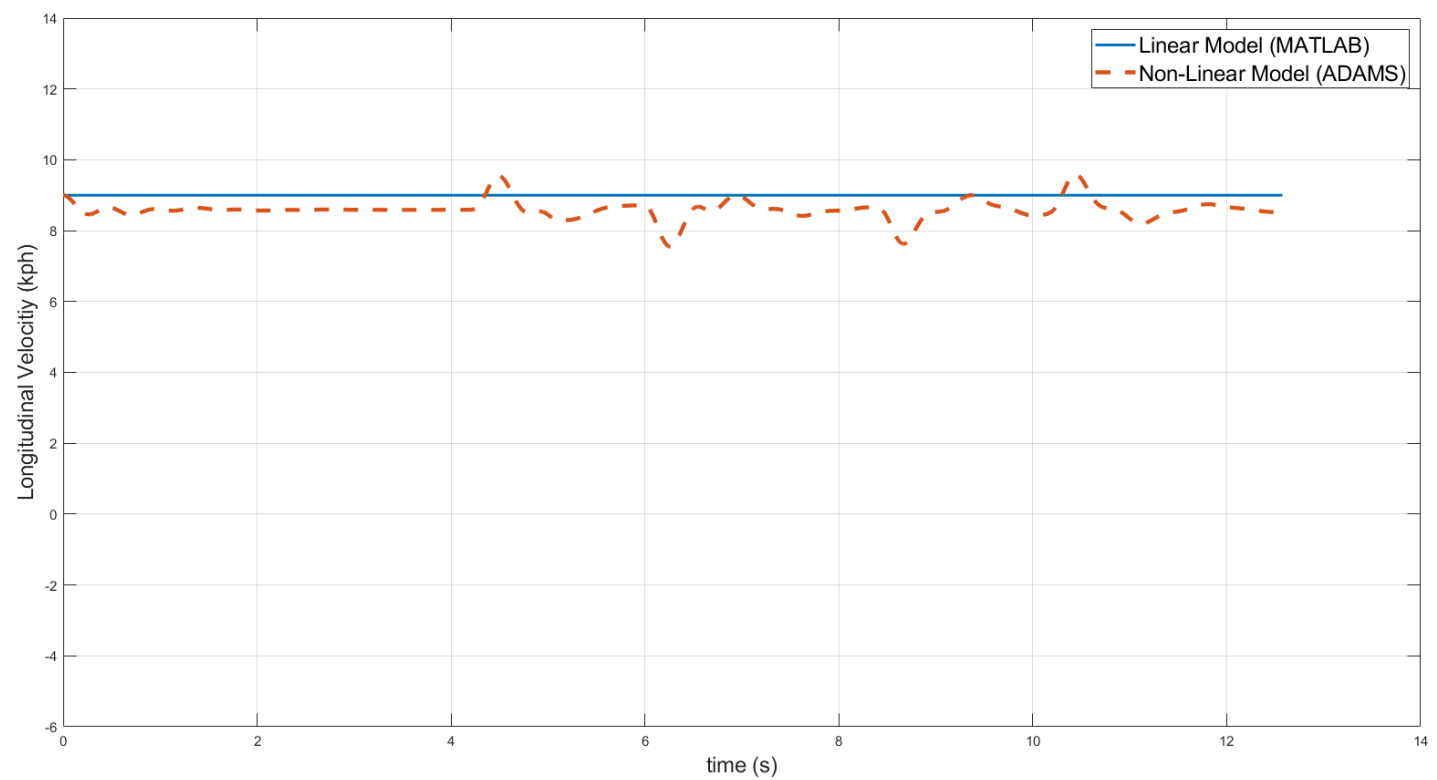

Figure 5.3. Linear Matlab model vs non-linear ADAMS model comparison, longitudinal velocity 


\subsection{Shallow Trench Simulations}

Various simulations are run with three different controllers to compare the performance of controllers in terms of disturbance rejection and reference tracking. These shallow trench simulations are run with linear state space model. The simulation matrix is given in the table below:

Table 5.1. Linear state-space simulation matrix

\begin{tabular}{|r|c|c|c|}
\hline & \multicolumn{3}{|c|}{ State Space Model } \\
\hline $5 \mathrm{kph}, 0.15 \mathrm{~m}$ depth & LQR & MPC & MPC + Preview \\
\hline $10 \mathrm{kph}, 0.15 \mathrm{~m}$ depth & LQR & MPC & MPC + Preview \\
\hline $20 \mathrm{kph}, 0.15 \mathrm{~m}$ depth & LQR & MPC & MPC + Preview \\
\hline $5 \mathrm{kph}, 0.25 \mathrm{~m}$ depth & LQR & MPC & MPC + Preview \\
\hline $10 \mathrm{kph}, 0.25 \mathrm{~m}$ depth & LQR & MPC & MPC + Preview \\
\hline $20 \mathrm{kph}, 0.25 \mathrm{~m}$ depth & LQR & MPC & MPC + Preview \\
\hline
\end{tabular}

In the first 10 seconds of simulations, vehicle passes through $0.15 \mathrm{~m}$ and $0.25 \mathrm{~m}$ shallow trenches. This is to test the disturbance rejection capacity of the controller. Between 15. and 25. seconds, the reference pitch angle is given to the vehicle, and the reference tracking performance is examined.

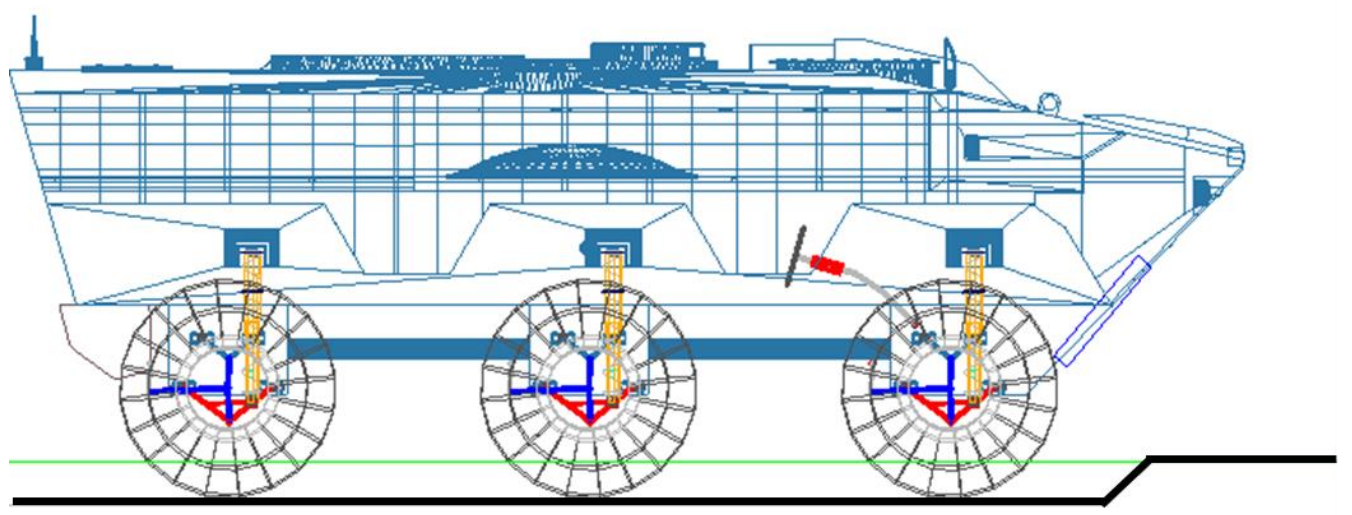

Figure 5.4. Linear system test road

The simulation results are given in the figures below. The primary purpose is to control the vehicle's pitch angle. So the results show the pitch response of uncontrolled and controlled systems. The controller performances are compared to each other with these plots. 


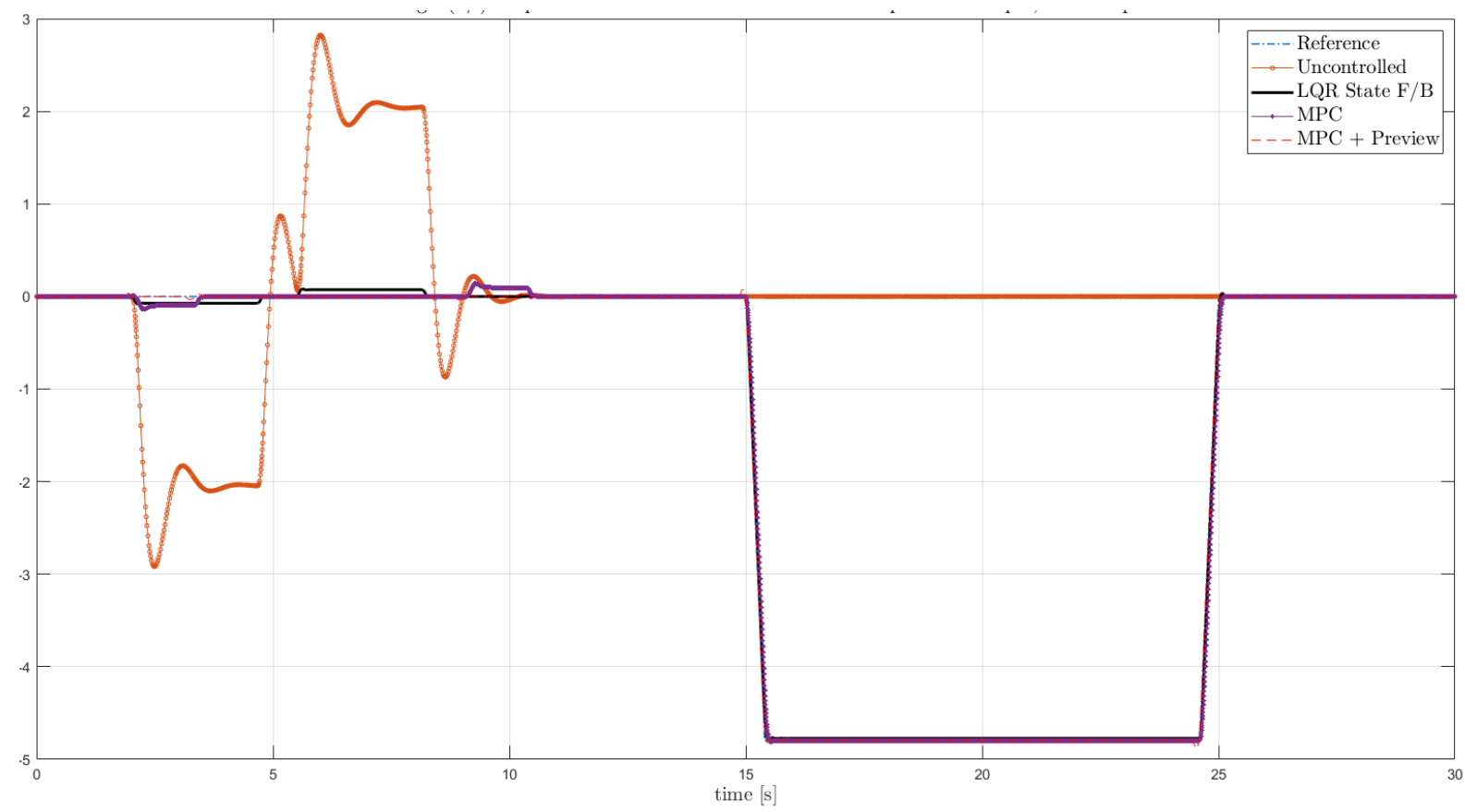

Figure 5.5. Uncontrolled vs controlled systems comparison, $5 \mathrm{kph}, 0.15 \mathrm{~m}$ depth, the horizontal axis is time, vertical axis is pitch angle [deg]

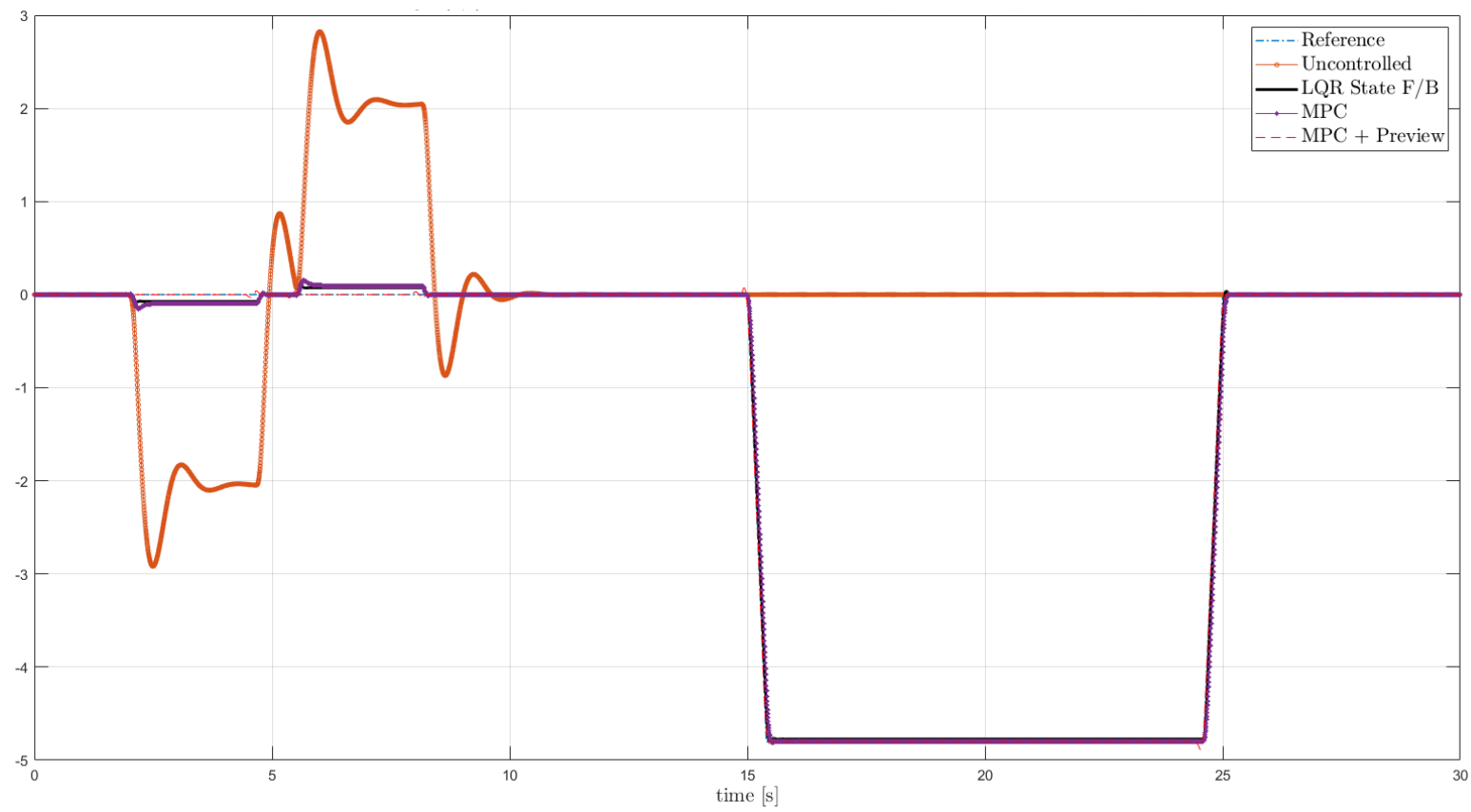

Figure 5.6. Uncontrolled vs controlled systems comparison, $10 \mathrm{kph}, 0.15 \mathrm{~m}$ depth, the horizontal axis is time, vertical axis is pitch angle [deg] 


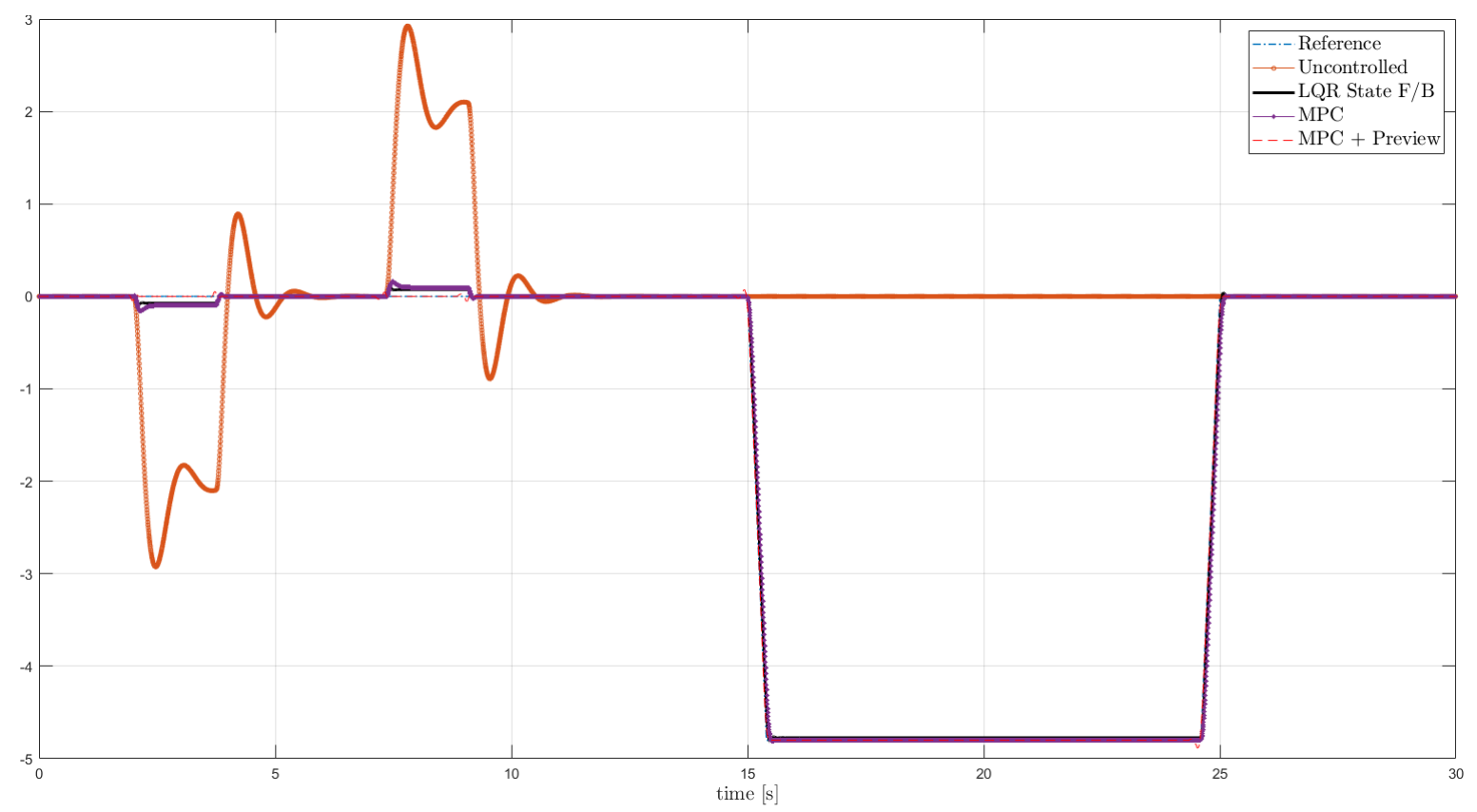

Figure 5.7. Uncontrolled vs controlled systems comparison, $20 \mathrm{kph}, 0.15 \mathrm{~m}$ depth, the horizontal axis is time, vertical axis is pitch angle [deg]

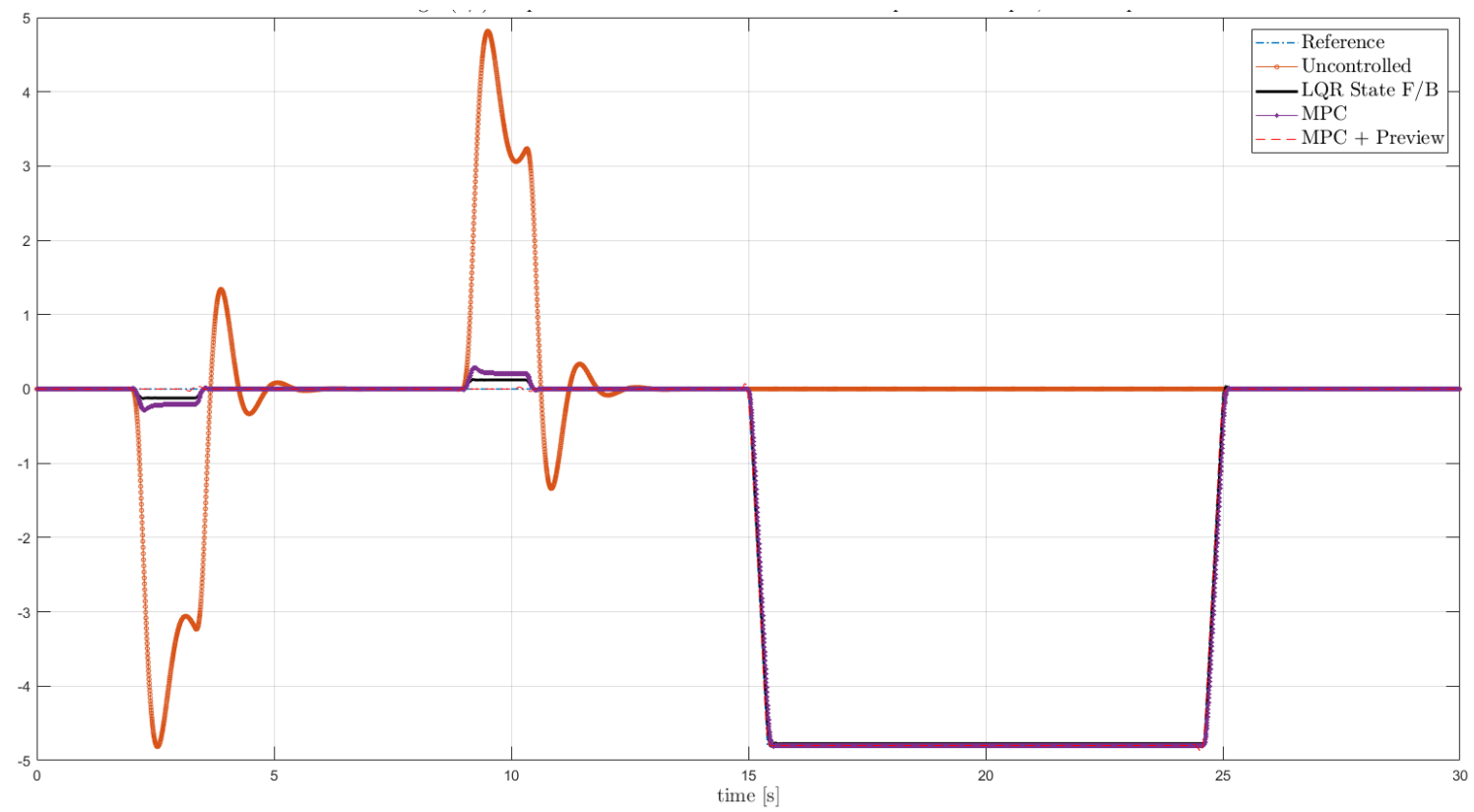

Figure 5.8. Uncontrolled vs controlled systems comparison, $5 \mathrm{kph}, 0.25 \mathrm{~m}$ depth, the horizontal axis is time, vertical axis is pitch angle [deg] 


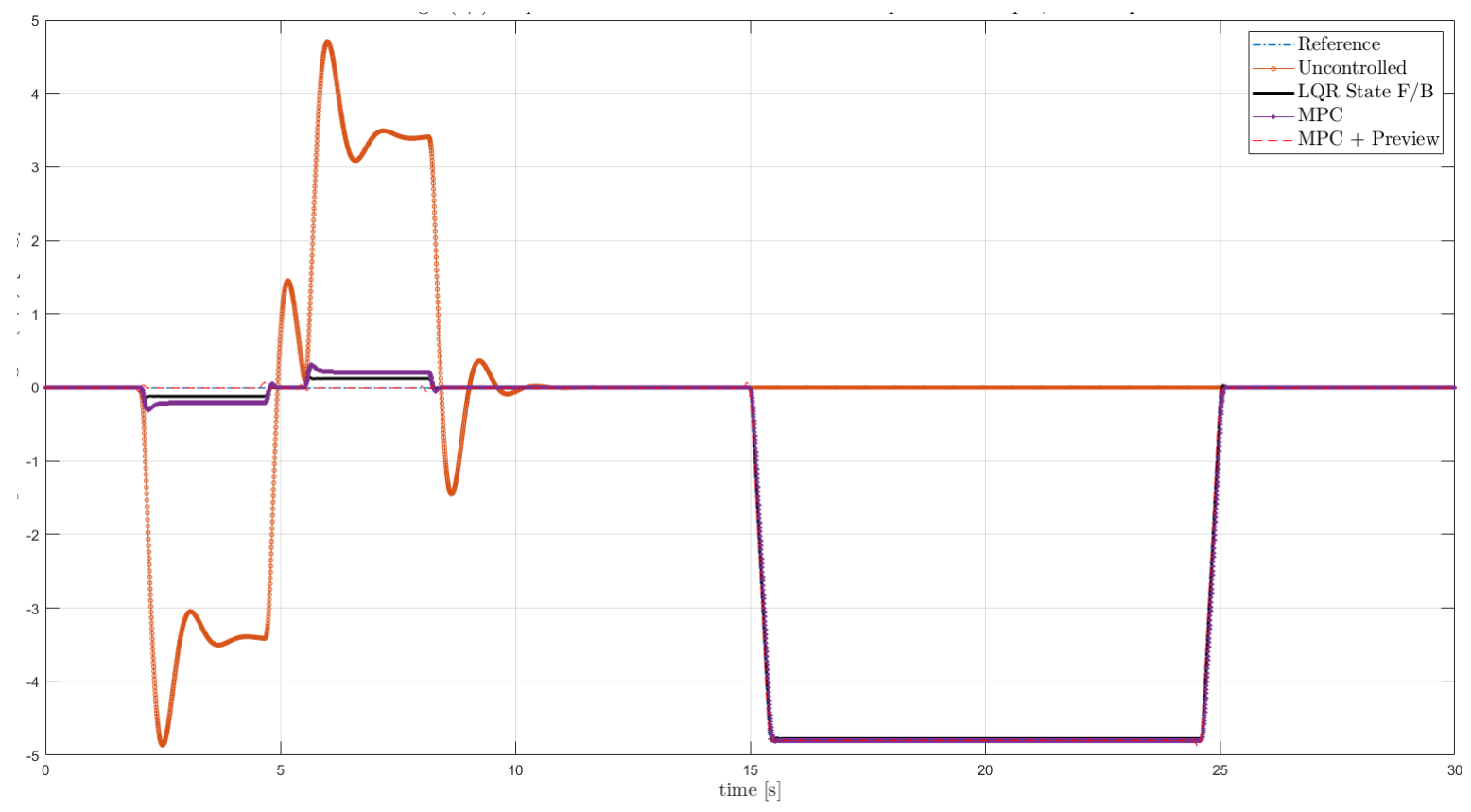

Figure 5.9. Uncontrolled vs controlled systems comparison, $10 \mathrm{kph}, 0.25 \mathrm{~m}$ depth, the horizontal axis is time, vertical axis is pitch angle [deg]

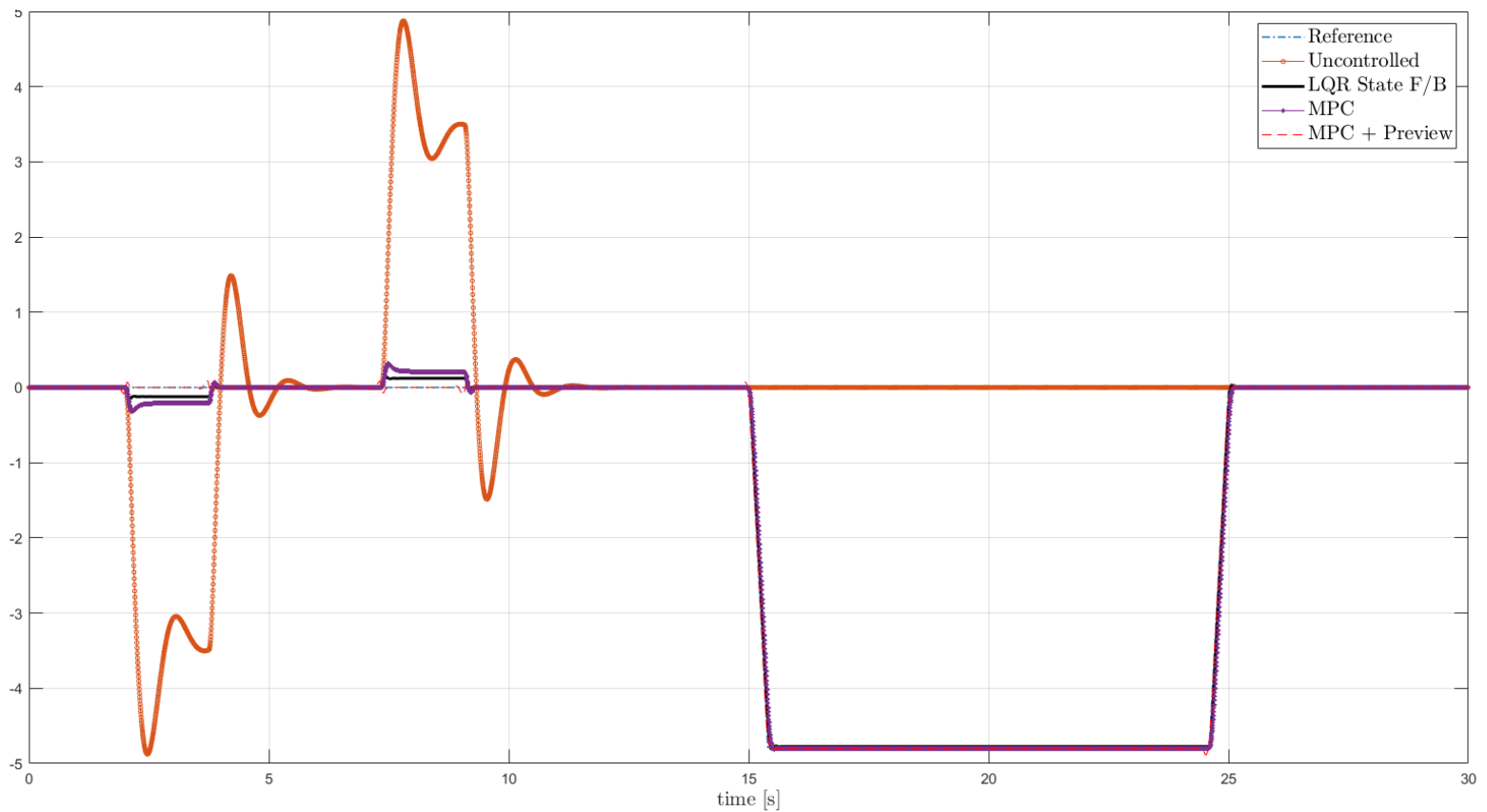

Figure 5.10. Uncontrolled vs controlled systems comparison, $10 \mathrm{kph}, 0.25 \mathrm{~m}$ depth, the horizontal axis is time, vertical axis is pitch angle [deg] 
Although values differ in simulations, the behavior is similar in almost all simulations. For this reason, the figures that show the behaviour of the controllers in more detail are given below for a sample result.(Figure 5.5)

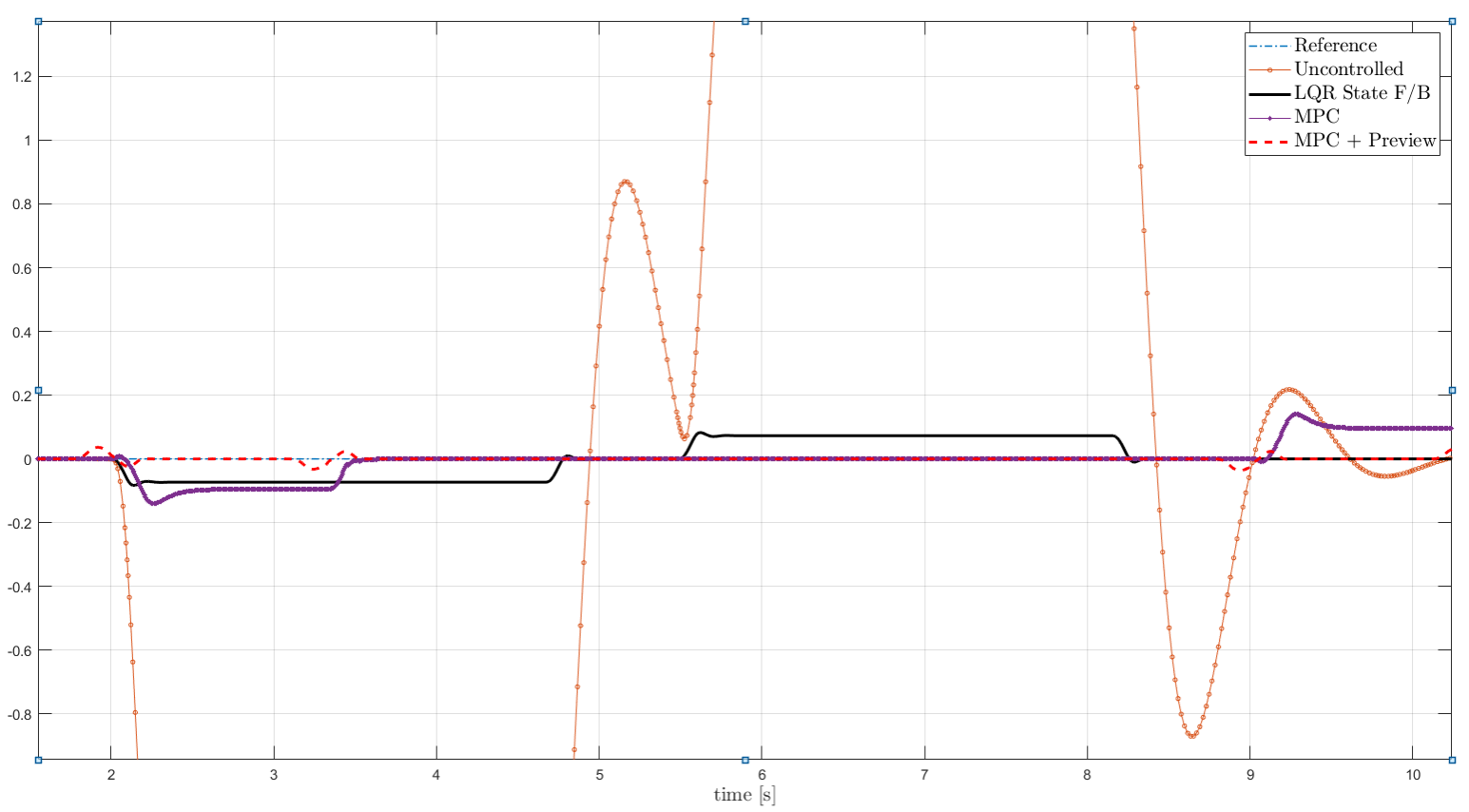

Figure 5.11. Uncontrolled vs controlled systems comparison, $5 \mathrm{kph}, 0.15 \mathrm{~m}$ depth, focused on disturbance section, the horizontal axis is time, vertical axis is pitch angle [deg]

It is evident that MPC+Preview control performs the best disturbance rejection. Another point to note is that MPC+Preview takes action for response before encountering the obstacle. LQR and MPC without preview are close to each other. 


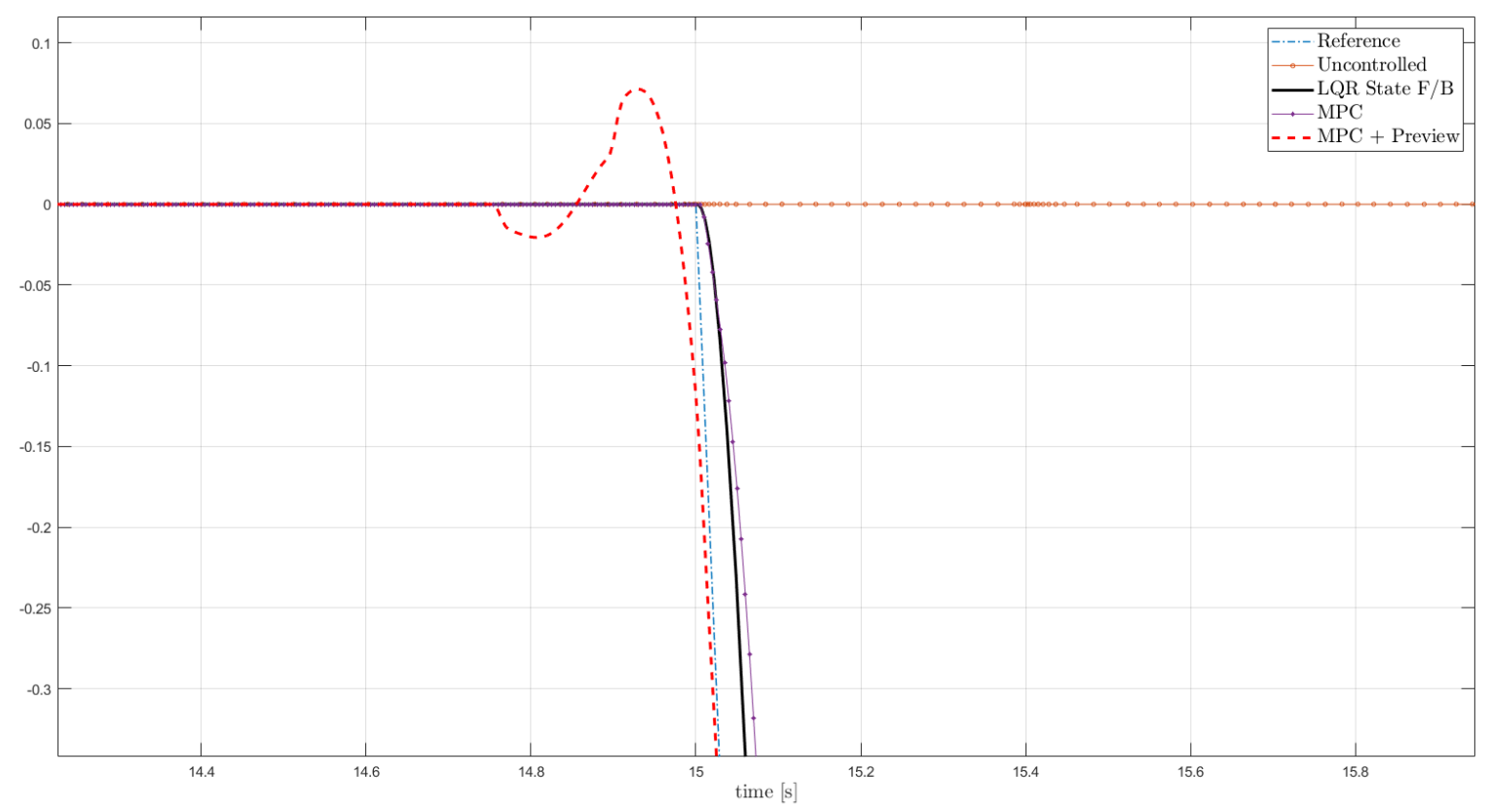

Figure 5.12. Uncontrolled vs controlled systems comparison, $5 \mathrm{kph}, 0.15 \mathrm{~m}$ depth, focused on reference tracking, 1

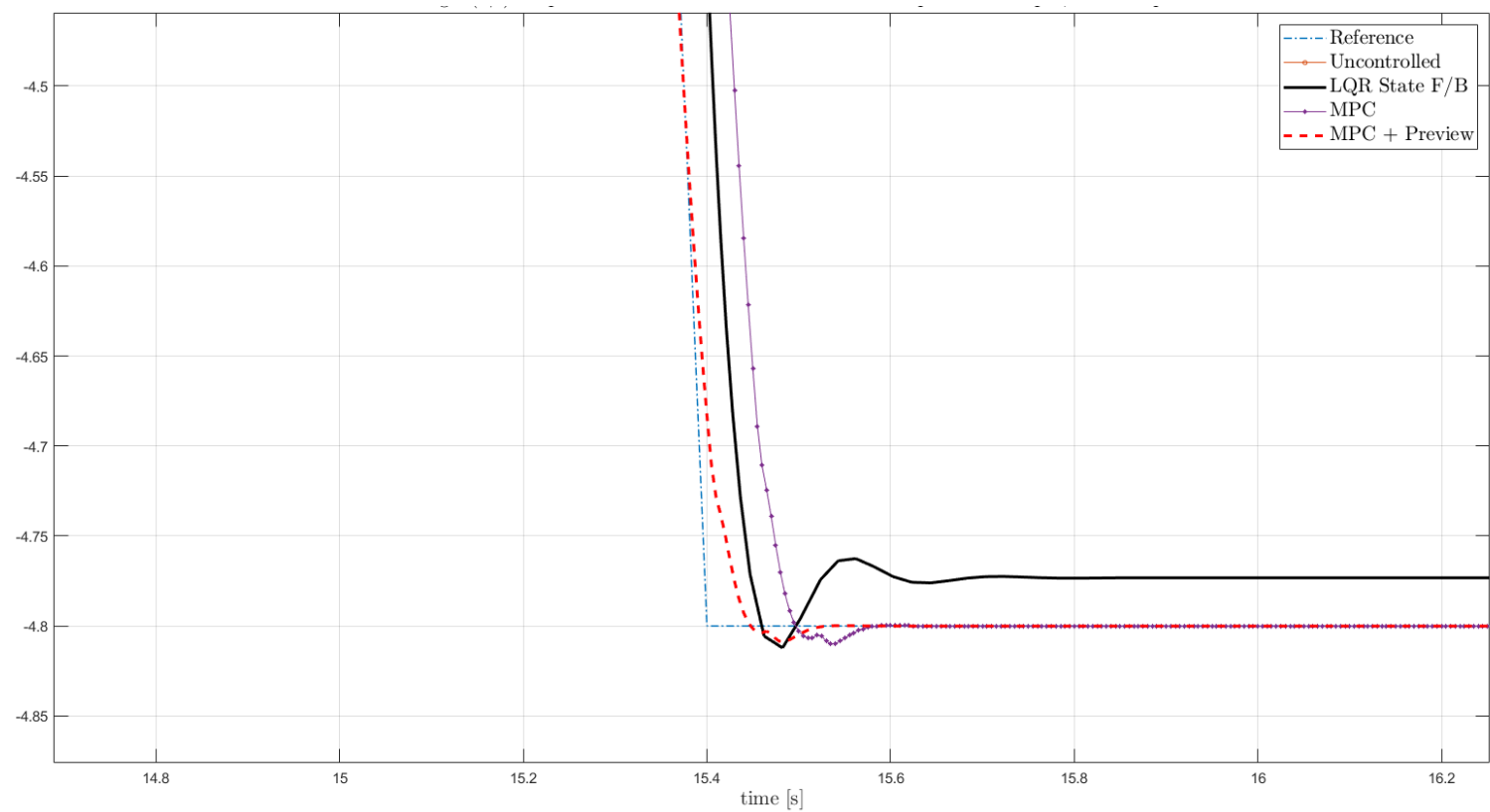

Figure 5.13. Uncontrolled vs controlled systems comparison, $5 \mathrm{kph}, 0.15 \mathrm{~m}$ depth, focused on reference tracking, 2, the horizontal axis is time, vertical axis is pitch angle [deg]

Figure 5.12 also shows that MPC+Preview controller takes action before the reference. The $\mathrm{MPC}+$ Preview is the most successful in tracking the ramp reference. Regarding others, LQR has a little bit better performance in tracking the ramp. 
However, when looking at the settling performance, both MPC controllers are sufficient in terms of steady-state errors. At this point, it can be said that LQR is more unsuccessful in terms of steady-state error.

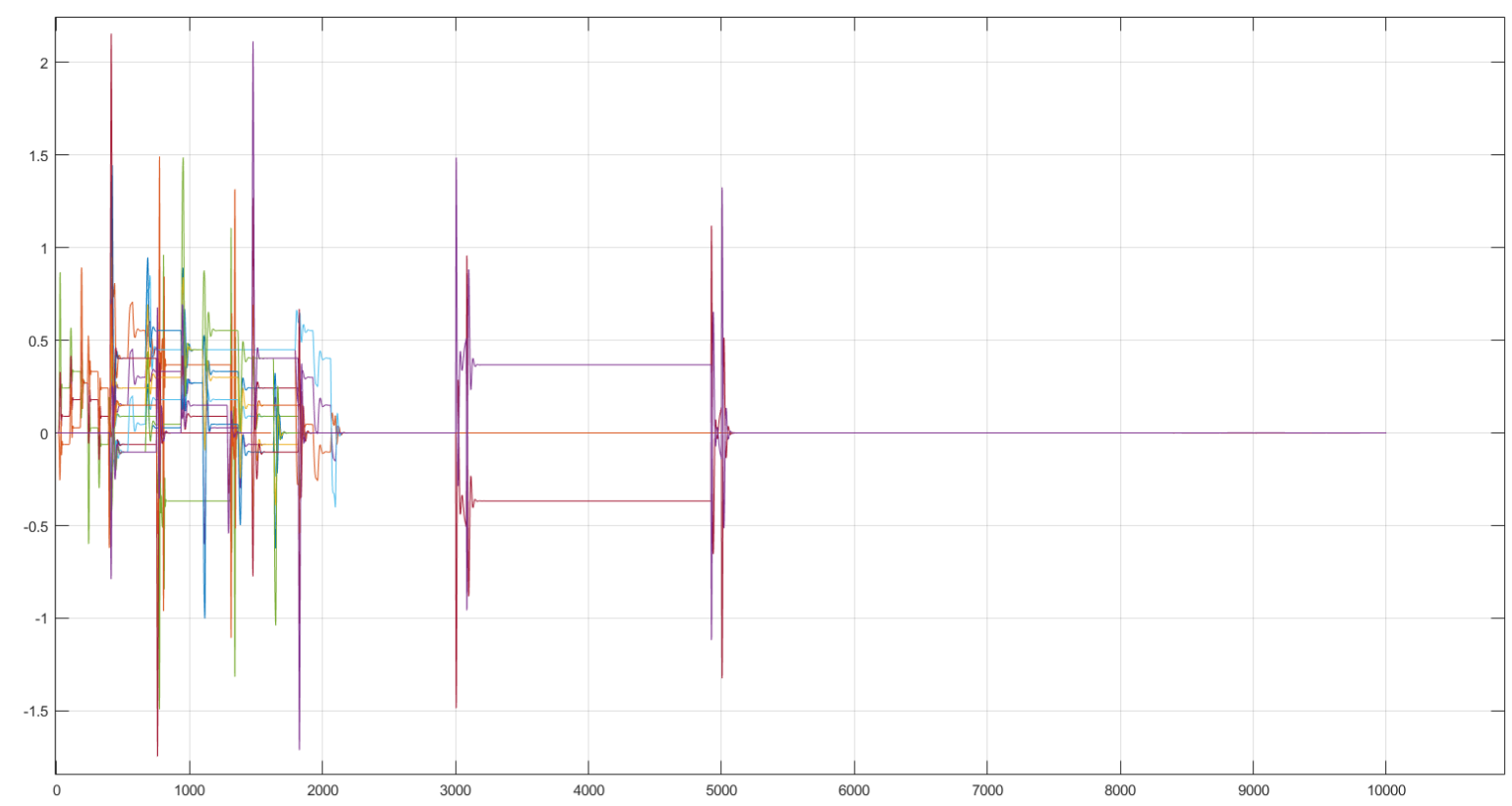

Figure 5.14. Control inputs comparison of the linear simulations, the vertical axis is Actuator Force [N], horizontal axis is data points

Figure 5.14 shows that almost all of the control inputs are in the actuator limits. There may be instant peaks; the softening constraints cause this. 


\subsection{Deep Trench Passive System Simulations}

The passive system simulation is given in Figure 5.15. It is shown that the $6 \times 6$ vehicle can only cross the trench of $1.4 \mathrm{~m}$ by hitting its front and rear bumpers.

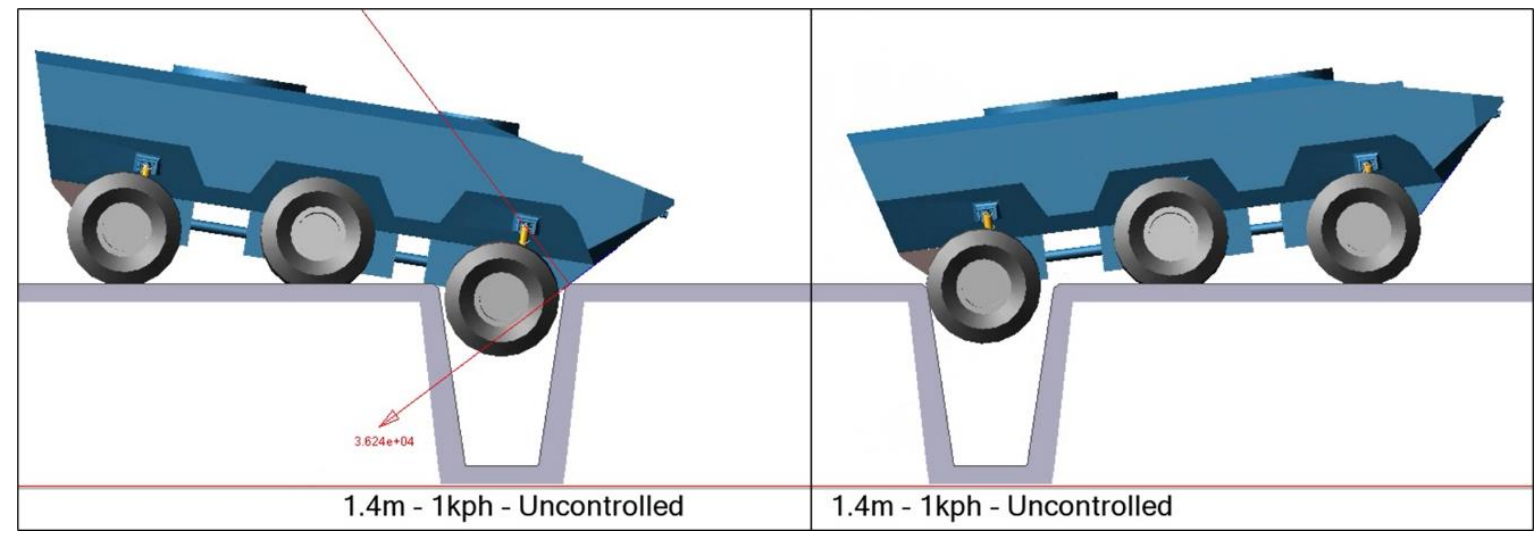

Figure 5.15. Uncontrolled trench crossing event, using bumpers $1.4 \mathrm{~m}, 1 \mathrm{kph}$

\begin{tabular}{|c|c|c|}
\hline 00:00:17 & 00:00:18 & 00:00:19 \\
\hline 00:00:20 & 00:00:21 & 00:00:23 \\
\hline 00:00:24 & 00:00:25 & 00:00:26 \\
\hline 00:00:27 & 00:00:28 & 00:00:30 \\
\hline 00:00:31 & 00:00:32 & 00:00:33 \\
\hline 00:00:34 & 00:00:37 & 00:00:40 \\
\hline
\end{tabular}

Figure 5.16. Uncontrolled vehicle, $1.4 \mathrm{~m}$ trench $-1 \mathrm{kph}$ 


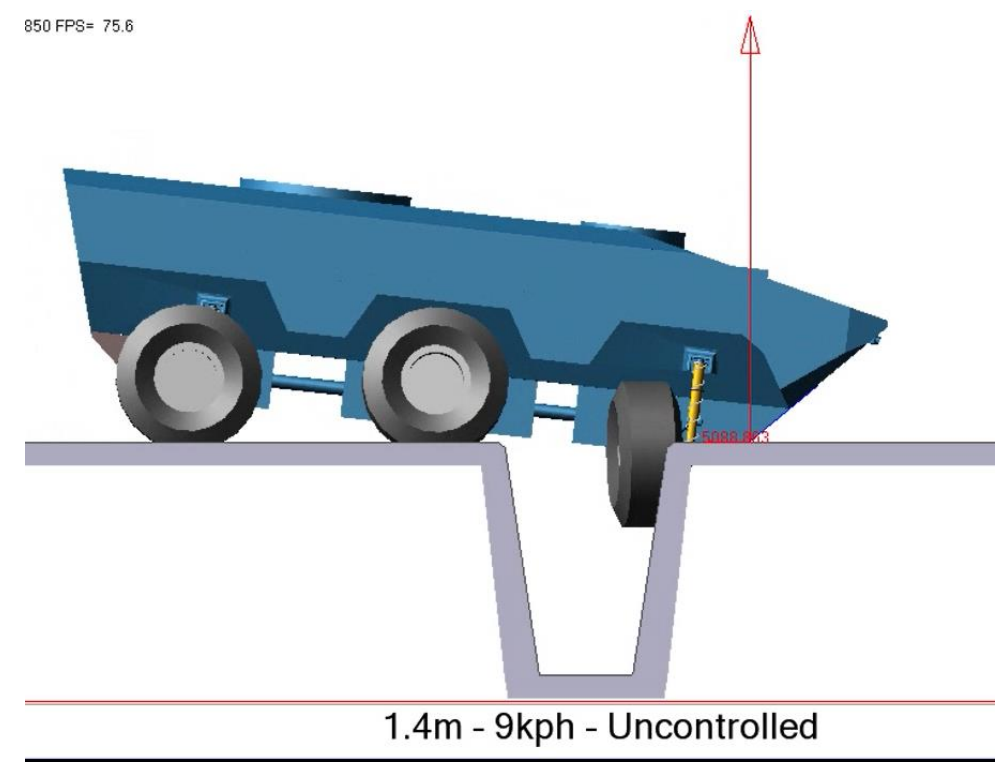

Figure 5.17. Uncontrolled vehicle, stuck in the trench, $1.4 m-9 k p h$

At first glance, it can be thought that vehicle can cross the obstacle by going faster. However, this may not always be the case. In the figure, an uncontrolled vehicle trying to pass through $1.4 \mathrm{~m}$ long trench is simulated at two different speeds. (Figure 5.16 and Figure 5.17) While the vehicle could pass the trench at $1 \mathrm{kph}$ speed, the first axle was stuck inside the trench at 9 $\mathrm{kph}$ and could not cross the trench. Here, there is an assumption that constant angular velocity is applied to the wheels of the vehicles.

The trench crossing algorithm with MPC + preview is simulated for different speeds and different trench lengths. These controlled systems are also simulated for the passive system with the same scenarios for comparison. The simulation matrix for uncontrolled systems is given in Table 5.2. 
Table 5.2. Uncontrolled system simulation matrix, ADAMS plant

\begin{tabular}{|c|c|c|c|c|c|c|c|c|c|}
\hline $\begin{array}{c}\text { Trench } \\
\text { Lengths }\end{array}$ & $\mathbf{1 . 2} \mathbf{m}$ & $\mathbf{1 . 3} \mathbf{~ m}$ & $\mathbf{1 . 4} \mathbf{~ m}$ & $\mathbf{1 . 5} \mathbf{~ m}$ & $\mathbf{1 . 6} \mathbf{~ m}$ & $\mathbf{1 . 7} \mathbf{m}$ & $\mathbf{1 . 8} \mathbf{~ m}$ & $\mathbf{1 . 9} \mathbf{~ m}$ & $\mathbf{2 . 0} \mathbf{~ m}$ \\
\hline \multirow{3}{*}{$\begin{array}{c}\text { Vehicle } \\
\text { Speed }\end{array}$} & $1 \mathrm{kph}$ & $1 \mathrm{kph}$ & $1 \mathrm{kph}$ & $1 \mathrm{kph}$ & $1 \mathrm{kph}$ & $1 \mathrm{kph}$ & $1 \mathrm{kph}$ & $1 \mathrm{kph}$ & $1 \mathrm{kph}$ \\
\cline { 2 - 10 } & $3 \mathrm{kph}$ & $3 \mathrm{kph}$ & $3 \mathrm{kph}$ & $3 \mathrm{kph}$ & $3 \mathrm{kph}$ & $3 \mathrm{kph}$ & $3 \mathrm{kph}$ & $3 \mathrm{kph}$ & $3 \mathrm{kph}$ \\
\cline { 2 - 10 } & $9 \mathrm{kph}$ & $9 \mathrm{kph}$ & $6 \mathrm{kph}$ & $6 \mathrm{kph}$ & $6 \mathrm{kph}$ & $6 \mathrm{kph}$ & $6 \mathrm{kph}$ & $6 \mathrm{kph}$ & $6 \mathrm{kph}$ \\
\hline
\end{tabular}

The trench crossing criteria of uncontrolled vehicles are divided into two. The first is that the sub-systems such as steering mechanisms, differentials, shafts located in the lower part of the vehicle should not hit the entrance or exit of the trench. Secondly, some acceleration and force values are determined as passing criteria and therefore, design criteria.

The sub-systems placed at the bottom of the vehicle are modelled as solid blocks in the vehicle body. There are differentials, shafts, steering gearboxes, and steering mechanisms. These dummy blocks are given in Figure 5.18.

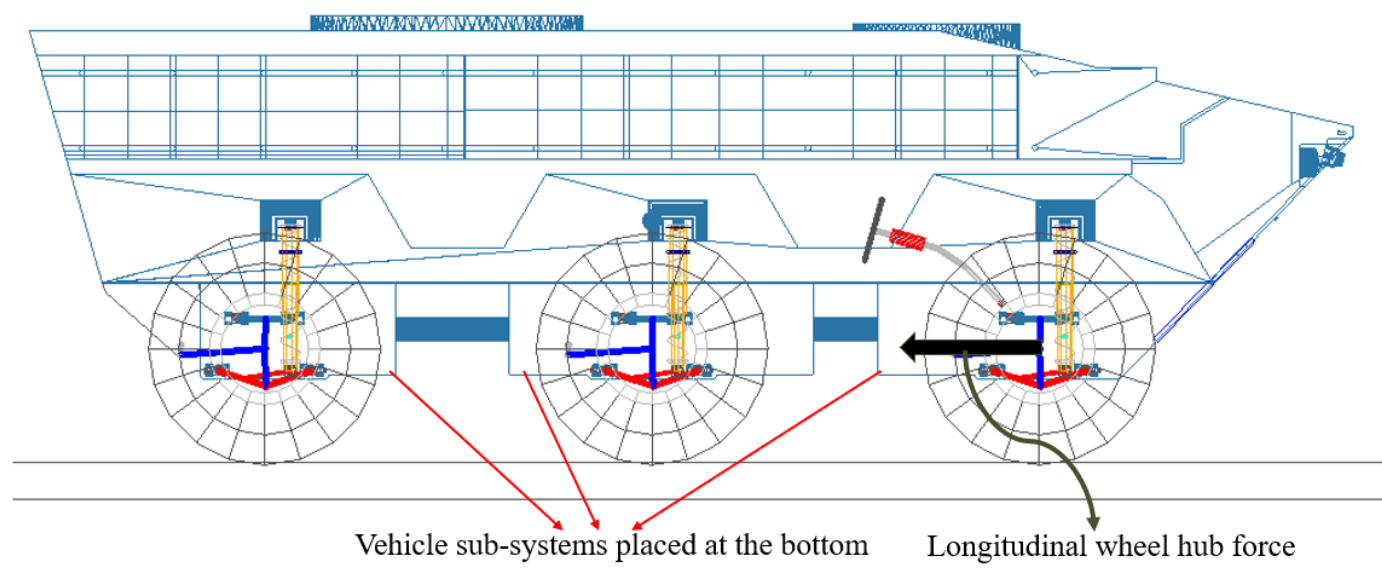

Figure 5.18. Vehicle sub-systems modelled as solid blocks and longitudinal wheel hub force 
In Table 5.3, the limit values of some parameters based on the trench transition of the vehicle are given. $\mathbf{a}$ and al are body vertical and longitudinal accelerations. RMS (Root Mean Square) values are based on PSD (Power Spectral Density) of the acceleration signals. $\gamma$ is the body pitch angle. $F_{\text {hub,f }}, F_{\text {hub,m }}$ and $F_{\text {hub,r }}$ are the longitudinal forces acting on wheel hubs respectively (Figure 5.18). These values are selected by considering the average structural design criteria of the real system and the approximate loads exposed in the actual trench crossing conditions.

Table 5.3. Uncontrolled system trench cross criteria

\begin{tabular}{|c|c|c|c|c|c|c|c|c|}
\hline $\begin{array}{c}\text { Paramete } \\
\mathbf{r}\end{array}$ & $\begin{array}{c}\text { Max. } \\
\mathbf{a z} \\
{\left[\mathbf{m} / \mathbf{s}^{2}\right]}\end{array}$ & $\begin{array}{c}\text { Max. } \\
\mathbf{a l} \\
{\left[\mathbf{m} / \mathbf{s}^{2}\right]}\end{array}$ & $\begin{array}{c}\text { RMS } \\
\mathbf{a z} \\
{\left[\mathbf{A}^{2} / \mathbf{H z}\right.} \\
]\end{array}$ & $\begin{array}{c}\text { RMS } \\
\mathbf{a l} \\
{\left[\mathbf{A}^{\mathbf{2}} / \mathbf{H z}\right.} \\
]\end{array}$ & $\begin{array}{c}\text { Max. } \\
\boldsymbol{\gamma} \\
{[\mathbf{d e g}]}\end{array}$ & $\begin{array}{c}\text { Max. } \\
\mathbf{F}_{\text {hub,f }} \\
{[\mathbf{N}]}\end{array}$ & $\begin{array}{c}\text { Max. } \\
\mathbf{F}_{\text {hub, }} \\
\mathbf{m} \\
{[\mathbf{N}]}\end{array}$ & $\begin{array}{c}\text { Max. } \\
\mathbf{F}_{\text {hub,r }} \\
{[\mathbf{N}]}\end{array}$ \\
\hline $\begin{array}{c}\text { Threshol } \\
\mathbf{d}\end{array}$ & 150 & 100 & 5 & 5 & 15 & 225 & 225 & 225 \\
\hline
\end{tabular}

The design parameters for all uncontrolled trench cross scenarios, their numerical values and whether the trench crossing is successful or not are shown in Table 5.4. 
Table 5.4. Comparison of all uncontrolled trench cross operations

\begin{tabular}{|c|c|c|c|c|c|c|c|c|c|c|}
\hline $\begin{array}{l}\text { Trench } \\
\text { Length }\end{array}$ & Speed & $\begin{array}{c}\text { Max. } \\
\mathbf{a}_{\mathbf{z}} \\
{\left[\mathbf{m} / \mathbf{s}^{2}\right]}\end{array}$ & $\begin{array}{c}\text { Max. } \\
\mathbf{a}_{1} \\
{\left[\mathrm{~m} / \mathrm{s}^{2}\right]}\end{array}$ & $\begin{array}{c}\text { RMS } \\
\mathbf{a}_{\mathbf{z}} \\
{\left[\mathrm{A}^{2} / \mathbf{H z}\right]}\end{array}$ & $\begin{array}{c}\text { RMS } \\
\mathbf{a}_{\mathbf{l}} \\
{\left[\mathrm{A}^{2} / \mathbf{H z}\right]}\end{array}$ & $\begin{array}{c}\text { Max. } \\
\gamma \\
{[\mathrm{deg}]}\end{array}$ & $\begin{array}{c}\text { Max. } \\
\text { F }_{\text {hub,f }} \\
{[k N]}\end{array}$ & $\begin{array}{c}\text { Max. } \\
\text { F }_{\text {hub,m }} \\
{[\mathrm{kN}]}\end{array}$ & $\begin{array}{c}\text { Max. } \\
\text { F }_{\text {hub,r }} \\
{[\mathrm{kN}]}\end{array}$ & STATUS \\
\hline \multirow{4}{*}{$1.2 \mathrm{~m}$} & $1 \mathrm{kph}$ & 49.6 & 24.3 & 0.8 & 0.5 & 9.6 & 64.9 & 38.2 & 56.8 & $\checkmark$ PASSED \\
\hline & $3 \mathrm{kph}$ & 56.7 & 22.9 & 1.1 & 1.0 & 8.9 & 85.5 & 40.0 & 48.1 & $\checkmark$ PASSED \\
\hline & $6 \mathrm{kph}$ & 10.4 & 14.3 & 1.5 & 2.4 & 10.8 & 146.2 & 42.7 & 46.1 & $\checkmark$ PASSED \\
\hline & $9 \mathrm{kph}$ & 6.1 & 5.6 & 1.8 & 1.5 & 4.9 & 89.3 & 23.9 & 28.4 & $\checkmark$ PASSED \\
\hline \multirow{4}{*}{$1.3 \mathrm{~m}$} & $1 \mathrm{kph}$ & 73.1 & 64.7 & 1.3 & 1.1 & 10.3 & 64.3 & 38.0 & 59.7 & $\checkmark$ PASSED \\
\hline & $3 \mathrm{kph}$ & 134.8 & 37.0 & 2.0 & 1.1 & 11.0 & 71.0 & 42.1 & 57.1 & $\checkmark$ PASSED \\
\hline & $6 \mathrm{kph}$ & 109.9 & 79.8 & 3.4 & 2.9 & 11.2 & 201.5 & 42.7 & 53.1 & $\checkmark$ PASSED \\
\hline & $9 \mathrm{kph}$ & 6.8 & 7.7 & 1.9 & 1.8 & 6.5 & 122.4 & 34.0 & 37.0 & $\checkmark$ PASSED \\
\hline \multirow{4}{*}{$1.4 \mathrm{~m}$} & $1 \mathrm{kph}$ & 71.5 & 66.5 & 1.3 & 1.2 & 11.1 & 63.1 & 39.3 & 59.7 & $\checkmark$ PASSED \\
\hline & $3 \mathrm{kph}$ & 193.3 & 284.3 & 3.9 & 4.4 & 10.8 & 150.1 & 42.7 & 53.8 & * FAILED \\
\hline & $6 \mathrm{kph}$ & 152.7 & 72.5 & 3.6 & 3.0 & 11.5 & 170.9 & 42.7 & \begin{tabular}{|l|}
142.4 \\
\end{tabular} & FAILED \\
\hline & $9 \mathrm{kph}$ & 191.8 & 119.4 & 8.2 & 5.8 & 10.9 & 378.9 & 42.6 & 38.3 & FAILED \\
\hline \multirow{4}{*}{1.5} & $1 \mathrm{kph}$ & 63.7 & 66.9 & 0.7 & 0.7 & 11.9 & 61.6 & 39.0 & 60.9 & $\checkmark$ PASSED \\
\hline & $3 \mathrm{kph}$ & 209.9 & 299.9 & 2.2 & 2.4 & 10.9 & 152.1 & 42.7 & 52.1 & FAILED \\
\hline & $6 \mathrm{kph}$ & 160.1 & 44.3 & 6.2 & 2.9 & 10.7 & 163.2 & 38.1 & 13.6 & $\times$ FAILED \\
\hline & $9 \mathrm{kph}$ & 165.3 & 66.4 & 5.3 & 4.9 & 13.9 & 324.9 & 42.6 & 139.0 & $\sim$ FAILED \\
\hline \multirow{4}{*}{$1.6 \mathrm{~m}$} & $1 \mathrm{kph}$ & 64.8 & 63.3 & 0.6 & 0.6 & 12.9 & 63.0 & 39.9 & 60.8 & $\checkmark$ PASSED \\
\hline & $3 \mathrm{kph}$ & 146.1 & 197.8 & 2.1 & 2.5 & 11.1 & 145.6 & 42.7 & 58.5 & FAILED \\
\hline & $6 \mathrm{kph}$ & 510.6 & \begin{tabular}{|l|}
392.7 \\
\end{tabular} & 4.1 & 4.0 & 11.6 & 43.5 & 42.7 & 148.2 & FAILED \\
\hline & $9 \mathrm{kph}$ & 152.6 & 71.3 & 5.2 & 4.3 & 14.2 & 295.1 & 42.6 & 53.8 & × FAILED \\
\hline \multirow{4}{*}{$1.7 \mathrm{~m}$} & $1 \mathrm{kph}$ & 68.3 & 86.4 & 1.2 & 1.6 & 88.0 & 63.3 & 45.6 & 126.1 & ₹ FAILED \\
\hline & $3 \mathrm{kph}$ & 182.9 & 223.0 & 1.9 & 2.1 & 11.9 & 123.9 & 42.0 & 61.7 & FAILED \\
\hline & $6 \mathrm{kph}$ & 348.0 & 387.3 & 4.0 & 5.9 & 15.6 & 39.5 & 42.7 & 144.9 & FAILED \\
\hline & $9 \mathrm{kph}$ & 147.2 & 74.6 & 5.4 & 4.2 & 14.4 & 282.9 & 42.6 & 89.9 & FAILED \\
\hline \multirow{4}{*}{$1.8 \mathrm{~m}$} & $1 \mathrm{kph}$ & 78.3 & 84.1 & 1.6 & 1.7 & 43.1 & 63.5 & 39.9 & 122.7 & × FAILED \\
\hline & $3 \mathrm{kph}$ & 144.9 & 182.8 & 3.1 & 3.5 & 13.2 & 74.2 & 42.5 & 58.2 & ₹ FAILED \\
\hline & $6 \mathrm{kph}$ & 425.6 & \begin{tabular}{|l|}
477.7 \\
\end{tabular} & 11.2 & 12.8 & 15.4 & 41.7 & 41.9 & 142.6 & FAILED \\
\hline & $9 \mathrm{kph}$ & 184.4 & 74.4 & 6.5 & 4.2 & 14.5 & 278.0 & 42.6 & 106.5 & ₹ FAILED \\
\hline \multirow{4}{*}{$1.9 \mathrm{~m}$} & $1 \mathrm{kph}$ & 68.0 & 88.3 & 2.0 & 1.7 & 89.9 & 205.9 & 53.4 & 144.0 & ₹ FAILED \\
\hline & $3 \mathrm{kph}$ & 203.9 & 264.3 & 2.7 & 2.7 & 14.6 & 62.5 & 42.7 & 56.6 & × FAILED \\
\hline & $6 \mathrm{kph}$ & 440.6 & 461.5 & 6.8 & 6.5 & 89.9 & 46.1 & 174.5 & 183.4 & FAILED \\
\hline & $9 \mathrm{kph}$ & 508.4 & 273.6 & 10.3 & 6.0 & 8.0 & 90.3 & 53.3 & 151.2 & ₹ FAILED \\
\hline \multirow{4}{*}{$2.0 \mathrm{~m}$} & $1 \mathrm{kph}$ & 83.8 & 160.6 & 1.3 & 2.2 & 16.1 & 97.5 & 37.8 & 18.9 & ₹ FAILED \\
\hline & $3 \mathrm{kph}$ & 153.0 & 204.5 & 4.3 & 4.9 & 90.0 & 57.0 & 42.9 & 164.6 & * FAILED \\
\hline & $6 \mathrm{kph}$ & 376.0 & 382.4 & 7.0 & 7.2 & 90.0 & 156.2 & 56.0 & 173.2 & * FAILED \\
\hline & $9 \mathrm{kph}$ & 543.1 & 513.0 & 15.0 & 18.0 & 27.9 & 38.1 & 42.7 & \begin{tabular}{|l|}
291.4 \\
\end{tabular} & FAILED \\
\hline
\end{tabular}


As mentioned in the previous sections, $6 \times 6$ vehicles can cross the trenches of limited lengths almost by stopping, or by hitting the front and rear bumpers on the ground at very low speeds. As can be seen in Table 5.4, the trenches of 1.2 and $1.3 \mathrm{~m}$ long, which are close to the vehicle wheel diameter, can pass smoothly at speeds up to $10 \mathrm{kph}$, which are considered as crosscountry speed. In trenches larger than the tire diameter, vehicles can cross trenches by hitting their bumpers to the ground at very low speeds depending on the vehicle's centre of gravity, axle layout and suspension characteristics. These trench lengths are $1.4 \mathrm{~m}, 1.5 \mathrm{~m}$ and $1.6 \mathrm{~m}$. The trench crossing operation of $1.4 \mathrm{~m}$ is given in Figure 5.15. Others are given below.

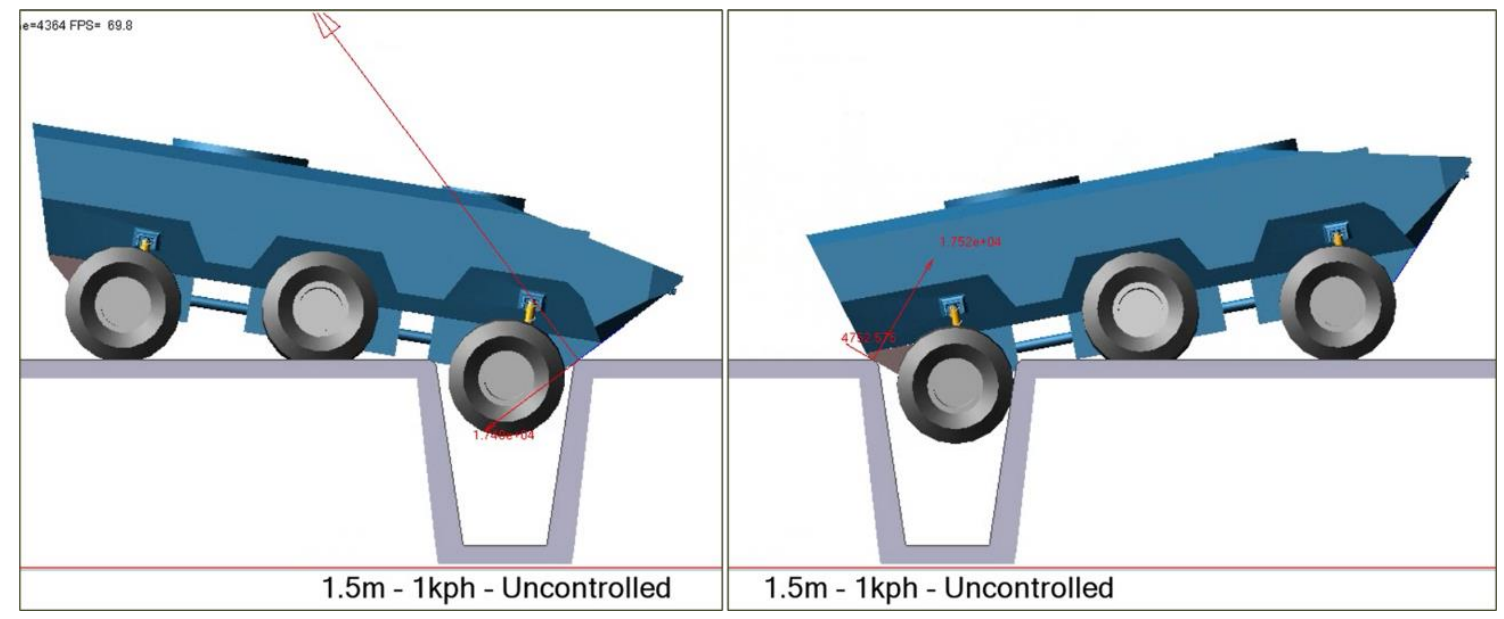

Figure 5.19. Uncontrolled trench crossing event, using bumpers $1.5 \mathrm{~m}-1 \mathrm{kph}$

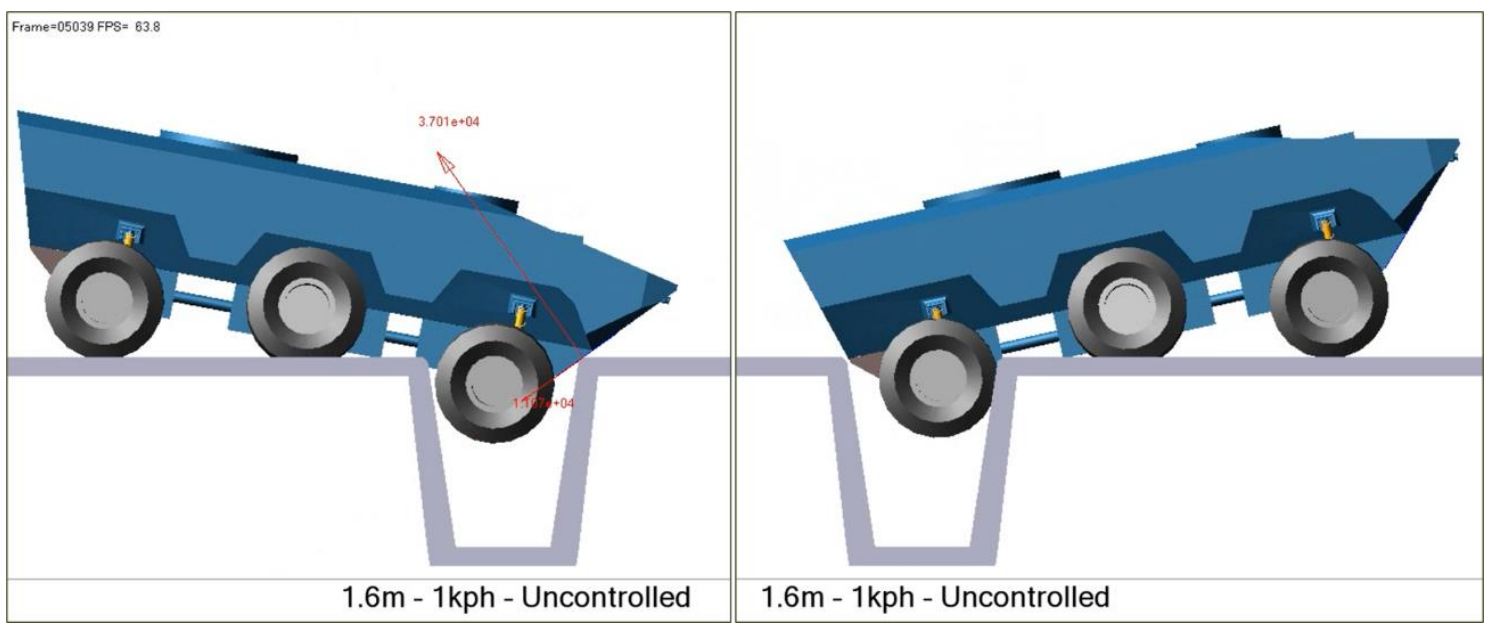

Figure 5.20. Uncontrolled trench crossing event, using bumpers $1.6 \mathrm{~m}-1 \mathrm{kph}$

As seen in Figure 5.19 and Figure 5.20, the trench limit for the specified vehicle at low speed is almost $1.5 \mathrm{~m}$ to $1.6 \mathrm{~m}$, both in terms of loads and accelerations on it and physically protecting the lower parts. 
At longer trenches, the vehicle crashes its lower sub-systems at low speeds. As the trench length increases a little more, it cannot go out from the trench by using the traction on the wheels. Again, as the trench length and speed increase, both the accelerations on the body and the forces acting on the suspension system are of harmful dimensions. The data, plots and figures of the trench crossings at these lengths and speeds are given in the next part. They are also compared with the controlled system simulations.

\subsection{Deep Trench Active System Simulations}

In this section, simulations of controlled and uncontrolled systems are given comparatively for different scenarios given in the table below. (Table 5.5) The plant used is the non-linear ADAMS plant, and the controller is a preview supported MPC controller.

Table 5.5. Controlled system simulation matrix

\begin{tabular}{|c|c|c|c|c|c|c|c|c|c|}
\hline $\begin{array}{c}\text { Trench } \\
\text { Lengths }\end{array}$ & $\mathbf{1 . 2} \mathbf{~ m}$ & $\mathbf{1 . 3} \mathbf{~ m}$ & $\mathbf{1 . 4} \mathbf{~ m}$ & $\mathbf{1 . 5} \mathbf{~ m}$ & $\mathbf{1 . 6} \mathbf{~ m}$ & $\mathbf{1 . 7} \mathbf{~ m}$ & $\mathbf{1 . 8} \mathbf{~ m}$ & $\mathbf{1 . 9} \mathbf{~ m}$ & $\mathbf{2 . 0} \mathbf{~ m}$ \\
\hline \multirow{3}{*}{$\begin{array}{c}\text { Vehicle } \\
\text { Speed }\end{array}$} & $1 \mathrm{kph}$ & $1 \mathrm{kph}$ & $1 \mathrm{kph}$ & $\mathbf{1} \mathbf{k p h}$ & $1 \mathrm{kph}$ & $1 \mathrm{kph}$ & $1 \mathrm{kph}$ & $\mathbf{1 ~ k p h}$ & $1 \mathrm{kph}$ \\
\cline { 2 - 10 } & $3 \mathrm{kph}$ & $3 \mathrm{kph}$ & $3 \mathrm{kph}$ & $\mathbf{3} \mathbf{k p h}$ & $3 \mathrm{kph}$ & $3 \mathrm{kph}$ & $3 \mathrm{kph}$ & $3 \mathrm{kph}$ & $3 \mathrm{kph}$ \\
\cline { 2 - 10 } & $9 \mathrm{kph}$ & $6 \mathrm{kph}$ & $6 \mathrm{kph}$ & $6 \mathrm{kph}$ & $6 \mathrm{kph}$ & $\mathbf{6} \mathbf{k p h}$ & $6 \mathrm{kph}$ & $6 \mathrm{kph}$ & $6 \mathrm{kph}$ \\
\hline
\end{tabular}

System behaviour at different speeds for each trench length has been studied separately and is presented below as subtitles.

The results of all controlled systems are not included in this section. Results for several critical situations showing controller performance are presented. The scenarios with the results in this section are shown in bold text in Table 5.5. Comparison tables and comments for all simulations made in the comparison and results section are presented.

All controlled and uncontrolled simulation results, visuals and graphics of the deep trenches are given in the appendix. 


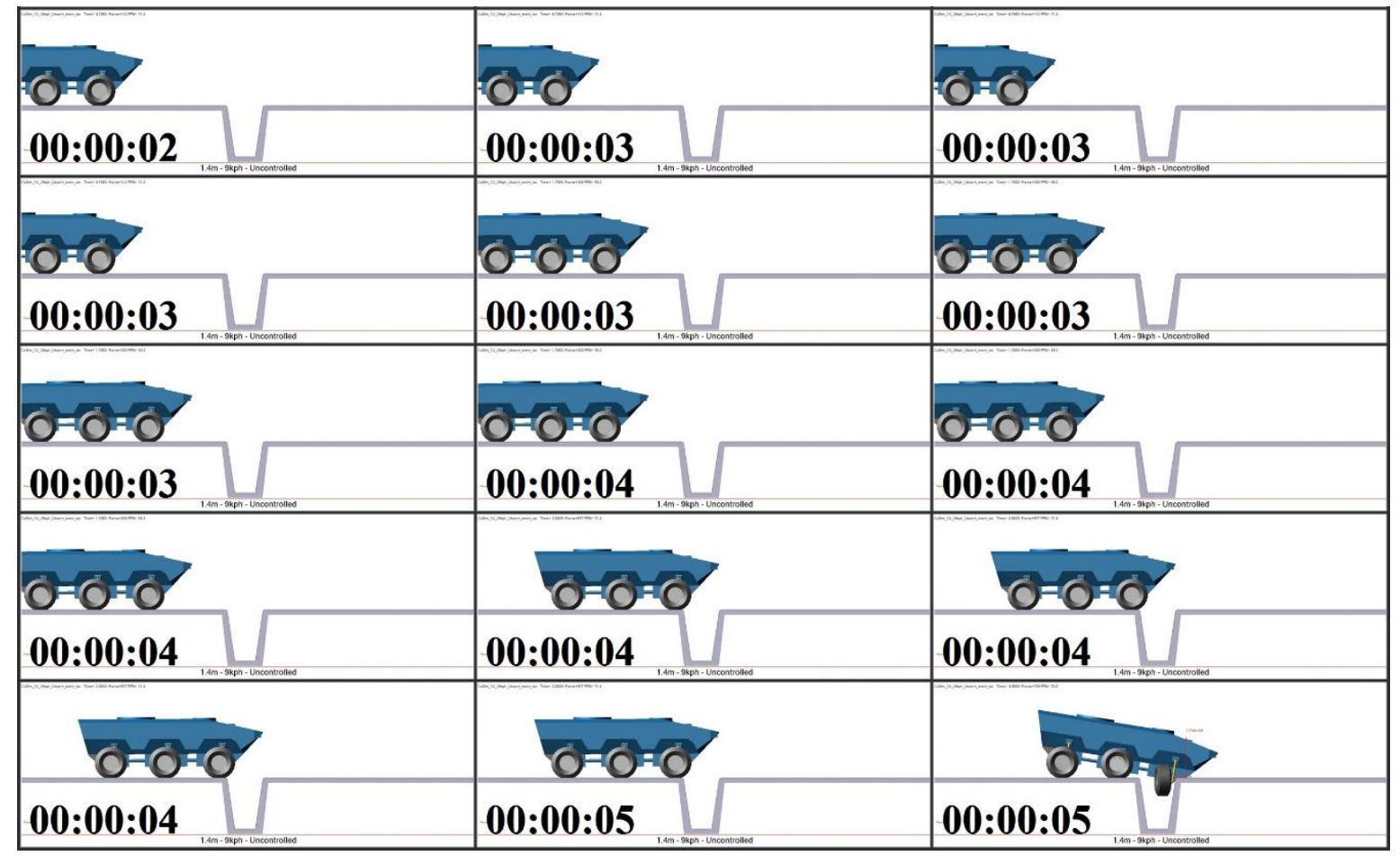

Figure 5.21. Trench crossing $1.4 \mathrm{~m}, 9 \mathrm{kph}$, uncontrolled

In the $1.4 \mathrm{~m}$ long trench, the vehicle cannot pass the trench as shown in Figure 5.21. At this speed, when the vehicle entered the trench, the force coming to the front axles caused the axles to break and the simulation failed.

At high speed (9 kph) and $1.4 \mathrm{~m}$ trench, the vehicle completes the operation without dropping the front and rear part into the trench as required by the trench cross algorithm. (Figure 5.22) 


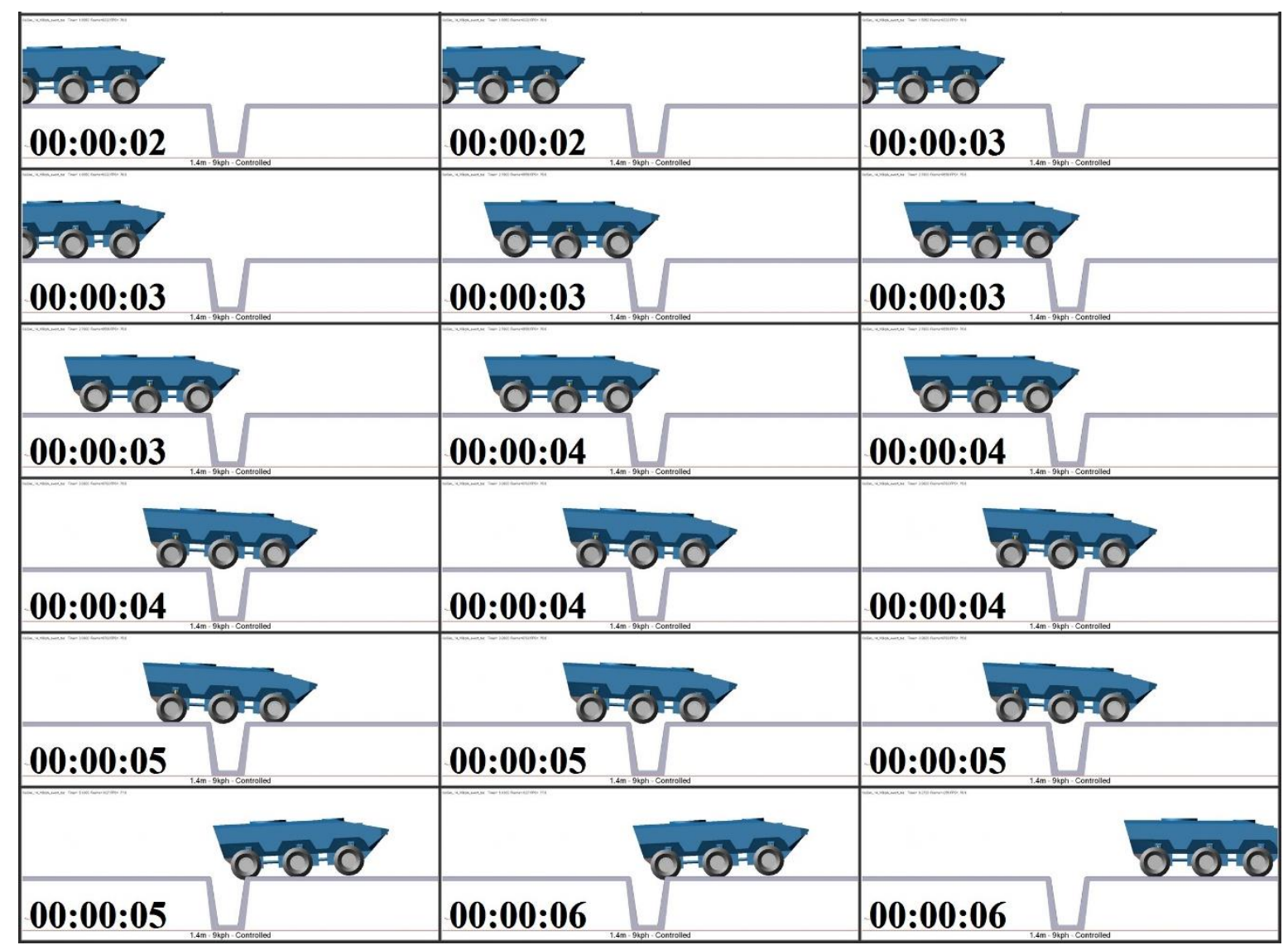

Figure 5.22. Trench crossing $1.4 \mathrm{~m}, 9 \mathrm{kph}$, controlled

Figure 5.23 shows the comparison of body acceleration response of controlled and uncontrolled systems. While the peak value of vertical acceleration in the uncontrolled system is $191.8 \mathrm{~m} / \mathrm{s}^{2}$, the maximum vertical acceleration in the controlled system is $7.1 \mathrm{~m} / \mathrm{s}^{2}$.

Similarly, regarding the longitudinal acceleration comparison, the peak values of uncontrolled and controlled systems are $119.4 \mathrm{~m} / \mathrm{s}^{2}$ and $8.6 \mathrm{~m} / \mathrm{s}^{2}$, respectively.

The figure also shows the comparison of pitch angles and pitch rate of controlled and uncontrolled systems when passing the trench. As can be seen in the plot, the maximum pitch angle is $10.9^{\circ}$ in the uncontrolled system, whereas it is $3.4^{\circ}$ in the controlled system. The vehicle cannot complete crossing the trench. 


\section{4 m Trench, 9 kph | Controlled vs Uncontrolled Output Comparison}

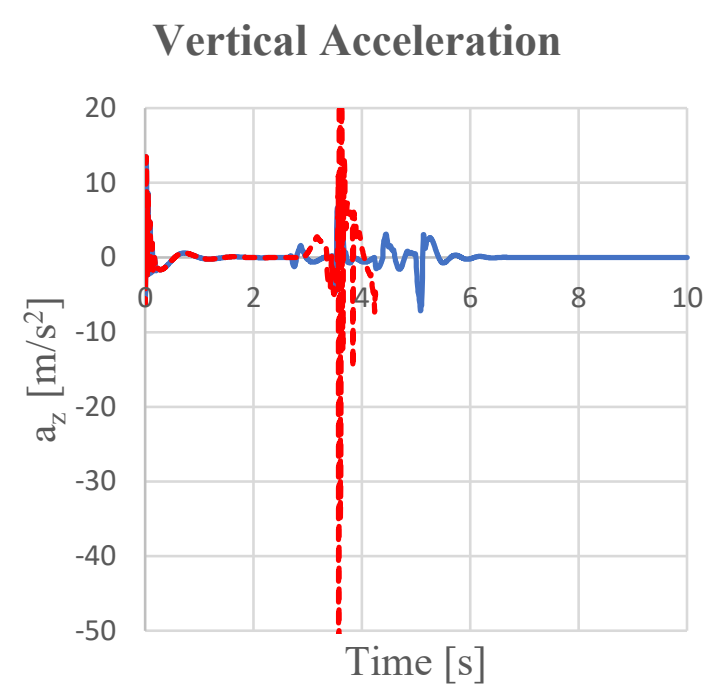

Longitudinal Acceleration

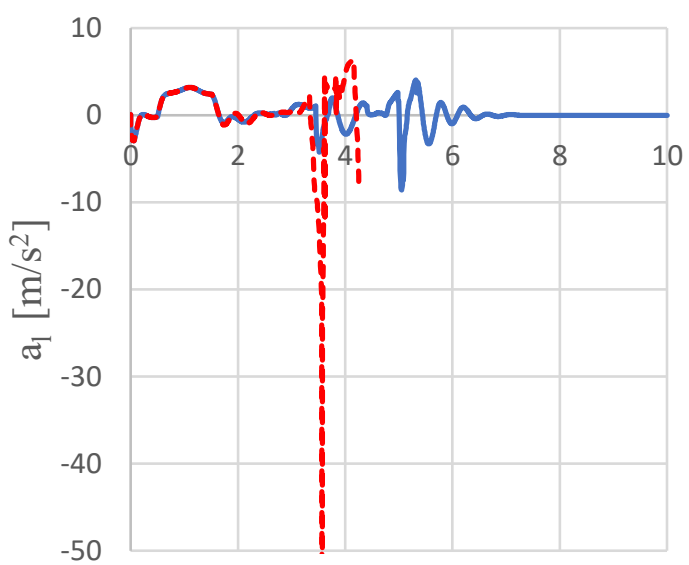

Time $[\mathrm{s}]$

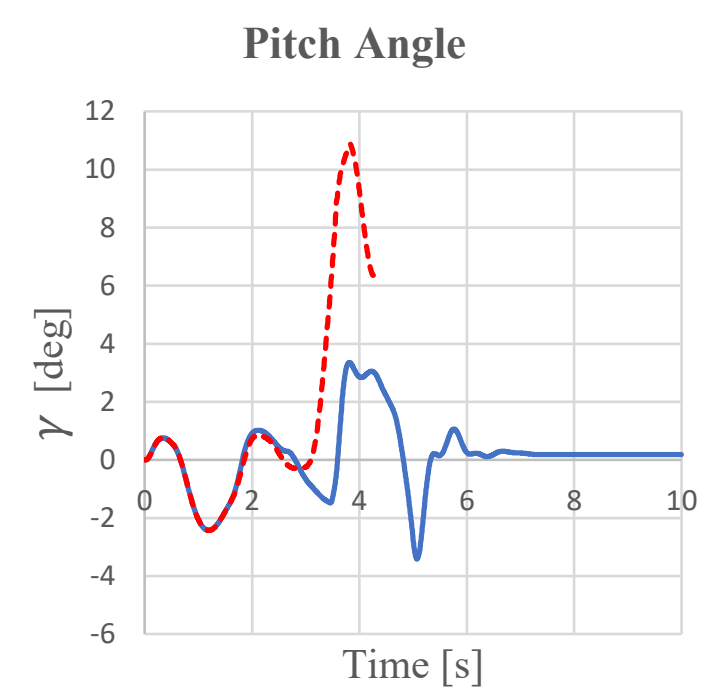

Pitch Angular Velocity

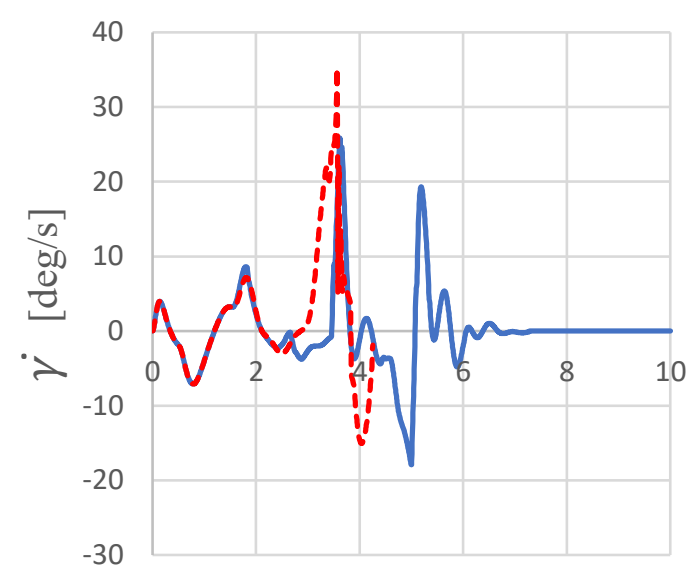

Time $[\mathrm{s}]$

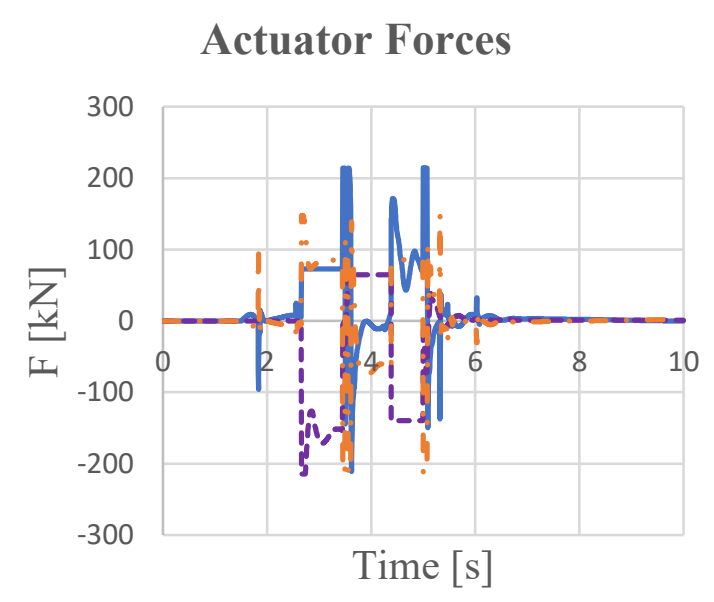

— Axle 1 ----A Axle 2 - - Axle 3

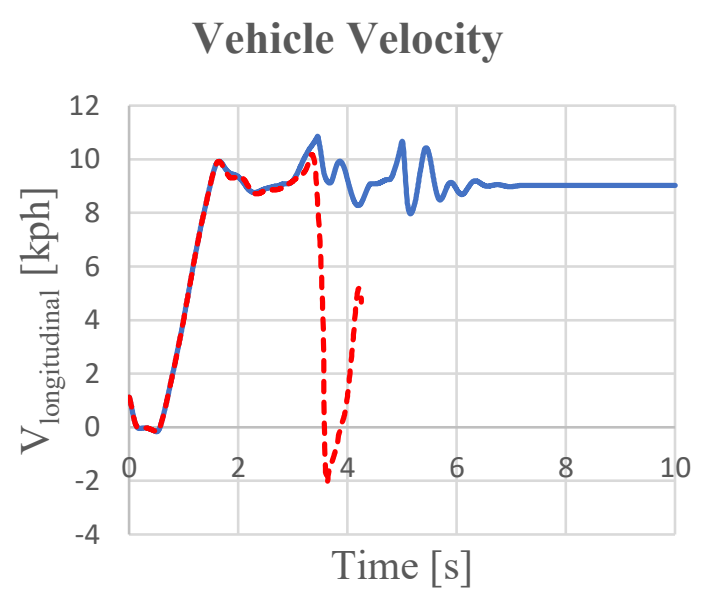

Controlled ------ Uncontrolled

Figure 5.23. Trench crossing $1.4 \mathrm{~m}, 9 \mathrm{kph}$, controlled $v$ s uncontrolled system results 
To make power spectral density comparisons, the RMS value in the uncontrolled system is $8.19 \mathrm{~A}^{2} / \mathrm{Hz}$, and it is $1.03 \mathrm{~A}^{2} / \mathrm{Hz}$ in the controlled system for body vertical acceleration. These RMS acceleration values for the longitudinal acceleration are $5.77 \mathrm{~A}^{2} / \mathrm{Hz}$ and 1.37 $\mathrm{A}^{2} / \mathrm{Hz}$, respectively where $\mathrm{A}$ is acceleration in $\mathrm{m} / \mathrm{s}^{2}$.

On the other hand, the maximum force acting on the wheel hub on the front axle during trench transition is about $379 \mathrm{kN}$ in the uncontrolled system and about $28 \mathrm{kN}$ in the controlled system.

5.5.2. $1.5 \mathrm{~m}$ Trench, $1 \mathrm{kph}$, Controlled vs Uncontrolled System Comparison

\begin{tabular}{|c|c|c|}
\hline 00:00:14 & 00:00:15 & 00:00:16 \\
\hline 00:00:17 & 00:00:19 & 00:00:20 \\
\hline 00:00:21 & 00:00:22 & 00:00:24 \\
\hline 00:00:25 & 00:00:26 & 00:00:27 \\
\hline 00:00:28 & 00:00:30 & 00:00:31 \\
\hline 00:00:32 & 00:00:33 & 00:00:35 \\
\hline 00:00:36 & 00:00:39 & 00:00:42 \\
\hline
\end{tabular}

Figure 5.24. Trench crossing $1.5 \mathrm{~m}, 1 \mathrm{kph}$, uncontrolled 
In the $1.5 \mathrm{~m}$ long trench, the vehicle can enter and exit the trench with hitting the bumpers as shown in Figure 5.24. This trench length has been determined as the maximum trench length for the specified $6 \times 6$ vehicle because this is the most extended length in this trench that the vehicle can pass the trench without hitting its sub-systems.

At low speed $(1 \mathrm{kph})$ and $1.5 \mathrm{~m}$ trench, the vehicle completes the operation without dropping the front and rear part into the trench as required by the trench cross algorithm.

(Figure 5.25)

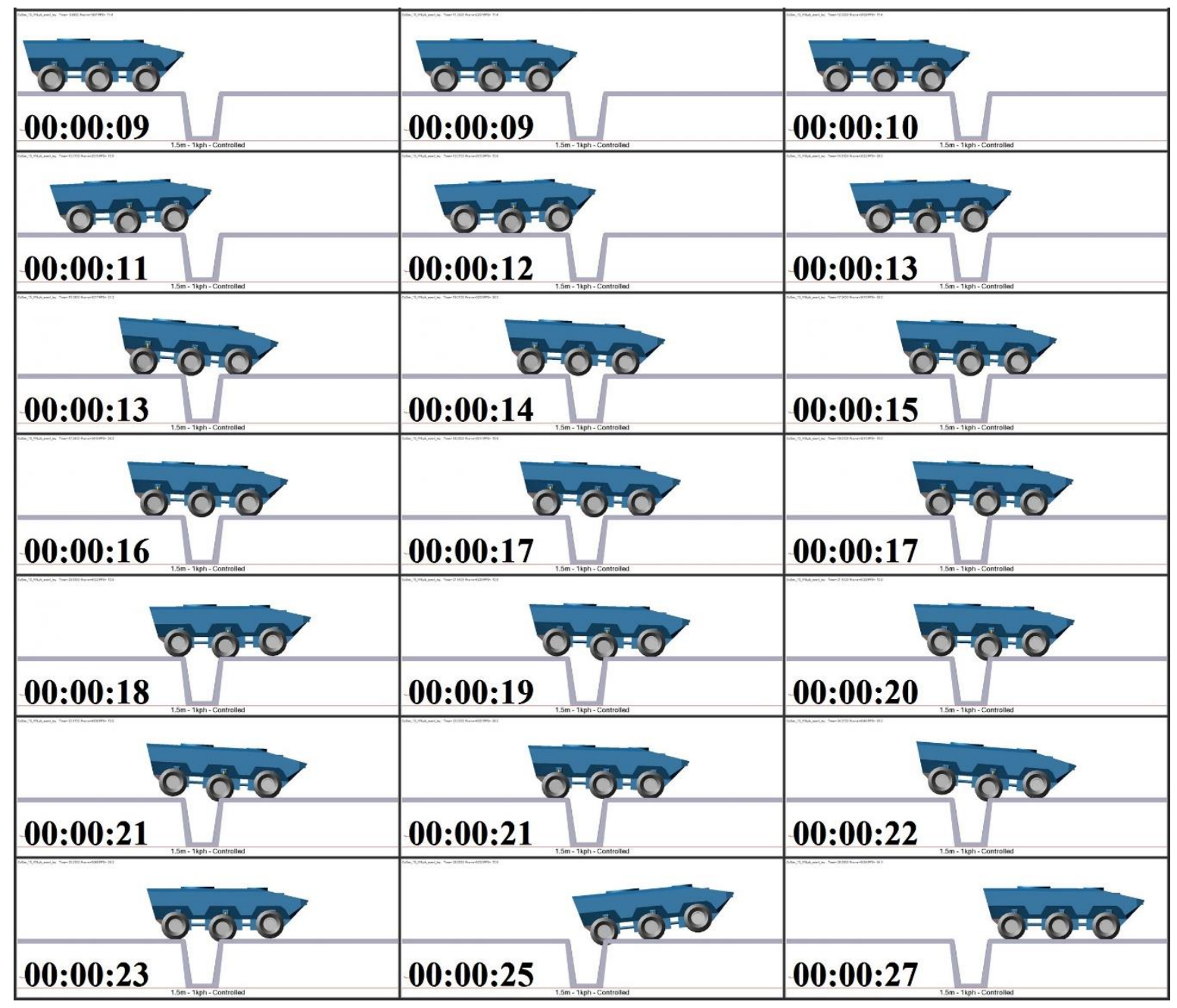

Figure 5.25. Trench crossing $1.5 \mathrm{~m}, 1 \mathrm{kph}$, controlled 
Figure 5.26 shows the comparison of body acceleration response of controlled and uncontrolled systems. While the peak value of vertical acceleration in the uncontrolled system is $63.7 \mathrm{~m} / \mathrm{s}^{2}$, the maximum vertical acceleration in the controlled system is $11.6 \mathrm{~m} / \mathrm{s}^{2}$.

Similarly, regarding the longitudinal acceleration comparison, the peak values of uncontrolled and controlled systems are $66.9 \mathrm{~m} / \mathrm{s}^{2}$ and $6.8 \mathrm{~m} / \mathrm{s}^{2}$, respectively.

The figure also shows the comparison of pitch angles and pitch rate of controlled and uncontrolled systems when passing the trench. As can be seen in the plot, the maximum pitch angle is $11.9^{\circ}$ in the uncontrolled system, whereas it is $6.2^{\circ}$ in the controlled system.

To make power spectral density comparisons, the RMS value in the uncontrolled system is $0.74 \mathrm{~A}^{2} / \mathrm{Hz}$, and it is $0.89 \mathrm{~A}^{2} / \mathrm{Hz}$ in the controlled system for body vertical acceleration. These RMS acceleration values for the longitudinal acceleration are $0.73 \mathrm{~A}^{2} / \mathrm{Hz}$ and 1.5 $\mathrm{A}^{2} / \mathrm{Hz}$, respectively where $\mathrm{A}$ is acceleration in $\mathrm{m} / \mathrm{s}^{2}$.

On the other hand, the maximum force acting on the wheel hub on the front axle during trench transition is about $62 \mathrm{kN}$ in the uncontrolled system and about $41 \mathrm{kN}$ in the controlled system. 


\section{5 m Trench, 1 kph | Controlled vs Uncontrolled Output Comparison}

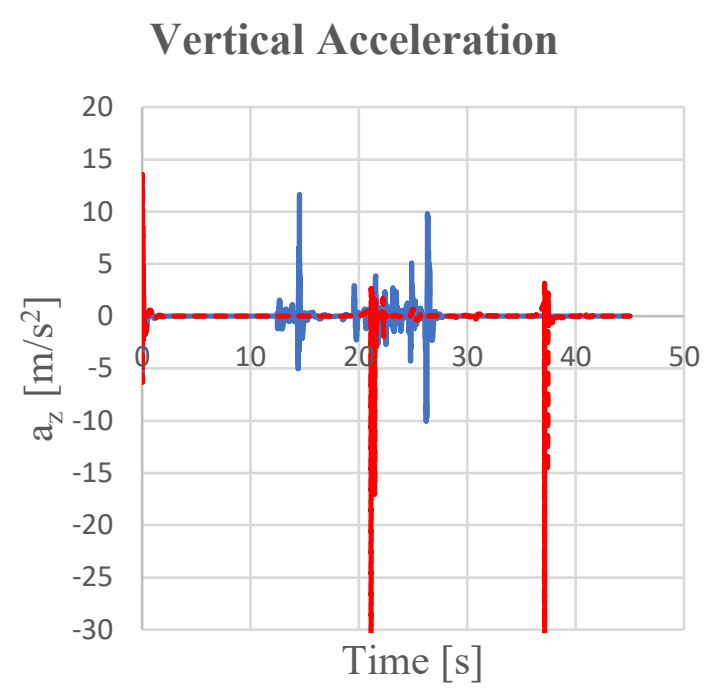

\section{Longitudinal Acceleration}
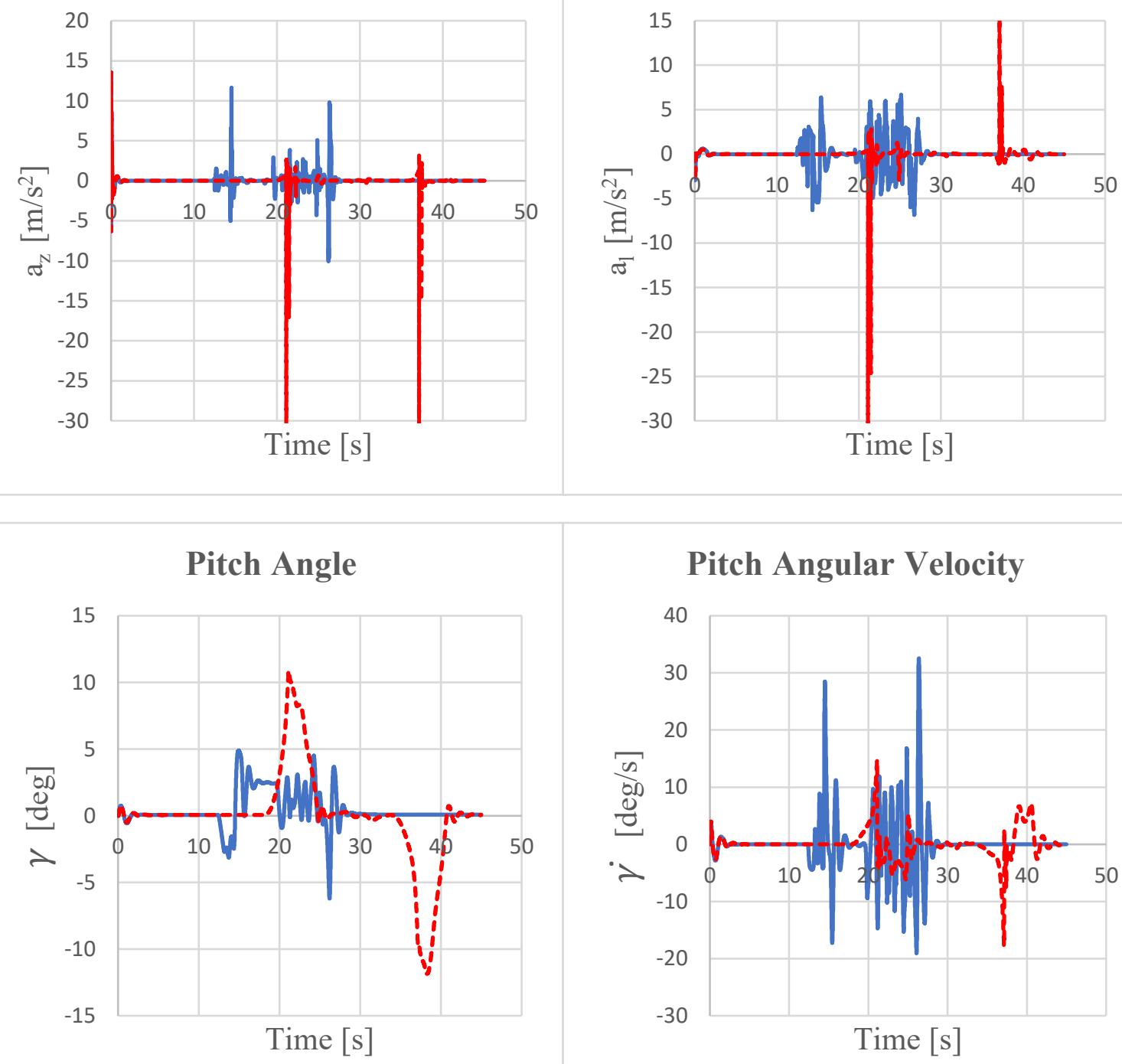

Pitch Angular Velocity

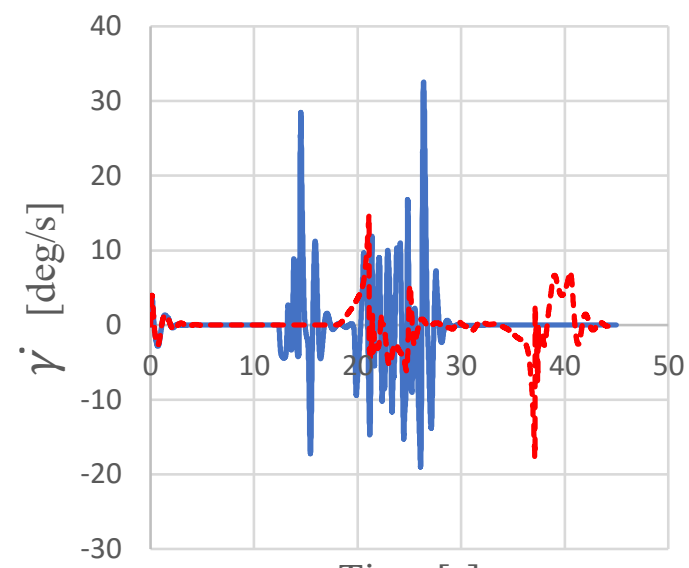

Time $[\mathrm{s}]$

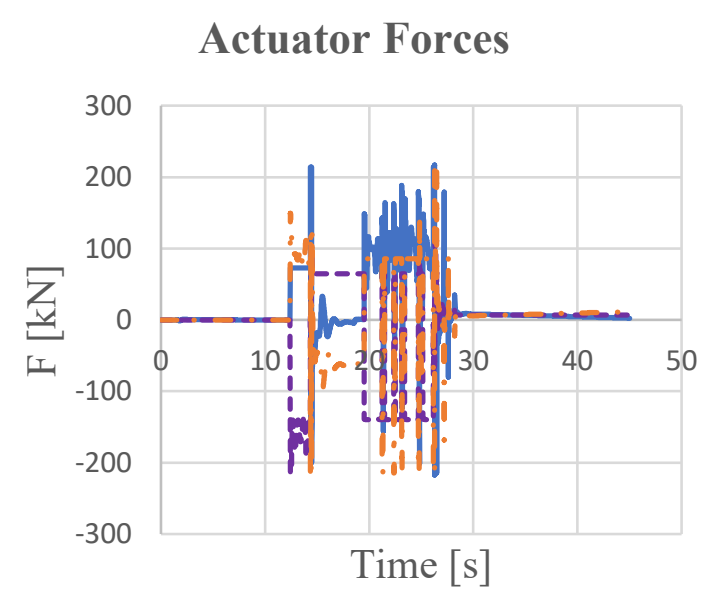

— Axle 1 ----A Axle 2 - - Axle 3

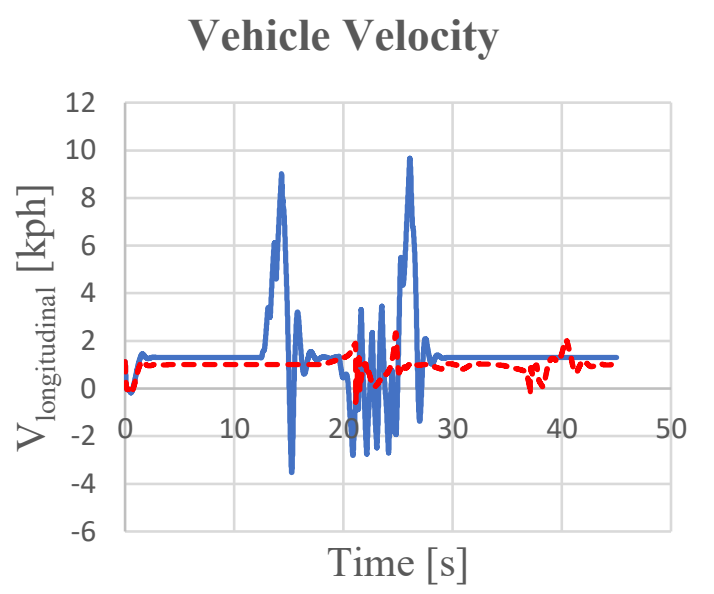

- Controlled ------Uncontrolled

Figure 5.26. Trench crossing $1.5 \mathrm{~m}, 1 \mathrm{kph}$, controlled $v$ s uncontrolled system results 
5.5.3. $1.5 \mathrm{~m}$ Trench, $3 \mathrm{kph}$, Controlled vs Uncontrolled System Comparison

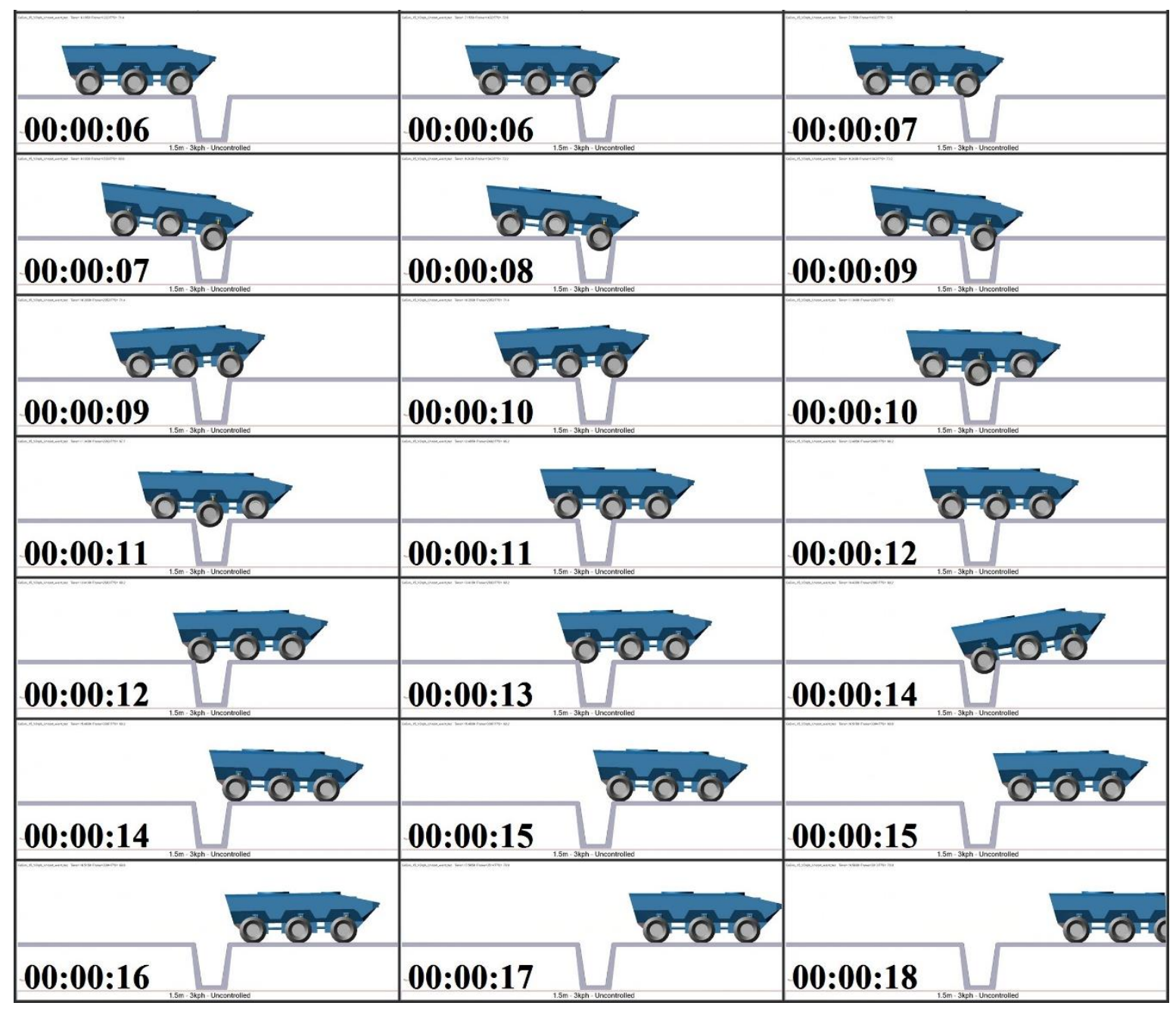

Figure 5.27 Trench crossing $1.5 \mathrm{~m}, 3 \mathrm{kph}$, uncontrolled

In the $1.5 \mathrm{~m}$ long trench, the vehicle can enter and exit the trench with hitting the bumpers as shown in Figure 5.27. 


\begin{tabular}{|c|c|c|}
\hline $0=0=0$ & $0=0=0$ & $0=0$ \\
\hline 00:00:06 & 00:00:07 & 00:00:07 \\
\hline 00:00:08 & 00:00:08 & 00:00:09 \\
\hline 00:00:09 & 00:00:10 & 00:00:10 \\
\hline 00:00:10 & 00:00:11 & 00:00:11 \\
\hline 00:00:12 & 00:00:12 & 00:00:13 \\
\hline 00:00:13 & 00:00:14 & 00:00:15 \\
\hline
\end{tabular}

Figure 5.28 Trench crossing $1.5 \mathrm{~m}, 3 \mathrm{kph}$, controlled

At relatively low speed $(3 \mathrm{kph})$ and $1.5 \mathrm{~m}$ trench, the vehicle completes the operation without dropping the front and rear part into the trench as required by the trench cross algorithm.

Figure 5.29 shows the comparison of body acceleration response of controlled and uncontrolled systems. While the peak value of vertical acceleration in the uncontrolled system is $209.9 \mathrm{~m} / \mathrm{s}^{2}$, the maximum vertical acceleration in the controlled system is $12.6 \mathrm{~m} / \mathrm{s}^{2}$. For the longitudinal acceleration comparison, the peak values of uncontrolled and controlled systems are $299.9 \mathrm{~m} / \mathrm{s}^{2}$ and $7.1 \mathrm{~m} / \mathrm{s}^{2}$, respectively.

Figure 5.29 also shows the comparison of pitch angles of controlled and uncontrolled systems when passing the trench. As can be seen in the plot, the maximum pitch angle is $10.9^{\circ}$ in the uncontrolled system, whereas it is $10.4^{\circ}$ in the controlled system. 
1.5 m Trench, 3 kph | Controlled vs Uncontrolled Output Comparison

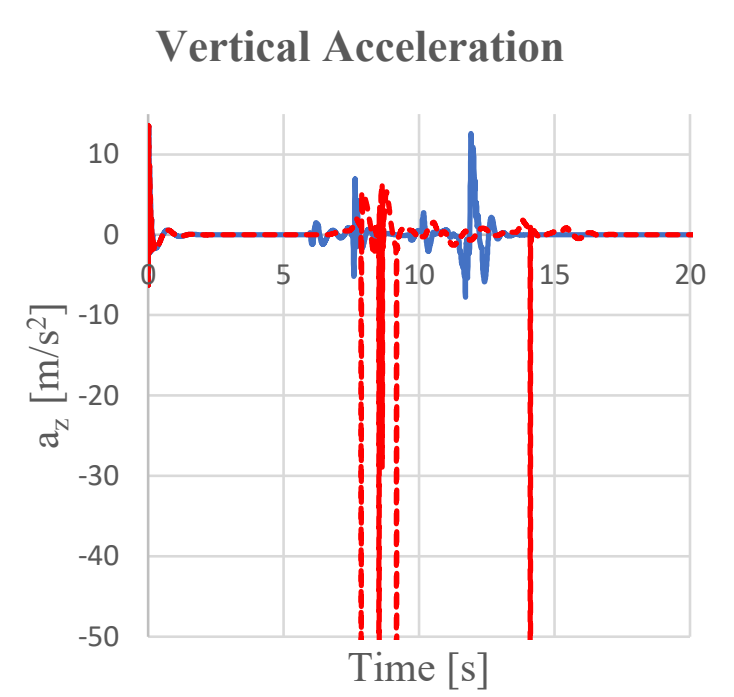

Longitudinal Acceleration
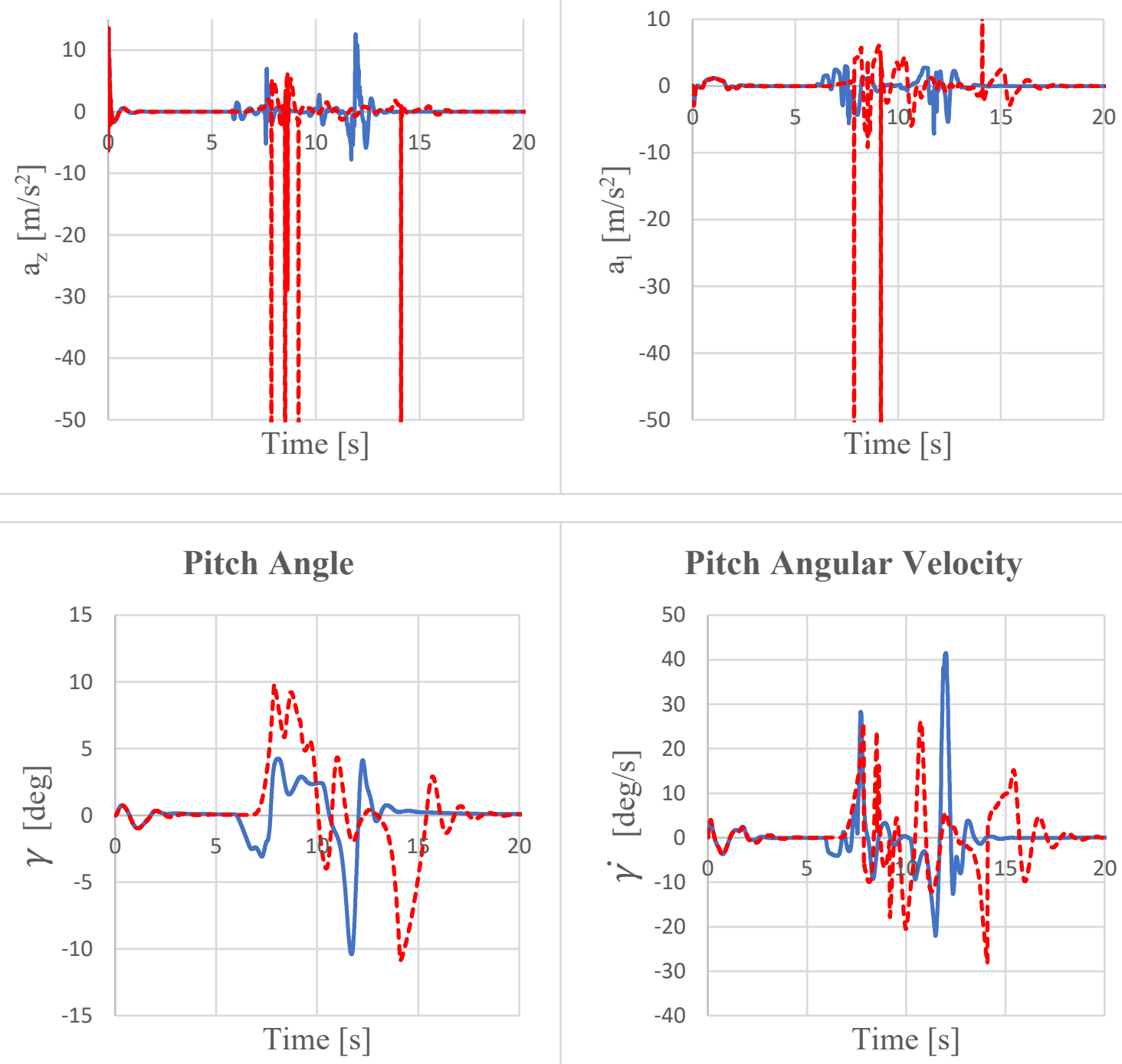

Pitch Angular Velocity

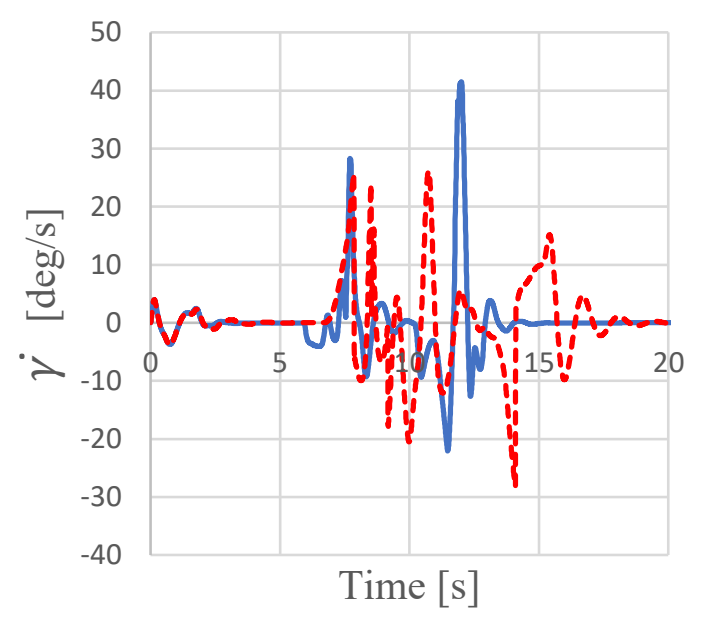

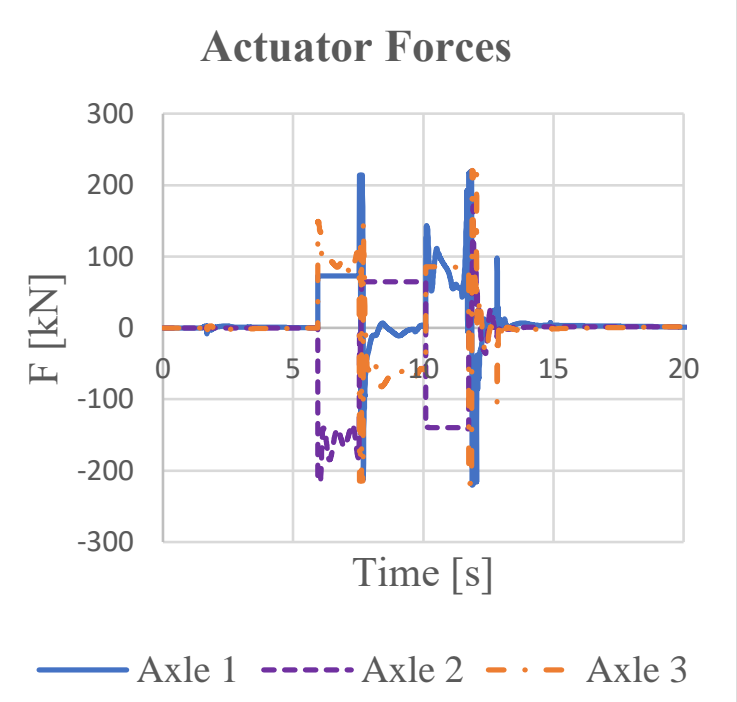

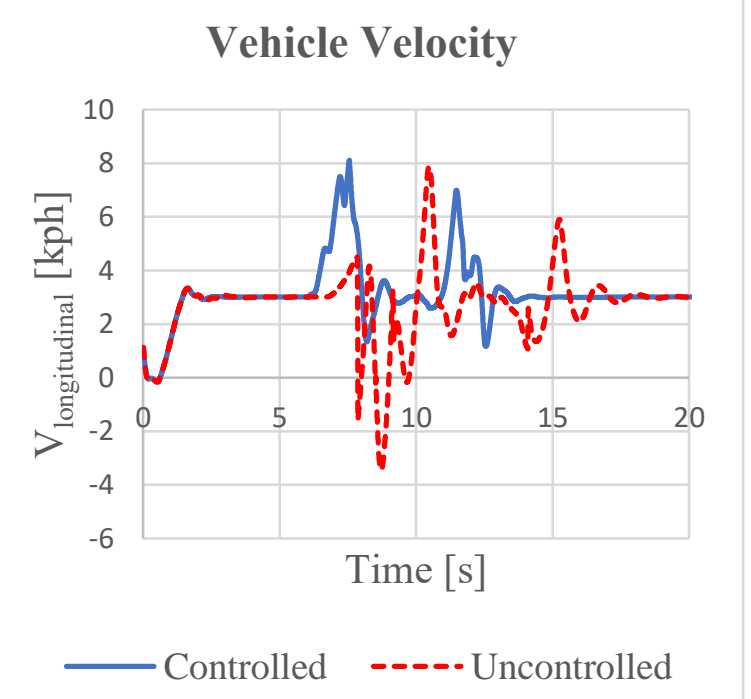

Figure 5.29. Trench crossing $1.5 \mathrm{~m}, 3 \mathrm{kph}$, controlled $v$ s uncontrolled system results 
To make power spectral density comparisons, the RMS value in the uncontrolled system is $2.19 \mathrm{~A}^{2} / \mathrm{Hz}$, and it is $1.07 \mathrm{~A}^{2} / \mathrm{Hz}$ in the controlled system for body vertical acceleration. These RMS acceleration values for the longitudinal acceleration are $2.37 \mathrm{~A}^{2} / \mathrm{Hz}$ and 0.88 $\mathrm{A}^{2} / \mathrm{Hz}$, respectively where $\mathrm{A}$ is acceleration in $\mathrm{m} / \mathrm{s}^{2}$.

On the other hand, the maximum force acting on the wheel hub on the front axle during trench transition is about $152 \mathrm{kN}$ in the uncontrolled system and about $29 \mathrm{kN}$ in the controlled system.

5.5.4. 1.7 m Trench, 6 kph, Controlled vs Uncontrolled System Comparison

\begin{tabular}{|c|c|c|}
\hline & & \\
\hline 00:00:03 & 00:00:04 & 00:00:04 \\
\hline 00:00:04 & 00:00:05 & 00:00:05 \\
\hline 00:00:05 & 00:00:06 & 00:00:06 \\
\hline 00:00:06 & 00:00:07 & 00:00:07 \\
\hline 00:00:07 & 00:00:08 & 00:00:08 \\
\hline 00:00:08 & 00:00:09 & 00:00:09 \\
\hline 00:00:09 & 00:00:10 & 00:00:11 \\
\hline
\end{tabular}

Figure 5.30 Trench crossing $1.7 \mathrm{~m}, 6 \mathrm{kph}$, uncontrolled 
In the scenario shown in Figure 5.30 above, the vehicle appears to pass the trench. However, the behaviour in the trench is bouncing back and forth. At the same time, accelerations on the vehicle in the vertical and longitudinal directions above the limits. Also, the RMS values of these accelerations are high. These values are given on the next page.

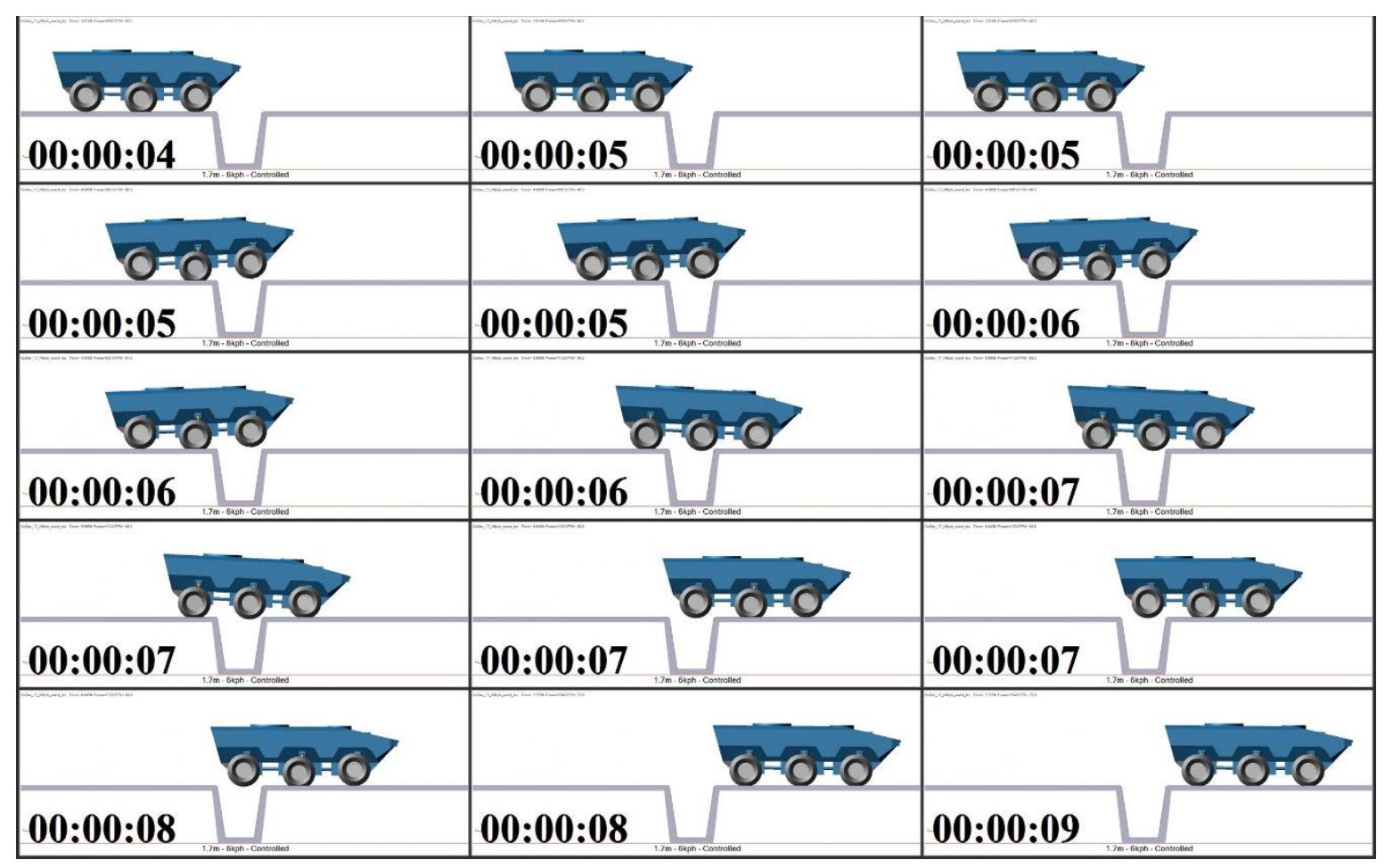

Figure 5.31 Trench crossing $1.7 \mathrm{~m}, 6 \mathrm{kph}$, controlled

At relatively high speed $(6 \mathrm{kph})$ and $1.7 \mathrm{~m}$ trench, the vehicle completes the operation without dropping the front and rear part into the trench as required by the trench cross algorithm. Also, medium (5-6 kph) speeds are the speeds that vehicle can cross the trench clearly in controlled systems as mentioned before.

Figure 5.32 shows the comparison of body acceleration response of controlled and uncontrolled systems. While the peak value of vertical acceleration in the uncontrolled system is $348.0 \mathrm{~m} / \mathrm{s}^{2}$, the maximum vertical acceleration in the controlled system is $11.3 \mathrm{~m} / \mathrm{s}^{2}$.

Similarly, regarding the longitudinal acceleration comparison, the peak values of uncontrolled and controlled systems are $387.3 \mathrm{~m} / \mathrm{s}^{2}$ and $9.4 \mathrm{~m} / \mathrm{s}^{2}$, respectively. Since the peak values are very high, these two graphics, which are given in order to see low acceleration levels more easily, are zoomed. 
1.7 m Trench, 6 kph | Controlled vs Uncontrolled Output Comparison

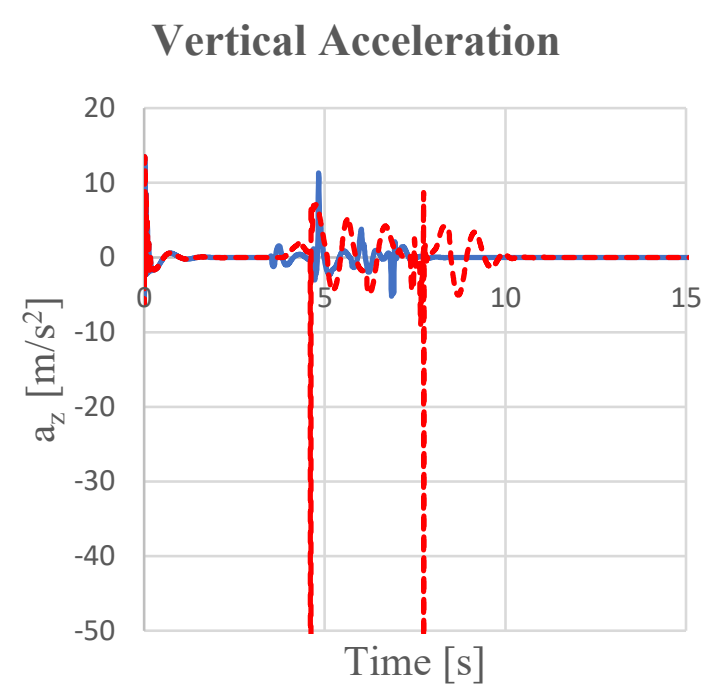

\section{Longitudinal Acceleration}

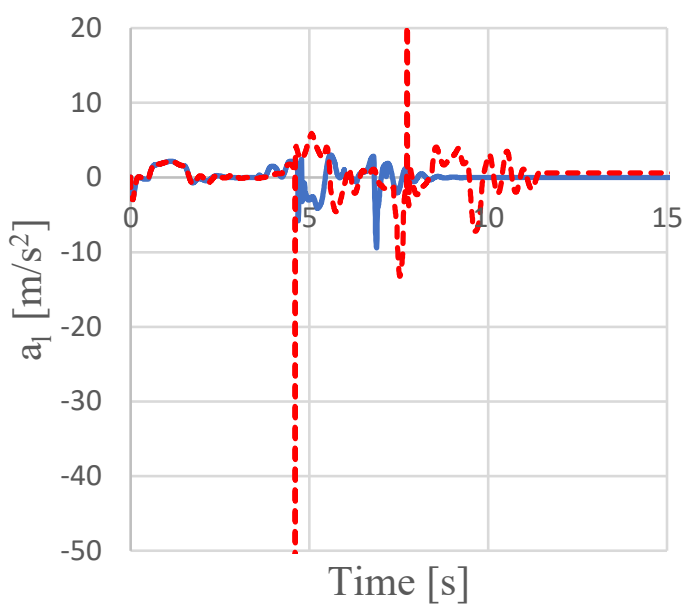

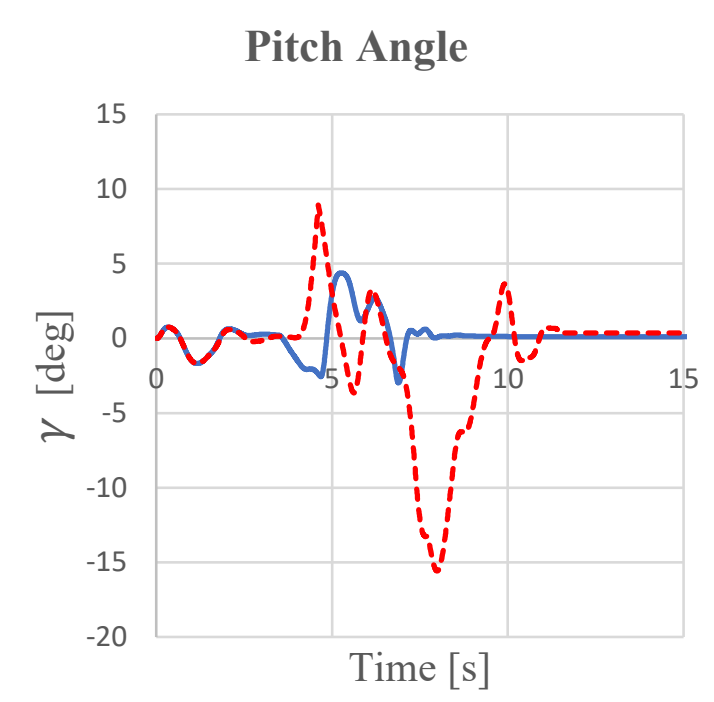

Pitch Angular Velocity

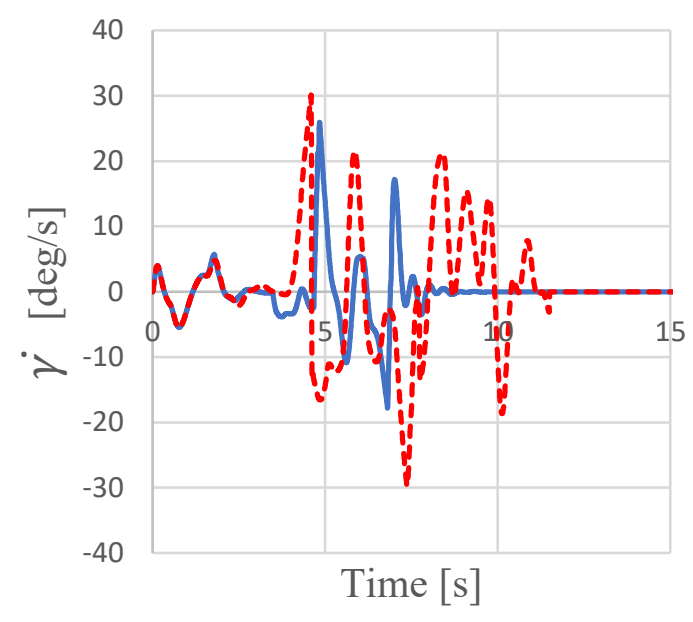

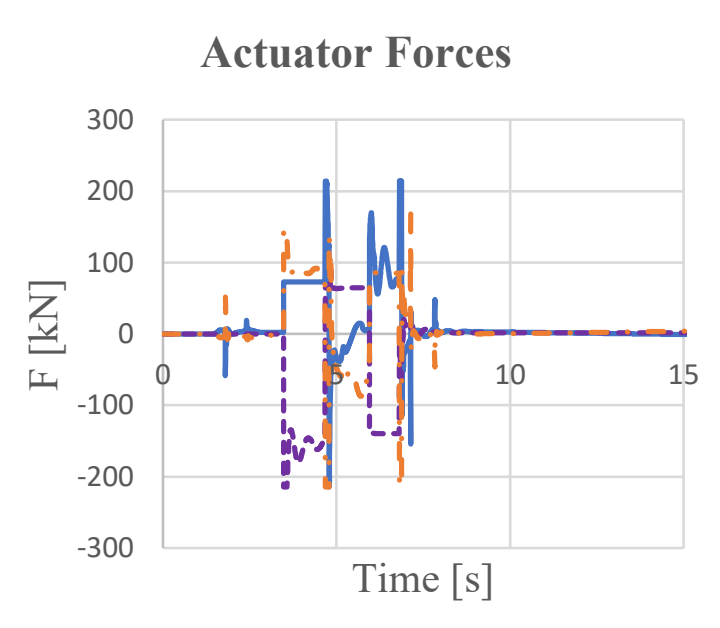

—Axle 1 ----A Axle 2 - - Axle 3

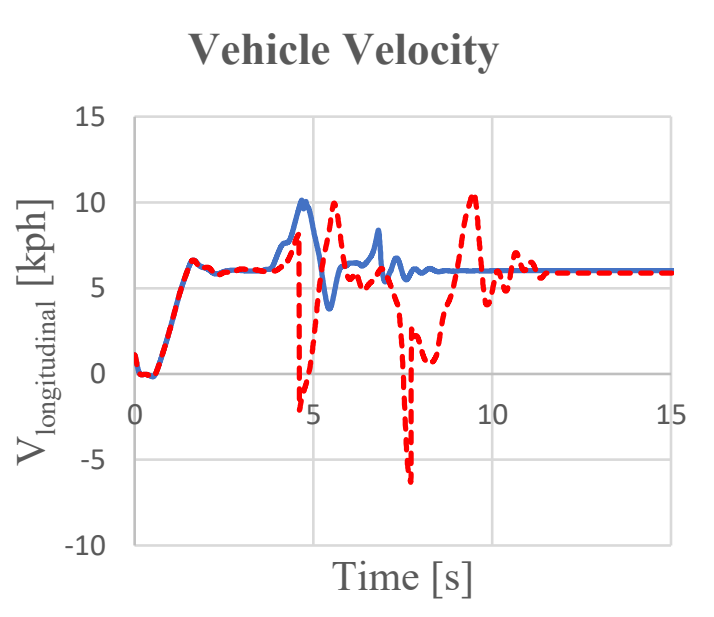

Controlled ------ Uncontrolled 
Figure 5.32 also shows the comparison of pitch angles of controlled and uncontrolled systems when passing the trench. As can be seen in the plot, the maximum pitch angle is $15.6^{\circ}$ in the uncontrolled system, whereas it is $4.4^{\circ}$ in the controlled system.

To make power spectral density comparisons, the RMS value in the uncontrolled system is $3.98 \mathrm{~A}^{2} / \mathrm{Hz}$, and it is $0.81 \mathrm{~A}^{2} / \mathrm{Hz}$ in the controlled system for body vertical acceleration. These RMS acceleration values for the longitudinal acceleration are $5.91 \mathrm{~A}^{2} / \mathrm{Hz}$ and 1.01 $\mathrm{A}^{2} / \mathrm{Hz}$, respectively where $\mathrm{A}$ is acceleration in $\mathrm{m} / \mathrm{s}^{2}$.

On the other hand, the maximum force acting on the wheel hub on the front axle during trench transition is about $36 \mathrm{kN}$ in the uncontrolled system and about $29 \mathrm{kN}$ in the controlled system. For the rear axles, these values are $145 \mathrm{kN}$ and $81 \mathrm{kN}$, respectively.

\subsubsection{9 m Trench, 1 kph, Controlled vs Uncontrolled System Comparison}

\begin{tabular}{|c|c|c|}
\hline 00:00:18 & 00:00:19 & 00:00:21 \\
\hline 00:00:22 & 00:00:23 & 00:00:25 \\
\hline 00:00:26 & 00:00:27 & 00:00:28 \\
\hline 00:00:30 & 00:00:31 & 00:00:32 \\
\hline 00:00:34 & 00:00:35 & 00:00:36 \\
\hline 00:00:37 & 00:00:40 & 00:00:43 \\
\hline
\end{tabular}

Figure 5.33 Trench crossing $1.9 \mathrm{~m}, 1 \mathrm{kph}$, uncontrolled 
The trench length where the passive system could pass was specified as $1.5 \mathrm{~m}$ in the previous sections. In Figure 5.33, it is seen that the vehicle cannot pass the trench. After second axle passes the trench the total traction is not enough to pull the vehicle out of trench. Also bottom sub-systems, axles and steering mechanisms are substantially in contact with ground.

\begin{tabular}{|c|c|c|}
\hline 00:00:14 & 00:00:15 & 00:00:16 \\
\hline 00:00:17 & 00:00:18 & 00:00:19 \\
\hline 00:00:20 & 00:00:21 & 00:00:22 \\
\hline 00:00:23 & 00:00:24 & 00:00:25 \\
\hline 00:00:26 & 00:00:27 & 00:00:28 \\
\hline 00:00:29 & 00:00:31 & 00:00:33 \\
\hline
\end{tabular}

Figure 5.34 Trench crossing $1.9 \mathrm{~m}, 1 \mathrm{kph}$, controlled

At low speed $(1 \mathrm{kph})$ and $1.9 \mathrm{~m}$ trench, the vehicle completes the operation without dropping the front and rear part into the trench as required by the trench cross algorithm. As the trench length increased too much and at the lowest speed, pitch and oscillation behaviour became evident during trench exit.

Figure 5.35 shows the comparison of body acceleration response of controlled and uncontrolled systems. While the peak value of vertical acceleration in the uncontrolled system is $68.0 \mathrm{~m} / \mathrm{s}^{2}$, the maximum vertical acceleration in the controlled system is $12.4 \mathrm{~m} / \mathrm{s}^{2}$.

Similarly, for the longitudinal acceleration comparison, the peak values of uncontrolled and controlled systems are $88.3 \mathrm{~m} / \mathrm{s}^{2}$ and $8.7 \mathrm{~m} / \mathrm{s}^{2}$, respectively. 


\section{9 m Trench, 1 kph | Controlled vs Uncontrolled Output Comparison}
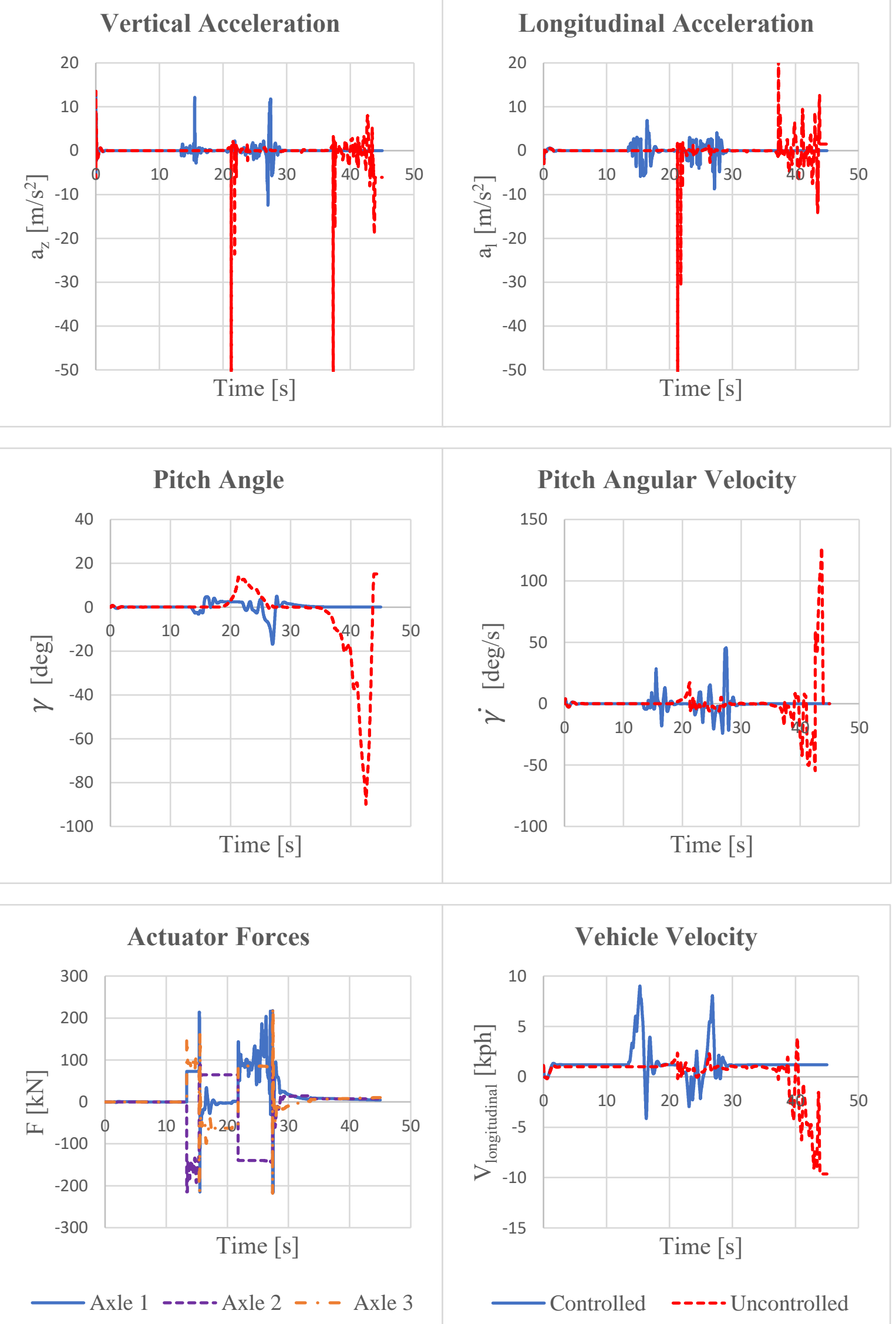

Figure 5.35 Trench crossing $1.9 \mathrm{~m}, 1 \mathrm{kph}$, body acceleration comparison of controlled vs uncontrolled systems 
Figure 5.35 also shows the comparison of pitch angles of controlled and uncontrolled systems when passing the trench. As can be seen in the plot, the maximum pitch angle is $89.9^{\circ}$ in the uncontrolled system, whereas it is $16.8^{\circ}$ in the controlled system.

To make power spectral density comparisons, the RMS value in the uncontrolled system is $1.96 \mathrm{~A}^{2} / \mathrm{Hz}$, and it is $1.12 \mathrm{~A}^{2} / \mathrm{Hz}$ in the controlled system for body vertical acceleration. These RMS acceleration values for the longitudinal acceleration are $1.70 \mathrm{~A}^{2} / \mathrm{Hz}$ and 1.22 $\mathrm{A}^{2} / \mathrm{Hz}$, respectively where $\mathrm{A}$ is acceleration in $\mathrm{m} / \mathrm{s}^{2}$.

On the other hand, the maximum force acting on the wheel hub on the front axle during trench transition is about $206 \mathrm{kN}$ in the uncontrolled system and about $40 \mathrm{kN}$ in the controlled system.

\subsubsection{0 m Trench, 9 kph, Controlled vs Uncontrolled System Comparison}

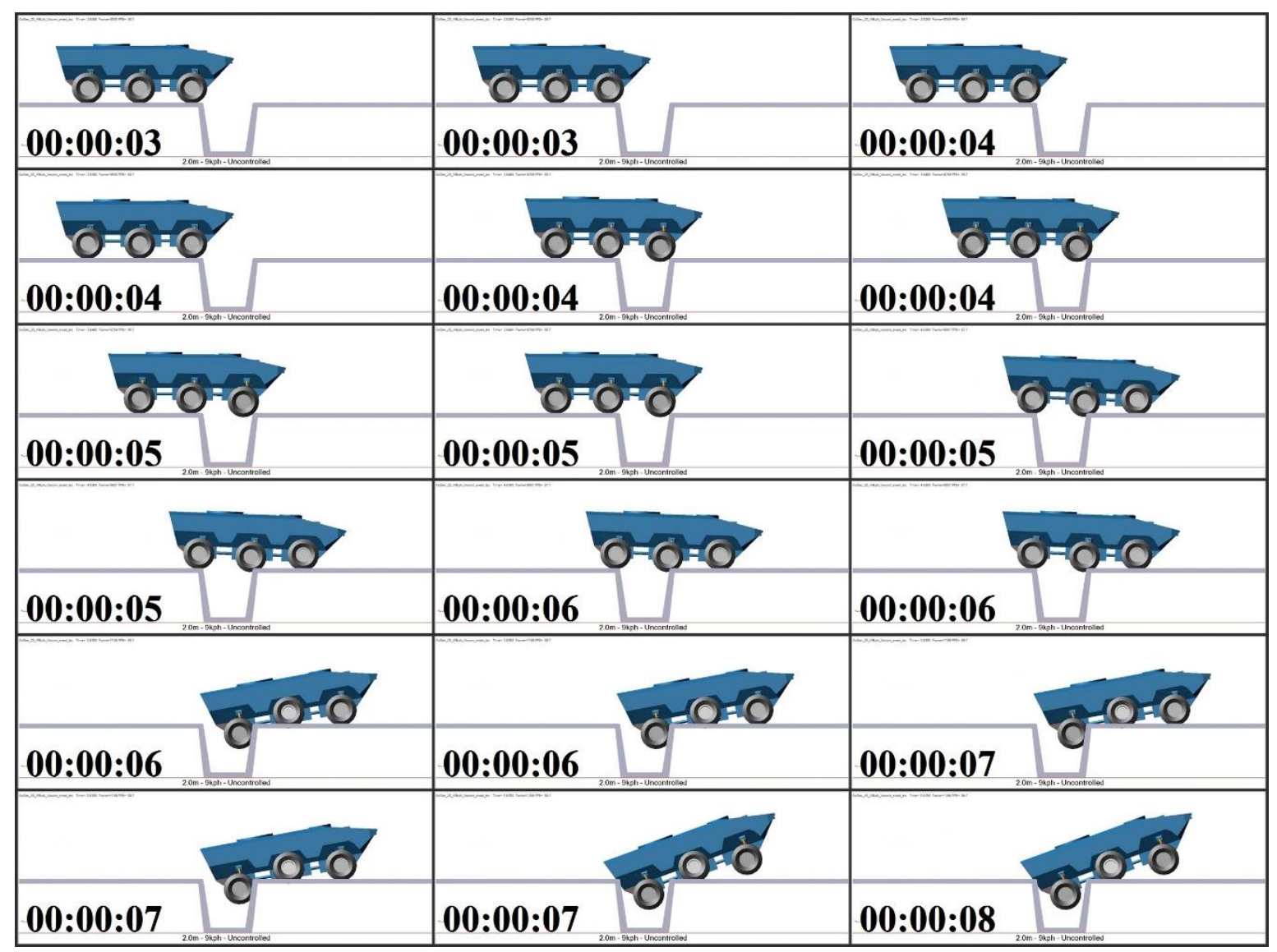

Figure 5.36 Trench crossing $2.0 \mathrm{~m}, 9 \mathrm{kph}$, uncontrolled 
In this scenario, the vehicle cannot pass the trench (Figure 5.36). It hits both its bottom subsystems and after the last axle falls into the trench, it cannot provide the necessary traction to get out of the trench.

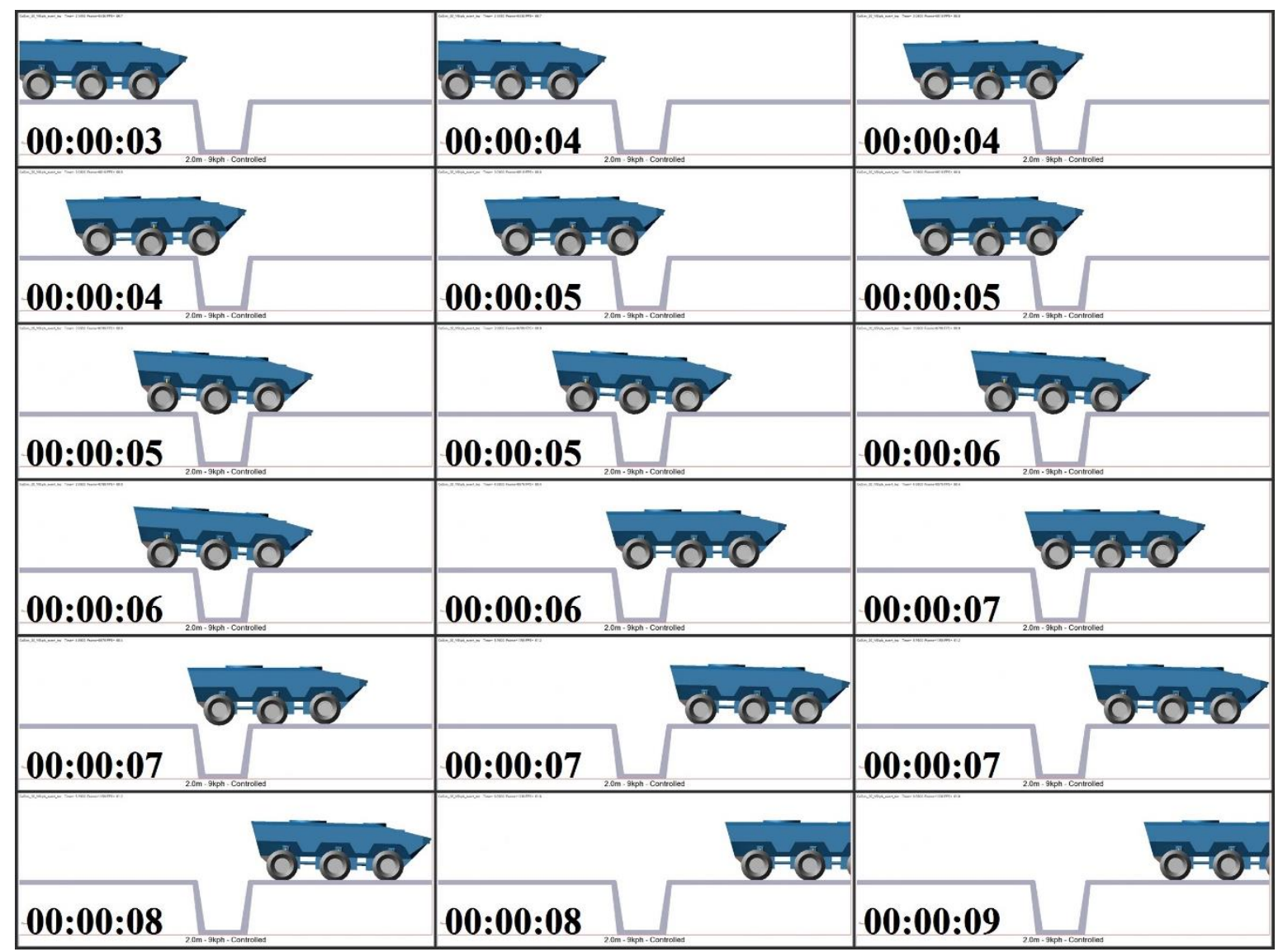

Figure 5.37 Trench crossing $2.0 \mathrm{~m}, 9 \mathrm{kph}$, controlled

At high speed $(9 \mathrm{kph})$ and $2.0 \mathrm{~m}$ trench, the vehicle completes the operation clearly without dropping the front and rear part into the trench as required by the trench cross algorithm. The crossing operation is very clear.

Figure 5.38 shows the comparison of body acceleration response of controlled and uncontrolled systems. While the peak value of vertical acceleration in the uncontrolled system is $543.1 \mathrm{~m} / \mathrm{s}^{2}$, the maximum vertical acceleration in the controlled system is $10.9 \mathrm{~m} / \mathrm{s}^{2}$. Regarding the longitudinal acceleration comparison, the peak values of uncontrolled and controlled systems are $513.0 \mathrm{~m} / \mathrm{s}^{2}$ and $4.6 \mathrm{~m} / \mathrm{s}^{2}$, respectively. 
2 m Trench, 9 kph | Controlled vs Uncontrolled Output Comparison

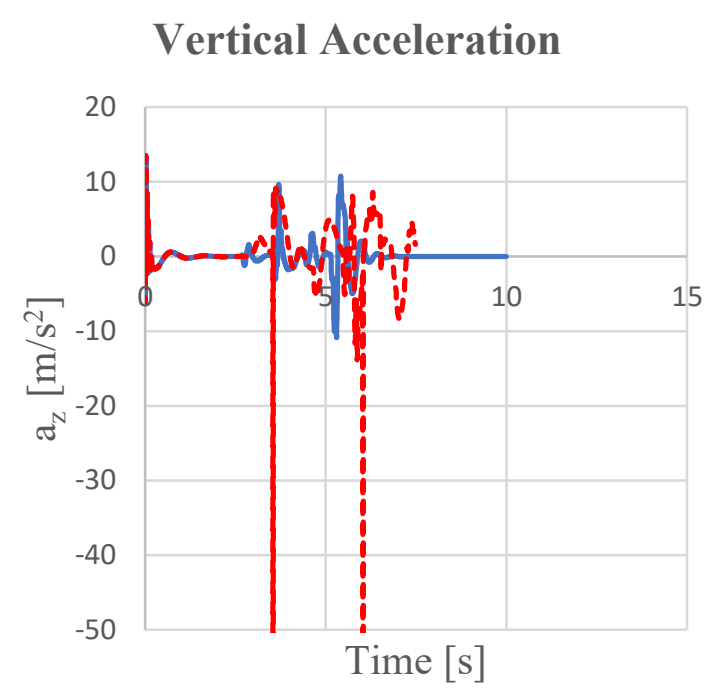

\section{Longitudinal Acceleration}

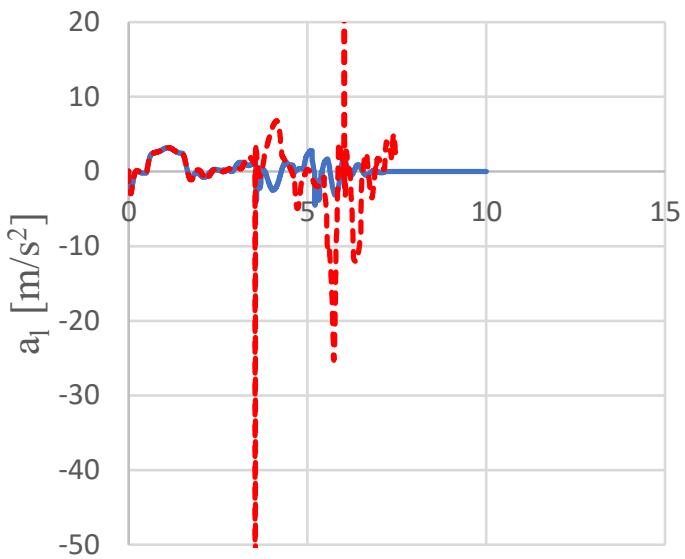

Time $[\mathrm{s}]$

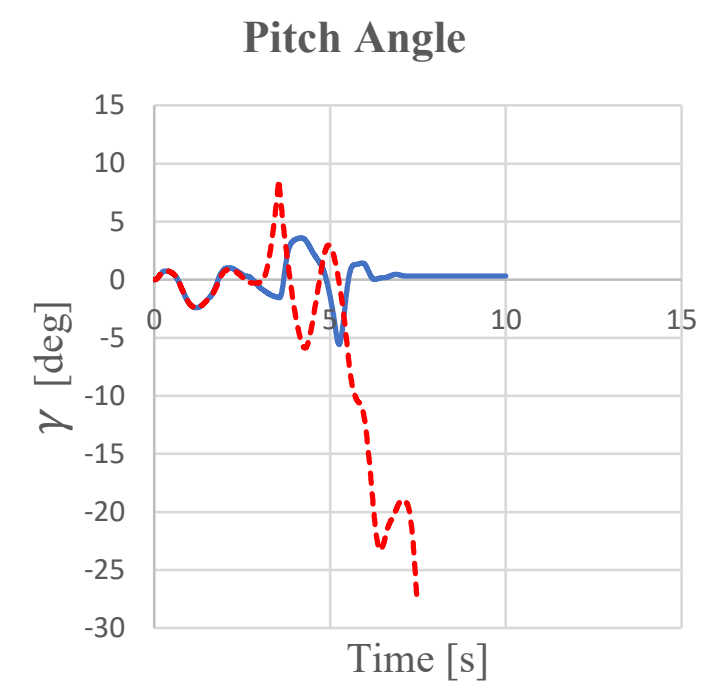

Pitch Angular Velocity

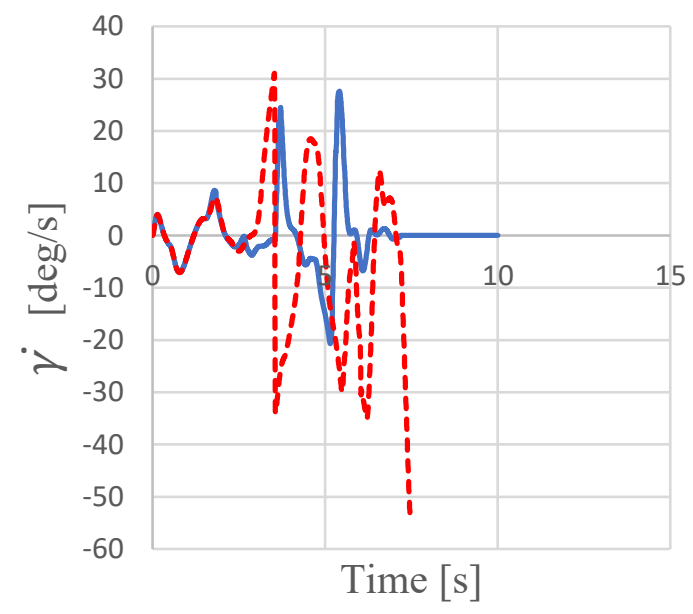

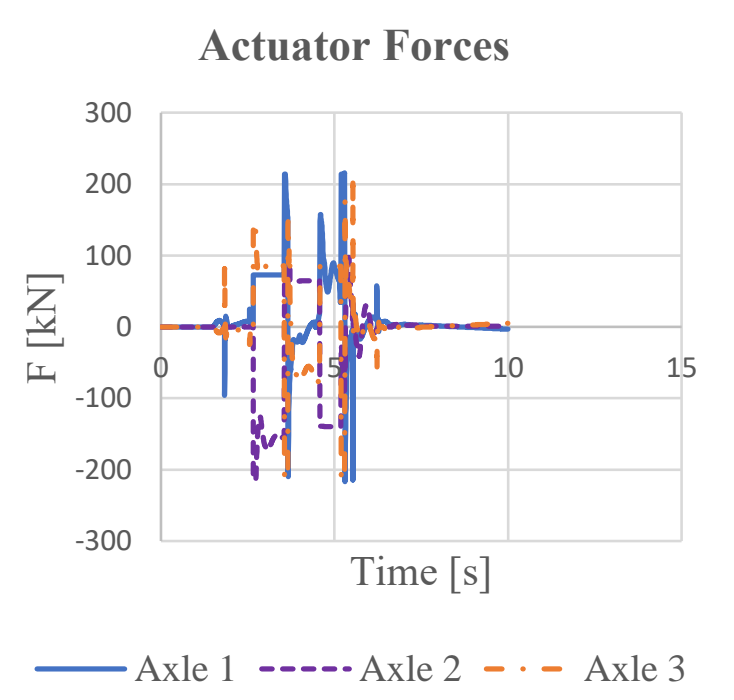

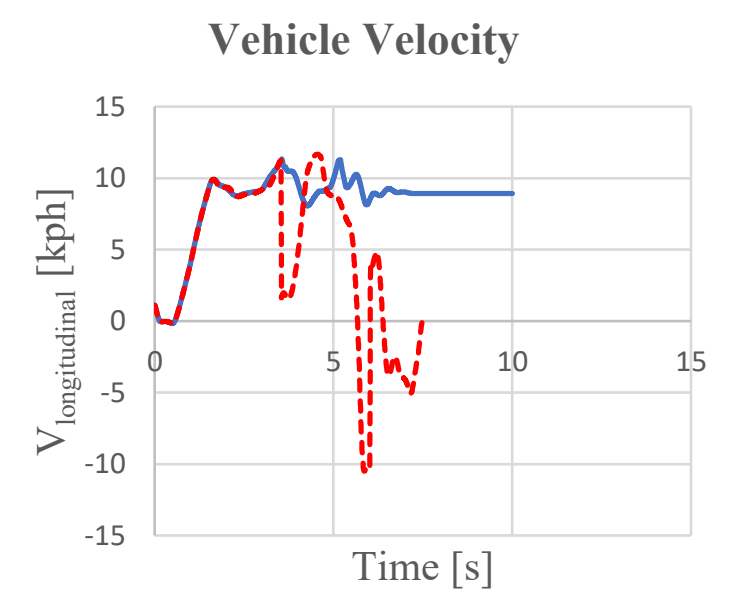

Controlled ------Uncontrolled

Figure 5.38 Trench crossing $2.0 \mathrm{~m}, 9 \mathrm{kph}$, body acceleration comparison of controlled vs uncontrolled systems 
Figure 5.38 also shows the comparison of pitch angles of controlled and uncontrolled systems when passing the trench. As can be seen in the plot, the maximum pitch angle is $27.9^{\circ}$ in the uncontrolled system, whereas it is $5.6^{\circ}$ in the controlled system. The vehicle cannot complete crossing the trench.

To make power spectral density comparisons, the RMS value in the uncontrolled system is $14.96 \mathrm{~A}^{2} / \mathrm{Hz}$, and it is $1.82 \mathrm{~A}^{2} / \mathrm{Hz}$ in the controlled system for body vertical acceleration. These RMS acceleration values for the longitudinal acceleration are $17.96 \mathrm{~A}^{2} / \mathrm{Hz}$ and 1.18 $\mathrm{A}^{2} / \mathrm{Hz}$, respectively where $\mathrm{A}$ is acceleration in $\mathrm{m} / \mathrm{s}^{2}$.

On the other hand, the maximum force acting on the wheel hub on the front axle during trench transition is about $38 \mathrm{kN}$ in the uncontrolled system and about $20 \mathrm{kN}$ in the controlled system. For the rear axles, these values are $291 \mathrm{kN}$ and $44 \mathrm{kN}$, respectively. 


\subsection{Comparison}

Some of simulation studies in the previous section are given. In this section, a comparison of these simulation studies and also all of the simulations are presented. The comparison can be divided into two primary sections: Linear system simulations and non-linear system simulations.

As stated before, the primary purpose of this thesis is to make a trend study whether the limits of mobility operations can be increased with MPC + preview. MPC is a control method that optimizes with the help of a specific prediction by containing linear or nonlinear models in it. In this thesis, the model used in MPC is a linear, 9 DOF full car ride model.

Firstly, in order to compare the MPC controller with some other control methods and to see its performance, simulations were made with pits that are not very deep with the linear system (where the wheel is not in contact with the ground). In these simulations, disturbance rejection and reference tracking capabilities of LQR, MPC and MPC + preview control methods were compared.

In the second part and as the main comparison, ADAMS model, which includes real trench conditions and non-linear, multi-body dynamics vehicle model closer to the real one, is used as a plant. With this plant, the MPC + preview controller was run in the Simulink environment as co-simulation.

Simulations using the ADAMS plant and MPC + preview control and passive system simulations are compared according to some criteria mentioned in the previous sections. From these comparisons, the body vertical and longitudinal acceleration comparison is given in Table 5.6, and the pitch angle comparison is given in Table 5.7. Additionally, the comparison of PSD RMS values of body vertical and longitudinal accelerations is also given in Table 5.8. 
Table 5.6. Body vertical and longitudinal acceleration comparison of controlled and uncontrolled systems

\begin{tabular}{|c|c|c|c|c|c|c|c|}
\hline & & \begin{tabular}{|l|} 
Controlled \\
\end{tabular} & Uncontrolled & & \begin{tabular}{|l|} 
Controlled \\
\end{tabular} & Uncontrolled & \\
\hline $\begin{array}{l}\text { Trench } \\
\text { Length }\end{array}$ & Speed & $\begin{array}{c}\text { Max. } \\
\mathbf{a}_{\mathbf{z}} \\
{\left[\mathbf{m} / \mathbf{s}^{2}\right]}\end{array}$ & $\begin{array}{c}\text { Max. } \\
\mathbf{a}_{\mathbf{z}} \\
{\left[\mathbf{m} / \mathbf{s}^{2}\right]}\end{array}$ & $\%$ & $\begin{array}{c}\text { Max. } \\
\mathbf{a}_{\mathbf{l}} \\
{\left[\mathrm{m} / \mathbf{s}^{2}\right]}\end{array}$ & $\begin{array}{c}\text { Max. } \\
\mathbf{a}_{\mathbf{l}} \\
{\left[\mathrm{m} / \mathrm{s}^{2}\right]}\end{array}$ & $\%$ \\
\hline & $1 \mathrm{kph}$ & 12.4 & 49.6 & $75 \%$ & 7.2 & 24.3 & $70 \%$ \\
\hline $12 \mathrm{~m}$ & $3 \mathrm{kph}$ & 8.9 & 56.7 & $84 \%$ & 7.8 & 22.9 & $66 \%$ \\
\hline $1.2 \mathrm{mI}$ & $6 \mathrm{kph}$ & 7.9 & 10.4 & $24 \%$ & 9.1 & 14.3 & $37 \%$ \\
\hline & $9 \mathrm{kph}$ & 13.4 & 6.1 & $-120 \%$ & 4.0 & 5.6 & $29 \%$ \\
\hline & $1 \mathrm{kph}$ & 14.1 & 73.1 & $81 \%$ & 7.2 & 64.7 & $89 \%$ \\
\hline $12 m$ & $3 \mathrm{kph}$ & 9.1 & 134.8 & $93 \%$ & 6.6 & 37.0 & $82 \%$ \\
\hline $1.5 \mathrm{~m}$ & $6 \mathrm{kph}$ & 12.9 & 109.9 & $88 \%$ & 5.4 & 79.8 & $93 \%$ \\
\hline & $9 \mathrm{kph}$ & 10.9 & 6.8 & $-61 \%$ & 4.1 & 7.7 & $47 \%$ \\
\hline & $1 \mathrm{kph}$ & 14.1 & 71.5 & $80 \%$ & 7.6 & 66.5 & $89 \%$ \\
\hline $11 \mathrm{~m}$ & $3 \mathrm{kph}$ & 14.0 & 193.3 & $93 \%$ & 5.4 & 284.3 & $98 \%$ \\
\hline $1.4 \mathrm{mI}$ & $6 \mathrm{kph}$ & 9.8 & 152.7 & $94 \%$ & 6.0 & 72.5 & $92 \%$ \\
\hline & $9 \mathrm{kph}$ & 7.1 & 191.8 & $96 \%$ & 8.6 & 119.4 & $93 \%$ \\
\hline & $1 \mathrm{kph}$ & 11.6 & 63.7 & $82 \%$ & 6.8 & 66.9 & $90 \%$ \\
\hline 15 & $3 \mathrm{kph}$ & 12.6 & 209.9 & $94 \%$ & 7.1 & 299.9 & $98 \%$ \\
\hline 1.5 & $6 \mathrm{kph}$ & 10.2 & 160.1 & $94 \%$ & 8.0 & 44.3 & $82 \%$ \\
\hline & $9 \mathrm{kph}$ & 8.3 & 165.3 & $95 \%$ & 8.4 & 66.4 & $87 \%$ \\
\hline & $1 \mathrm{kph}$ & 13.7 & 64.8 & $79 \%$ & 9.8 & 63.3 & $84 \%$ \\
\hline $16 \mathrm{~m}$ & $3 \mathrm{kph}$ & 14.6 & 146.1 & $90 \%$ & 7.1 & 197.8 & $96 \%$ \\
\hline 1.0111 & $6 \mathrm{kph}$ & 11.2 & 510.6 & $98 \%$ & 9.4 & 392.7 & $98 \%$ \\
\hline & $9 \mathrm{kph}$ & 9.6 & 152.6 & $94 \%$ & 8.3 & 71.3 & $88 \%$ \\
\hline & $1 \mathrm{kph}$ & 14.6 & 68.3 & $79 \%$ & 9.5 & 86.4 & $89 \%$ \\
\hline 17 & $3 \mathrm{kph}$ & 15.2 & 182.9 & $92 \%$ & 6.6 & 223.0 & $97 \%$ \\
\hline $1.7 \mathrm{III}$ & $6 \mathrm{kph}$ & 11.3 & 348.0 & $97 \%$ & 9.4 & 387.3 & $98 \%$ \\
\hline & $9 \mathrm{kph}$ & 9.2 & 147.2 & $94 \%$ & 8.0 & 74.6 & $89 \%$ \\
\hline & $1 \mathrm{kph}$ & 12.9 & 78.3 & $83 \%$ & 8.0 & 84.1 & $90 \%$ \\
\hline 18 & $3 \mathrm{kph}$ & 15.8 & 144.9 & $89 \%$ & 6.5 & 182.8 & $96 \%$ \\
\hline $1.0 \mathrm{III}$ & $6 \mathrm{kph}$ & 11.3 & 425.6 & $97 \%$ & 9.2 & 477.7 & $98 \%$ \\
\hline & $9 \mathrm{kph}$ & 9.1 & 184.4 & $95 \%$ & 8.2 & 74.4 & $89 \%$ \\
\hline & $1 \mathrm{kph}$ & 12.4 & 68.0 & $82 \%$ & 8.7 & 88.3 & $90 \%$ \\
\hline $19 \mathrm{~m}$ & $3 \mathrm{kph}$ & 13.7 & 203.9 & $93 \%$ & 6.6 & 264.3 & $98 \%$ \\
\hline & $6 \mathrm{kph}$ & 11.3 & 440.6 & $97 \%$ & 8.3 & 461.5 & $98 \%$ \\
\hline & $9 \mathrm{kph}$ & 10.1 & 508.4 & $98 \%$ & 6.5 & 273.6 & $98 \%$ \\
\hline & $1 \mathrm{kph}$ & 14.8 & 83.8 & $82 \%$ & 7.3 & 160.6 & $95 \%$ \\
\hline $2.0 \mathrm{~m}$ & $3 \mathrm{kph}$ & 16.0 & 153.0 & $90 \%$ & 6.3 & 204.5 & $97 \%$ \\
\hline & $6 \mathrm{kph}$ & 11.5 & 376.0 & $97 \%$ & 5.8 & 382.4 & $98 \%$ \\
\hline & $9 \mathrm{kph}$ & 10.9 & 543.1 & $98 \%$ & 4.6 & 513.0 & $99 \%$ \\
\hline
\end{tabular}


Table 5.7. Vehicle pitch angle comparison of controlled and uncontrolled systems

\begin{tabular}{|c|c|c|c|c|}
\hline & \multirow{2}{*}{\begin{tabular}{|c|} 
Controlled \\
\\
Maximum \\
Pitch Angle \\
[deg]
\end{tabular}} & \multirow{2}{*}{$\begin{array}{c}\text { Uncontrolled } \\
\\
\text { Maximum } \\
\text { Pitch Angle } \\
\text { [deg] }\end{array}$} & \multirow[b]{2}{*}{$\%$} \\
\hline $\begin{array}{l}\text { Trench } \\
\text { Length }\end{array}$ & Speed & & & \\
\hline \multirow{4}{*}{$1.2 \mathrm{~m}$} & $1 \mathrm{kph}$ & 7.6 & 9.6 & $21 \%$ \\
\hline & $3 \mathrm{kph}$ & 5.5 & 8.9 & $38 \%$ \\
\hline & $6 \mathrm{kph}$ & 4.8 & 10.8 & $56 \%$ \\
\hline & $9 \mathrm{kph}$ & 7.8 & 4.9 & $-59 \%$ \\
\hline \multirow{4}{*}{$1.3 \mathrm{~m}$} & $1 \mathrm{kph}$ & 9.3 & 10.3 & $10 \%$ \\
\hline & $3 \mathrm{kph}$ & 5.1 & 11.0 & $53 \%$ \\
\hline & $6 \mathrm{kph}$ & 7.5 & 11.2 & $33 \%$ \\
\hline & $9 \mathrm{kph}$ & 6.0 & 6.5 & $8 \%$ \\
\hline \multirow{4}{*}{$1.4 \mathrm{~m}$} & $1 \mathrm{kph}$ & 8.4 & 11.1 & $24 \%$ \\
\hline & $3 \mathrm{kph}$ & 8.8 & 10.8 & $18 \%$ \\
\hline & $6 \mathrm{kph}$ & 5.4 & 11.5 & $53 \%$ \\
\hline & $9 \mathrm{kph}$ & 3.4 & 10.9 & $69 \%$ \\
\hline \multirow{4}{*}{1.5} & $1 \mathrm{kph}$ & 6.2 & 11.9 & $48 \%$ \\
\hline & $3 \mathrm{kph}$ & 10.4 & 10.9 & $5 \%$ \\
\hline & $6 \mathrm{kph}$ & 5.0 & 10.7 & $53 \%$ \\
\hline & $9 \mathrm{kph}$ & 3.5 & 13.9 & $75 \%$ \\
\hline \multirow{4}{*}{$1.6 \mathrm{~m}$} & $1 \mathrm{kph}$ & 10.5 & 12.9 & $19 \%$ \\
\hline & $3 \mathrm{kph}$ & 12.4 & 11.1 & $-12 \%$ \\
\hline & $6 \mathrm{kph}$ & 4.3 & 11.6 & $63 \%$ \\
\hline & $9 \mathrm{kph}$ & 4.0 & 14.2 & $72 \%$ \\
\hline \multirow{4}{*}{$1.7 \mathrm{~m}$} & $1 \mathrm{kph}$ & 10.7 & 88.0 & $88 \%$ \\
\hline & $3 \mathrm{kph}$ & 14.8 & 11.9 & $-24 \%$ \\
\hline & $6 \mathrm{kph}$ & 4.4 & 15.6 & $72 \%$ \\
\hline & $9 \mathrm{kph}$ & 4.2 & 14.4 & $71 \%$ \\
\hline \multirow{4}{*}{$1.8 \mathrm{~m}$} & $1 \mathrm{kph}$ & 13.0 & 43.1 & $70 \%$ \\
\hline & $3 \mathrm{kph}$ & 15.0 & 13.2 & $-14 \%$ \\
\hline & $6 \mathrm{kph}$ & 4.4 & 15.4 & $72 \%$ \\
\hline & $9 \mathrm{kph}$ & 4.2 & 14.5 & $71 \%$ \\
\hline \multirow{4}{*}{$1.9 \mathrm{~m}$} & $1 \mathrm{kph}$ & 16.8 & 89.9 & $81 \%$ \\
\hline & $3 \mathrm{kph}$ & 15.6 & 14.6 & $-7 \%$ \\
\hline & $6 \mathrm{kph}$ & 4.4 & 89.9 & $95 \%$ \\
\hline & $9 \mathrm{kph}$ & 4.7 & 8.0 & $41 \%$ \\
\hline \multirow{4}{*}{$2.0 \mathrm{~m}$} & $1 \mathrm{kph}$ & 13.6 & 16.1 & $15 \%$ \\
\hline & $3 \mathrm{kph}$ & 15.2 & 90.0 & $83 \%$ \\
\hline & $6 \mathrm{kph}$ & 6.0 & 90.0 & $93 \%$ \\
\hline & $9 \mathrm{kph}$ & 5.6 & 27.9 & $80 \%$ \\
\hline
\end{tabular}


Table 5.8. Body vertical and longitudinal PSD RMS acceleration comparison of controlled and uncontrolled systems

\begin{tabular}{|c|c|c|c|c|c|c|c|}
\hline & & Controlled & Uncontrolled & & Controlled & \begin{tabular}{|l|} 
Uncontrolled \\
\end{tabular} & \\
\hline $\begin{array}{l}\text { Trench } \\
\text { Length }\end{array}$ & Speed & $\begin{array}{c}\mathbf{a}_{\mathrm{z}} \\
\mathbf{R M S} \\
{\left[\mathbf{A}^{2} / \mathbf{H z}\right]}\end{array}$ & $\begin{array}{c}\mathbf{a}_{\mathbf{z}} \\
\mathbf{R M S} \\
{\left[\mathbf{A}^{2} / \mathbf{H z}\right]}\end{array}$ & $\%$ & $\begin{array}{c}\mathbf{a}_{\mathbf{l}} \\
\mathbf{R M S} \\
{\left[\mathbf{A}^{2} / \mathbf{H z}\right]}\end{array}$ & $\begin{array}{c}\mathbf{a}_{\mathbf{l}} \\
\mathbf{R M S} \\
{\left[\mathbf{A}^{2} / \mathbf{H z}\right]}\end{array}$ & $\%$ \\
\hline & $1 \mathrm{kph}$ & 0.86 & 0.78 & $-10 \%$ & 0.92 & 0.46 & $-100 \%$ \\
\hline & $3 \mathrm{kph}$ & 0.84 & 1.08 & $22 \%$ & 0.74 & 1.02 & $27 \%$ \\
\hline $1.2 \mathrm{~m}$ & $6 \mathrm{kph}$ & 0.94 & 1.47 & $36 \%$ & 1.02 & 2.43 & $58 \%$ \\
\hline & $9 \mathrm{kph}$ & 2.02 & 1.75 & $-15 \%$ & 1.24 & 1.52 & $18 \%$ \\
\hline & $1 \mathrm{kph}$ & 0.91 & 1.25 & $27 \%$ & 0.99 & 1.10 & $10 \%$ \\
\hline $13 \mathrm{~m}$ & $3 \mathrm{kph}$ & 0.84 & 1.99 & $58 \%$ & 0.74 & 1.12 & $34 \%$ \\
\hline $1.3 \mathrm{~m}$ & $6 \mathrm{kph}$ & 1.40 & 3.44 & $59 \%$ & 0.92 & 2.85 & $68 \%$ \\
\hline & $9 \mathrm{kph}$ & 1.88 & 1.89 & $1 \%$ & 1.22 & 1.78 & $31 \%$ \\
\hline & $1 \mathrm{kph}$ & 0.92 & 1.34 & $31 \%$ & 1.16 & 1.15 & $-1 \%$ \\
\hline & $3 \mathrm{kph}$ & 1.11 & 3.91 & $72 \%$ & 0.79 & 4.35 & $82 \%$ \\
\hline $1.4 \mathrm{~m}$ & $6 \mathrm{kph}$ & 1.15 & 3.63 & $68 \%$ & 0.88 & 3.03 & $71 \%$ \\
\hline & $9 \mathrm{kph}$ & 1.03 & 8.19 & $87 \%$ & 1.37 & 5.77 & $76 \%$ \\
\hline & $1 \mathrm{kph}$ & 0.89 & 0.74 & $-20 \%$ & 1.50 & 0.73 & $-105 \%$ \\
\hline 15 & $3 \mathrm{kph}$ & 1.07 & 2.19 & $51 \%$ & 0.88 & 2.37 & $63 \%$ \\
\hline 1.5 & $6 \mathrm{kph}$ & 1.09 & 6.22 & $82 \%$ & 0.94 & 2.91 & $68 \%$ \\
\hline & $9 \mathrm{kph}$ & 1.13 & 5.26 & $79 \%$ & 1.36 & 4.86 & $72 \%$ \\
\hline & $1 \mathrm{kph}$ & 0.99 & 0.57 & $-74 \%$ & 1.38 & 0.56 & $-146 \%$ \\
\hline $16 \mathrm{~m}$ & $3 \mathrm{kph}$ & 1.18 & 2.06 & $43 \%$ & 1.01 & 2.53 & $60 \%$ \\
\hline $1.0 \mathrm{~m}$ & $6 \mathrm{kph}$ & 0.85 & 4.06 & $79 \%$ & 1.02 & 3.96 & $74 \%$ \\
\hline & $9 \mathrm{kph}$ & 1.24 & 5.24 & $76 \%$ & 1.37 & 4.30 & $68 \%$ \\
\hline & $1 \mathrm{kph}$ & 1.04 & 1.20 & $13 \%$ & 2.06 & 1.56 & $-32 \%$ \\
\hline $17 \mathrm{~m}$ & \begin{tabular}{|l|l|}
$\mathrm{kph}$ \\
\end{tabular} & 1.29 & 1.92 & $33 \%$ & 1.01 & 2.11 & $52 \%$ \\
\hline $1 . / \mathrm{m}$ & $6 \mathrm{kph}$ & 0.81 & 3.98 & $80 \%$ & 1.01 & 5.91 & $83 \%$ \\
\hline & $9 \mathrm{kph}$ & 1.29 & 5.39 & $76 \%$ & 1.36 & 4.15 & $67 \%$ \\
\hline & $1 \mathrm{kph}$ & 1.27 & 1.58 & $20 \%$ & 1.51 & 1.67 & $10 \%$ \\
\hline $18 \mathrm{~m}$ & $3 \mathrm{kph}$ & 1.32 & 3.09 & $57 \%$ & 0.99 & 3.47 & $71 \%$ \\
\hline $1.8 \mathrm{~m}$ & $6 \mathrm{kph}$ & 0.83 & 11.19 & $93 \%$ & 1.04 & 12.75 & $92 \%$ \\
\hline & $9 \mathrm{kph}$ & 1.28 & 6.47 & $80 \%$ & 1.34 & 4.16 & $68 \%$ \\
\hline & $1 \mathrm{kph}$ & 1.12 & 1.96 & $43 \%$ & 1.22 & 1.70 & $28 \%$ \\
\hline $10 \mathrm{~m}$ & $3 \mathrm{kph}$ & 1.43 & 2.72 & $47 \%$ & 0.96 & 2.73 & $65 \%$ \\
\hline $1.9 \mathrm{~m}$ & $6 \mathrm{kph}$ & 1.00 & 6.83 & $85 \%$ & 1.00 & 6.46 & $85 \%$ \\
\hline & $9 \mathrm{kph}$ & 1.50 & 10.26 & $85 \%$ & 1.22 & 5.95 & $79 \%$ \\
\hline & $1 \mathrm{kph}$ & 1.19 & 1.27 & $6 \%$ & 1.05 & 2.22 & $53 \%$ \\
\hline $20 n$ & $3 \mathrm{kph}$ & 1.34 & 4.29 & $69 \%$ & 0.96 & 4.88 & $80 \%$ \\
\hline $2.0 \mathrm{~m}$ & \begin{tabular}{|l}
$6 \mathrm{kph}$ \\
\end{tabular} & 1.32 & 7.03 & $81 \%$ & 1.01 & 7.19 & $86 \%$ \\
\hline & $9 \mathrm{kph}$ & 1.82 & 14.96 & $88 \%$ & 1.18 & 17.96 & $93 \%$ \\
\hline
\end{tabular}


As can be seen in Table 5.6, the controlled system has provided significant improvements in terms of longitudinal peak acceleration compared to the uncontrolled system. It is possible to say the same for vertical acceleration with two exceptions. Only at the lowest trench lengths, which are almost close to the wheel diameter, occurred in the highest speed scenario. As seen in Table 5.6, only 1.2 and $1.3 \mathrm{~m}$ trench lengths and $9 \mathrm{kph}$ speed, the body vertical maximum accelerations seem better in the uncontrolled vehicle. In all other cases, the controlled system has provided severe improvements.

Table 5.7 showing the comparison of pitch angles shows that, in general, the controlled system has improved the pitch behaviour of the system. Here, rather than pitch correction in the trench crossing algorithm, suppressing body accelerations is more prominent. However, it is the main target that the vehicle passes the trench without hitting any sub-systems to the ground. The pitch angle can be seen as high in some scenarios. The main reason for this is simulations with the same controller parameters at speeds from $1 \mathrm{kph}$ to $9 \mathrm{kph}$. However, in general, trench transitions have been completed in controlled systems without problems such as hitting sub-systems, crossing bumpers to the trench start and finish or very high accelerations, despite high pitch angles in some situations.

When Table 5.8 is examined, it can be seen that the controlled systems have higher RMS value in some scenarios in terms of PSD RMS values. This limited number of situations generally occurred in scenarios where short and medium-length trenches were passed at low speed. Although the percentage differences seem to be excessive in these scenarios, it can be seen that their absolute values are still within limits. In all cases except these few scenarios, the controlled system has made significant improvements to PSD RMS acceleration values.

Figure 5.39 shows an example of PSD plot. RMS acceleration values are found by the square root of the area under the envelope lines drawn for response in these PSD plots. 


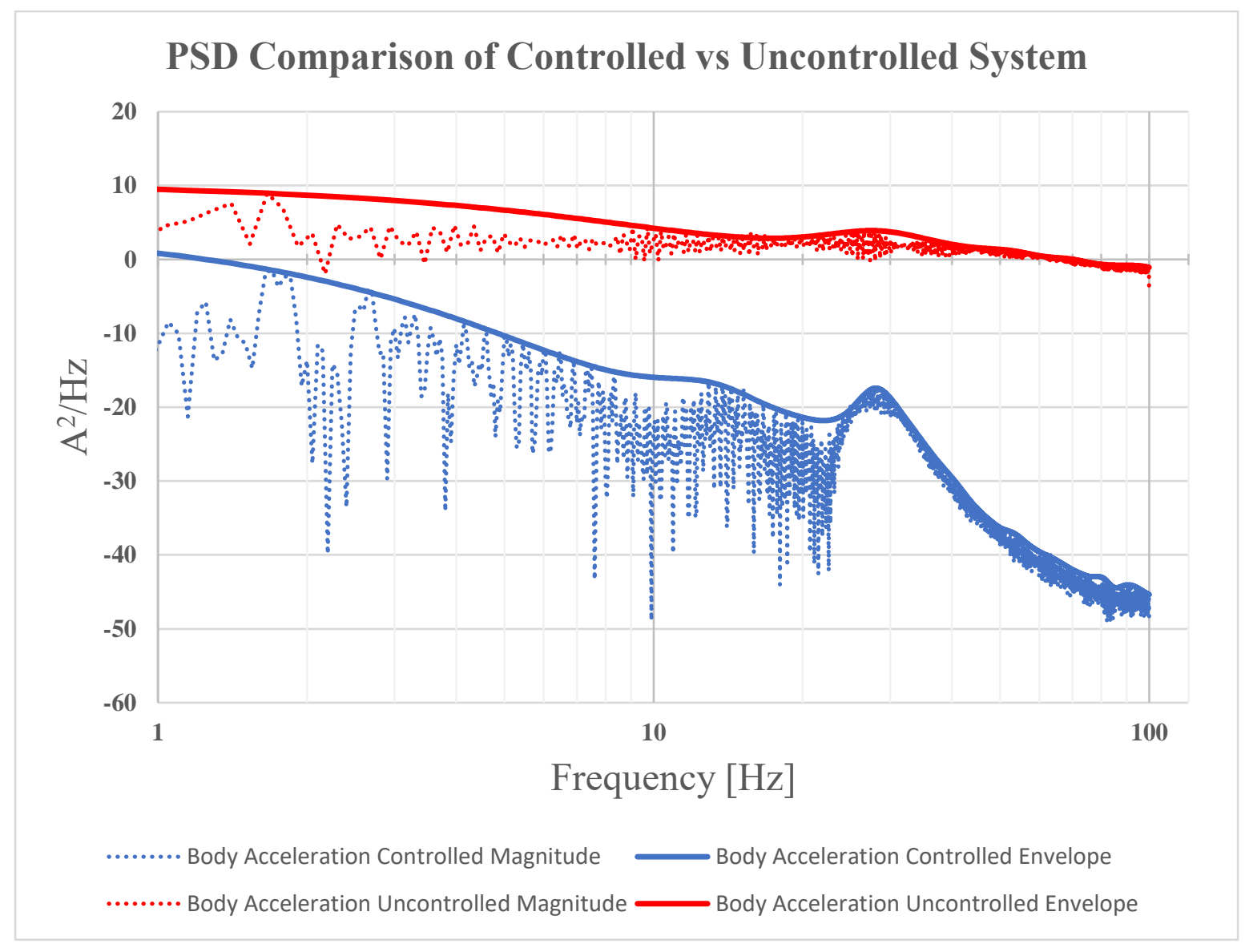

Figure 5.39. PSD Comparison example of controlled vs uncontrolled system, 1.8m, $6 \mathrm{kph}$

In Table 5.9, a comparison table is given for each parameter over cost functions calculated with specific weights. Each given scenario is scored from 1 to 5 . Here, 1 is the lowest, 5 is the highest-rated area scenario. Again, in all these scenarios with a controlled system, trench transition has been determined successfully. This scoring can be considered as the performance evaluation of the controller and the trenching algorithm under different conditions. 
Table 5.9. Controlled system simulations rating

\begin{tabular}{|c|c|c|c|c|c|c|c|c|}
\hline \multirow{2}{*}{$\begin{array}{l}\text { Trench } \\
\text { Length }\end{array}$} & \multirow{2}{*}{ Speed } & \multirow{2}{*}{$\begin{array}{l}\text { Cost } \\
\text { Function }\end{array}$} & \multicolumn{6}{|c|}{ Rating } \\
\hline & & & Score & 1 & 2 & 3 & 4 & J \\
\hline \multirow{4}{*}{$1.2 \mathrm{~m}$} & $1 \mathrm{kph}$ & 93.7 & 3.3 & $\bar{t}$ & $\bar{\hbar}$ & 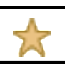 & $\bar{t}$ & $\underline{\underline{w}}$ \\
\hline & $3 \mathrm{kph}$ & 1616.4 & 3.5 & $\bar{t}$ & $\bar{t}$ & $\sqrt{2}$ & th & 23 \\
\hline & $6 \mathrm{kph}$ & 1638.5 & 3.5 & $\sqrt{t}$ & $\bar{t}$ & $\sqrt{t}$ & $i s$ & $\bar{t}$ \\
\hline & $9 \mathrm{kph}$ & 1184.2 & 5.0 & th & t & th & th & 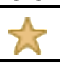 \\
\hline \multirow{4}{*}{$1.3 \mathrm{~m}$} & $1 \mathrm{kph}$ & 92.4 & 2.5 & $\bar{t}$ & tל & 项 & $\sqrt{2}$ & 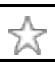 \\
\hline & $3 \mathrm{kph}$ & 1294.5 & 4.6 & th & $\hbar$ & $\sqrt{t}$ & t & {$[3$} \\
\hline & $6 \mathrm{kph}$ & 1347.5 & 4.4 & th & $\sqrt{t}$ & $\hbar$ & th & $i s$ \\
\hline & $9 \mathrm{kph}$ & 99.8 & 4.9 & th & $\frac{1}{5}$ & $\hbar$ & $\sqrt{t}$ & th \\
\hline \multirow{4}{*}{$1.4 \mathrm{~m}$} & $1 \mathrm{kph}$ & 2.4 & 3.0 & $\bar{t}$ & th & th & $\underline{\hbar t}$ & $\underline{t}$ \\
\hline & $3 \mathrm{kph}$ & 52.8 & 4.1 & t & th & $\hbar$ & t & $\underline{t}$ \\
\hline & $6 \mathrm{kph}$ & 76.0 & 4.7 & t & th & $\hbar$ & th & 级 \\
\hline & $9 \mathrm{kph}$ & 163.6 & 4.0 & th & th & $\hbar$ & th & is \\
\hline \multirow{4}{*}{1.5} & $1 \mathrm{kph}$ & 27.8 & 3.2 & t & t & $\hbar$ & $\hat{s}$ & $t^{2}$ \\
\hline & $3 \mathrm{kph}$ & 1651.1 & 3.4 & t & 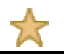 & $\hbar$ & $\hat{s}$ & $t^{2}$ \\
\hline & $6 \mathrm{kph}$ & 453.5 & 4.1 & t & t & th & 放 & 2 \\
\hline & $9 \mathrm{kph}$ & 65.0 & 4.0 & t & t & $\hbar$ & t & 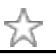 \\
\hline \multirow{4}{*}{$1.6 \mathrm{~m}$} & $1 \mathrm{kph}$ & 92.6 & 2.3 & t & t & 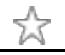 & 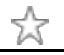 & 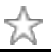 \\
\hline & $3 \mathrm{kph}$ & 1896.2 & 2.6 & $\sqrt{t}$ & $\hbar$ & 项 & 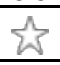 & $\varepsilon$ \\
\hline & $6 \mathrm{kph}$ & 43.0 & 3.4 & t & t & $\hbar$ & 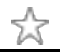 & 2 \\
\hline & $9 \mathrm{kph}$ & 02.8 & 3.9 & t & t & th & th & 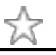 \\
\hline \multirow{4}{*}{$1.7 \mathrm{~m}$} & $1 \mathrm{kph}$ & 80.1 & $2 . c$ & t & t & $\underline{t}$ & $\omega^{2}$ & $\underline{n}$ \\
\hline & $3 \mathrm{kph}$ & 89.1 & 2.3 & t & t & $\hat{\omega}$ & 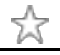 & ह \\
\hline & $6 \mathrm{kph}$ & 521.2 & 3.5 & t & t & ts & th & in \\
\hline & $9 \mathrm{kph}$ & 177.5 & 4.0 & t & th & $\hbar$ & t & th \\
\hline \multirow{4}{*}{$1.8 \mathrm{~m}$} & $1 \mathrm{kph}$ & 65.7 & 2.0 & $t^{2}$ & $\bar{t}$ & $\hbar$ & $\bar{t}$ & $\underline{2}$ \\
\hline & $3 \mathrm{kph}$ & 2009.0 & 2.2 & $\bar{t}$ & $\bar{t}$ & $\hat{\omega}$ & $\hat{\hbar}$ & $\underline{t}$ \\
\hline & $6 \mathrm{kph}$ & 1615.5 & 3.5 & th & th & th & th & $\frac{1}{n}$ \\
\hline & $9 \mathrm{kph}$ & 91.3 & 4.0 & th & th & $\hbar$ & th & $x^{2}$ \\
\hline \multirow{4}{*}{$1.9 \mathrm{~m}$} & $1 \mathrm{kph}$ & 2.9 & 1.0 & t & $i s$ & $\hbar$ & $i s$ & $\frac{1}{2}$ \\
\hline & $3 \mathrm{kph}$ & 1989.0 & 2.3 & 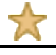 & $\hbar$ & $\sqrt{2}$ & 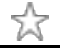 & 过 \\
\hline & $6 \mathrm{kph}$ & 4 & 3.9 & t & t & $\hbar$ & th & $\underline{n}$ \\
\hline & $9 \mathrm{kph}$ & 51.7 & 4.8 & t & t & $\hbar$ & t & 功 \\
\hline \multirow{4}{*}{$2.0 \mathrm{~m}$} & $1 \mathrm{kph}$ & 8 & 1. & t & th & 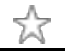 & $t^{2}$ & 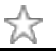 \\
\hline & $3 \mathrm{kph}$ & 2014.4 & 2.2 & t & t & $\hbar$ & 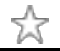 & $\underline{\omega}$ \\
\hline & $6 \mathrm{kph}$ & 12924 & 4. & t & th & ts & t & 占 \\
\hline & $9 \mathrm{kph}$ & 1177.5 & 5.0 & $\sqrt{t}$ & $\sqrt{t}$ & th & $\sqrt{k}$ & \\
\hline
\end{tabular}


Weights determined for the cost function given in Table 5.9 are given in Table 5.10 given below.

Table 5.10. Controlled system simulations rating weights

\begin{tabular}{|c|c|c|c|c|c|c|c|c|}
\hline Parameter & $\begin{array}{c}\text { Max. } \\
\mathbf{a}_{\mathbf{z}} \\
{\left[\mathbf{m} / \mathbf{s}^{2}\right]}\end{array}$ & $\begin{array}{c}\text { Max. } \\
\text { al } \\
{\left[\mathbf{m} / \mathbf{s}^{2}\right]}\end{array}$ & $\begin{array}{c}\text { RMS } \\
\mathbf{a}_{\mathbf{z}} \\
{\left[\mathbf{A}^{\mathbf{2}} / \mathbf{H z}\right]}\end{array}$ & $\begin{array}{c}\text { RMS } \\
\text { al } \\
{\left[\mathbf{A}^{2} / \mathbf{H z}\right]}\end{array}$ & $\begin{array}{c}\text { Max. } \\
\gamma \\
{[\mathrm{deg}]}\end{array}$ & $\begin{array}{c}\text { Max. } \\
\text { Fhub,f } \\
{[k \mathbf{N}]}\end{array}$ & $\begin{array}{c}\text { Max. } \\
\text { Fhub,m } \\
{[\mathrm{kN}]}\end{array}$ & $\begin{array}{c}\text { Max. } \\
\text { F hub,r } \\
{[\mathrm{kN}]}\end{array}$ \\
\hline $\begin{array}{c}\text { Cost } \\
\text { Function } \\
\text { Weight } \\
{[\%]}\end{array}$ & 12.5 & 17.5 & 12.5 & 15.0 & 17.5 & 10.0 & 7.5 & 7.5 \\
\hline
\end{tabular}

As can be seen in Table 5.9, the trench crossings in the middle and upper-speed bands were scored higher in almost all trench lengths. If the weighted average of trench crossing speeds is to be taken according to the rating points, the average speed is approximately $5.5 \mathrm{kph}$. This can be regarded as the optimum trench cross speed with trenches from $1.2 \mathrm{~m}$ to $2.0 \mathrm{~m}$ and the controller designed for $6 \times 6$ vehicles of the specified specifications.

Cost functions for both controlled system and uncontrolled system was calculated based on the specified parameters and their percentile weights. System behaviour improves as these values go to small value.

Cost functions calculated for active and passive systems are normalized between 0 and 1 and shown in Table 5.11. Next, Figure 5.40 shows the contour plot for these normalized cost functions, and Figure 5.41 shows the surface plot. Both the table values and the two plots provided show that the MPC controller with preview significantly improves system output. A reference normalized cost is calculated and showed in Figure 5.41. This reference value is calculated based on the limit values shown in Table 5.3. The vehicles that passively pass the trench are below the determined cost, but in some cases, they do not cross the trench even though they are below the cost. The main reason for this is that rather than the numerical values obtained from simulations, the vehicle subsystems have physical contact with ground or cannot provide enough traction. 
Table 5.11. Cost function comparison of controlled vs uncontrolled systems

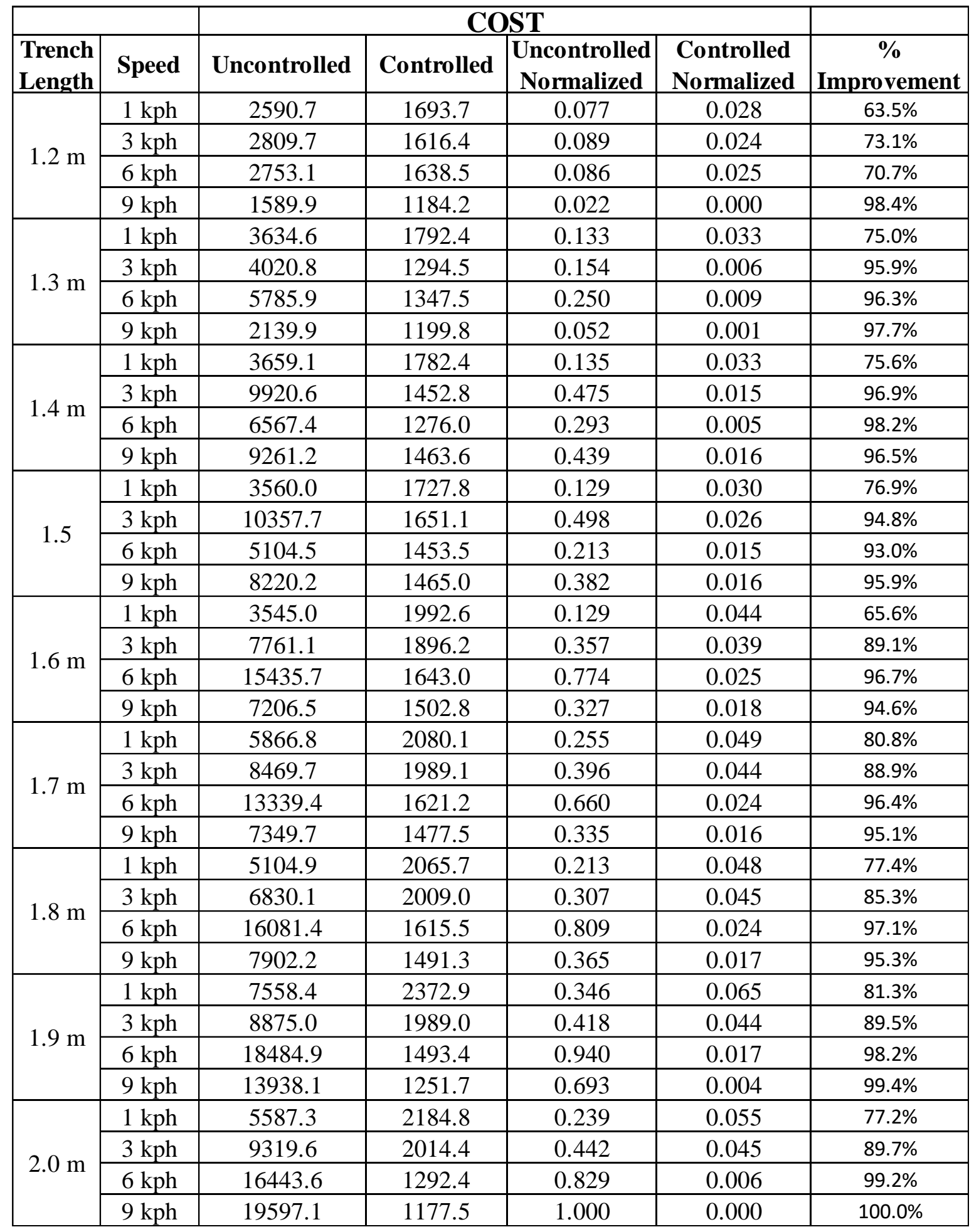




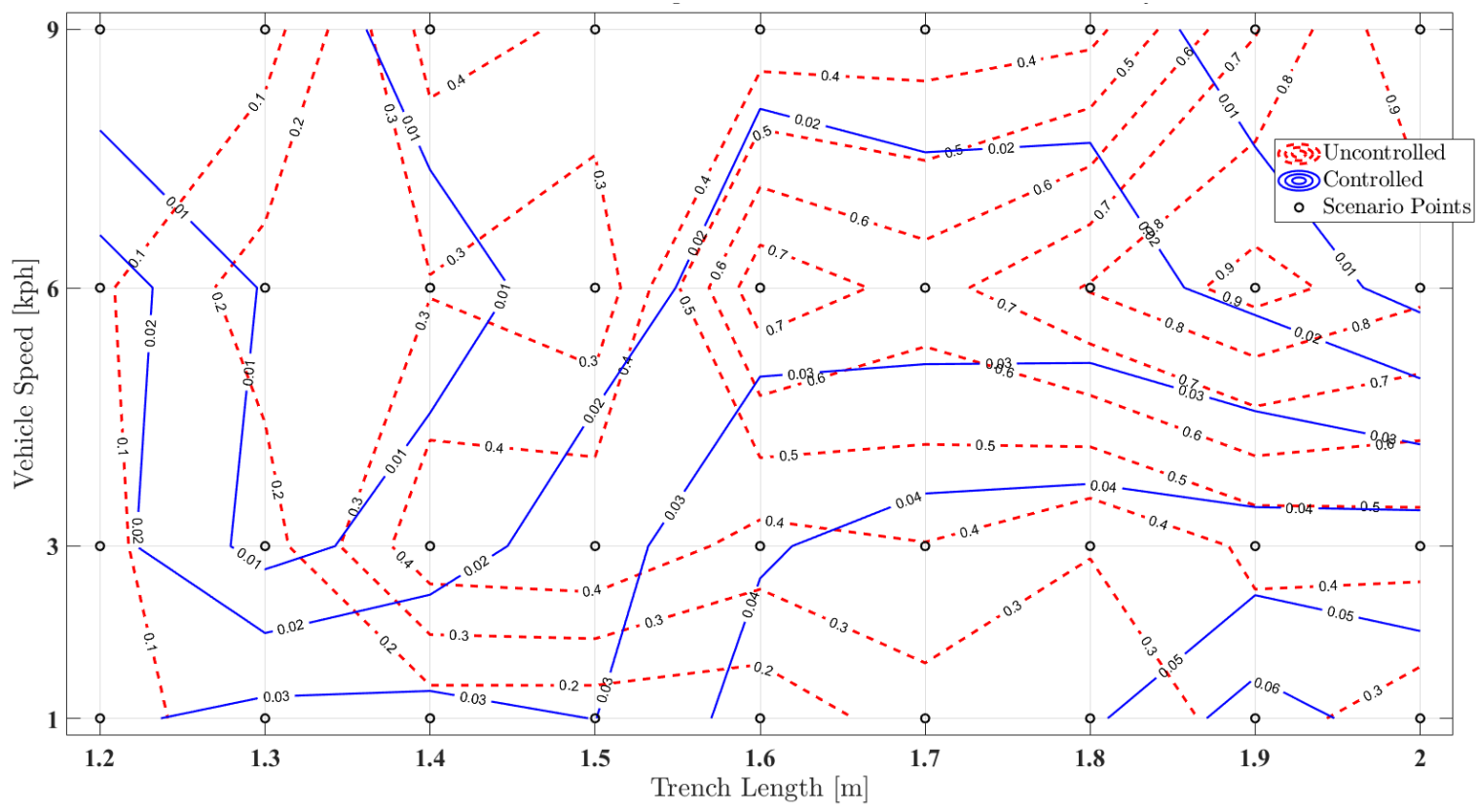

Figure 5.40. Contour plot of normalized cost function comparison of controlled vs uncontrolled systems

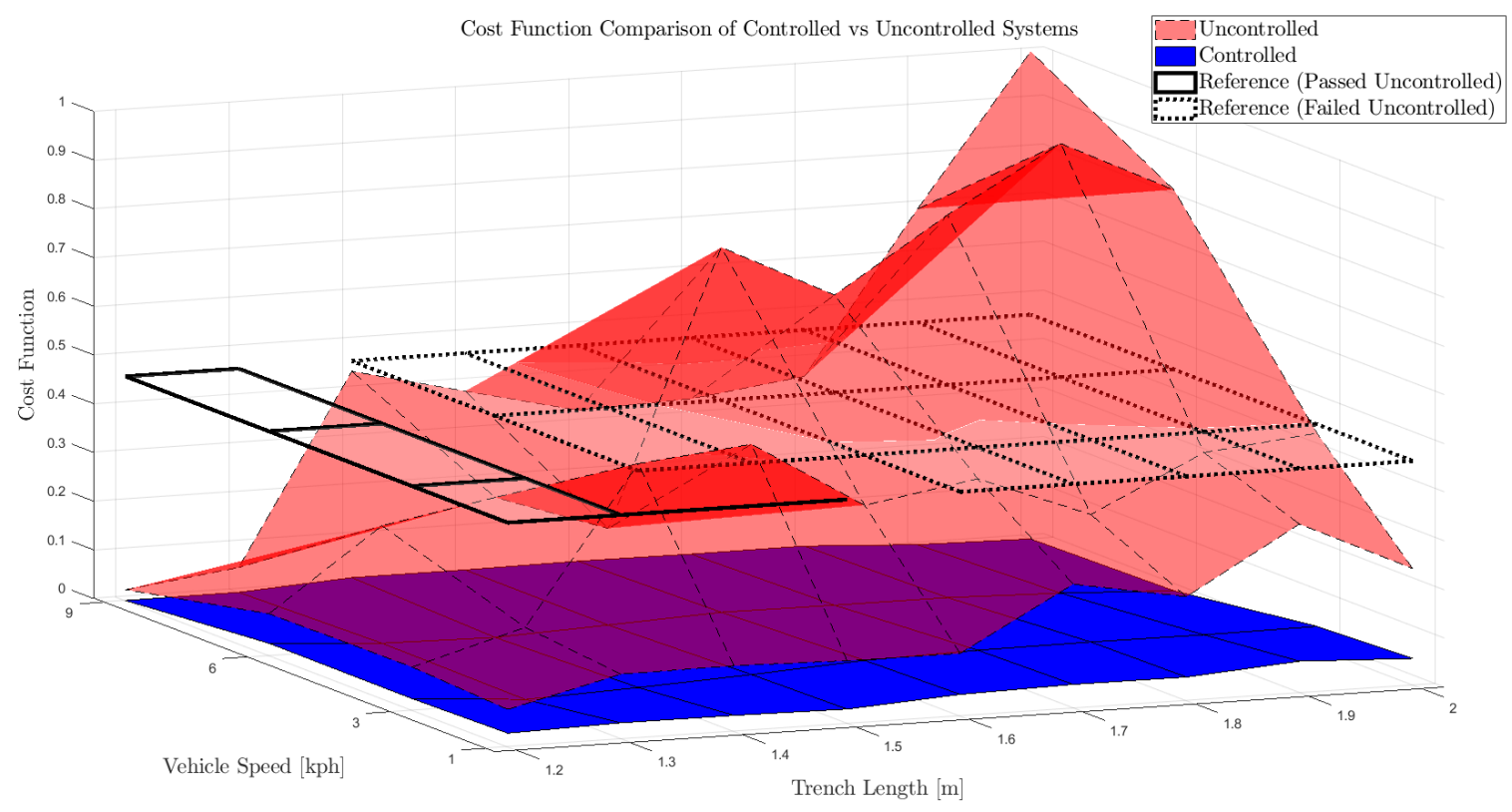

Figure 5.41. Surface plot of normalized cost function comparison of controlled vs uncontrolled systems 


\section{CONCLUSION AND FUTURE WORK}

\subsection{Conclusion}

In this section, the general summary of the studies done for the thesis and the point reached has been mentioned. As stated in the previous sections, this thesis aims to increase the limits of trench crossing operations. In line with this goal, passive system simulations and simulations using several different controllers are presented. How much the mobility capabilities of a $6 \times 6$ wheeled military vehicle can be increased is given in the results (Section 5) and comparison ( $\underline{\text { Section 5.6) }}$ sections.

As the control method to realize the trench cross algorithm, which is the target of the thesis, MPC with preview support, has been determined. How MPC works is explained in previous sections.

It can accommodate different system models to be linear or non-linear or adaptive within different MPC variants. Optimization and prediction are made thanks to this model. In this thesis, 9 DOF linear full car ride models were found to be sufficient for MPC since it was aimed to control the acceleration and pitch values of the vehicle in the vertical direction and to perform this control with the actuators located in the suspension part.

Controller was designed on this linear model and ultimately this controller was tested with the non-linear model modelled in the ADAMS program. The convergence of the linear model and the multi-body ADAMS model is shown in Section 5.2. This linear model used in the controller for prediction was found to be adequate for the non-linear model. Meanwhile, the system linearized with the small angle assumption generally gives more convergent results in most of controlled systems or systems that can cross the trench with lower pitch angles. 
Compared to LQR, MPC and MPC + preview controllers, MPC + preview controller showed better performance in both disturbance rejection and reference tracking. MPC and MPC + preview are close to each other and clearly better than LQR controllers in terms of steadystate error. However, LQR looks better on ramp track with a little difference from MPC without preview.

Although MPC and LQR controllers follow the reference input from behind according to MPC + preview, in other words, MPC + preview controller starts moving before the input or disturbance; there is more pre-overshoot in the MPC + preview controller. After all reference tracking and disturbance rejection capabilities of the designed controller have been tested and found sufficient for this system.

In the studies with linear model, LQR, MPC and MPC + preview controllers have been designed over the linear model. These are tuned according to the linear model. The LQR controller is used for comparison with the MPC designed as the primary target. However, neither MPC without preview nor LQR can cross alone deep trenches. Regardless of these controllers, a reference pitch angle or contingent and momentary changing constraints/weights should be given according to the position of the vehicle relative to the trench, and the axles of the vehicle should be placed in the most suitable position according to the trench.

When it comes to the part that forms the primary basis of the thesis work, MPC controller, with its most general expression, has significantly increased the trench crossing ability with the support of the preview and the trench cross algorithm.

Numerically speaking, it was seen in the data in Table 5.4 that the passive vehicle can cross the maximum trench of $1.5 \mathrm{~m}$. Moreover, trenches such as 1.5 and $1.4 \mathrm{~m}$ can only pass at very low speeds, such as $1 \mathrm{kph}$. It can also pass shorter trenches at higher speeds due to wheels with diameters close to the trench length of 1.2 or $1.3 \mathrm{~m}$. However, with the increase in speed from 1.4-1.5 m length, the passive vehicle was not successful in any scenario. 
When looking at the controlled system, it can be seen that the trench crossing is completed under the specified criteria in all scenarios from $1.2 \mathrm{~m}$ length to $2.0 \mathrm{~m}$ length and from $1 \mathrm{kph}$ speed to $9 \mathrm{kph}$ speed. Here, as well as the MPC controller optimizing around specific parameters, the algorithm used in conjunction with the preview road signal has a critical role in feeding the required parameters to MPC instantly.

As a result, it has been demonstrated that the ability of a $6 \times 6$ wheeled military vehicle to trench cross operation can be significantly increased.

\subsection{Future Work}

Vehicle parameters and features used in this thesis are not used directly for privacy reasons. The geometric dimensions are at a level to compare a class equivalent to a particular class. The spring and damper characteristics used were linearized, and a thesis study was carried out to be different at a certain level. The primary purpose of the thesis is to show that the trench cross capabilities can be increased by using the hydro-struts on the axles actively, with the MPC controller with preview support. In this thesis, it has been shown that there can be a severe improvement.

Hydro-pneumatic suspensions are used in most military vehicle applications that adjust the ride height. These suspensions, as mentioned in the literature, are essential system components with an internal structure and sophisticated characteristics. In this thesis, these characteristics are used by linearizing around a specific working condition. In cases where the study presented in the thesis will be used in real applications, the internal structure and dynamics of these hydro-pneumatic suspensions should also be included in the model. At this point, the use of non-linear MPC or adaptive MPC may be inevitable instead of simple MPC used in the thesis.

Another point is that the use of actuator limits used in this thesis may be limited in real applications. However, it can be considered as a trend study to increase mobility. After it has been demonstrated that the mobility of $6 x 6$ vehicles can be improved significantly with the active ride height control, this obstacle crossing operation will be possible with a loop that also controls the speed of the vehicle because the actuator limits in real vehicles are not as 
high as seen in this study. However, this is possible thanks to the lower limit actuators or hydropneumatic suspensions that can reach the required ride height and pitch angle by making much lower speeds or by stop-and-go.

For applications that can be developed prospectively, MPC and preview assisted control mechanisms in providing a basis for autonomous driving or support systems. The vehicle used in this thesis is a typical armoured personnel carrier or similar vehicle. However, considering the weights and thus the components to be used in unmanned ground vehicle applications, many full or semi-autonomous driving operations that will be the basis of the study in this thesis do not seem far away. 


\section{REFERENCES}

[1] W. Bauer, Hydropneumatic Suspension Systems, Springer, Heidelberg, Chapter 1, 2011, [2] Products, FNSS Savunma Sistemleri A.Ş., https://www.fnss.com.tr/en/products, (Date of Access: 02 June 2020)

[3] D. Simner, Unpublished "Military Vehicle Design, Wheels vs Tracks" lecture notes, Cranfield University, 2019.

[4] F. Saglam, Y.S. Unlusoy, Hidro-Pnömatik Süspansiyonlu Araçlar İçin Tümleşik Sürüş Konforu ve Yükseklik Kontrolü, 7. Otomotiv Teknolojileri Kongresi, OTEKON, Bursa, 2014.

[5] M. Oscarsson, A Hydropneumatic Suspension Parameter Study on Heavy Multi-Axle Vehicle Handling, Master Thesis, KTH, School of Engineering Sciences (SCI), Aeronautical and Vehicle Engineering, Stockholm, Sweden, 2015.

[6] D.A. B., K. SenthilKumar, P.D. H.V.Vankudre, Modeling and Simulation of Multi-Axle Combat Vehicle Model over Different Terrain Obstacles, International Journal of Emerging Technology and Advanced Engineering, Volume 4, Issue 12 (2014).

[7] M. Trikande, V. Jagirdar, V. Rajamohan, P.R. Rao, Investigation on Semi-active Suspension System for Multi-axle Armoured Vehicle using Co-simulation, Defence Science Journal, 67 (2017) 269-275.

[8] W. Hao, X. Xu, H. Xu, F. Zhou, Enhancing the Obstacle-crossing Performance of AllTerrain Vehicle Based on Variable-wheelbase Chassis *, 2019 IEEE/ASME International Conference on Advanced Intelligent Mechatronics (AIM), 2019, pp. 1329-1334.

[9] V.V. Jagirdar, M.W. Trikande, Terrain Accessibility Prediction for a New Multi axle Armoured Wheeled Vehicle, 2019.

[10] A.V. Papunin, V.V. Belyakov, V.S. Makarov, Calculation of trench width to be overcome by multi-axis wheeled vehicle, IOP Conference Series: Earth and Environmental Science, 194 (2018) 062027.

[11] G. Thomas, V.V. Vantsevich, Wheel-terrain-obstacle interaction in vehicle mobility analysis, Vehicle System Dynamics, 48 (2010) 139-156.

[12] R.N. Jazar, Vehicle Dynamics: Theory and Applicaiton 3rd ed., Springer, New York, NY, 2017.

[13] Thomas D. Gillespie, Fundamentals of Vehicle Dynamics, Society of Automotive Engineers Inc., Warrendale, PA, 1992. 
[14] K. Bayar, Modeling of the Dynamics of Multi-Axle Steered Vehicles, Master Thesis, The Graduate School of Natural and Applied Sciences of Middle East Technical University, Ankara, 2006.

[15] E.T. Önder, S.Ç. Başlamışlı, Dynamic Modeling and Simulation of Subcomponents of a 8x8 Four Axle Vehicle, OTEKON 2016 8. Otomotiv Teknolojileri Kongresi,Bursa, 2016. [16] E. Shoesmith, G.E.P. Box, N.R. Draper, Empirical Model-Building and Response Surfaces, The Statistician, 37 (1987) 82.

[17] MSC Software Corporation, MSC ADAMS/Car ${ }^{\mathrm{TM}}$ user guide, 2019.

[18] MSC Software Corporation, MSC ADAMS/Controls ${ }^{\mathrm{TM}}$ user guide, 2019.

[19] Ö. Yazar, 6x6 Taktik Tekerlekli Askeri Aracın Taşıt Dinamiği Modellemesi ve Testler ile Modelin Doğrulaması, Yüksek Lisans Tezi, Gazi Üniversitesi Fen Bilimleri Enstitüsü, Ankara, 2013.

[20] S. Li, L. He, Co-simulation study of vehicle ESP system based on ADAMS and MATLAB, JSW, 6 (2011) 866-872.

[21] Z. Xiu-qin, Y. Bo, Y. Chao, X. Guan-neng, Research on ABS of Multi-axle Truck Based on ADAMS/Car and Matlab/Simulink, Procedia Engineering, 37 (2012) 120-124.

[22] Y. Zhang, M.-m. Xia, J.-y. Qin, H. Zhang, Research on co-simulation using ADAMS and MATLAB for automobile active suspension system, 2010 International Conference on Computer Application and System Modeling (ICCASM 2010), 2010, pp. V14-366-V314-370. [23] J. Richalet, A. Rault, J.L. Testud, J. Papon, Model algorithmic control of industrial processes, IFAC Proceedings Volumes, 10 (1977) 103-120.

[24] A. Bemporad, Model Predictive Control Lecture Notes, IMT School for Advanced Studies Lucca, 2019.

[25] D.Q. Mayne, J.B. Rawlings, C.V. Rao, P.O.M. Scokaert, Constrained model predictive control: Stability and optimality, Automatica, 36 (2000) 789-814.

[26] E.F. Camacho, C. Bordons, Model Predictive Control, Springer, Sevilla, Spain, 1999.

[27] S. Frank, Model Predictive Control, 2018, pp. 91-94

[28] S.V. Raković, W. Levine, Handbook of Model Predictive Control, Springer International Publishing, Birkhäuser, 2019.

[29] J. Maciejowski, Predictive Control With Constraints, Prentice Hall,2002.

[30] T.J.J.v.d. Boom, Model Based Predictive Control: status and perspective, Symposium on Control, Optimization and Supervision CESA 96' IMACS MulticonferenceLille, 1996, pp. 112. 
[31] Y. Rasekhipour, I. Fadakar, A. Khajepour, Autonomous driving motion planning with obstacles prioritization using lexicographic optimization, Control Engineering Practice, 77 (2018) 235-246.

[32] Lane Change Assist Using Nonlinear Model Predictive Control, Mathworks Inc., 2019.

[33] W. Wang, X. Chen, J. Wang, Unsprung Mass Effects on Electric Vehicle Dynamics based on Coordinated Control Scheme, 2019 American Control Conference (ACC), 2019, pp. 971-976.

[34] H.B. Pacejka, Tyre and Vehicle Dynamics 2nd edition, Butterworth-Heinemann, Burlington, MA, 2006.

[35] A. Bemporad, M. Morari, N.L. Ricker, Model Predictive Control Toolbox ${ }^{\mathrm{TM}}$, MathWorks, Inc, Natick, MA, 2015.

[36] L. Wang, Model Predictive Control System Design and Implementation Using MATLAB, Springer, London, 2009. 


\section{APPENDIX A}

Trench Length $1.2 \mathrm{~m}$, Controlled vs Uncontrolled System Simulations

Trench Length $1.2 \mathrm{~m}, 1 \mathrm{kph}$

\begin{tabular}{|c|c|c|}
\hline 00:00:08 & 00:00:09 & 00:00:10 \\
\hline 00:00:11 & 00:00:12 & 00:00:13 \\
\hline 00:00:14 & 00:00:15 & 00:00:16 \\
\hline 00:00:17 & 00:00:18 & 00:00:19 \\
\hline 00:00:20 & 00:00:21 & 00:00:22 \\
\hline 00:00:23 & 00:00:24 & 00:00:25 \\
\hline 00:00:26 & 00:00:27 & 00:00:28 \\
\hline 00:00:29 & 00:00:32 & 00:00:34 \\
\hline
\end{tabular}

Figure A.1. Trench length $1.2 \mathrm{~m}, 1 \mathrm{kph}$, uncontrolled system 


\begin{tabular}{|c|c|c|}
\hline $0=0=0$ & $a=0=0$ & $0=0=c$ \\
\hline 00:00:08 & 00:00:09 & 00:00:10 \\
\hline 00:00:11 & 00:00:12 & 00:00:13 \\
\hline 00:00:14 & 00:00:15 & 00:00:16 \\
\hline 00:00:17 & 00:00:18 & 00:00:19 \\
\hline 00:00:20 & 00:00:21 & 00:00:22 \\
\hline 00:00:23 & 00:00:24 & 00:00:25 \\
\hline 00:00:26 & 00:00:27 & 00:00:28 \\
\hline 00:00:29 & 00:00:31 & 00:00:33 \\
\hline
\end{tabular}

Figure A.2. Trench length $1.2 \mathrm{~m}, 1 \mathrm{kph}$, controlled system 


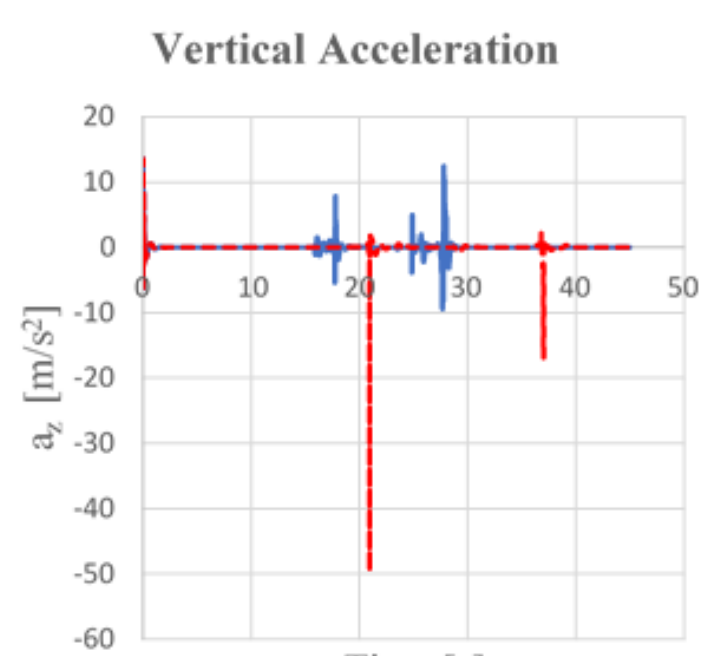

Time $[\mathrm{s}]$

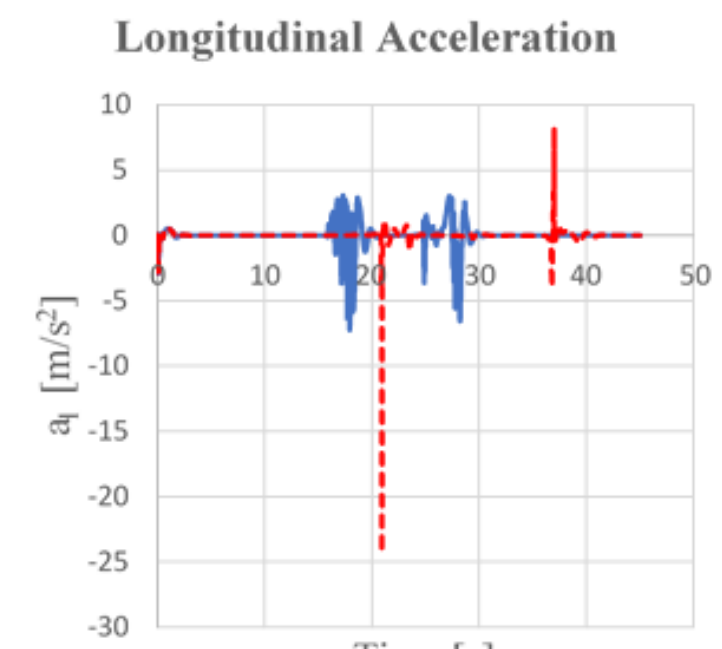

Time $[\mathrm{s}]$

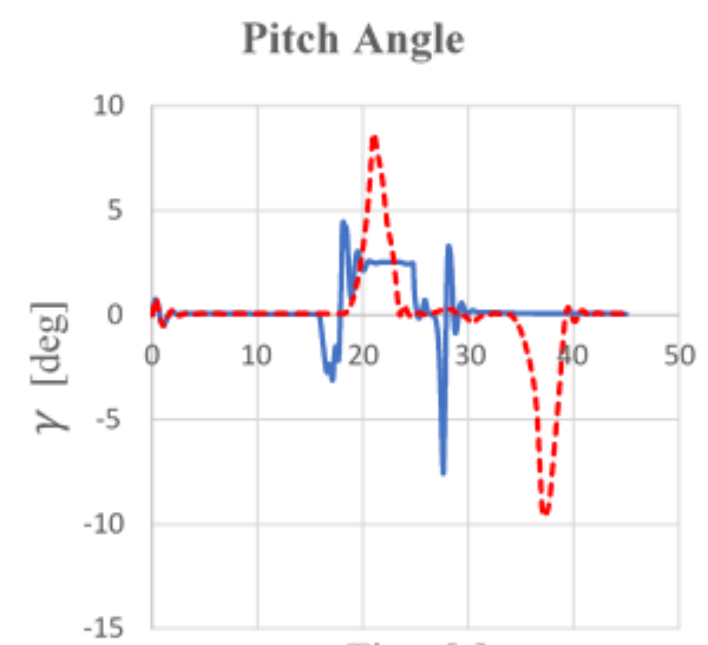

Time $[\mathrm{s}]$
Pitch Angular Velocity

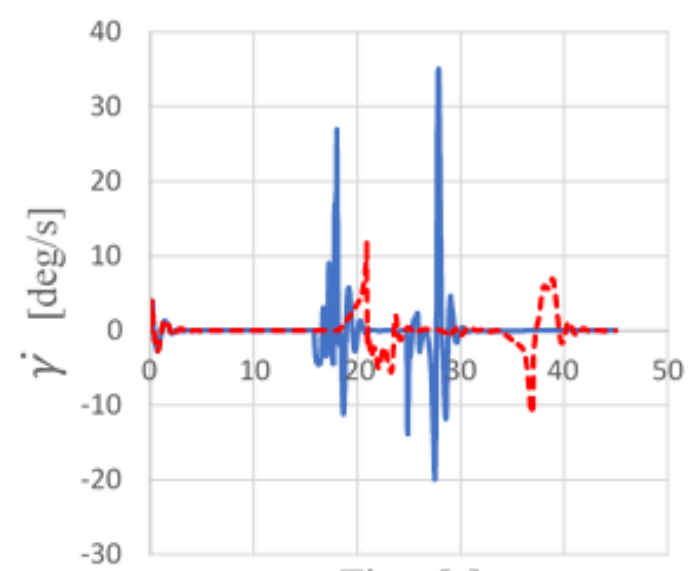

Time $[\mathrm{s}]$

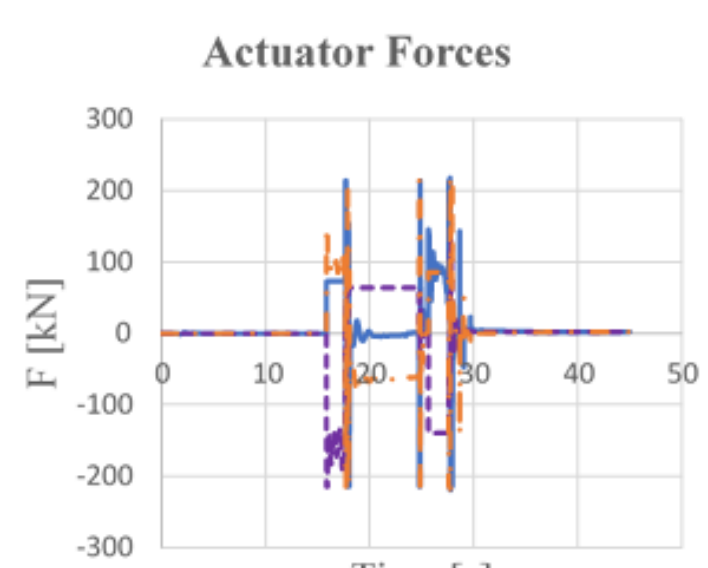

Time $[\mathrm{s}]$

—Axle 1 - - Axle 2 - - Axle 3

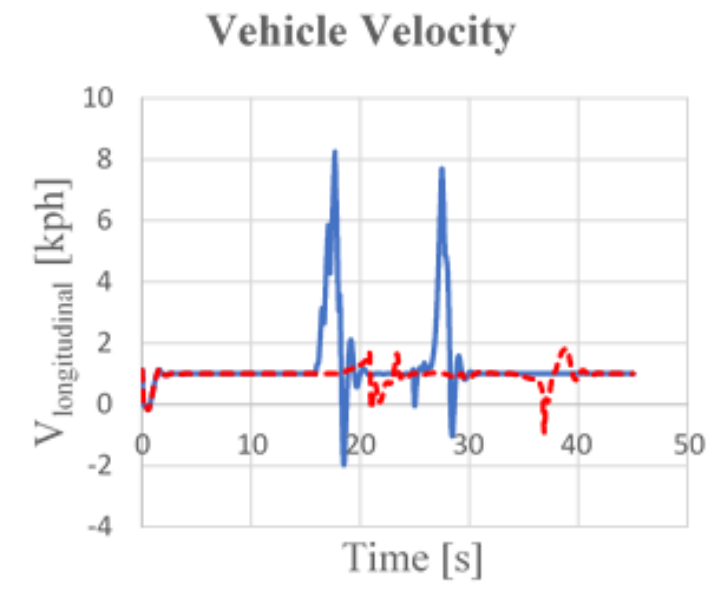

Controlled ----.-Uncontrolled

Figure A.3. Trench length $1.2 \mathrm{~m}, 1 \mathrm{kph}$, controlled vs uncontrolled output comparison 
Trench Length 1.2 m, 3 kph

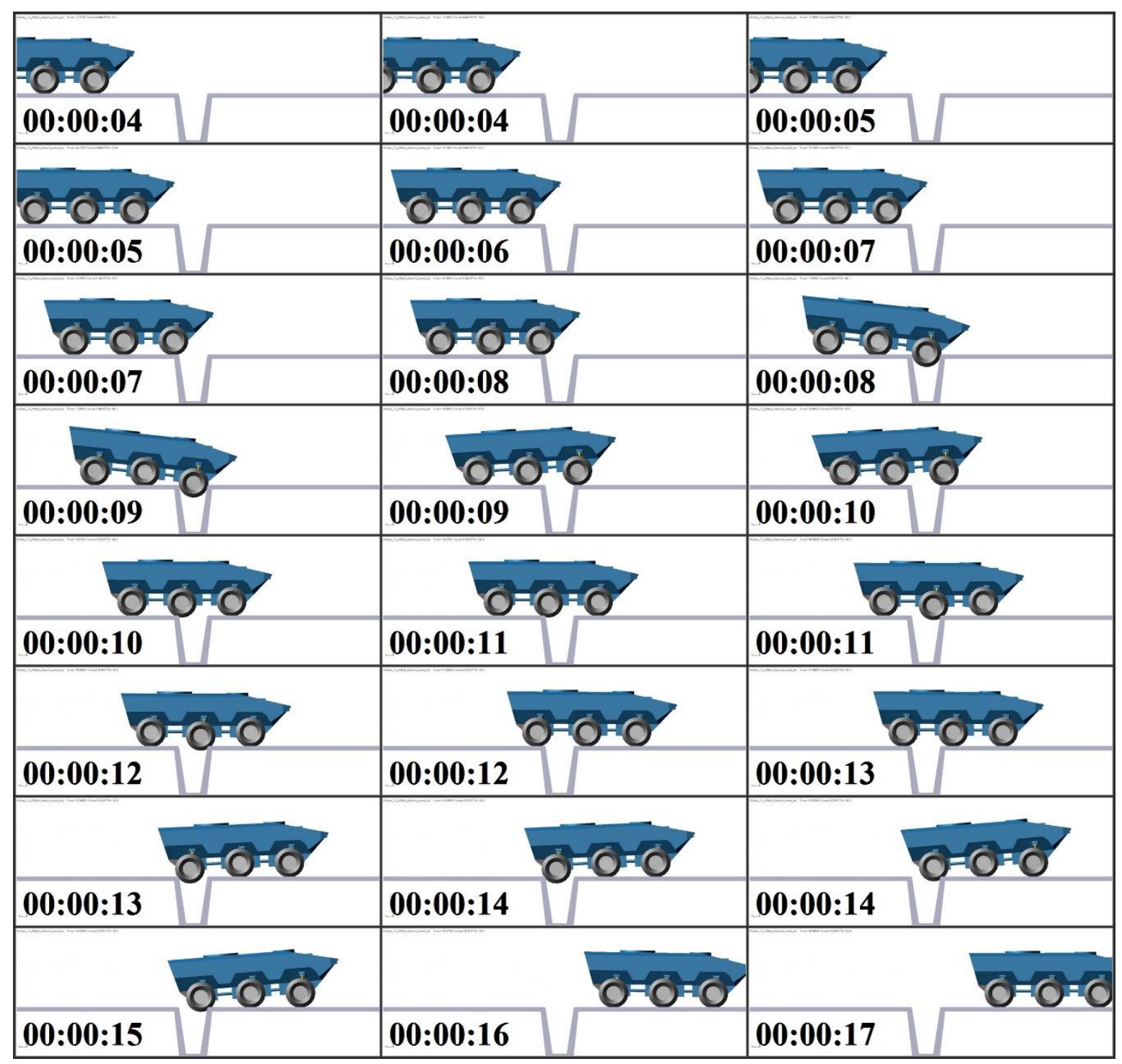

Figure A.4. Trench length $1.2 \mathrm{~m}, 3 \mathrm{kph}$, uncontrolled system 


\begin{tabular}{|c|c|c|}
\hline$=0=0$ & $=0=0$ & $=0=0$ \\
\hline 00:00:04 & 00:00:05 & 00:00:05 \\
\hline 00:00:06 & 00:00:06 & 00:00:07 \\
\hline 00:00:07 & 00:00:08 & 00:00:09 \\
\hline 00:00:09 & 00:00:10 & 00:00:10 \\
\hline 00:00:11 & 00:00:11 & 00:00:12 \\
\hline 00:00:12 & 00:00:13 & 00:00:14 \\
\hline 00:00:14 & 00:00:15 & 00:00:15 \\
\hline 00:00:16 & 00:00:17 & 00:00:18 \\
\hline
\end{tabular}

Figure A.5. Trench length $1.2 \mathrm{~m}, 3 \mathrm{kph}$, controlled system 

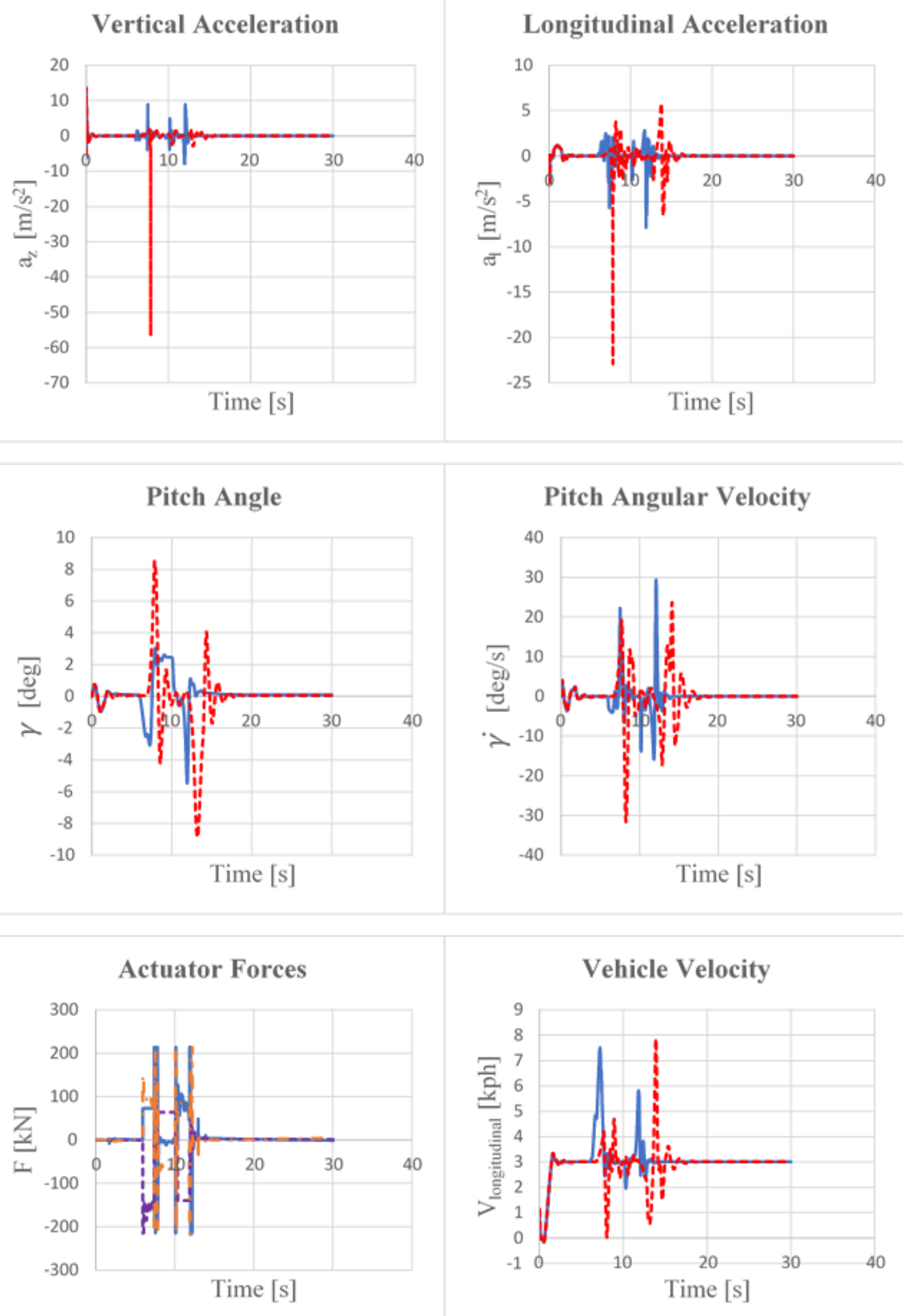

Axle 1 ----A Axle 2 - - Axle 3

Controlled -----Uncontrolled

Figure A.6. Trench length $1.2 \mathrm{~m}, 3 \mathrm{kph}$, controlled vs uncontrolled output comparison 
Trench Length $1.2 \mathrm{~m}, 6 \mathrm{kph}$

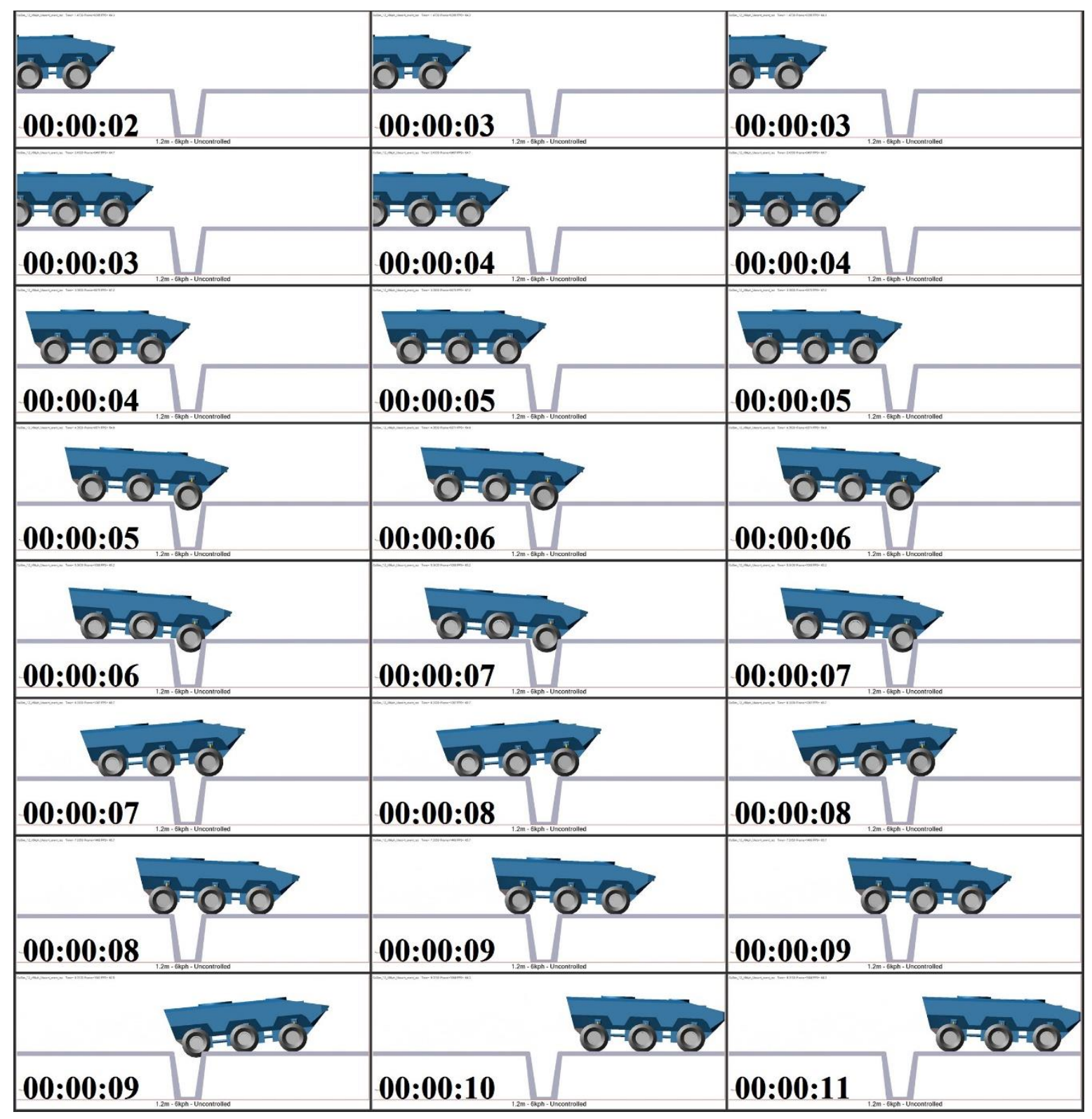

Figure A.7. Trench length $1.2 \mathrm{~m}, 6 \mathrm{kph}$, uncontrolled system 


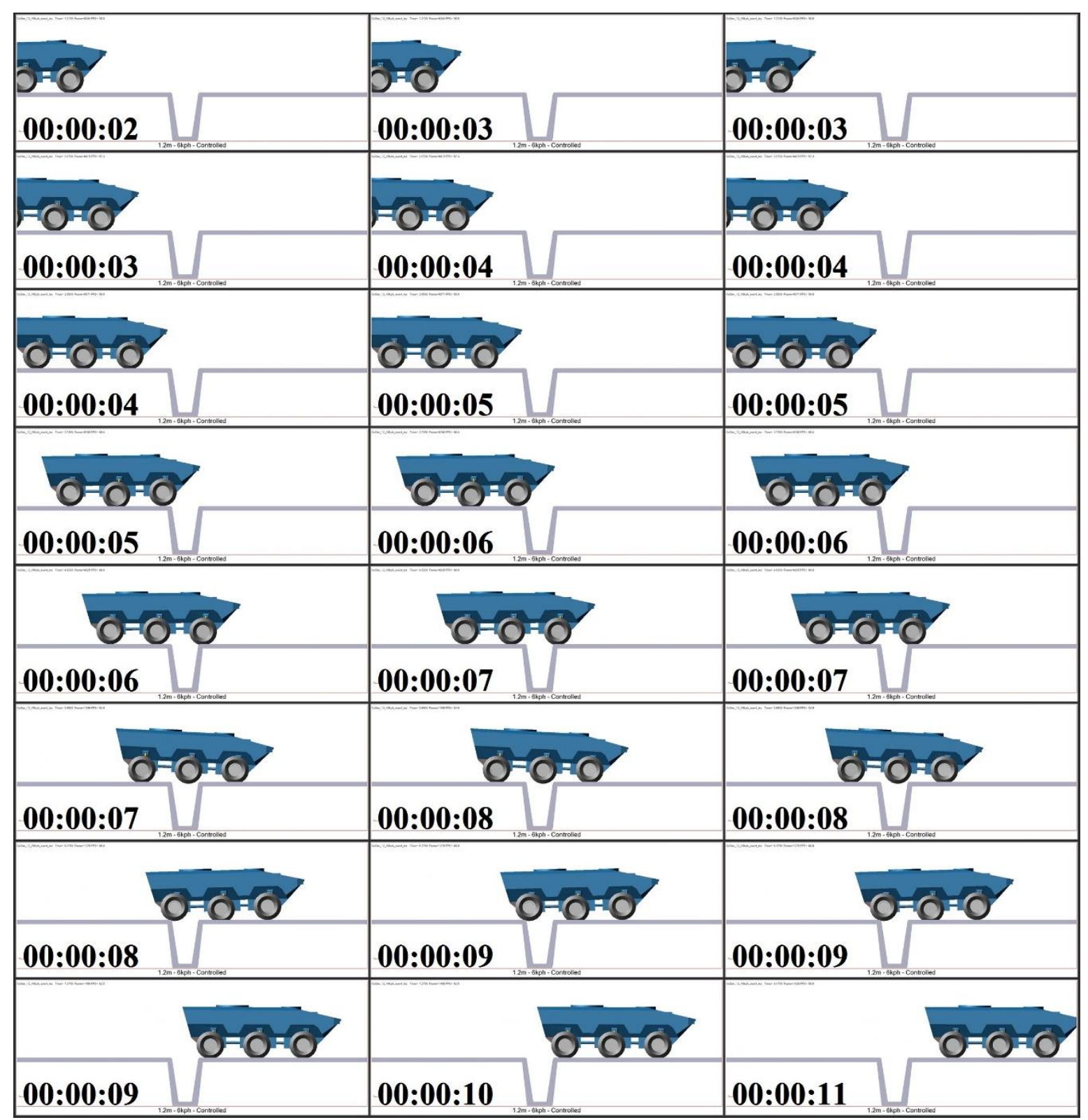

Figure A.8. Trench length $1.2 \mathrm{~m}, 6 \mathrm{kph}$, controlled system 


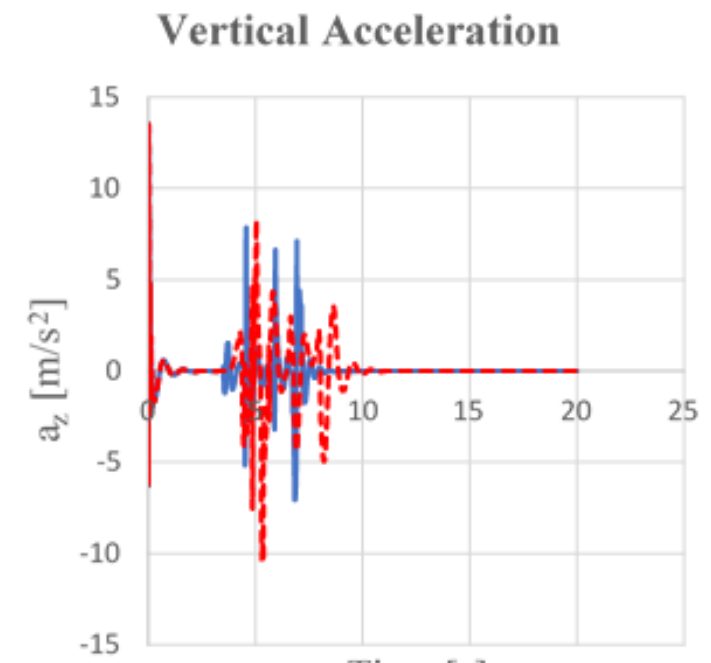

Time $[\mathrm{s}]$

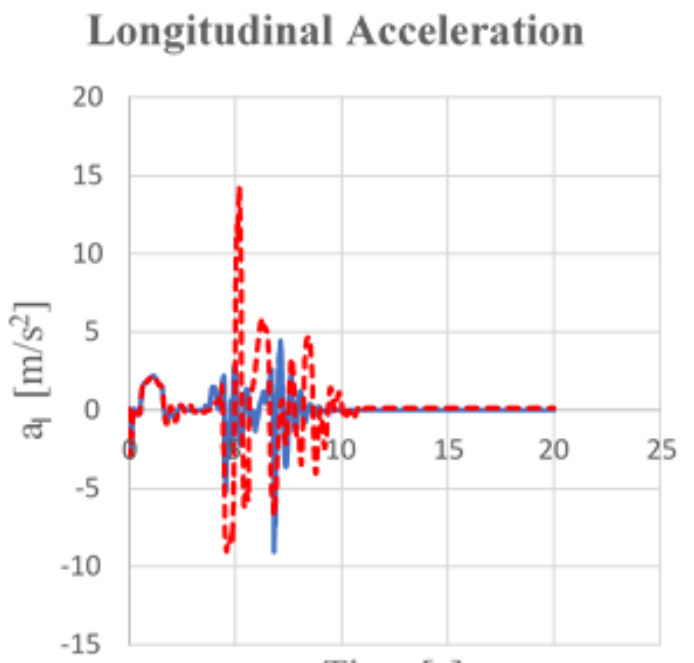

Time $[\mathrm{s}]$

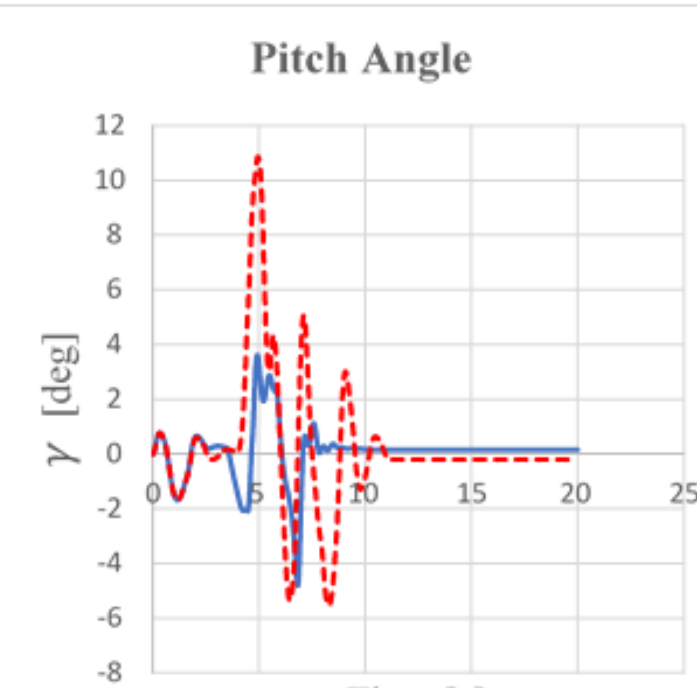

Time [s]

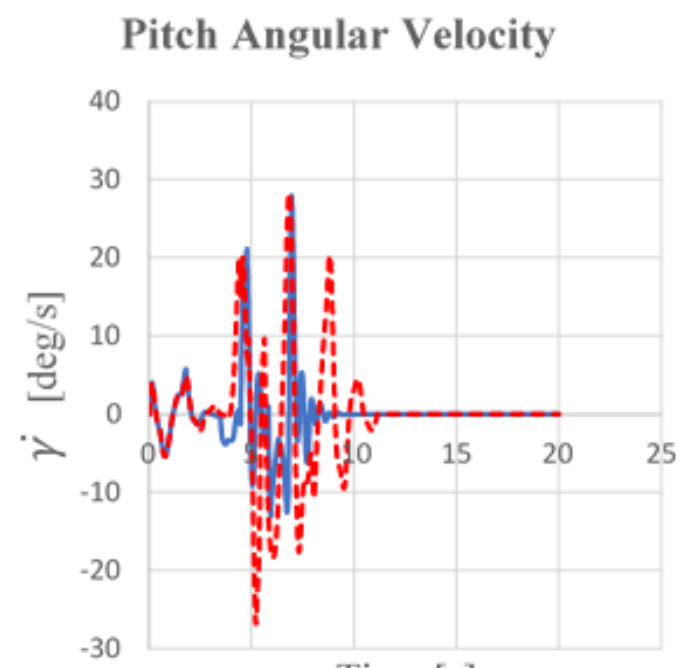

Time [s]

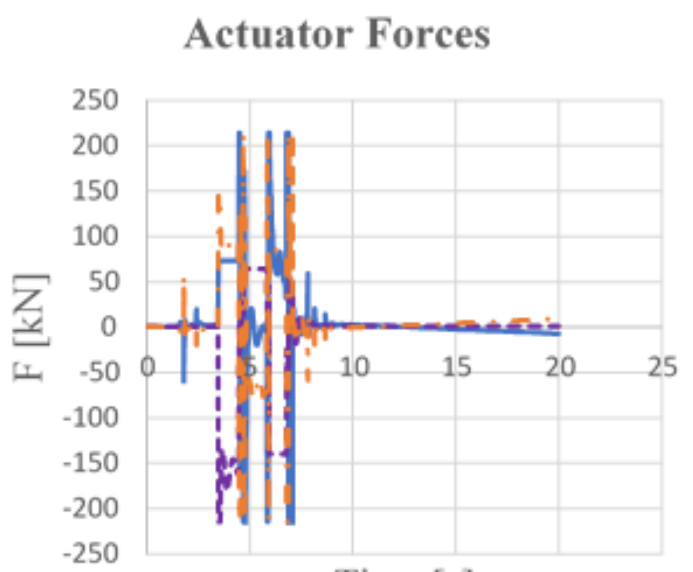

Time $[\mathrm{s}]$

Axle 1 --.-Axle 2 - Axle 3

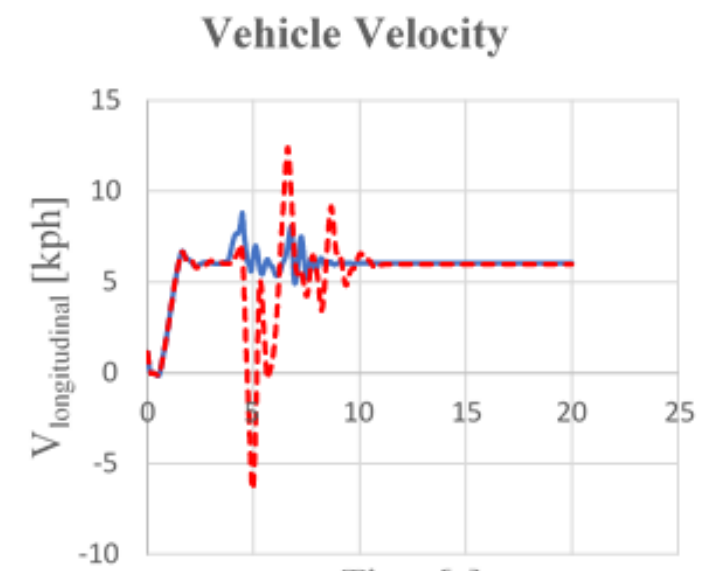

Time $[\mathrm{s}]$

Controlled ----Uncontrolled

Figure A.9. Trench length $1.2 \mathrm{~m}, 6 \mathrm{kph}$, controlled vs uncontrolled output comparison 
Trench Length $1.2 \mathrm{~m}, 9 \mathrm{kph}$

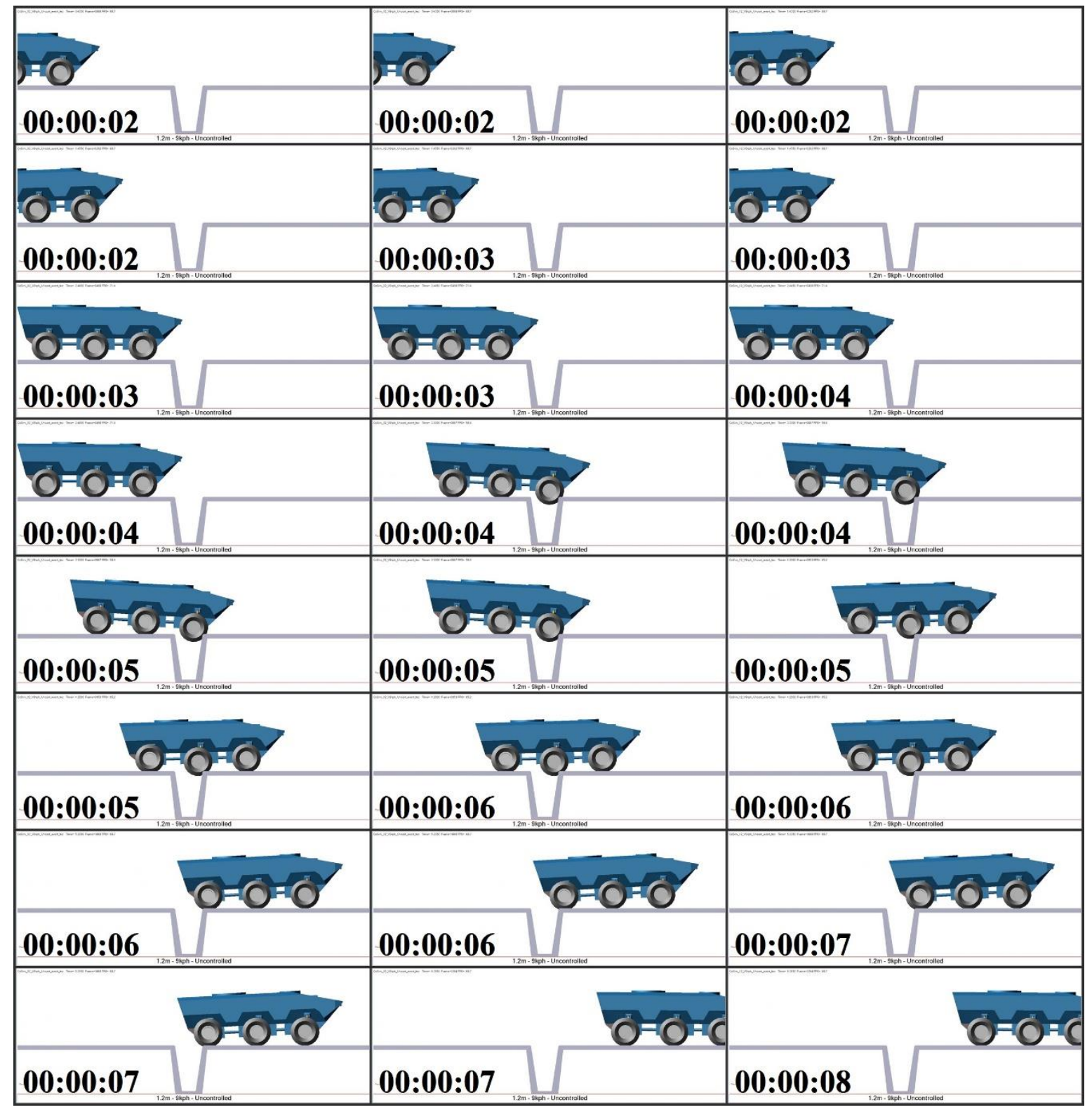

Figure A.10. Trench length $1.2 \mathrm{~m}, 9 \mathrm{kph}$, uncontrolled system 


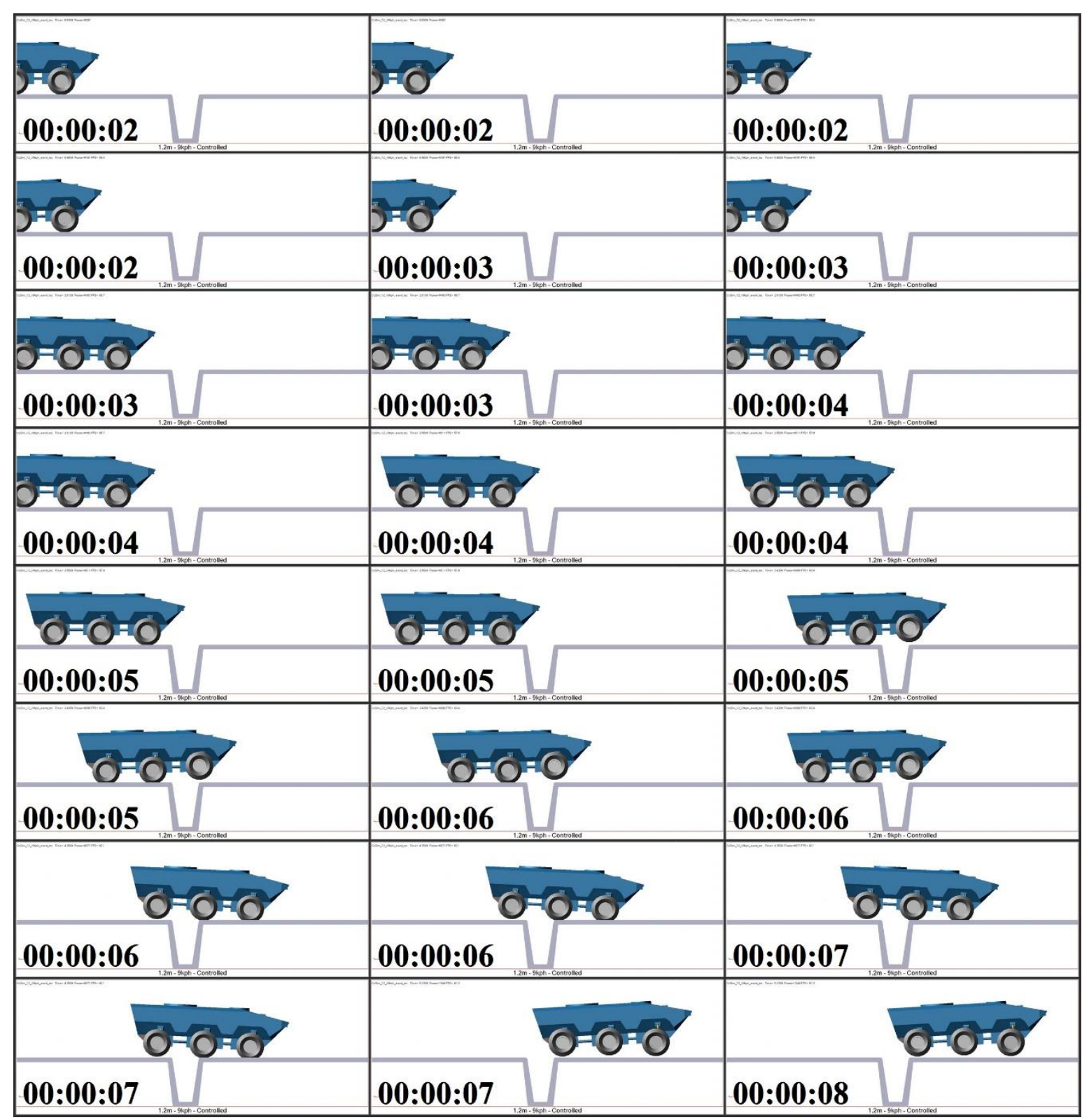

Figure A.11. Trench length $1.2 \mathrm{~m}, 9 \mathrm{kph}$, controlled system 

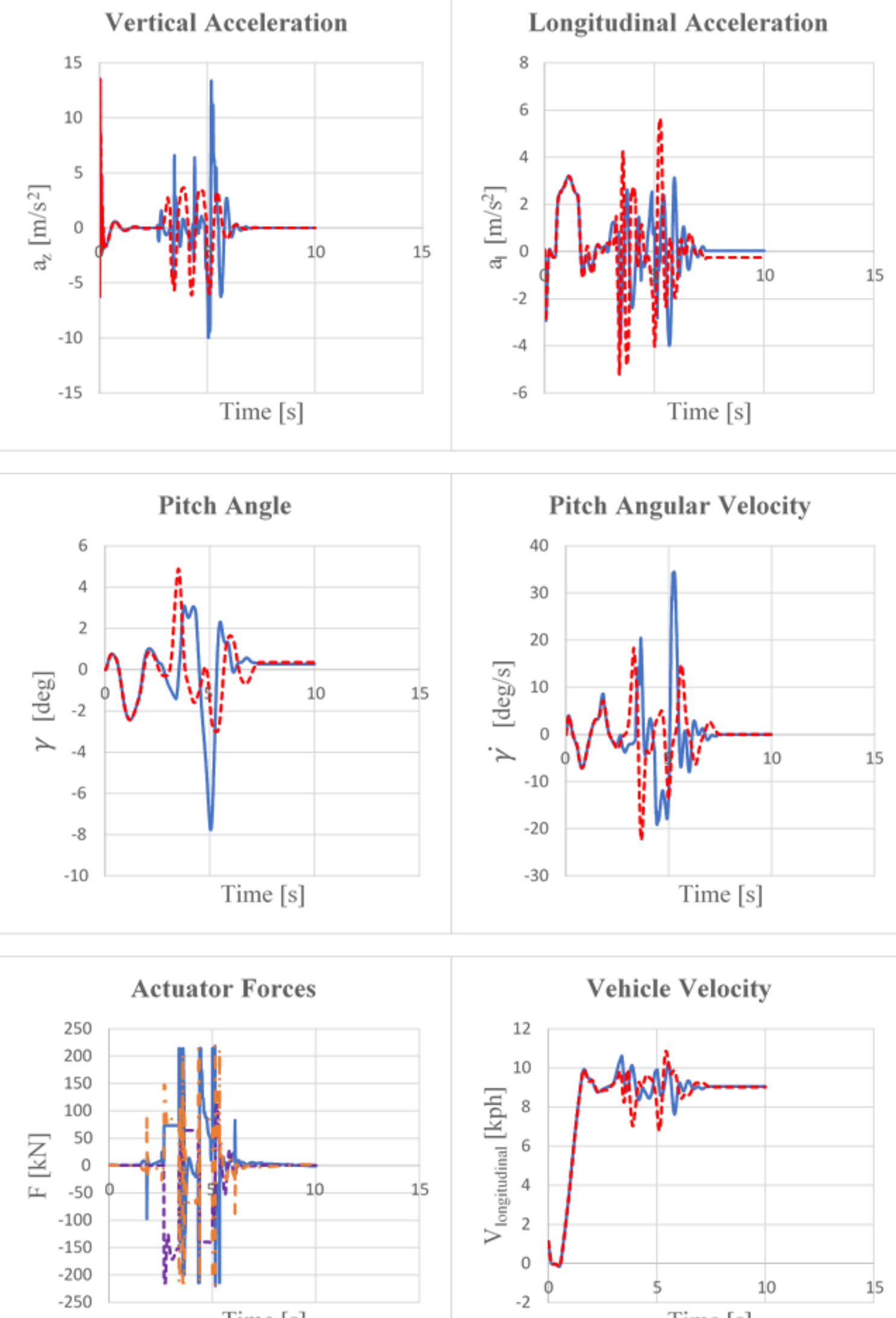

Time $[\mathrm{s}]$

- Axle 1 ----Axle 2 - - Axle 3

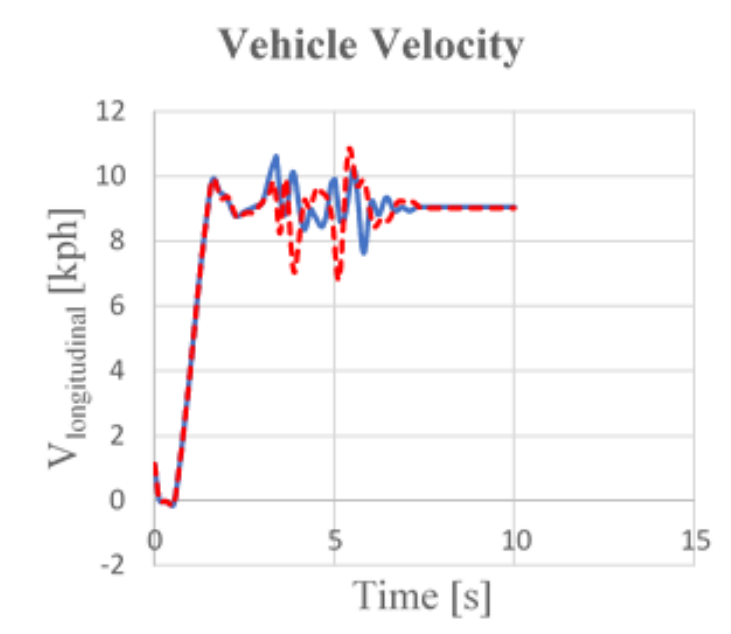

Controlled -----. Uncontrolled

Figure A.12. Trench length $1.2 \mathrm{~m}, 9 \mathrm{kph}$, controlled vs uncontrolled output comparison 
Trench Length $1.3 \mathrm{~m}$, Controlled vs Uncontrolled System Simulations

Trench Length $1.3 \mathrm{~m}, 1 \mathrm{kph}$

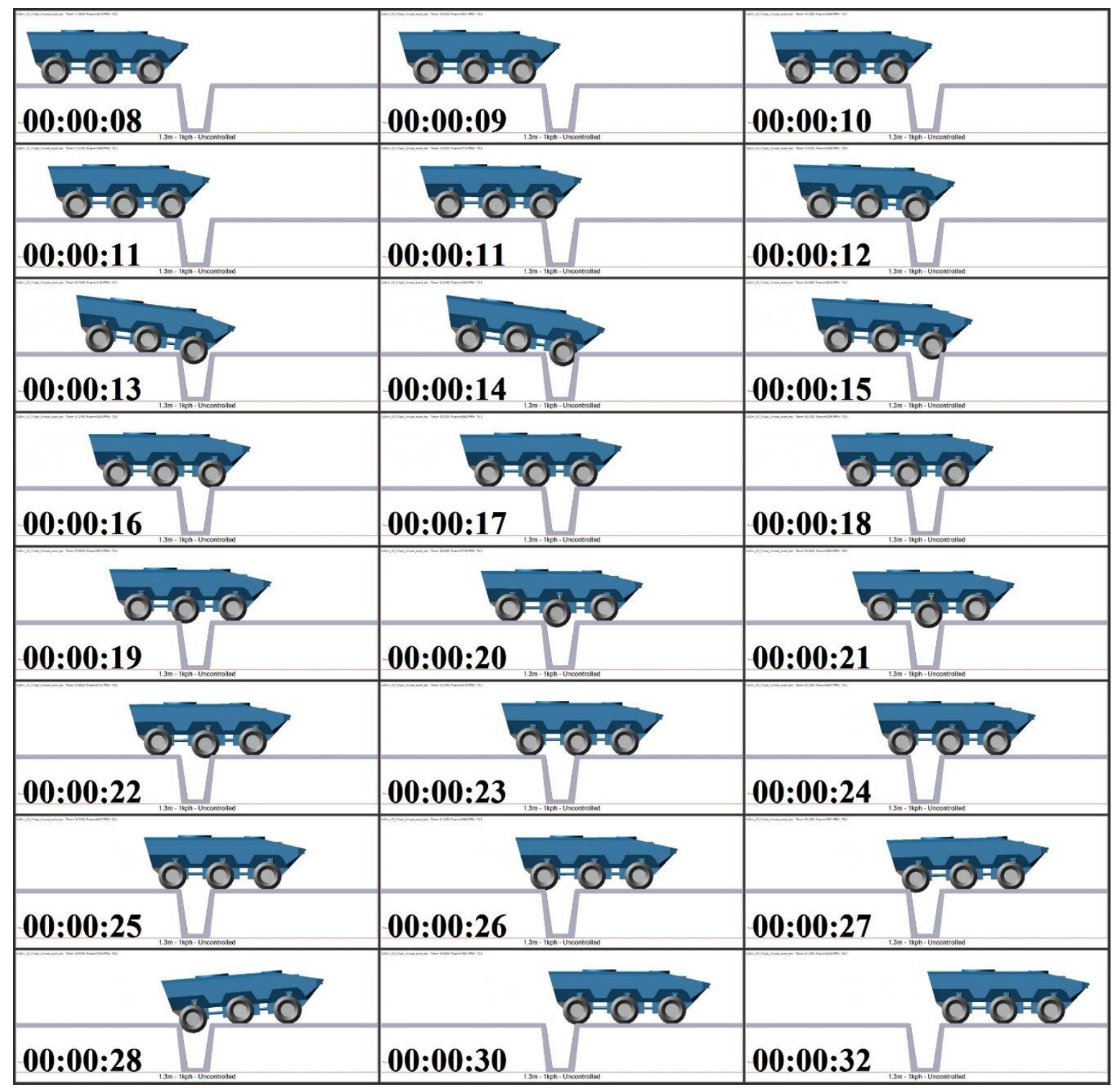

Figure A.13. Trench length $1.3 \mathrm{~m}, 1 \mathrm{kph}$, uncontrolled system 


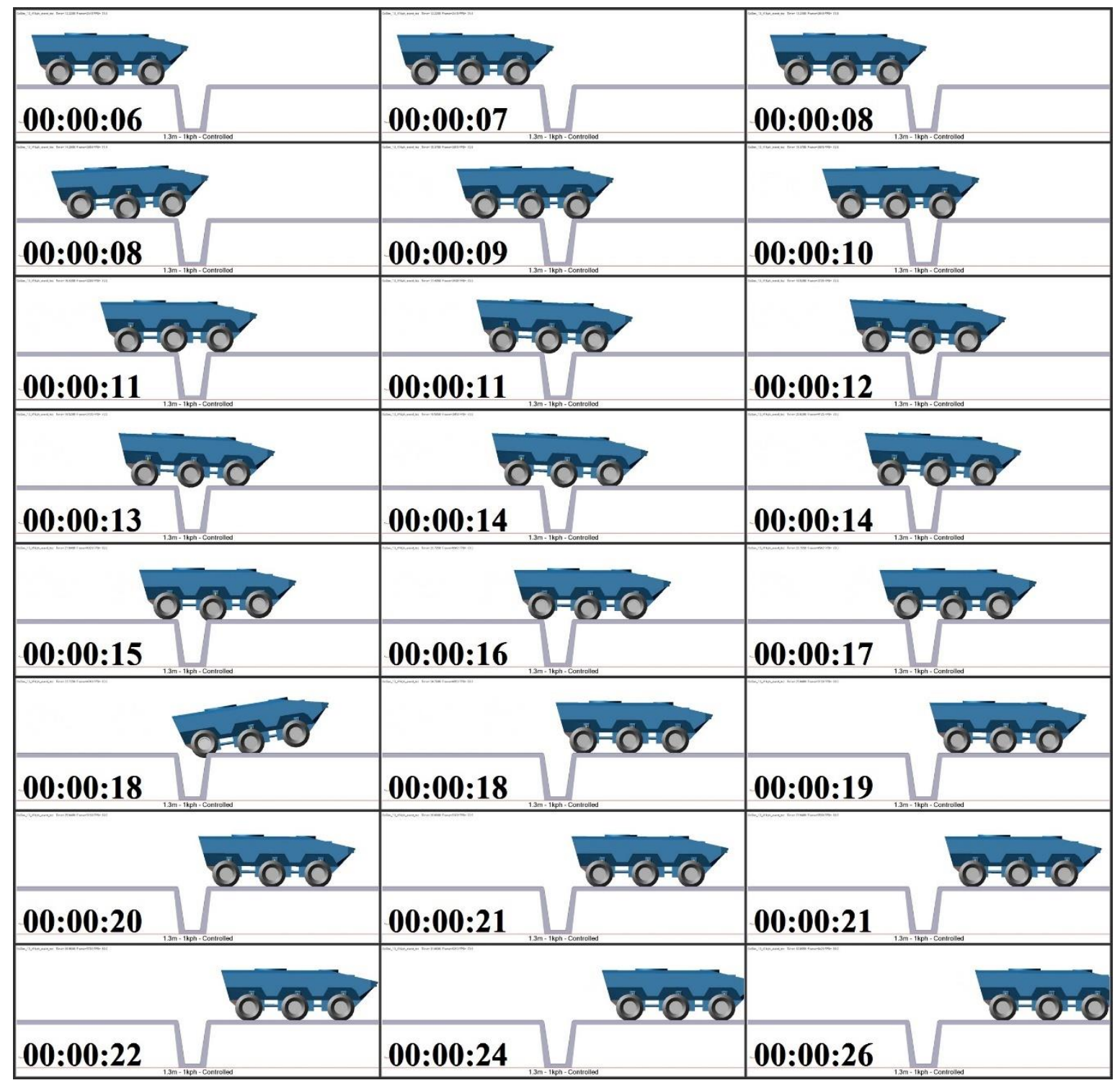

Figure A.14. Trench length $1.3 \mathrm{~m}, 1 \mathrm{kph}$, controlled system 


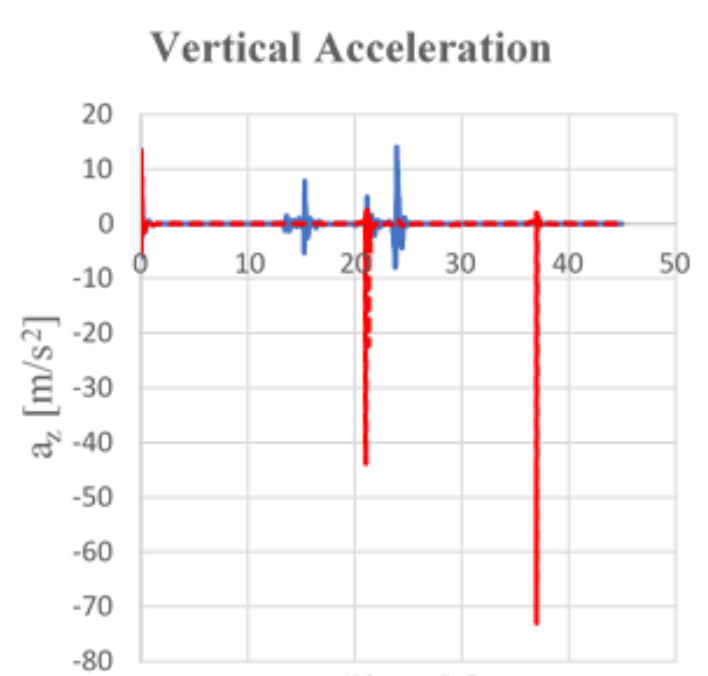

Time [s]

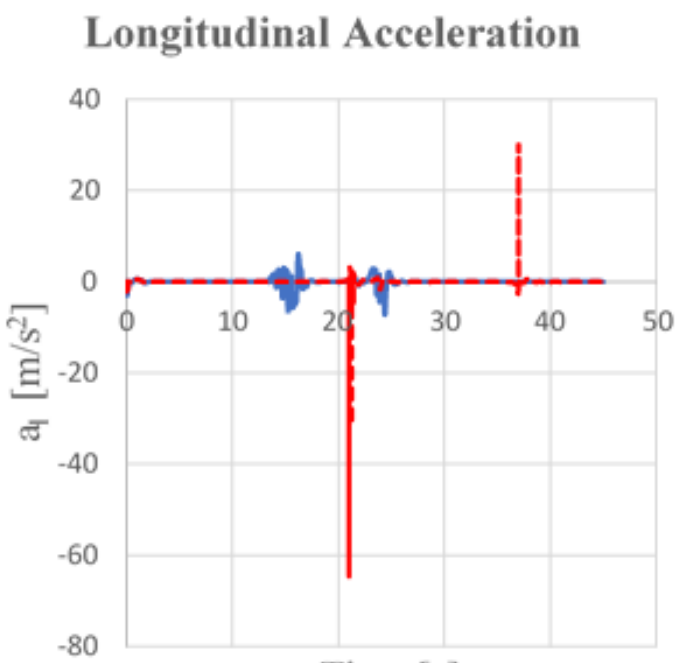

Time [s]
Pitch Angle

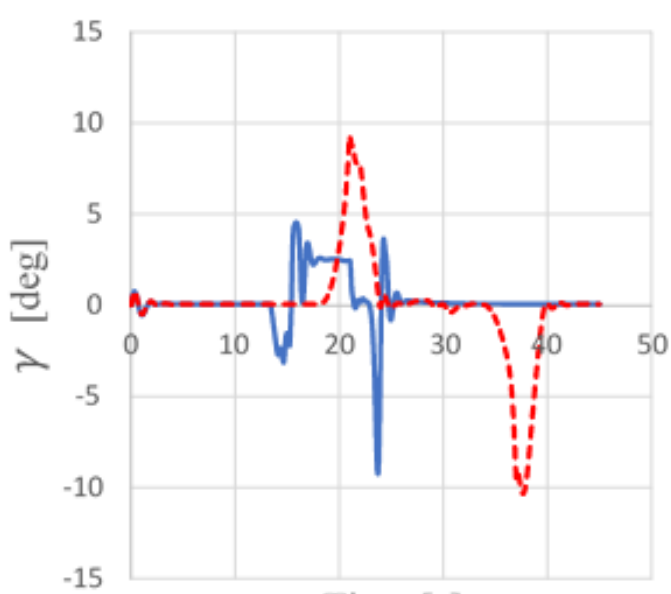

Time [s]
Pitch Angular Velocity

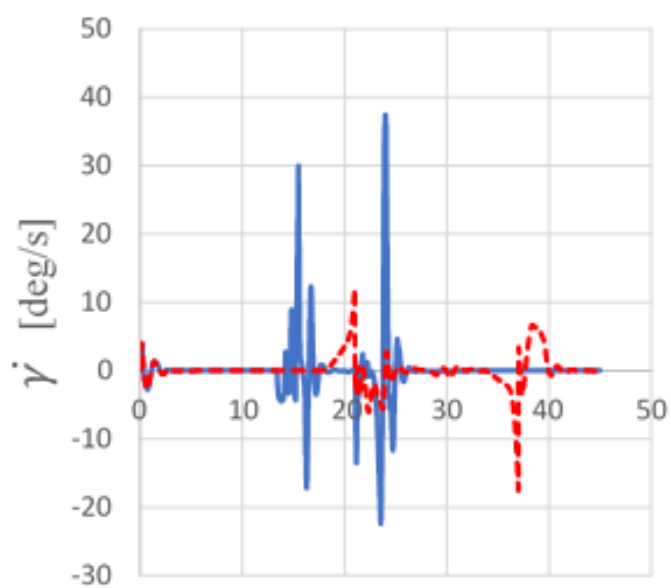

Time [s]

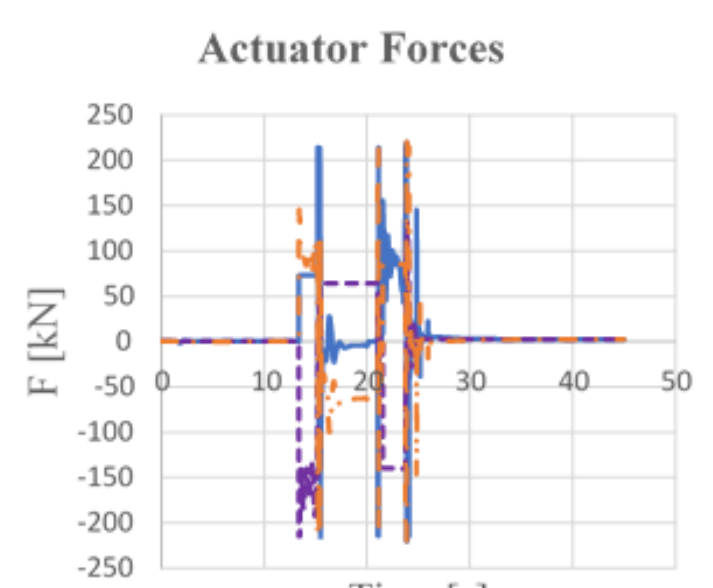

Time [s]

—Axle 1 - - Axle 2 - - Axle 3

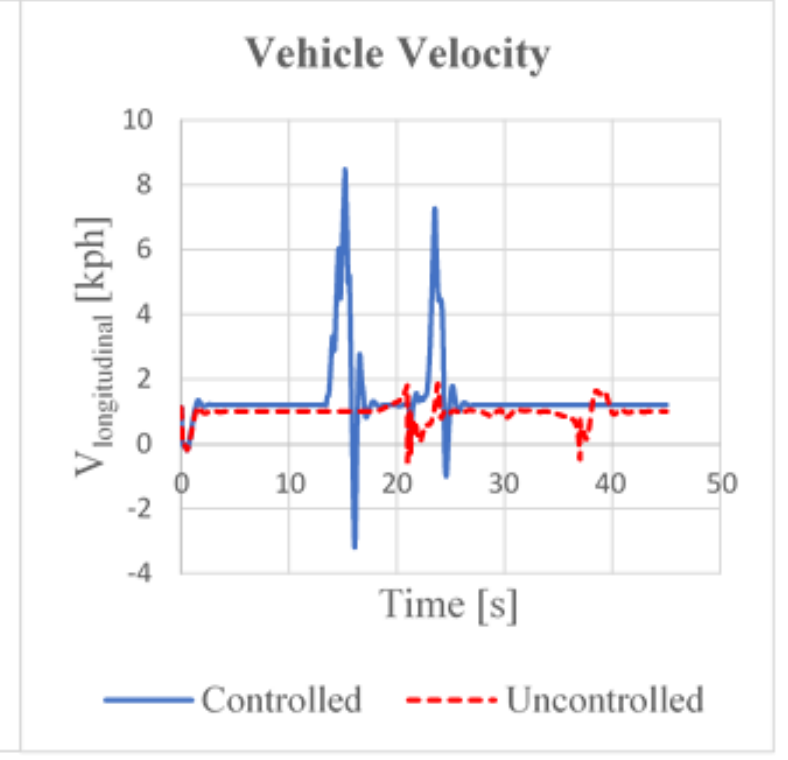

Figure A.15. Trench length $1.3 \mathrm{~m}, 1 \mathrm{kph}$, controlled vs uncontrolled output comparison 
Trench Length 1.3 m, 3 kph

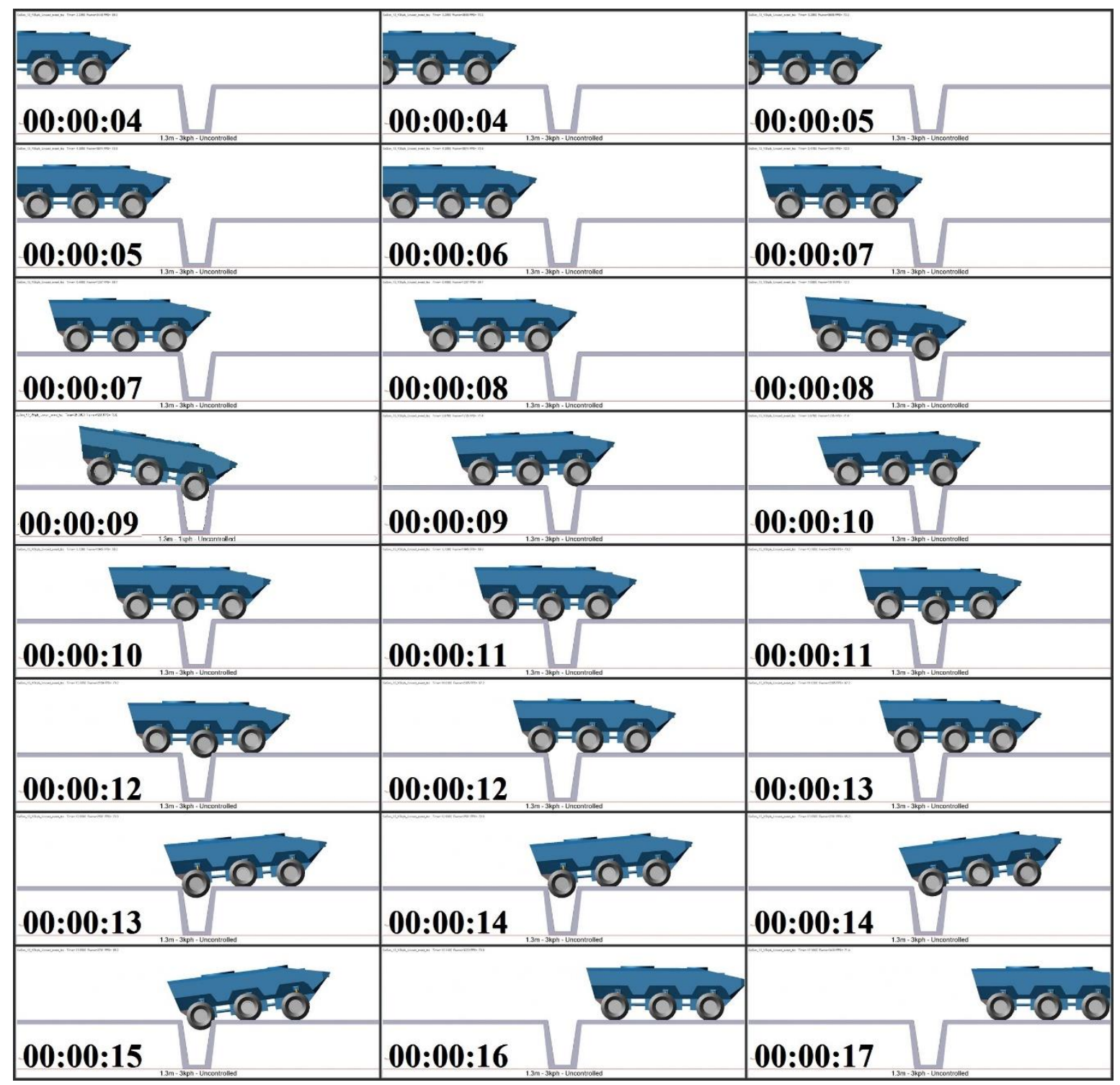

Figure A.16. Trench length $1.3 \mathrm{~m}, 3 \mathrm{kph}$, uncontrolled system 


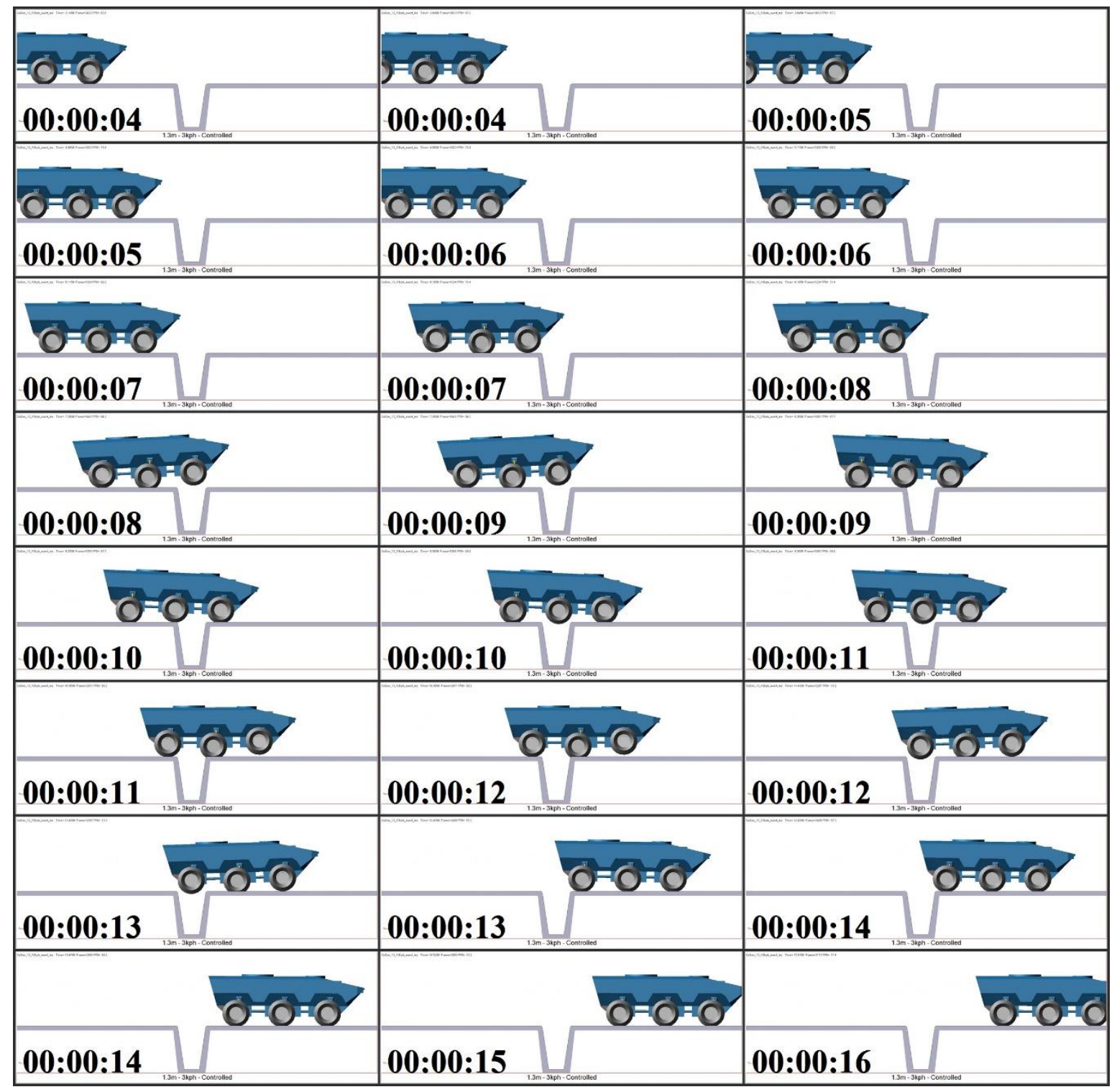

Figure A.17. Trench length $1.3 \mathrm{~m}, 3 \mathrm{kph}$, uncontrolled system 


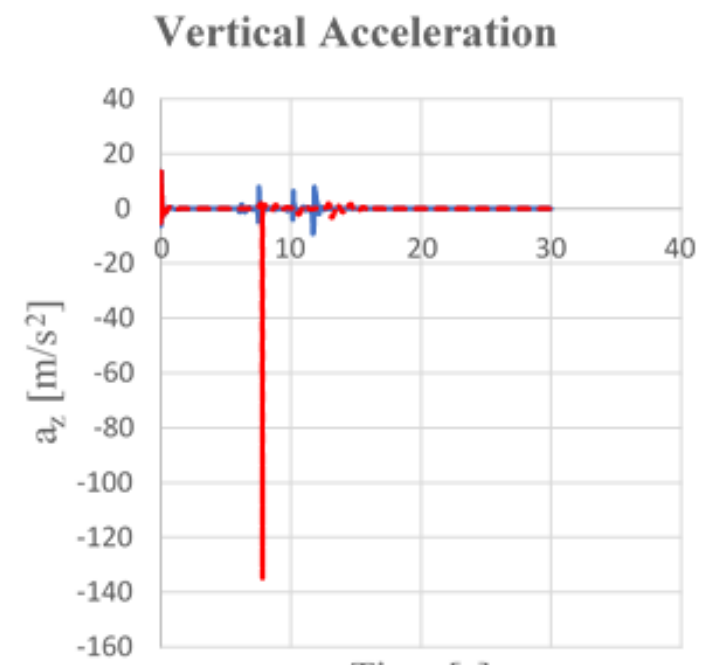

Time $[\mathrm{s}]$

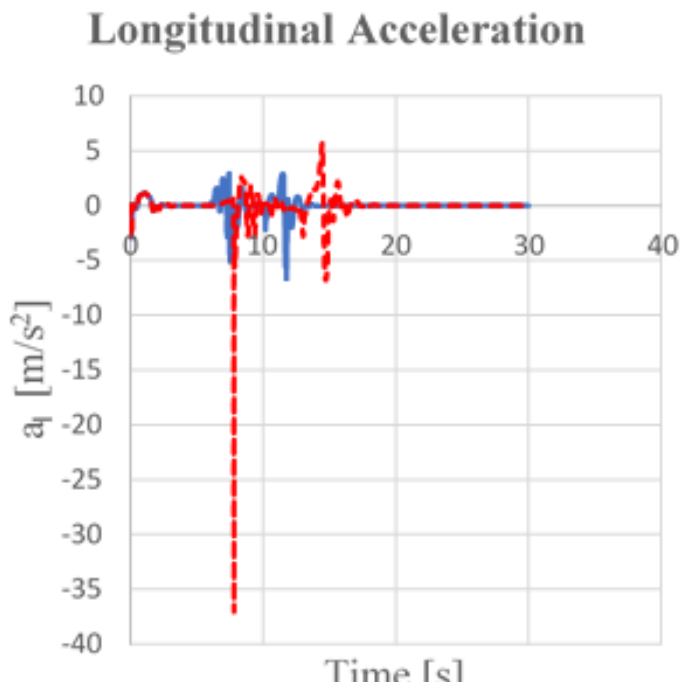

Time [s]

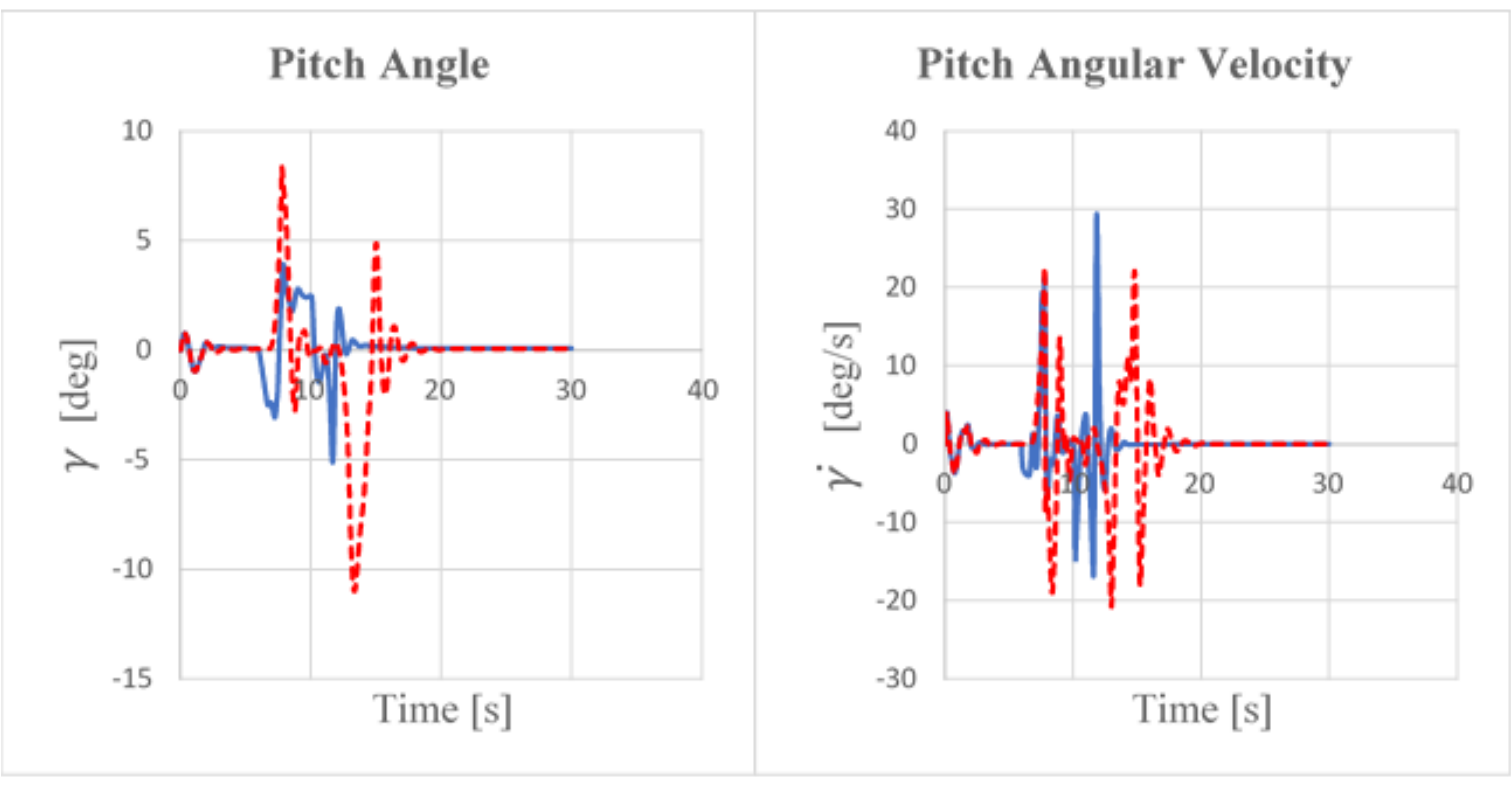

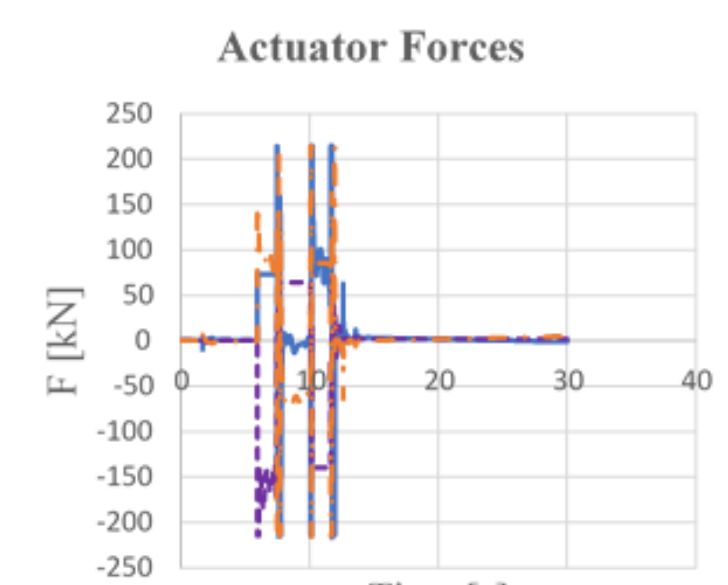

Time [s]

— Axle 1 - - Axle 2 - Axle 3

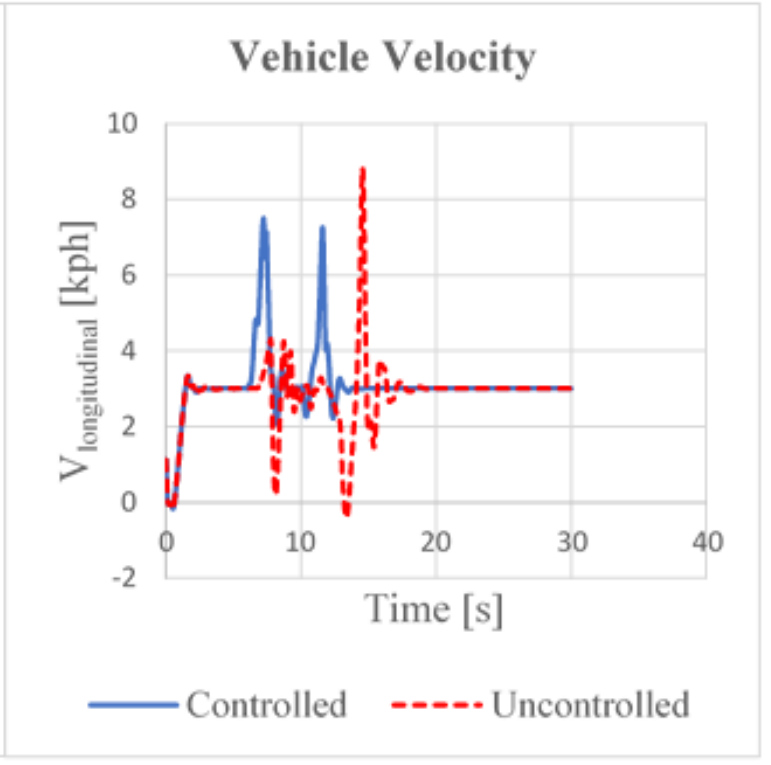

Figure A.18. Trench length $1.3 \mathrm{~m}, 3 \mathrm{kph}$, controlled vs uncontrolled output comparison 
Trench Length $1.3 \mathrm{~m}, 6 \mathrm{kph}$

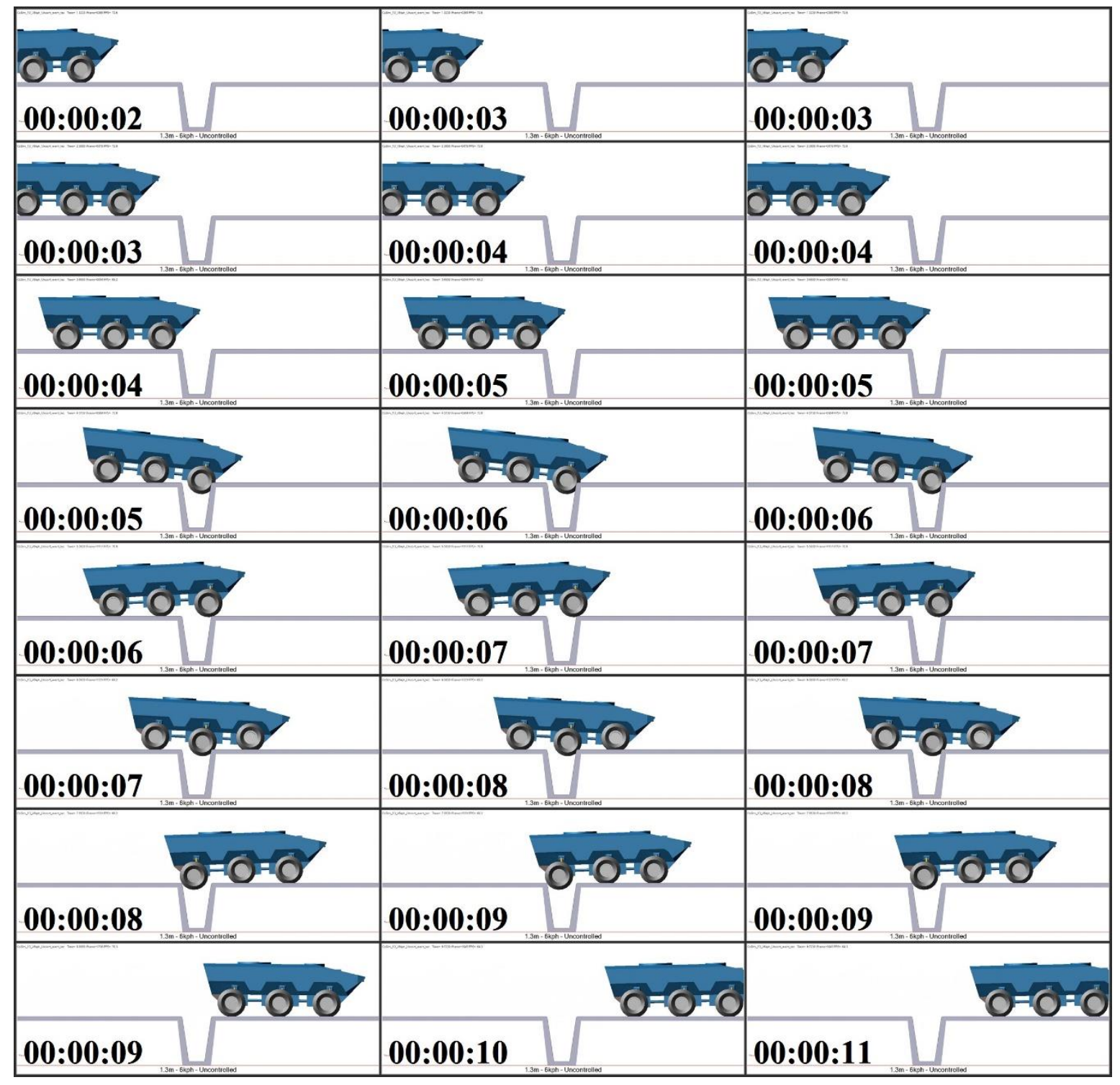

Figure A.19. Trench length $1.3 \mathrm{~m}, 6 \mathrm{kph}$, uncontrolled system 


\begin{tabular}{|c|c|c|}
\hline $0=0$ & $0=0$ & $0=0$ \\
\hline 00:00:02 & 00:00:02 & 00:00:03 \\
\hline $0=0$ & $0=0=0$ & $0=0=0$ \\
\hline 00:00:03 & 00:00:03 & 00:00:04 \\
\hline$b=0=0$ & & $0=0=$ \\
\hline 00:00:04 & 00:00:04 & 00:00:04 \\
\hline 00:00:05 & 00:00:05 & 00:00:05 \\
\hline 00:00:06 & 00:00:06 & 00:00:06 \\
\hline 00:00:07 & 00:00:07 & 00:00:07 \\
\hline 00:00:08 & 00:00:08 & 00:00:08 \\
\hline 00:00:08 & 00:00:09 & 00:00:10 \\
\hline
\end{tabular}

Figure A.20. Trench length $1.3 \mathrm{~m}, 6 \mathrm{kph}$, controlled system 

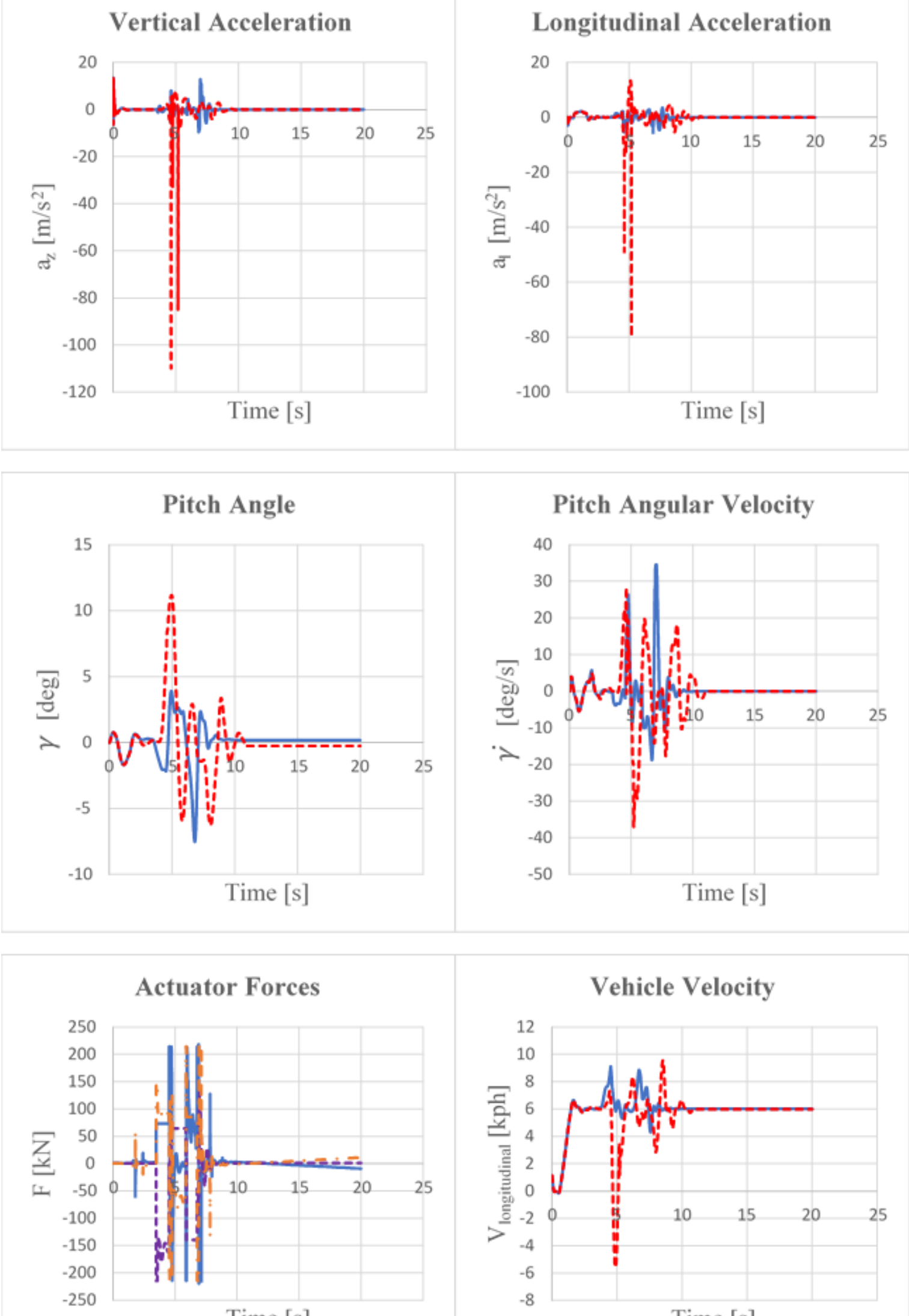

Time [s]

— Axle 1 ----Axle 2 - - Axle 3

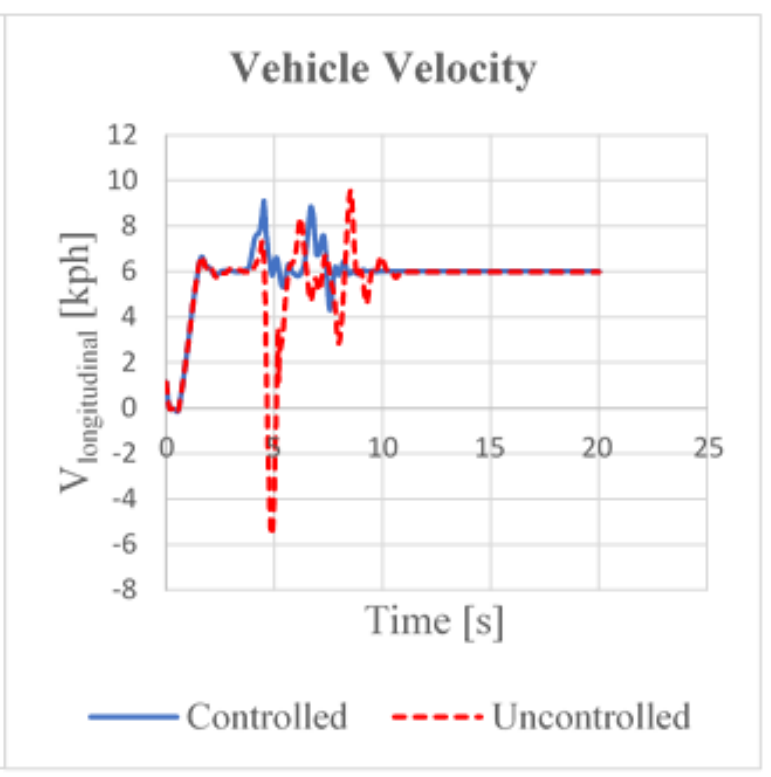

Figure A.21. Trench length $1.3 \mathrm{~m}, 6 \mathrm{kph}$, controlled vs uncontrolled output comparison 
Trench Length $1.3 \mathrm{~m}, 9 \mathrm{kph}$

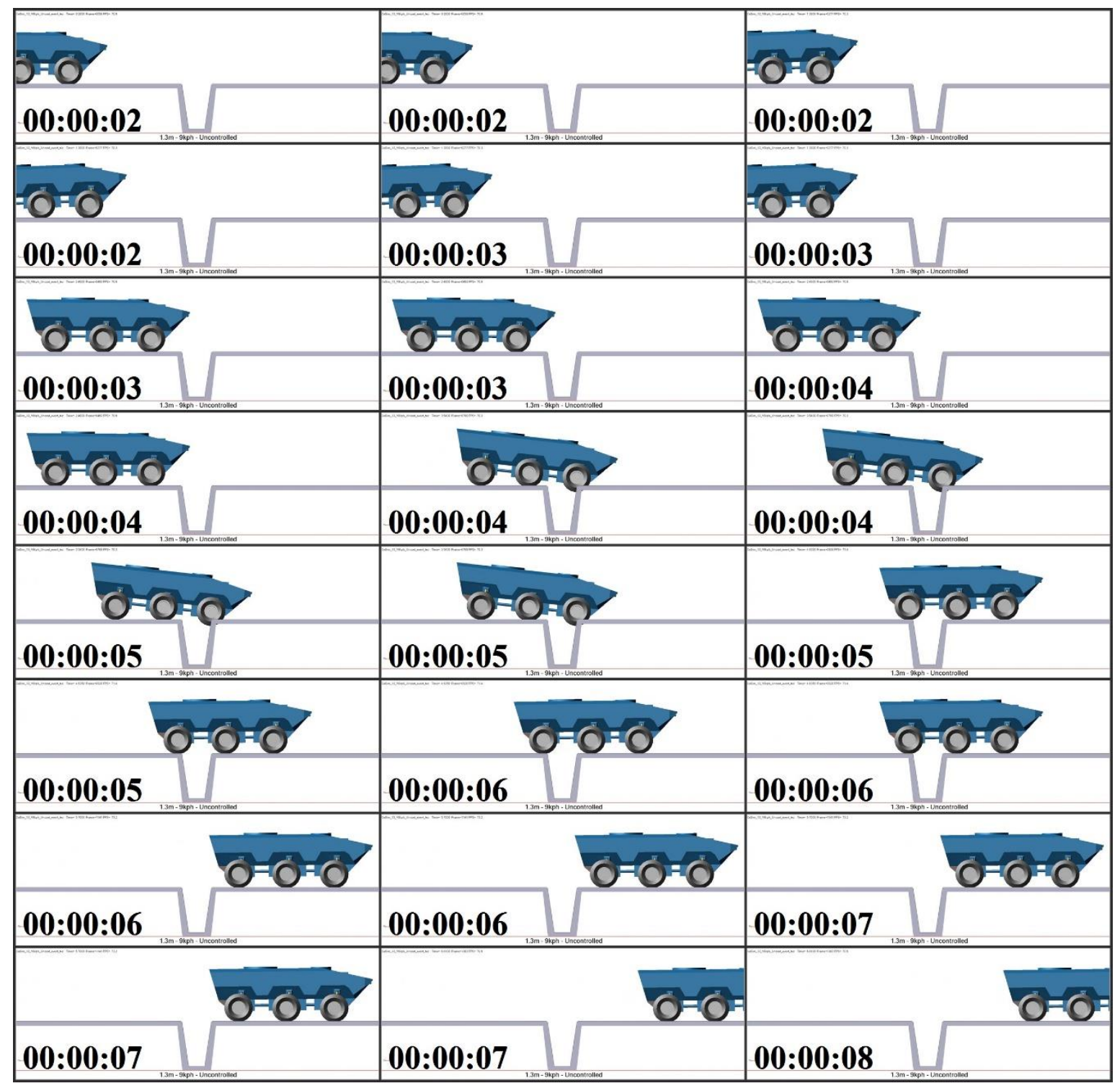

Figure A.22. Trench length $1.3 \mathrm{~m}, 9 \mathrm{kph}$, uncontrolled system 


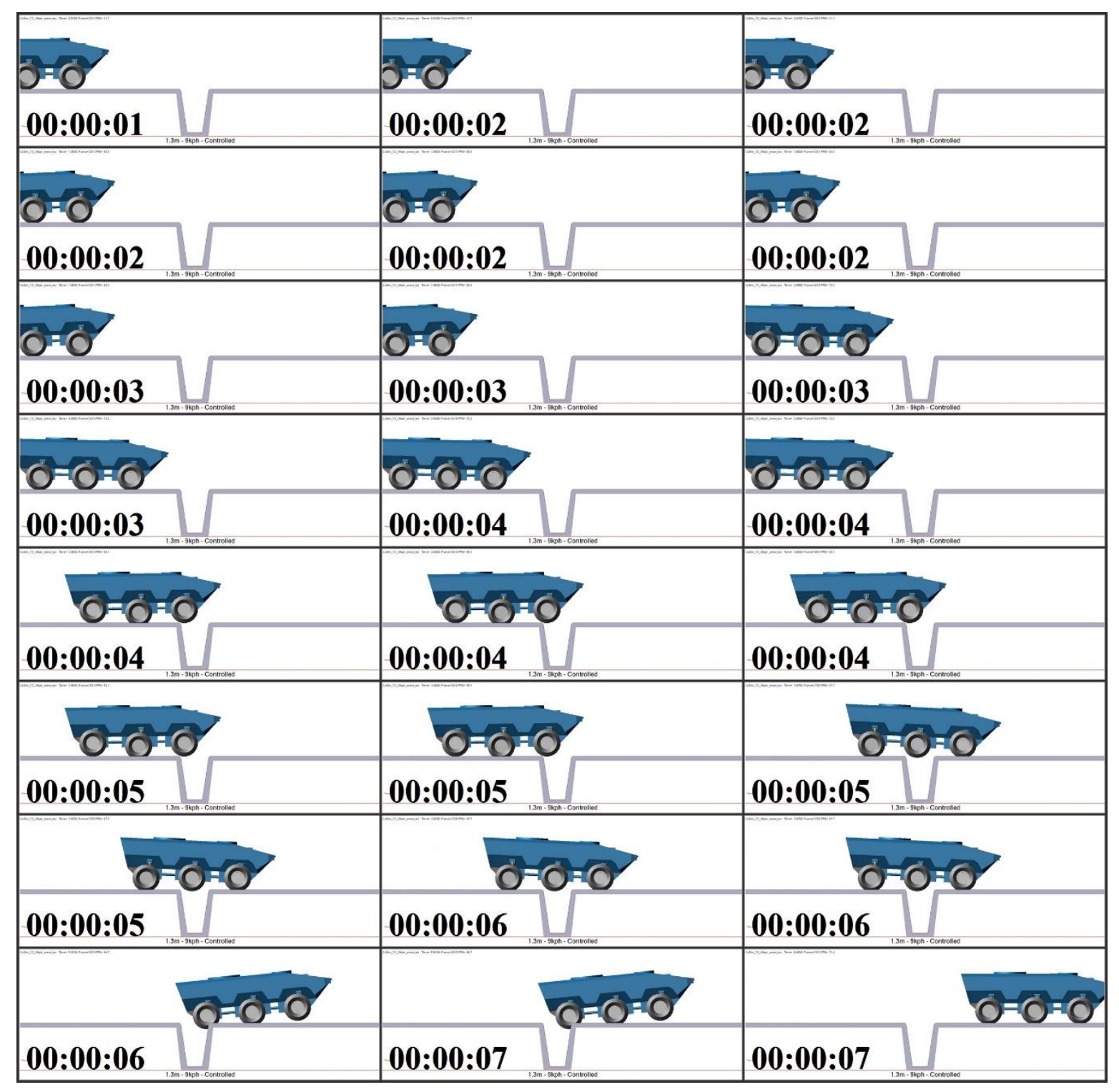

Figure A.23. Trench length $1.3 \mathrm{~m}, 9 \mathrm{kph}$, controlled system 

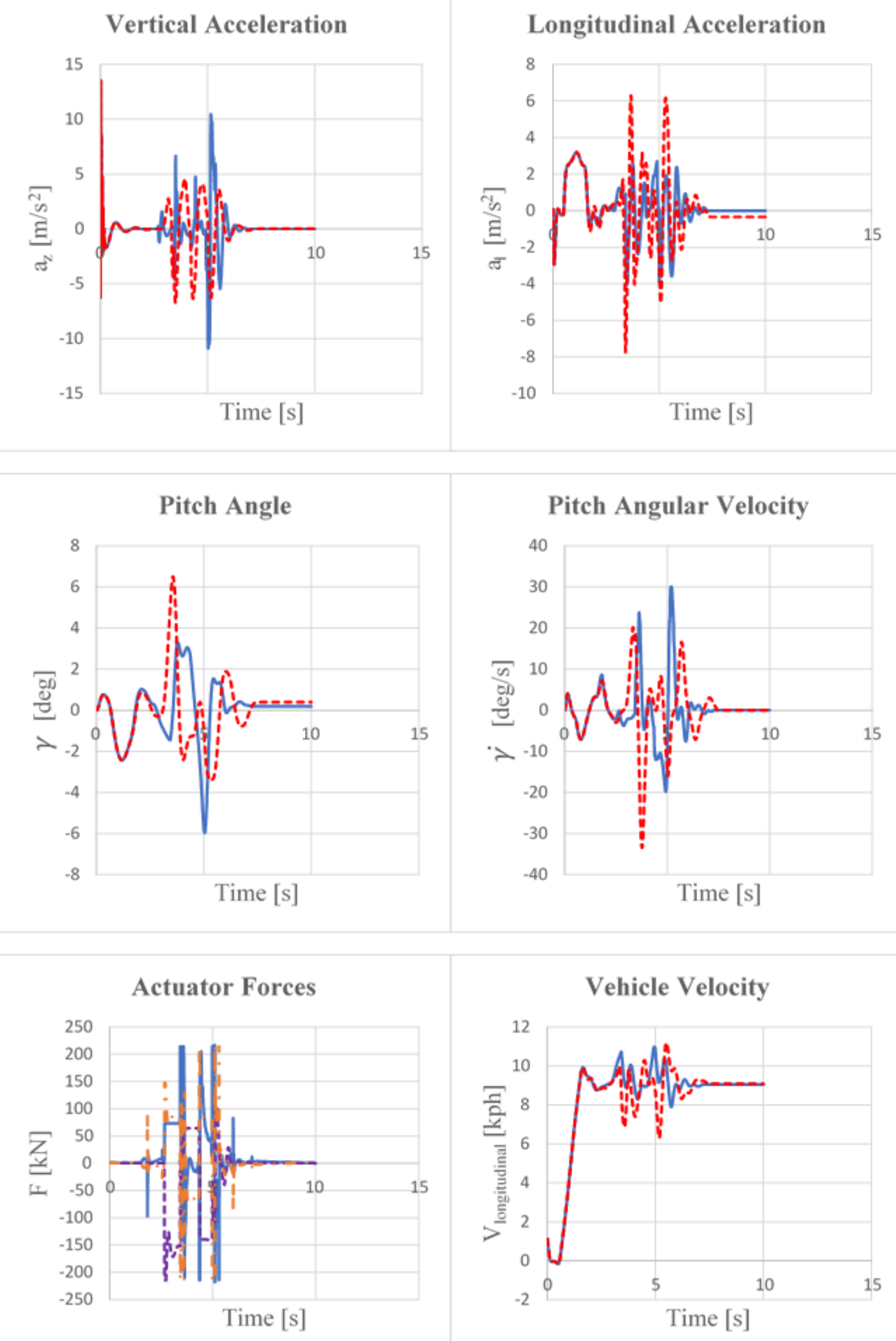

— Axle 1 ----A Axle 2 - - Axle 3

Controlled -----. Uncontrolled

Figure A.24. Trench length $1.3 \mathrm{~m}, 9 \mathrm{kph}$, controlled vs uncontrolled output comparison 
Trench Length 1.4 m, Controlled vs Uncontrolled System Simulations

Trench Length $1.4 \mathrm{~m}, 1 \mathrm{kph}$

\begin{tabular}{|c|c|c|}
\hline $0=0=0$ & $\theta=0=0$ & $0=0=0$ \\
\hline 00:00:09 & 00:00:11 & 00:00:12 \\
\hline 00:00:13 & 00:00:14 & 00:00:15 \\
\hline 00:00:17 & 00:00:18 & 00:00:19 \\
\hline 00:00:20 & 00:00:21 & 00:00:23 \\
\hline 00:00:24 & 00:00:25 & 00:00:26 \\
\hline 00:00:27 & 00:00:28 & 00:00:30 \\
\hline 00:00:31 & 00:00:32 & 00:00:33 \\
\hline 00:00:34 & 00:00:37 & 00:00:40 \\
\hline
\end{tabular}

Figure A.25. Trench length $1.4 \mathrm{~m}, 1 \mathrm{kph}$, uncontrolled system 


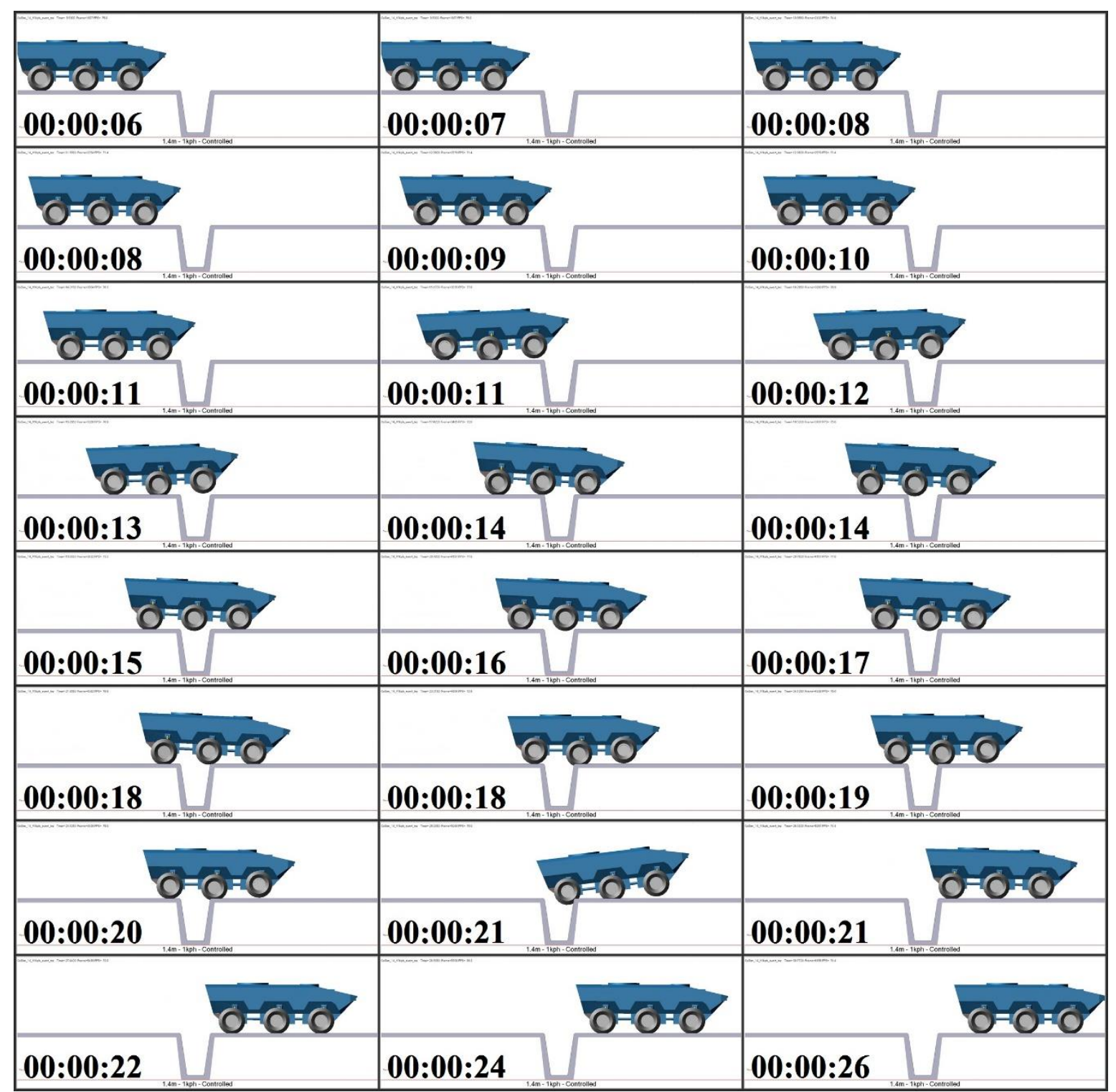

Figure A.26. Trench length $1.4 \mathrm{~m}, 1 \mathrm{kph}$, controlled system 

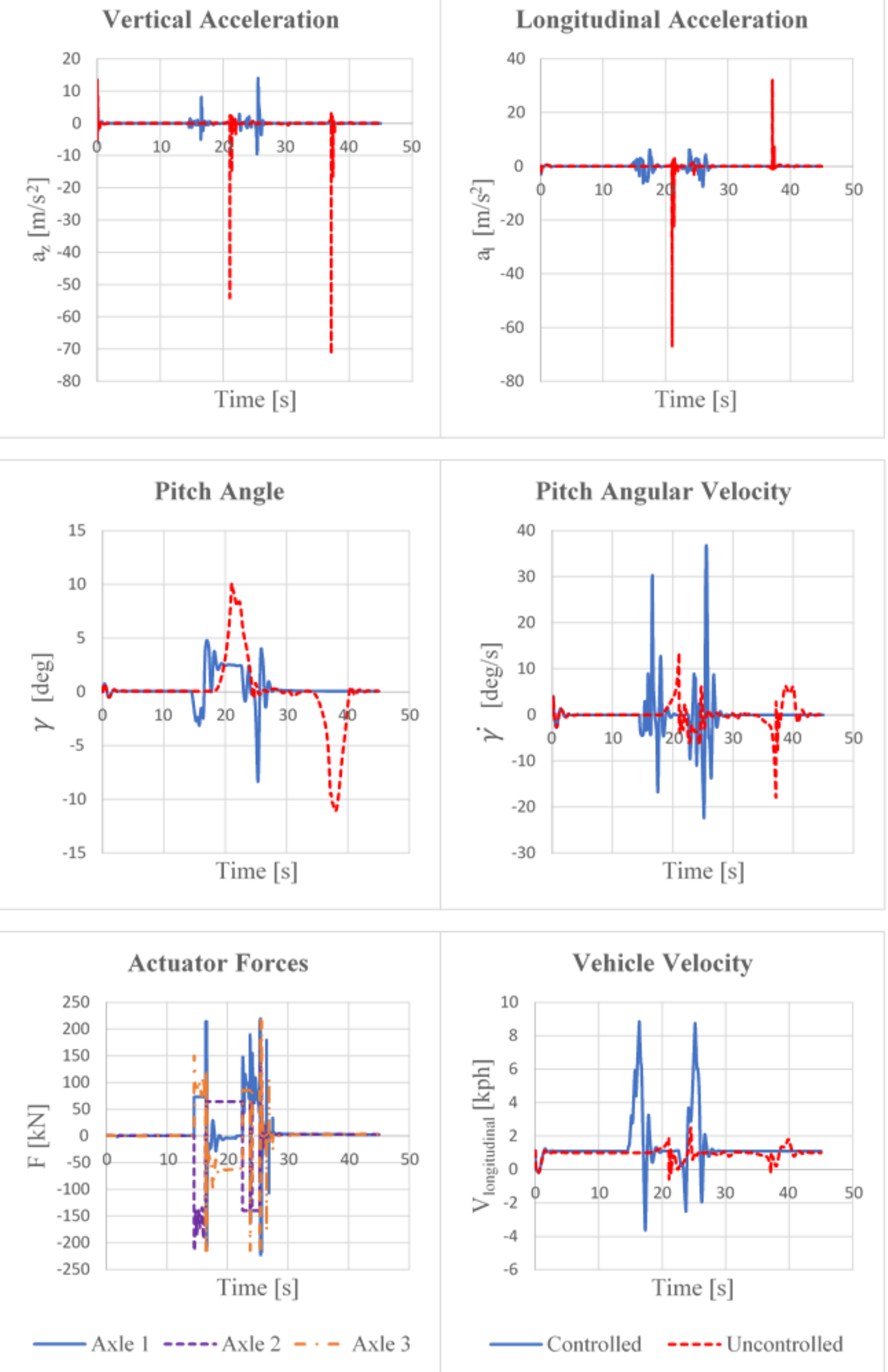

Figure A.27. Trench length $1.4 \mathrm{~m}, 1 \mathrm{kph}$, controlled vs uncontrolled output comparison 
Trench Length 1.4 m, 3 kph

\begin{tabular}{|c|c|c|}
\hline 00:00:04 & 00:00:05 & 00:00:05 \\
\hline 00:00:06 & 00:00:06 & 00:00:07 \\
\hline 00:00:07 & 00:00:08 & 00:00:09 \\
\hline 00:00:09 & 00:00:10 & 00:00:10 \\
\hline 00:00:11 & 00:00:11 & 00:00:12 \\
\hline 00:00:12 & 00:00:13 & 00:00:13 \\
\hline 00:00:14 & 00:00:15 & 00:00:15 \\
\hline 00:00:16 & 00:00:17 & 00:00:18 \\
\hline
\end{tabular}

Figure A.28. Trench length $1.4 \mathrm{~m}, 3 \mathrm{kph}$, uncontrolled system 


\begin{tabular}{|c|c|c|}
\hline$=0$ & $=0=0$ & $=0=0$ \\
\hline 00:00:03 & 00:00:03 & 00:00:04 \\
\hline$=0=0$ & $0=0=0$ & $0=0=0$ \\
\hline 00:00:04 & 00:00:04 & 00:00:05 \\
\hline $00: 00: 05$ & (angone & angarar \\
\hline & & \\
\hline 00:00:06 & 00:00:07 & 00:00:07 \\
\hline 00:00:07 & 00:00:08 & 00:00:08 \\
\hline 00:00:09 & 00:00:09 & 00:00:09 \\
\hline 00:00:10 & 00:00:10 & 00:00:11 \\
\hline 00:00:11 & 00:00:12 & 00:00:13 \\
\hline
\end{tabular}

Figure A.29. Trench length 1.4 m, 3 kph, controlled system 

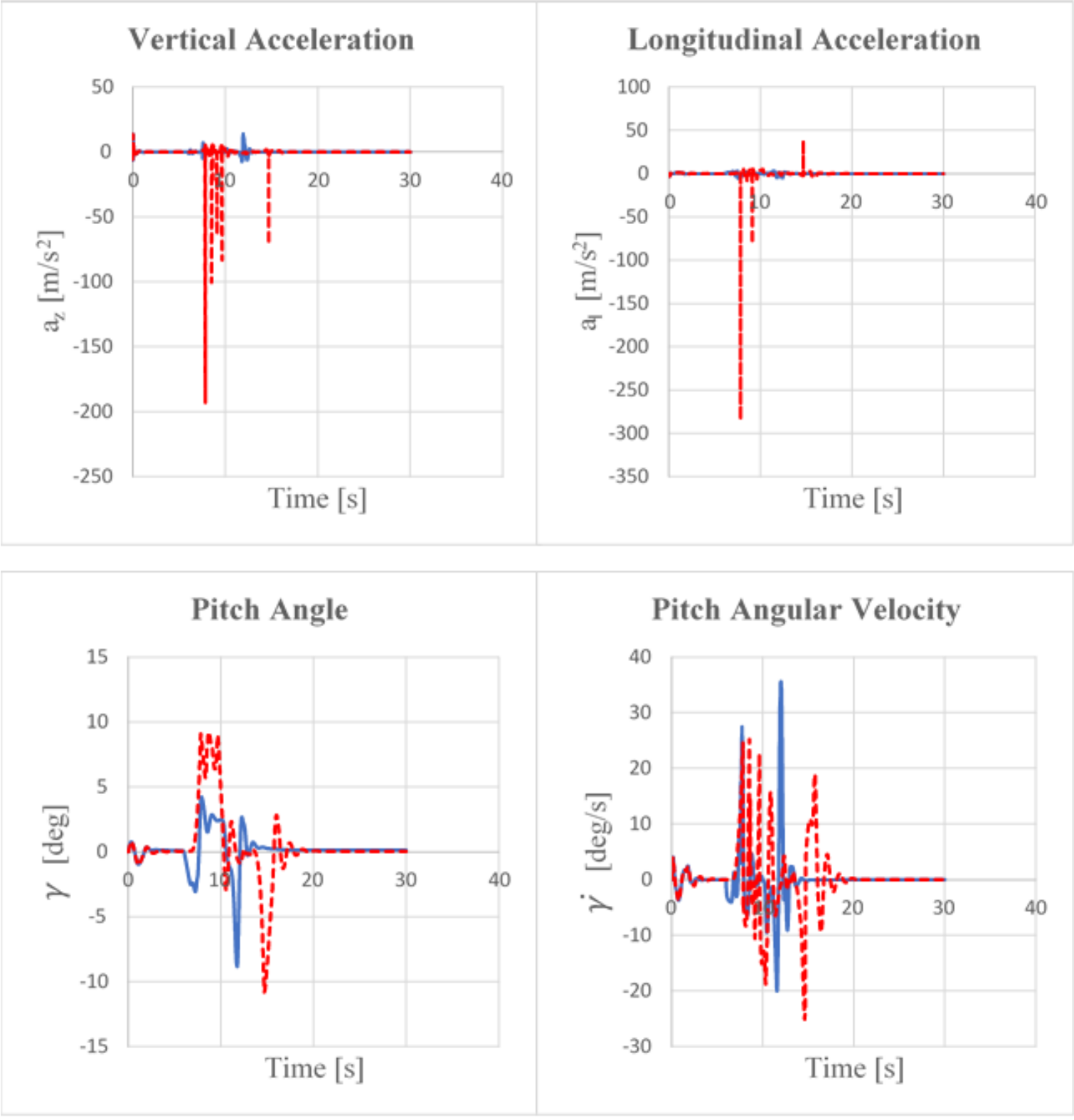

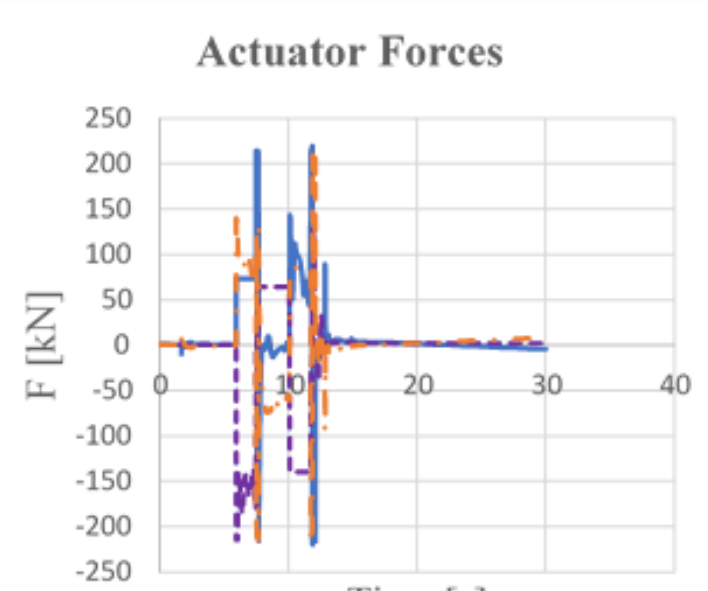

Time $[\mathrm{s}]$

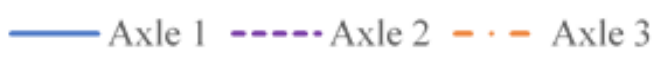

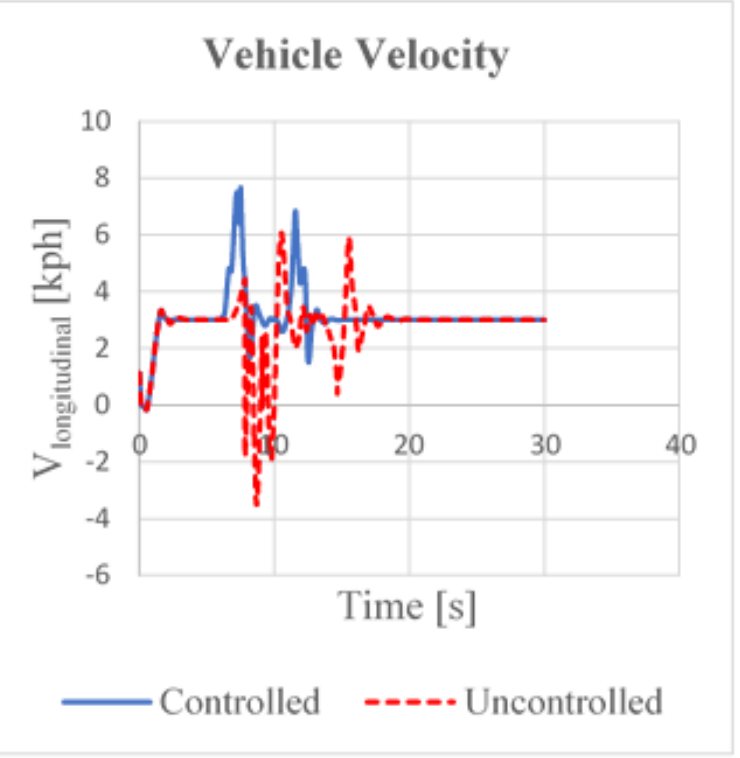

Vehicle Velocity

Controlled -----. Uncontrolled

Figure A.30. Trench length $1.4 \mathrm{~m}, 3 \mathrm{kph}$, controlled vs uncontrolled output comparison 
Trench Length 1.4 m, 6 kph

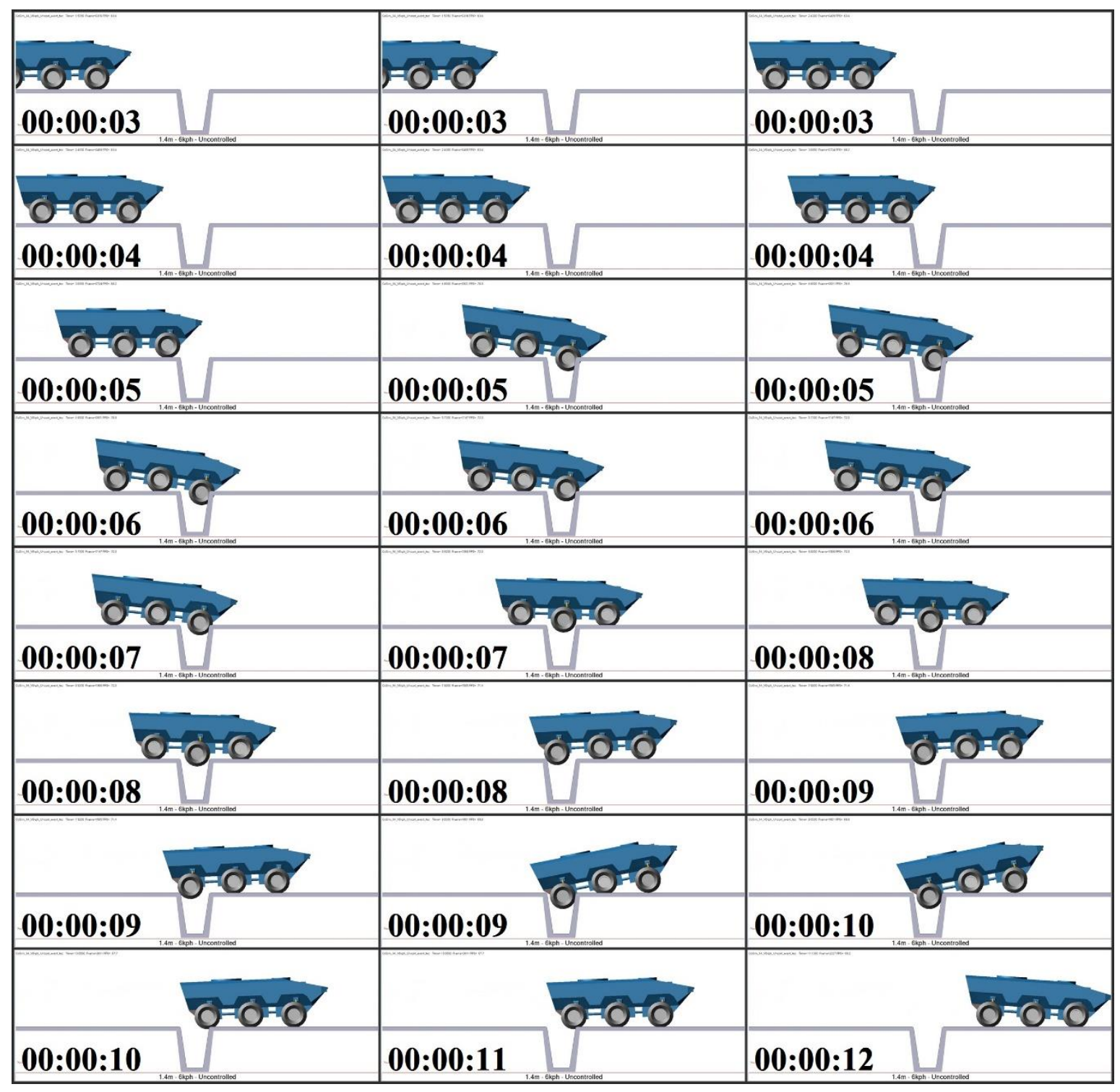

Figure A.31. Trench length 1.4 m, 6 kph, uncontrolled system 


\begin{tabular}{|c|c|c|}
\hline $0=0$ & $=0=0$ & $=0=0$ \\
\hline 00:00:02 & 00:00:02 & 00:00:02 \\
\hline$=0=0$ & $=0=0$ & $0=0=0$ \\
\hline 00:00:03 & 00:00:03 & 00:00:03 \\
\hline $0=0=0$ & $0=0=0$ & c \\
\hline 00:00:03 & 00:00:04 & 00:00:04 \\
\hline 00:00:04 & 00:00:05 & 00:00:05 \\
\hline 00:00:05 & 00:00:05 & 00:00:06 \\
\hline 00:00:06 & 00:00:06 & 00:00:06 \\
\hline 00:00:07 & 00:00:07 & 00:00:07 \\
\hline 00:00:08 & 00:00:08 & 00:00:09 \\
\hline
\end{tabular}

Figure A.32. Trench length $1.4 \mathrm{~m}, 6 \mathrm{kph}$, controlled system 

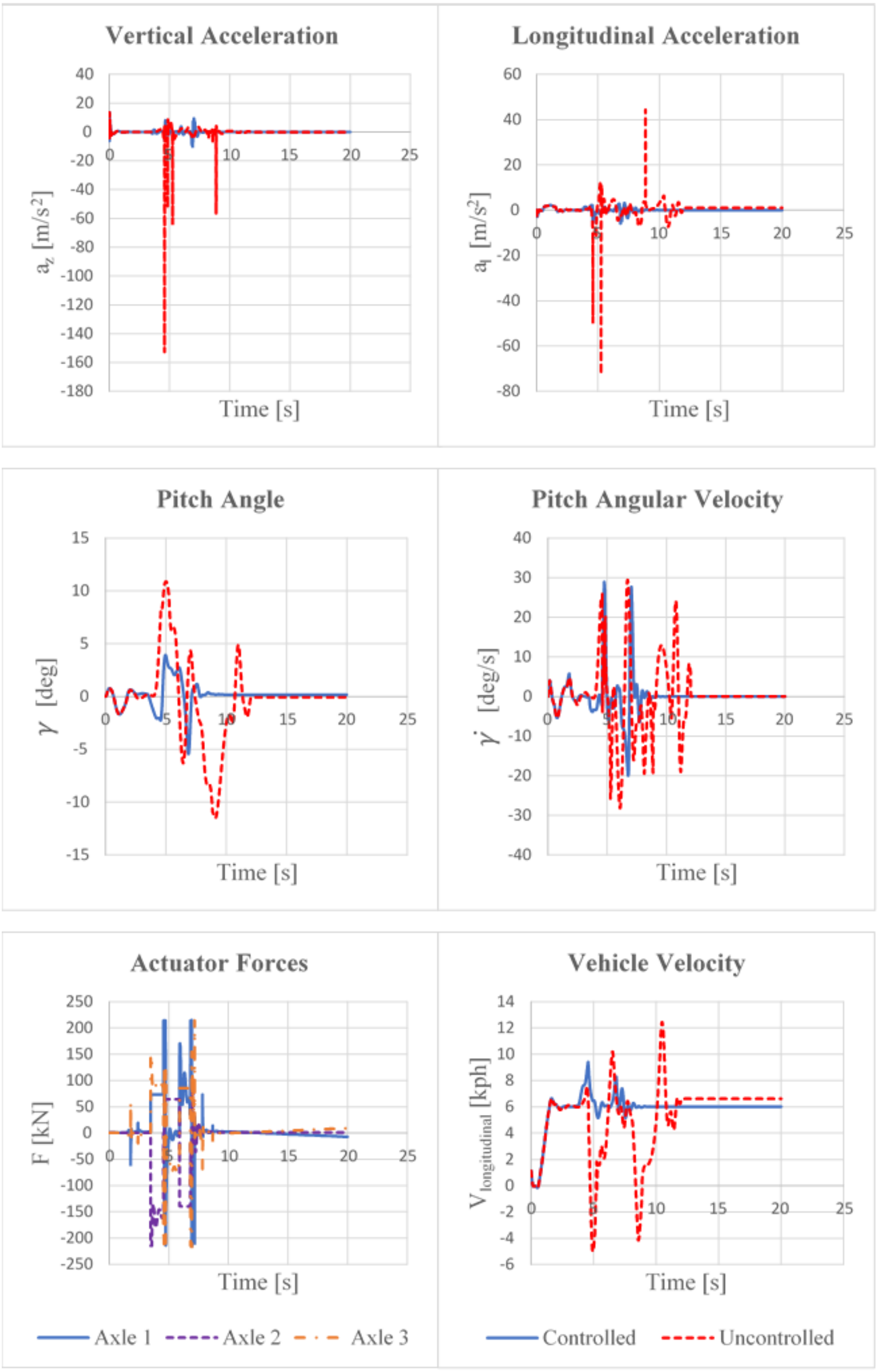

Figure A.33. Trench length $1.4 \mathrm{~m}$, $6 \mathrm{kph}$, controlled vs uncontrolled output comparison 
Trench Length 1.4 m, 9 kph

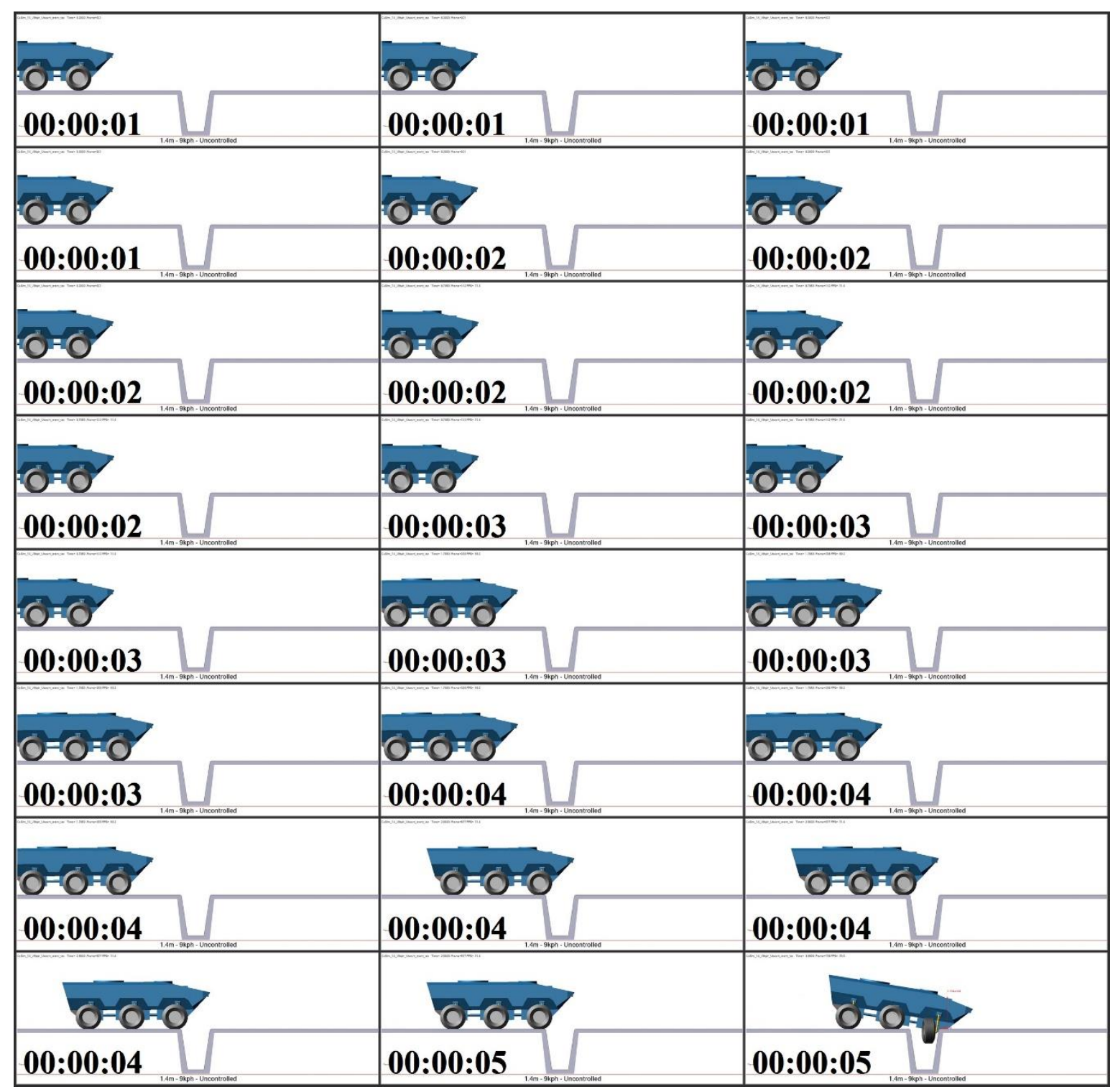

Figure A.34. Trench length $1.4 \mathrm{~m}, 9 \mathrm{kph}$, uncontrolled system 


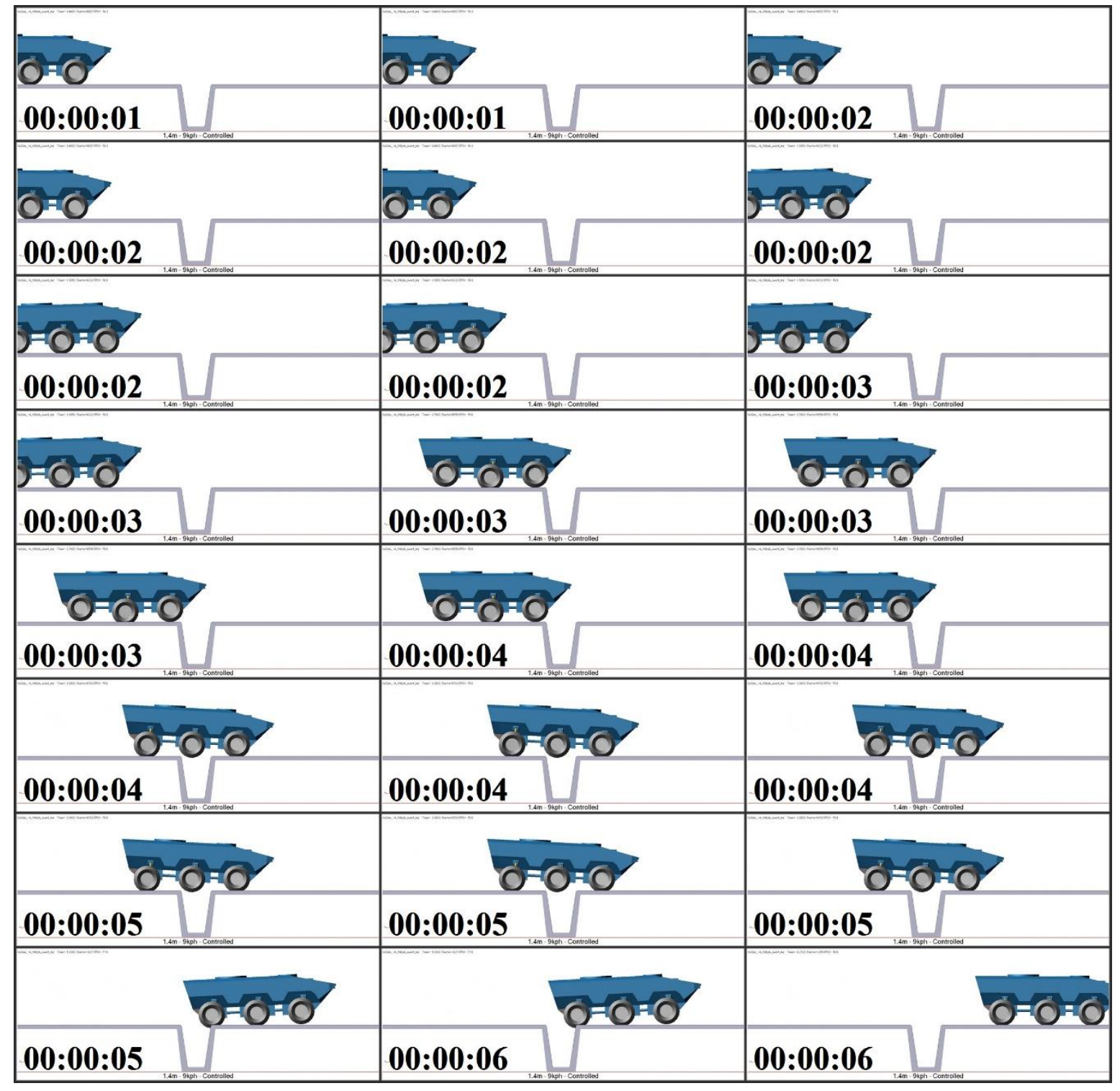

Figure A.35. Trench length $1.4 \mathrm{~m}, 9 \mathrm{kph}$, controlled system 

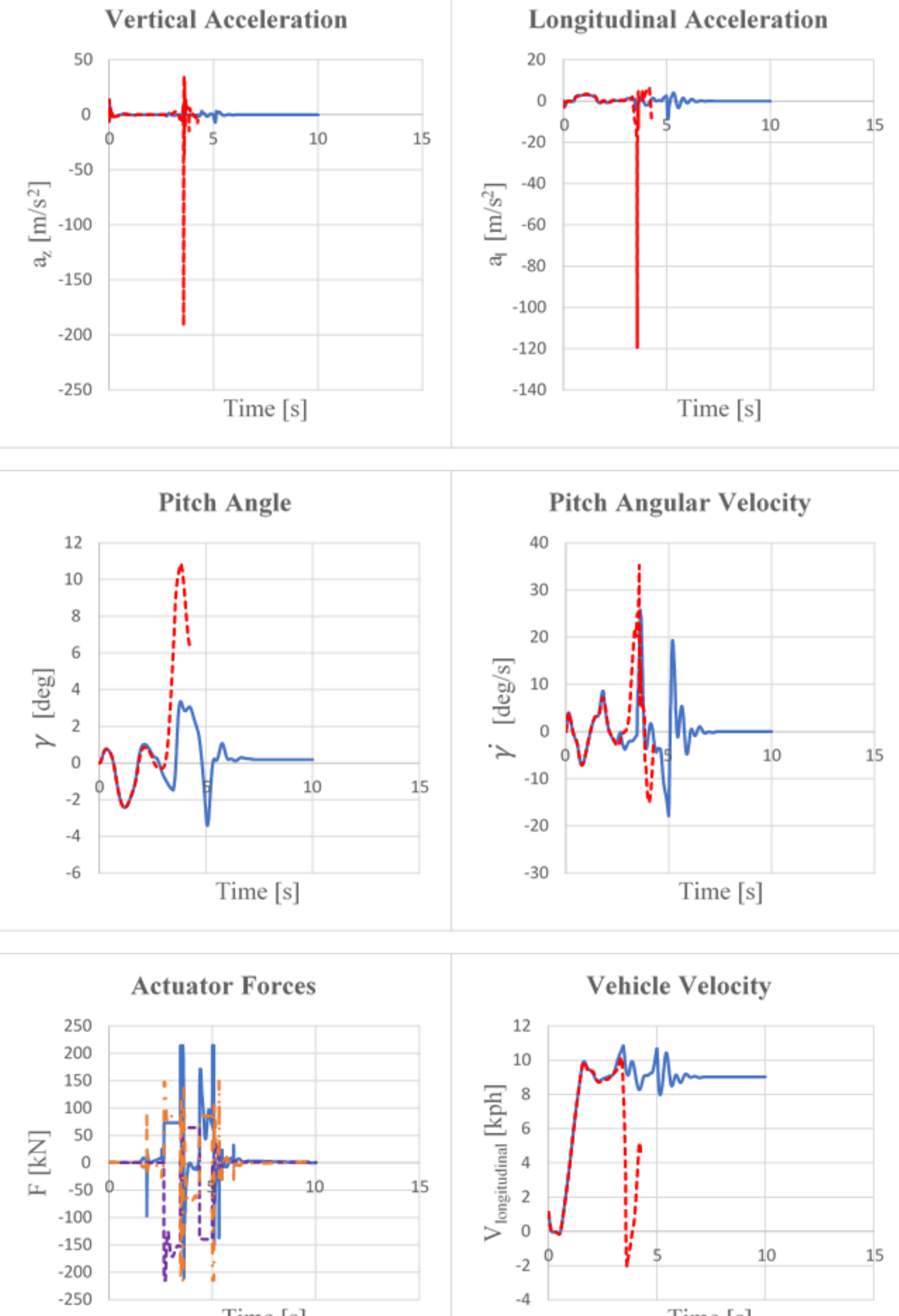

Time [s]

— Axle 1 ----Axle 2 - - Axle 3

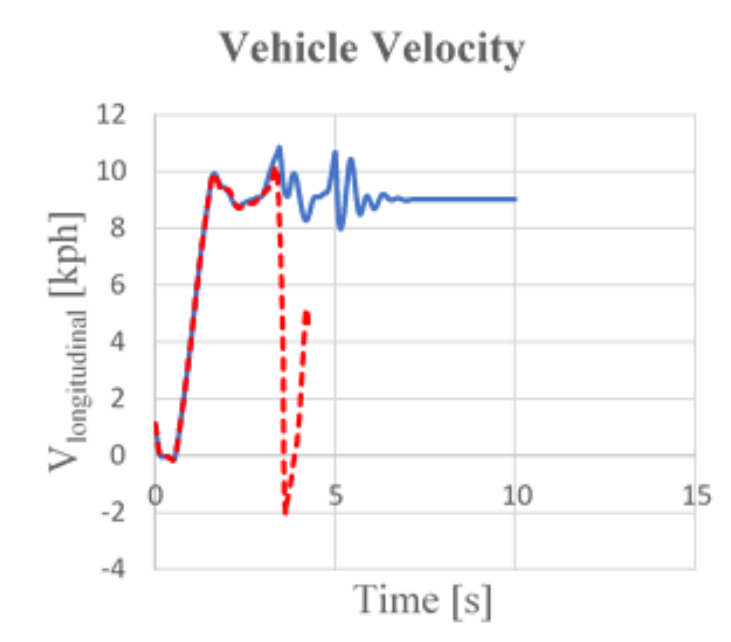

Controlled -----. Uncontrolled

Figure A.36. Trench length $1.4 \mathrm{~m}, 9 \mathrm{kph}$, controlled vs uncontrolled output comparison 
Trench Length $1.5 \mathrm{~m}$, Controlled vs Uncontrolled System Simulations

Trench Length $1.5 \mathrm{~m}, 1 \mathrm{kph}$

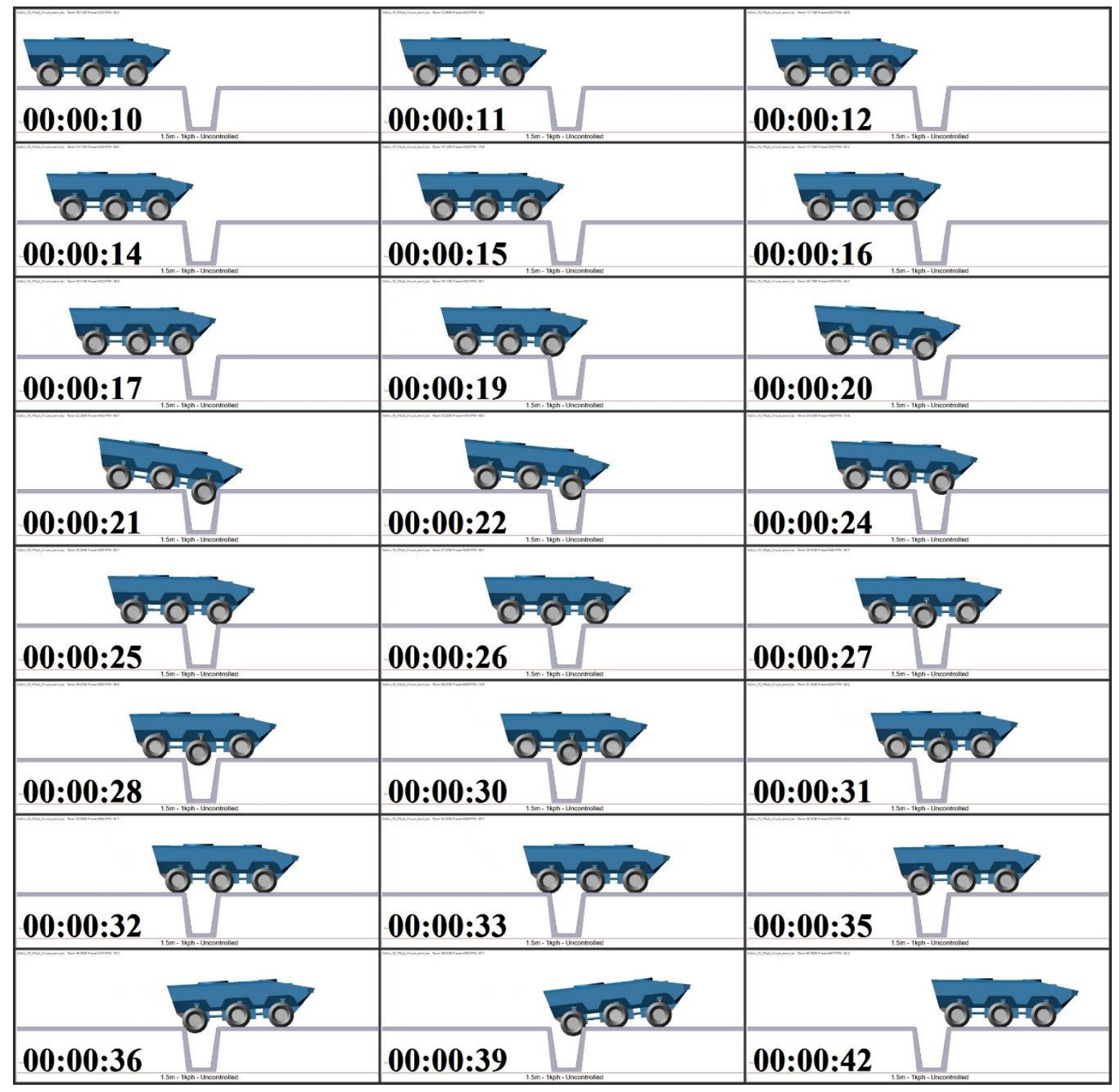

Figure A.37. Trench length $1.5 \mathrm{~m}, 1 \mathrm{kph}$, uncontrolled system 


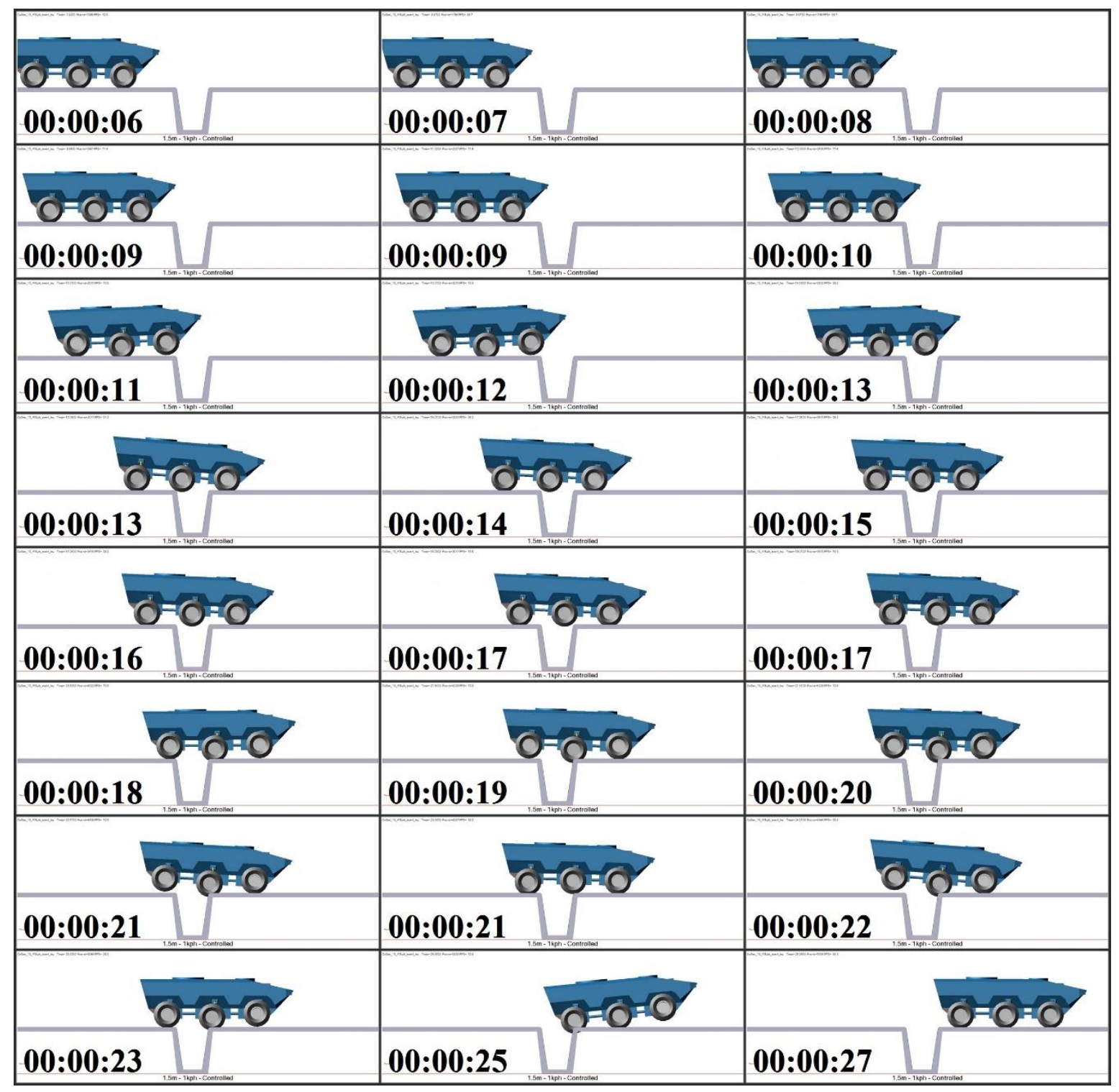

Figure A.38. Trench length $1.5 \mathrm{~m}, 1 \mathrm{kph}$, controlled system 

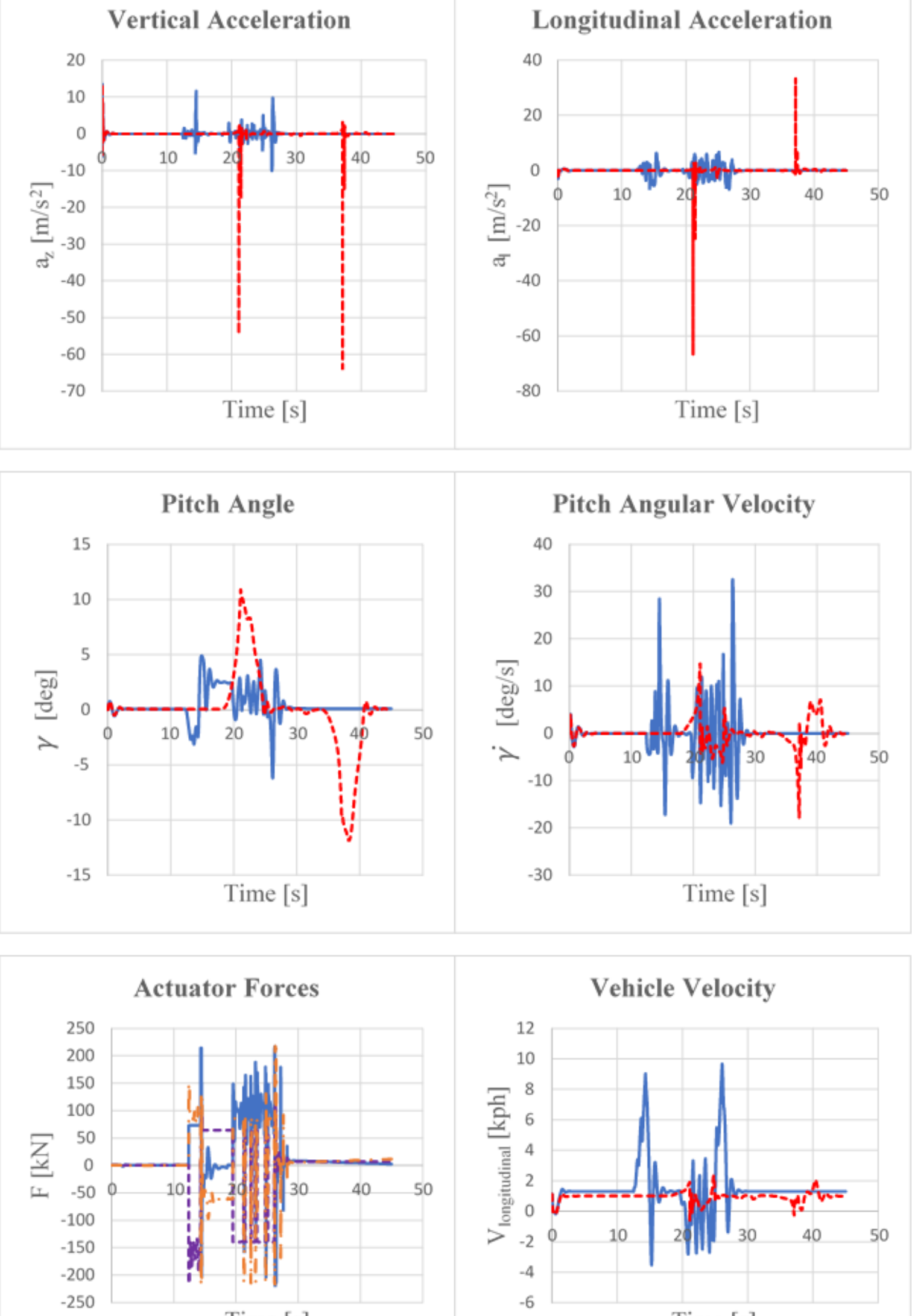

Time [s]

— Axle 1 ----A Axle 2 - - Axle 3

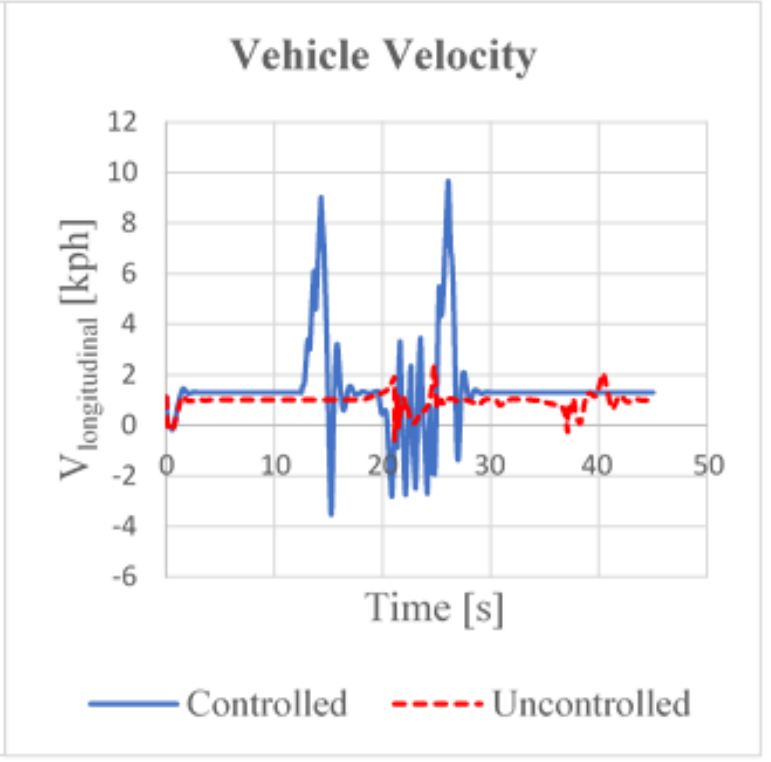

Figure A.39. Trench length $1.5 \mathrm{~m}, 1 \mathrm{kph}$, controlled vs uncontrolled output comparison 
Trench Length 1.5 m, 3 kph

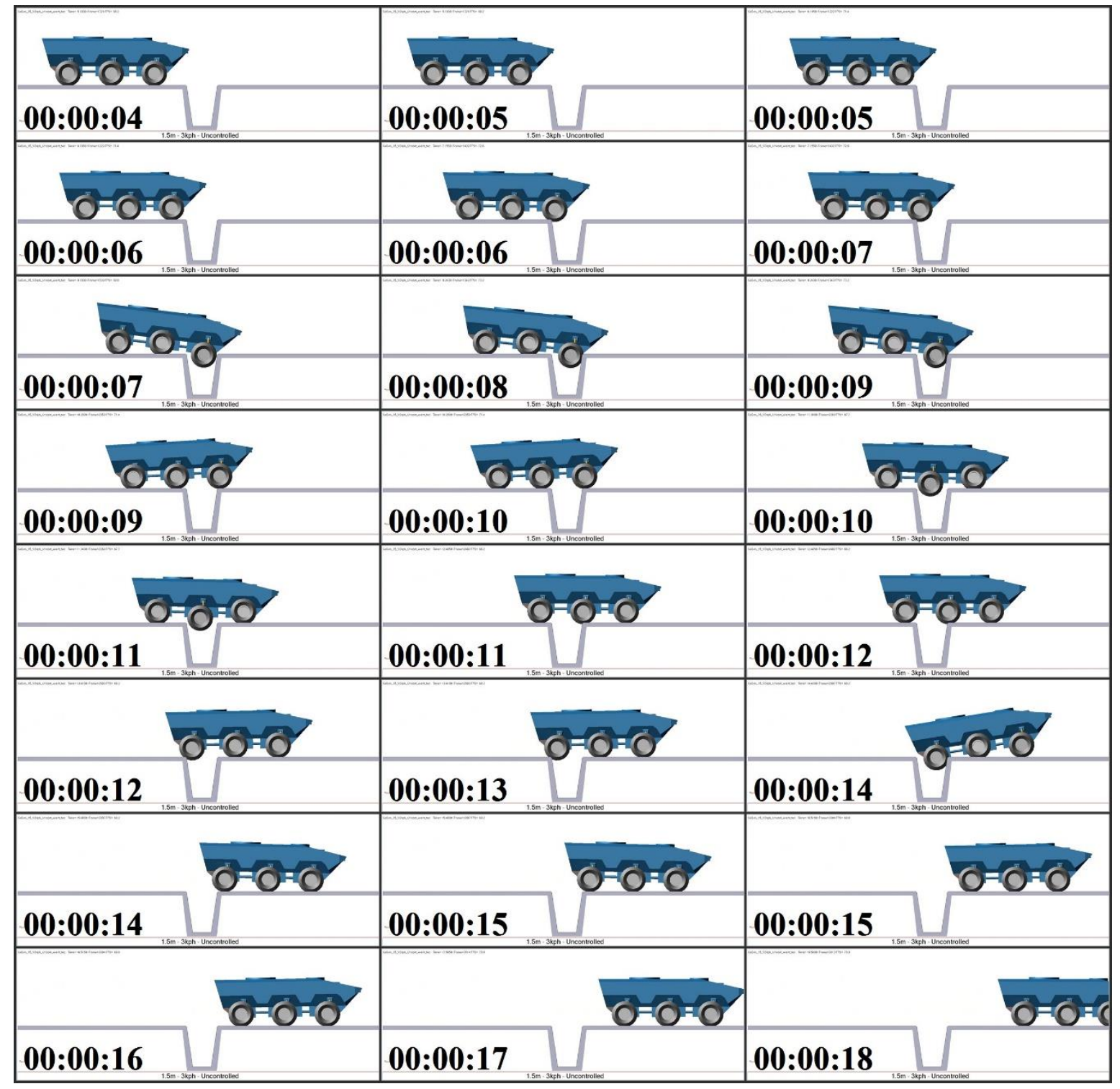

Figure A.40. Trench length $1.5 \mathrm{~m}, 3 \mathrm{kph}$, uncontrolled system 


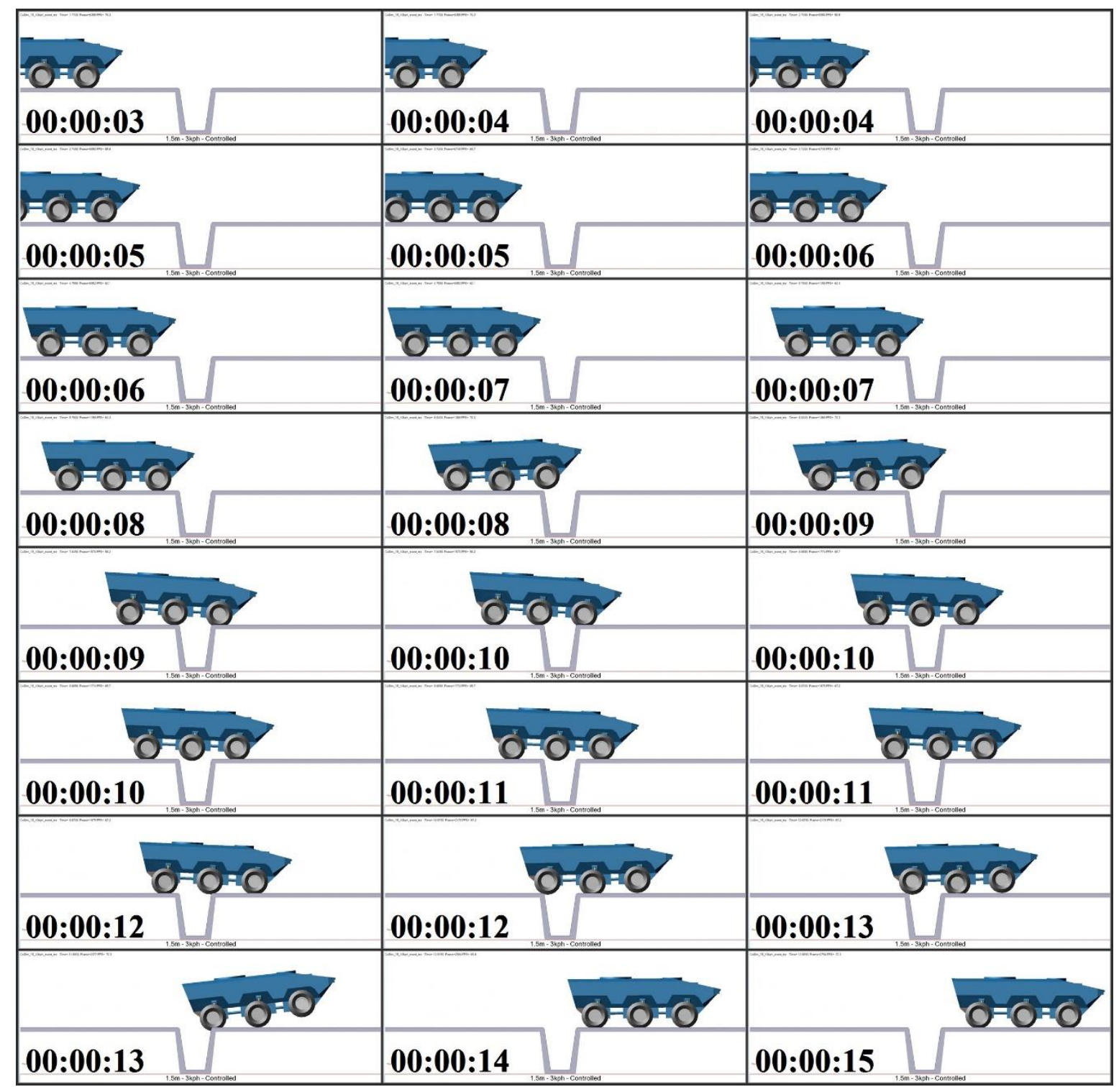

Figure A.41. Trench length $1.5 \mathrm{~m}, 3 \mathrm{kph}$, controlled system 


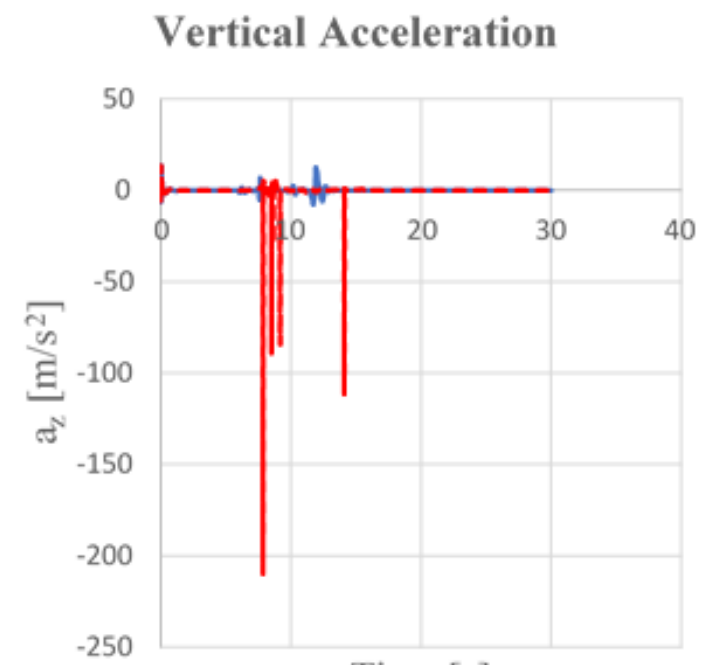

Time $[\mathrm{s}]$

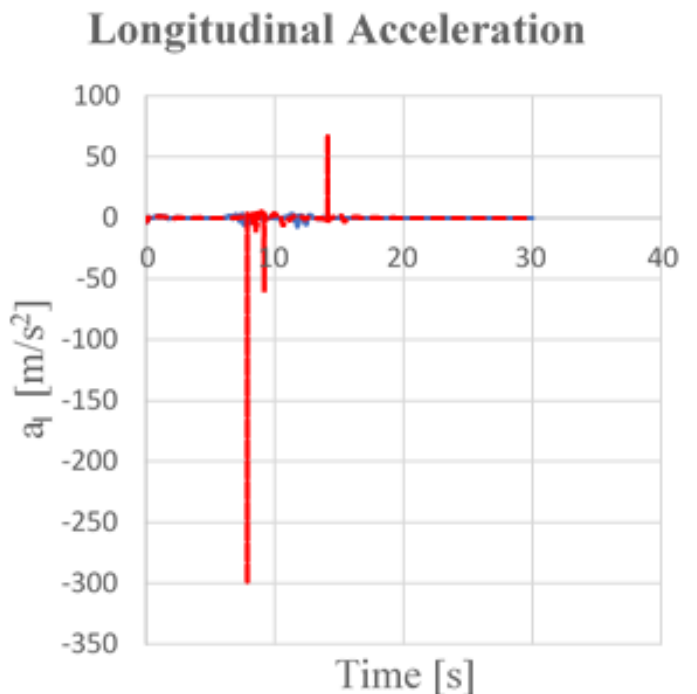

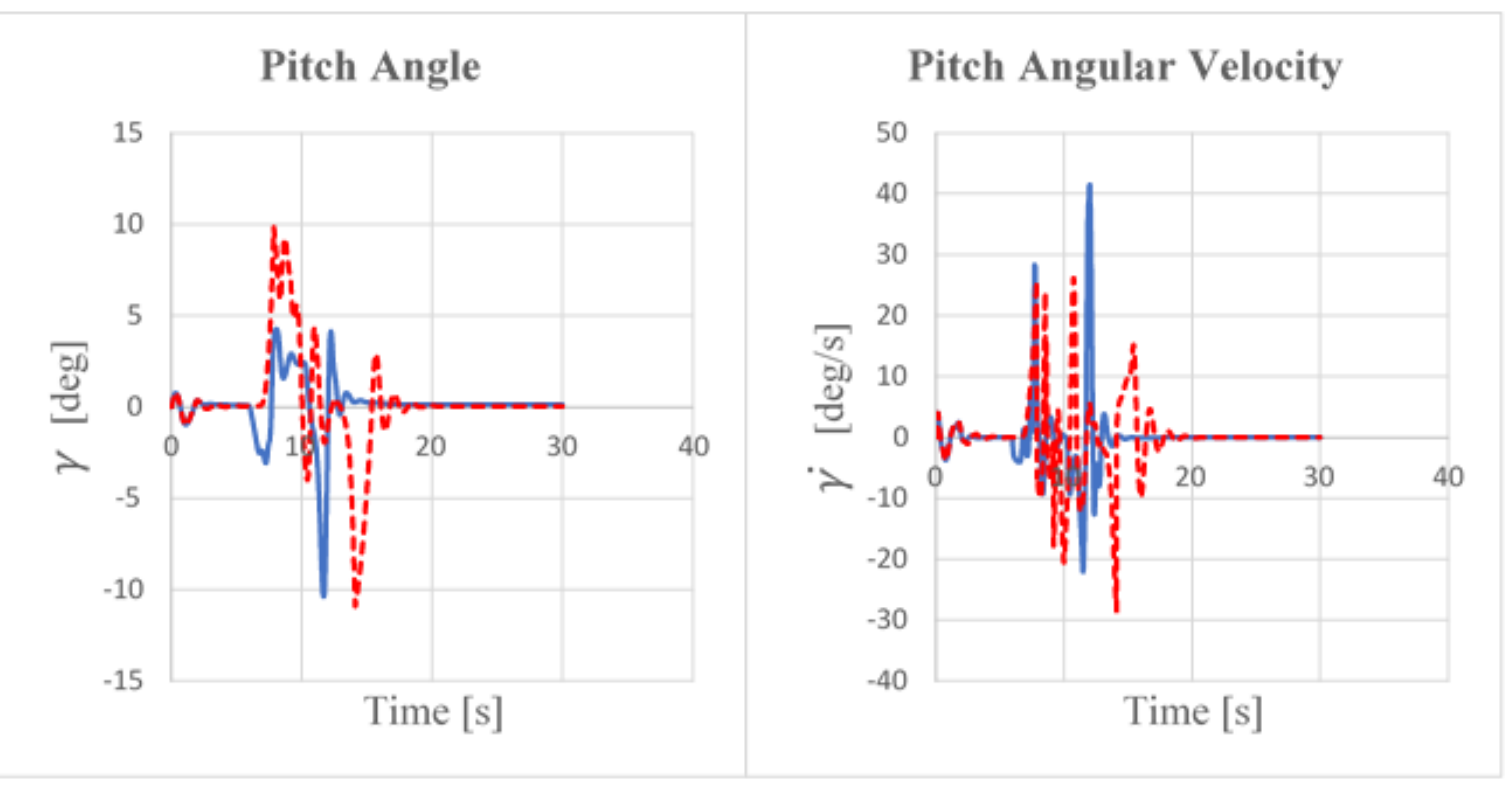

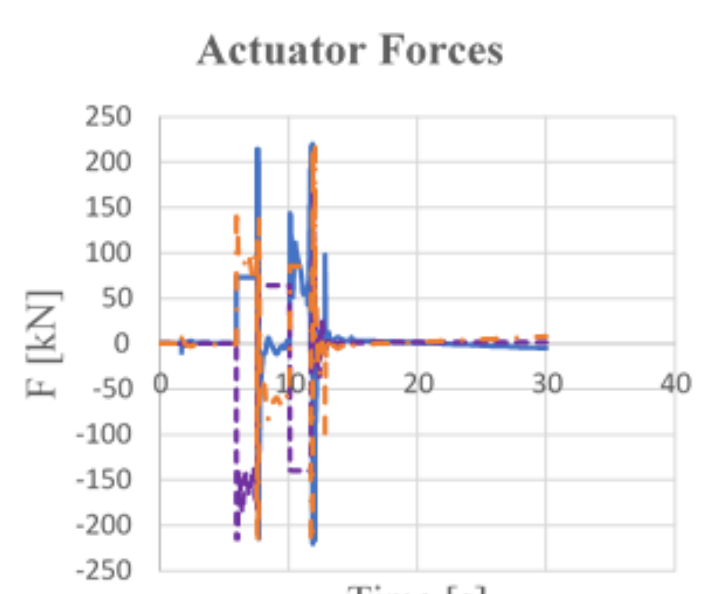

Time [s]

Axle 1 ---. Axle 2 - - Axle 3

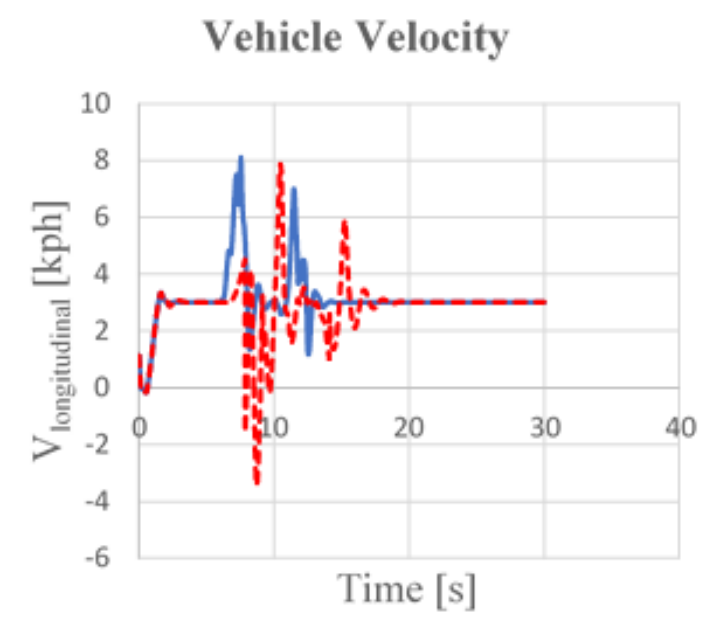

—Controlled -----・ Uncontrolled

Figure A.42. Trench length $1.5 \mathrm{~m}, 3 \mathrm{kph}$, controlled vs uncontrolled output comparison 
Trench Length 1.5 m, 6 kph

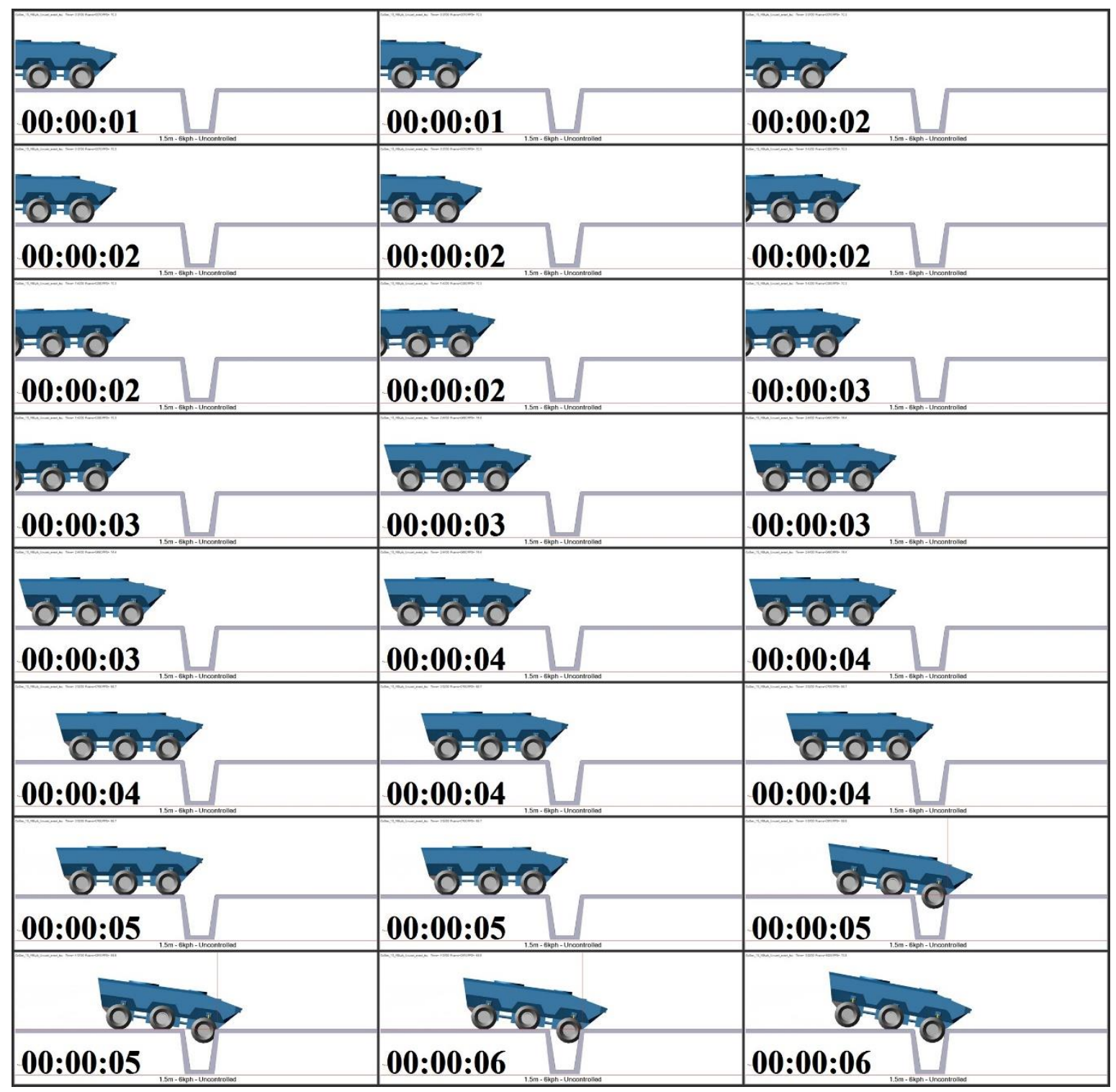

Figure A.43. Trench length $1.5 \mathrm{~m}, 6 \mathrm{kph}$, uncontrolled system 


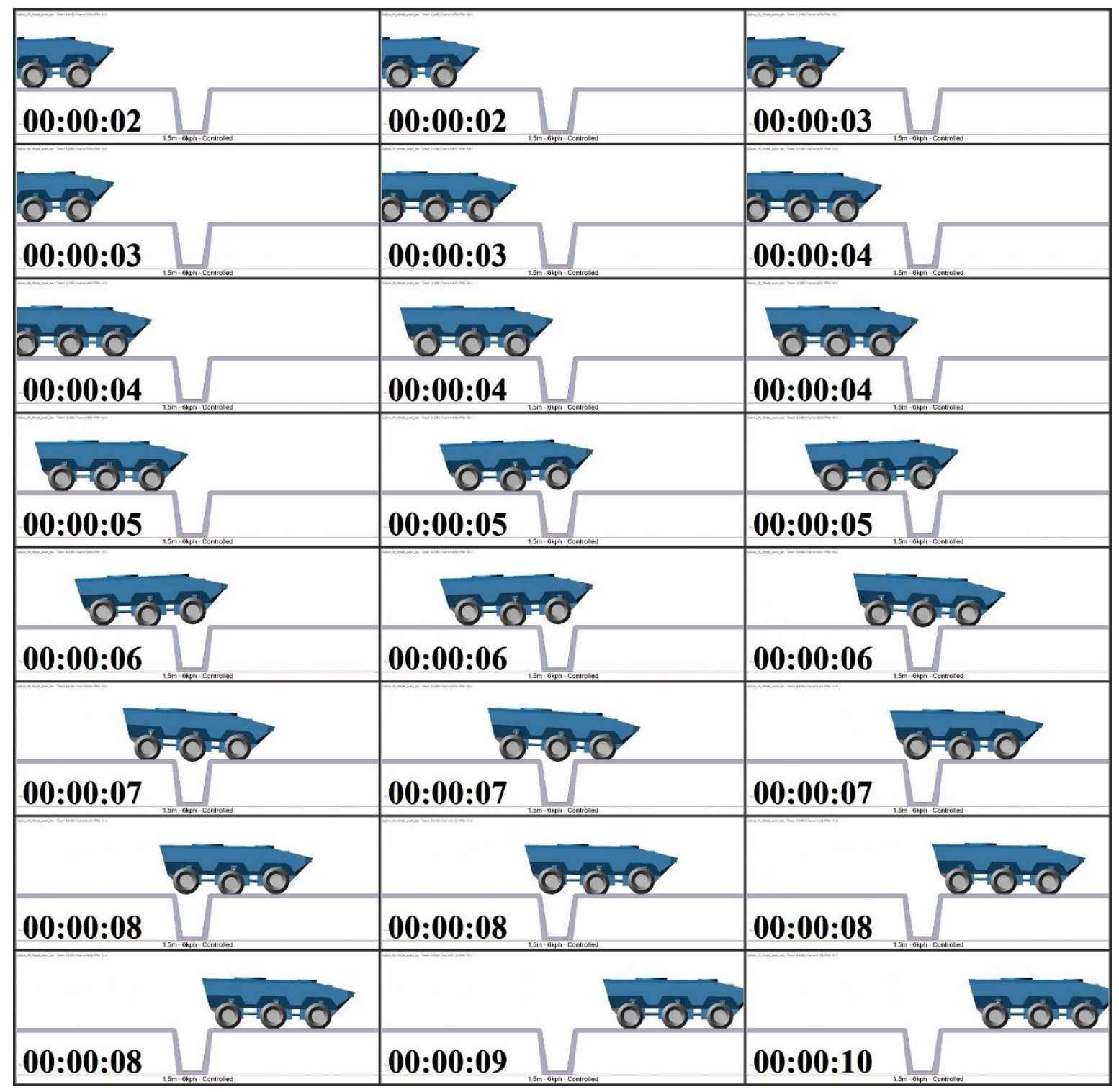

Figure A.44. Trench length $1.5 \mathrm{~m}, 6 \mathrm{kph}$, controlled system 

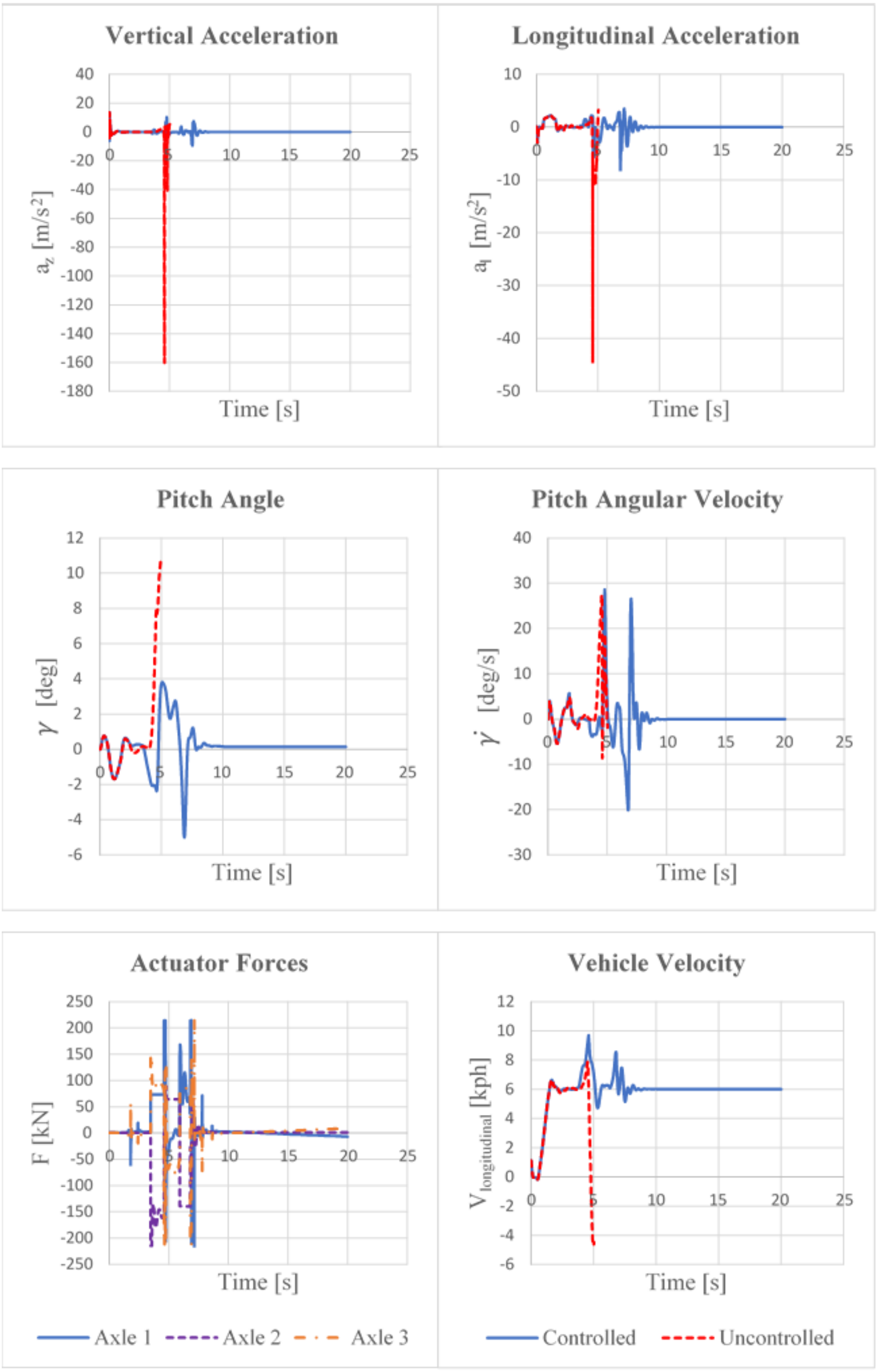

Figure A.45. Trench length $1.5 \mathrm{~m}$, $6 \mathrm{kph}$, controlled vs uncontrolled output comparison 
Trench Length 1.5 m, 9 kph

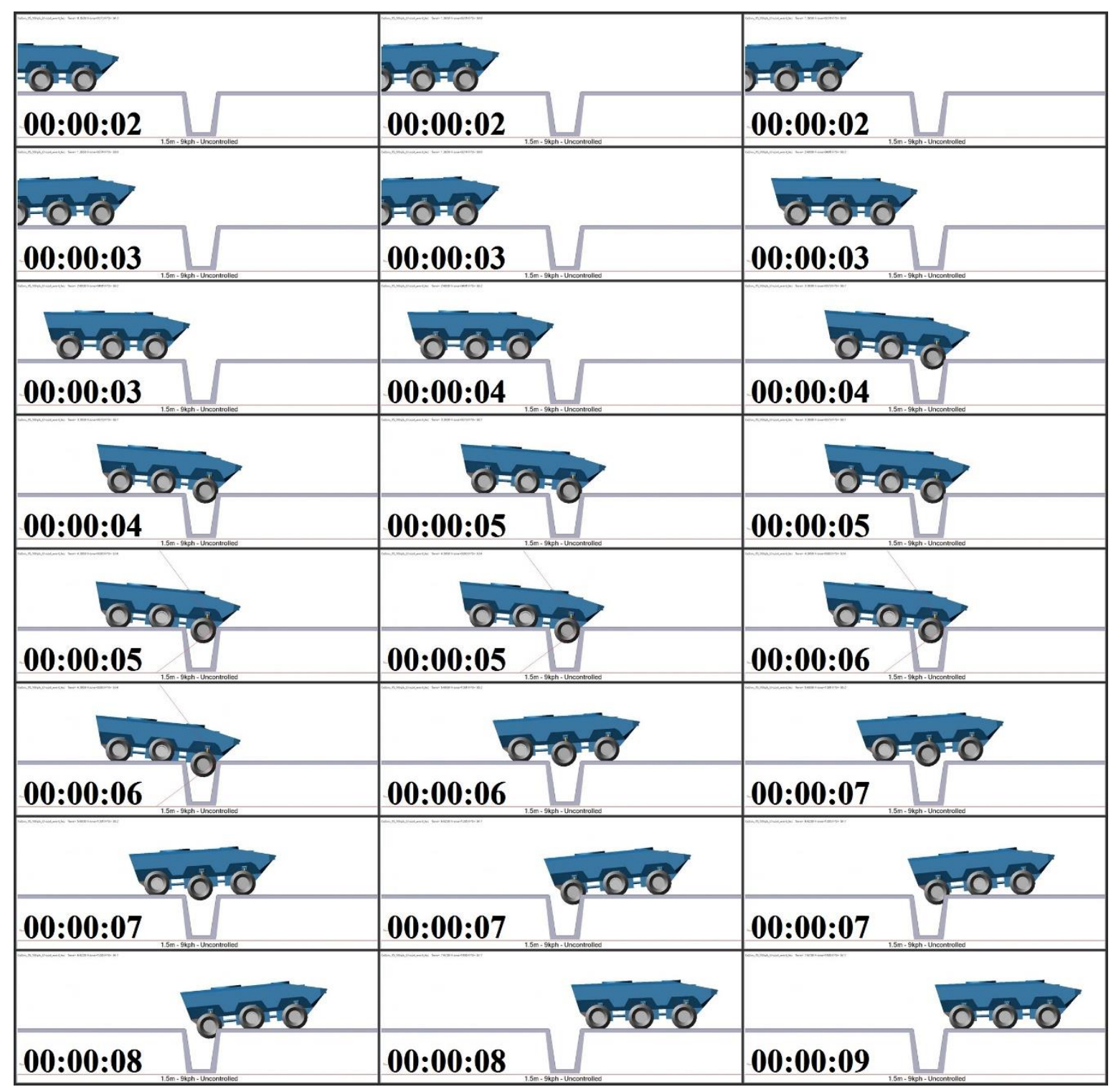

Figure A.46. Trench length $1.5 \mathrm{~m}, 9 \mathrm{kph}$, uncontrolled system 


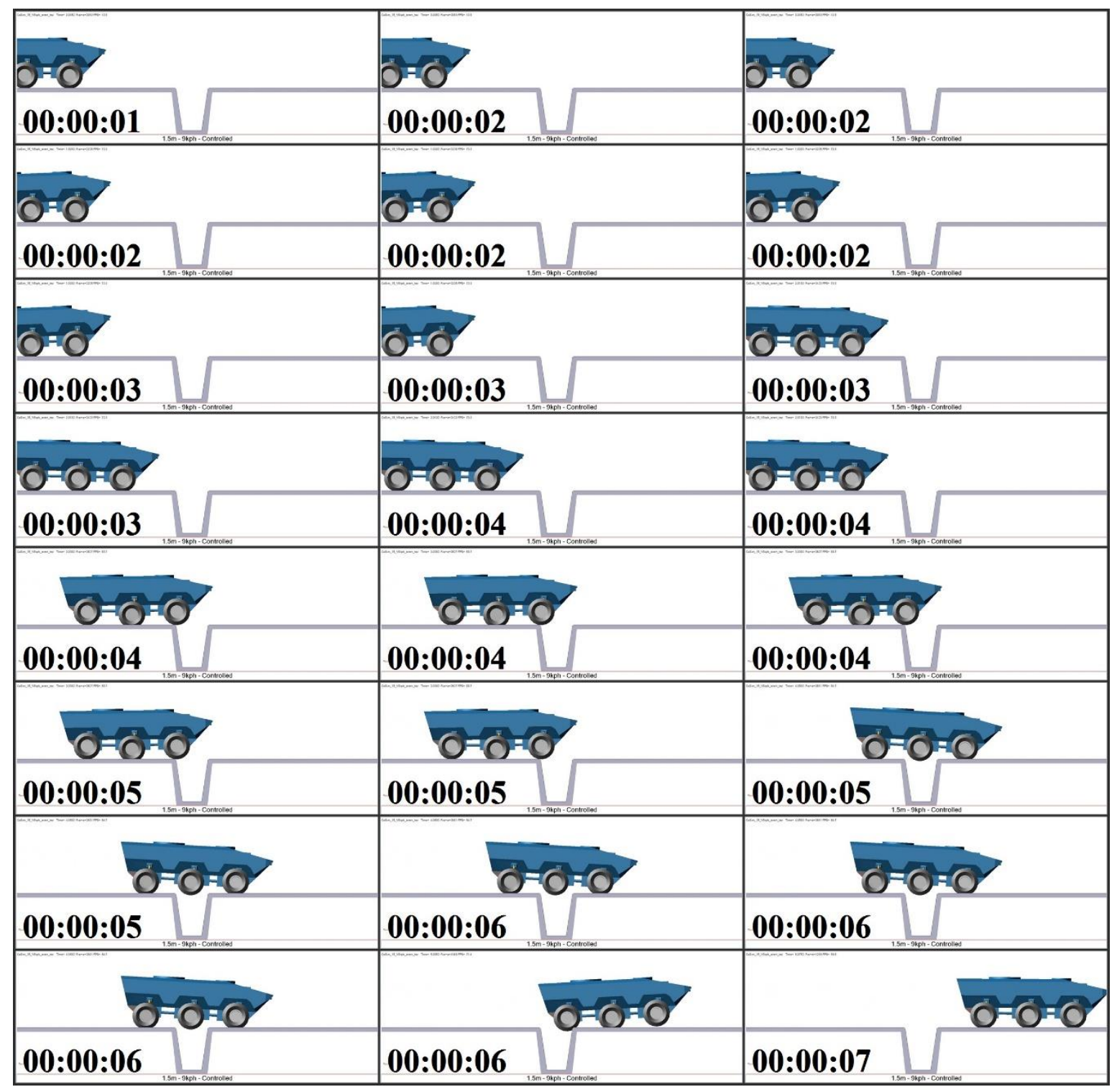

Figure A.47. Trench length $1.5 \mathrm{~m}, 9 \mathrm{kph}$, uncontrolled system 

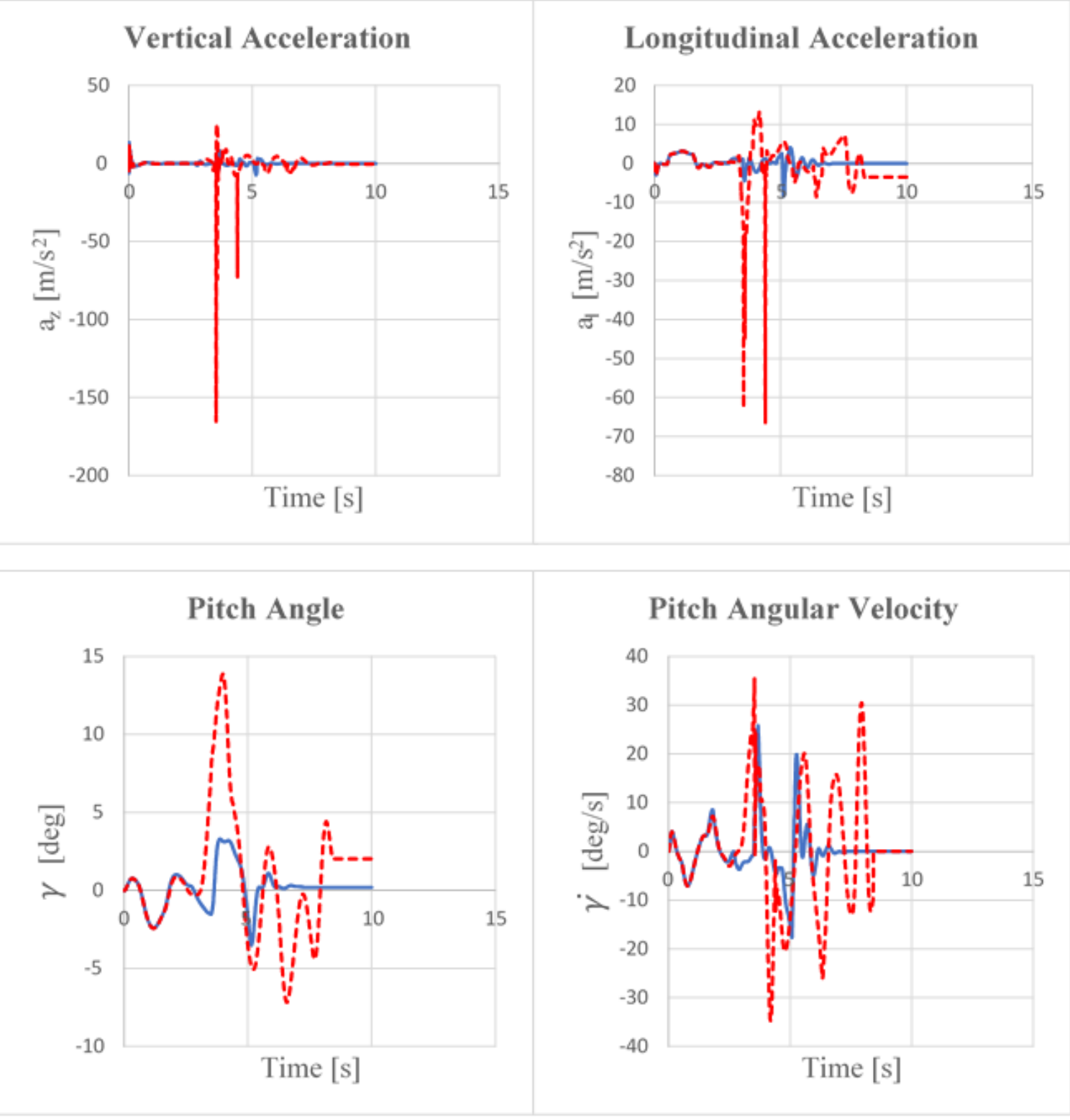

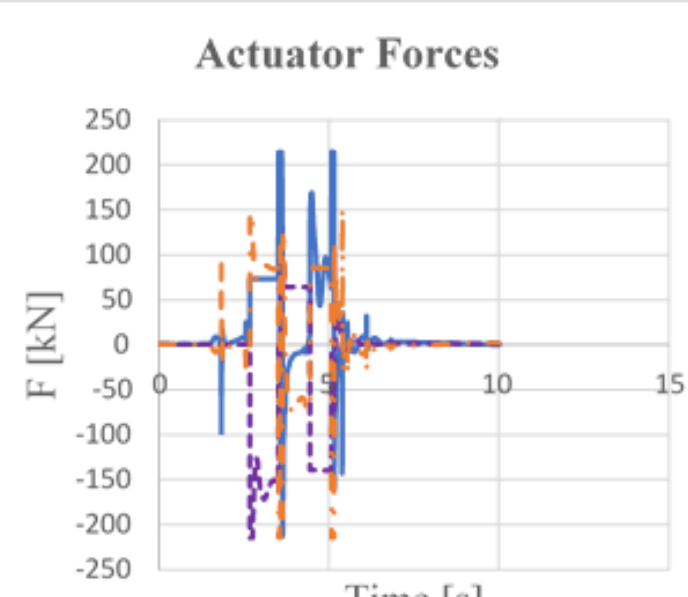

Time $[\mathrm{s}]$

- Axle 1 ----. Axle 2 - - Axle 3

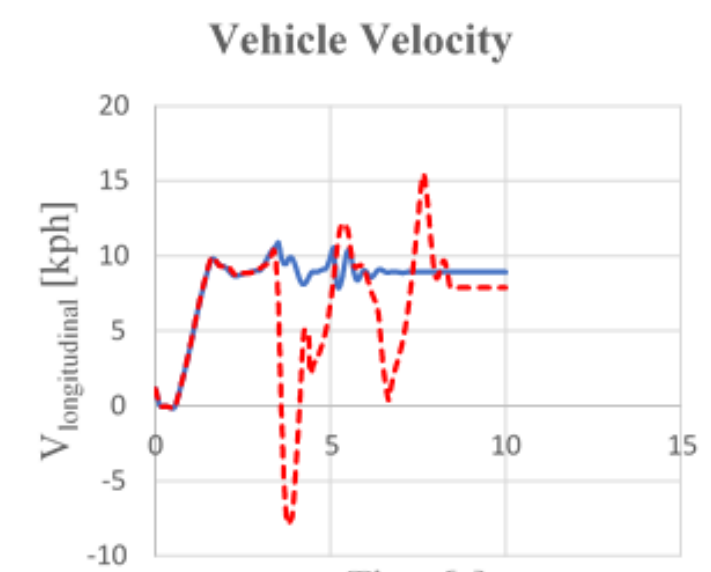

Time $[\mathrm{s}]$

Controlled ----.- Uncontrolled

Figure A.48. Trench length $1.5 \mathrm{~m}, 9 \mathrm{kph}$, controlled vs uncontrolled output comparison 
Trench Length $1.6 \mathrm{~m}$, Controlled vs Uncontrolled System Simulations

Trench Length $1.6 \mathrm{~m}, 1 \mathrm{kph}$

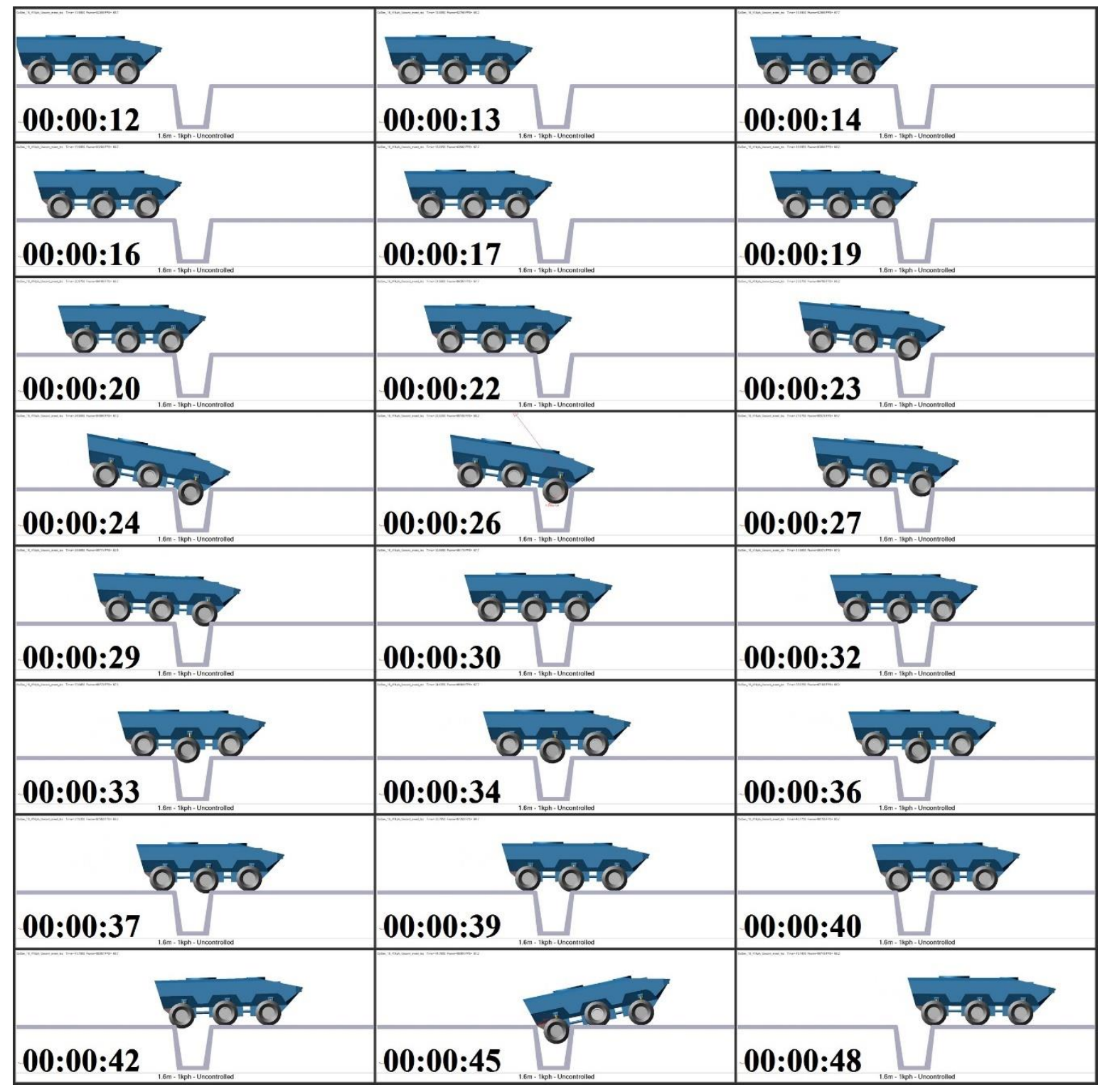

Figure A.49. Trench length $1.6 \mathrm{~m}, 1 \mathrm{kph}$, uncontrolled system 


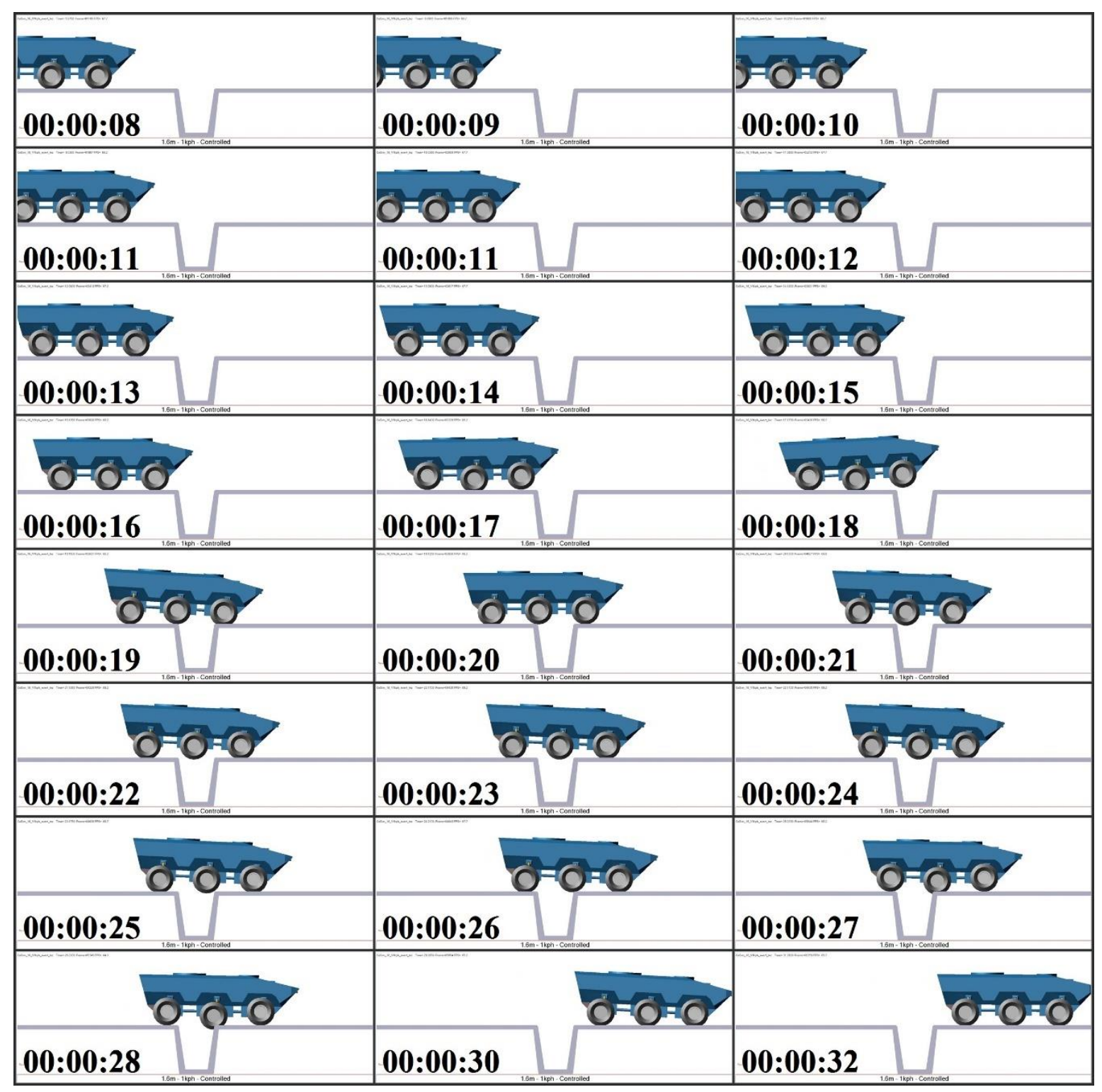

Figure A.50. Trench length $1.6 \mathrm{~m}, 1 \mathrm{kph}$, controlled system 


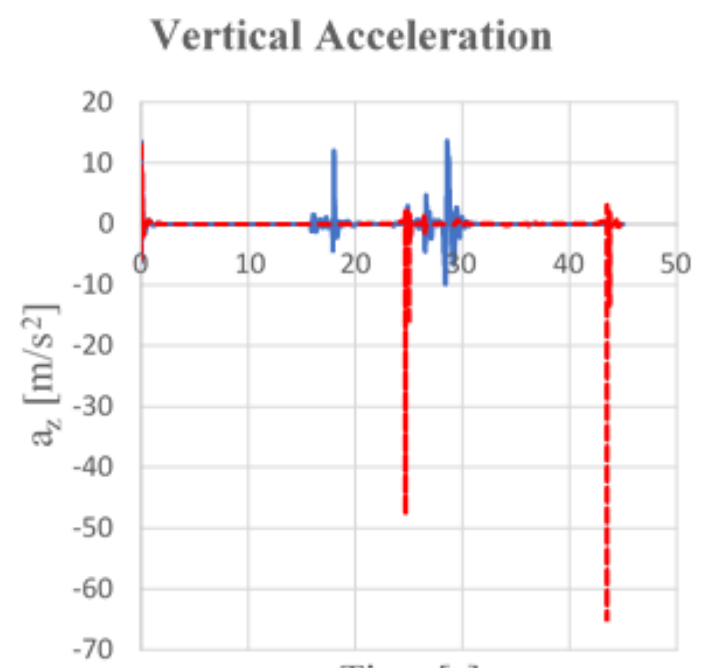

Time [s]

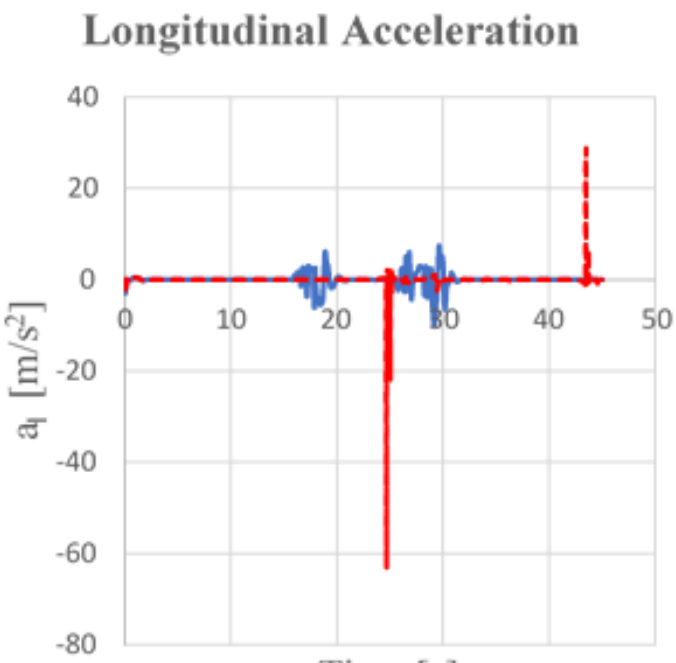

Time [s]

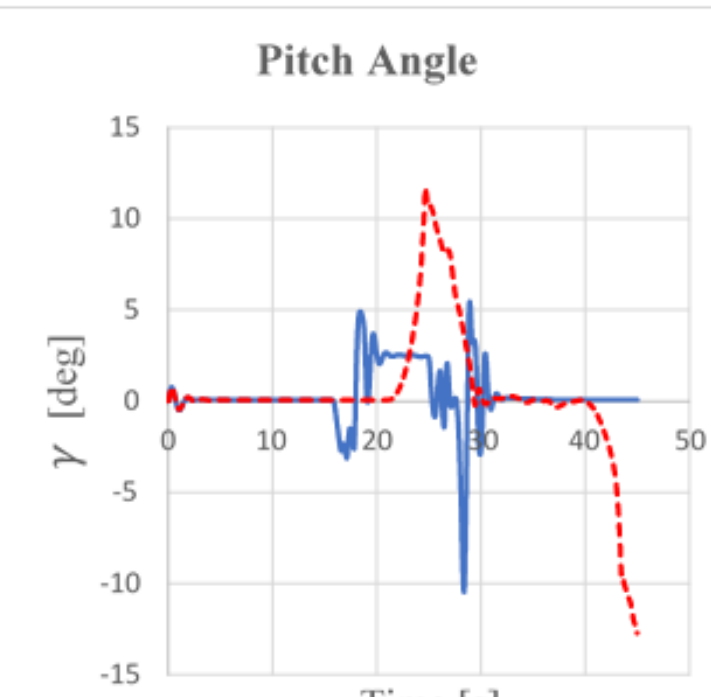

Time [s]

\section{Pitch Angular Velocity}

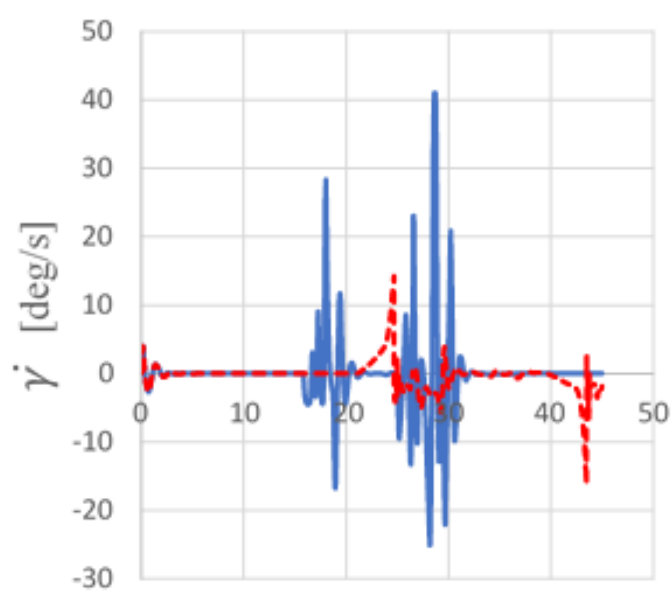

Time [s]

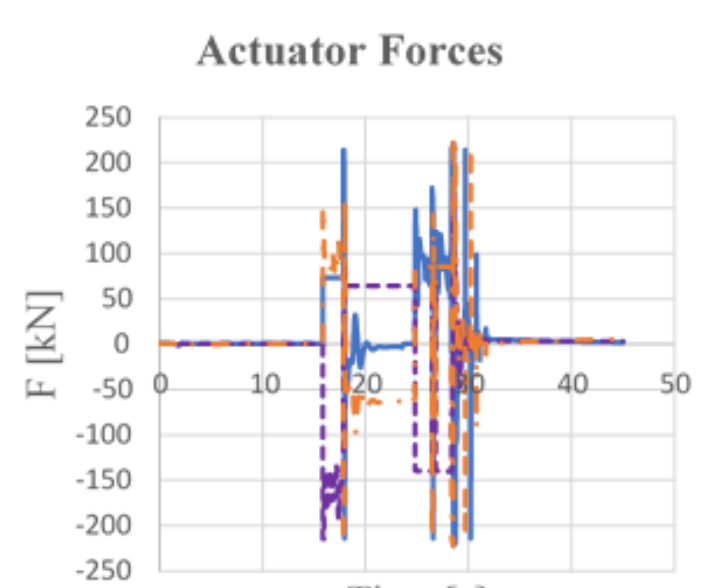

Time [s]

—Axle 1 - - Axle 2 - Axle 3

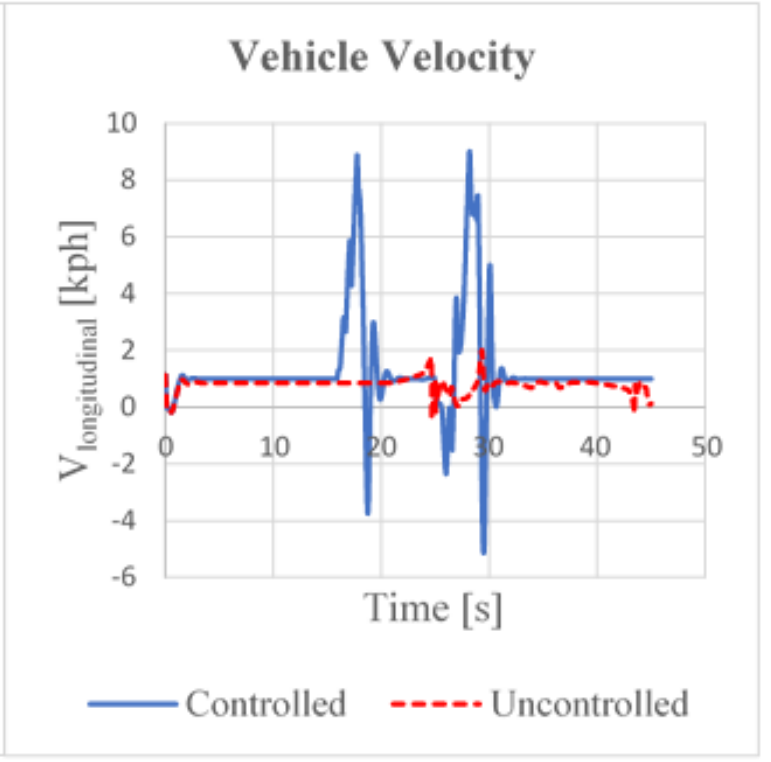

Figure A.51. Trench length $1.6 \mathrm{~m}, 1 \mathrm{kph}$, controlled vs uncontrolled output comparison 
Trench Length 1.6 m, 3 kph

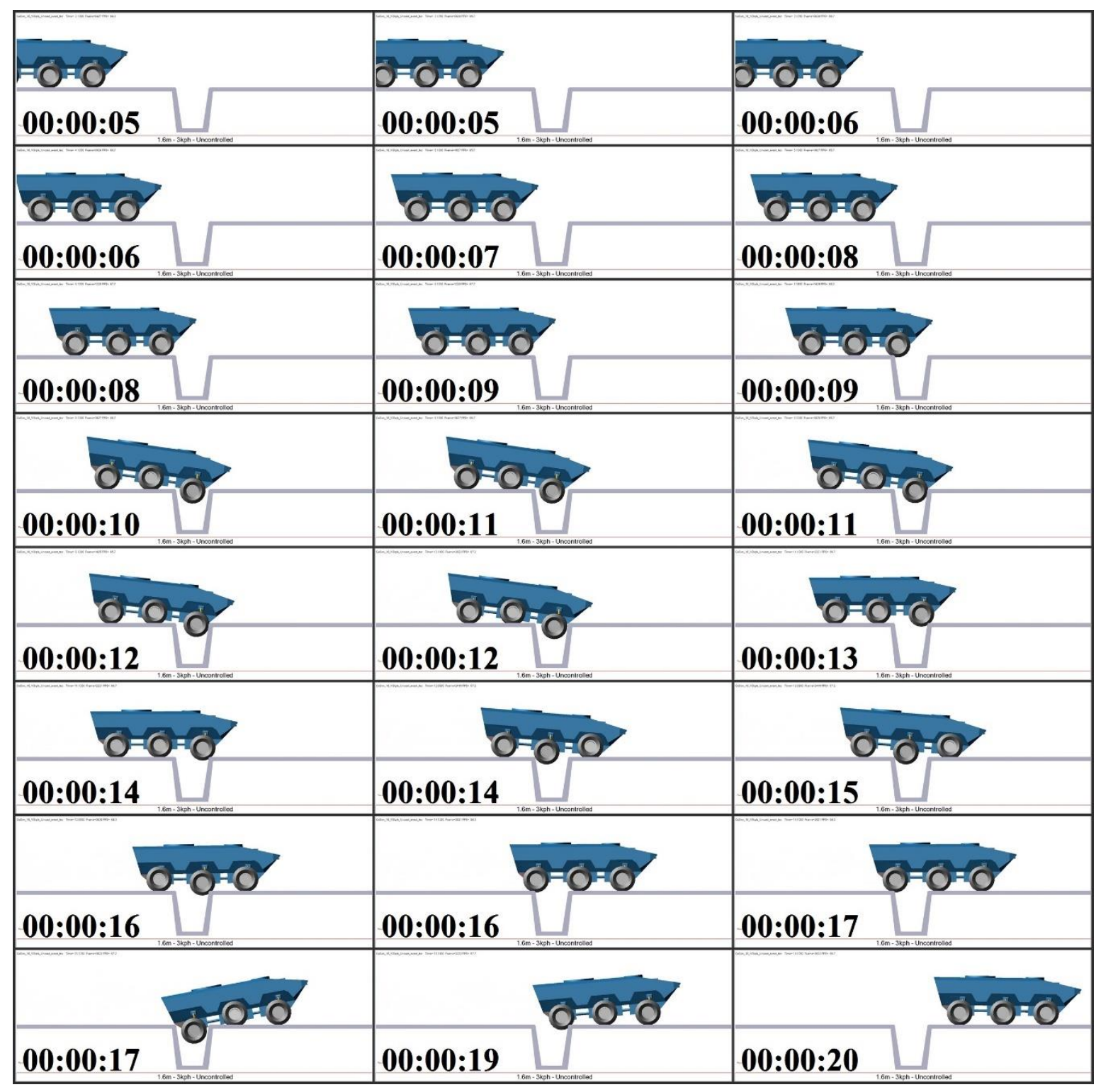

Figure A.52. Trench length $1.6 \mathrm{~m}, 3 \mathrm{kph}$, uncontrolled system 


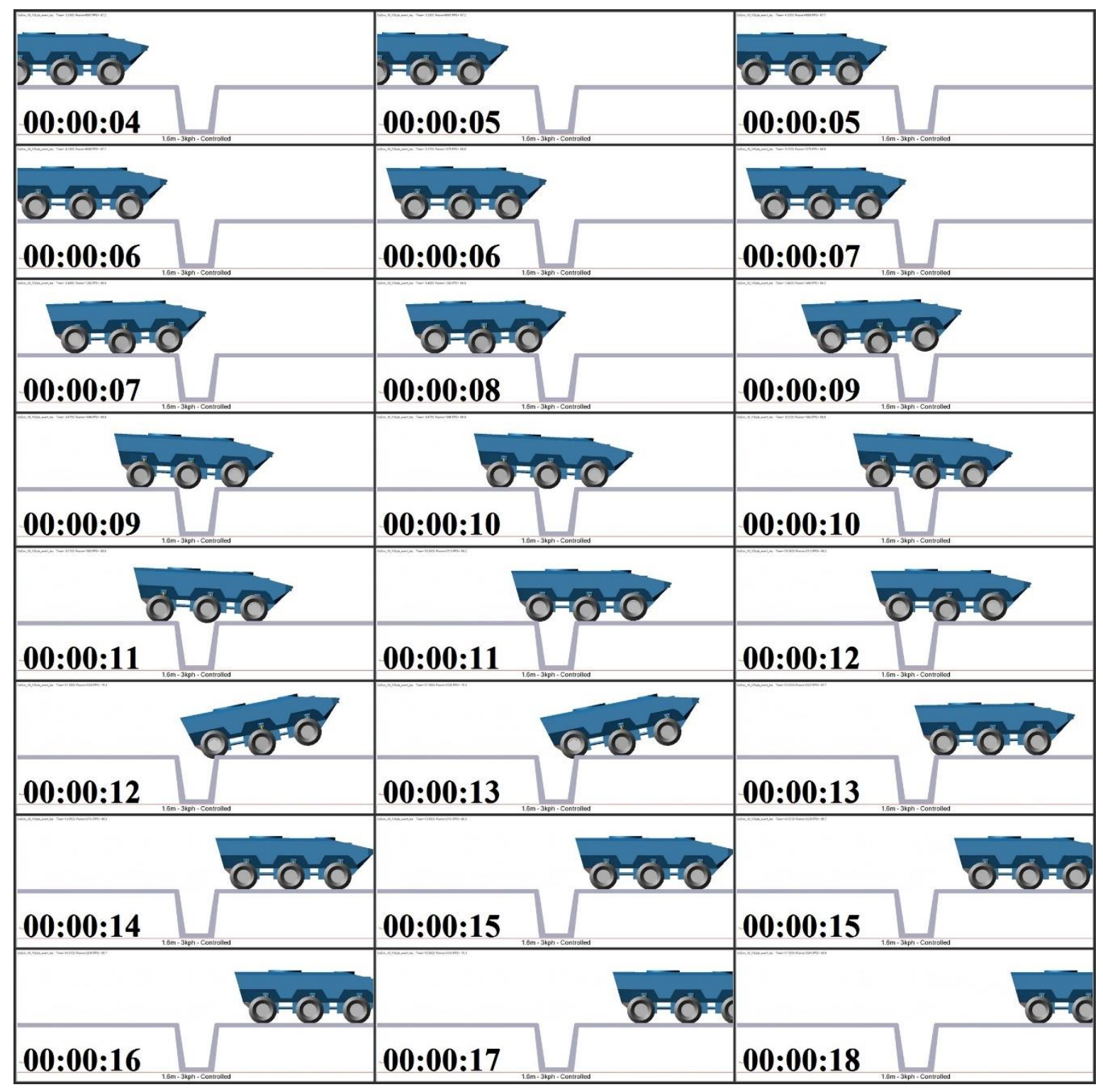

Figure A.53. Trench length $1.6 \mathrm{~m}, 3 \mathrm{kph}$, controlled system 


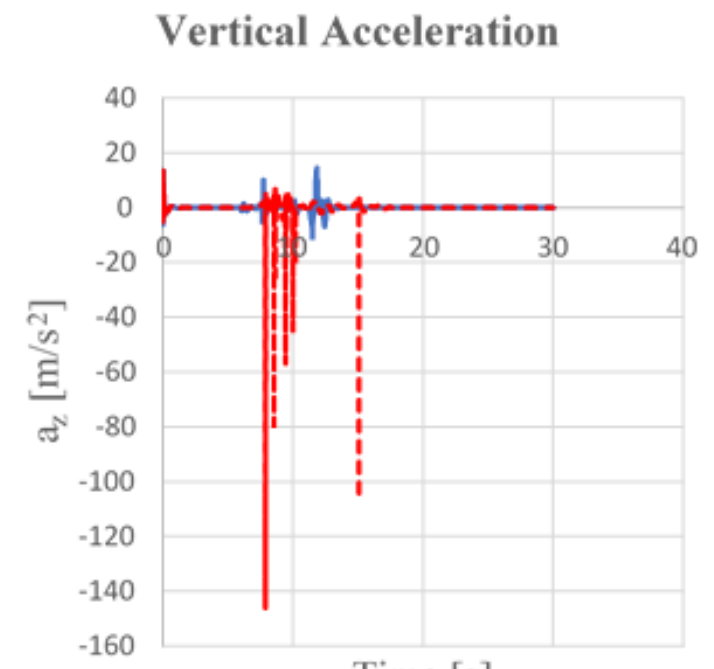

Time $[\mathrm{s}]$

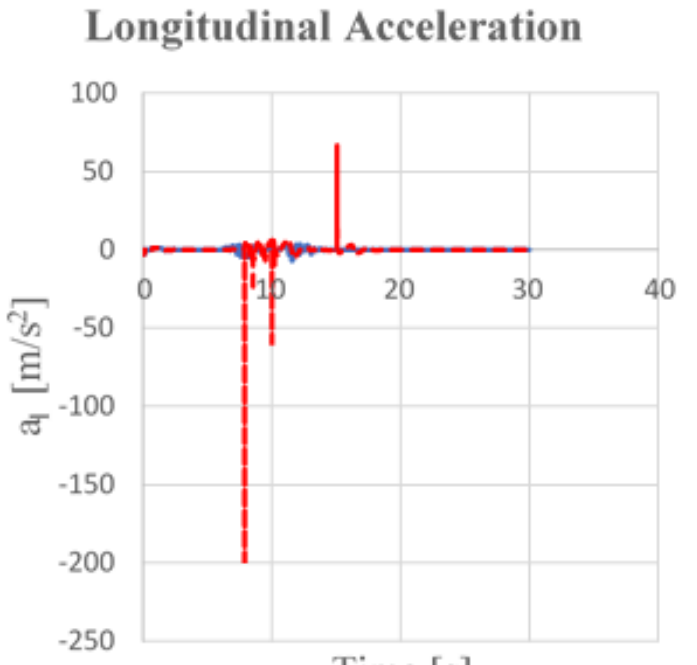

Time $[\mathrm{s}]$

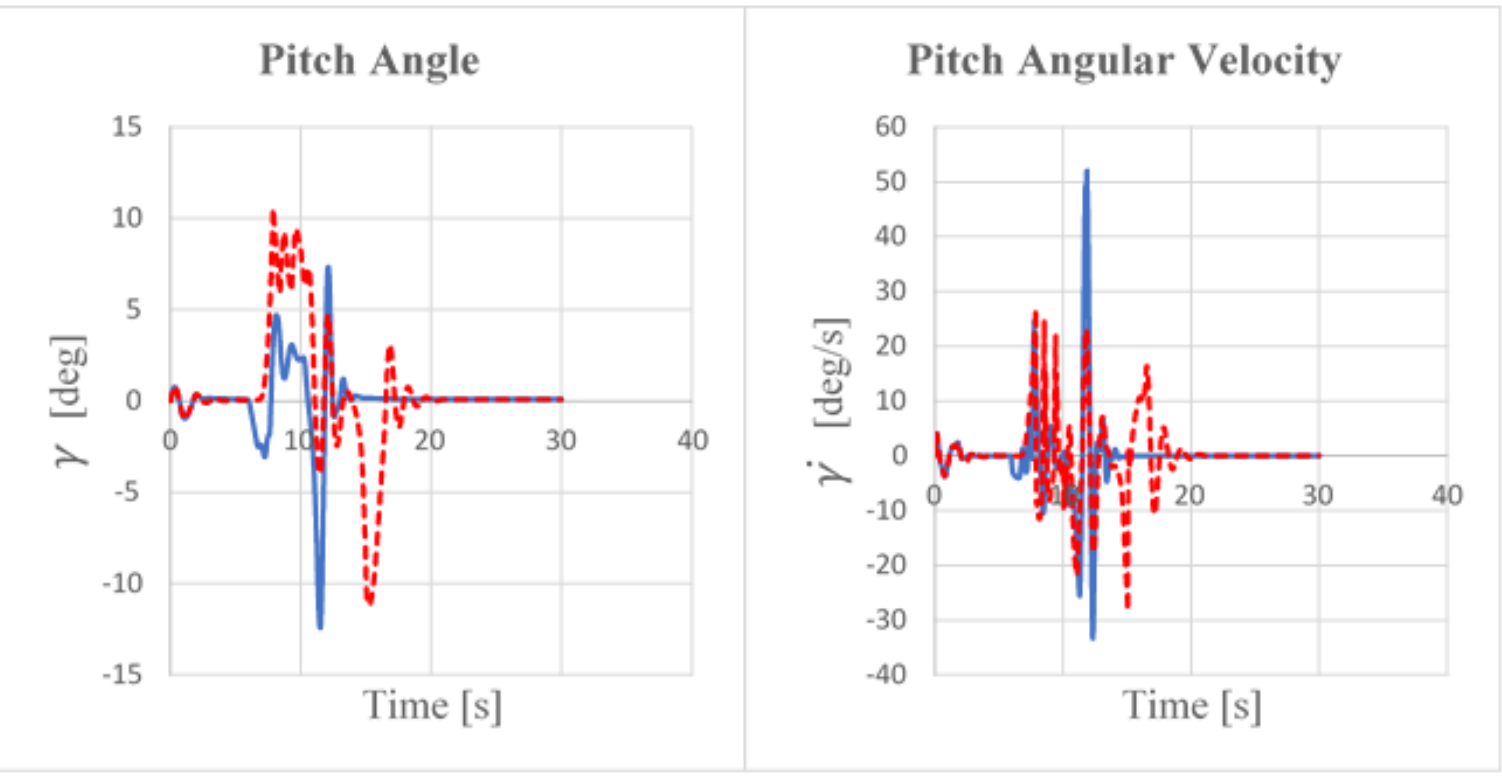

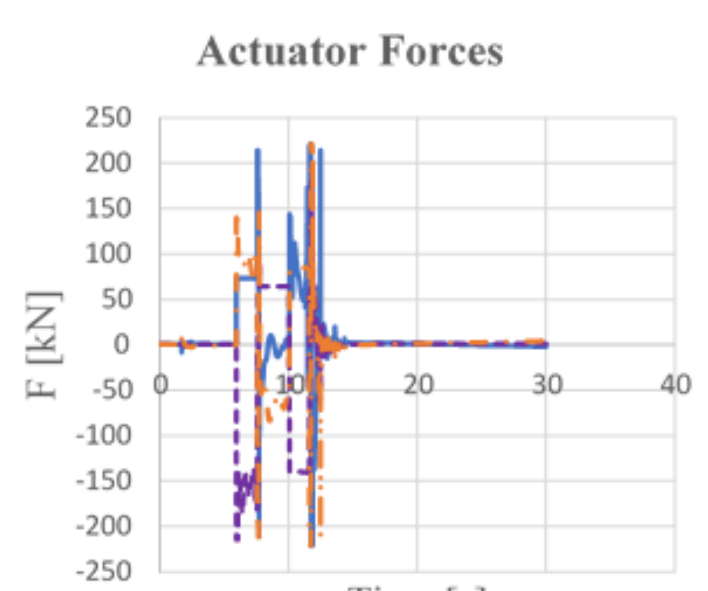

Time [s]

Axle 1 ---A Axle 2 - - Axle 3

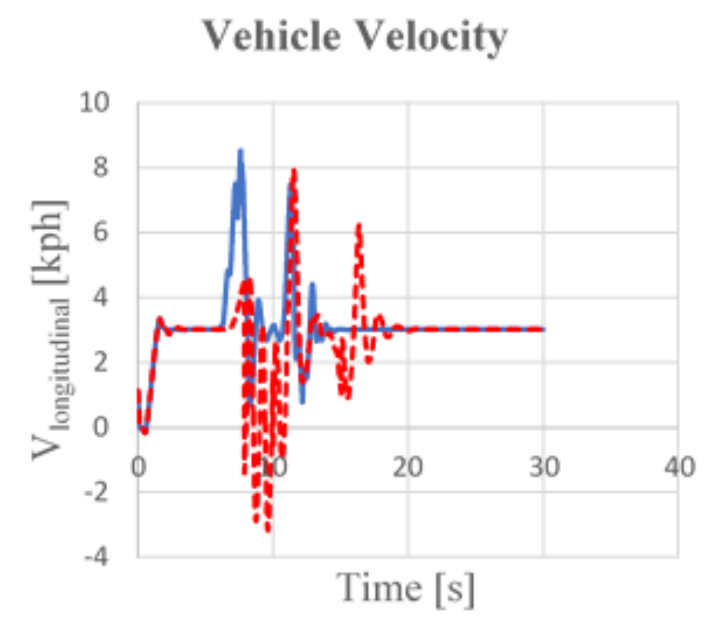

—Controlled -----・ Uncontrolled

Figure A.54. Trench length $1.6 \mathrm{~m}, 3 \mathrm{kph}$, controlled vs uncontrolled output comparison 
Trench Length 1.6 m, 6 kph

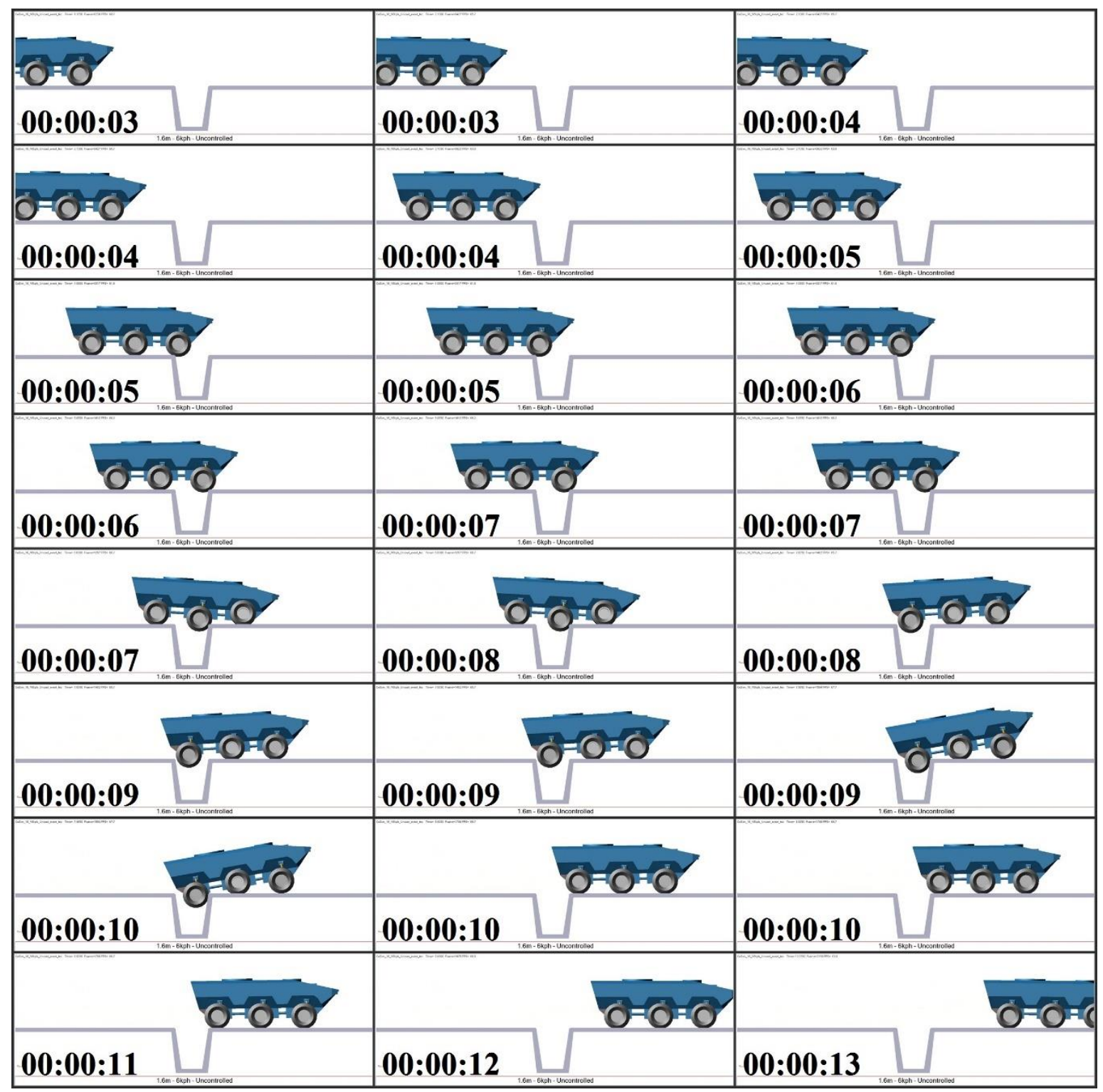

Figure A.55. Trench length $1.6 \mathrm{~m}, 6 \mathrm{kph}$, uncontrolled system 


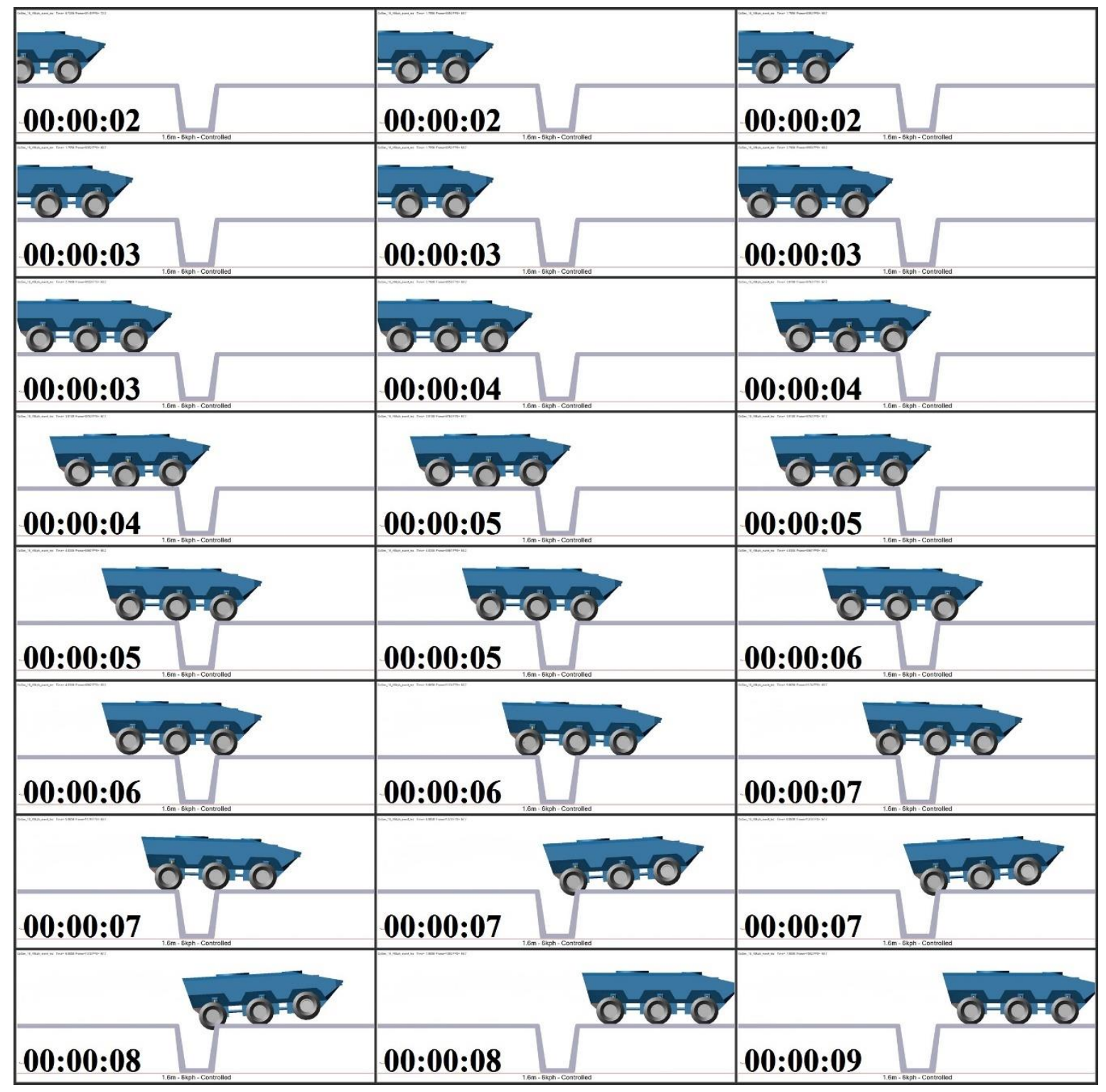

Figure A.56. Trench length $1.6 \mathrm{~m}, 6 \mathrm{kph}$, controlled system 

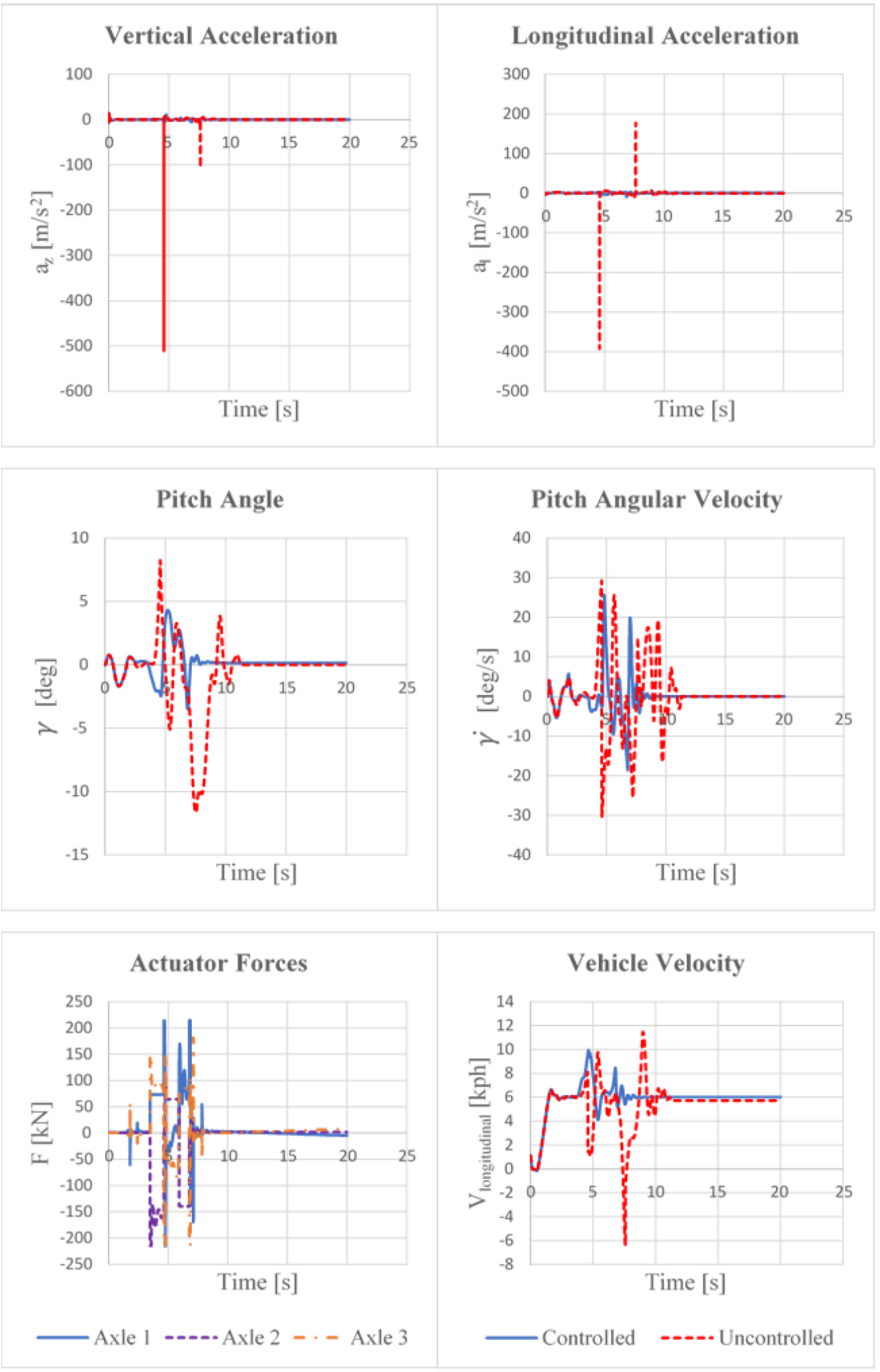

Figure A.57. Trench length $1.6 \mathrm{~m}$, $6 \mathrm{kph}$, controlled vs uncontrolled output comparison 
Trench Length 1.6 m, 9 kph

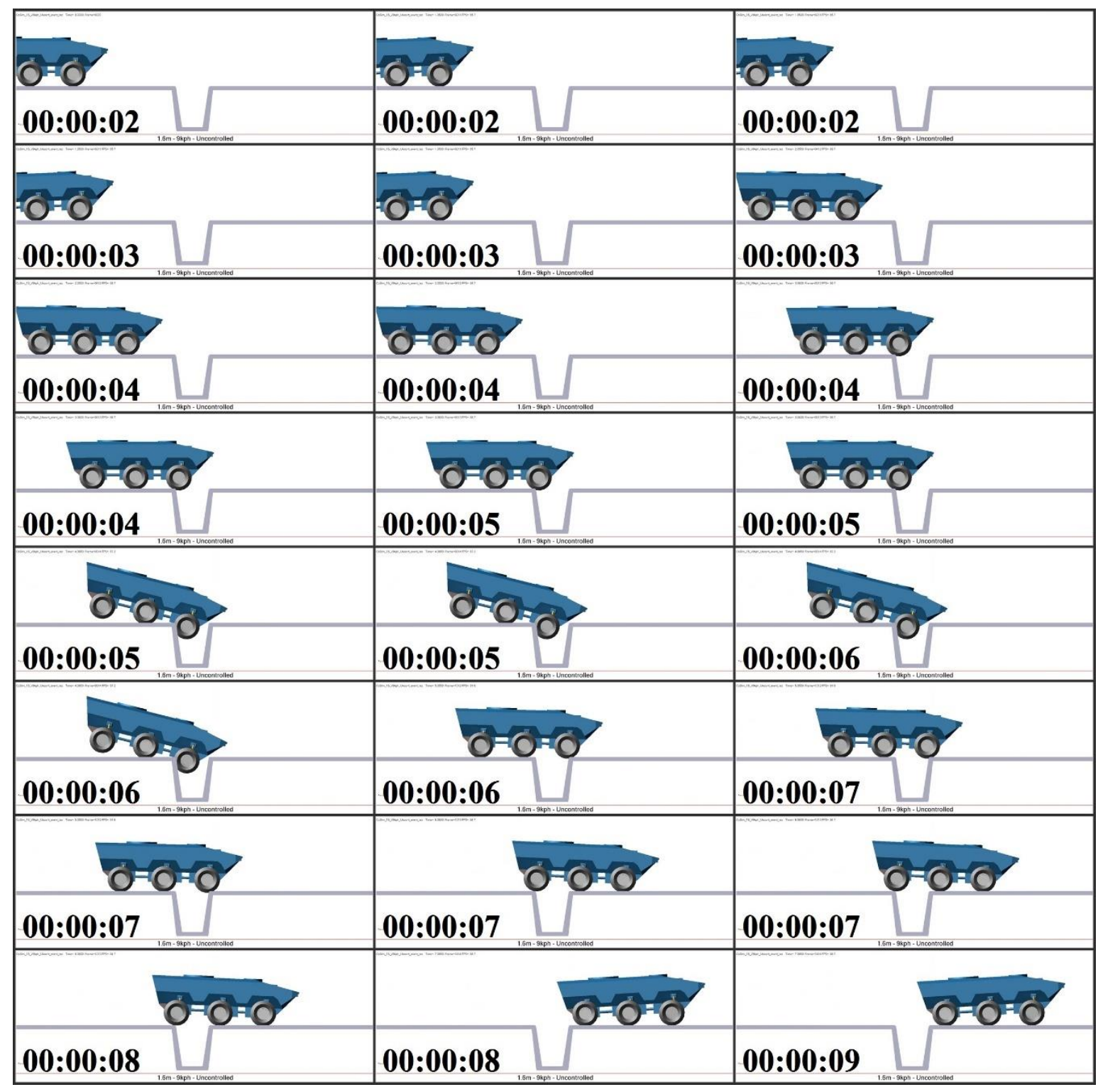

Figure A.58. Trench length $1.6 \mathrm{~m}, 9 \mathrm{kph}$, uncontrolled system 


\begin{tabular}{|c|c|c|}
\hline$a=0$ & $0=0$ & $0=0$ \\
\hline 00:00:01 & 00:00:02 & 00:00:02 \\
\hline$a=0$ & $0=0$ & $0=0$ \\
\hline 00:00:02 & 00:00:02 & 00:00:02 \\
\hline $0=0$ & $0=0$ & $0=0=0$ \\
\hline 00:00:03 & 00:00:03 & 00:00:03 \\
\hline $0=0=0$ & $0=0=0$ & $0=0=0$ \\
\hline 00:00:03 & 00:00:04 & 00:00:04 \\
\hline 00:00:04 & 00:00:04 & 00:00:04 \\
\hline 00:00:05 & 00:00:05 & 00:00:05 \\
\hline 00:00:05 & 00:00:06 & 00:00:06 \\
\hline 00:00:06 & 00:00:06 & 00:00:07 \\
\hline
\end{tabular}

Figure A.59. Trench length $1.6 \mathrm{~m}, 9 \mathrm{kph}$, controlled system 

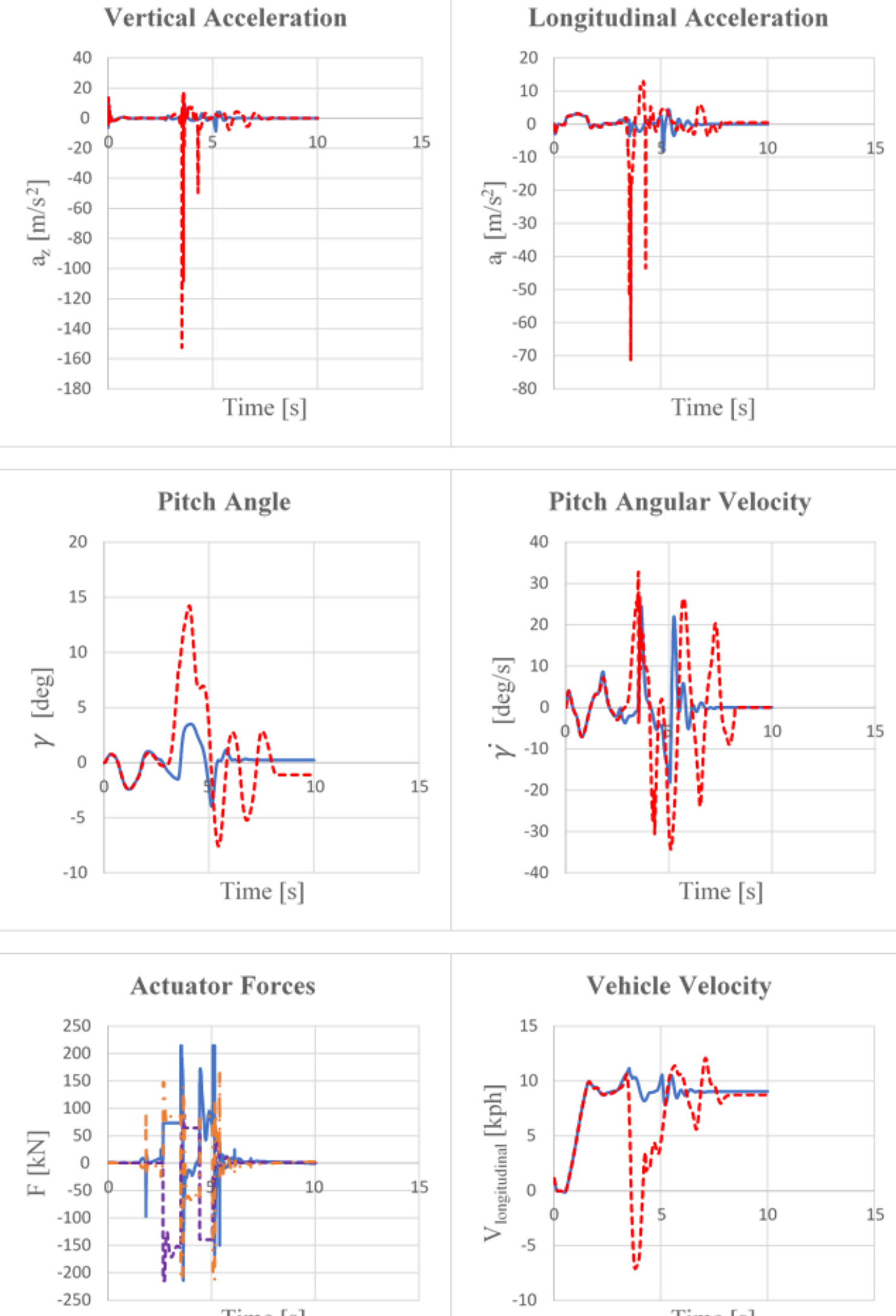

Time [s]

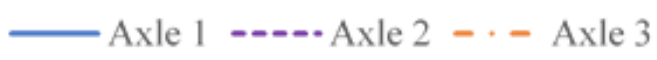

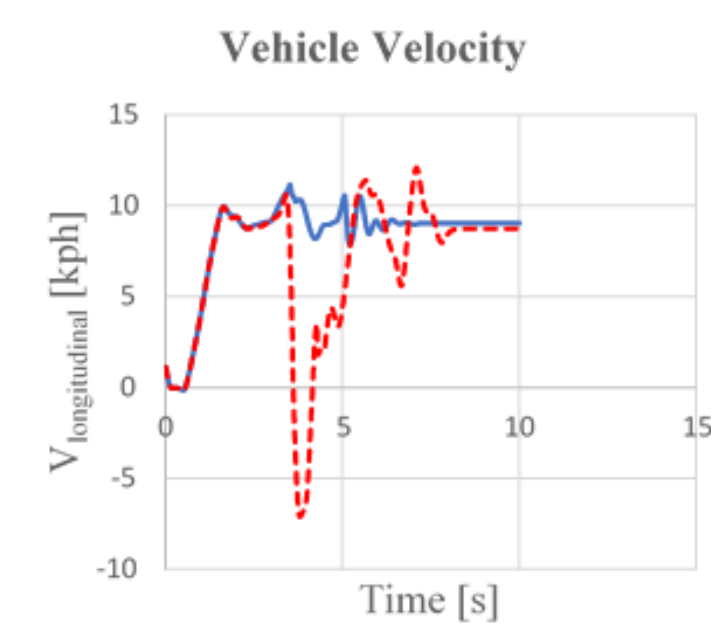

Controlled ----.. Uncontrolled

Figure A.60. Trench length $1.6 \mathrm{~m}, 9 \mathrm{kph}$, controlled vs uncontrolled output comparison 
Trench Length $1.7 \mathrm{~m}$, Controlled vs Uncontrolled System Simulations

Trench Length $1.7 \mathrm{~m}, 1 \mathrm{kph}$

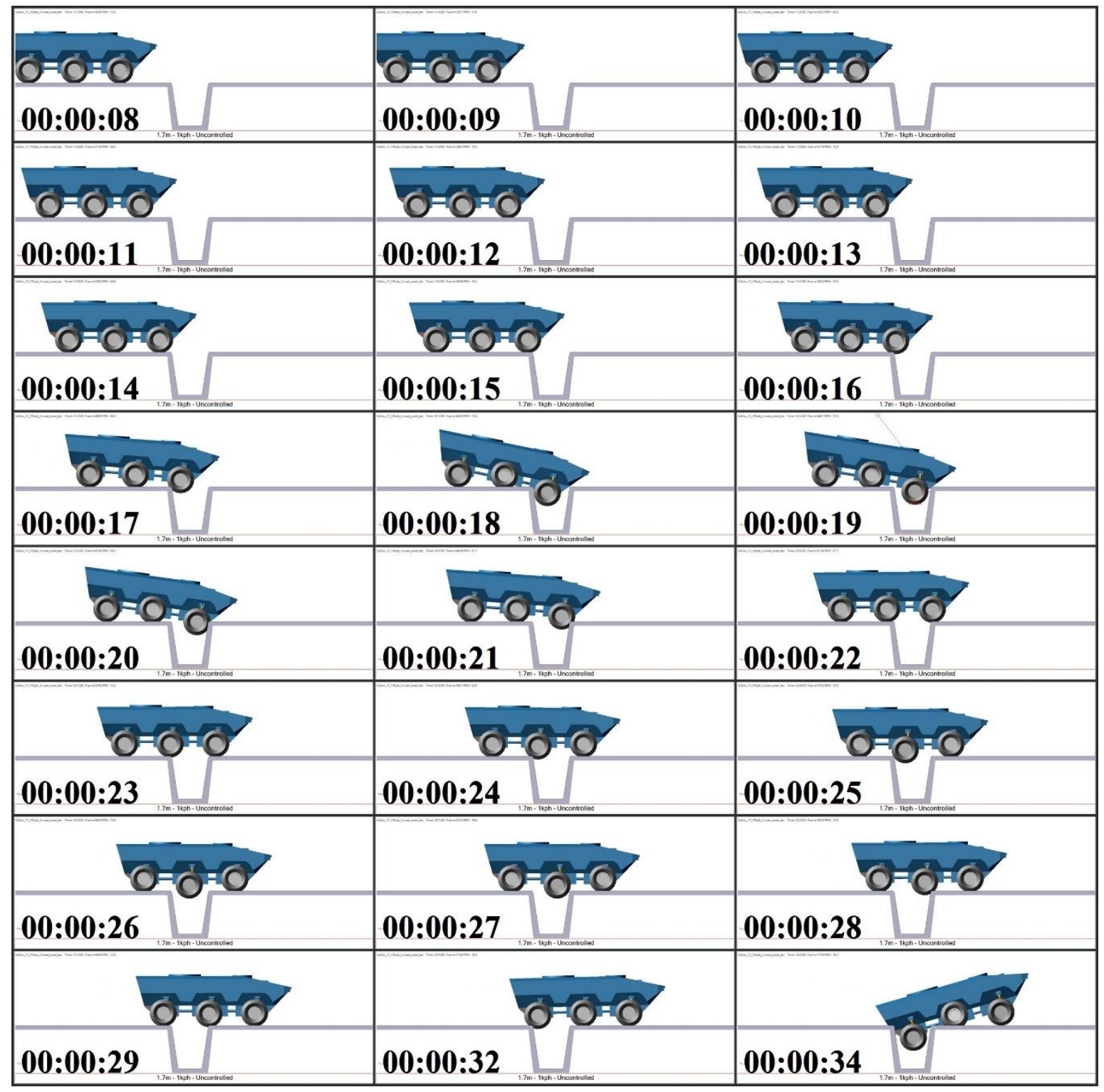

Figure A.61. Trench length $1.7 \mathrm{~m}, 1 \mathrm{kph}$, uncontrolled system 


\begin{tabular}{|c|c|c|}
\hline$=0=0$ & $3=0=0$ & $3=0=0$ \\
\hline 00:00:07 & 00:00:08 & 00:00:09 \\
\hline $0=0$ & $0=0=0$ & $0=0=0$ \\
\hline 00:00:10 & 00:00:11 & 00:00:12 \\
\hline $00 \cdot 00 \cdot 13$ & $00 \cdot 00 \cdot 14$ & $00 \cdot 00 \cdot 14$ \\
\hline & & \\
\hline 00:00:15 & 00:00:16 & 00:00:17 \\
\hline 00:00:18 & 00:00:19 & 00:00:20 \\
\hline 00:00:21 & 00:00:22 & 00:00:23 \\
\hline 00:00:23 & 00:00:24 & 00:00:25 \\
\hline 00:00:26 & 00:00:28 & 00:00:30 \\
\hline
\end{tabular}

Figure A.62. Trench length $1.7 \mathrm{~m}, 1 \mathrm{kph}$, controlled system 

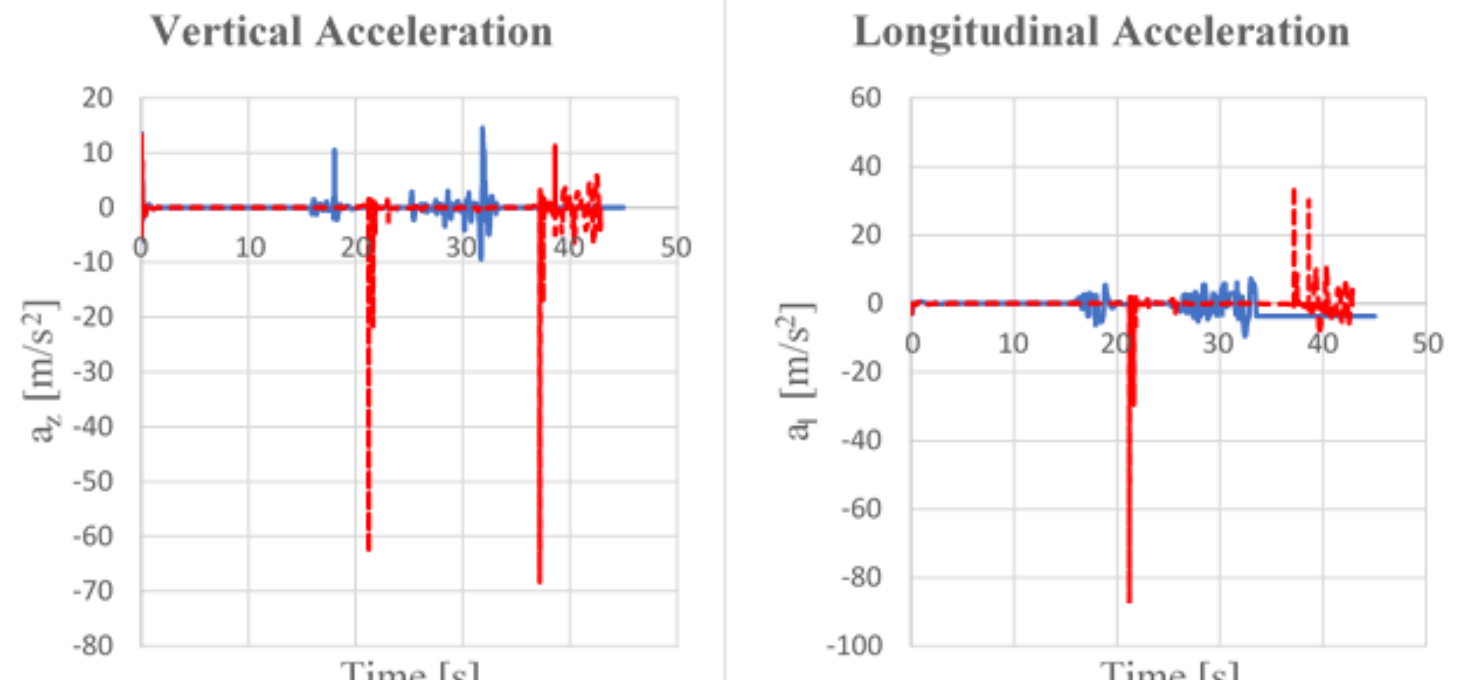

Time [s]

Time [s]

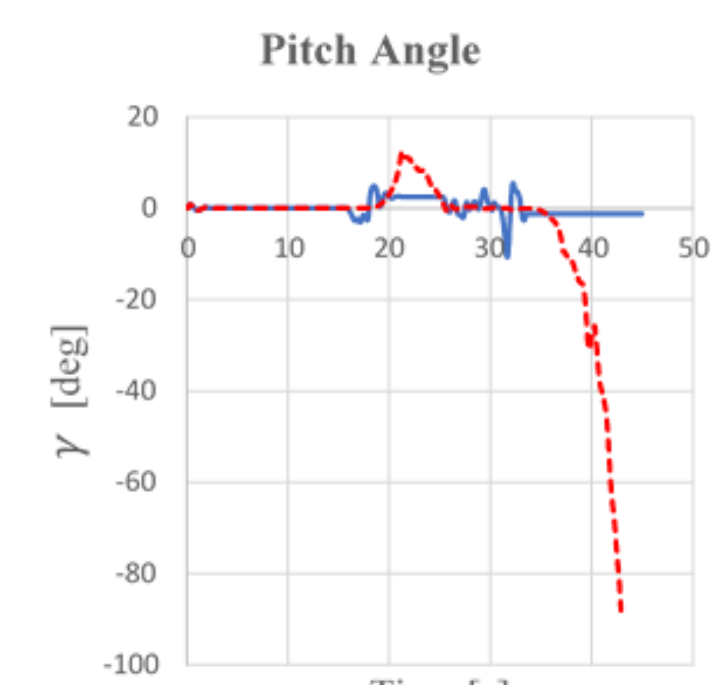

Time $[\mathrm{s}]$

\section{Pitch Angular Velocity}

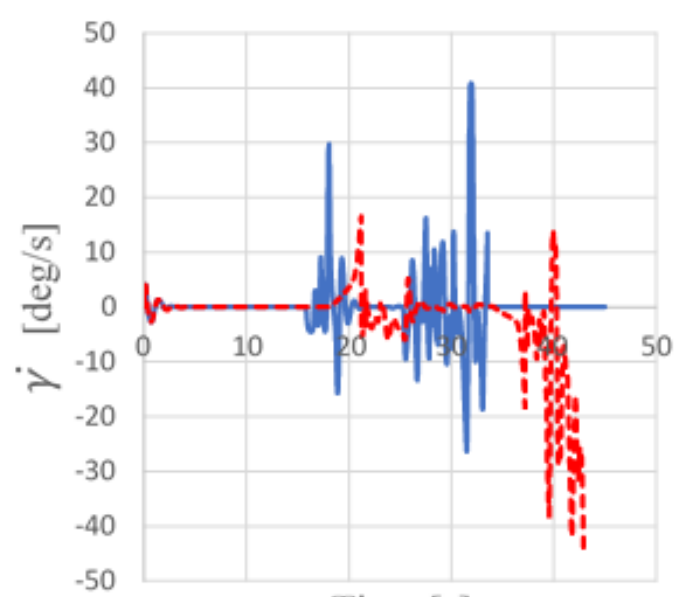

Time $[\mathrm{s}]$

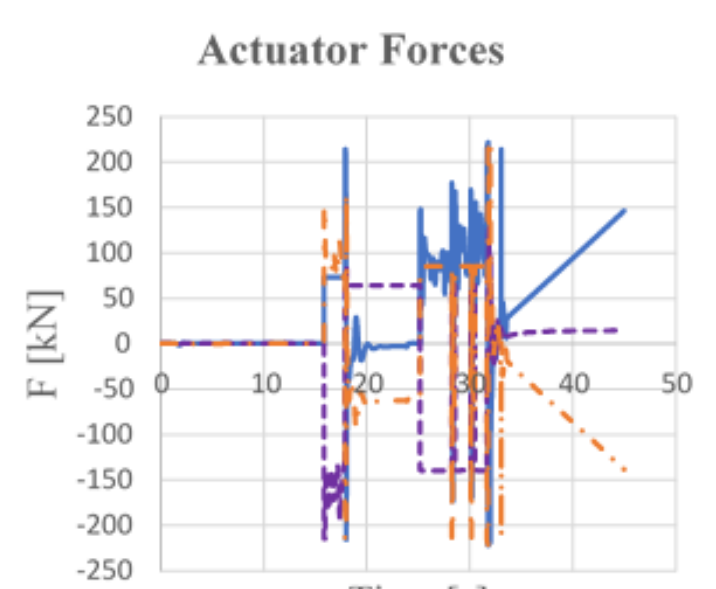

Time [s]

—Axle 1 - - Axle 2 - - Axle 3

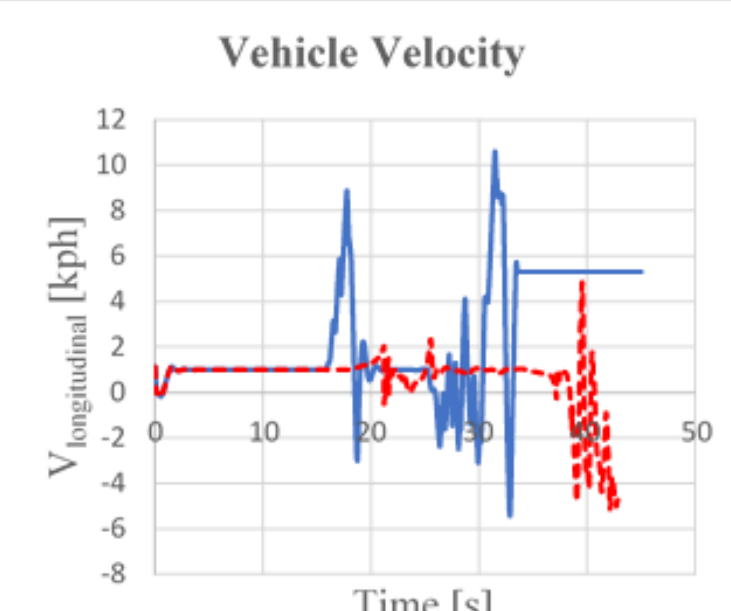

Time [s]

Controlled ----.Uncontrolled

Figure A.63. Trench length $1.7 \mathrm{~m}, 1 \mathrm{kph}$, controlled vs uncontrolled output comparison 
Trench Length 1.7 m, 3 kph

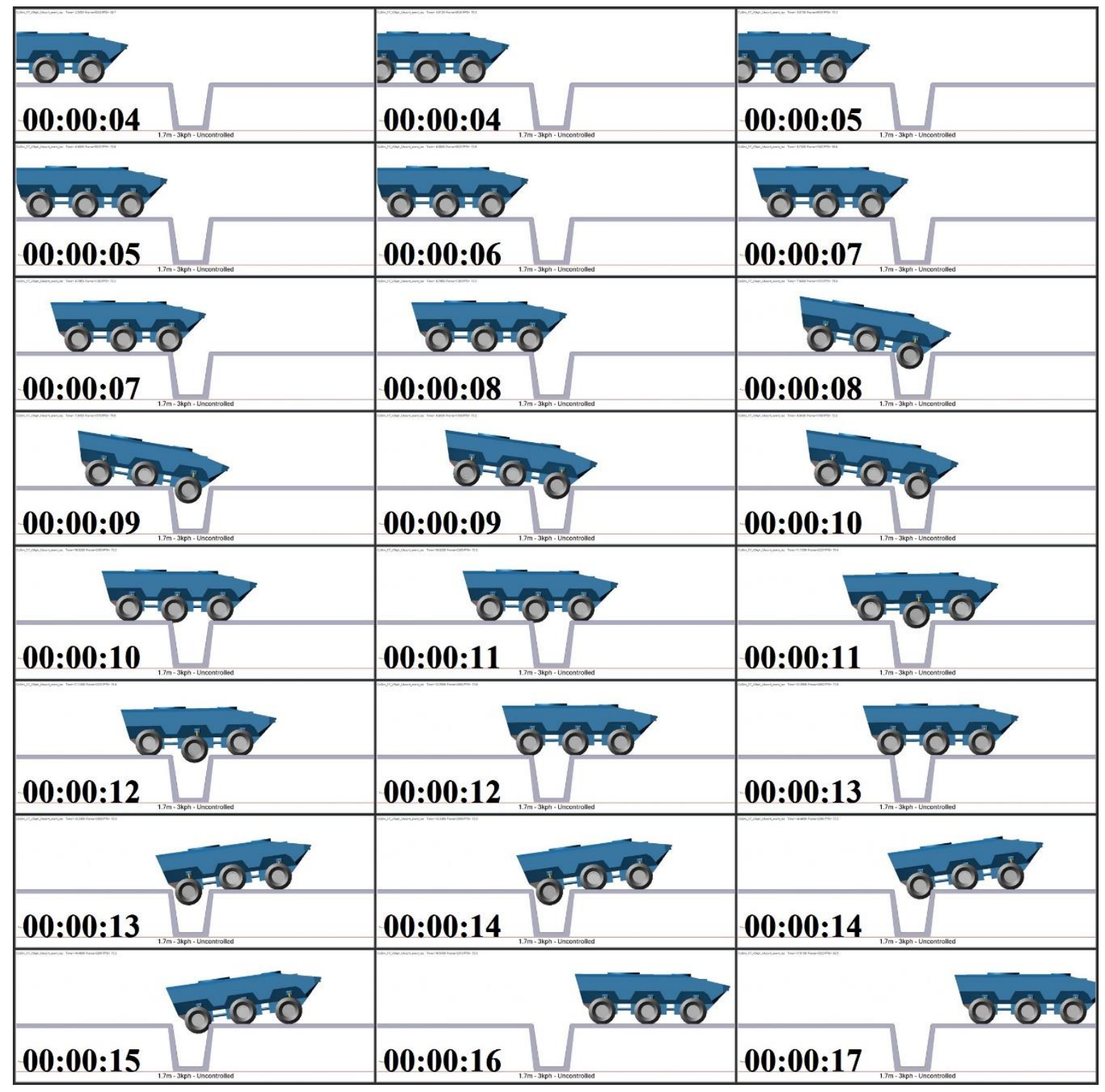

Figure A.64. Trench length $1.7 \mathrm{~m}, 3 \mathrm{kph}$, uncontrolled system 


\begin{tabular}{|c|c|c|}
\hline $0=0$ & $=0=0$ & $=0=0$ \\
\hline 00:00:03 & 00:00:03 & 00:00:04 \\
\hline $0^{-}$ & $b=0=0$ & $0=0=0$ \\
\hline 00:00:04 & 00:00:05 & 00:00:05 \\
\hline $0=0$ & 0 & \\
\hline 00:00:05 & 00:00:06 & 00:00:06 \\
\hline 00:00:07 & 00:00:07 & 00:00:08 \\
\hline 00:00:08 & 00:00:08 & 00:00:09 \\
\hline 00:00:09 & 00:00:10 & 00:00:10 \\
\hline 00:00:10 & 00:00:11 & 00:00:11 \\
\hline 00:00:12 & 00:00:13 & 00:00:14 \\
\hline
\end{tabular}

Figure A.65. Trench length $1.7 \mathrm{~m}, 3 \mathrm{kph}$, controlled system 


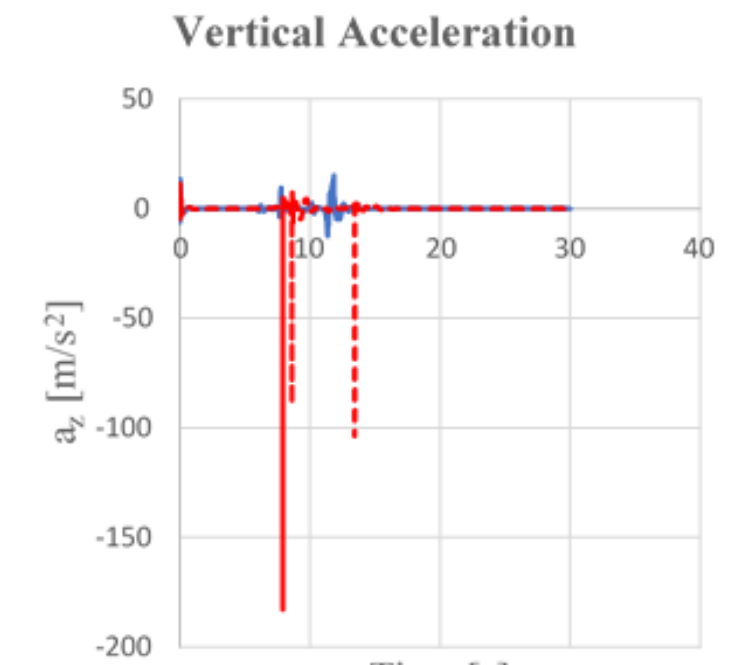

Time $[\mathrm{s}]$

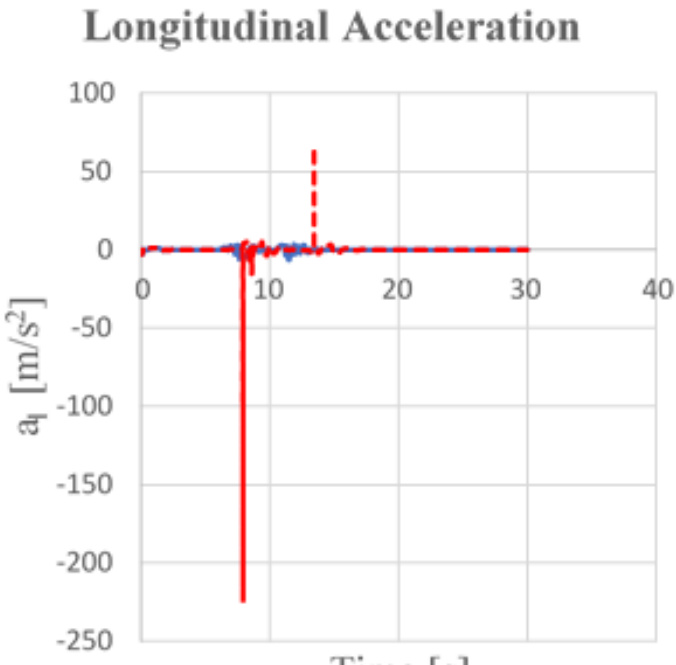

Time $[\mathrm{s}]$

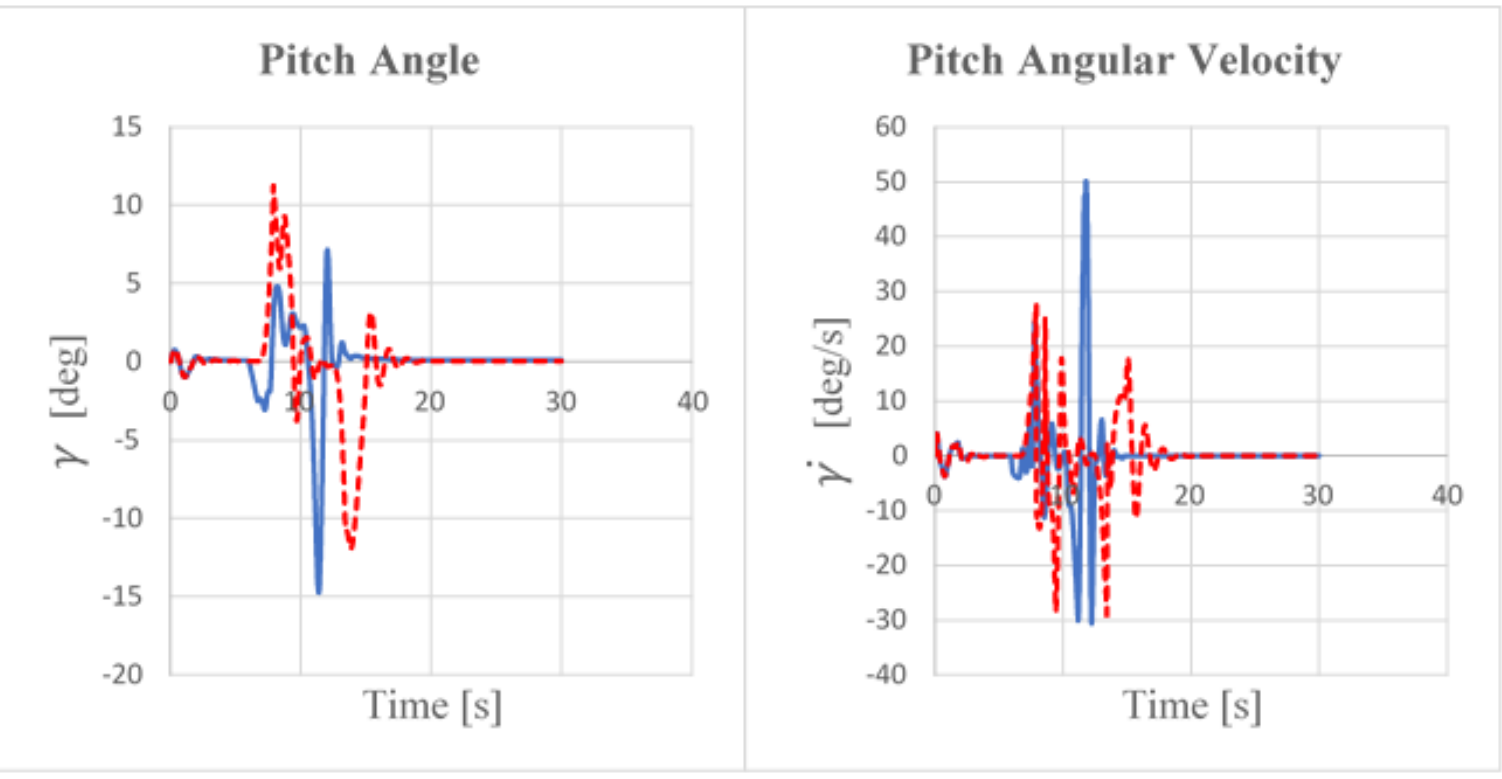

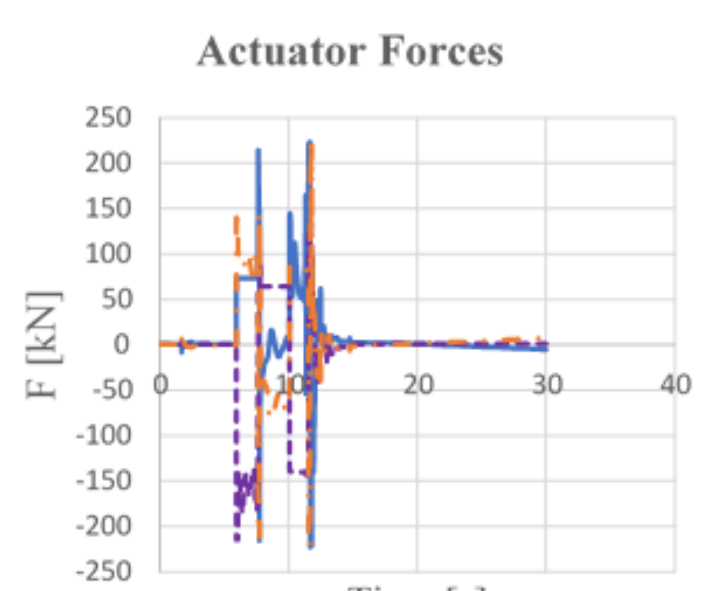

Time [s]

Axle 1 ---A Axle 2 - - Axle 3

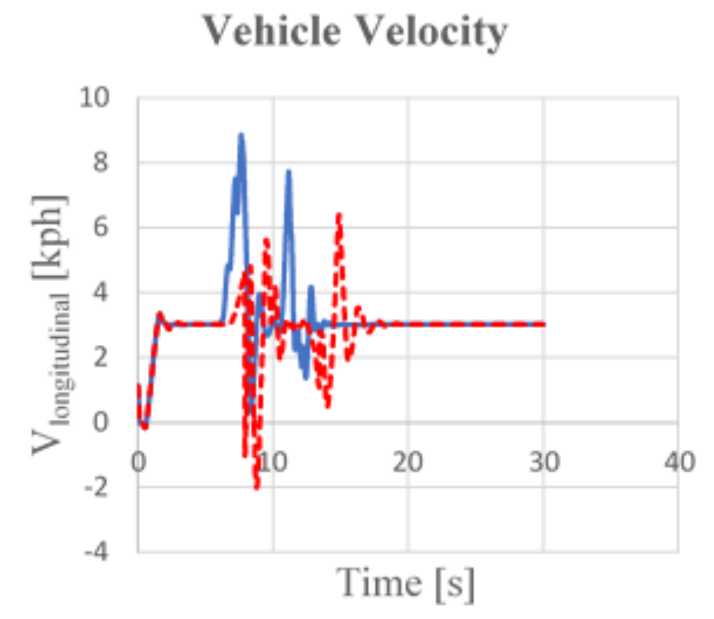

—Controlled -----・ Uncontrolled

Figure A.66. Trench length $1.7 \mathrm{~m}, 3 \mathrm{kph}$, controlled vs uncontrolled output comparison 
Trench Length 1.7 m, 6 kph

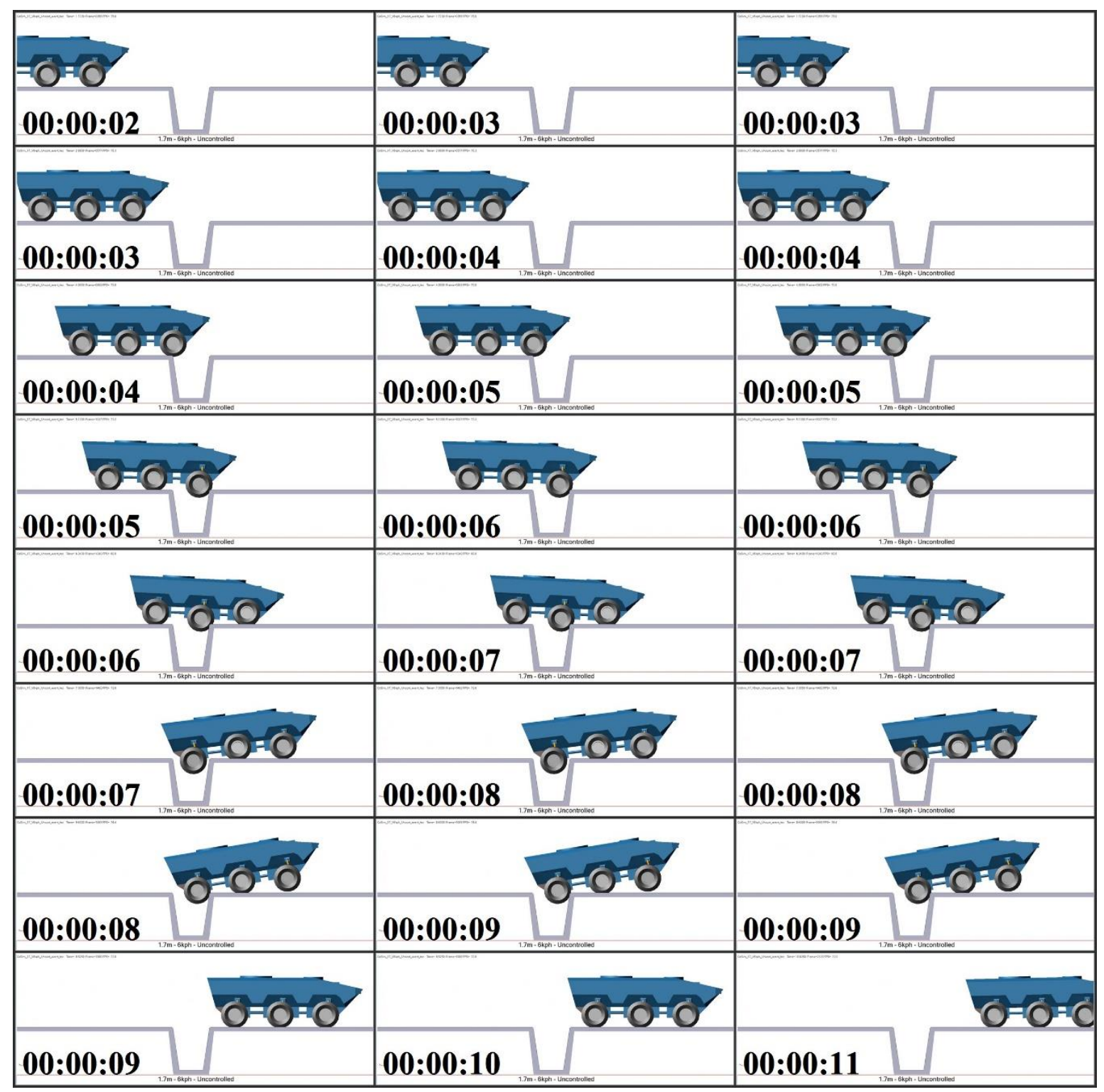

Figure A.67. Trench length $1.7 \mathrm{~m}, 6 \mathrm{kph}$, uncontrolled system 


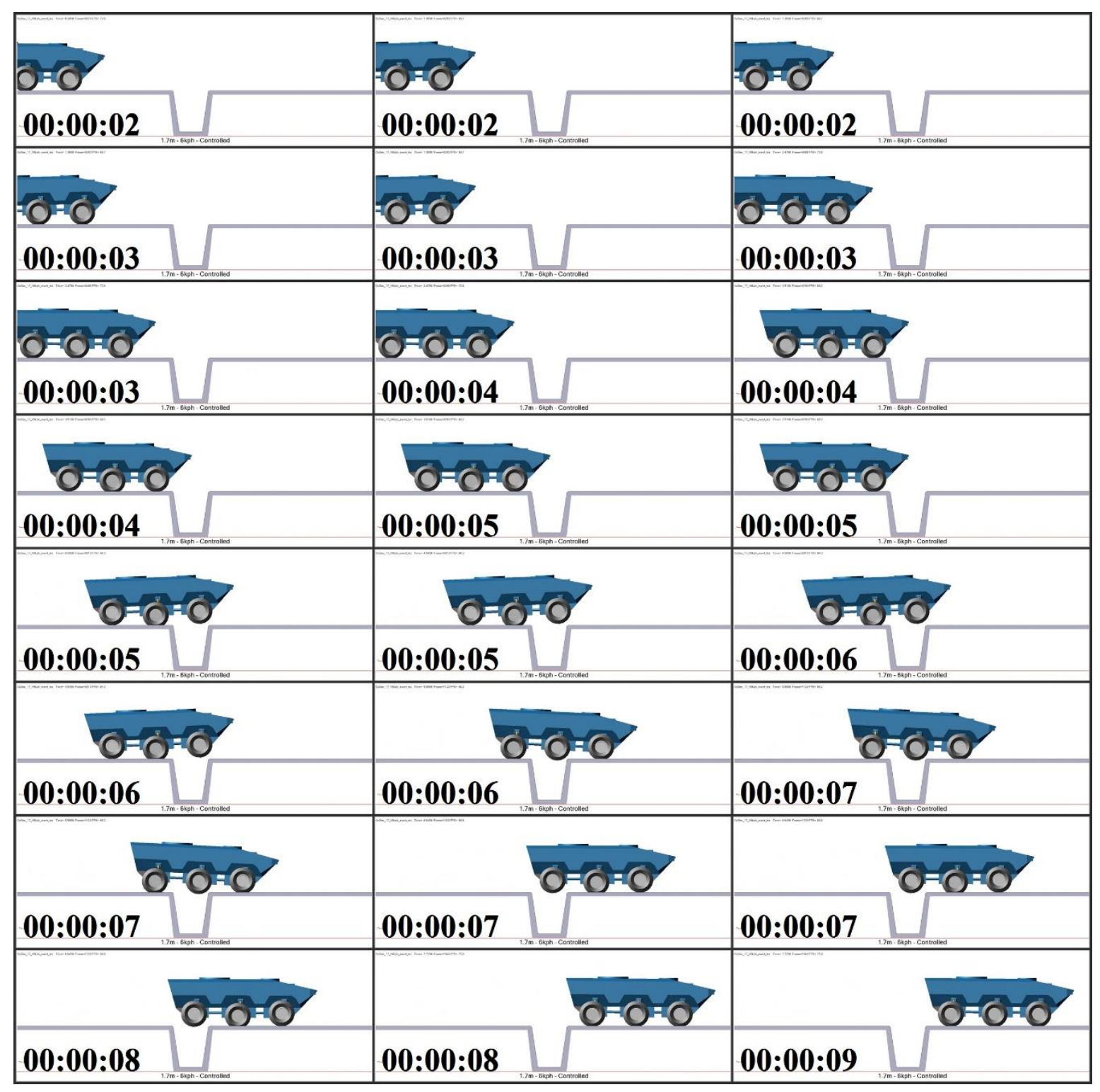

Figure A.68. Trench length $1.7 \mathrm{~m}, 6 \mathrm{kph}$, controlled system 

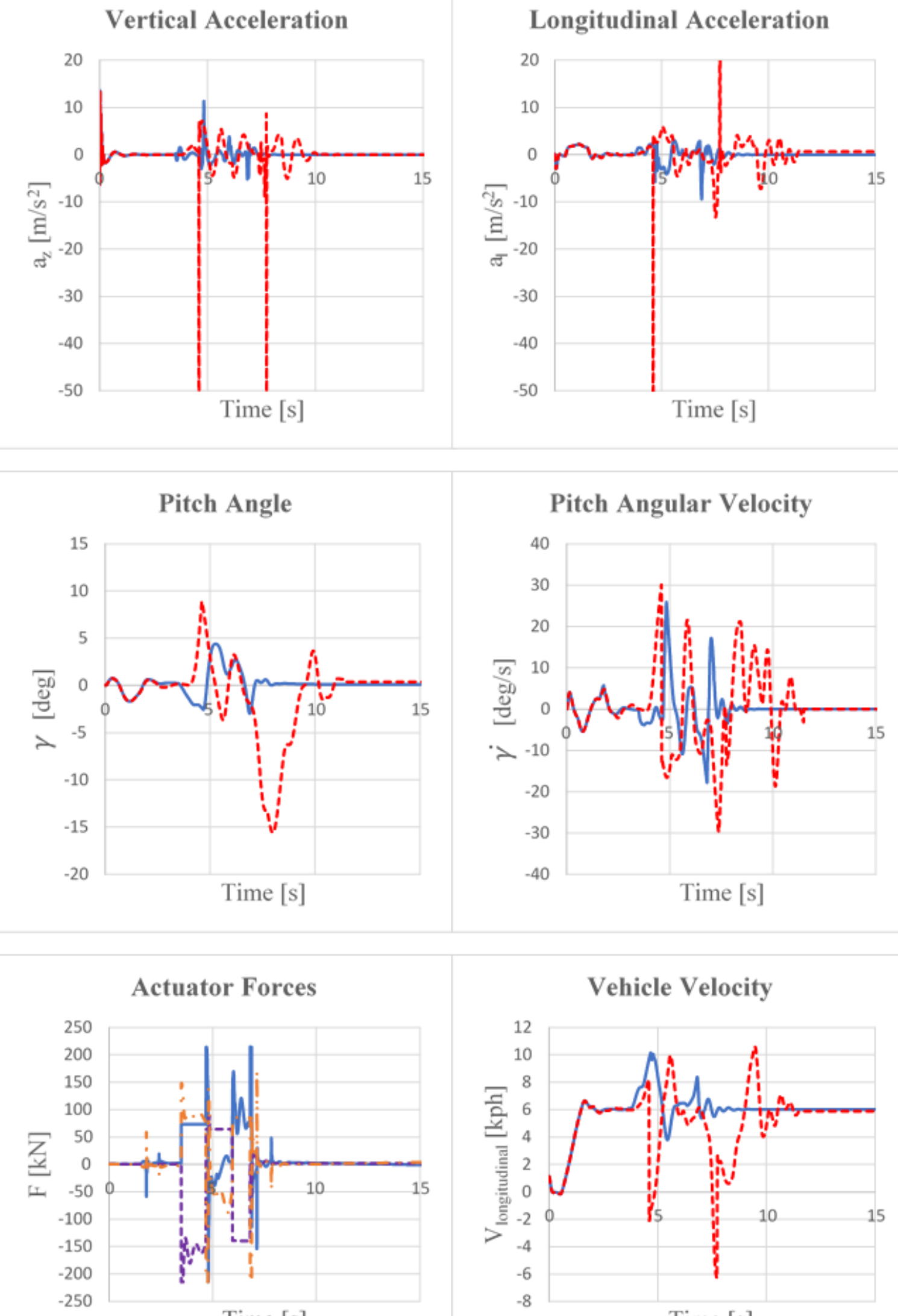

Time $[\mathrm{s}]$
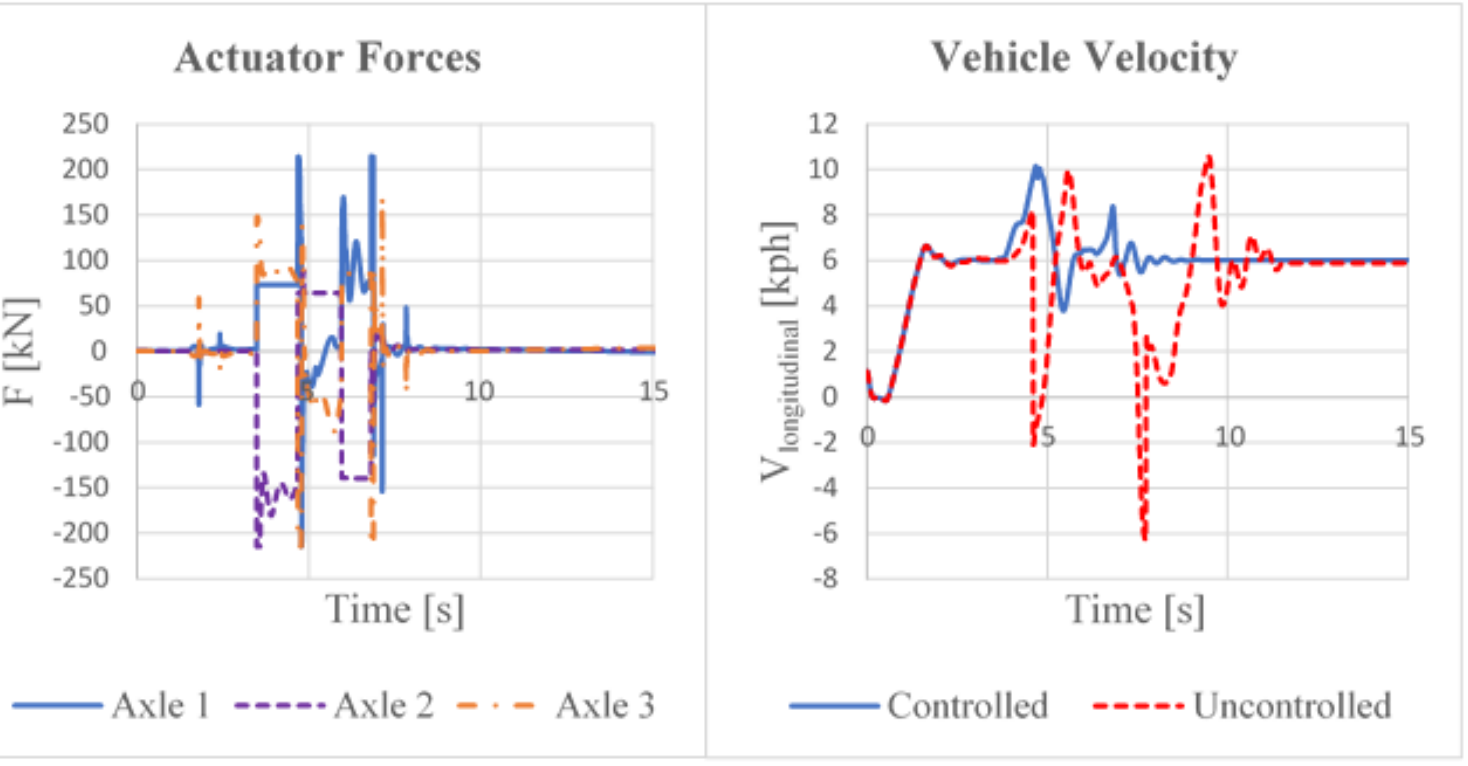

Figure A.69. Trench length $1.7 \mathrm{~m}, 6 \mathrm{kph}$, controlled vs uncontrolled output comparison 
Trench Length 1.7 m, 9 kph

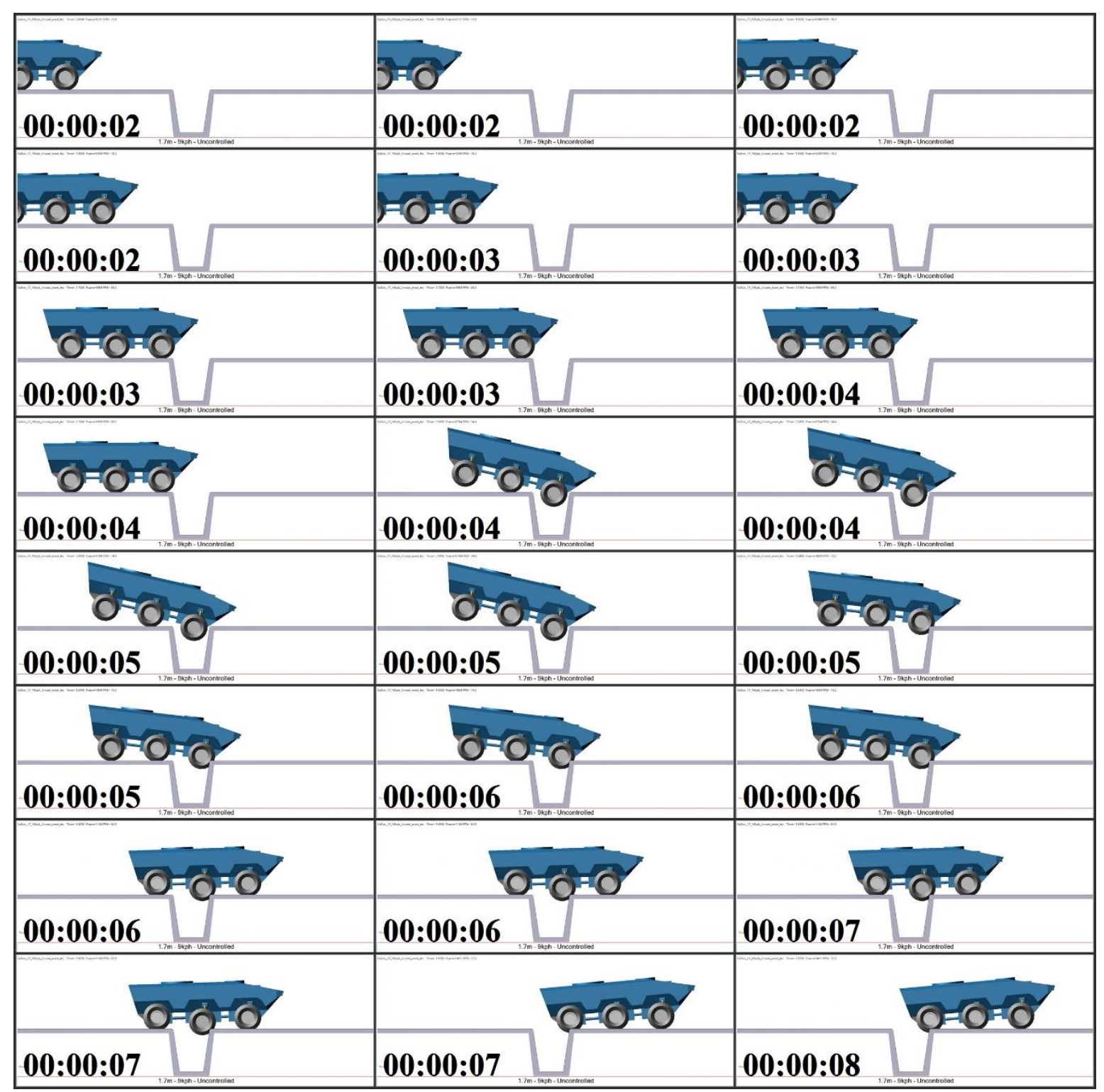

Figure A.70. Trench length $1.7 \mathrm{~m}, 9 \mathrm{kph}$, uncontrolled system 


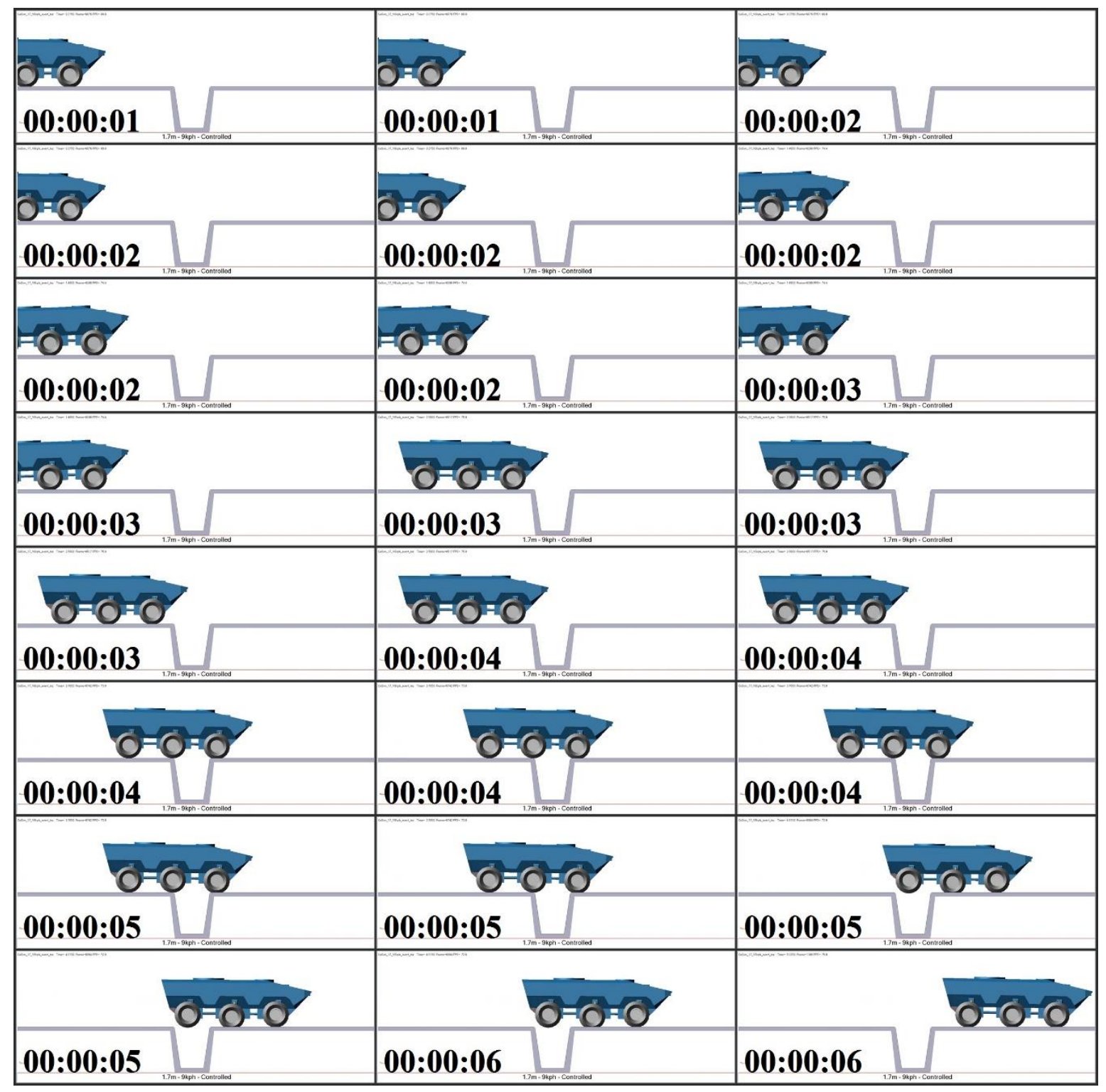

Figure A.71. Trench length $1.7 \mathrm{~m}, 9 \mathrm{kph}$, controlled system 

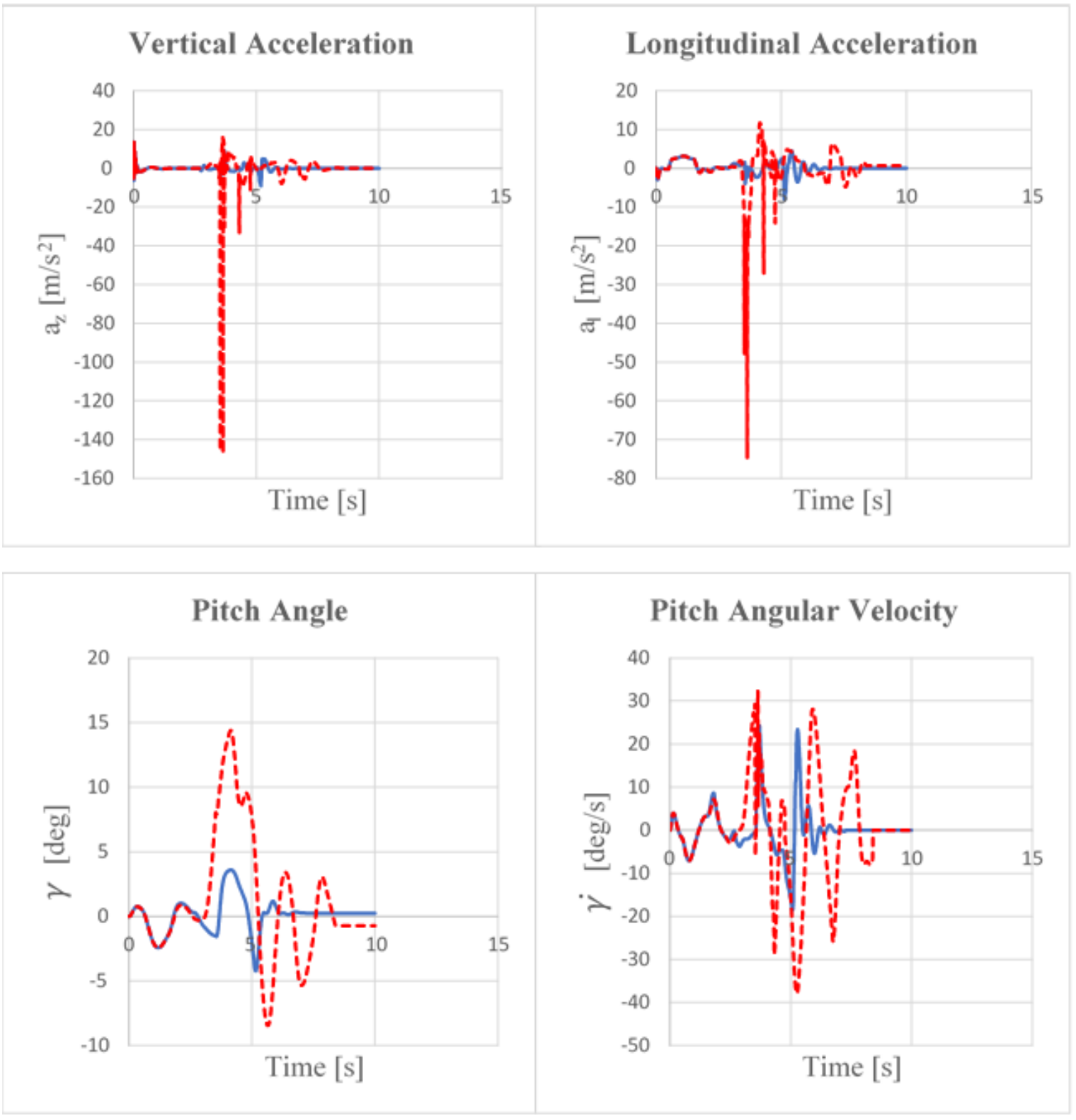

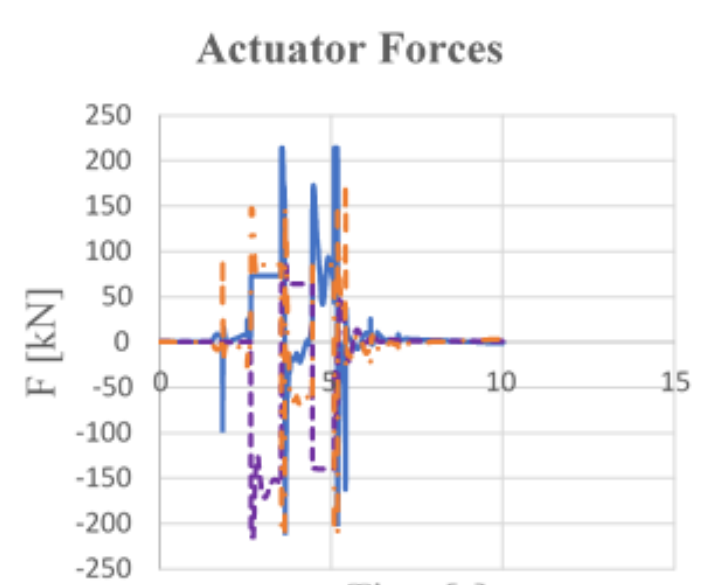

Time [s]

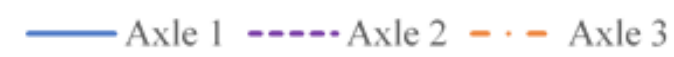

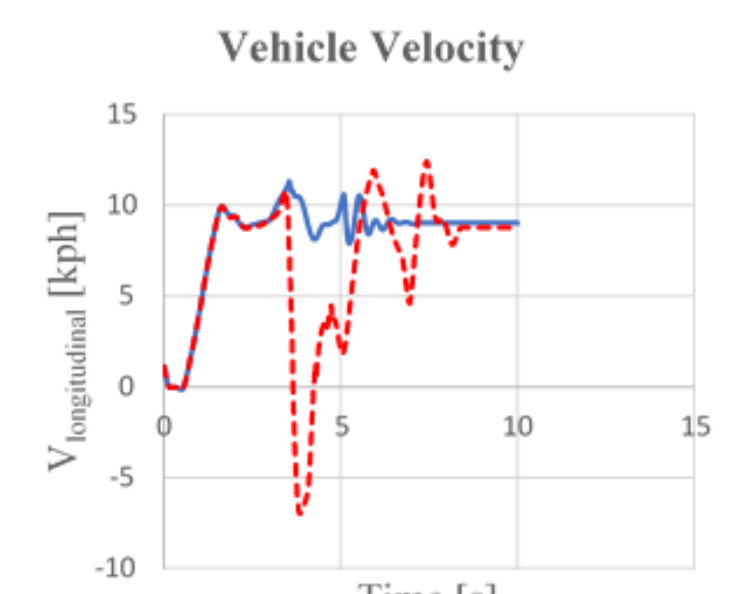

Time [s]

Controlled ----.. Uncontrolled

Figure A.72. Trench length $1.7 \mathrm{~m}, 9 \mathrm{kph}$, controlled vs uncontrolled output comparison 
Trench Length $1.8 \mathrm{~m}$, Controlled vs Uncontrolled System Simulations

Trench Length $1.8 \mathrm{~m}, 1 \mathrm{kph}$

\begin{tabular}{|c|c|c|}
\hline $0=0=0$ & $0=0=0$ & $0=0=0$ \\
\hline 00:00:10 & 00:00:11 & 00:00:12 \\
\hline & & \\
\hline 00:00:13 & 00:00:15 & 00:00:16 \\
\hline 00:00:17 & 00:00:18 & 00:00:19 \\
\hline 00:00:21 & 00:00:22 & 00:00:23 \\
\hline 00:00:24 & 00:00:25 & 00:00:27 \\
\hline 00:00:28 & 00:00:29 & 00:00:30 \\
\hline 00:00:31 & 00:00:33 & 00:00:34 \\
\hline 00:00:35 & 00:00:38 & 00:00:41 \\
\hline
\end{tabular}

Figure A.73. Trench length $1.8 \mathrm{~m}, 1 \mathrm{kph}$, uncontrolled system 


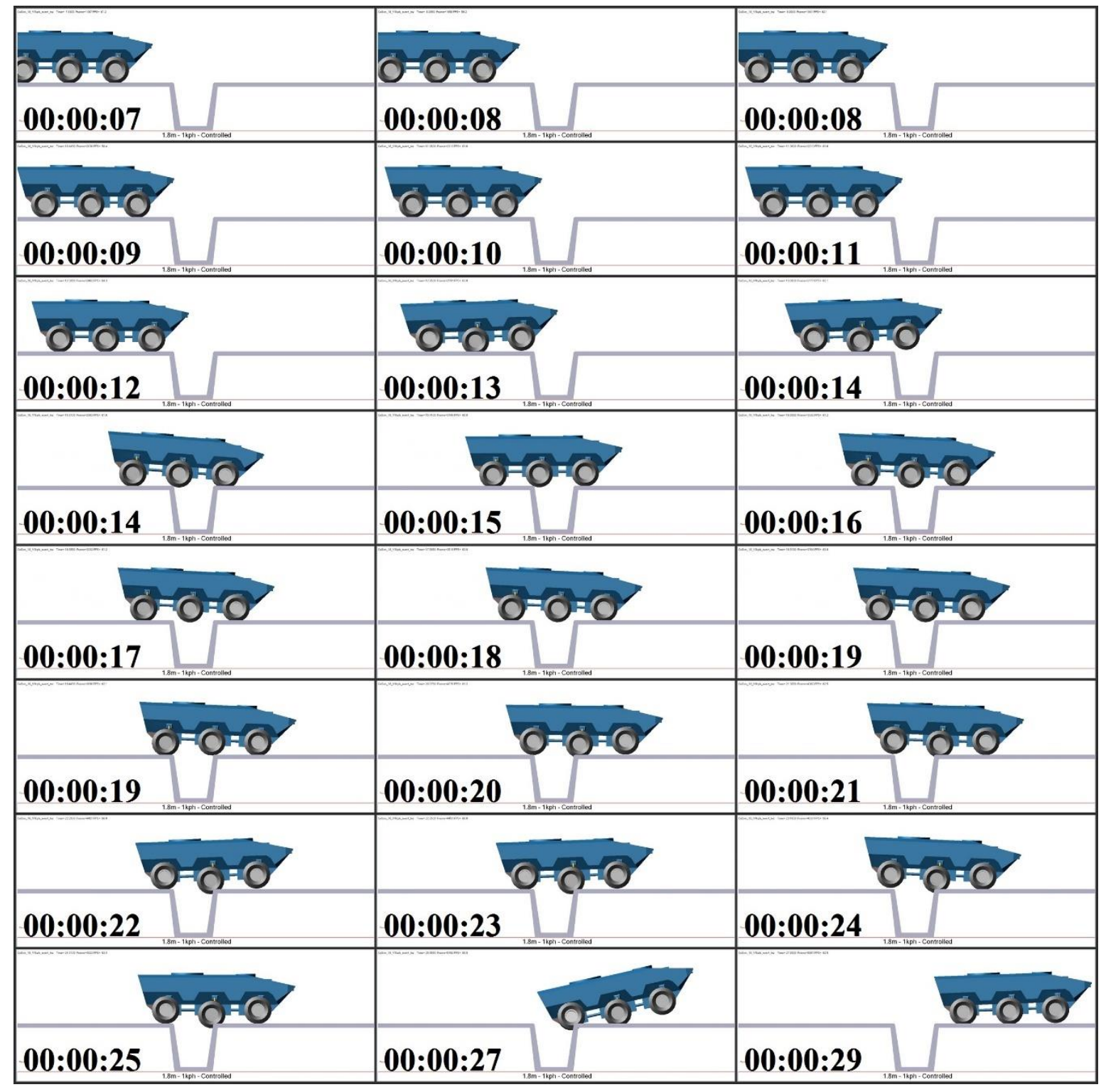

Figure A.74. Trench length $1.8 \mathrm{~m}, 1 \mathrm{kph}$, controlled system 

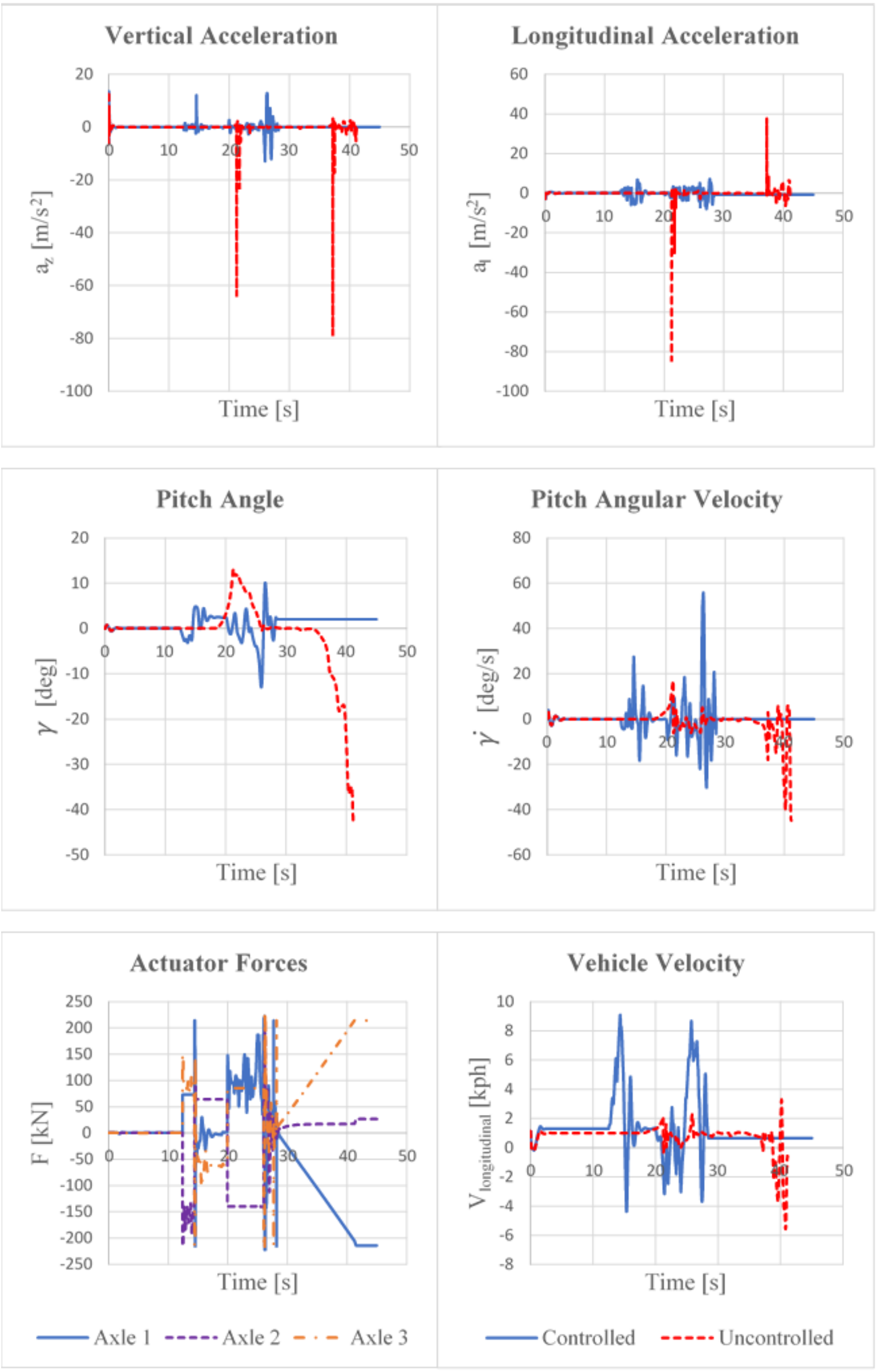

Figure A.75. Trench length $1.8 \mathrm{~m}, 1 \mathrm{kph}$, controlled vs uncontrolled output comparison 
Trench Length 1.8 m, 3 kph

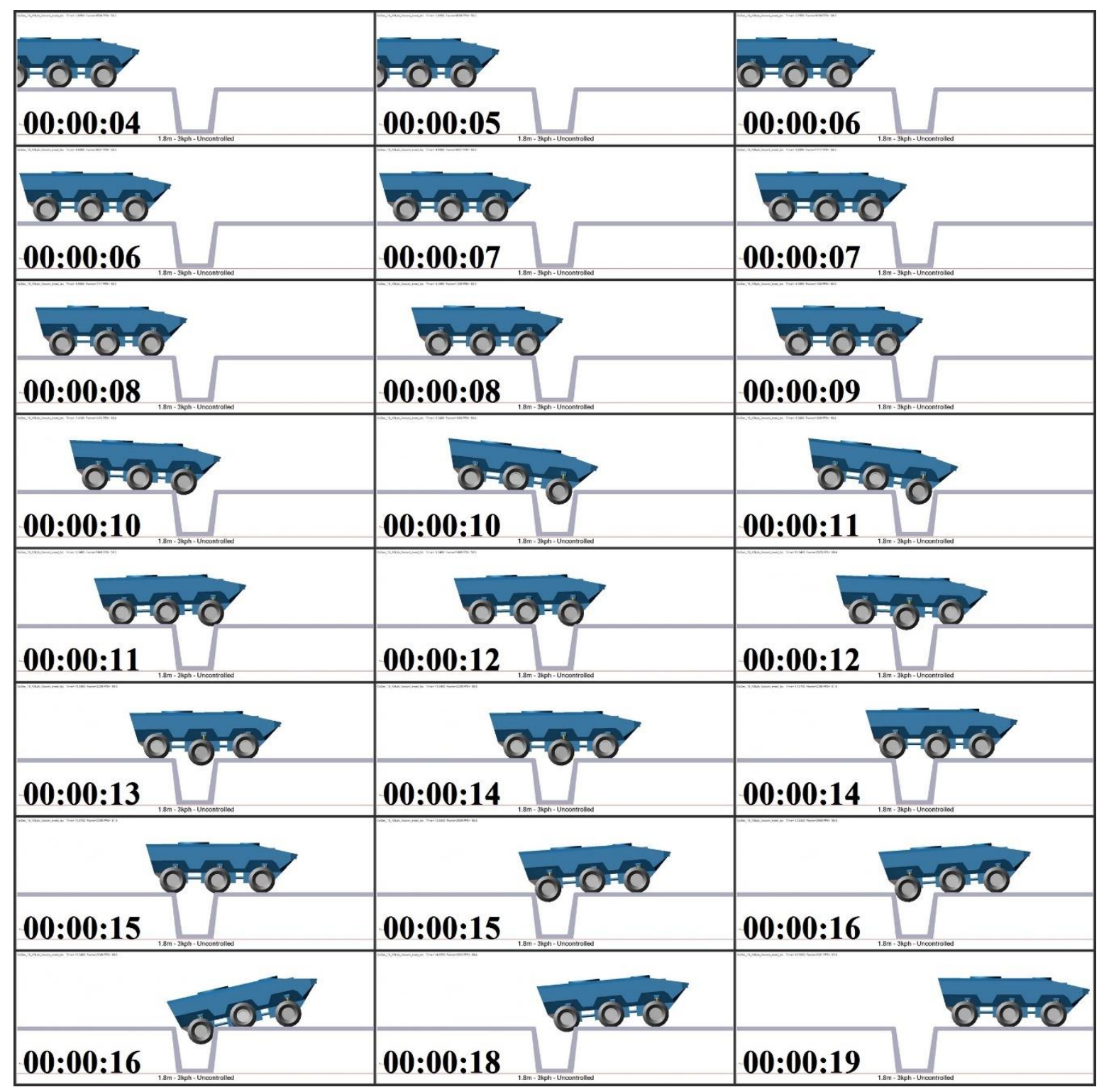

Figure A.76. Trench length $1.8 \mathrm{~m}, 3 \mathrm{kph}$, uncontrolled system 


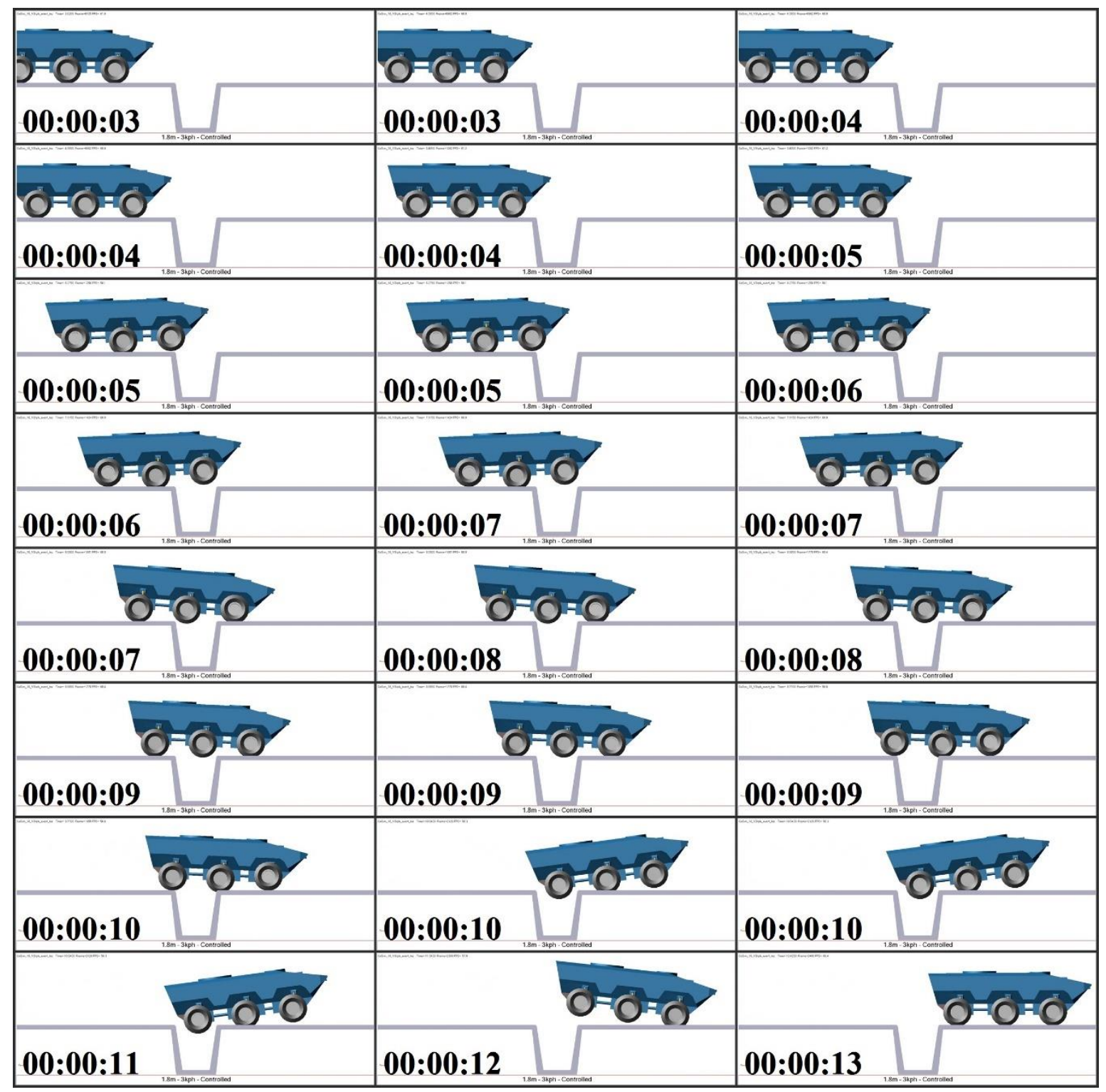

Figure A.77. Trench length $1.8 \mathrm{~m}, 3 \mathrm{kph}$, controlled system 

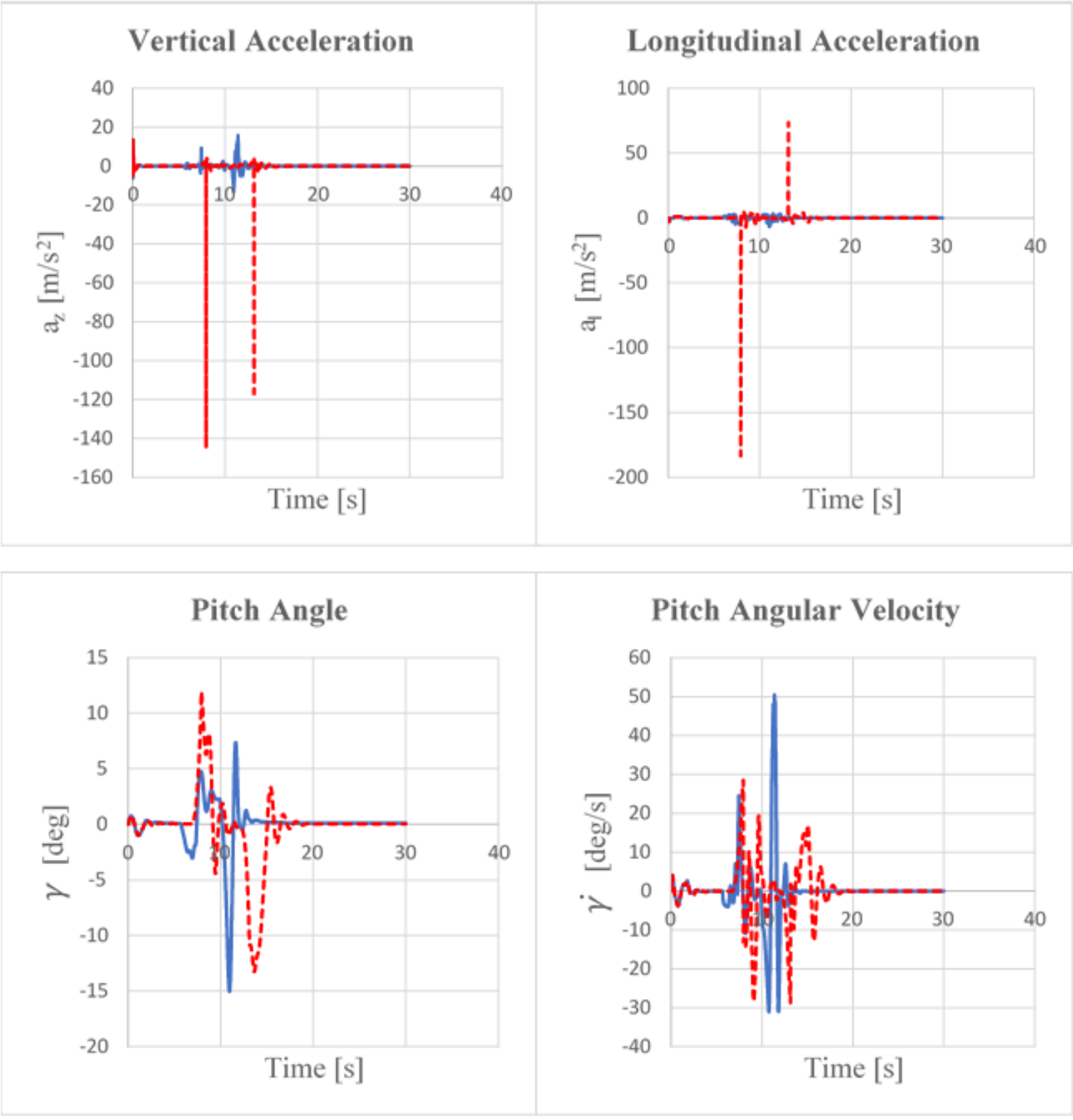

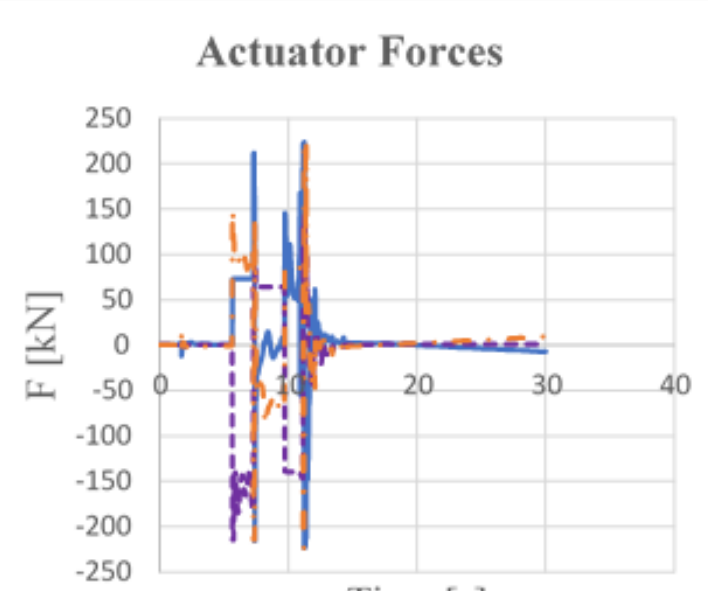

Time $[\mathrm{s}]$

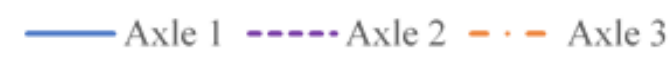

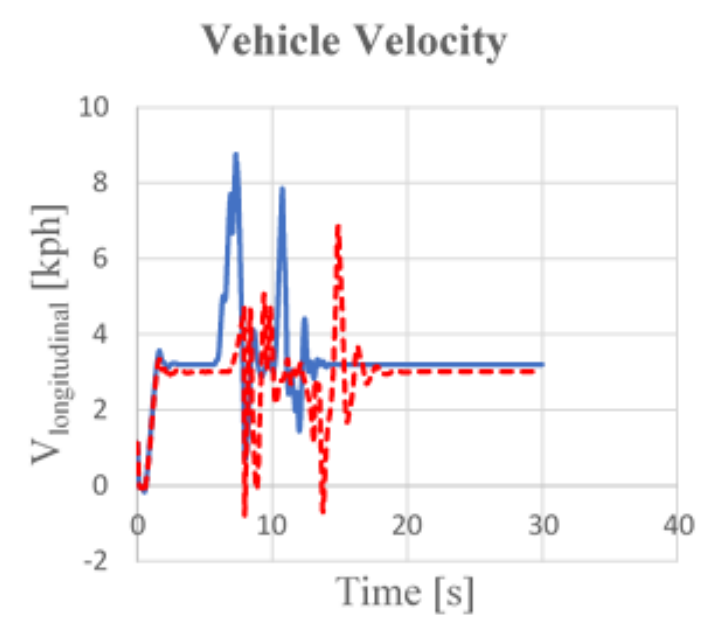

Controlled ----.. Uncontrolled

Figure A.78. Trench length $1.8 \mathrm{~m}, 3 \mathrm{kph}$, controlled vs uncontrolled output comparison 
Trench Length $1.8 \mathrm{~m}, 6 \mathrm{kph}$

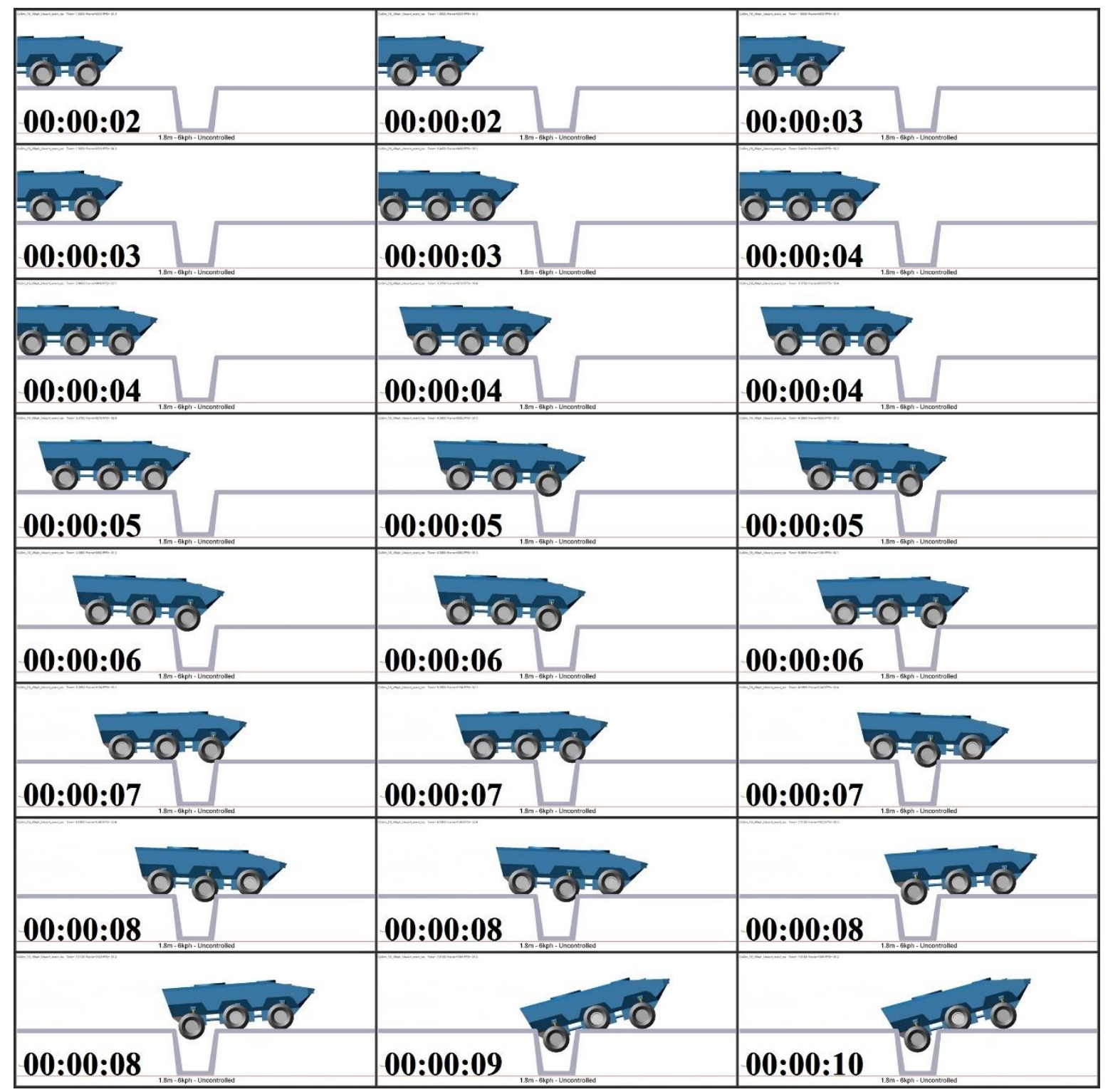

Figure A.79. Trench length $1.8 \mathrm{~m}, 6 \mathrm{kph}$, uncontrolled system 


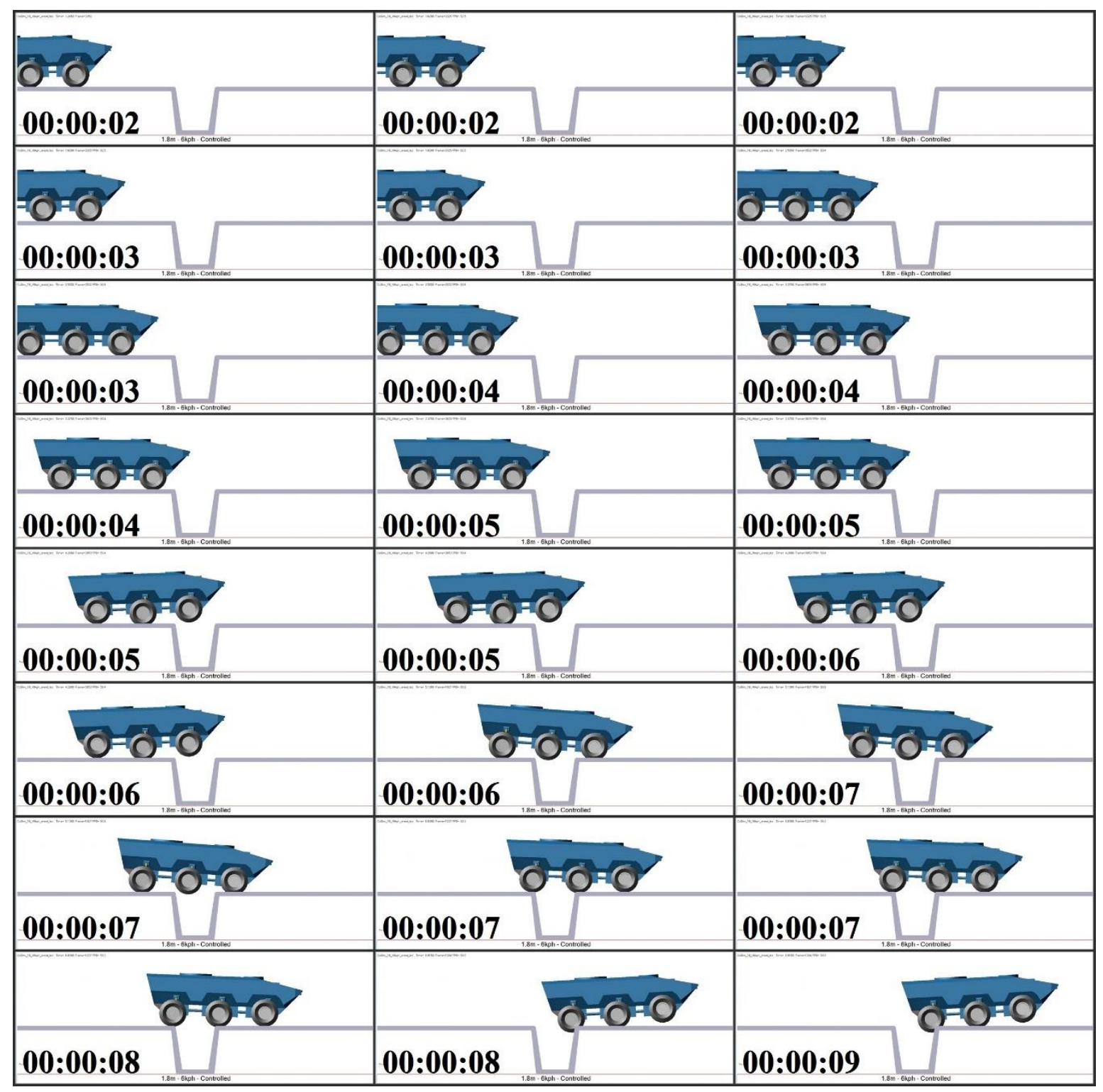

Figure A.80. Trench length $1.8 \mathrm{~m}, 6 \mathrm{kph}$, controlled system 


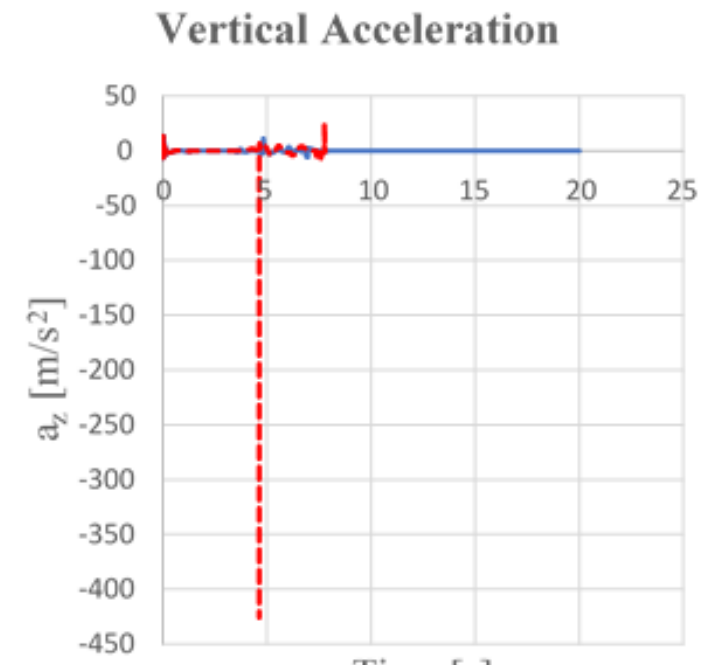

Time [s]

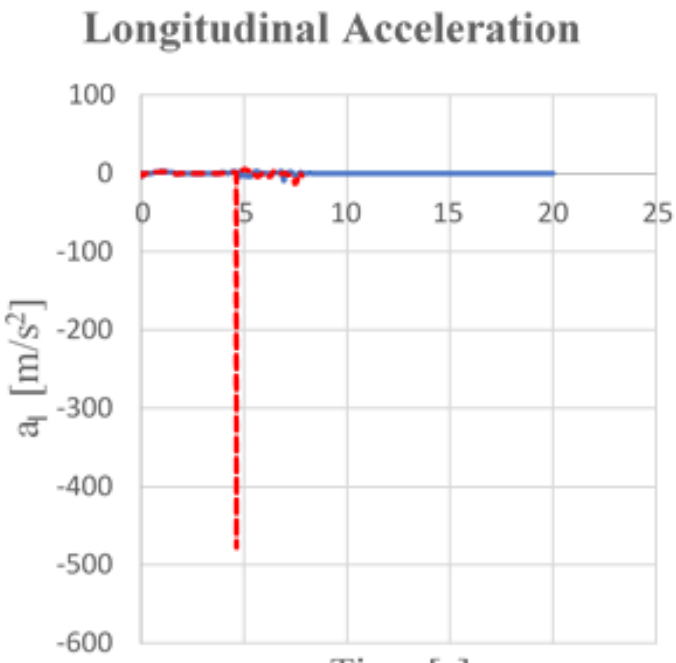

Time [s]

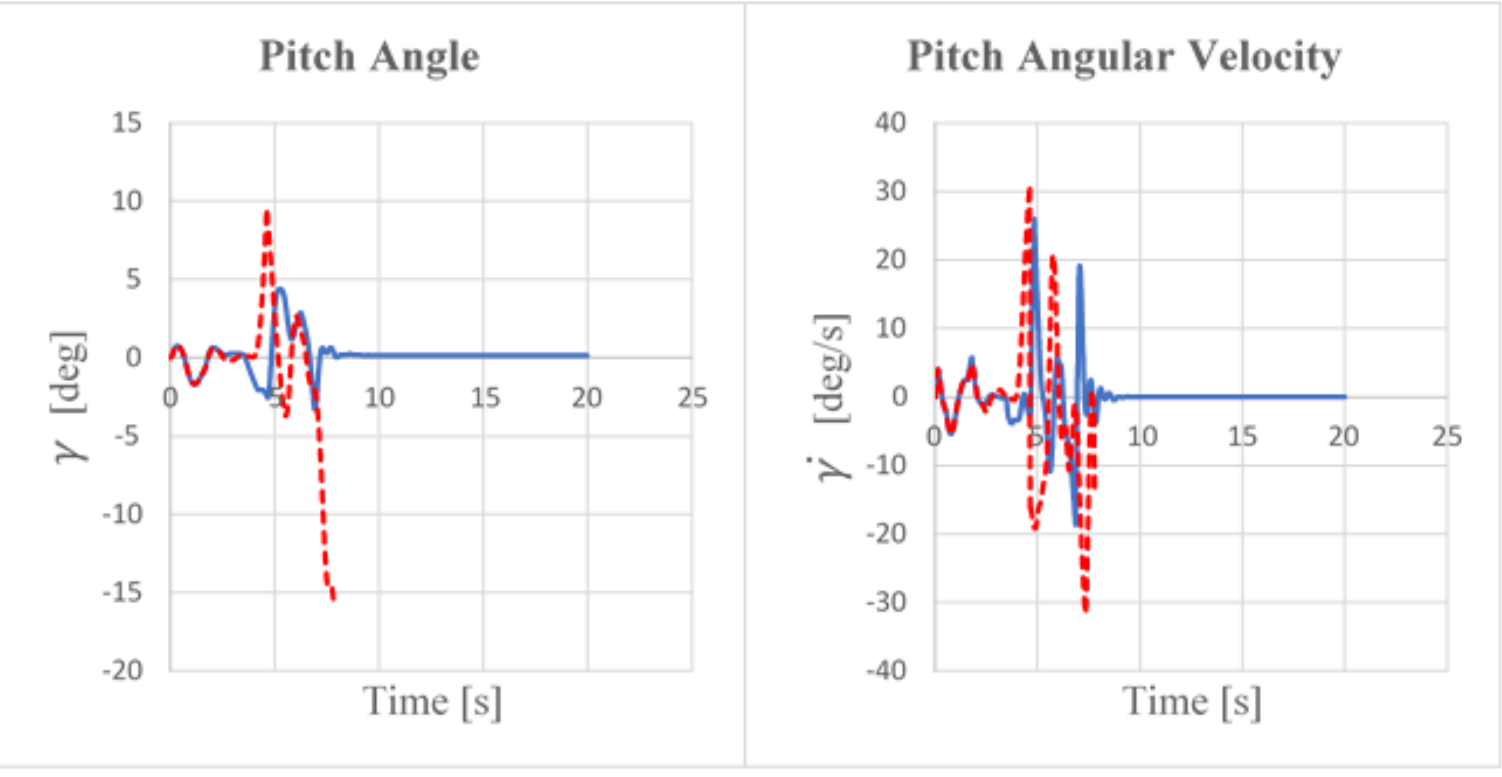

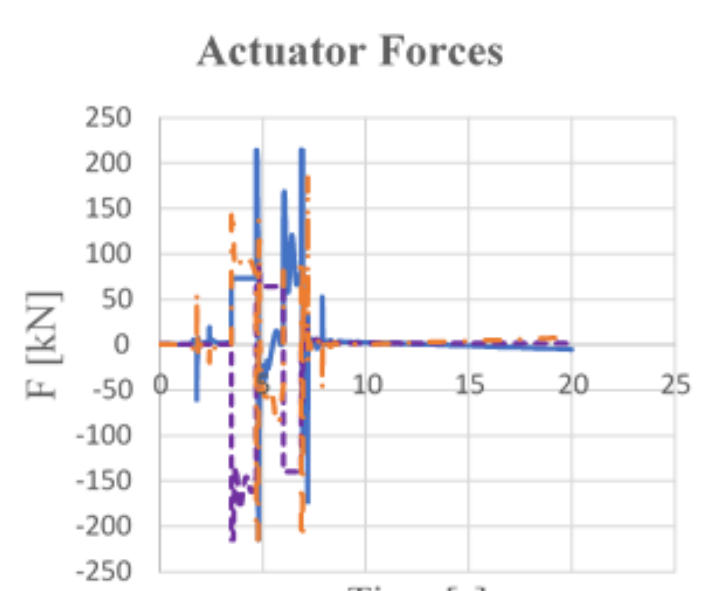

Time [s]

- Axle 1 - - Axle $2 \ldots$ Axle 3

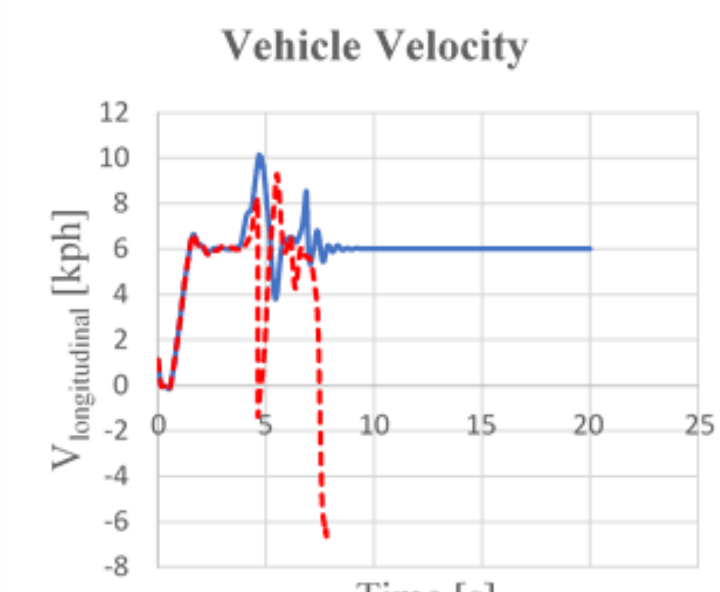

Time [s]

Controlled ----.Uncontrolled

Figure A.81. Trench length $1.8 \mathrm{~m}, 6 \mathrm{kph}$, controlled vs uncontrolled output comparison 
Trench Length $1.8 \mathrm{~m}, 9 \mathrm{kph}$

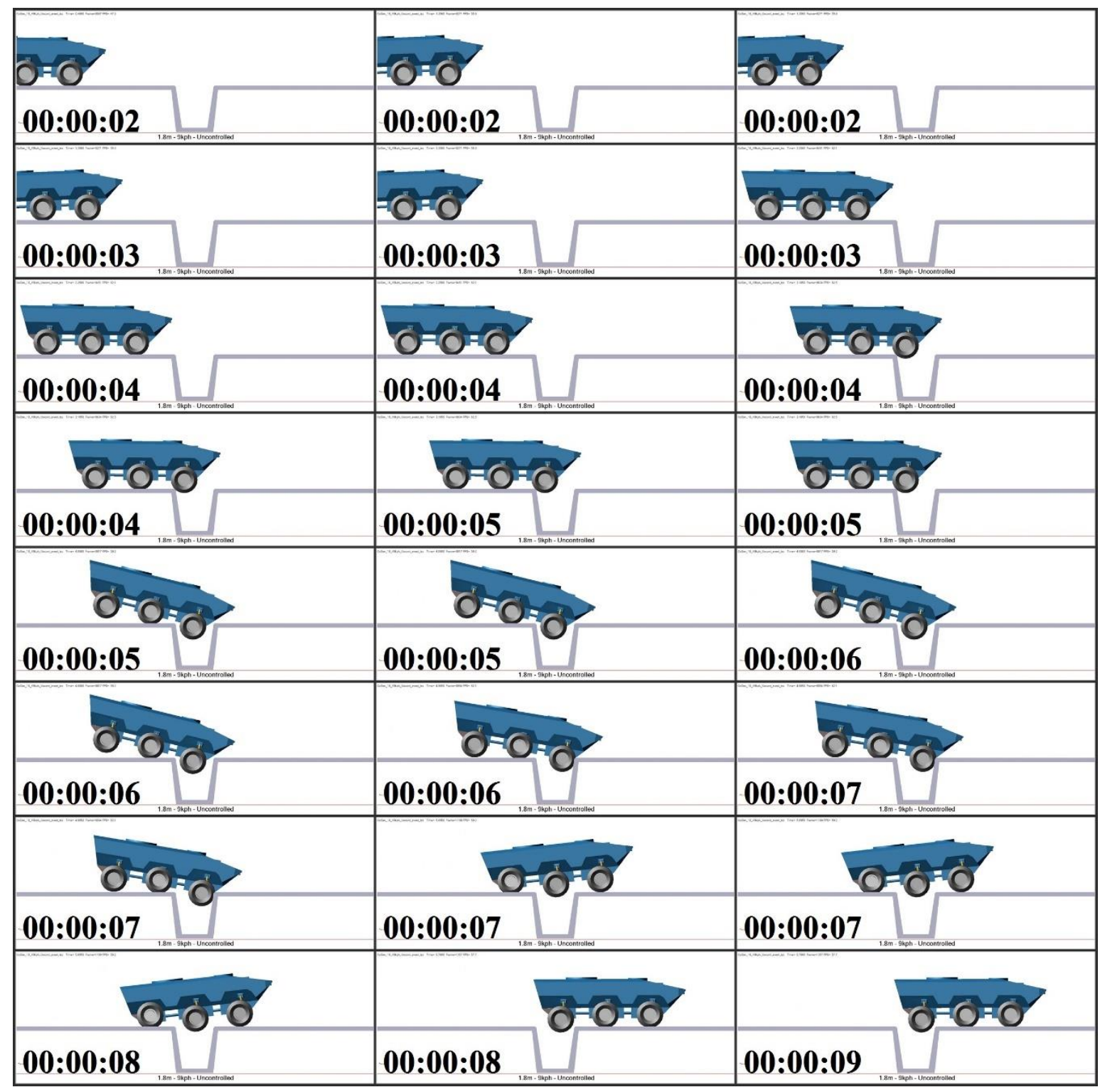

Figure A.82. Trench length $1.8 \mathrm{~m}, 9 \mathrm{kph}$, uncontrolled system 


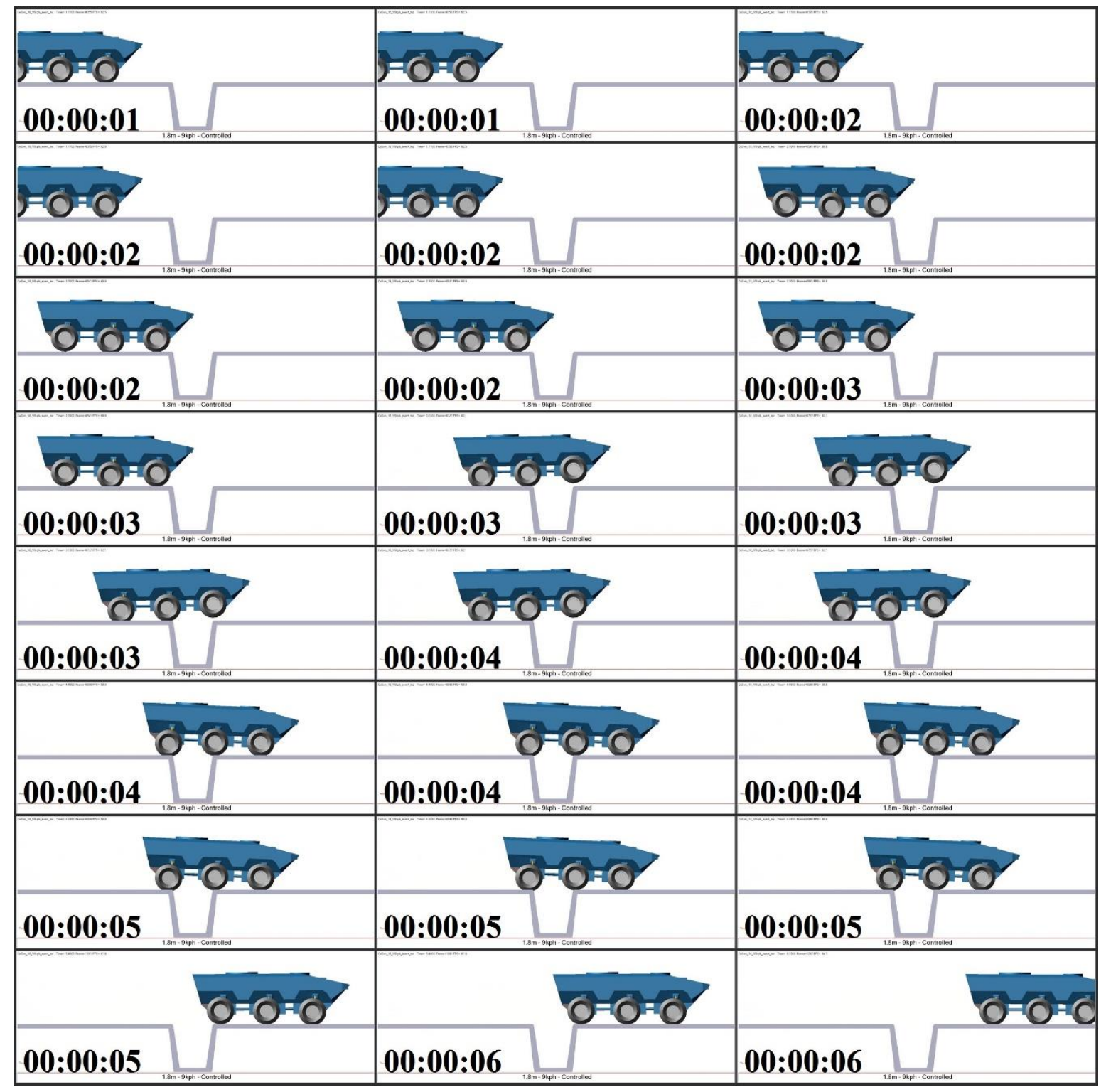

Figure A.83. Trench length $1.8 \mathrm{~m}, 9 \mathrm{kph}$, controlled system 

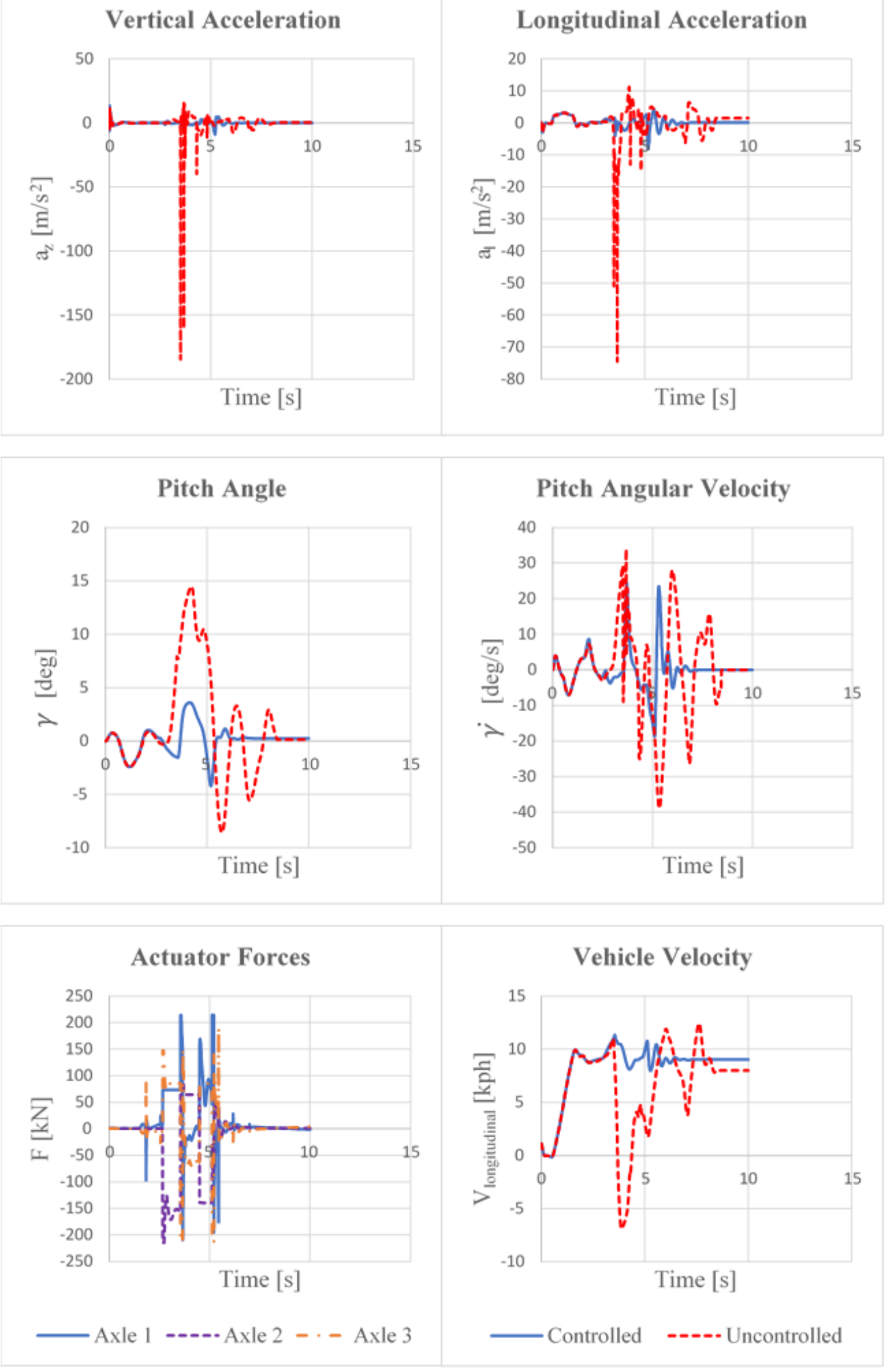

Figure A.84. Trench length $1.8 \mathrm{~m}, 9 \mathrm{kph}$, controlled vs uncontrolled output comparison 
Trench Length $1.9 \mathrm{~m}$, Controlled vs Uncontrolled System Simulations

Trench Length 1.9 m, 1 kph

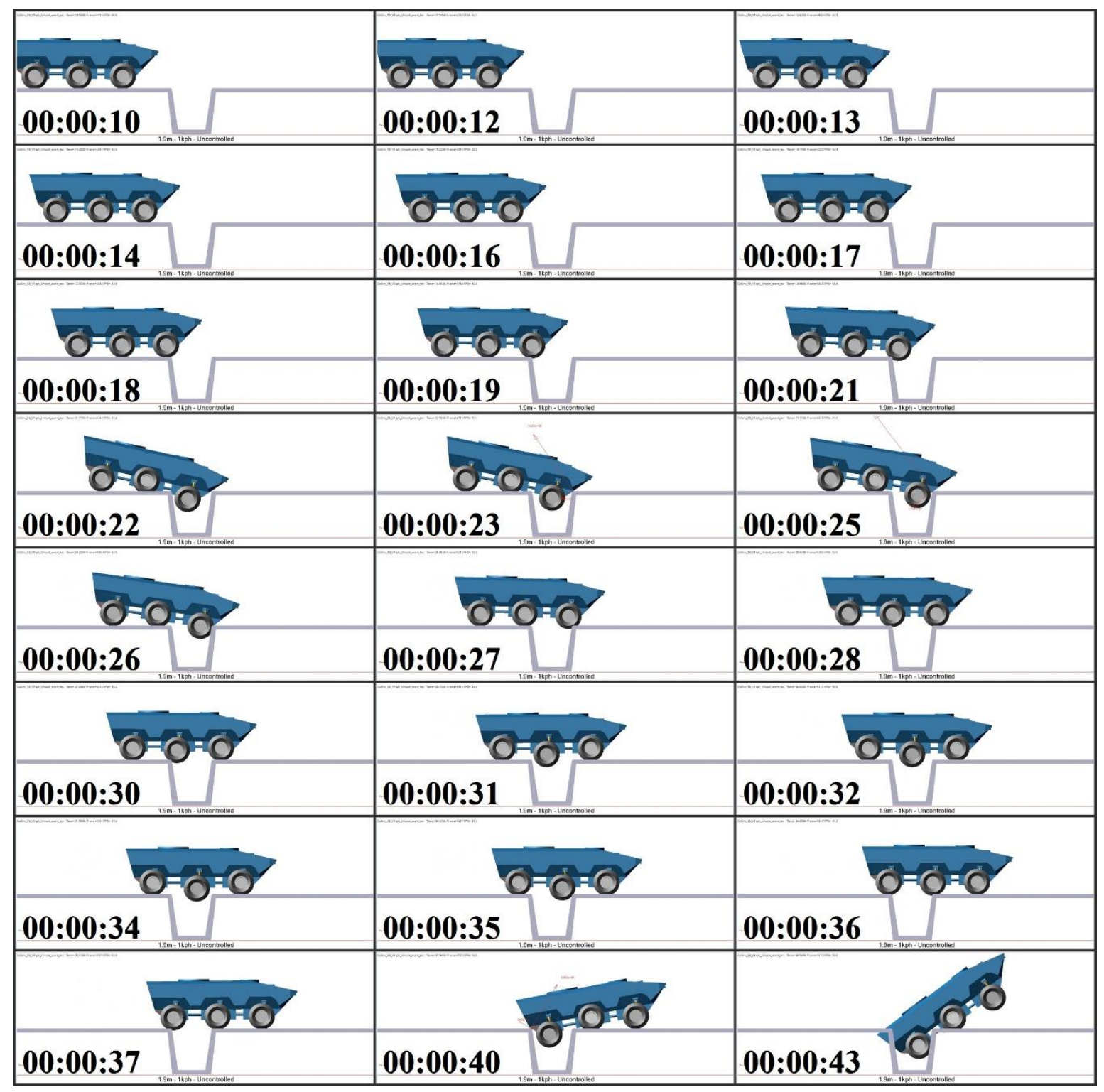

Figure A.85. Trench length $1.9 \mathrm{~m}, 1 \mathrm{kph}$, uncontrolled system 


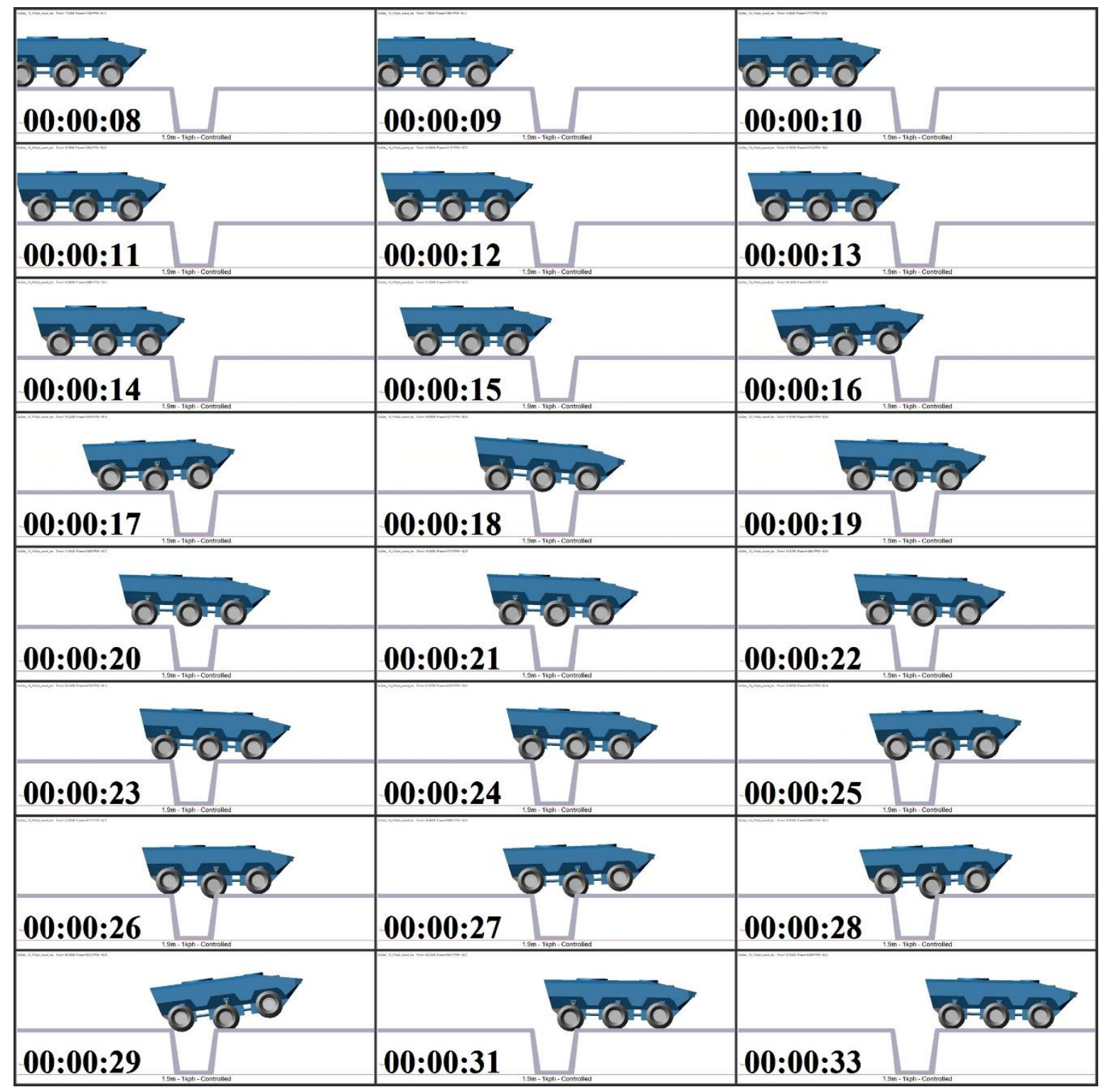

Figure A.86. Trench length $1.9 \mathrm{~m}, 1 \mathrm{kph}$, controlled system 

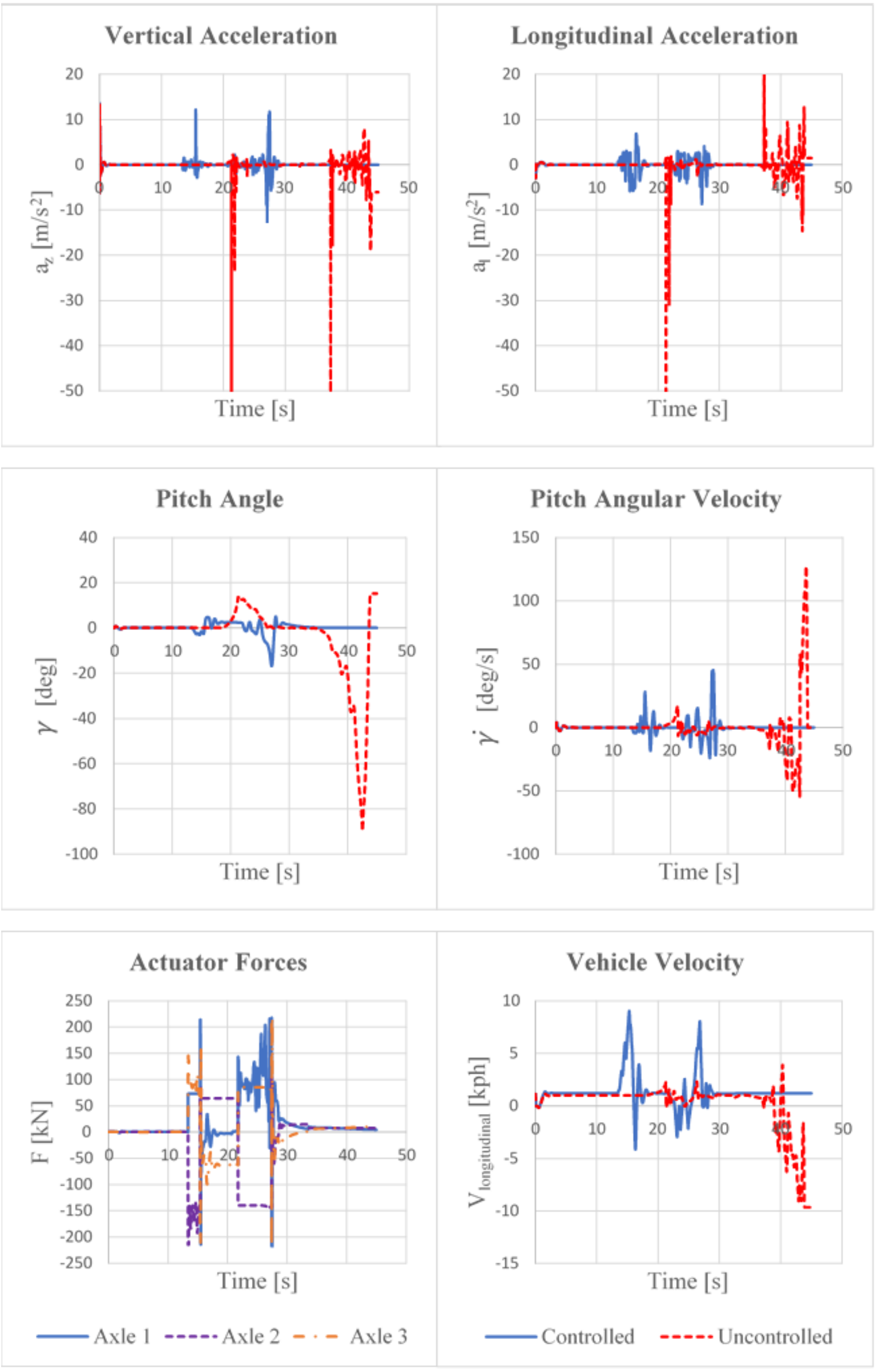

Figure A.87. Trench length $1.9 \mathrm{~m}, 1 \mathrm{kph}$, controlled vs uncontrolled output comparison 
Trench Length 1.9 m, 3 kph

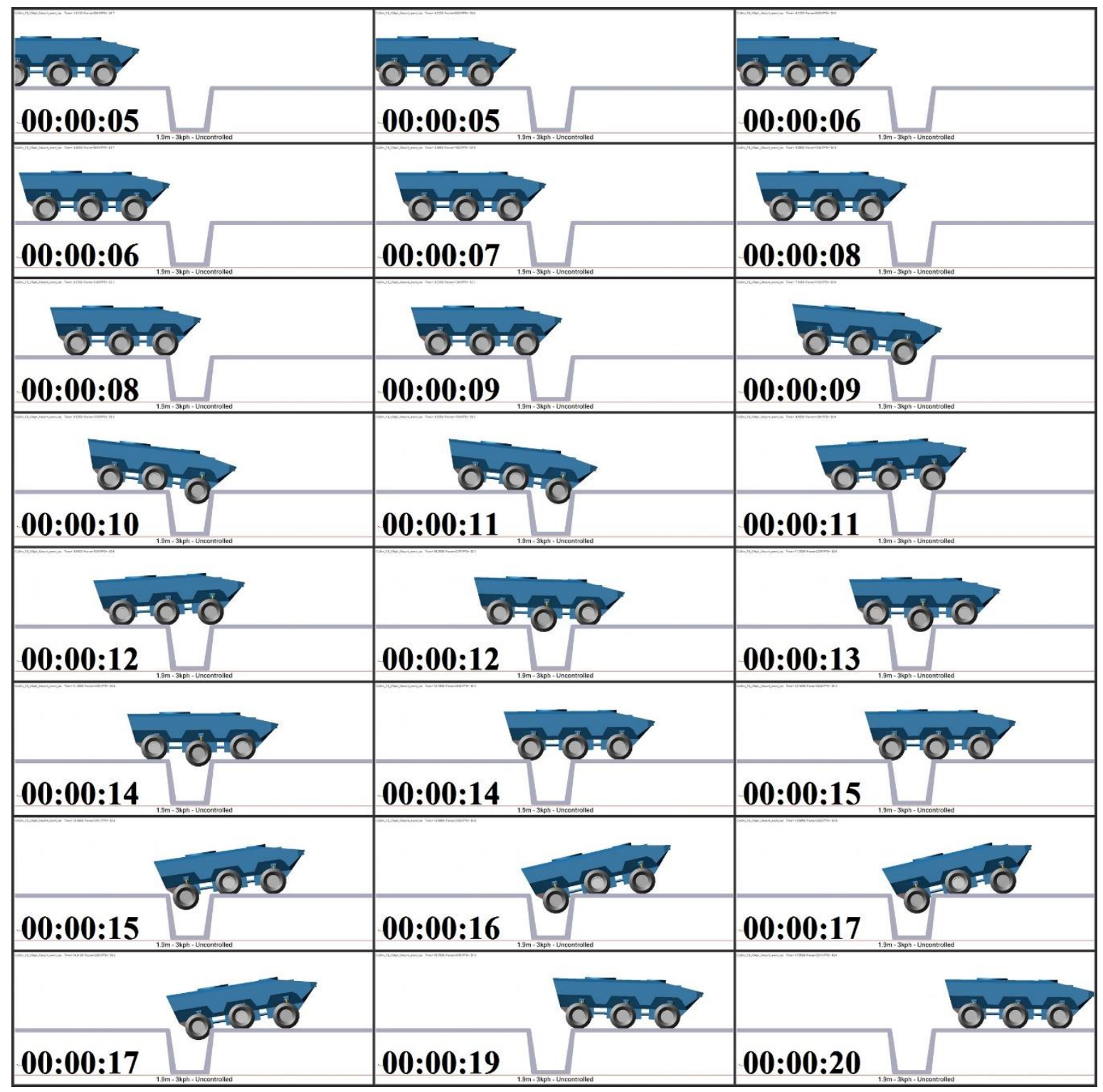

Figure A.88. Trench length $1.9 \mathrm{~m}, 3 \mathrm{kph}$, uncontrolled system 


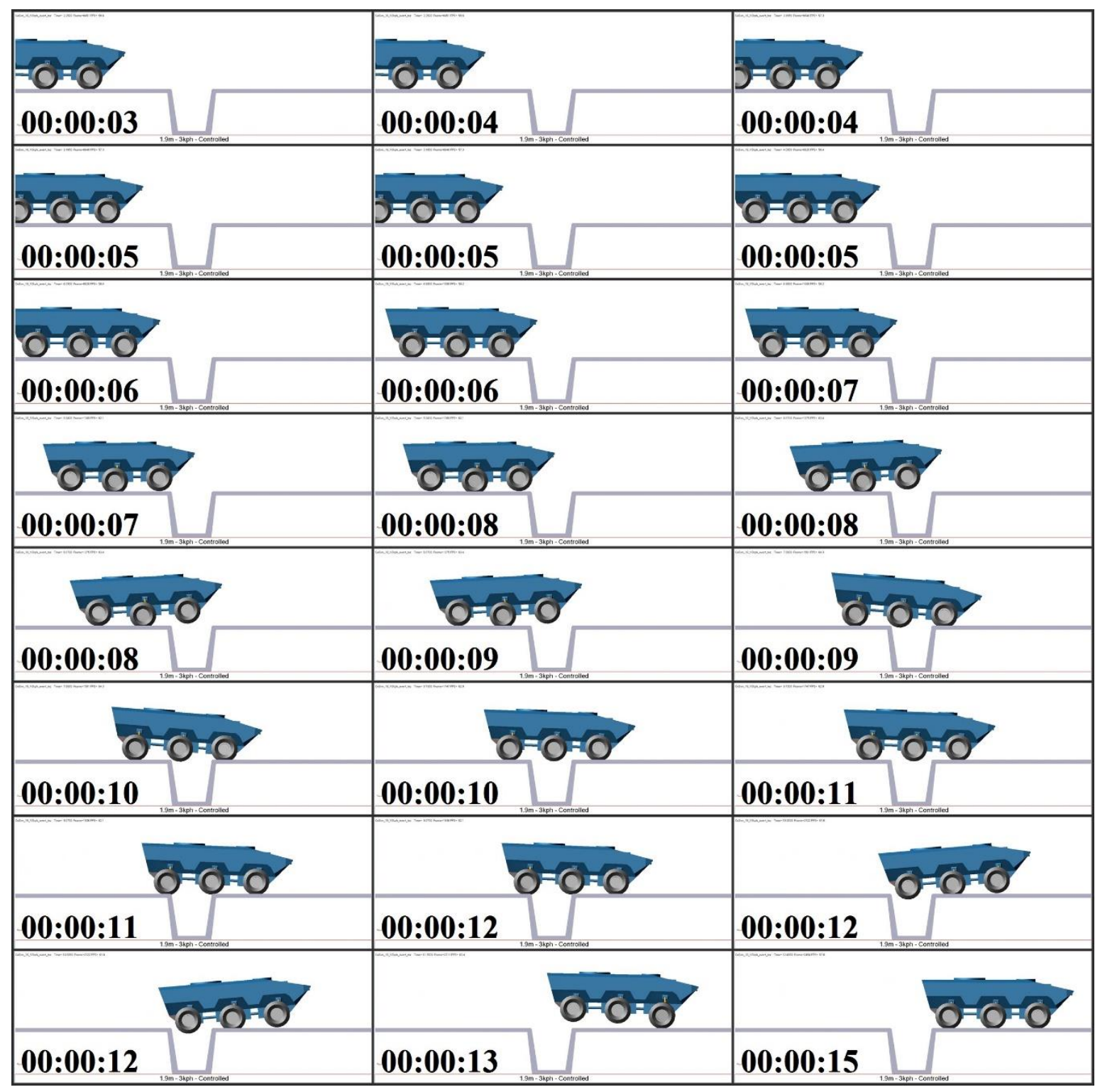

Figure A.89. Trench length $1.9 \mathrm{~m}, 3 \mathrm{kph}$, controlled system 

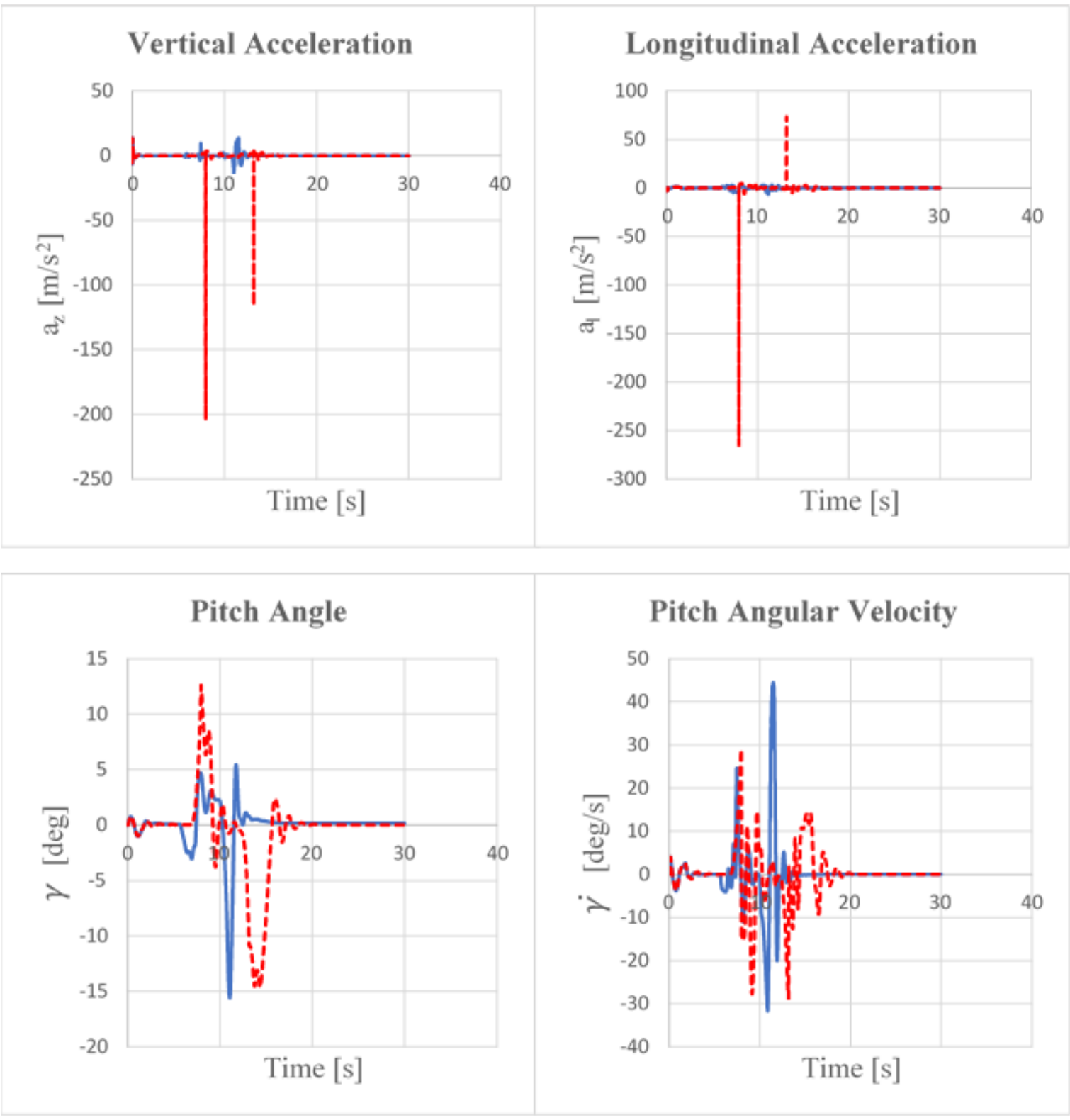

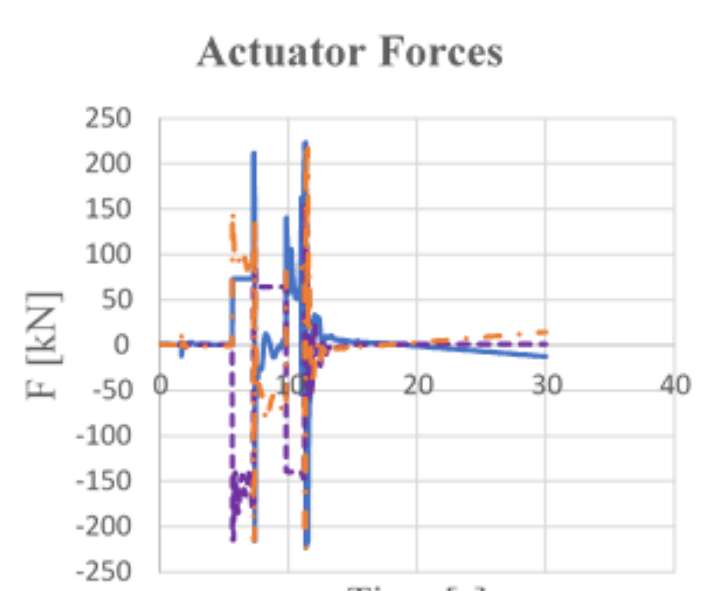

Time $[\mathrm{s}]$

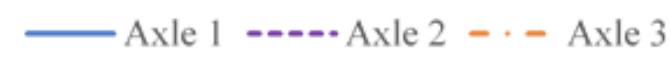

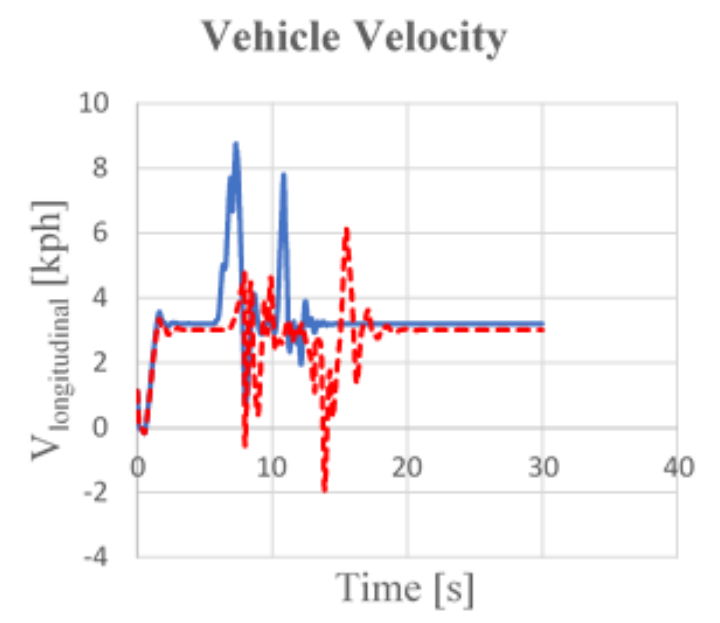

Controlled ----.. Uncontrolled

Figure A.90. Trench length $1.9 \mathrm{~m}, 3 \mathrm{kph}$, controlled vs uncontrolled output comparison 
Trench Length 1.9 m, 6 kph

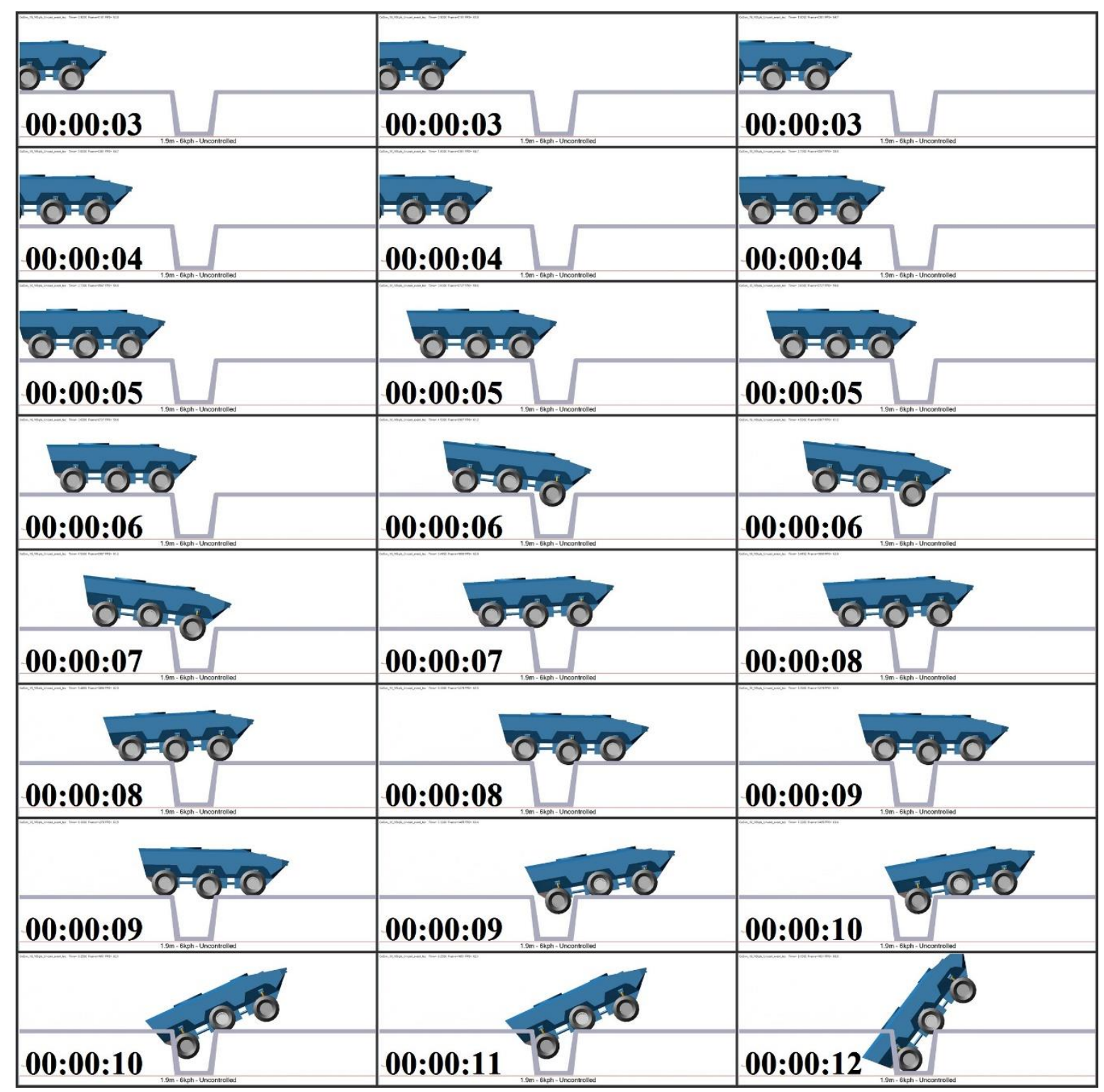

Figure A.91. Trench length $1.9 \mathrm{~m}, 6 \mathrm{kph}$, uncontrolled system 


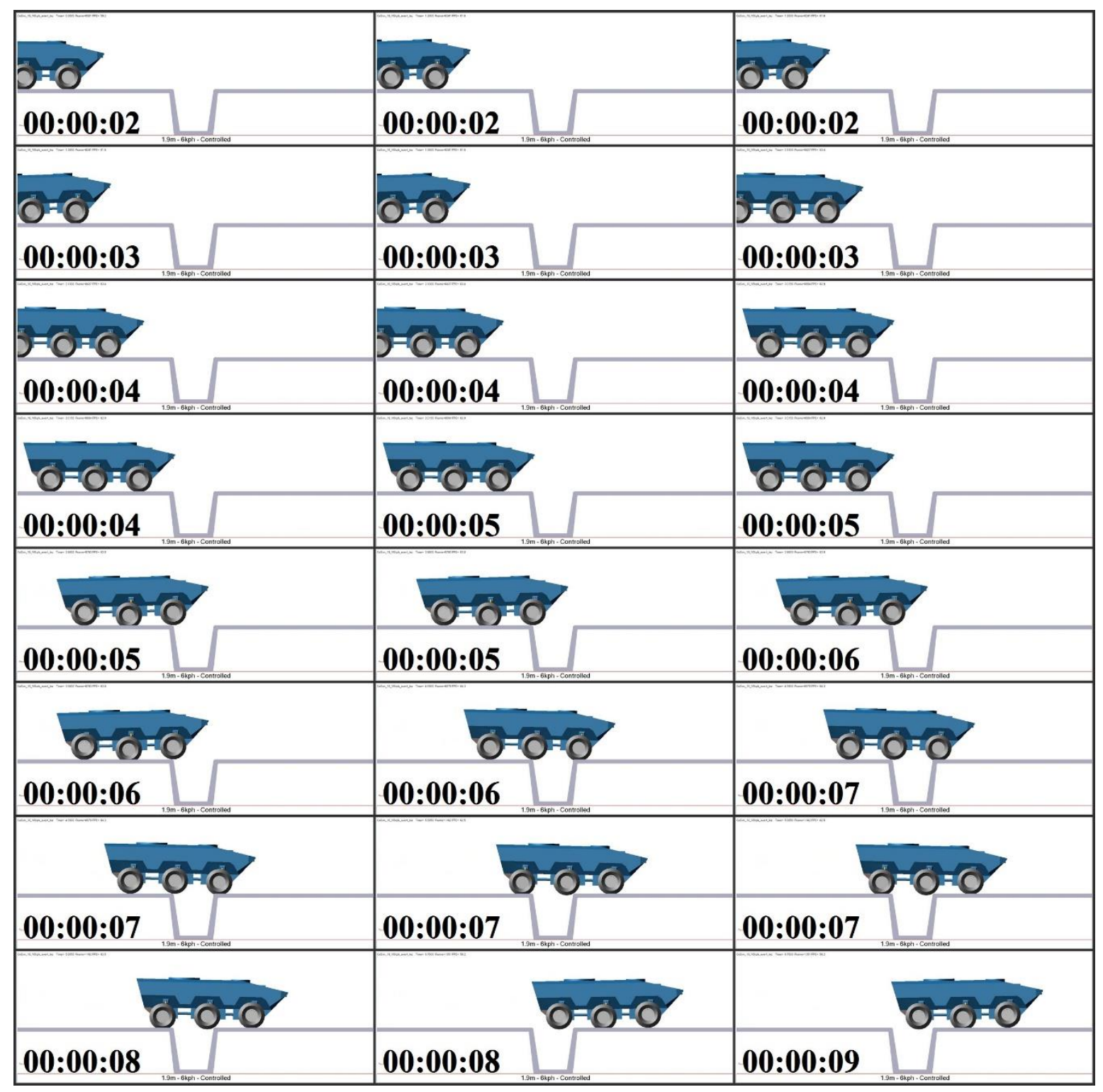

Figure A.92. Trench length $1.9 \mathrm{~m}, 6 \mathrm{kph}$, controlled system 

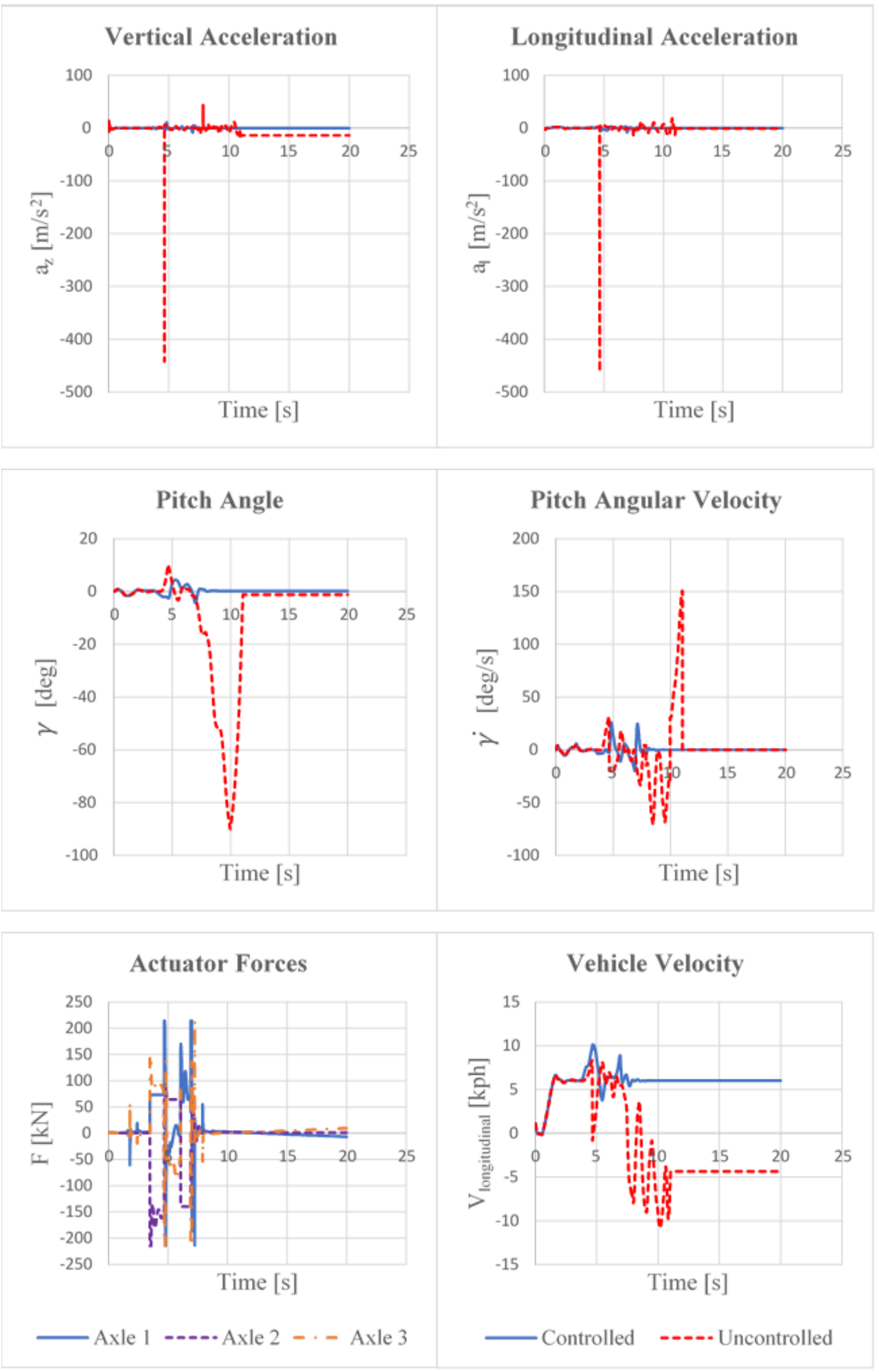

Figure A.93. Trench length $1.9 \mathrm{~m}$, $6 \mathrm{kph}$, controlled vs uncontrolled output comparison 
Trench Length 1.9 m, 9 kph

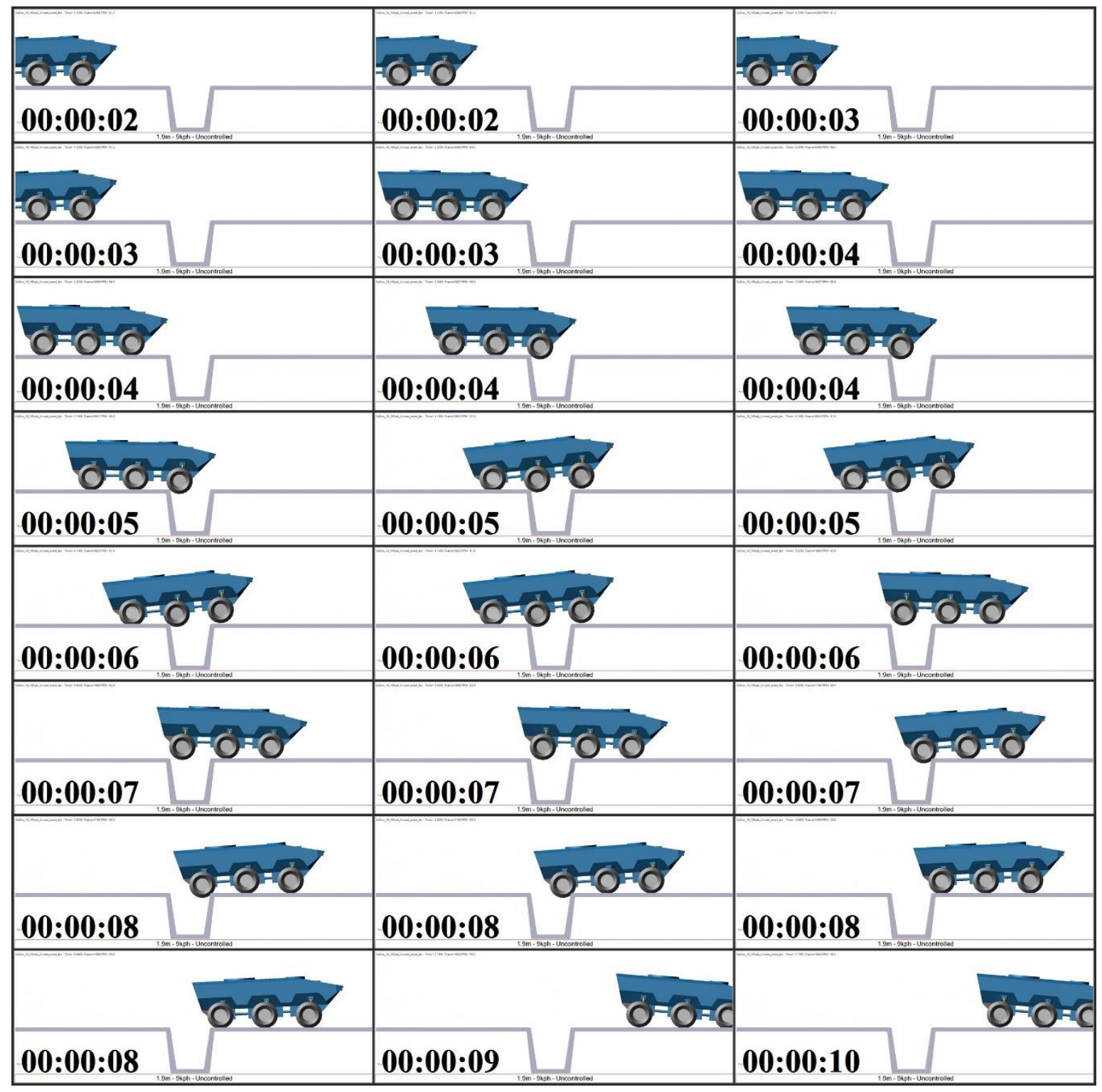

Figure A.94. Trench length $1.9 \mathrm{~m}, 9 \mathrm{kph}$, uncontrolled system 


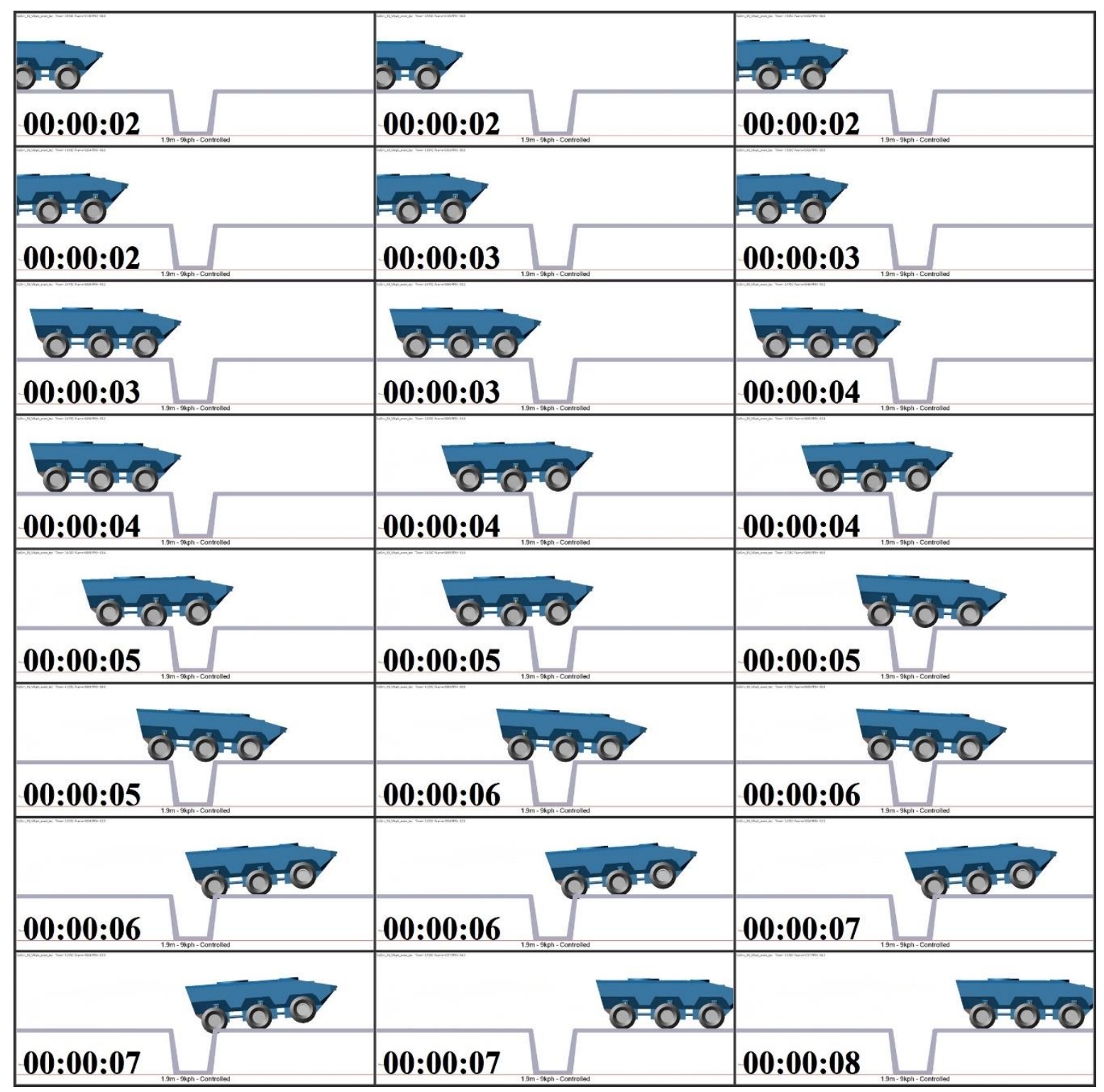

Figure A.95. Trench length $1.9 \mathrm{~m}, 9 \mathrm{kph}$, controlled system 

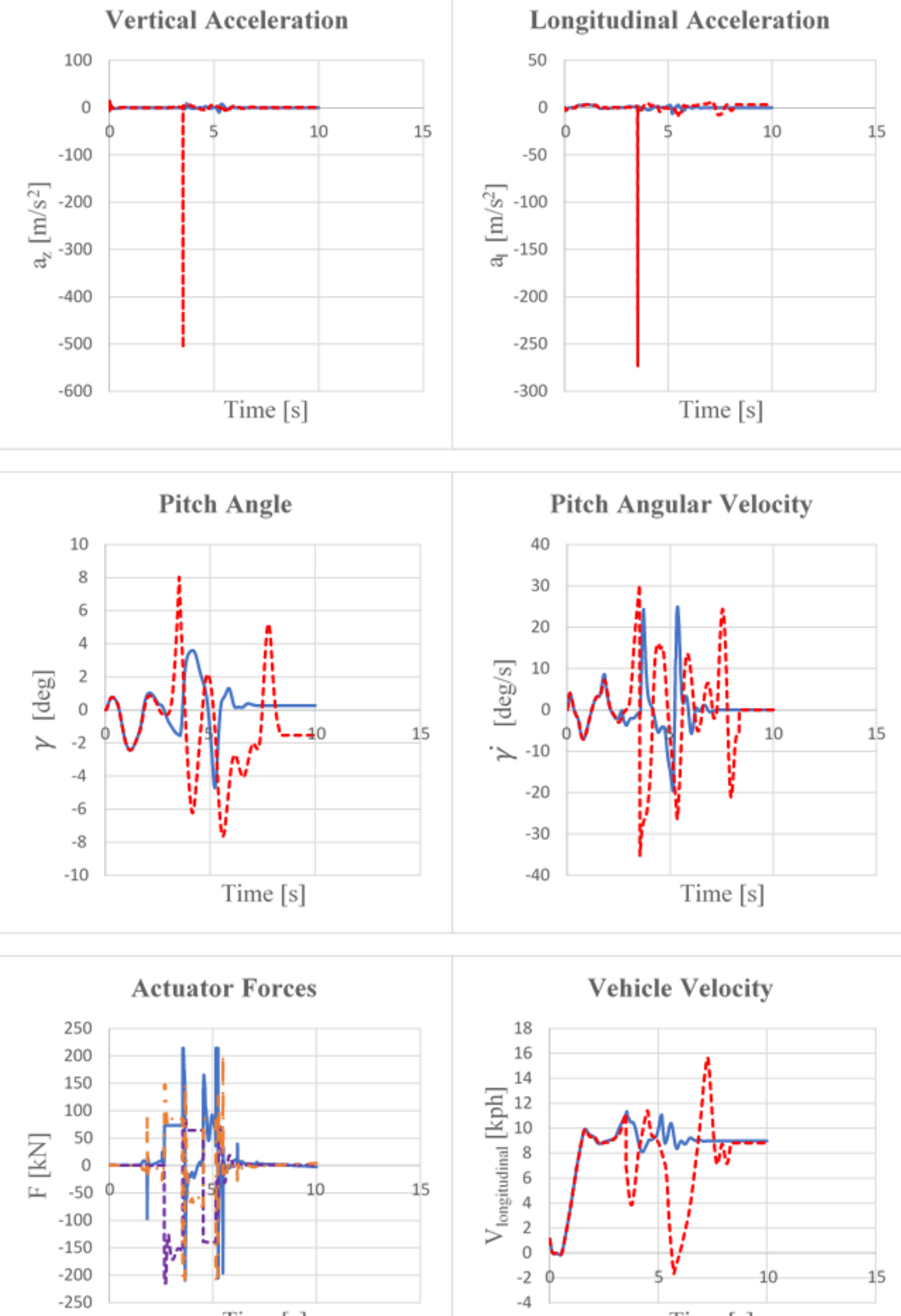

Time $[\mathrm{s}]$

- Axle 1 ----Axle 2 - - Axle 3

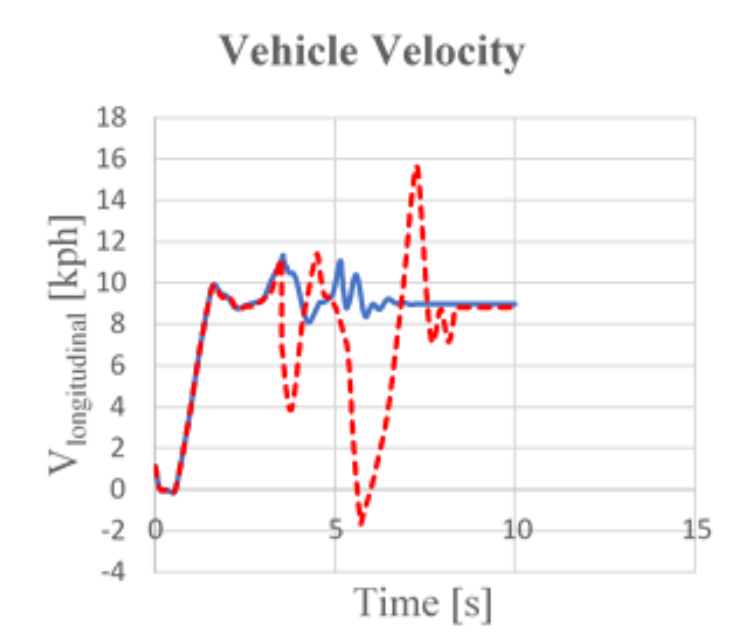

—Controlled -----Uncontrolled

Figure A.96. Trench length $1.9 \mathrm{~m}, 9 \mathrm{kph}$, controlled vs uncontrolled output comparison 
Trench Length $2.0 \mathrm{~m}$, Controlled vs Uncontrolled System Simulations

Trench Length $2.0 \mathrm{~m}, 1 \mathrm{kph}$

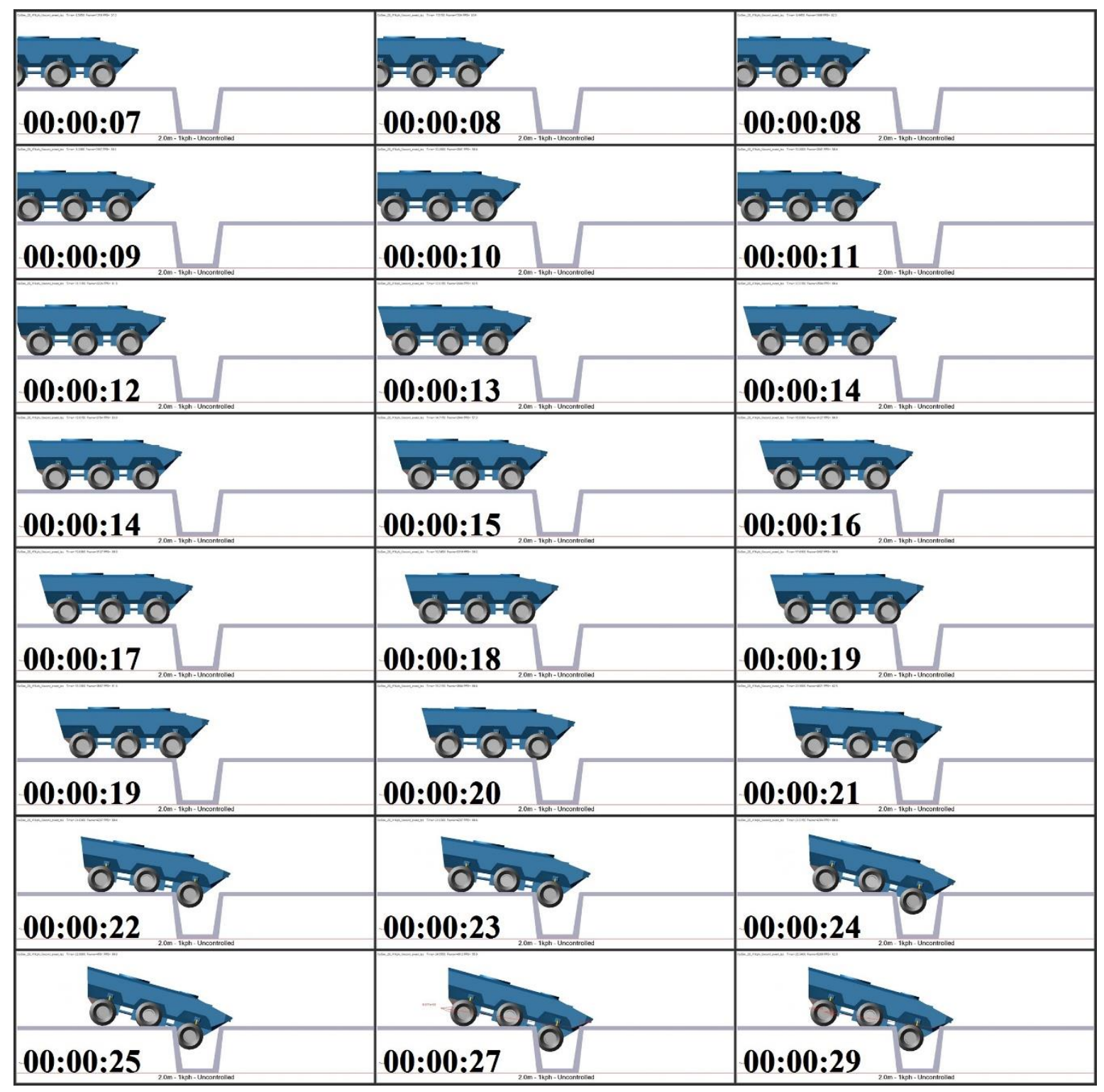

Figure A.97. Trench length $2.0 \mathrm{~m}, 1 \mathrm{kph}$, uncontrolled system 


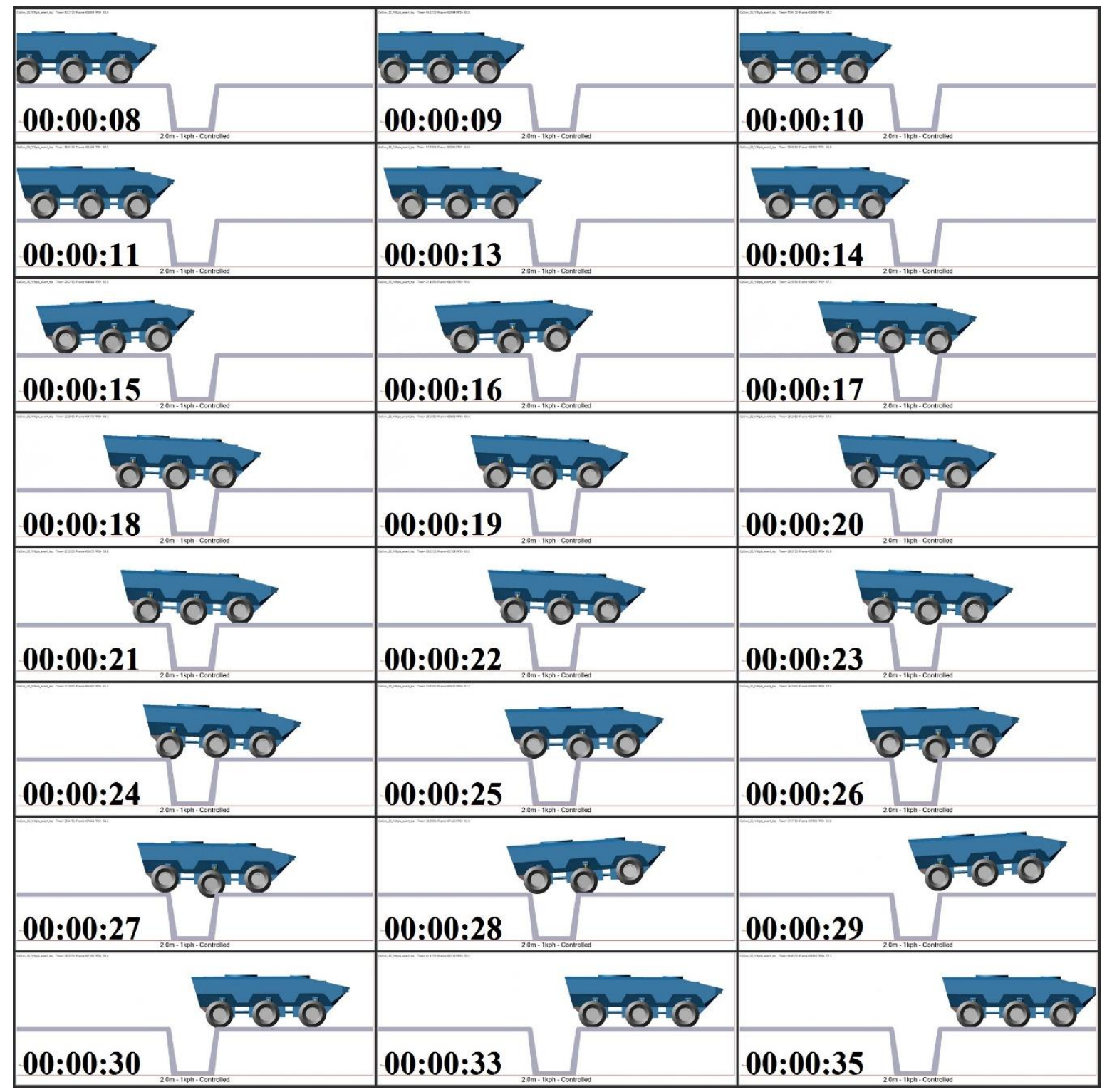

Figure A.98. Trench length $2.0 \mathrm{~m}, 1 \mathrm{kph}$, controlled system 


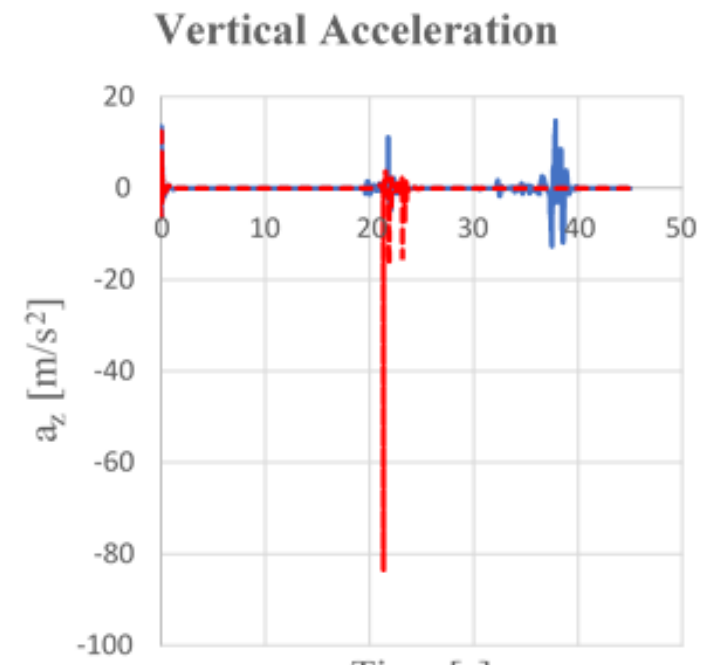

Time [s]

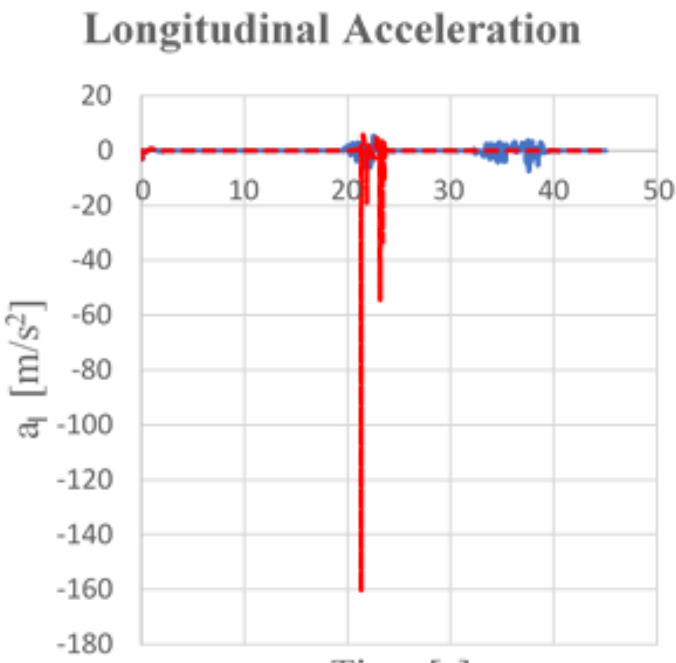

Time $[\mathrm{s}]$
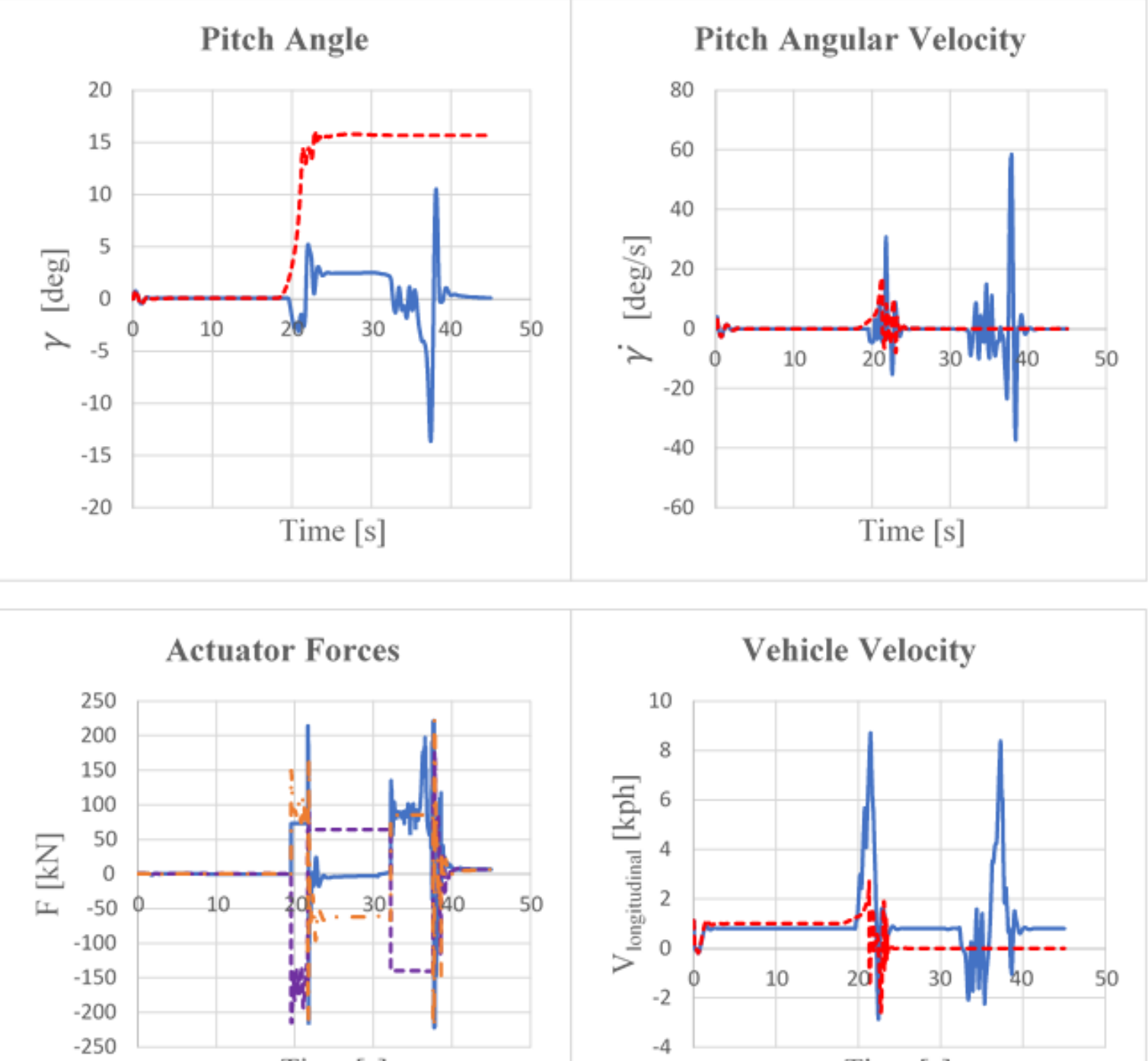

Time [s]

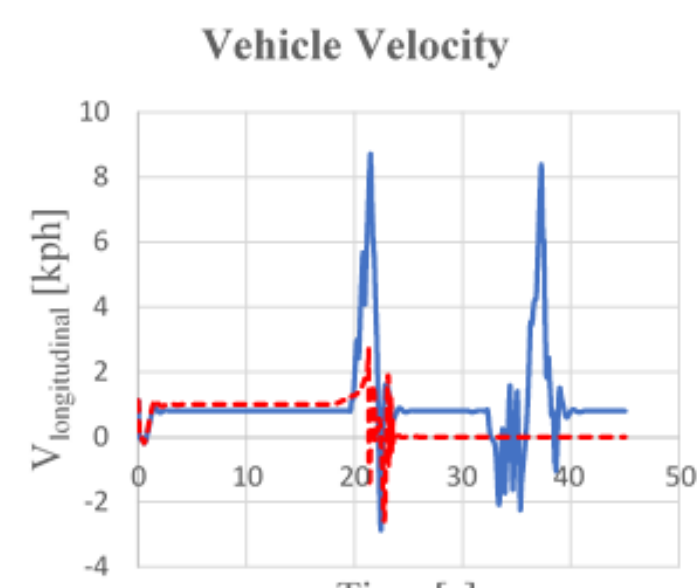

Time [s]

Axle 1 ---. Axle 2 - - Axle 3

Controlled -.-.-Uncontrolled

Figure A.99. Trench length $2.0 \mathrm{~m}, 1 \mathrm{kph}$, controlled vs uncontrolled output comparison 
Trench Length 2.0 m, 3 kph

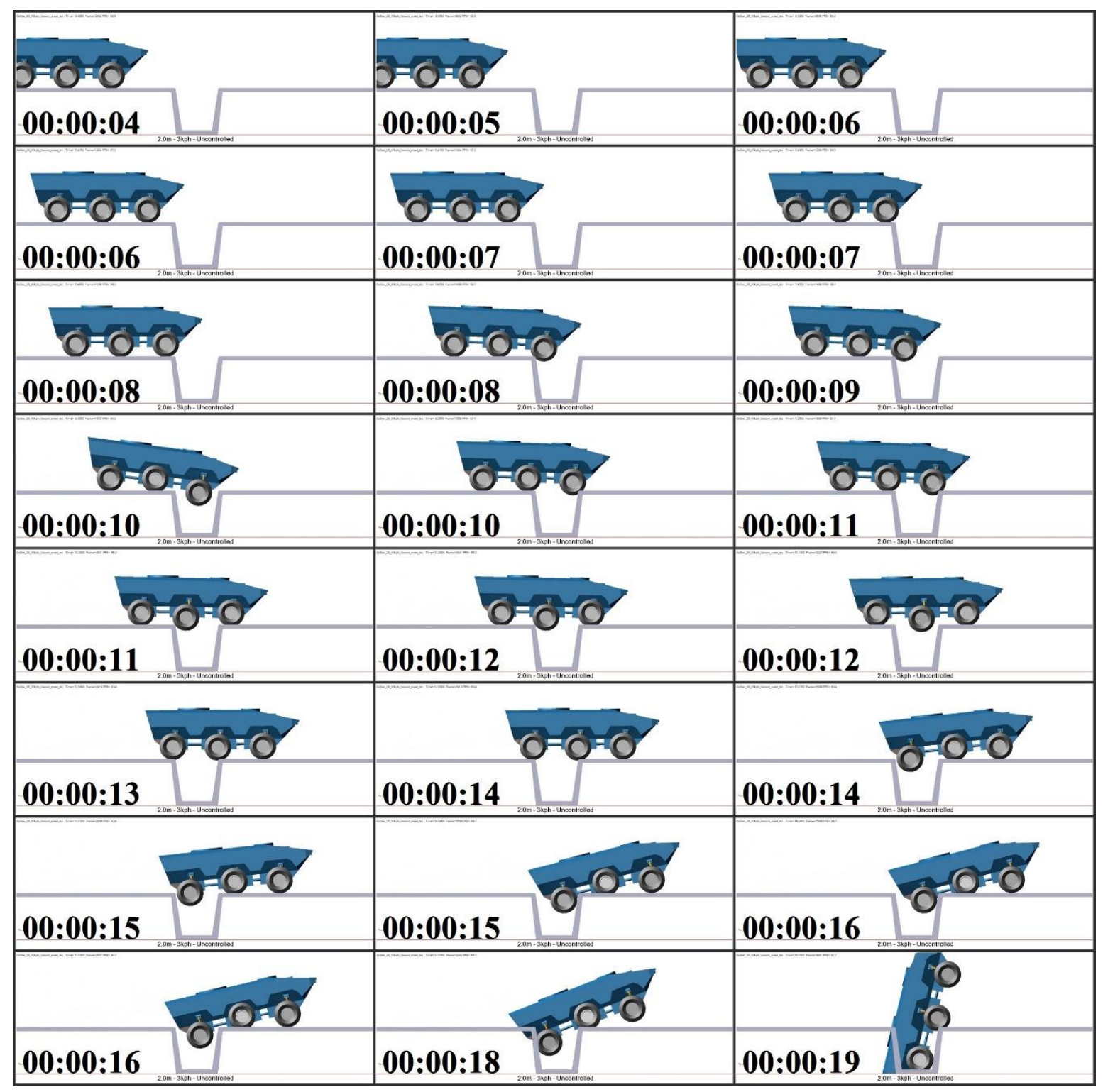

Figure A.100. Trench length $2.0 \mathrm{~m}, 3 \mathrm{kph}$, uncontrolled system 


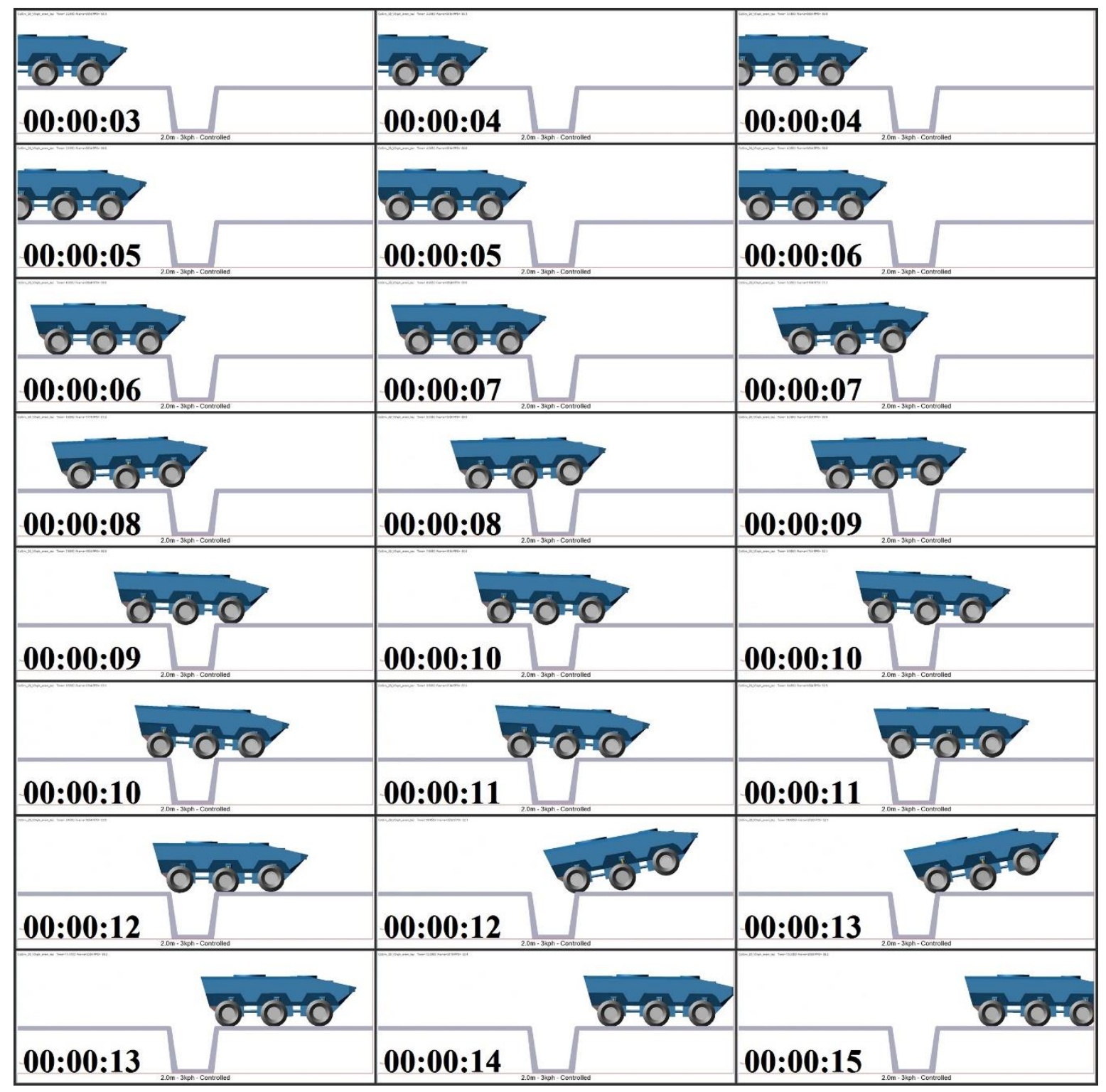

Figure A.101. Trench length $2.0 \mathrm{~m}, 3 \mathrm{kph}$, controlled system 

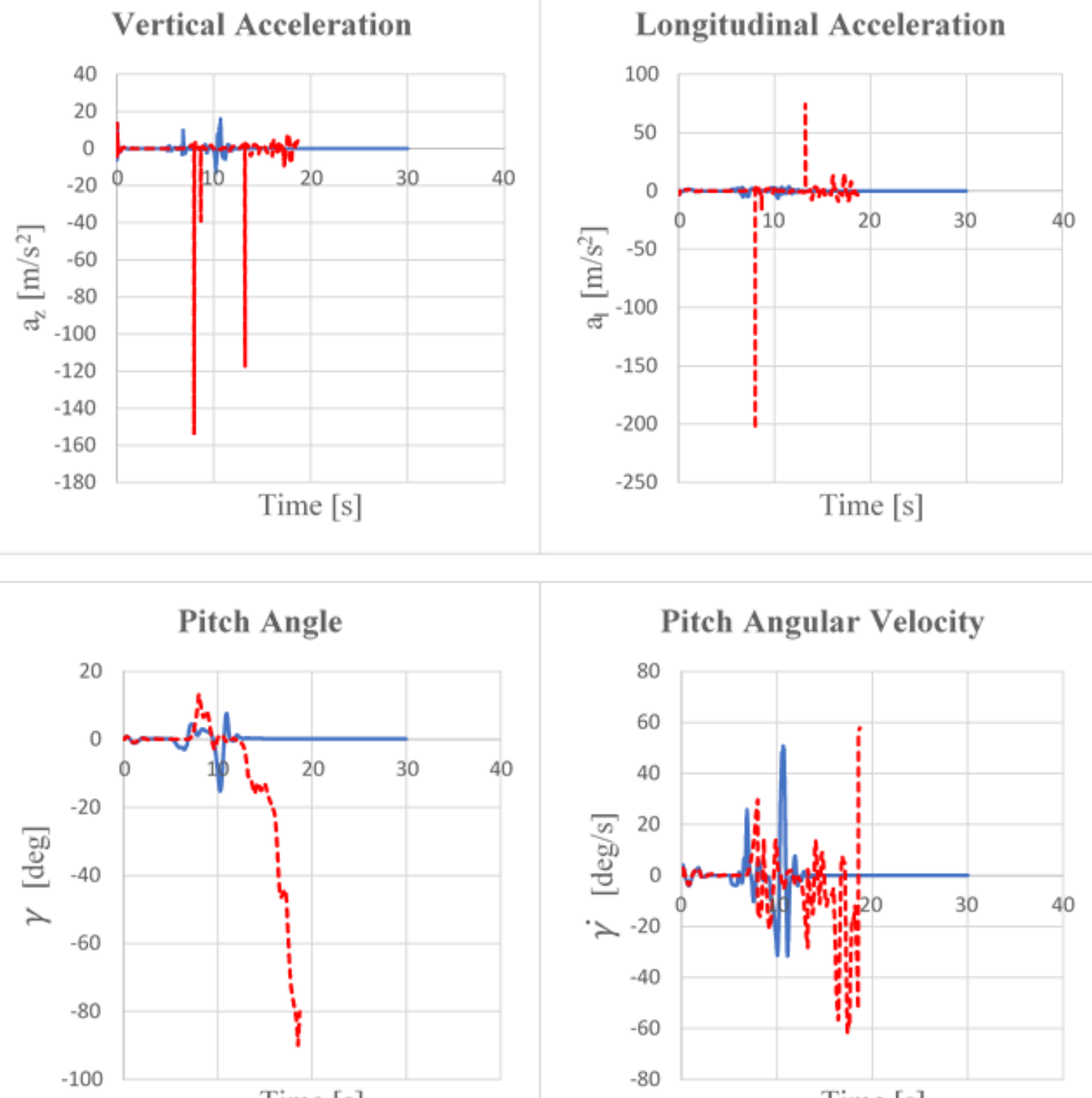

Time [s]
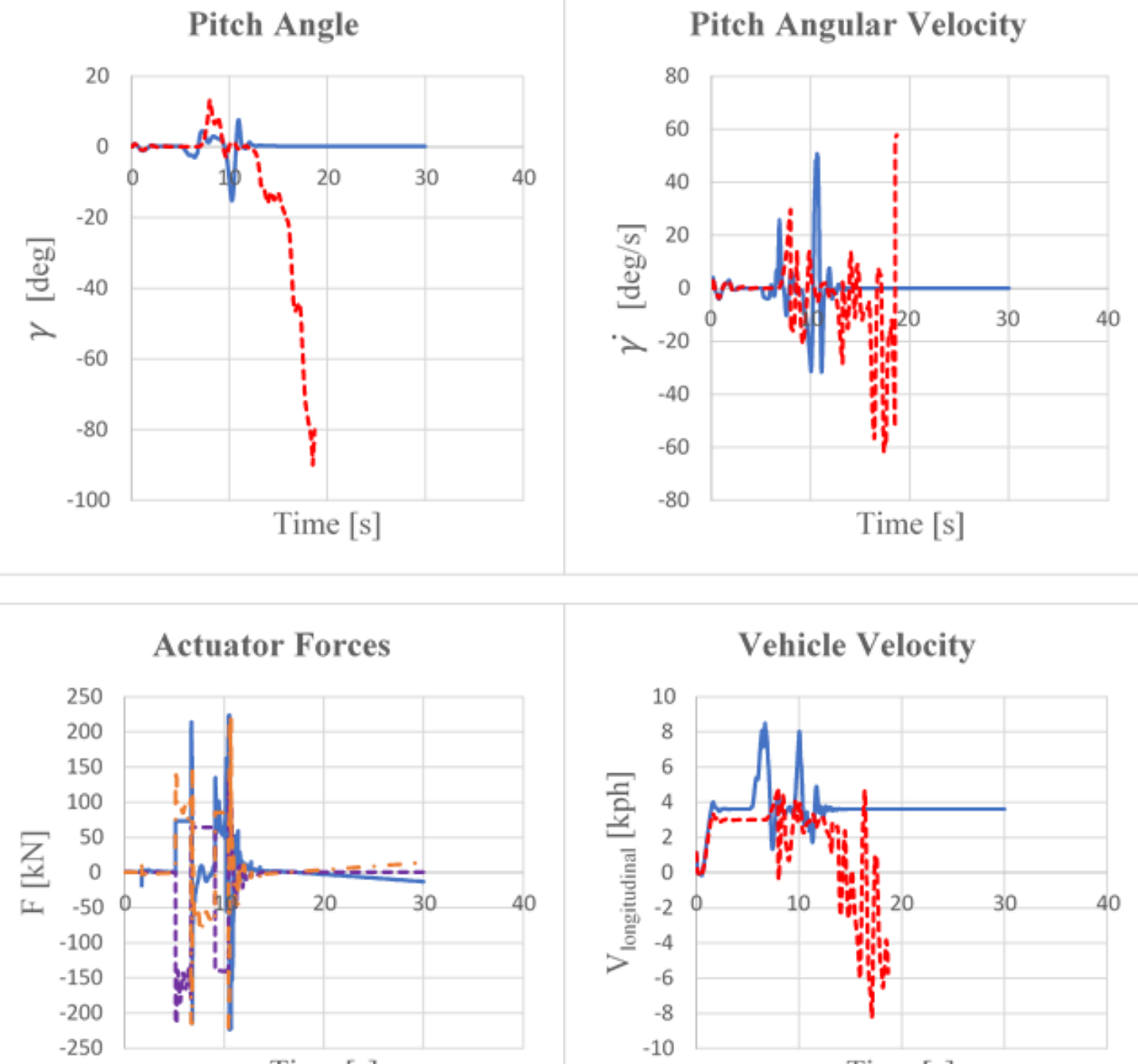

Time [s]

Axle 1 ---. Axle 2 - - Axle 3

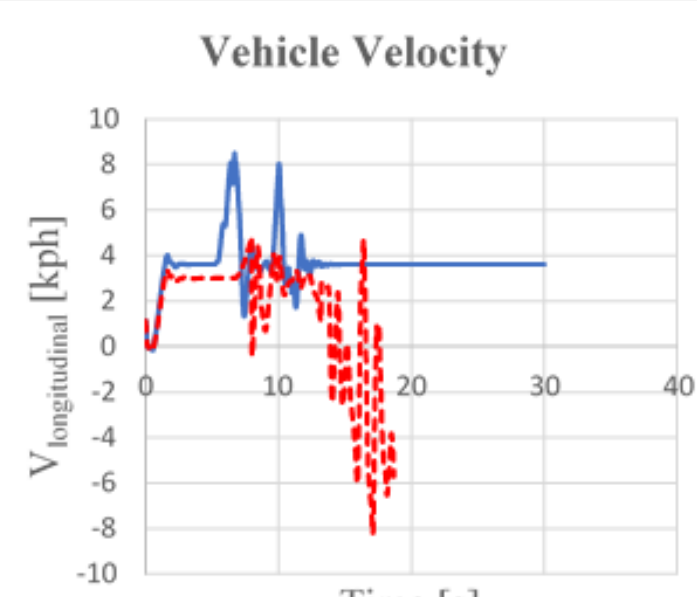

Time [s]

Controlled ----.- Uncontrolled

Figure A.102. Trench length $2.0 \mathrm{~m}, 3 \mathrm{kph}$, controlled vs uncontrolled output comparison 
Trench Length $2.0 \mathrm{~m}, 6 \mathrm{kph}$

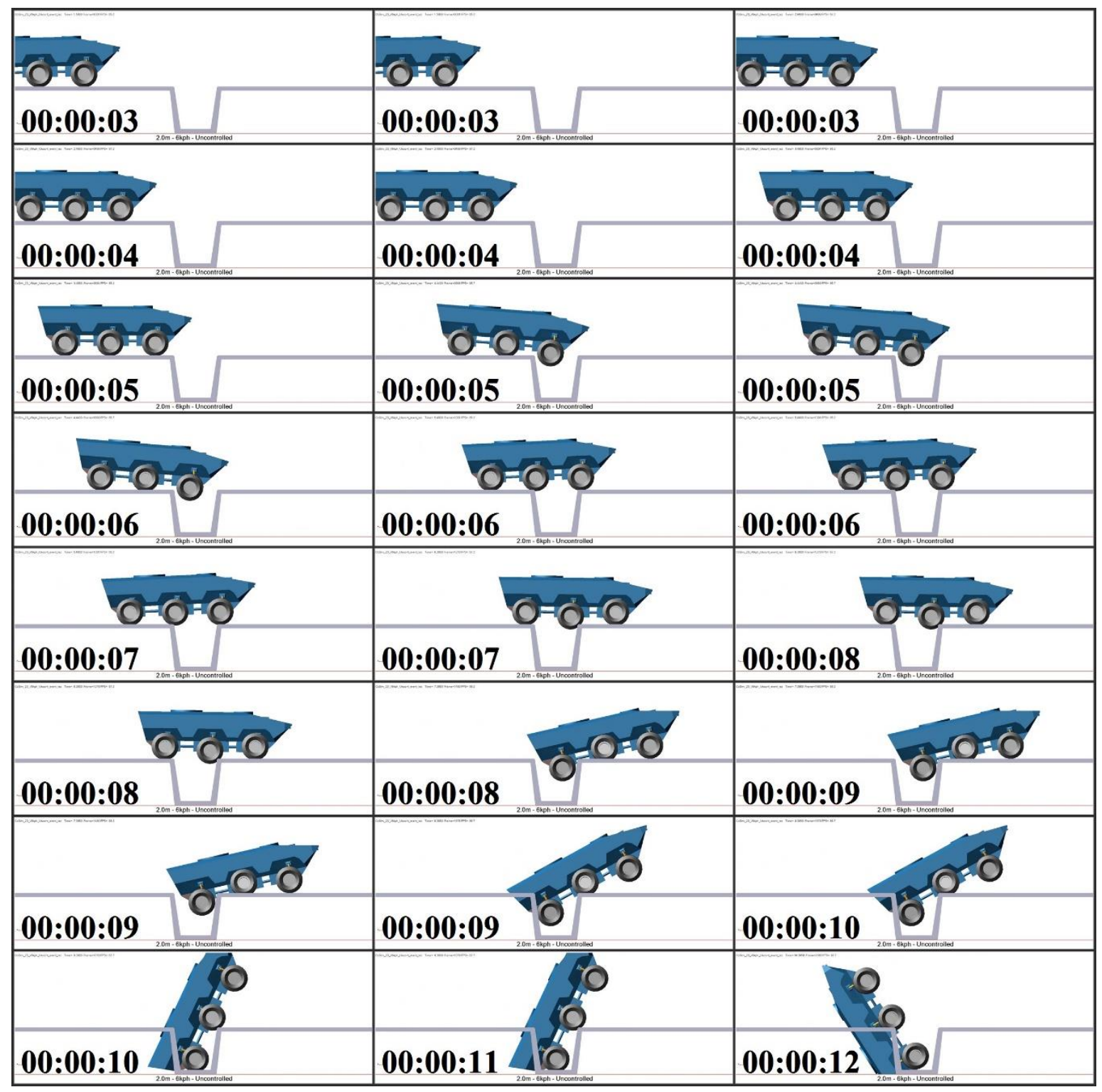

Figure A.103. Trench length $2.0 \mathrm{~m}, 6 \mathrm{kph}$, uncontrolled system 


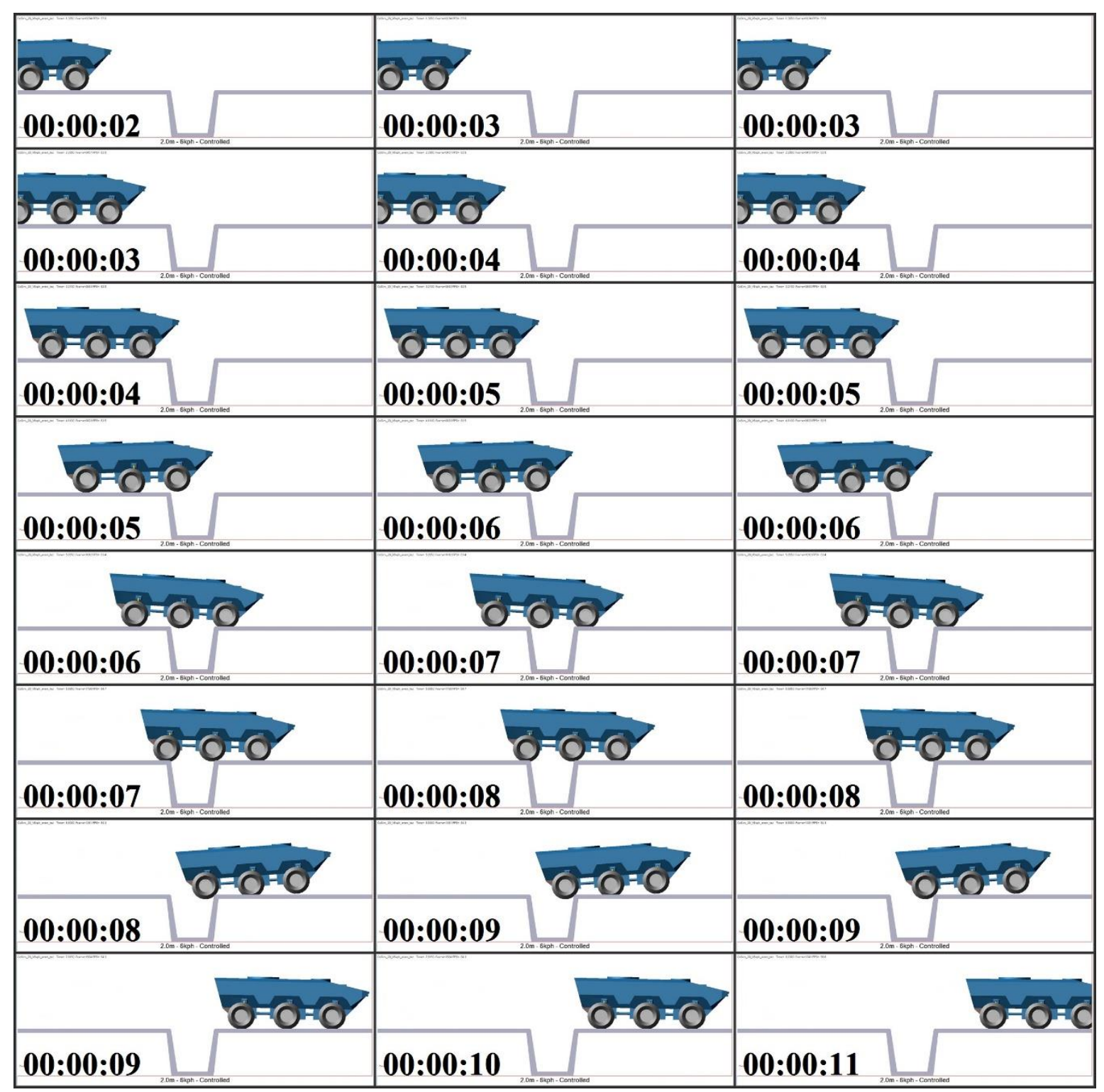

Figure A.104. Trench length 2.0 m, 6 kph, controlled system 

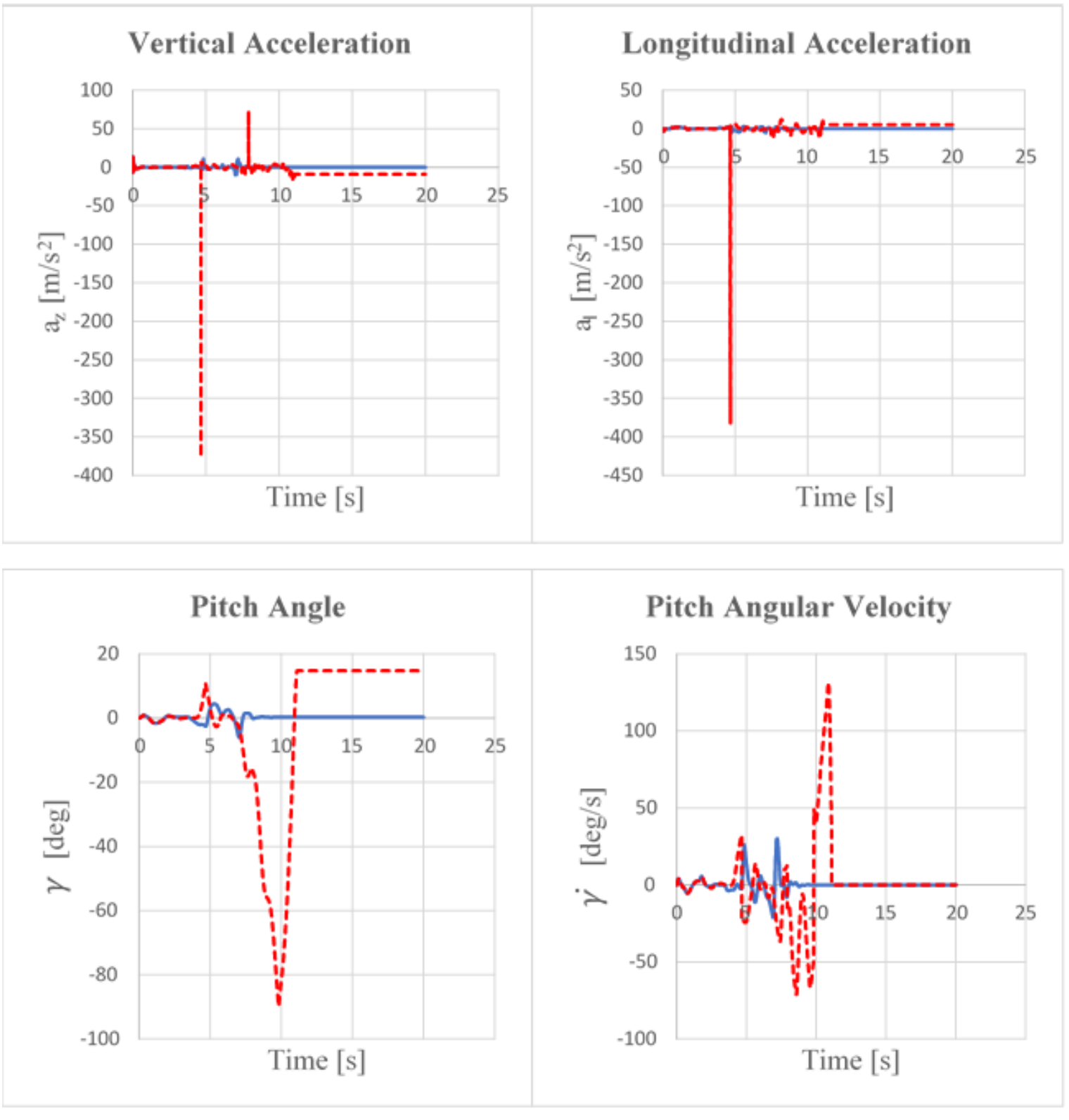

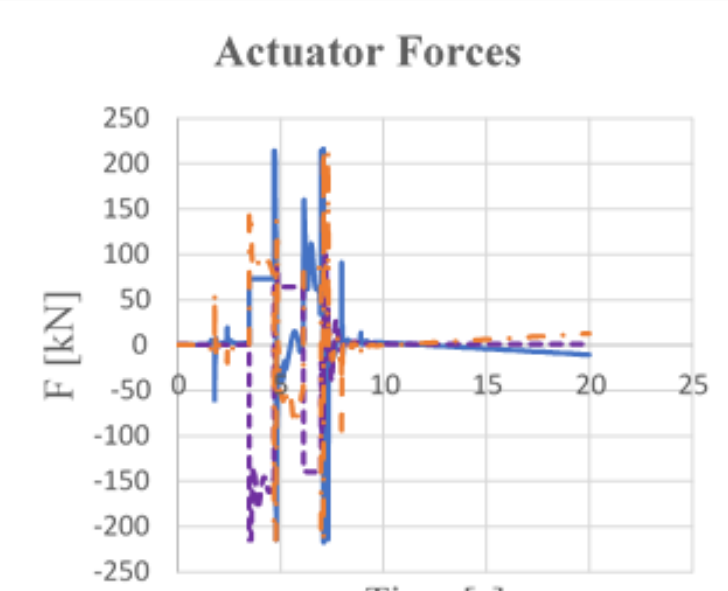

Time $[\mathrm{s}]$

- Axle 1 ----. Axle 2 - - Axle 3

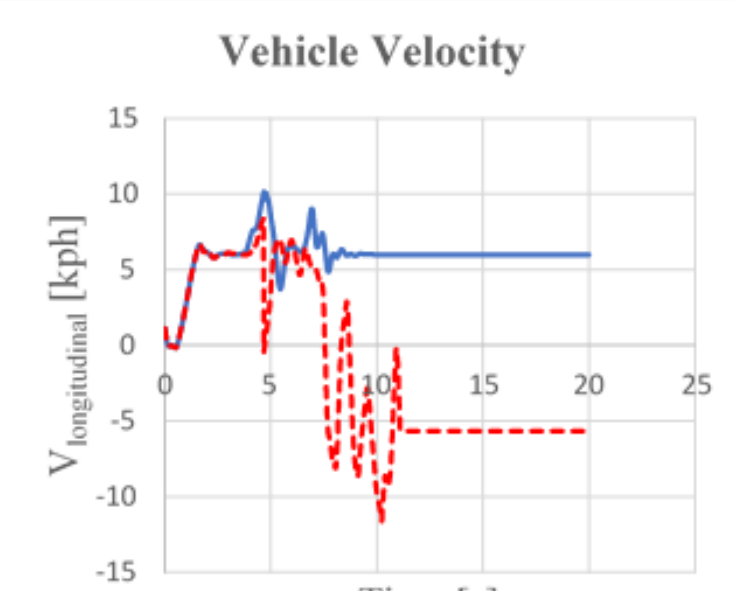

Time [s]

Controlled ----.. Uncontrolled

Figure A.105. Trench length $2.0 \mathrm{~m}, 6 \mathrm{kph}$, controlled vs uncontrolled output comparison 
Trench Length $2.0 \mathrm{~m}, 9 \mathrm{kph}$

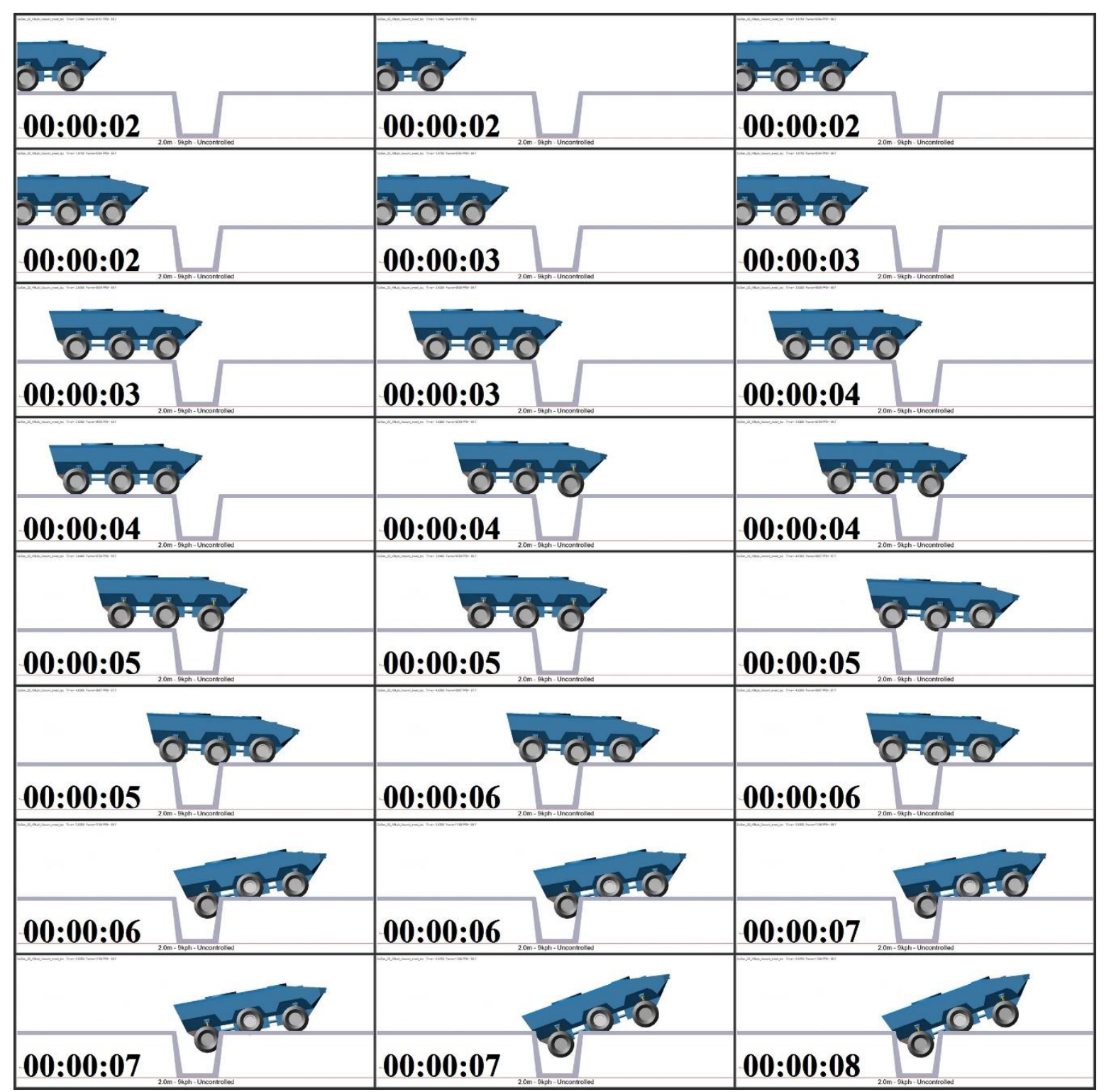

Figure A.106. Trench length $2.0 \mathrm{~m}, 9 \mathrm{kph}$, uncontrolled system 


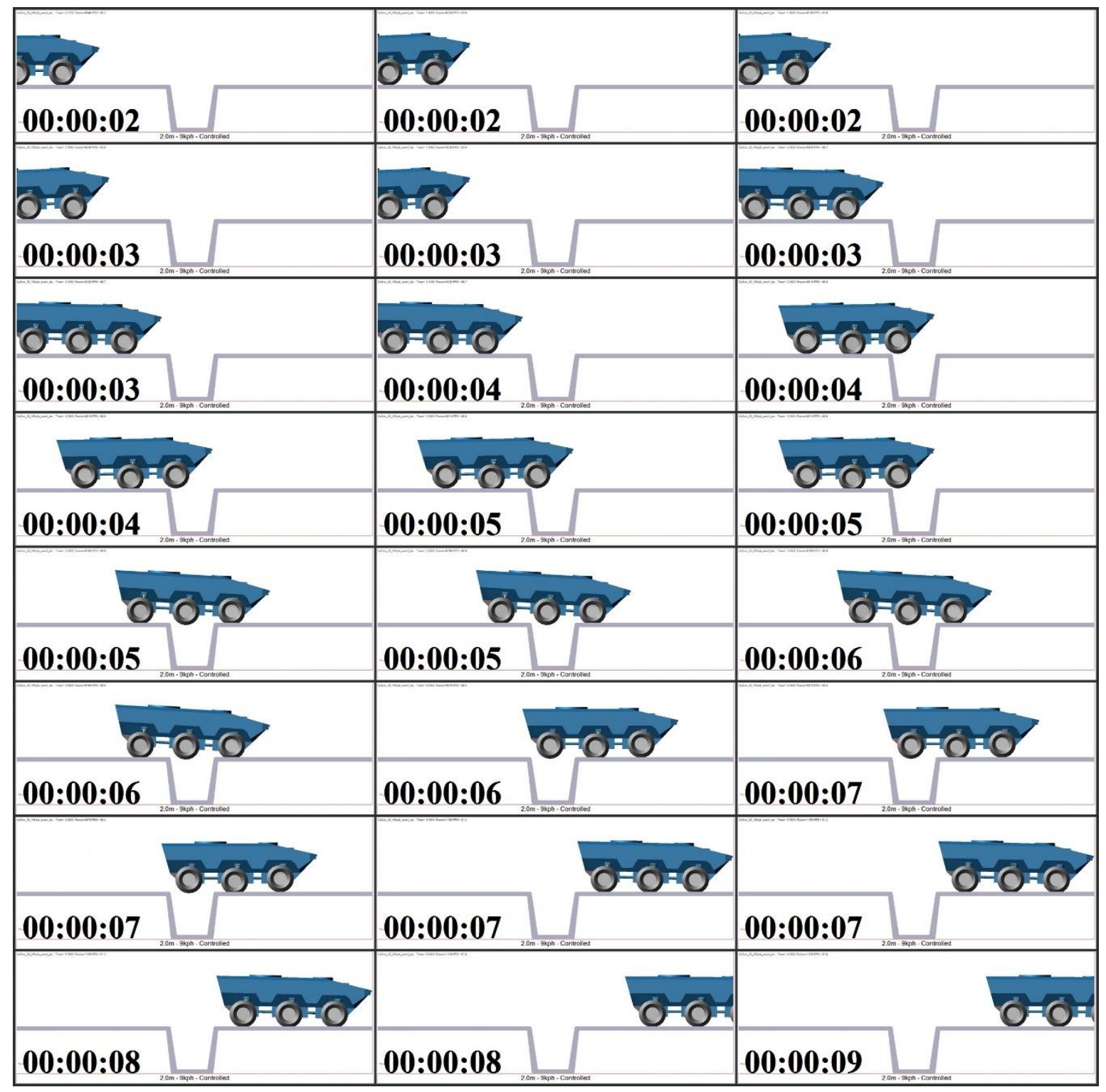

Figure A.107. Trench length $2.0 \mathrm{~m}, 9 \mathrm{kph}$, controlled system 

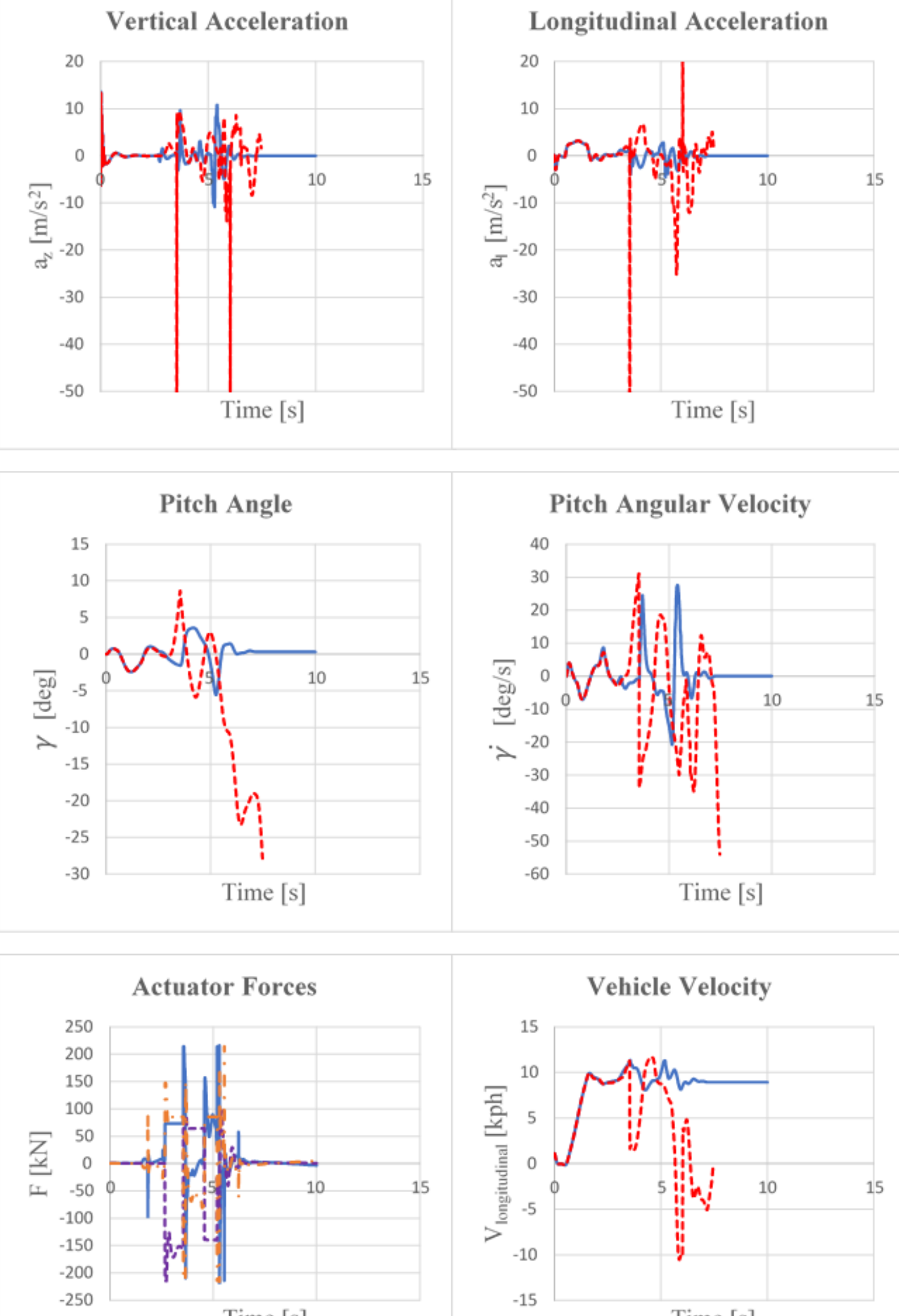

Time $[\mathrm{s}]$

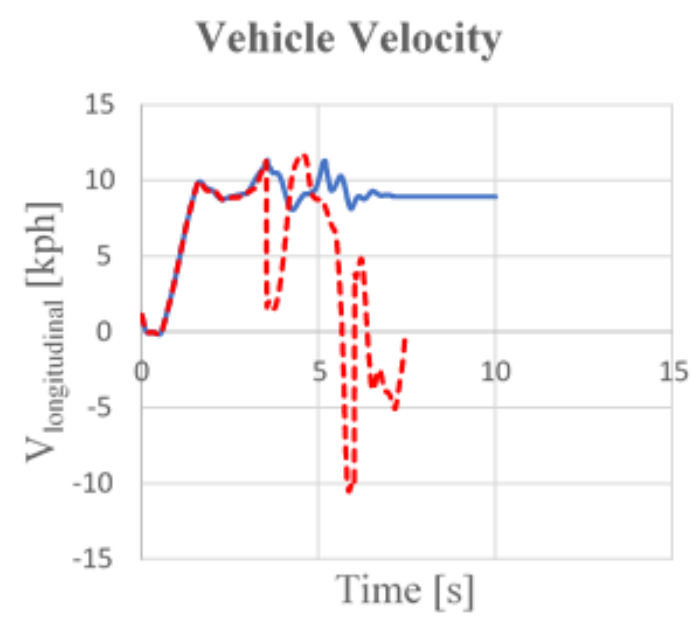

—Axle 1 ----. Axle 2 - - Axle 3

Controlled -----. Uncontrolled

Figure A.108. Trench length $2.0 \mathrm{~m}, 9 \mathrm{kph}$, controlled vs uncontrolled output comparison 


\section{APPENDIX B}

\section{CREATING THE LINEAR SYSTEM MODEL}

\section{Parameter Definition}

$\%$ TRACK WIDTH DEFINITIONS

syms tw1r tw1l tw2r tw2l tw3r tw31

\% tw1r : 1st Axle Right Half Track Width

$\%$ tw1l : 1st Axle Left Half Track Width

$\%$ tw2r : 2nd Axle Right Half Track Width

$\%$ tw2l : 2nd Axle Left Half Track Width

$\%$ tw3r : 3rd Axle Right Half Track Width

$\%$ tw3l : 3rd Axle Left Half Track Width

$\%$ WHEELBASE DEFINITIONS

syms CoGx Wb12 Wb23

\% CoGx : Distance from 1st Axle to Center of Gravity

$\%$ Wb12 : Distance from 1st Axle to 2nd Axle

$\%$ Wb23 : Distance from 2nd Axle to 3rd Axle

$\%$ WEIGHT DEFINITIONS

syms ms Jx Jy

syms mus1r mus1l mus2r mus21 mus3r mus31

$\% \mathrm{~ms}$ : Sprung Mass

$\%$ Jx : Inertia about $X$ axis (Roll)

$\%$ Jy : Inertia about $Y$ axis (Pitch)

\% mus1r : 1st Axle Right Unsprung Mass

$\%$ mus1l : 1st Axle Left Unsprung Mass

$\%$ mus2r : 2nd Axle Right Unsprung Mass

\% mus2l : 2nd Axle Left Unsprung Mass

$\%$ mus3r : 3rd Axle Right Unsprung Mass

$\%$ mus31 : 3rd Axle Left Unsprung Mass

$\%$ BODY BOUNCE \& EULER ANGLES

syms zs theta gamma

$\%$ zS : Body Bounce (+) for upwards

\% theta: Body Roll (+) for lean left

\% gamma: Body Pitch (+) for pitch up front 
$\%$ These are temporary variables, not state variables. $\%$ Will be defined below.

syms zs1r zs1l zs2r zs2l zs3r zs31

\% zs1r : 1st Axle Right Sprung Mass Vertical Displacement \% zs1l : 1st Axle Left Sprung Mass Vertical Displacement \% zs2r : 2nd Axle Right Sprung Mass Vertical Displacement \% zs2l : 2nd Axle Left Sprung Mass Vertical Displacement \% zs3r : 3rd Axle Right Sprung Mass Vertical Displacement \% zs31 : 3rd Axle Left Sprung Mass Vertical Displacement

syms zs1r_dot zs1l_dot zs2r_dot zs21_dot zs3r_dot zs31_dot

\% zs1r_dot : 1st Axle Right Sprung Mass Vertical Velocity

\% zs1l_dot : 1st Axle Left Sprung Mass Vertical Velocity

\% zs2r_dot : 2nd Axle Right Sprung Mass Vertical Velocity

\% zs2l_dot : 2nd Axle Left Sprung Mass Vertical Velocity

\% zs3r_dot : 3rd Axle Right Sprung Mass Vertical Velocity

\% zs3l_dot : 3rd Axle Left Sprung Mass Vertical Velocity

syms zs1r_ddot zs11_ddot zs2r_ddot zs21_ddot zs3r_ddot zs31_ddot

\% zs1r_ddot : 1st Axle Right Sprung Mass Vertical Acceleration

\% zs1l_ddot : 1st Axle Left Sprung Mass Vertical Acceleration

\% zs2r_ddot : 2nd Axle Right Sprung Mass Vertical Acceleration

\% zs2l_ddot : 2nd Axle Left Sprung Mass Vertical Acceleration

\% zs3r_ddot : 3rd Axle Right Sprung Mass Vertical Acceleration

\% zs3l_ddot : 3rd Axle Left Sprung Mass Vertical Acceleration

syms zus1r zus1l zus2r zus2l zus3r zus3l

\% zus1r : 1st Axle Right Unsprung Mass Vertical Displacement

\% zus1l : 1st Axle Left Unsprung Mass Vertical Displacement

\% zus2r : 2nd Axle Right Unsprung Mass Vertical Displacement

$\%$ zus2l : 2nd Axle Left Unsprung Mass Vertical Displacement

\% zus3r : 3rd Axle Right Unsprung Mass Vertical Displacement

\% zus3l : 3rd Axle Left Unsprung Mass Vertical Displacement

syms zus1r_dot zus1l_dot zus2r_dot zus2l_dot zus3r_dot zus31_dot

\% zus1r_dot : 1st Axle Right Unsprung Mass Vertical Velocity

\% zus1l_dot : 1st Axle Left Unsprung Mass Vertical Velocity

\% zus2r_dot : 2nd Axle Right Unsprung Mass Vertical Velocity

\% zus2l_dot : 2nd Axle Left Unsprung Mass Vertical Velocity

\% zus3r_dot : 3rd Axle Right Unsprung Mass Vertical Velocity

\% zus3l_dot : 3rd Axle Left Unsprung Mass Vertical Velocity 
syms zus1r_ddot zus1l_ddot zus2r_ddot zus21_ddot zus3r_ddot zus31_ddot

\% zus1r_ddot : 1st Axle Right Unsprung Mass Vertical Acceleration

\% zus1l_ddot : 1st Axle Left Unsprung Mass Vertical Acceleration

$\%$ zus2r_ddot : 2nd Axle Right Unsprung Mass Vertical Acceleration

\% zus21_ddot : 2nd Axle Left Unsprung Mass Vertical Acceleration

$\%$ zus3r_ddot : 3rd Axle Right Unsprung Mass Vertical Acceleration

\% zus31_ddot : 3rd Axle Left Unsprung Mass Vertical Acceleration

syms zs_dot theta_dot gamma_dot

$\%$ zs_dot : Body Bounce velocity

\% theta_dot: Body Roll Angular Velocity

\% gamma_dot: Body Pitch Angular Velocity

syms zs_ddot theta_ddot gamma_ddot

$\%$ zs_ddot : Body Bounce Acceleration

\% theta_ddot: Body Roll Angular Acceleration

\% gamma_ddot: Body Pitch Angular Acceleration

syms z01r z011 z02r z021 z03r z031

\% z01r : 1st Axle Right Road Input Displacement

$\%$ z011 : 1st Axle Left Road Input Displacement

$\%$ zO2r : 2nd Axle Right Road Input Displacement

$\%$ z021 : 2nd Axle Left Road Input Displacement

\% z03r : 3rd Axle Right Road Input Displacement

\% z031 : 3rd Axle Left Road Input Displacement

syms z01r_dot z011_dot z02r_dot z021_dot z03r_dot z031_dot

$\%$ z01r_dot : 1st Axle Right Road Input Velocity

\% z011_dot : 1st Axle Left Road Input Velocity

$\%$ z02r_dot : 2nd Axle Right Road Input Velocity

$\%$ z02I_dot : 2nd Axle Left Road Input Velocity

$\%$ z03r_dot : 3rd Axle Right Road Input Velocity

\% z031_dot : 3rd Axle Left Road Input Velocity

syms z01r_ddot z011_ddot z02r_ddot z021_ddot z03r_ddot z031_ddot

\% z01r_ddot : 1st Axle Right Road Input Acceleration

\% z011_ddot : 1st Axle Left Road Input Acceleration

$\%$ z02r_ddot : 2nd Axle Right Road Input Acceleration

$\%$ z02l_ddot : 2nd Axle Left Road Input Acceleration

$\%$ z03r_ddot : 3rd Axle Right Road Input Acceleration

\% z031_ddot : 3rd Axle Left Road Input Acceleration

$\%$ FORCE DEFINITIONS 
\% Ft1r : 1st Axle Right Tire Force

\% Ft1l : 1st Axle Left Tire Force

$\%$ Ft2r : 2nd Axle Right Tire Force

$\%$ Ft2l : 2nd Axle Left Tire Force

$\% \mathrm{Ft} 3 \mathrm{r}$ : 3rd Axle Right Tire Force

$\%$ Ft3l : 3rd Axle Left Tire Force

syms Fs1r Fs1l Fs2r Fs2l Fs3r Fs31

\% Fs1r : 1st Axle Right Suspension Force

\% Fs1l : 1st Axle Left Suspension Force

$\%$ Fs2r : 2nd Axle Right Suspension Force

$\%$ Fs2l : 2nd Axle Left Suspension Force

$\% \mathrm{Fs3r}$ : 3rd Axle Right Suspension Force

\% Fs31 : 3rd Axle Left Suspension Force

g_eqn=0; $\quad \%$ Gravity for equations

$\%$ _TIRE STIFFNESS

syms kt1r kt1l kt2r kt2l kt3r kt3l

$\%$ kt1r : 1st Axle Right Tire Stiffness [N/m]

$\%$ kt1l : 1st Axle Left Tire Stiffness [N/m]

$\%$ kt2r : 2nd Axle Right Tire Stiffness [N/m]

$\%$ kt2l : 2nd Axle Left Tire Stiffness [N/m]

$\%$ kt3r : 3rd Axle Right Tire Stiffness [N/m]

$\%$ kt3l : 3rd Axle Left Tire Stiffness [N/m]

syms ct1r ct1l ct2r ct2l ct3r ct3l

\% ct1r : 1st Axle Right Tire Damping Coefficient [Ns/m]

\% ct1l : 1st Axle Left Tire Damping Coefficient [Ns/m]

\% ct2r : 2nd Axle Right Tire Damping Coefficient [Ns/m]

\% ct21 : 2nd Axle Left Tire Damping Coefficient [Ns/m]

\% ct3r : 3rd Axle Right Tire Damping Coefficient [Ns/m]

\% ct3l : 3rd Axle Left Tire Damping Coefficient [Ns/m]

syms ks1r ks1l ks2r ks2l ks3r ks3l

\% ks1r : 1st Axle Right Suspension Stiffness [N/m]

$\%$ ks1l : 1st Axle Left Suspension Stiffness [N/m]

$\% \mathrm{ks} 2 \mathrm{r}$ : 2nd Axle Right Suspension Stiffness [N/m]

$\%$ ks21 : 2nd Axle Left Suspension Stiffness [N/m]

$\% \mathrm{ks} 3 \mathrm{r}$ : 3rd Axle Right Suspension Stiffness [N/m]

$\%$ ks3l : 3rd Axle Left Suspension Stiffness [N/m]

syms cs1r cs1l cs2r cs2l cs3r cs31 
\% cs1r : 1st Axle Right Suspension Damping Coefficient [Ns/m]

\% cs1l : 1st Axle Left Suspension Damping Coefficient [Ns/m]

\% cs2r : 2nd Axle Right Suspension Damping Coefficient [Ns/m]

\% cs21 : 2nd Axle Left Suspension Damping Coefficient [Ns/m]

\% cs3r : 3rd Axle Right Suspension Damping Coefficient [Ns/m]

\% cs31 : 3rd Axle Left Suspension Damping Coefficient [Ns/m]

syms F_HS_1r F_HS_1l F_HS_2r F_HS_2l F_HS_3r F_HS_3I

\% F_HS_1r : 1st Axle Right Hydrostrut Force [N]

\% F_HS_1l : 1st Axle Left Hydrostrut Force [N]

$\%$ F_HS_2r : 2nd Axle Right Hydrostrut Force [N]

$\%$ F_HS_21 : 2nd Axle Left Hydrostrut Force [N]

$\%$ F_HS_3r : 3rd Axle Right Hydrostrut Force [N]

\% F_HS_31 : 3rd Axle Left Hydrostrut Force [N]

$\%$

STATES

states $=[$ zus1r zus1r_dot zus1l zus11_dot...

zus2r zus2r_dot zus2l zus2l_dot...

zus3r zus3r_dot zus31 zus3l_dot...

zs zs_dot theta theta_dot gamma gamma_dot];

disturbances $=[z 01 r$ z01r_dot z011 z011_dot...

z02r z02r_dot z021 z021_dot...

z03r z03r_dot z031 z031_dot];

inputs $=[$ F_HS_1r F_HS_1l F_HS_2r F_HS_2l F_HS_3r F_HS_3l]; 


\section{Equations of Motion}

Linearization regarding small angle assumption:

$\sin ($ theta $)=$ theta

$\sin ($ gamma $)=$ gamma

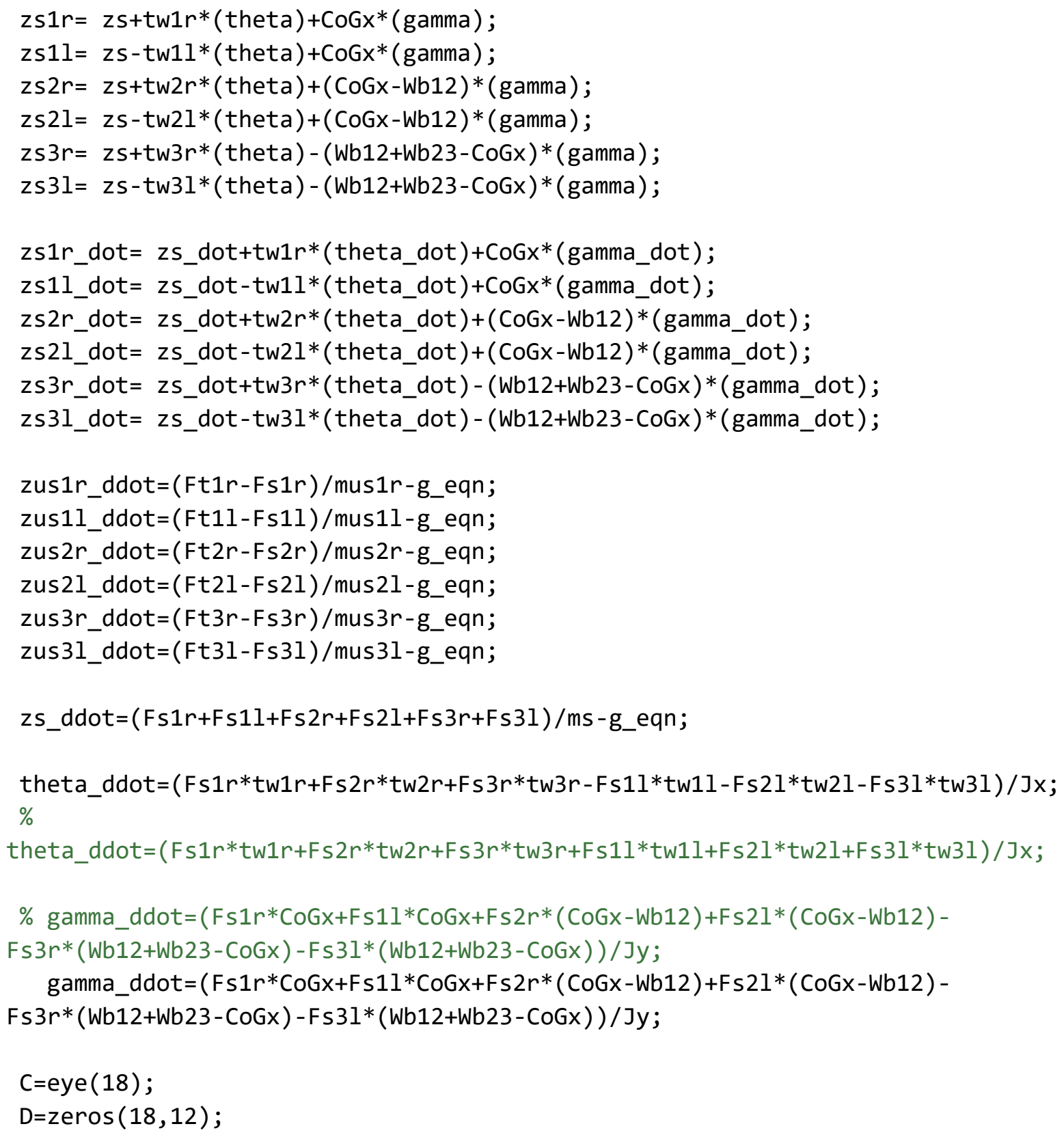




\section{Force Expressions}

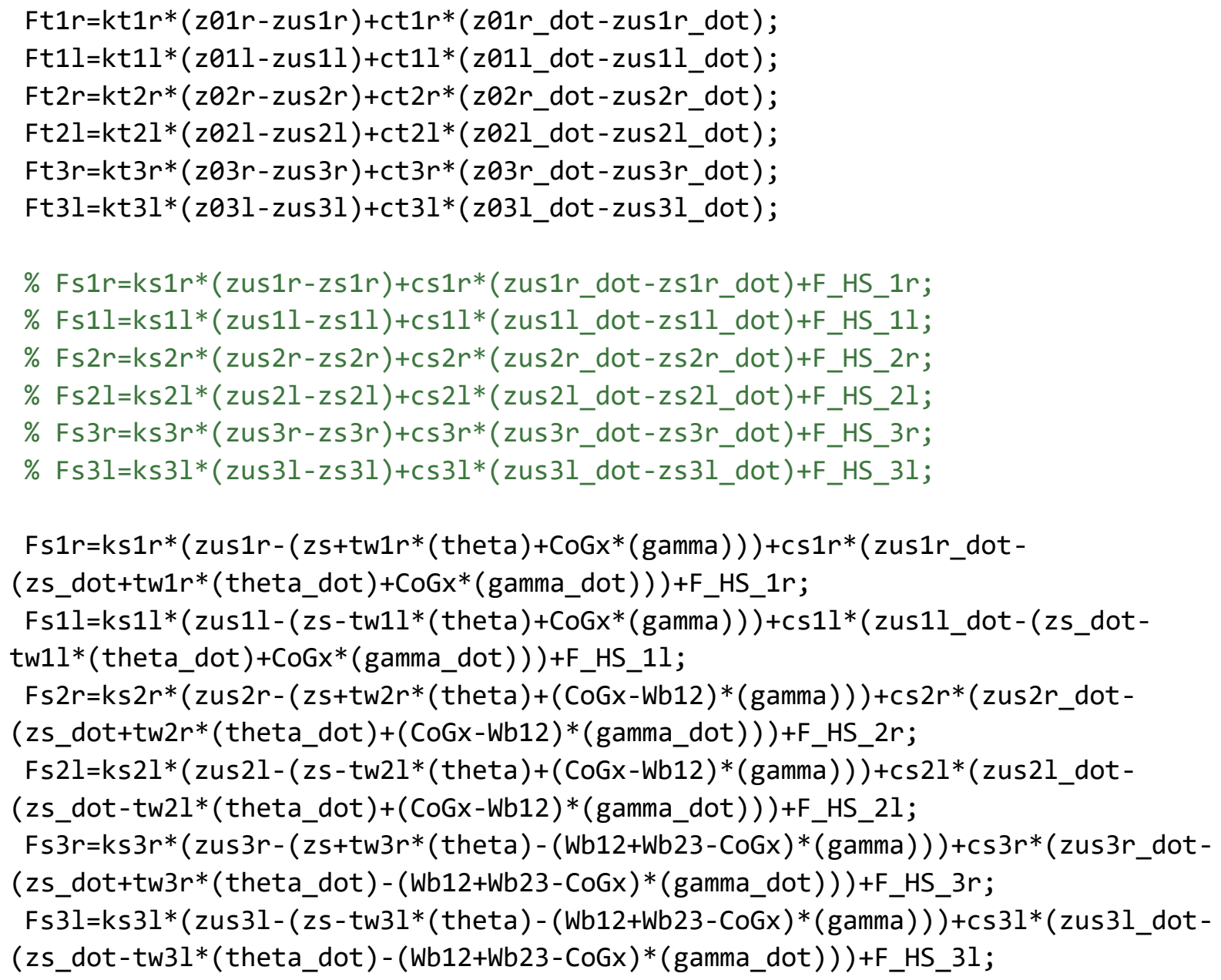

\section{Expand Expressions}

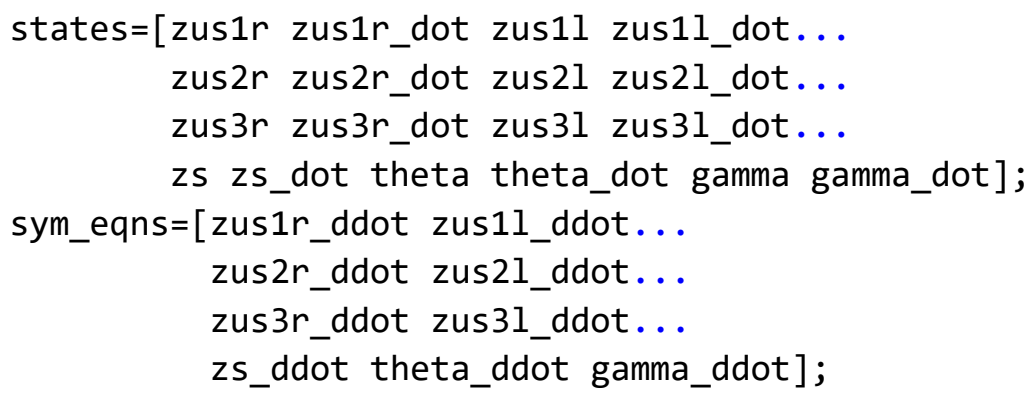




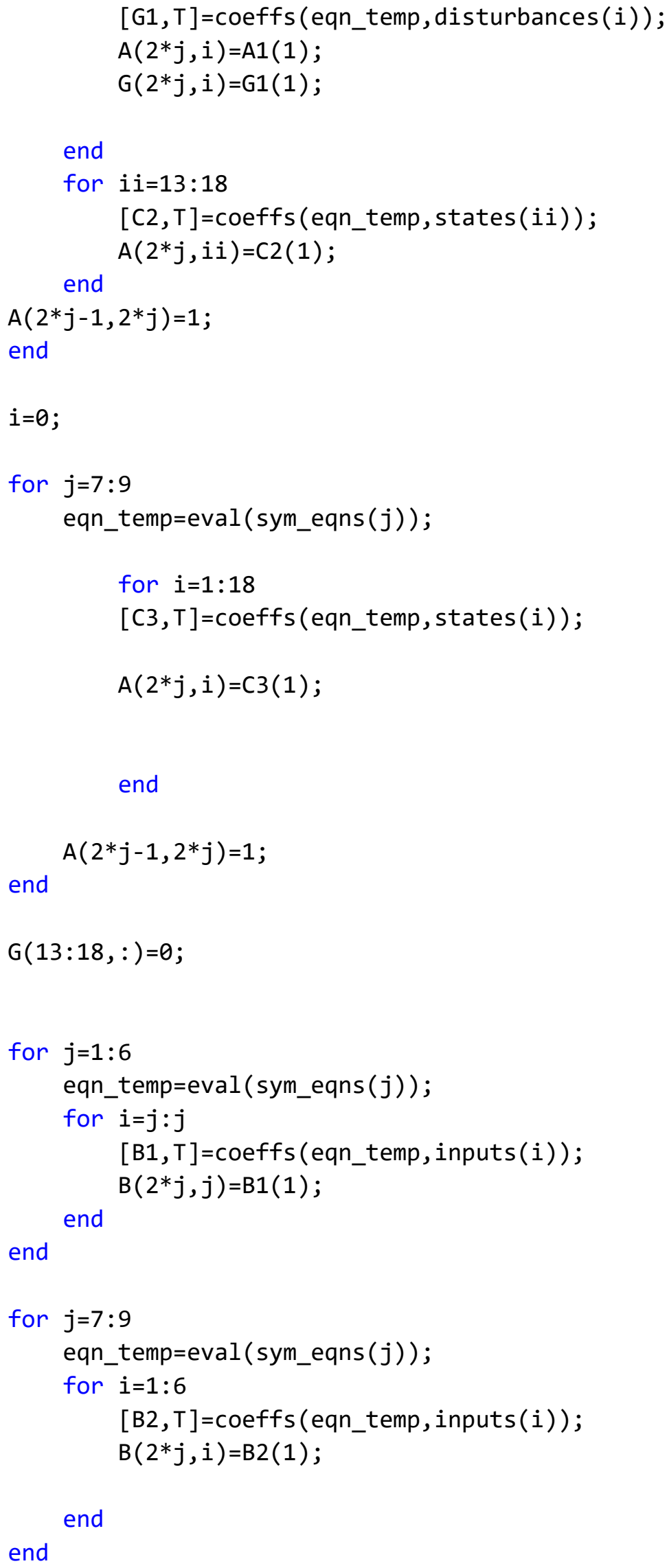




\section{Numeric Evaluation of "A" Matrix}

$\% \%$ PART A. VEHICLE PARAMETERS

$\%$ Tire Stiffness

kt1r $=-($ Confidential $)$;

kt1l= - (Confidential);

$k t 2 r=-($ Confidential)

kt2l= - (Confidential);

$k t 3 r=-($ Confidential);

kt3l= - (Confidential);

\% Tire Damping Coeffs

ct1r $=$ - (Confidential);

ct1l= - (Confidential);

ct $2 r=-($ Confidential $)$;

ct2l= - (Confidential);

ct3r $=$ - (Confidential);

ct3l= - (Confidential);

\% Strut Stiffness

ks1r = - (Confidential);

ks1l= - (Confidential);

$k s 2 r=-($ Confidential);

ks2l= - (Confidential);

$\mathrm{ks} 3 \mathrm{r}=-($ Confidential $)$;

ks3l= - (Confidential);

\% Strut Damping Coeffs

cs1r = - (Confidential);

cs1l= - (Confidential);

$\operatorname{cs} 2 r=-($ Confidential);

$\operatorname{cs} 21=-($ Confidential);

cs3r = - (Confidential);

cs3l= - (Confidential);

\% Track Widths

tw1r = - (Confidential);

tw1l= - (Confidential);

tw2r $=$ - (Confidential);

tw2l= - (Confidential);

tw3r = - (Confidential);

tw $31=-($ Confidential);

\% Masses

mus1r = - (Confidential);

mus1l= - (Confidential);

mus2r $=$ - (Confidential);

mus2l= - (Confidential);

mus3r = - (Confidential); 


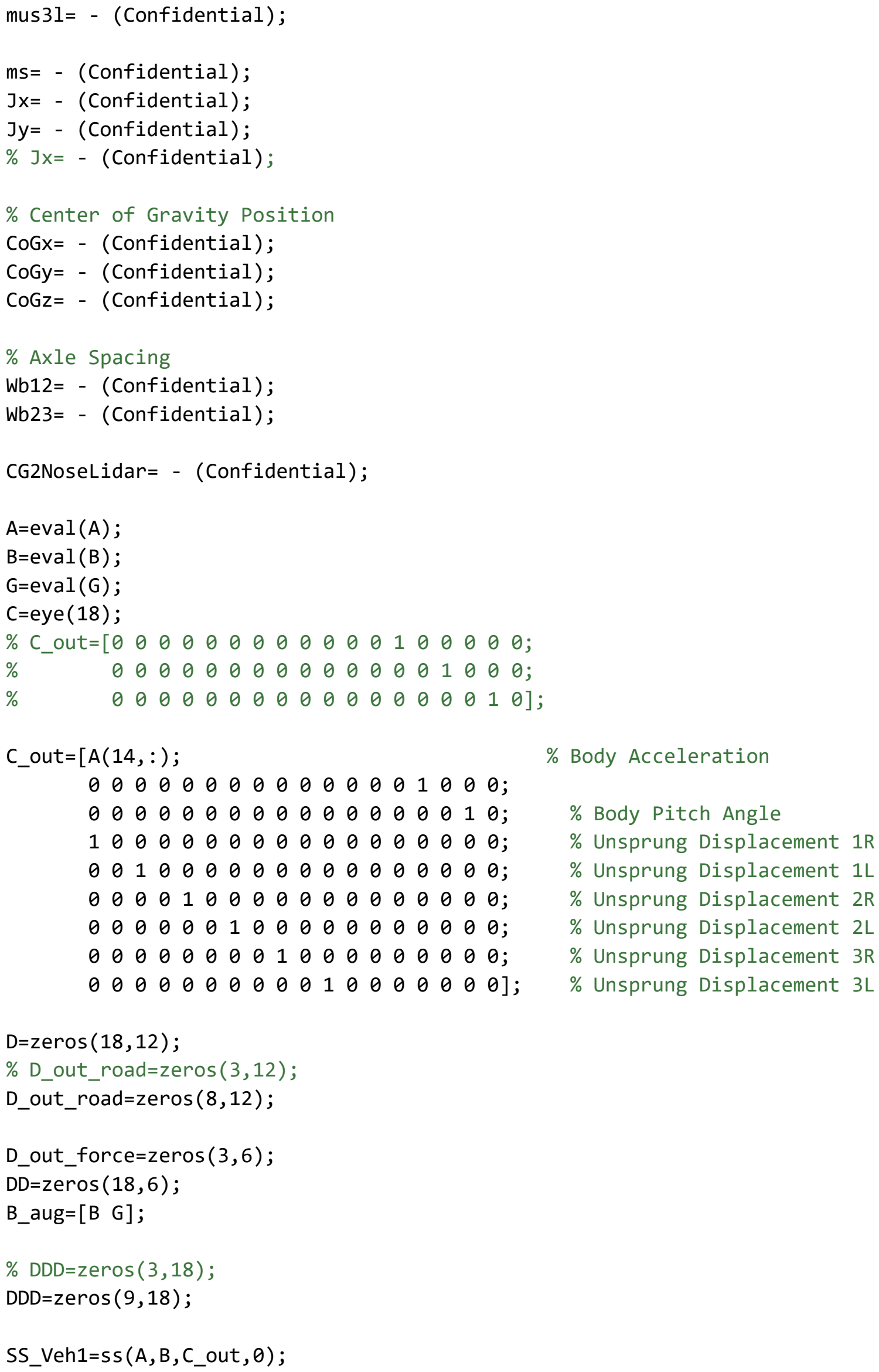




\section{Discrete Time}

$\% \mathrm{Ts}=0.01$;

$\%[A d, B d, C d, D d]=c 2 d m\left(A, B \_a u g, C \_o u t, D D D, T s\right)$;

$\%$ sys_plant=ss (A, B, C_out, $0, T s)$;

$\%$ sys=minreal(sys_plant);

$\%$ [Ad_norm, Bd_norm, Cd_norm, Dd_norm] $=c 2 d m(A, B$, eye (18), DD, Ts );

$\%$ sys_norm $=s s(A, B$, eye $(18), D D, T s)$;

$\%$ [Ad_norm, Bd_norm, Cd_norm, Dd_norm $]=c 2 d m\left(A, B, C \_o u t, D \_o u t \_f o r c e, T s\right)$;

$\%$ LTI_sys=ss (Ad_norm, Bd_norm,Cd_norm,Dd_norm, Ts) ;

\% TF_LTI_sys=tf(LTI_sys);

\section{Run Simulation}

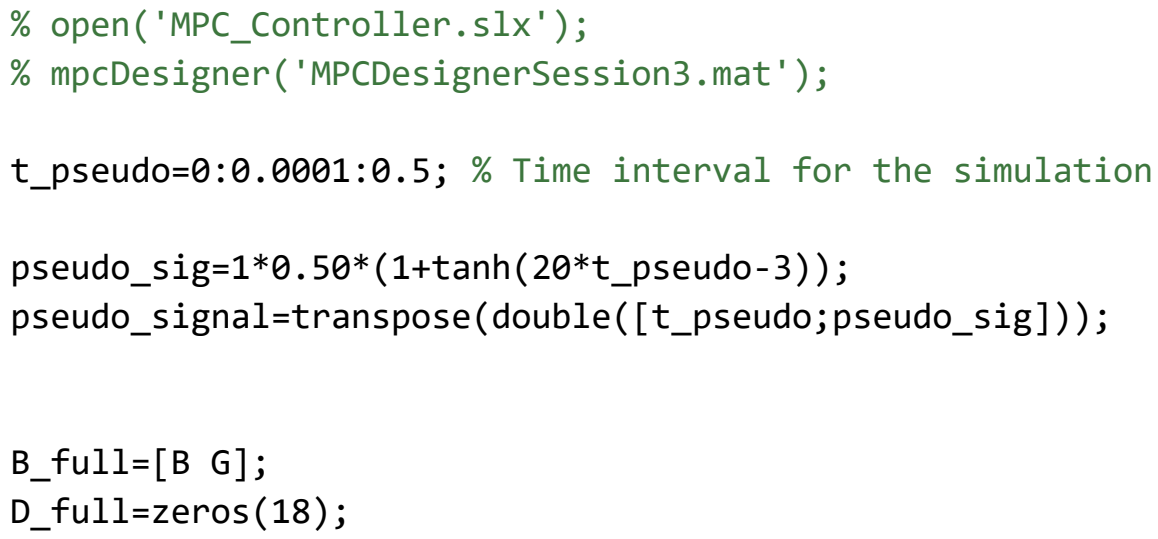




\section{CREATING MPC CONTROLLER}

\section{MPC Code}

$\%$ run SS_VehicleModel.mlx

clc;

\section{Load Files}

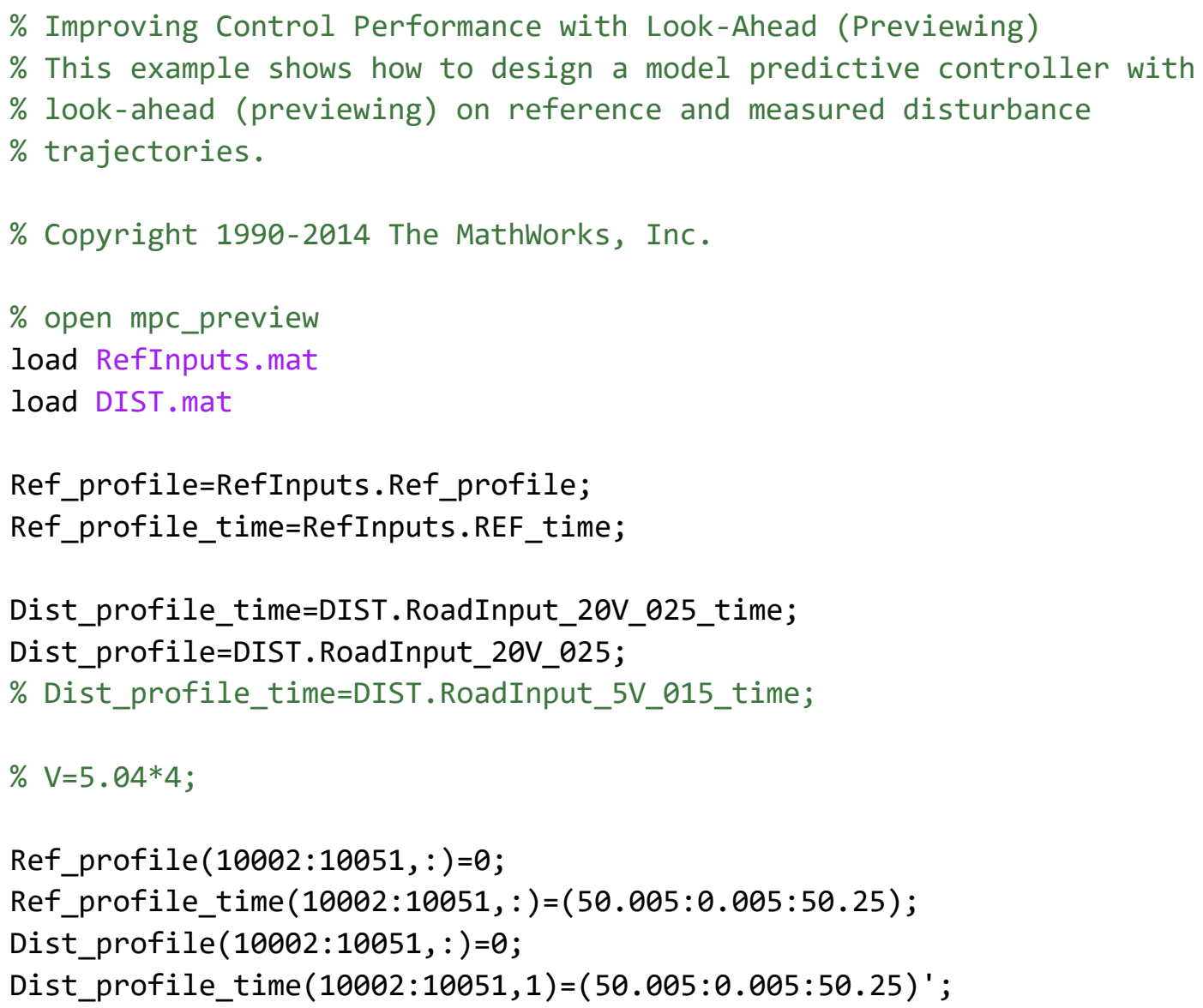

\section{Define Plant Model}

\% Define the plant model as a linear time invariant system with two inputs $\%$ (one manipulated variable and one measured disturbance) and one output. 


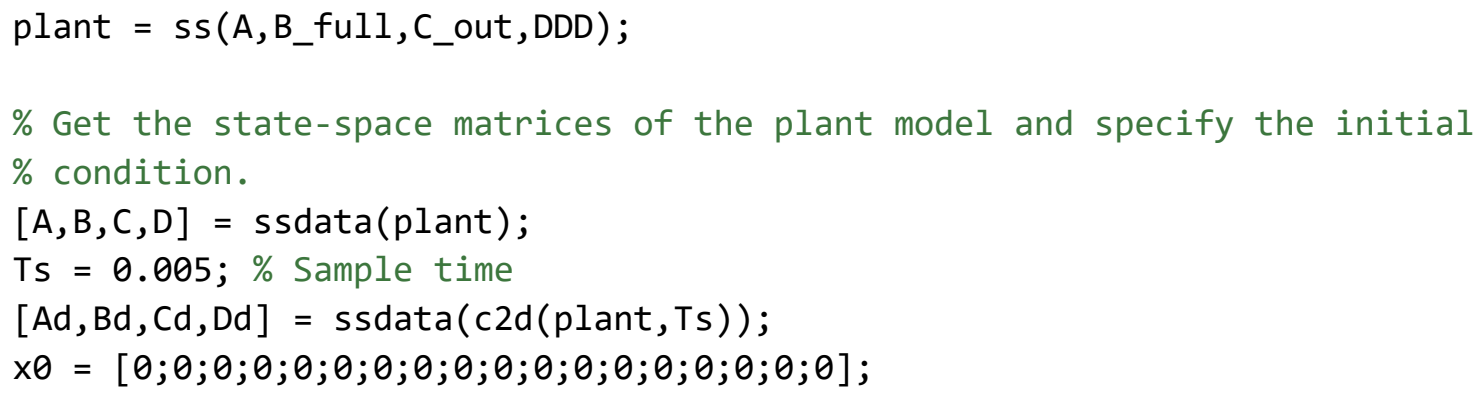

\section{Design Model Predictive Controller}

$\%$ Define type of input signals.

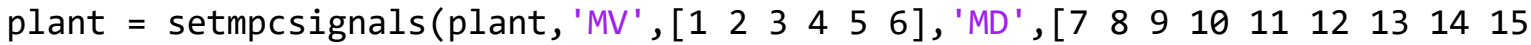
1617 18]);

\section{Create MPC Object}

$\%$ Create the MPC object.

$\mathrm{p}=50 ; \quad \%$ prediction horizon

$m=3 ; \quad \%$ control horizon

mpcobj $=m p c(p l a n t, T s, p, m)$;

\% Specify MV constraints.

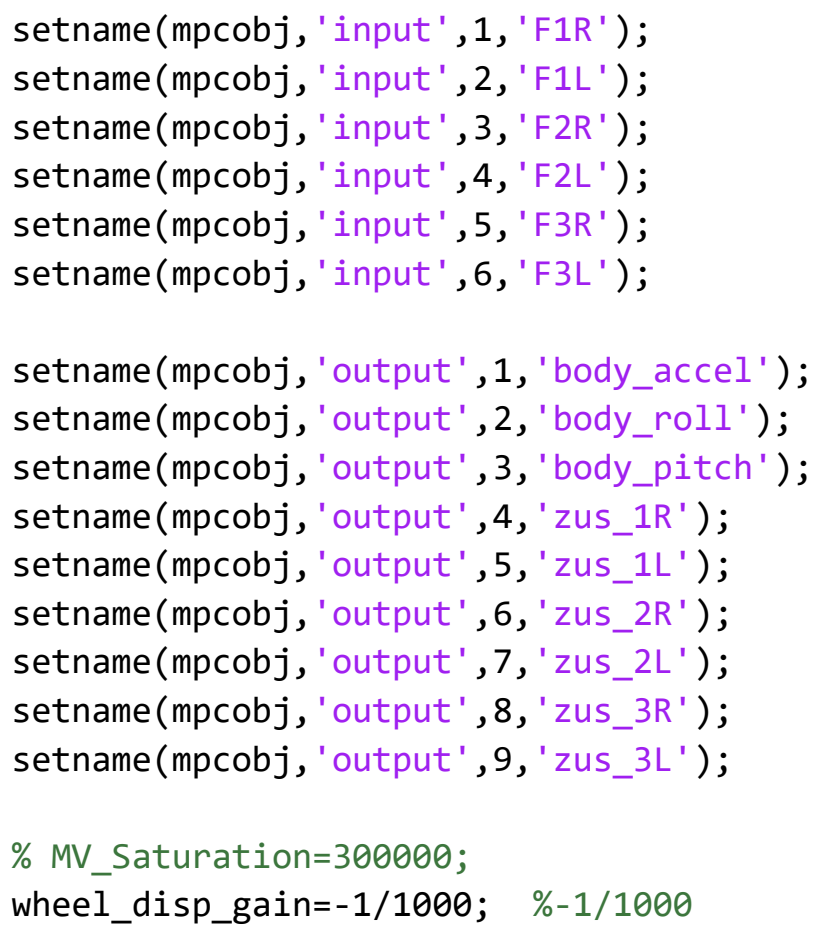




\section{Parameters}

MV_Limit $=$ - (Confidential);

MV_Limit_Rate $=$ - (Confidential);

MV_Limit_ECR $=0.001$;

MV_RateLimit_ECR $=0.001$;

Z_us_disp_limit $=0.3$;

OV_1_Limit=2; \% Body Accel $\mathrm{m} / \mathrm{s}^{\wedge} 2$

OV_2_Limit $=0.01$; \% Body Roll rad

OV_3_Limit $=0.1$; \% Body Pitch rad

OV_4_Limit=Z_us_disp_limit; $\%$ Z_us_1R

OV_5_Limit=Z_us_disp_limit; \% Z_us_1L

OV_6_Limit=Z_us_disp_limit; $\%$ Z_us_2R

OV_7_Limit $=Z$ _us_disp_limit; $\%$ Z_us_2L

OV_8_Limit $=Z$ _us_disp_limit; $\%$ Z_us_3R

ov_9_Limit=Z_us_disp_limit; $\%$ Z_us_3L

OV_1_LimitECR $=0.1 ; \%$ Body Accel m/s^2

OV_2_LimitECR $=0.01 ; \quad \%$ Body Roll rad

OV_3_LimitECR $=0.5$; $\%$ Body Pitch rad

OV_4_LimitECR $=0.1 ; \%$ Z_us_1R

OV_5_LimitECR $=0.1$; \% Z_us_1L

OV_6_LimitECR $=0.1 ; \%$ Z_us_2R

OV_7_LimitECR $=0.1 ; \%$ Z_us_2L

OV_8_LimitECR $=0.1 ; \%$ Z_us_3R

OV_9_LimitECR $=0.1 ; \%$ Z_us_3L

OV_1_ScaleFactor $=2 * 0 V$ 1_Limit; \% Body Accel m/ $\mathrm{s}^{\wedge} 2$

OV_2_ScaleFactor $=2 * 0 V \_2$ _imit; \% Body Roll rad

OV_3_ScaleFactor $=2 * 0 V$ _3_Limit; \% Body Pitch rad

OV_4_ScaleFactor $=1$; \% Z_us_1R

OV_5_ScaleFactor $=1 ; \%$ Z_us_1L

OV_6_ScaleFactor $=1$; \% Z_us_2R

OV_7_ScaleFactor $=1$; \% Z_us_2L

OV_8_ScaleFactor $=1$; \% Z_us_3R

OV_9_ScaleFactor $=1$; \% Z_us_3L

MV_Weight $=0.1$;

MV_RateWeight $=0.1$;

OV_1_Weight $=10 ; \%$ Body Accel $\mathrm{m} / \mathrm{s}^{\wedge} 2$

OV_2_Weight $=0.5$; \% Body Roll rad

OV_3_Weight $=50 * 2$; \% Body Pitch rad

OV_4_Weight $=0.4 ; \%$ Z_us_1R

OV_5_weight $=0.4 ; \%$ Z_us_1L 
OV_6_Weight $=0.4 ; \%$ Z_us_2R

OV_7_Weight $=0.4 ; \%$ Z_us_2L

OV_8_Weight $=0.4 ; \%$ Z_us_3R

OV_9_Weight $=0.4 ; \%$ Z_us_3L

WeightECR=1e3;

$\% * * * * * * * * * * * * * * * * * * * * * * * * * * * * * * * * * * * * * *$

mpcobj.MV(1).Min=-MV_Limit;

mpcobj.MV(2).Min=-MV_Limit;

mpcobj.MV(3).Min=-MV_Limit;

mpcobj.MV(4). Min=-MV_Limit;

mpcobj.MV(5).Min=-MV_Limit;

mpcobj.MV(6).Min=-MV_Limit;

mpcobj.MV(1).Max=MV_Limit;

mpcobj.MV (2).Max=MV_Limit;

mpcobj.MV (3).Max=MV_Limit;

mpcobj.MV(4).Max=MV_Limit;

mpcobj.MV(5).Max=MV_Limit;

mpcobj.MV(6).Max=MV_Limit;

mpcobj.MV(1).ScaleFactor=2*MV_Limit;

mpcobj.MV (2).ScaleFactor=2*MV_Limit;

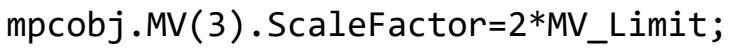

mpcobj.MV(4). ScaleFactor $=2 * M V \_L i m i t$;

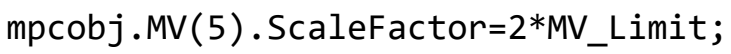

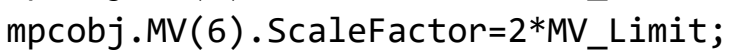

mpcobj.MV(1). RateMin=-MV_Limit_Rate;

mpcobj.MV(2). RateMin=-MV_Limit_Rate;

mpcobj.MV(3). RateMin=-MV_Limit_Rate;

mpcobj.MV (4). RateMin=-MV_Limit_Rate;

mpcobj.MV(5). RateMin=-MV_Limit_Rate;

mpcobj.MV (6). RateMin=-MV_Limit_Rate;

mpcobj.MV(1). RateMax=MV_Limit_Rate;

mpcobj.MV(2). RateMax=MV_Limit_Rate;

mpcobj.MV (3). RateMax=MV_Limit_Rate;

mpcobj.MV (4). RateMax=MV_Limit_Rate;

mpcobj.MV(5). RateMax=MV_Limit_Rate;

mpcobj.MV(6). RateMax=MV_Limit_Rate;

mpcobj.MV(1).MinECR=MV_Limit_ECR;

mpcobj.MV(2).MinECR=MV_Limit_ECR;

mpcobj.MV(3).MinECR=MV_Limit_ECR;

mpcobj.MV(4). MinECR=MV_Limit_ECR;

mpcobj.MV(5).MinECR=MV_Limit_ECR;

mpcobj.MV (6).MinECR=MV_Limit_ECR;

mpcobj.MV (1).MaxECR=MV_Limit_ECR;

mpcobj.MV(2).MaxECR=MV_Limit_ECR; 
mpcobj.MV (3).MaxECR=MV_Limit_ECR;

mpcobj.MV (4).MaxECR=MV_Limit_ECR;

mpcobj.MV(5).MaxECR=MV_Limit_ECR;

mpcobj.MV(6).MaxECR=MV_Limit_ECR;

mpcobj.MV (1).RateMinECR=MV_RateLimit_ECR;

mpcobj.MV (2). RateMinECR=MV_RateLimit_ECR;

mpcobj.MV (3). RateMinECR=MV_RateLimit_ECR;

mpcobj.MV (4). RateMinECR=MV_RateLimit_ECR;

mpcobj.MV (5). RateMinECR=MV_RateLimit_ECR;

mpcobj.MV (6). RateMinECR=MV_RateLimit_ECR;

mpcobj.MV (1).RateMaxECR=MV_RateLimit_ECR;

mpcobj.MV (2). RateMaxECR=MV_RateLimit_ECR;

mpcobj.MV (3).RateMaxECR=MV_RateLimit_ECR;

mpcobj.MV (4). RateMaxECR=MV_RateLimit_ECR;

mpcobj.MV (5). RateMaxECR=MV_RateLimit_ECR;

mpcobj.MV(6). RateMaxECR=MV_RateLimit_ECR;

mpcobj.OutputVariables(1).Min=-OV_1_Limit; mpcobj. OutputVariables (2). Min $=-0 V_{-}{ }_{2}$ Limit; mpcobj.OutputVariables (3). Min=-OV_3_Limit; mpcobj. OutputVariables (4). Min=-OV_4_Limit; mpcobj.OutputVariables(5).Min=-OV_5_Limit; mpcobj.OutputVariables (6).Min=-OV_6_Limit; mpcobj.OutputVariables (7).Min=-OV_7_Limit; mpcobj.OutputVariables (8).Min=-OV_8_Limit; mpcobj.OutputVariables (9).Min=-OV_9_Limit;

mpcobj . OutputVariables (1).Max=0V_1_Limit; mpcobj. OutputVariables(2). Max=0V_2_Limit; mpcobj. OutputVariables (3). Max $=0$ __3_Limit; mpcobj. OutputVariables (4).Max=0V_4_Limit; mpcobj.OutputVariables (5).Max=0V_5_Limit; mpcobj. OutputVariables (6). Max=0V_6_Limit; mpcobj. OutputVariables (7). Max=0V_7_Limit; mpcobj. OutputVariables (8). Max=0V_8_Limit; mpcobj . OutputVariables (9).Max=0V_9_Limit;

mpcobj.OutputVariables(1).MinECR=OV_1_LimitECR; mpcobj.OutputVariables (2).MinECR $=0 \mathrm{~V} \_2$ LimitECR; mpcobj.OutputVariables (3).MinECR $=0 V$ _3_LimitECR; mpcobj. OutputVariables (4). MinECR=OV_4_LimitECR; mpcobj. OutputVariables (5).MinECR=0V_5_LimitECR; mpcobj.OutputVariables (6).MinECR=OV_6_LimitECR; mpcobj. OutputVariables (7).MinECR=0V_7_LimitECR; mpcobj. OutputVariables (8). MinECR=OV_8_LimitECR; mpcobj.OutputVariables (9).MinECR=0V_9_LimitECR;

$\%$ Body Accel m/s^2

\% Body Roll rad

\% Body Pitch rad

$\%$ Z_us_1R

$\%$ Z_us_1L

$\%$ Z_us_2R

$\%$ Z_us_2L

$\%$ Z_us_3R

$\%$ Z_us_3L

$\%$ Body Accel m/s^2

$\%$ Body Roll rad 
mpcobj.OutputVariables (3).MaxECR=0V_3_LimitECR; mpcobj.OutputVariables (4).MaxECR=OV_4_LimitECR; mpcobj.OutputVariables (5).MaxECR=OV_5_LimitECR; mpcobj . OutputVariables (6). MaxECR=OV_6_LimitECR; mpcobj.OutputVariables (7). MaxECR=0V_7_LimitECR; mpcobj. OutputVariables (8). MaxECR=OV_8_LimitECR; mpcobj . OutputVariables (9).MaxECR=0V_9_LimitECR;

\% Body Pitch rad

$\%$ Z_us_1R

$\%$ Z_us_ 1 L

$\%$ Z_us_2R

$\%$ Z_us_2L

$\%$ Z_us_3R

$\%$ Z_us_3L

mpcobj.OutputVariables(1).ScaleFactor=0V_1_ScaleFactor; mpcobj.OutputVariables (2).ScaleFactor=0V_2_ScaleFactor; mpcobj. OutputVariables (3).ScaleFactor=0V_3_ScaleFactor; mpcobj. OutputVariables (4).ScaleFactor=0V_4_ScaleFactor; mpcobj.OutputVariables(5).ScaleFactor=0V_5_ScaleFactor; mpcobj.OutputVariables (6).ScaleFactor=0V_6_ScaleFactor; mpcobj.OutputVariables (7).ScaleFactor=0V_7_ScaleFactor; mpcobj.OutputVariables (8).ScaleFactor=0V_8_ScaleFactor; mpcobj.OutputVariables (9).ScaleFactor=0V_9_ScaleFactor;

\author{
$\%$ Body Accel m/s^2 \\ $\%$ Body Roll rad \\ \% Body Pitch rad \\ $\%$ Z_us_1R \\ $\%$ Z_us_1L \\ $\%$ Z_us_2R \\ $\%$ Z_us_2L \\ $\%$ Z us_3R \\ $\%$ Z_us_3L
}

mpcobj . Weights . ManipulatedVariables (1)=MV_Weight; mpcobj . Weights. ManipulatedVariables (2)=MV_Weight; mpcobj . Weights. ManipulatedVariables (3)=MV_Weight; mpcobj . Weights . ManipulatedVariables (4)=MV_Weight; mpcobj . Weights. ManipulatedVariables (5)=MV_Weight; mpcobj . Weights . ManipulatedVariables (6)=MV_Weight;

mpcobj . Weights . ManipulatedVariablesRate(1)=MV_RateWeight; mpcobj. Weights. ManipulatedVariablesRate (2)=MV_RateWeight; mpcobj. Weights. ManipulatedVariablesRate(3)=MV_RateWeight; mpcobj . Weights. ManipulatedVariablesRate (4)=MV_RateWeight; mpcobj. Weights. ManipulatedVariablesRate (5)=MV_RateWeight; mpcobj. Weights. ManipulatedVariablesRate(6)=MV_RateWeight;

mpcobj . Weights . OutputVariables (1)=0V_1_Weight; mpcobj. Weights. OutputVariables (2)=0V_2_Weight; mpcobj. Weights. OutputVariables (3)=0V_3_Weight; mpcobj. Weights. OutputVariables (4)=0V_4_Weight; mpcobj. Weights . OutputVariables $(5)=0$ V_5_Weight; mpcobj. Weights. OutputVariables $(6)=0$ V_6_Weight; mpcobj. Weights . OutputVariables (7)=0V_7_Weight; mpcobj. Weights . OutputVariables $(8)=0$ V_8_Weight; mpcobj . Weights . OutputVariables $(9)=0$ V_9_Weight; $\%$ Body Accel m/s^2 \% Body Roll rad $\%$ Body Pitch rad $\%$ Z_us_1R $\%$ Z_us_1L $\%$ Z_us_2R $\%$ Z_us_2L $\%$ Z_us_3R \% Z_us_3L

mpcobj. Weights. ECR=WeightECR;

$\% \operatorname{review}(m p c o b j)$ 


\section{Simulate with Simulink}

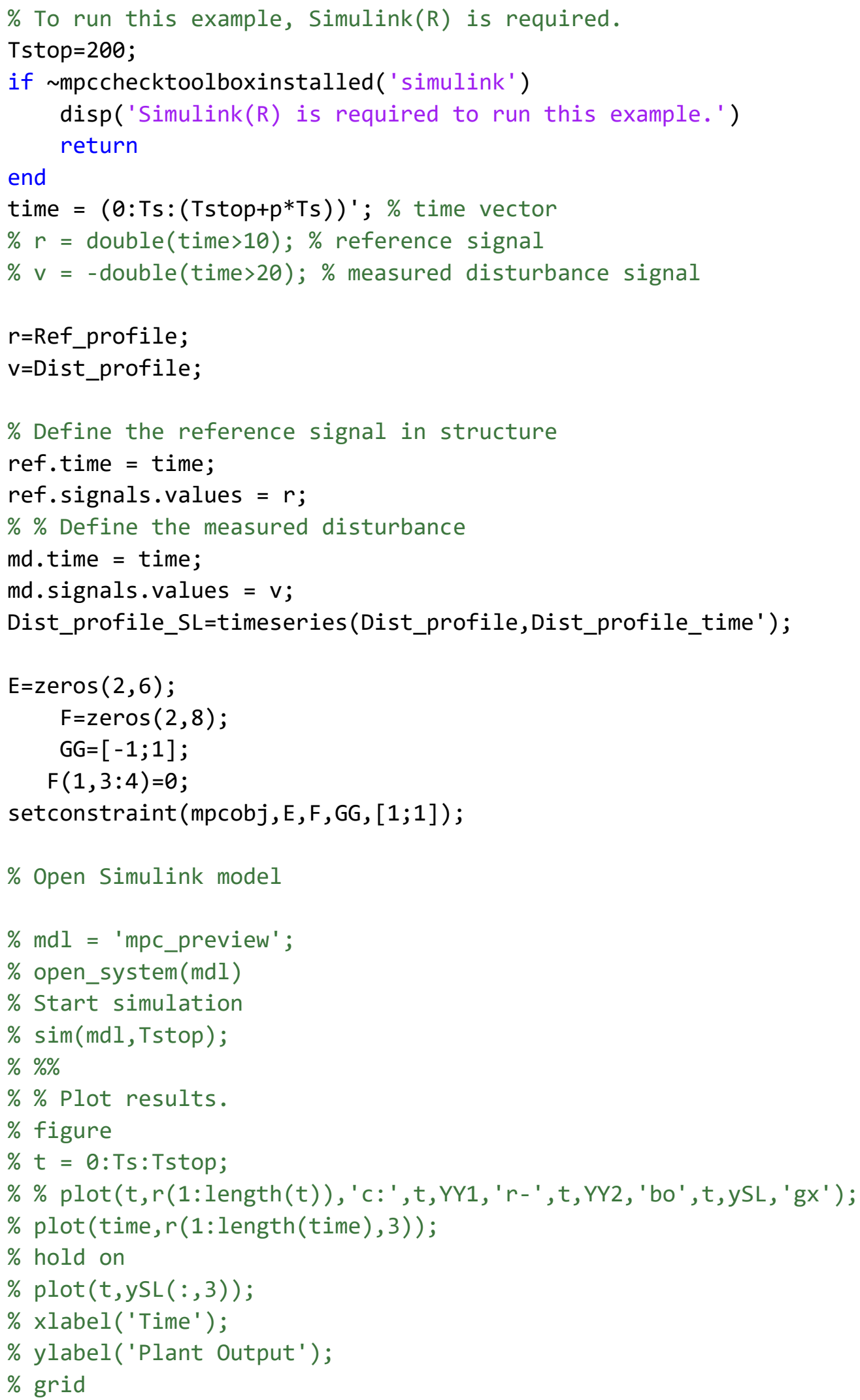




\section{Run}

\% For Uncontrolled Simulation set to $\theta$

$\%$ For Controlled Simulation set to 1

ControlSelection $=1$;

DesiredVel_sim=9;

\% switch (DesiredVel_sim)

$\% \quad$ case 1

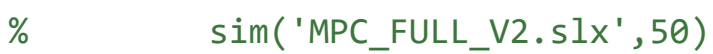

$\%$

$\%$ case 3

$\%$ sim('MPC_FULL_V2.slx', 30)

$\%$

$\%$ case 6

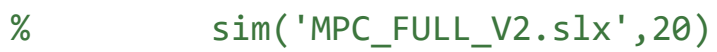

$\%$

$\%$ case 9

$\% \quad \operatorname{sim}($ 'MPC_FULL_V3.slx',10)

$\%$ case 1

$\%$ datestr(now)

$\%$ sim('REF_DIST_MPC_FULL_V2.slx', 180)

$\%$ datestr(now)

$\%$ end 


\section{APPENDIX C}

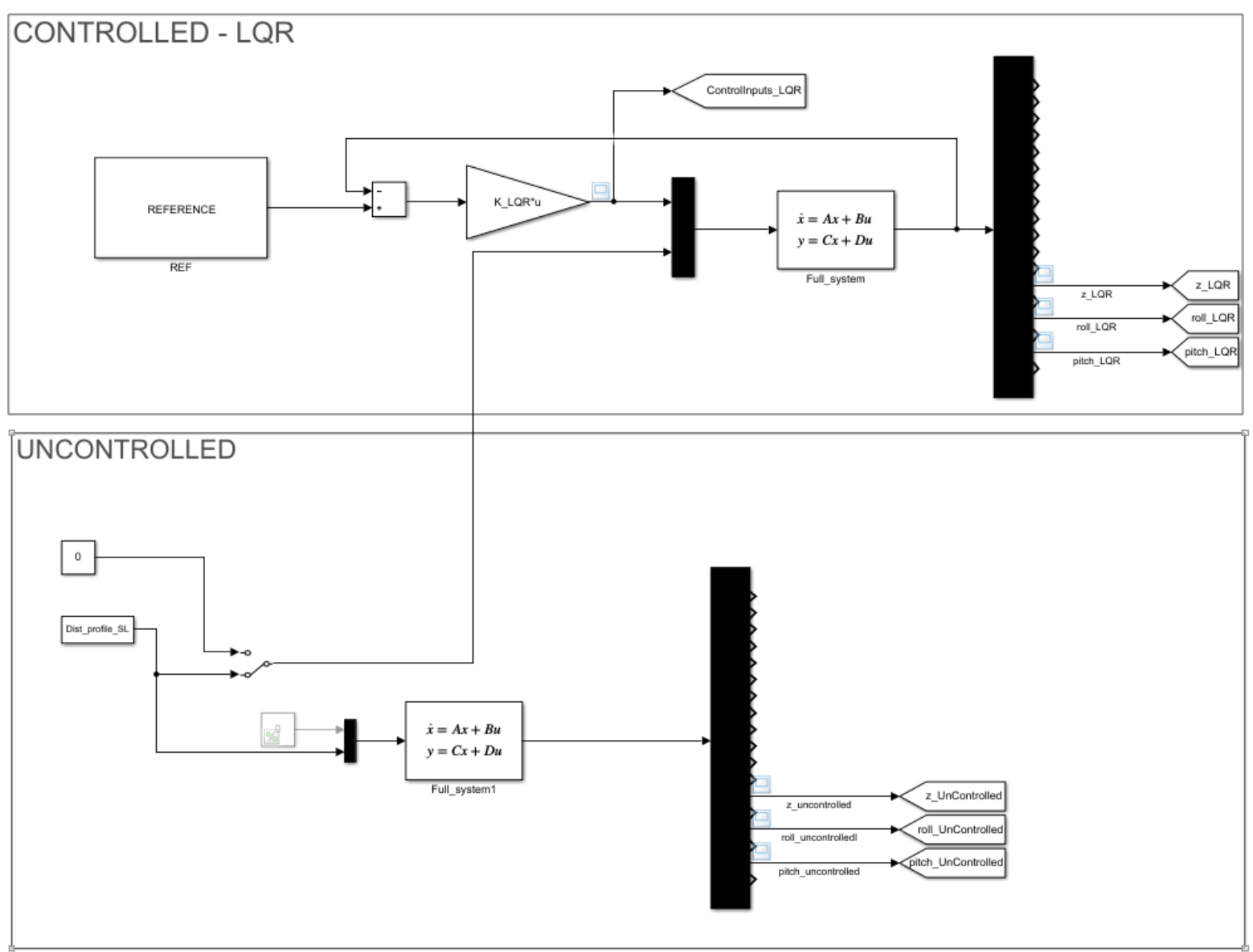

Figure C.1. Controlled LQR block diagram vs uncontrolled system

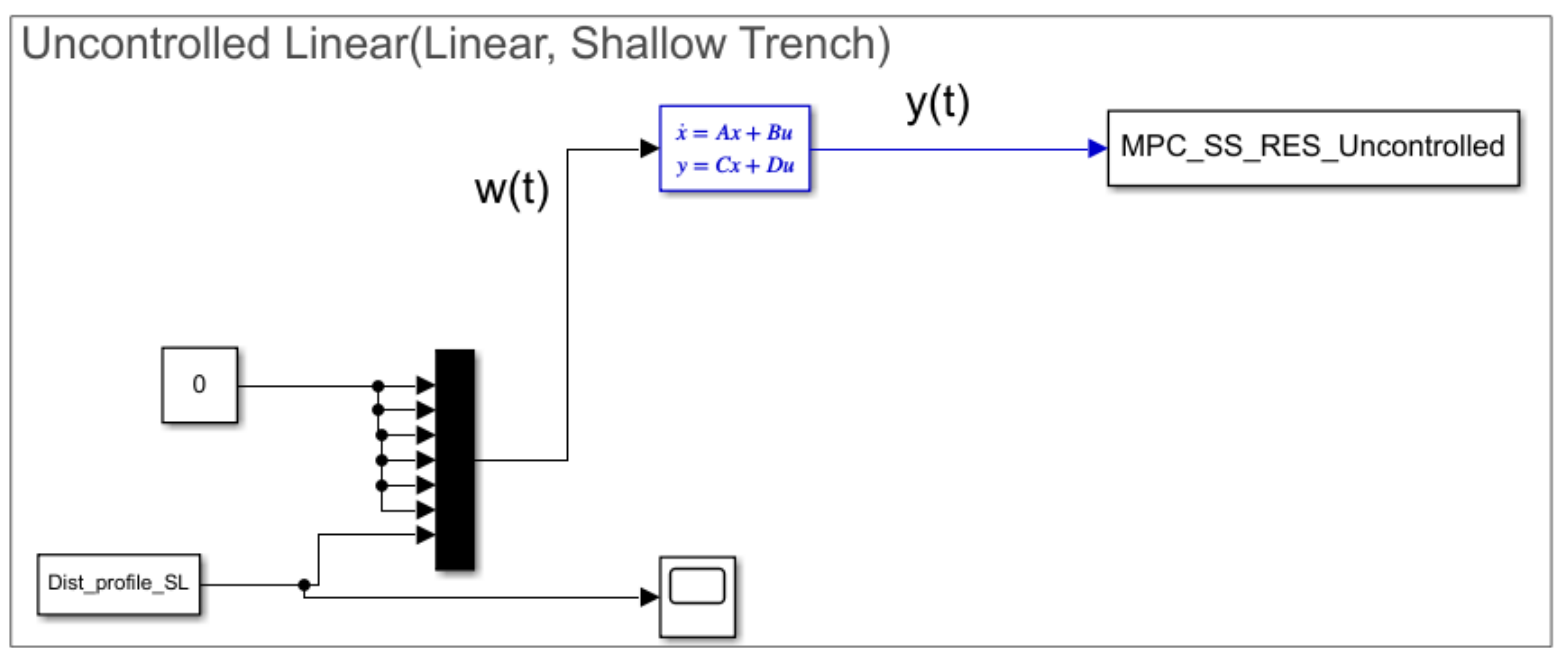

Figure C.2. Linear Uncontrolled system block diagram 


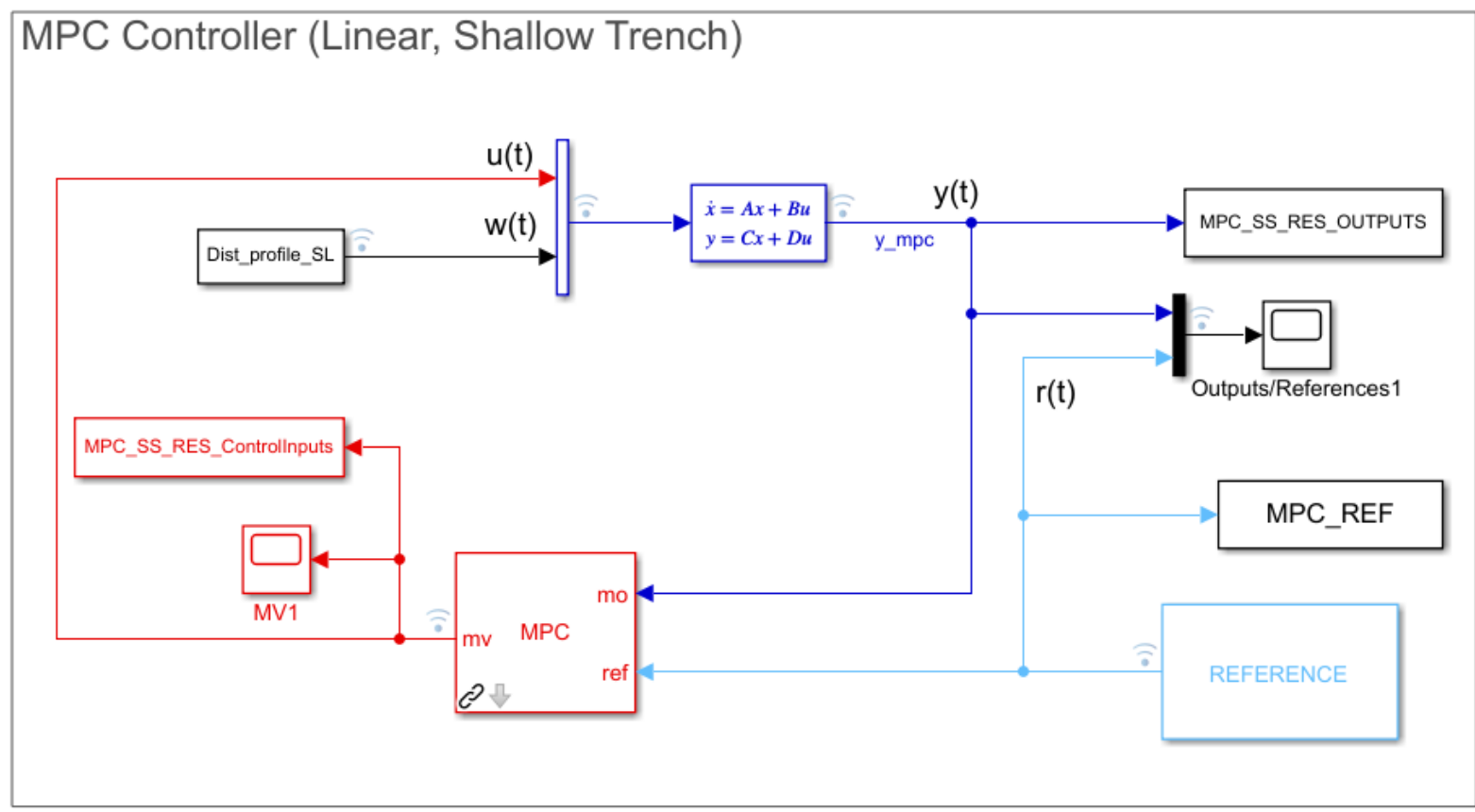

Figure C.3. MPC Controller block diagram, linear shallow trench

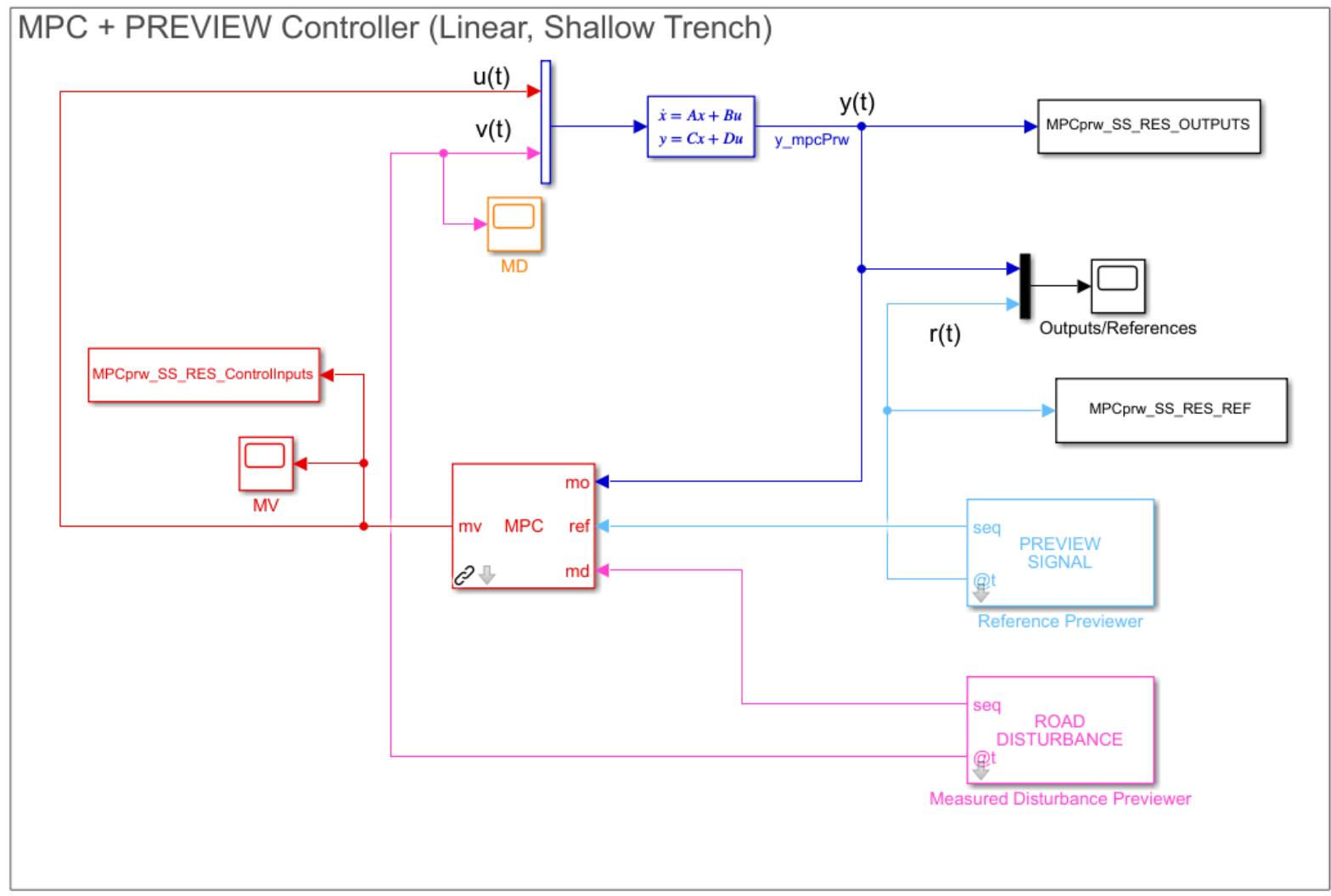

Figure C.4. MPC Controller with preview block diagram, linear shallow trench 


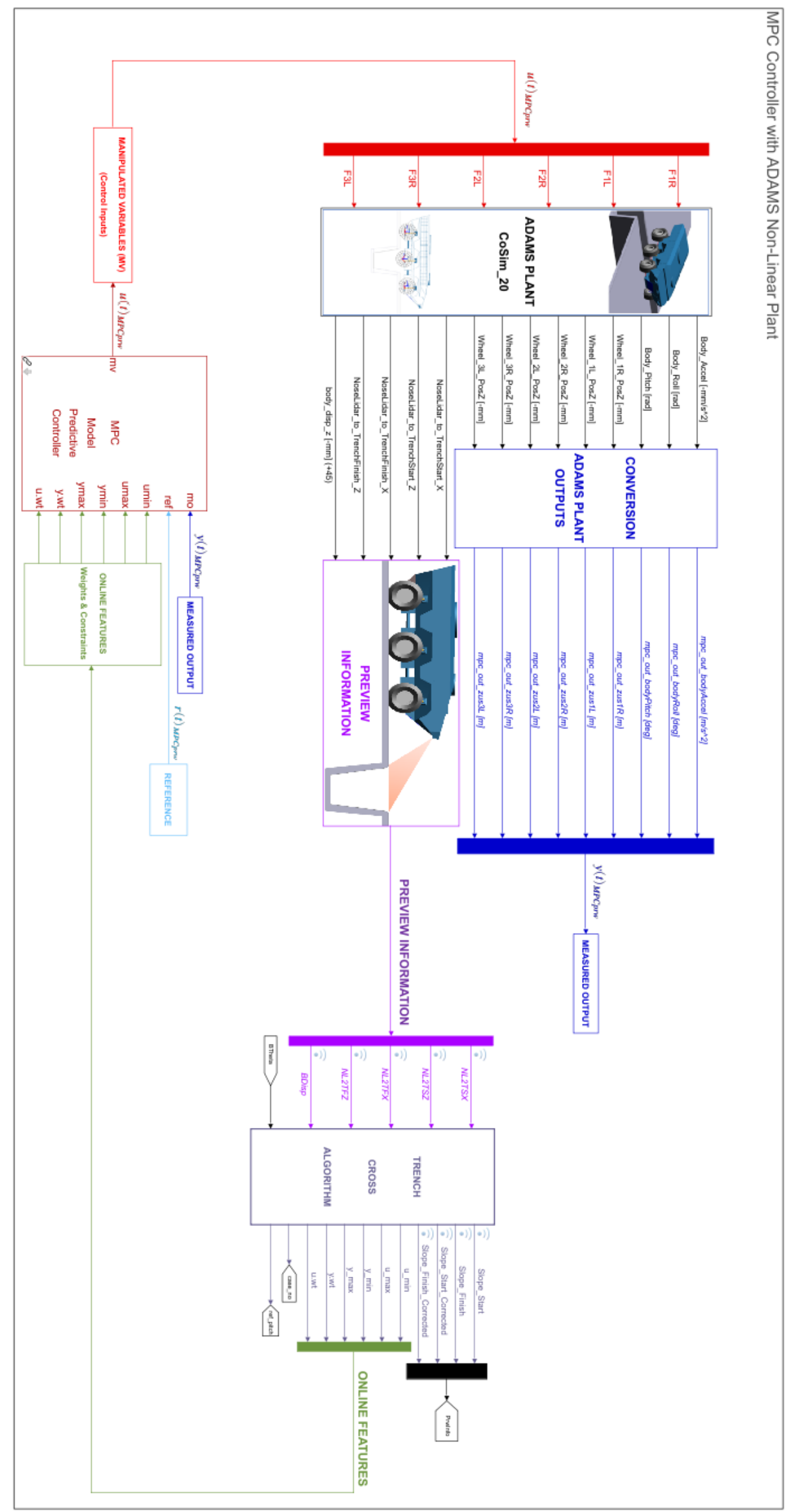

Figure C.5. Non-linear controlled system block diagram with MPC+preview 


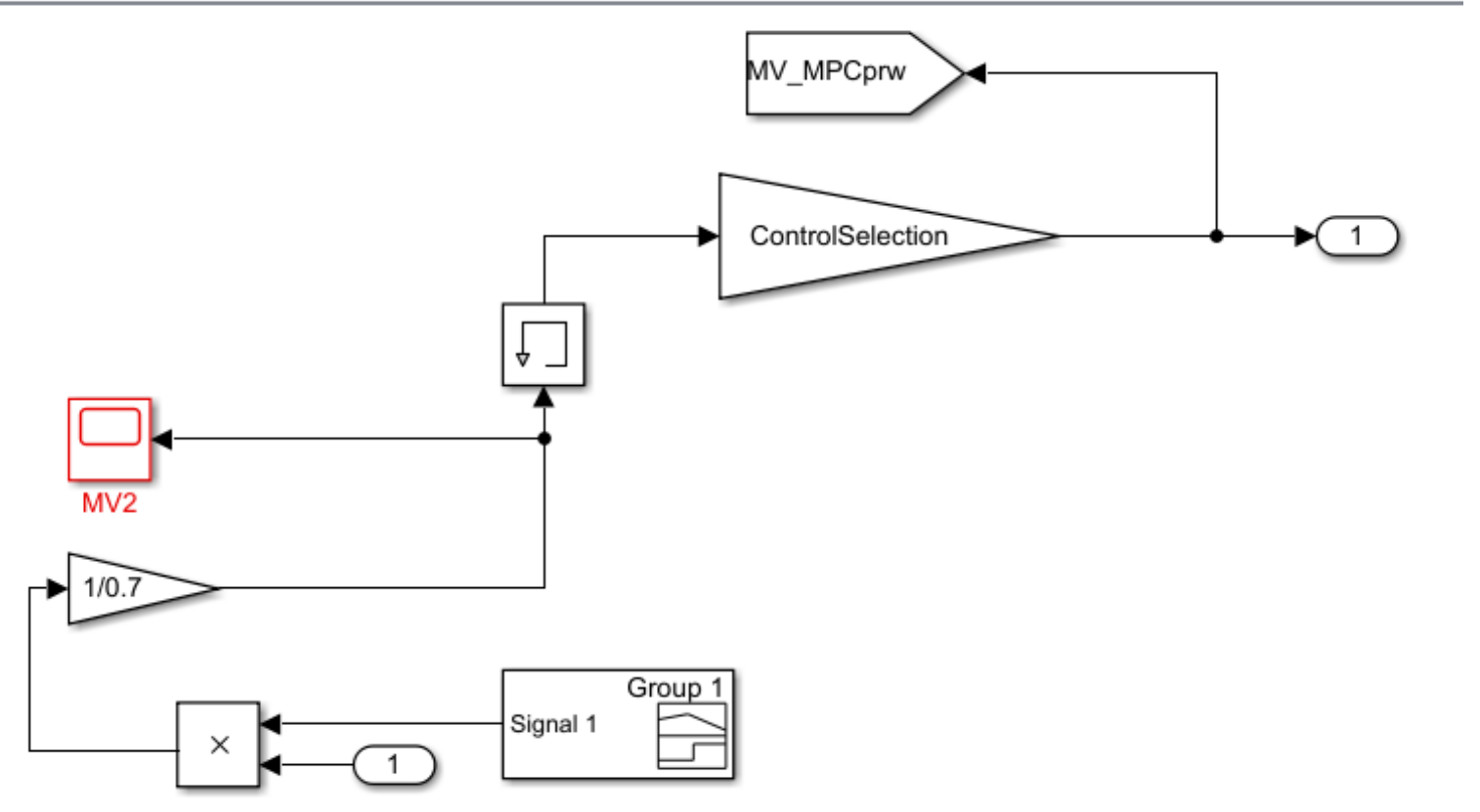

Figure C.6. Non-linear controlled system, manipulated variables sub-block 

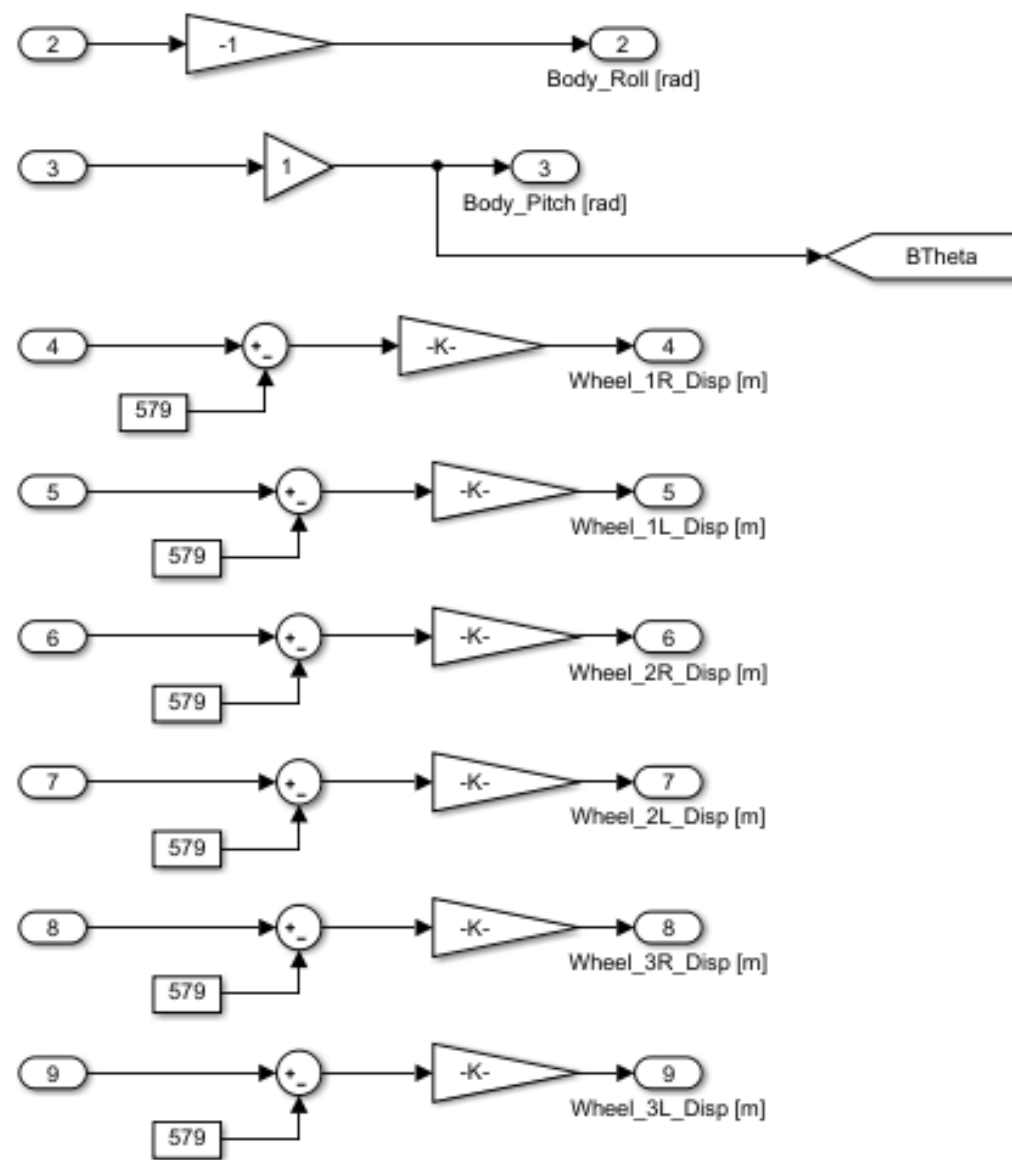

Figure C.7. Non-linear controlled system, ADAMS conversion sub-block 


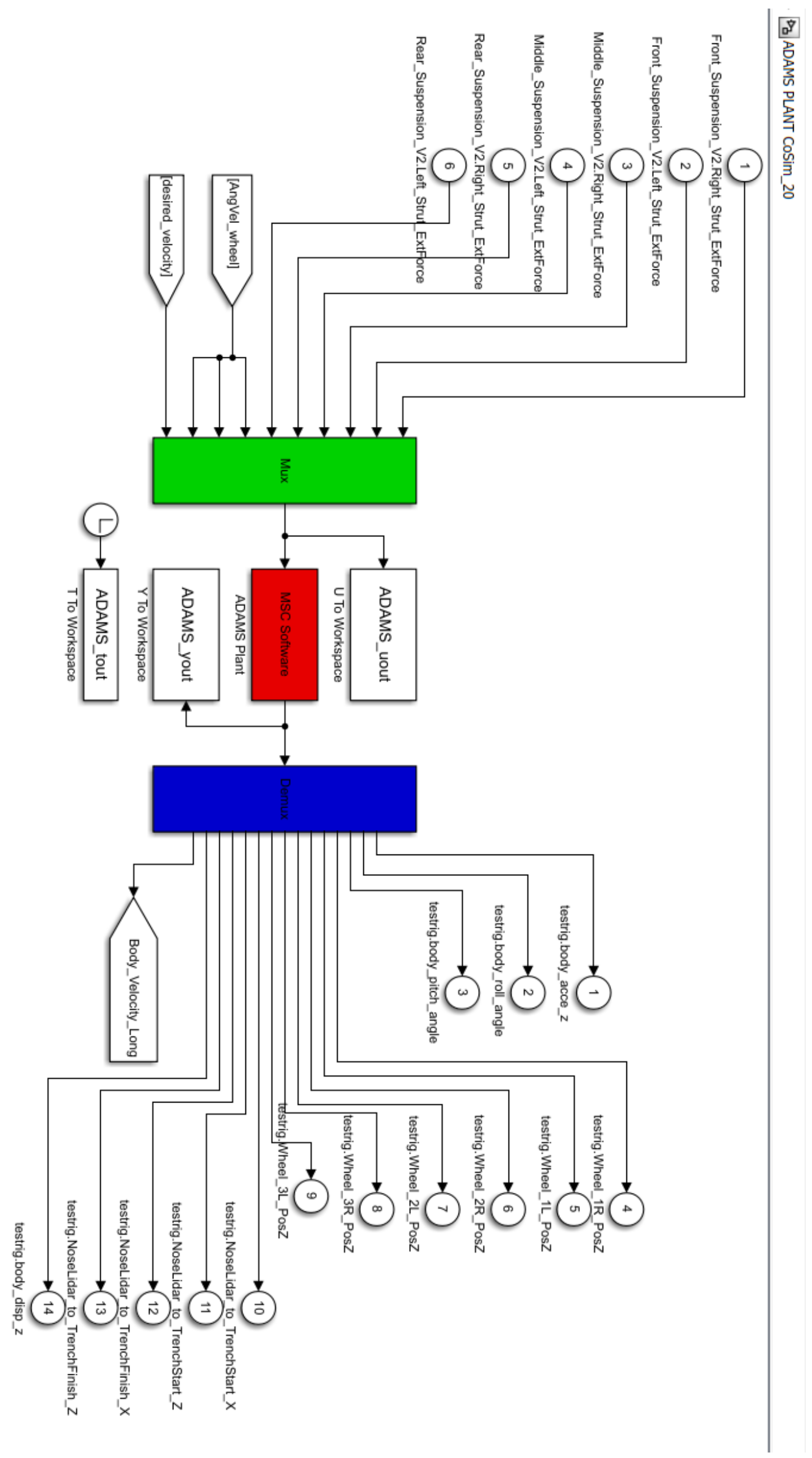

Figure C.8. Non-linear controlled system, ADAMS plant sub-block 


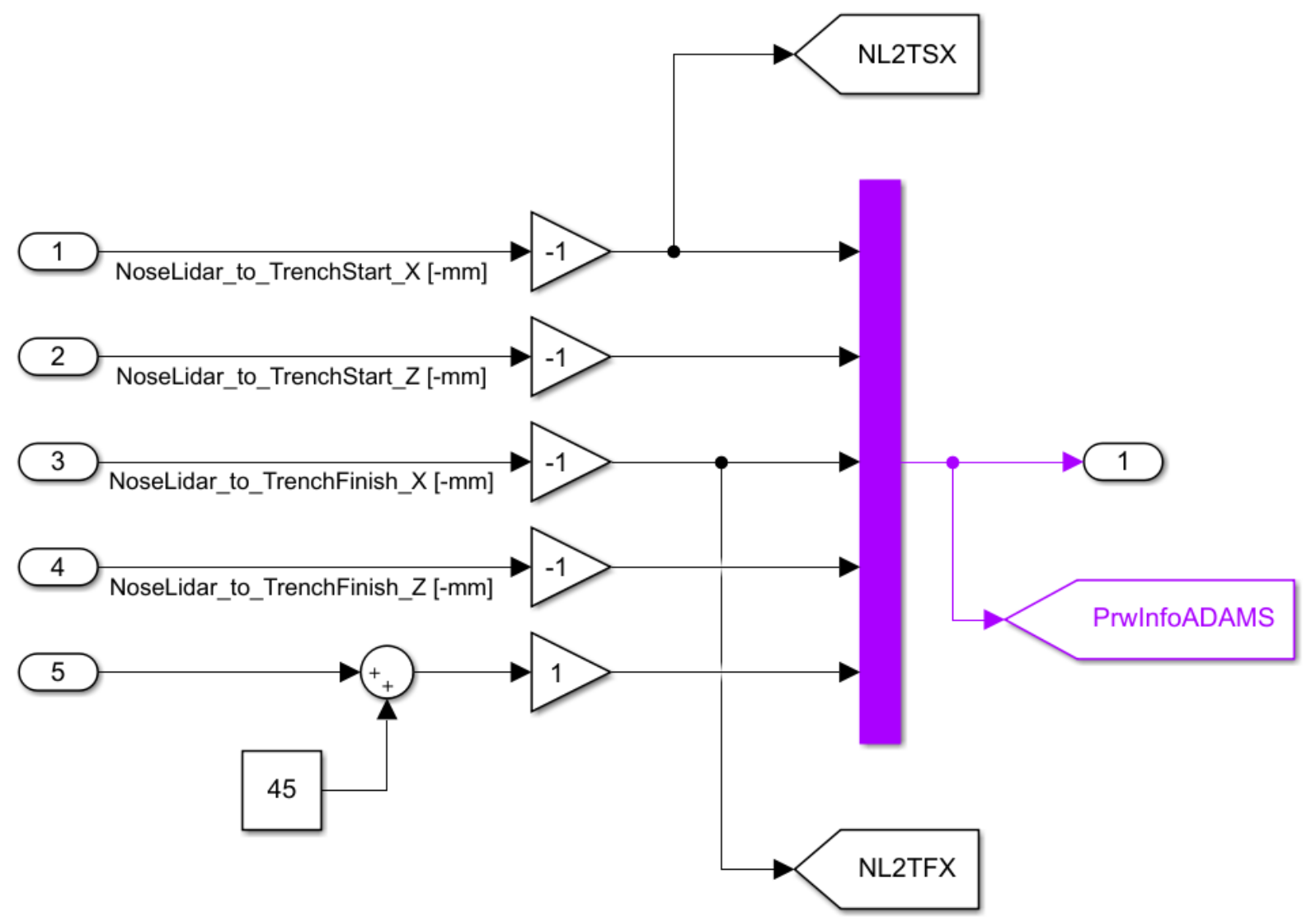

Figure C.9. Non-linear controlled system, preview information sub-block 